\title{
Chances and challenges of home-based bimanual training in children with unilateral cerebral palsy
}

Citation for published version (APA):

Beckers, L. W. M. E. (2019). Chances and challenges of home-based bimanual training in children with unilateral cerebral palsy. [Doctoral Thesis, Maastricht University]. Maastricht University. https://doi.org/10.26481/dis.20190515lb

Document status and date:

Published: 01/01/2019

DOI:

10.26481/dis.20190515lb

Document Version:

Publisher's PDF, also known as Version of record

\section{Please check the document version of this publication:}

- A submitted manuscript is the version of the article upon submission and before peer-review. There can be important differences between the submitted version and the official published version of record.

People interested in the research are advised to contact the author for the final version of the publication, or visit the DOI to the publisher's website.

- The final author version and the galley proof are versions of the publication after peer review.

- The final published version features the final layout of the paper including the volume, issue and page numbers.

Link to publication

\footnotetext{
General rights rights.

- You may freely distribute the URL identifying the publication in the public portal. please follow below link for the End User Agreement:

www.umlib.nl/taverne-license

Take down policy

If you believe that this document breaches copyright please contact us at:

repository@maastrichtuniversity.nl

providing details and we will investigate your claim.
}

Copyright and moral rights for the publications made accessible in the public portal are retained by the authors and/or other copyright owners and it is a condition of accessing publications that users recognise and abide by the legal requirements associated with these

- Users may download and print one copy of any publication from the public portal for the purpose of private study or research.

- You may not further distribute the material or use it for any profit-making activity or commercial gain

If the publication is distributed under the terms of Article $25 \mathrm{fa}$ of the Dutch Copyright Act, indicated by the "Taverne" license above, 


\begin{abstract}
Chances and challenges of home-based bimanual training in children with unilateral cerebral palsy
\end{abstract}

Laura Beckers 
ISBN (printed edition): 978-90-903-1740-3

ISBN (electronic edition): 978-90-903-1775-5

Cover design: *studio Michał Sławiński and Laura Beckers

Layout and typesetting: *studio Michał Sławiński, thesisprint.eu

(C) 2019 Laura Beckers, Maastricht, the Netherlands

All rights reserved. No part of this publication may be reproduced or transmitted in any form or by any means, electronic or mechanical, including photocopying, recording or any other storage or retrieval system, without written permission from the copyright owner.

The research presented in this dissertation was conducted at the department of Rehabilitation Medicine, Care and Public Health Research Institute (CAPHRI), Maastricht University (Maastricht, the Netherlands) and Adelante Centre of Expertise in Rehabilitation and Audiology (Hoensbroek, the Netherlands).

The COAD-project was funded by Johanna Kinderfonds, HandicapNL (former Revalidatiefonds), and Stichting Rotterdams Kinderrevalidatie Fonds Adriaanstichting in the 3rd Program Rehabilitation Research of ZonMw (the Netherlands Organization for Health Research and Development). Additional grants were provided by Revalidatiefonds and Stichting Vooruit.

The printing of this thesis was financially supported by the 'Phelps Stichting voor spastici'. 


\section{Chances and challenges of home-based bimanual training in children with unilateral cerebral palsy}

Dissertation

to obtain the degree of Doctor at the Maastricht University, on the authority of the Rector Magnificus, Prof. dr. R.M. Letschert in accordance with the decision of the Board of Deans, to be defended in public on Wednesday I5 May 2019, at I6.00 hours

by

Laura Wilma Maria Elisabeth Beckers 


\section{Promotor}

Prof. dr. R.J.E.M. Smeets

\section{Co-promotores}

Dr. Y.J.M. Janssen-Potten

Dr. E.A.A. Rameckers

Dr. J.J.W. van der Burg (Radboud University)

\section{Assessment Committee}

Prof. dr. R.J. Vermeulen (chair)

Prof. dr. J.W. Gorter (McMaster University, Canada)

Prof. dr. M.J. Jongmans (Utrecht University)

Dr. E.P.E. Mesters

Prof. dr. J.A.M.C.F. Verbunt 


\section{TABLE OF CONTENTS}

ChAPTER 1 General introduction I

CHAPTER 2 Feasibility and effect of home-based therapy programmes for II children with cerebral palsy: a protocol for a systematic review

Chapter 3 Feasibility and effectiveness of home-based therapy programs 25 for children with cerebral palsy: a systematic review

ChAPTER 4 Home-based bimanual training based on motor learning II7 principles in children with unilateral cerebral palsy and their parents (the COAD-study): rationale and protocols

CHAPTER 5 Therapy-related stress in parents of children with 133 a physical disability: a conceptual analysis

Chapter 6 Cross-cultural Translation and Adaptation of the Lifestyle I47 Assessment Questionnaire (LAQ-CP) Into Dutch: A Brief Report

ChAPTER 7 Barriers to recruitment of children with cerebral I57 palsy in a trial on home-based training

Chapter 8 Effect of home-based bimanual training in children with $\quad$ I79 unilateral cerebral palsy (the COAD-study): a case series programs in children with unilateral cerebral palsy (the COAD-study): protocol for a mixed methods study

ChAPTER 10 Process evaluation of two home-based bimanual training programs in children with unilateral cerebral palsy (the COAD-study): a mixed methods study

Chapter 11 Single-case experimental design studies in children 26I with cerebral palsy: a scoping review

Chapter 12 General discussion

VALORIZATION ADDENDUM

SUMMARY 313

Nederlandse Samenvatting (Dutch SUmmary) $32 \mathrm{I}$

Aвout the AUthor $\quad 327$

Publication list $\quad 329$

DANKWOORD (ACKNOWLEDGEMENTS) 33 I 

Chapter I

General introduction 



\section{Cerebral palsy}

Cerebral palsy (CP) is the most common cause of motor disability in children, affecting approximately 2 per 1,000 live births. ${ }^{1,2}$ According to the internationally accepted definition, CP can be described as "(..) a group of permanent disorders of the development of movement and posture, causing activity limitation, that are attributed to non-progressive disturbances that occurred in the developing fetal or infant brain. The motor disorders of $C P$ are often accompanied by disturbances of sensation, perception, cognition, communication, behavior, by epilepsy and by secondary musculoskeletal problems.13 $\mathrm{CP}$ is heterogeneous, with different types, topographical distribution, and severity. The Surveillance of Cerebral Palsy in Europe (SCPE) distinguishes three main types of CP: spastic, ataxic, and dyskinetic. ${ }^{4}$ Spastic CP is characterized by at least two neurological signs: (1) abnormal pattern of posture and/or movement, (2) increased tone, and/or (3) pathological reflexes.4 The SCPE supports the distinction between unilateral and bilateral spastic $C P, 4$ though the traditional terms hemiplegia, diplegia, and quadriplegia are still used to describe topographical distribution. ${ }^{5}$ About $30 \%$ of children with CP have unilateral spastic CP (UCP). ${ }^{6}$ The focus of this dissertation is on this subtype.

\section{Arm-hand FunCtioning}

The motor deficits of children with uCP are often most prominent in the upper extremity. Motor skills are compromised by impairments in motor execution, sensorimotor integration, motor planning, and bimanual coordination beyond unimanual dexterity impairments? In addition, certain children with UCP disregard the remaining capacity of the affected arm and hand for spontaneous use in daily life, which is known as developmental disregard. ${ }^{.}$The typical manual performance can be described by means of the Manual Ability Classification System (MACS). This five-point rating scale classifies how children with CP use their hands to handle objects in daily activities. Children with UCP generally have MACS level I, II, or III. Level I indicates that a child handles objects easily and successfully, whereas a child classified in level III handles objects with difficulty and needs help to prepare and/or modify activities. ${ }^{9}$ The arm-hand impairments of CP can manifest themselves in all three components of functioning of the Children \& Youth Version of the theoretical framework International Classification of Functioning, Disability, and Health (ICF-CY): body functions and structures, activities, and participation. ${ }^{10}$ Within the activity domain, three separate but related constructs can be distinguished: motor capacity (the child's ability to perform a task in a standardized, controlled environment), motor capability (the child's ability to perform a task in his/her daily environment), and motor performance (what the child actually does do in his/her daily environment).." As most daily functional tasks are bimanual in nature, the difficulties that children with UCP experience in planning and performing bimanual activities negatively affect their independence, participation, and quality of life. ${ }^{12,13}$ 


\section{UPPER EXTREMITY THERAPY APPROACHES}

In former times, traditional neurodevelopmental therapy models targeted tone reduction and movement normalization. ${ }^{14}$ For about the last two decades, this focus has increasingly shifted from the body structures and functions level of the ICF-CY to enhancing activities. ${ }^{14}$ Different therapy approaches have been developed. Constraint-induced movement therapy (CIMT) and bimanual intensive movement therapy (BIMT) are the most frequently reported in the literature. Both therapy approaches are based on motor learning theory and entail intensive structured practice. $^{14-17}$

CIMT aims to increase spontaneous use of the affected upper extremity by physically restraining the less-affected arm, while the child performs repetitive unimanual task practice. In children with UCP, modified protocols of CIMT are often applied, using age-appropriate therapy dosage, schedule, and tasks. $12,17,18$

BIMT targets the use of the affected hand as a functional (nondominant) assisting hand by practicing bimanual activities that require coordinated use of both hands together. ${ }^{14,16}$ This dissertation will use the definition suggested by Klepper et al., who describe BIMT as "an intervention that (1) incorporates structured, intensive bimanual training; (2) follows the principles of motor control and motor learning, and (3) is aimed at improving upper extremity function in children with UCP".17 Alternative terms used to describe this therapy approach include bimanual intensive therapy (BIT), and hand-arm bimanual intensive therapy (HABIT).

Abundant research has demonstrated the effectiveness of both CIMT and BIMT on upper extremity outcomes at the ICF-CY activity level, although fewer studies had investigated BIMT. ${ }^{12,14-}$

${ }^{18}$ Compared to each other, neither of the therapy approaches is superior. ${ }^{12,16-18}$ However, BIMT leads to more improvements in bimanual coordination during functional activities, achievement of goals meaningful to children and parents, and bilateral spontaneous use of the hand. ${ }^{14,16-18}$ This is in accordance with the specificity of practice hypothesis that assumes more transfer of learning with increased similarity of skill components. ${ }^{16}$ In addition, activities children are engaged in during BIMT may be more diverse and motivating to the child than are unimanual CIMT activities. ${ }^{16,19}$ Nevertheless, research indicates that meaningful improvements are more strongly associated with training dose than with the therapy approach. ${ }^{12,17}$

The Dutch guideline for treatment for children with spastic CP gives six criteria for functional BIMT and CIMT interventions. First, the therapy should be goal-directed. Goals should be defined in consultation with the parents and/or child, and preferably determined using a valid and reliable instrument, such as the Canadian Occupational Performance Measure (COPM) or Goal Attainment Scale (GAS). Second, the therapy should always target the activity and participation components of the ICF-CY. Third, the therapy should be task-specific: based on a task analysis aiming at practicing (sub-)activities that have been formulated in the goals. Fourth, the child and its parents should have an active role in learning, discovering, and finding solutions. Fifth, for the result of the intervention, the focus should be on functionality (that the child can and actually does perform the activity) rather than normality (that the child performs the activity according to qualitative or motor norms). Sixth and last, the therapy should be context-specific. ${ }^{20}$ A meta-analysis by Sakzewski et al. ${ }^{12}$ has revealed two additional recommendations for upper 
extremity interventions in children with UCP: (1) contemporary motor learning approaches that use activity-based therapy should be used; and (2) therapy should be adequately dosed.12 Still, there are some important issues for future research. The minimum and optimal dosage required, and the effectiveness of intensive short-duration interventions compared to distributed training schedules are still unknown. ${ }^{12,14,16-18}$ Other remaining questions relate to therapy costs, ${ }^{15}$ as well as the target population, timing, and content of BIMT. ${ }^{18}$ Last, the feasibility and effects of parents delivering home-based training should be investigated. ${ }^{12,17}$

\section{MOTOR LEARNING STRATEGIES}

A challenge in bimanual training is that children tend to compensate using only the less-affected hand. ${ }^{14,16}$ Functional use and motor skills of the affected hand can be learned implicitly and/or explicitly. A Delphi study by Kleynen et al. ${ }^{21}$ proposes definitions for implicit and explicit motor learning. Implicit learning is defined as "learning which progresses with no or minimal increase in verbal knowledge of movement performance (e.g., facts and rules) and without awareness", whereas explicit motor learning is regarded as "learning which generates verbal knowledge of movement performance (e.g., facts and rules), involves cognitive stages within the learning process and is dependent on working memory involvement". ${ }^{\prime 1}$ In this dissertation, these types of motor learning are considered to represent opposite ends of a continuum rather than a dichotomy. Explicit motor learning depends on the working memory to temporarily store and process declarative knowledge. ${ }^{22}$ Children with CP are at risk of working memory and executive function deficits. ${ }^{23}$ It may be hypothesized that this restricts their ability to learn motor skills in an explicit way. The capacity for explicit motor learning is particularly reduced in children with right UCP, as explicit learning seems to be associated with left hemisphere activity. ${ }^{24}$ Hence, although at this time in interventions the emphasis is primarily on explicit approaches, children with UCP may benefit from including more implicit learning principles. ${ }^{22}$ For home-based interventions, it is additionally hypothesized that implicit skill acquisition requires less prompting from parents. This, in turn, might be advantageous in terms of motivation and self-efficacy of the child, parent-child interaction, and compliance.

\section{HOME-BASED INTERVENTIONS}

A home-based intervention is, according to Novak and Barry, a mode of therapy which comprises "therapeutic activities that the child performs with parental assistance in the home environment with the goal of achieving desired health outcomes".25 Parents of children with CP introduced a slightly different definition: "Home programs are a form of guidance and advice which become a way of life for parents and children. Through regular practice of activities at home, parents maximize their child's potential. Parents use the guidance and support that they gain from home programs to build confidence about how to help their child".25

The aim of rehabilitation is for children to perform acquired skills in their daily living environment. The principle of practice specificity indicates that the practice and actual performance situation need to be comparable. The more similar the context, the better transfer of learning occurs. This implies that generalization of bimanual performance is fostered when the child prac- 
tices in the natural home environment. ${ }^{26}$ In addition, as can be derived from the last sentence of the second definition, parent-delivered home-based interventions may also increase self-efficacy, empowerment, and self-reliance of parents.

There is a shift towards self-reliance of families being a priority, professionals fulfilling a coaching role, and the use of e-health technologies, as key aspects of future health care. ${ }^{27}$ In pediatric rehabilitation, family-centered care is generally accepted and increasingly adopted as a framework for development of rehabilitation programs. Key elements of family-centered care include recognizing the family as central and primary source of support to the child, encouraging the development of true collaborative relationships and partnership between families and health-care providers, acknowledging the parents' expertise, and focusing on enhancing their competence. ${ }^{28}$ Home-based interventions fit in well with this mode of care. Parents being coached by the rehabilitation team, supported by e-health applications where needed, may contribute to increased self-reliance in parents.

Despite the importance of home-based interventions, there is scant information on intervention's content, and evidence of best practices is still lacking. Moreover, there remains a paucity of evidence on home-based BIMT, hereafter referred to as home-based bimanual training.

In previous studies of parent-delivered home-based interventions, several unfavorable consequences have been found, partly related to the high training intensity. These include limited compliance, parents feeling pressured to adhere to therapeutic activities, parents sacrificing other activities, and a negative impact on parent-child emotional availability and interaction, as well as on family relationships and wellbeing. ${ }^{29-32}$ These consequences may induce or increase parental stress..$^{30}$ Unfortunately, these aspects are often ignored in the design and evaluation of homebased interventions.

Bringing the aforementioned together, optimal home-based bimanual training programs must (1) be goal-directed; (2) target the activity and participation domains of the ICF-CY; (3) focus on functionality rather than normality; (4) facilitate an active role for both the child and the parents; (5) be task-specific; (6) be context-specific; (7) adopt motor learning approaches; (8) have a dose that is adequate to generate therapy effects and at the same time be feasible for parent-delivered training at home; and (9) implement strategies to prevent or limit therapy-related parental stress and other unintended psychological and social effects. We developed two variants of a home-based bimanual training program (one implicitly and one explicitly oriented) according to these requirements.

\section{Aims and OUthine Of This dissertation}

This dissertation presents the results of the research project COAD ("CO-creation At hanD: the road to independence"). The overall aim of this dissertation was to generate scientific knowledge on the chances and challenges of home-based bimanual training in children with UCP. Two home-based training programs were developed that are novel in the motor learning principles applied (implicit versus explicit) and in the focus on support of parents through an interdisciplinary coaching process. 
This dissertation continues with the protocol (Chapter 2) and results (Chapter 3) of a systematic review regarding the feasibility and effects of home-based therapy programs for children with CP. In Chapter 4, the rationale and protocols of the home-based bimanual training programs of the COAD-study are described in detail. This includes one program based on implicit motor learning principles and another on explicit motor learning principles. Parents delivering interventions in children with a physical disability are potentially at risk of therapy-related parental stress. Chapter 5 provides a conceptual analysis of this topic. In addition to activity-measures, participation is an important outcome to focus on in intervention research in children with CP. In the Dutch guideline for children with spastic CP, four participation outcome measures are recommended..$^{20}$ Given the targeted age groups and ease of administration, in the COAD-study the Lifestyle Assessment Questionnaire for children with cerebral palsy (LAQ-CP) was considered most appropriate to evaluate participation. Since no Dutch version of this measure was then available, Chapter 6 is concerned with the cross-cultural translation and adaptation of the LAQ-CP into Dutch. Besides, a multicenter randomized controlled trial (RCT) was designed to evaluate the effectiveness of the home-based training programs. However, as many problems arose, the trial had to be terminated earlier than planned, and the study was transformed into a case-series. Therefore, Chapter 7 elaborates on the barriers to the recruitment of participants in this trial through a multidimensional evaluation to facilitate future research in this field. The effects of the home-based training programs are eventually presented as a case series in Chapter 8. Parallel to the effect study, we conducted a preplanned mixed-methods process evaluation of the homebased training programs. Chapter 9 outlines the protocol for this study, while in Chapter 10 the results are reported. Given the drawbacks of RCTs in the population of children with CP, more recognition should be given to alternative interventional research designs. Hence, Chapter 11 gives an overview of single-case experimental design studies performed in children with CP by means of a scoping review. Finally, Chapter 12 synthesizes the findings in a general discussion and provides recommendations for clinical practice and future research. 


\section{REFERENCES}

1. Oskoui M, Coutinho F, Dykeman J, Jette N, Pringsheim T. An update on the prevalence of cerebral palsy: a systematic review and meta-analysis. Dev Med Child Neurol. 2013;55(6):509-519.

2. Sellier E, Platt MJ, Andersen GL, Krageloh-Mann I, De La Cruz J, Cans C. Decreasing prevalence in cerebral palsy: a multi-site European population-based study, 1980 to 2003. Dev Med Child Neurol. 2016;58(1):85-92.

3. Rosenbaum P, Paneth N, Leviton A, et al. A report: the definition and classification of cerebral palsy April 2006. Dev Med Child Neurol. Supplement. 2007;109:8-14.

4. Surveillance of cerebral palsy in Europe: a collaboration of cerebral palsy surveys and registers. Surveillance of Cerebral Palsy in Europe (SCPE). Dev Med Child Neurol. 2000;42(12):816-824.

5. Rethlefsen SA, Ryan DD, Kay RM. Classification systems in cerebral palsy. Orthop Clin North Am. 2010;41(4):457-467.

6. Krageloh-Mann I, Cans C. Cerebral palsy update. Brain Dev. 2009;31(7):537-544.

7. Gordon AM, Bleyenheuft Y, Steenbergen B. Pathophysiology of impaired hand function in children with unilateral cerebral palsy. Dev Med Child Neurol. Nov 2013;55 Suppl 4:32-37.

8. Zielinski IM, Steenbergen B, Baas CM, Aarts P, Jongsma ML. Event-related Potentials During Target-response Tasks to Study Cognitive Processes of Upper Limb Use in Children with Unilateral Cerebral Palsy. JVis Exp. 2016(107) 53420.

9. Eliasson AC, Krumlinde-Sundholm L, Rosblad B, et al. The Manual Ability Classification System (MACS) for children with cerebral palsy: scale development and evidence of validity and reliability. Dev Med Child Neurol. 2006;48(7):549-554.

10. Arnould C, Bleyenheuft Y, Thonnard JL. Hand functioning in children with cerebral palsy. Front Neurol. 2014;5:48.

11. Holsbeeke L, Ketelaar M, Schoemaker MM, Gorter JW. Capacity, capability, and performance: different constructs or three of a kind? Arch Phys Med Rehabil. 2009;90(5):849-855.

12. Sakzewski L, Ziviani J, Boyd RN. Efficacy of upper limb therapies for unilateral cerebral palsy: a meta-analysis. Pediatrics. 2014;133(1):e175-204.

13. Skold A, Josephsson S, Eliasson AC. Performing bimanual activities: the experiences of young persons with hemiplegic cerebral palsy. Am J Occup Ther. 2004;58(4):416-425.

14. Sakzewski L, Gordon A, Eliasson AC. The state of the evidence for intensive upper limb therapy approaches for children with unilateral cerebral palsy. J Child Neurol. 2014;29(8):1077-1090.

15. Novak I, Mclntyre S, Morgan C, et al. A systematic review of interventions for children with cerebral palsy: state of the evidence. Dev Med Child Neurol. 2013;55(10):885-910.

16. Andersen JC, Majnemer A, O'Grady K, Gordon AM. Intensive upper extremity training for children with hemiplegia: from science to practice. Semin Pediatr Neurol. 2013;20(2):100-105.

17. Klepper SE, Clayton Krasinski D, Gilb MC, Khalil N. Comparing Unimanual and Bimanual Training in Upper Extremity Function in Children With Unilateral Cerebral Palsy. Pediatr Phys Ther. 2017;29(4):288-306.

18. Dong VA, Tung IH, Siu HW, Fong KN. Studies comparing the efficacy of constraint-induced movement therapy and bimanual training in children with unilateral cerebral palsy: a systematic review. Dev Neurorehabil. 2013;16(2):133-143. 
19. Gordon AM, Schneider JA, Chinnan A, Charles JR. Efficacy of a hand-arm bimanual intensive therapy (HABIT) in children with hemiplegic cerebral palsy: a randomized control trial. Dev Med Child Neurol. 2007;49(11):830-838.

20. De Nederlandse Vereniging van Revalidatieartsen. Richtlijn Spastische cerebrale parese bij kinderen. Utrecht 2015.

21. Kleynen M, Braun SM, Bleijlevens MH, et al. Using a Delphi technique to seek consensus regarding definitions, descriptions and classification of terms related to implicit and explicit forms of motor learning. PloS one. 2014;9(6):e100227.

22. Steenbergen B, van der Kamp J, Verneau M, Jongbloed-Pereboom M, Masters RS. Implicit and explicit learning: applications from basic research to sports for individuals with impaired movement dynamics. Disabil Rehabil. 2010;32(18):1509-1516.

23. Jenks KM, de Moor J, van Lieshout EC. Arithmetic difficulties in children with cerebral palsy are related to executive function and working memory. J Child Psychol Psychiatry. 2009;50(7):824-833.

24. van der Kamp J, Steenbergen B, Masters RSW. Explicit and implicit motor learning in children with unilateral cerebral palsy. Disabil Rehabil. 2018;40(23):2790-2797.

25. Novak I, Berry J. Home program intervention effectiveness evidence. Phys Occup Ther Pediatr. 2014;34(4):384-389.

26. Palisano RJ, Campbell SK, Orlin M. Physical Therapy for Children-E-Book: Elsevier Health Sciences; 2014.

27. Dutch Association of Medical Specialists. Vision document: Medical Specialist 2025 - Ambition, trust, cooperation 2017.

28. Mackean GL, Thurston WE, Scott CM. Bridging the divide between families and health professionals' perspectives on family-centred care. Health Expect. 2005;8(1):74-85.

29. McConnell D, Parakkal M, Savage A, Rempel G. Parent-mediated intervention: adherence and adverse effects. Disabil Rehabil. 2015;37(10):864-872.

30. Peplow UC, Carpenter C. Perceptions of parents of children with cerebral palsy about the relevance of, and adherence to, exercise programs: a qualitative study. Phys Occup Ther Pediatr. 2013;33(3):285-299.

31. Lin KC, Wang TN, Wu CY, et al. Effects of home-based constraint-induced therapy versus dose-matched control intervention on functional outcomes and caregiver well-being in children with cerebral palsy. Res Dev Disabil. 2011;32(5):1483-1491.

32. Barfoot J, Meredith P, Ziviani J, Whittingham K. Parent-child interactions and children with cerebral palsy: An exploratory study investigating emotional availability, functional ability, and parent distress. Child Care Health Dev. 2017;43(6):812-822. 

Chapter 2

\section{Feasibility and effect of home-based therapy programmes for children with cerebral palsy: a protocol for a systematic review}

LWME Beckers*, MLAP Schnackers*, YJM Janssen-Potten, J Kleijnen, B Steenbergen

*contributed equally 


\begin{abstract}
Introduction Given the promising advantages of upper extremity home-based programmes in children with cerebral palsy (CP), a systematic review of the available literature on this topic is warranted. The purpose of the systematic review described in this protocol is to investigate currently available home-based occupational therapy and physiotherapy programmes regarding both their feasibility and effect.
\end{abstract}

Methods and analysis This protocol describes a systematic review, developed in accordance with the Preferred Reporting Items for Systematic Review and Meta-Analysis Protocols (PRISMA-P) 2015. Studies will be included in which primary data are collected, participants are children aged $<18$ years with any type of CP and the intervention of interest is a home-based occupational therapy or physiotherapy intervention. Comparators of interest are: no therapy, care as usual, centre-based occupational therapy or physiotherapy, an alternative home-based programme and a medical intervention. Studies will be included that report either on feasibility (ie, acceptability, demand, implementation, practicality, adaptation, expansion or integration) or on efficacy/ effectiveness (ie, child-related upper extremity outcomes within all International Classification of Functioning, Disability and Health levels or parent-related/caregiver-related outcomes on the psychological and social domain). Relevant studies will be identified by searching the databases MEDLINE, EMBASE, CINAHL, PsycINFO, PEDro, OTSeeker and CPCI-S as well as the trial registers ICTRP and CENTRAL, the reference lists of included records and by circulating a bibliography of the included records to authors of included studies. There will be no restrictions on language or year of publication. The search strategy consists of terms related to the population and intervention. Data will be extracted in duplicate using a digital data extraction form.

Ethics and dissemination The proposed study does not involve collection of primary data. Accordingly, no ethical approval is required. The authors will disseminate the findings of this systematic review through publication in a peer-reviewed journal and conference presentation(s).

Trial registration number CRD42016043743; pre-results. 


\section{INTRODUCTION}

Cerebral palsy (CP) is one of the most common causes of physical disability in children. The majority of children with CP have impaired arm-hand function. ${ }^{1}$ Abundant research has shown the effectiveness of centre-based therapies in children with $\mathrm{CP}$, including upper extremity interventions such as constraint-induced movement therapy and bimanual training. ${ }^{2-5}$

In recent years, home-based programmes have received increasing attention in rehabilitation of children with CP. These programmes are a useful addition to centre-based occupational therapy and physiotherapy for a number of reasons. First, home-based programmes provide a unique opportunity to continue aspects of therapy, either in between centre-based sessions or after centre-based therapy has ended. This benefits the retention of established intervention effects. Second, they increase parental involvement and empowerment, in turn contributing to reciprocal partnerships between parents and health professionals. This enables parents and health professionals to learn from each other and share each other's perspectives on the rehabilitation of the child. Third, home-based programmes anticipate changes in the healthcare system by providing a treatment approach that is considered to be cost-effective. Fourth, and finally, home-based programmes may be the preferred or even the only feasible option in specific contexts, for example, in cases where long distances need to be travelled from the child's home to the institution. Given these promising advantages of home-based programmes in children with $\mathrm{CP}$, a systematic review of the available literature on this topic is warranted.

In a recent study, Sakzewski et a/ provided a systematic overview of non-surgical upper extremity therapies in children with unilateral CP and argued that treatment at home may be an effective supplement to centre-based interventions. In the same year, Novak and Berry ${ }^{7}$ focused on the effectiveness of home-based programmes in children with CP. In contrast to the study of Sakzewski et al, the review of Novak et al was not systematic. Similar to Sakzewski et al however, they concluded that home-based programmes using goal directed training are effective for improving motor outcomes. ${ }^{7}$ To extend the two previous studies, the review described in this protocol will be systematic as well as specifically focused on home-based interventions in children with CP. The review will be distinctive because its purpose is to investigate currently available homebased occupational therapy and physiotherapy programmes regarding both their feasibility and their effect, and to evaluate both child-related and parent-related outcomes.

Feasibility will be addressed, as this is a prerequisite for effective home-based programmes. Since feasibility is a general facet of home-based programmes per se, the review will not be limited to feasibility of upper extremity programmes. Feasibility of a variety of home-based occupational therapy and physiotherapy programmes will be reviewed. In contrast, the review of the effect will indeed be limited to home-based programmes that focus on the upper extremity.

Both efficacy and effectiveness will be addressed. Efficacy and effectiveness are related concepts, but with an important difference between them. Efficacy relates to results of an intervention under ideal circumstances (ie, explanatory study). Effectiveness, on the other hand, relates to the beneficial effect of an intervention under 'real world' clinical settings (ie, pragmatic study). ${ }^{8}$ Parents play a key role in a home-based programme for their child. Hence, their contribution to the programme might affect their psychosocial health, either positive or negative, which 
may influence the adherence to the programme. Therefore, next to child-related outcomes, parent-related outcomes are of primary interest in our evaluation of efficacy and effectiveness of home-based programmes.

Comparison of effectiveness of different upper extremity home-based programmes is critical for clinical decision-making when considering options for individual treatment plans, or for institutional decision-making. Moreover, detailed insight into different home-based programmes regarding feasibility, efficacy as well as effectiveness will result in recommendations to improve existing home-based programmes and to develop and design of new programmes.

\section{OвJectives}

The aim of the systematic review described in this protocol is to provide a clear view on the available home-based occupational therapy and physiotherapy programmes in children with CP (aged $<18$ years), specifically home-based programmes that focus on the upper extremity. Two objectives will be addressed by the systematic review:

1. To assess the feasibility of home-based occupational therapy and physiotherapy programmes in children with CP.

2. To assess the efficacy and effectiveness of home-based occupational therapy and physiotherapy programmes that focus on the upper extremity in children with $C P$, on child-related and parent-related outcomes.

\section{METHODS AND ANALYSIS}

\section{DESIGN}

The protocol for the systematic review was developed in accordance with the Preferred Reporting Items for Systematic Review and Meta-Analysis Protocols (PRISMA-P) 2015.,10

\section{ELIGIBILITY CRITERIA}

\section{STUDY DESIGNS}

Studies in which primary data were collected will be included. That is, (systematic) reviews and meta-analyses will be excluded. A relatively small number of available studies are expected. ${ }^{7}$ Since it is strived for an overview of all available evidence, all types of study designs will be included regarding feasibility as well as efficacy and effectiveness. However, the hierarchy of evidence will be taken into consideration for reporting of the results of the review. 


\section{PARTICIPANTS}

Studies on children aged $<18$ years with any type of CP will be included. Studies in which adult patients participated in the home-based programme next to children will also be included, provided that data were reported separately for children and adults (aged $\geq 18$ years). Likewise, studies in which both children with CP and children with other disorders were studied will also be included, provided that data of children with CP were reported separately.

\section{INTERVENTIONS AND SETTING}

Studies that report on at least one home-based occupational therapy or physiotherapy intervention will be included. An intervention is considered to be home-based if treatment is performed in the home setting without a healthcare provider being physically present. Studies that only include therapy provided at a healthcare facility, (pre)school or day care will be excluded. In case the intervention takes place in different settings, studies will be included if treatment of the child in the home setting is a fundamental, prespecified element of the intervention. Regarding the research objective on efficacy/effectiveness, exclusively studies that report on interventions targeting the upper extremity will be included. Hence, studies that report on general gross motor function will be excluded.

We consider home-based programmes as complex interventions, composed of several interacting components. Possible components could be, for example, training of the parents, exercises or supervision by a healthcare professional. Where possible, feasibility, efficacy or effectiveness of components will be described separately.

\section{COMPARATORS}

The nature of the comparator (ie, the intervention against which the home-based programme is compared) is unrelated to the feasibility of the home-based programme. Hence, to describe the feasibility of home-based programmes, studies on all possible control interventions will be included as well as studies without one.

To assess efficacy/effectiveness, comparators of interest are: no therapy, care as usual, centre-based occupational therapy or physiotherapy, and medical intervention (ie, drugs or a surgical procedure such as botulinum toxin injections). Studies in which two or more home-based programmes were compared with each other will also be included. 


\section{Outcomes}

To determine feasibility, studies will be included that reported on outcomes within the areas acceptability, demand, implementation, practicality, adaptation, expansion and integration. ${ }^{11}$ These terms will be elaborated on in the paragraph on 'Outcomes and prioritisation'.

With regard to efficacy and effectiveness, the outcomes of interest are:

- Child-related outcomes within all levels of the International Classification of Functioning, Disability and Health (ICF), that is, body functions and structures, activity and participation. ${ }^{12}$

- Parent-related or caregiver-related outcomes on the psychological and social domain.

Studies reporting on at least one of the aforementioned outcomes regarding feasibility, efficacy or effectiveness will be included.

\section{TIMING}

There will be no restrictions on the length of follow-up to assess outcomes.

\section{LANGUAGE}

There will be no language restrictions.

\section{INFORMATION SOURCES}

Several complementary information sources will be used to identify relevant studies, applying the strategy described in the next paragraph. Also, unpublished studies will be searched for. There will be no restriction on the year of publication. First, the following electronic databases will be searched for literature: MEDLINE (Ovid interface), EMBASE (Ovid interface) CINAHL (EBSCO interface), PsycINFO (EBSCO interface), CPCI-S (Web of Science interface), OTseeker and PEDro. The search will be supplemented by searching for trial protocols through the trial register ICTRP. Until reaching a point of literature saturation (ie, the moment when no new relevant studies emerge), the reference lists of included records will be scrutinised, as well as the reference lists of (systematic) reviews and meta-analysis that were found during the search. Titles and abstracts of the references will be compared with the eligibility criteria to decide on inclusion in the selection process. Finally, a bibliography of the included records will be circulated to all corresponding and last authors of included studies. They will be asked to provide details of any other related study either by their research group or associates.

\section{SEARCH STRATEGY}

The search strategy includes keywords (eg, MESH-terms) and text words (ie, within title and abstract), combining population and intervention-related search terms, for example: 


\section{MEDLINE SEARCH-OVID INTERFACE}

1. Cerebral Palsy/

2. ((cerebral adj2 pals\$) or encephalopathia infantalis or spastic diplegia\$ or little\$ disease). ti,ab.

3. or/1-2

4. exp Self Care/

5. Home Care Services/

6. (Home or in?home or home?based or self care or residence or domiciliary).ti,ab.

7. or/4-6

8. exp Exercise Therapy/

9. Physical Therapy Modalities/

10. (Exercise\$ or therapy or therapies or program\$ or train\$ or physiotherapy\$ or occupational or (physical adj2 therap\$)).ti,ab.

11. or/8-10

12. 3 and 7 and 11

Two members of the research team (LWMEB and JK) developed the search strategy for each database and trial register. JK will perform the search.

\section{STUDY RECORDS}

\section{DATA MANAGEMENT}

Literature search results and corresponding PDF files will be uploaded to the reference management software EndNote. EndNote will be used to remove duplicate records. All records will be uploaded in Covidence. This program will be used for selection, data collection and maintenance of the review.

\section{SELECTION PROCESS}

Before the selection process starts, search results of the various information sources will be merged and duplicate records will be removed. The first phase of the selection process consists of the screening of titles and abstracts on eligibility criteria such that irrelevant records are removed. In case of any doubt regarding relevance, the record will remain included at this stage. Subsequently, full texts will be retrieved for all potentially relevant records. Full-text records will be examined for compliance of studies with the eligibility criteria. Records for which the full text cannot be retrieved may also be included, although results will be reported separately. Multiple records of the same study will be identified and handled as described in the paragraph 'data collection process! 
The first phase of the selection process (ie, screening of titles and abstracts) will be performed in duplicate by two independent reviewers (LWMEB and MLAPS). Examination of the fulltext records will be performed in duplicate and independently by the same reviewers (LWMEB and MLAPS), who will not be blinded to any study information. Inter-rater agreement will be calculated. In case of discrepancies in any phase of the selection process, a third and fourth reviewer (YJJ-P and BS) will mediate to reach consensus.

\section{DATA COLLECTION PROCESS}

Data extraction will be carried out by use of Covidence, in duplicate by two independent reviewers (LWMEB and MLAPS). To increase consistency between the reviewers, the data extraction form will be pilot tested before the start of the data collection process of the review. This will be carried out by duplicate completion of the form for two CP-related records (one feasibility study and one randomised controlled trial), which are not eligible for inclusion in this review (LWMEB and MLAPS). Adaptations will be processed based on discrepancy between the data extraction results of the reviewers, evaluated by a third reviewer (YJJ-P) and the reviewers' experiences during the pilot test. In case of discrepancies between the reviewers during the review, a third and fourth reviewer (YJJ-P and BS) will arbitrate to reach consensus.

In the absence of complete descriptions of essential information, the reviewers will contact the corresponding author to collect the required information. The decision to contact an author will be made in mutual agreement between the four reviewers (LWMEB, MLAPS, YJJ-P and BS). On the basis of an earlier study, it is expected to receive additional information for $\sim 50 \%$ of the incompletely described studies. ${ }^{13}$

Multiple records of a single study will be searched for by comparing author names, intervention locations, intervention characteristics, sample sizes and outcomes of eligible records. If multiple records present different outcome variables or time points, they will be combined into one record within the review. If overlapping records on the same outcome variable(s) as well as the same time points are found, only the one reporting on the largest sample size will be included in the review. This will avoid double-counting of participants. In case of inconsistencies between reports, the reviewers will contact the authors for clarification, using the same approach mentioned earlier in this paragraph.

\section{DATA ITEMS}

From all records, the following information will be extracted: author(s), publication date, study design, country, comparator, number of participants (in total and per study arm), outcomes, duration of follow-up and time points of measurements.

The following treatment characteristics of the home-based programmes will be extracted: objective, therapy provider(s), duration of the programme, frequency and duration of sessions, treatment approach (eg, task-specific training). Furthermore, demographics of participating children will be extracted: age, gender, diagnosis (including type and topographical distribution of (P), level on the Manual Ability Classification System, level on the Gross Motor Function Classifi- 
cation System and level on the Communication Function Classification System. Finally, the demographics of parents of participating children will be extracted: age, gender and educational level. The data extracted from the included studies will be summarised and tabulated.

\section{OUTCOMES AND PRIORITISATION}

Concerning the feasibility objective, the following areas of outcomes will be applied: ${ }^{11}$ Primary outcome:

- Acceptability: 'the extent to which programme deliverers or programme recipients judge the programme as suitable, satisfying or attractive (eg, satisfaction)'.

Secondary outcomes:

- Demand: 'the extent to which a programme is likely to be used (eg, expressed interest to use)'.

- Implementation:'the extent to which a programme can successfully be delivered to intended participants in some defined, but not fully controlled, context (eg, success or failure of execution).'

- Practicality: 'the extent to which a programme can be carried out with intended participants using existing means, resources and circumstances and without outside intervention (eg, ability of participants to carry out intervention activities)'.

- Adaptation: 'the extent to which an existing programme performs when changes are made regarding format or population (eg, degree to which similar outcomes are obtained in a new format)'.

- Integration: 'the extent to which a programme can be integrated within an existing system (eg, perceived sustainability)'.

- Expansion:'the extent to which a previously tested programme can be expanded to provide a new programme or service (eg, positive or negative effects on organisation)'.

For the efficacy and effectiveness studies, the following outcomes will be used:

Primary outcomes:

- Child-related upper extremity outcomes within the level activity of the ICF (eg, Assisting Hand Assessment).

Secondary outcomes:

- Child-related upper extremity outcomes within the levels body functions and structures and participation of the ICF (eg, spasticity assessment and Children's Assessment of Participation and Enjoyment, respectively).

- Parent-related or caregiver-related outcomes within the psychological and social domain (eg, Parenting Stress Index).

If available, results from an intention-to-treat analysis will be used. All outcomes may be measured quantitatively or qualitatively. 


\section{RISK OF BIAS INDIVIDUAL STUDIES}

Risk of bias of predominantly quantitative studies will be assessed by the Checklist for Measuring Quality by Downs and Black. ${ }^{14}$ The checklist contains 27 yes/no questions across five constructs: study quality (the overall quality of the study); external validity (the ability to generalise findings of the study); study bias (to assess bias in the intervention and outcome measure(s)); confounding and selection bias (to determine bias from sampling or group assignment); and power of the study (to determine if findings are due to chance)..$^{14}$ Risk of bias of predominantly qualitative studies will be assessed by the JBI Critical Appraisal Checklist for Qualitative Research, which consists of 10 questions that can be answered with yes, no, unclear or not applicable..$^{15}$

The risk of bias will be assessed for all applicable studies in duplicate by two independent reviewers (LWMEB and MLAPS), without blinding. To increase consistency between the reviewers, the checklists will be pilot tested before the start of the risk of bias assessment of the review. This will be carried out by duplicate assessment of two CP-related records (one predominantly quantitative study and one predominantly qualitative study), which are not eligible for inclusion in this review. In case of disagreement between reviewers during the review, a third and fourth reviewer (YJJ-P and BS) will arbitrate. Risk of bias will be assessed on the study level. Results across studies will be presented graphically. Additionally, the reviewers will state how the risk of bias may have influenced their review findings.

\section{DATA SYNTHESIS}

Based on the results of the article of Novak et al, ${ }_{1}^{7}$ a limited number of efficacy and effectiveness studies are expected to be found by the systematic search. Additionally, the studies are expected to be both clinically and methodologically heterogeneous. Hence, it is anticipated that conducting a meta-analysis will not be appropriate. Therefore, a method for meta-analysis is not included in this protocol. If the assumption emerges to be wrong, this will result in an amendment of the protocol, in which a method for meta-analysis will be included. An amendment will be made if at least three efficacy and effectiveness studies are comparable regarding treatment, comparator(s) and outcome(s). ${ }^{16}$ The reviewers (LWMEB, MLAPS, YJJ-P and BS) will decide whether a meta-analysis is appropriate and report the rationale.

\section{META-BIASES}

For the efficacy and effectiveness studies included in the review, the risk of selective reporting (outcome reporting bias) will be determined. This will be assessed by comparing the records on study results with previously published study protocols and registrations. The studies of which no study protocol or trial registration was found will also be listed. This procedure will be performed by one reviewer (LWMEB). 
In case of $\geq 10$ homogeneous efficacy and effectiveness studies (according to the criteria described in the paragraph 'data synthesis'), risk of publication bias will be assessed by graphing a funnel plot and, if appropriate, supplemented by a statistical test. In the plot, it will be indicated which studies have been published in a journal and which ones have not been (yet).

\section{CONFIDENCE IN CUMULATIVE ESTIMATE}

For each efficacy and effectiveness outcome, the Grading of Recommendations Assessment, Development and Evaluation (GRADE) guidelines will be used to judge the quality of evidence within the domains risk of bias, publication bias, imprecision, inconsistency and indirectness. ${ }^{17}$ The quality of the evidence can be scored as high quality (very confident that the true effect lies close to that of the estimate of the effect), moderate quality (moderately confident in the effect estimate: the true effect is likely to be close to the estimate of the effect, but there is a possibility that it is substantially different), low quality (confidence in the effect estimate is limited: the true effect may be substantially different from the estimate of the effect) or very low quality (very little confidence in the effect estimate: the true effect is likely to be substantially different from the estimate of effect). The same approach as described in the paragraph 'risk of bias individual studies' will be used for pilot testing and scoring.

\section{Amendments}

In case of protocol amendments, the authors will document the date, description of the changes and rationale for each amendment.

\section{ETHICS AND DISSEMINATION}

\section{ETHICAL CONSIDERATIONS}

The proposed study does not involve collection of primary data. Accordingly, no ethical approval is required.

\section{Dissemination PLAN}

This systematic review protocol was registered in the International Prospective Register of Systematic Reviews (PROSPERO) on 29 July 2016 (registration number CRD42016043743).

The authors will disseminate the findings of this review through publication in a peer-reviewed journal and conference presentation(s). The results will be reported according to the most recent version of the Preferred Reporting Items for Systematic Reviews and Meta-Analyses (PRISMA). ${ }^{18}$ 


\section{Contributors}

LWMEB designed the study protocol and drafted the initial manuscript. LWMEB and JK developed the search strategy. MLAPS, YJJ-P, JK and BS provided critical insights and reviewed the protocol and manuscript, making important intellectual contributions. All authors read and approved the final version.

\section{FUNDING}

This work was supported by ZonMw (the Netherlands Organisation for Health Research and Development), grant number 630000001, as part of the research project COAD (Co-creation at hand: The road to independence). The clinical trial that is part of the COAD-project is registered under number NTR5743.

\section{Competing InTERESTS}

None declared. 


\section{REFERENCES}

1. Arner M, Eliasson AC, Nicklasson S, et al. Hand function in cerebral palsy. Report of children in a population-based longitudinal healthcare program. J Hand Surg 2008;33:1337-47.

2. Novak I, McIntyre S, Morgan C, et al. A systematic review of interventions for children with cerebral palsy: state of the evidence. Dev Med Child Neurol 2013;55:885-910.

3. Aarts PB, Jongerius PH, Geerdink YA, et al. Effectiveness of modified constraint-induced movement therapy in children with unilateral spastic cerebral palsy: a randomized controlled trial. Neurorehabil Neural Repair 2010;24:509-18.

4. Gordon AM, Schneider JA, Chinnan A, et al. Efficacy of a hand-arm bimanual intensive therapy (HABIT) in children with hemiplegic cerebral palsy: a randomized control trial. Dev Med Child Neurol 2007:49:830-8.

5. de Brito Brandao M, Gordon AM, Mancini MC. Functional impact of constraint therapy and bimanual training in children with cerebral palsy: a randomized controlled trial. Am J Occup Ther 2012;66:672-81.

6. Sakzewski L, Ziviani J, Boyd RN. Efficacy of upper limb therapies for unilateral cerebral palsy: a meta-analysis. Pediatrics 2014;133:e175-204.

7. Novak I, Berry J. Home program intervention effectiveness evidence. Phys Occup Ther Pediatr 2014;34:384-9.

8. Gartlehner G, Hansen RA, Nissman D, et al. Criteria for distinguishing effectiveness from efficacy trials in systematic reviews. Technical Review 12 (Prepared by the RTI-International-University of North Carolina Evidence-based Practice Center under Contract No. 290-02-0016.) AHRQ Publication No. 06-0046. Rockville, MD: Agency for Healthcare Research and Quality, 2006.

9. Moher D, Shamseer L, Clarke M, et al. Preferred reporting items for systematic review and meta-analysis protocols (PRISMA-P) 2015 statement. Syst Rev 2015;4:1.

10. Shamseer L, Moher D, Clarke M, et al. Preferred reporting items for systematic review and meta-analysis protocols (PRISMA-P) 2015:elaboration and explanation. BMJ 2015;349:97647.

11. Bowen DJ, Kreuter M, Spring B, et al. How we design feasibility studies. Am J Prev Med 2009;36:452-7.

12. World Health Organization. Towards a common language for functioning, disability and health. ICF: the international classification of functioning, disability and health. Geneva: World Health Organization, 2002. http://www.who.int/classifications/icf/icfbeginnersguide.pdf (accessed Jul 2016).

13. Selph SS, Ginsburg AD, Chou R. Impact of contacting study authors to obtain additional data for systematic reviews: diagnostic accuracy studies for hepatic fibrosis. Syst Rev 2014;3:107.

14. Downs SH, Black N. The feasibility of creating a checklist for the assessment of the methodological quality both of randomised and non-randomised studies of healthcare interventions. J Epidemiol Community Health 1998;52:377-84.

15. The Joanna Briggs Institute. The Joanna Briggs Institute Critical Appraisal tools for use in JBI systematic reviews. Checklist for qualitative research. The Joanna Briggs Institute, 2016. http://joannabriggs.org/ assets/docs/critical-appraisal-tools/JBI_Critical_Appraisal-Checklist_for_Qualitative_Research.pdf (accessed Jul 2016). 
16. Higgins JPT, Green S, eds. Cochrane handbook for systematic reviews of interventions version 5.1.0 [updated March 2011]. The Cochrane Collaboration, 2011. http://handbook.cochrane.org/(accessed Jul 2016).

17. Guyatt GH, Oxman AD, Schunemann HJ, et al. GRADE guidelines: a new series of articles in the journal of clinical epidemiology. J Clin Epidemiol 2011;64:380-2.

18. Moher D, Liberati A, Tetzlaff J, et al. Preferred reporting items for systematic reviews and meta-analyses: the PRISMA statement. Int J Surg 2010;8:336-41. 
Chapter 3

\title{
Feasibility and effectiveness of home- based therapy programs for children with cerebral palsy: a systematic review
}

\author{
LWME Beckers*, MME Geijen*, J Kleijnen, EAA Rameckers, \\ MLAP Schnackers, RJEM Smeets, YJM Janssen-Potten \\ * contributed equally
}

In preparation for submission 



\section{INTRODUCTION}

Over the last years, despite an increased survival rate of low birth weight infants, the overall prevalence of cerebral palsy (CP) has remained constant at 2.11 per 1000 live births. ${ }^{2}$ CP is the largest diagnostic group treated in pediatric rehabilitation. Social participation, independence and self-efficacy are restricted in children with CP as they experience limitations in the execution of daily activities. ${ }^{3}$ About $60 \%$ of the children between the age of 4 and 16 years has problems with the effective use of the arm and hand during reach, grasp, release, and manipulation of objects, resulting in limitations in performance of activities of daily life. ${ }^{45}$ Most currently applied upper extremity interventions aim at improving functionality and abilities toward independence. Studies examining these interventions have shown that the key ingredients for effective treatment constitute a high training intensity combined with meaningful goal-directed and task-specific training. ${ }^{6}$ The relevant context for the child to learn new activities of daily living is usually the home environment and interventions provided in this context are called home-based programs.? ${ }^{8}$ Home-based programs are defined as "therapeutic activities that the child performs with parental assistance in the home environment with the goal to achieve desired health outcomes".

Home-based programs are thought to be a useful addition or even replacement of center-based therapy in the rehabilitation of children with CP. ${ }^{6}$ Home-based programs provide a unique opportunity to train the child continuously and specific tasks are trained in a relevant context for the child. Furthermore, these programs enable parents to incorporate training into their daily routine with the child, so no separate training moments are necessary, generalization is fostered and the intensity and repetition of trained tasks can be high, which all enhance effective motor learning. ${ }^{9}$ In addition, the increased amount of training may facilitate the retention of established intervention effects. Furthermore, it may also increase parental involvement and empowerment, in turn contributing to reciprocal partnerships between parents and health professionals. ${ }^{10}$

Despite consensus on the importance of home-based training programs for children with $\mathrm{CP}$, there is scarce information regarding program characteristics that may influence family participation. ${ }^{11}$ For example, parents can be either therapy provider in collaboration with a health professional (partnership home-program) or supervised by a health professional (therapist-directed home program). ${ }^{12}$ When parents become therapy providers, the relationship between the parents and the health professional changes: the health professional becomes the coach of the parents. Depending on the role of parents and their specific needs, the way and amount of coaching can vary from limited instruction only at the beginning of the program, to extensive demonstration, feedback and coaching throughout the entire program. The mode of coaching can vary from home visits by the therapist to remote coaching by e-mail or telephone consultation.

Parents are of great importance in home-based programs. Although a survey among parents has shown that they do not have an unfavorable opinion concerning home programs these home programs may induce or enhance stress in parents. ${ }^{12}$ Parents may experience pressure to comply, especially when the program is demanding. Furthermore, the altered parent-child interaction during training may cause additional tension. ${ }^{13}$ As the role of the parents changes to therapy provider, this may cause a conflict between their parenting style and their approach as a therapy provider. Consequently, loss of motivation by the parents and/or the child to complete 
the training activities may affect compliance and probably the effectiveness of the intervention. Because of the aforementioned factors, home-based interventions need to be carefully developed and implemented.

Feasibility is an important aspect that needs to be considered when implementing homebased programs. Feasibility studies are used to determine whether an intervention is relevant, sustainable, and appropriate for further testing. ${ }^{14}$ Several studies have investigated the feasibility of home-based programs for children with CP and indicated that the programs were feasible in terms of compliance and adherence. ${ }^{15} 16$ However, up until now no systematic overview is available of relevant feasibility components, such as satisfaction, acceptability or practicality of home-based programs and even when these treatments appear feasible they are not necessarily effective. So far, the effectiveness of home-based programs in children with CP has been reviewed by Novak and Berry. They concluded that home-based programs using goal directed training are effective in improving motor and functional outcomes. ${ }^{8}$ Another systematic review by Sakzewski et al. on non-surgical upper extremity therapies in children with unilateral $\mathrm{CP}$, concluded that home-based programs are an effective supplement next to center-based interventions. ${ }^{6}$

Supplementary to these two reviews, this systematic review aims to provide a clear summary on both feasibility and effectiveness of currently available home-based programs in children with CP (aged $<18$ years), specifically focusing on the upper extremity. The effectiveness will be investigated on both child-related and parent-related outcomes, as parent involvement has received little research attention.

The following two objectives will be addressed by the systematic review:

1. To assess the feasibility of home-based occupational therapy and physiotherapy programs in children with $C P$.

2. To assess the effectiveness and efficacy of home-based occupational therapy and physiotherapy programs that focus on the upper extremity in children with CP, on child-related and parent-related outcomes.

\section{Methods}

\section{Protocol AND Registration}

Objectives and methods of this systematic review were pre-specified and registered in the International Prospective Register of Systematic Reviews (PROSPERO), registration number CRD42016043743, as well as published in a protocol. ${ }^{17}$

\section{ELIGIBILITY CRITERIA}

1. Types of studies: All types of original studies concerning feasibility or effectiveness of home-based therapy in children with CP. An intervention was considered to be homebased if treatment was performed in the home setting without a healthcare provider being 
physically present. Studies that only included therapy provided at a healthcare facility, (pre) school or day care were excluded. In case the intervention took place in different settings, studies were only included if treatment of the child in the home setting was a fundamental, pre-specified element of the intervention. The studies included in this systematic review were categorized using the scale published by the American Academy for Cerebral Palsy and Development Medicine (AACPDM) to hierarchize studies based on research design types of either intervention (group) studies or single subject design studies. ${ }^{18}$

2. Types of participants: Children aged $<18$ years with any type of CP. In case of a more heterogeneous study population, results of the target population must have been reported separately.

3. Types of intervention: A home-based occupational therapy or physiotherapy intervention, in which treatment is performed in the home setting without (continuous) physical presence of a healthcare provider. To investigate effectiveness, only upper extremity interventions were included.

4. Types of comparators: Concerning feasibility, studies comprising all types of comparators or no control intervention were considered. In order to determine effectiveness: no therapy, care as usual, center-based occupational therapy or physiotherapy, a distinct home-based program, a pharmacological intervention, and a surgical procedure.

5. Types of outcome measures: To review feasibility, studies reporting on one of the key areas of focus for feasibility studies, as proposed by Bowen et al.: acceptability, demand, implementation, practicality, adaptation, expansion, or integration. ${ }^{14}$ Regarding effectiveness, child-related outcome measures related to any level of the International Classification of Functioning, Disability and Health (ICF), or parent-related outcomes within the psychological and social domain including parenting. ${ }^{19}$

6. Report criteria: No restrictions regarding language, publication status or publication date were applied.

\section{INFORMATION SOURCES}

Records were identified using the electronic databases MEDLINE (Ovid interface; 1946-present), EMBASE (Ovid interface; 1974-present), CINAHL (EBSCO interface; 1981-present), PsycINFO (EBSCO interface), OTseeker, and PEDro. Moreover, reference lists of included papers and excluded reviews and meta-analyses were scanned.

\section{SEARCH}

Search terms for population and for intervention were combined for MESH-terms and text words in titles and abstracts. The search strategies were created by LB and revised after peer review by JK. A data search expert from Kleijnen Systematic Reviews Ltd conducted the search on 10 October 2016. 


\section{STUDY SELECTION}

The software platform Covidence was used to complete the eligibility assessment. LB and MS independently executed screening of titles and abstracts as well as the unblinded evaluation of full text publications in duplicate. Any disagreements between the reviewers were resolved through consensus and arbitrated by YJ-P, when necessary.

\section{Data COLlection process}

LB and MG collected data independently for each study. A data extraction form was developed a priori, pilot tested on two records that were not eligible for this review, and refined accordingly. During data collection the reviewers discussed any discrepancies and consulted YJ-P to mediate when necessary. Authors were contacted if essential information was missing from a study. Author names, intervention locations, intervention characteristics, sample sizes and outcomes were compared to identify duplicate publications. If reports were inconsistent, the reviewers contacted the authors to obtain clarification. Multiple records reporting on different outcomes or time points of one study were combined. For records investigating the same outcomes and time points, only the record reporting the largest sample size was included.

\section{DATA ITEMS}

General information was extracted from each included study: 1) study characteristics (author(s), publication year, study design, country, comparator, number of participants (in total and per study arm), outcomes, duration of follow-up, and time points of measurements, 2) intervention characteristics (objective, therapy provider(s), approach of coaching parents, duration of the program, frequency and duration of sessions, treatment approach, and motor learning approach), 3) demographics of participating children: (age, gender, diagnosis (including type and topographical distribution of (P), level on the Manual Ability Classification System, level on the Gross Motor Function Classification System, and level on the Communication Function Classification System), and 4) demographics of parents of participating children (age, gender, and educational level).

Feasibility was assessed primarily by outcomes related to the feasibility area, whereas the areas demand, implementation, practicality, adaptation, integration, and expansion were of secondary interest. Definitions of these constructs are provided in the protocol ${ }^{17}$. Concerning the effectiveness objective, child-related upper extremity outcomes within the level activity of the ICF were primary. Outcomes assessing body functions and structures, participation, and parent-related outcomes were of secondary interest.

Home-based programs are often complex interventions, formed by multiple interacting components. For that reason, if results were reported separately for particular components of the intervention, this was also recorded. 


\section{RISK OF BIAS IN INDIVIDUAL STUDIES}

The Joanna Briggs Institute (JBI) Critical Appraisal Checklist for Qualitative Research was used to determine risk of bias of qualitative studies. ${ }^{20}$ Studies with a primary focus on intervention effectiveness were assessed by criteria based on the Checklist for Measuring Quality by Downs and Black. ${ }^{21}$ The construct power was not included, since this item estimates precision rather than bias. Furthermore, the single items were summarized into overall scores and each study was classified into excellent (24-28 points), good (19-23 points), fair (14-18 points) or poor (<14 points). ${ }^{22}$ All assessments were done at the study level. LB and MG performed the unblinded assessment independently. In case the reviewers could not come to an agreement, YJ-P interceded.

\section{Results}

The systematic literature search resulted in 1328 records. After screening title and abstract 1140 of these records were marked irrelevant. One hundred and eighty-eight records were full-text assessed for eligibility. One hundred and nineteen records did not meet the eligibility criteria. The search resulted in a total of 69 records, some reporting on the same study. The flowchart of the search is depicted in Figure 1.

2111 records through database searching MEDLINE: 457

EMBASE: 895

Psyclnfo: 402

OTseeker: 8

ICTRP: 10

Cochrane: 284

PEDro: 55
810 duplicates removed

1140 records irrelevant

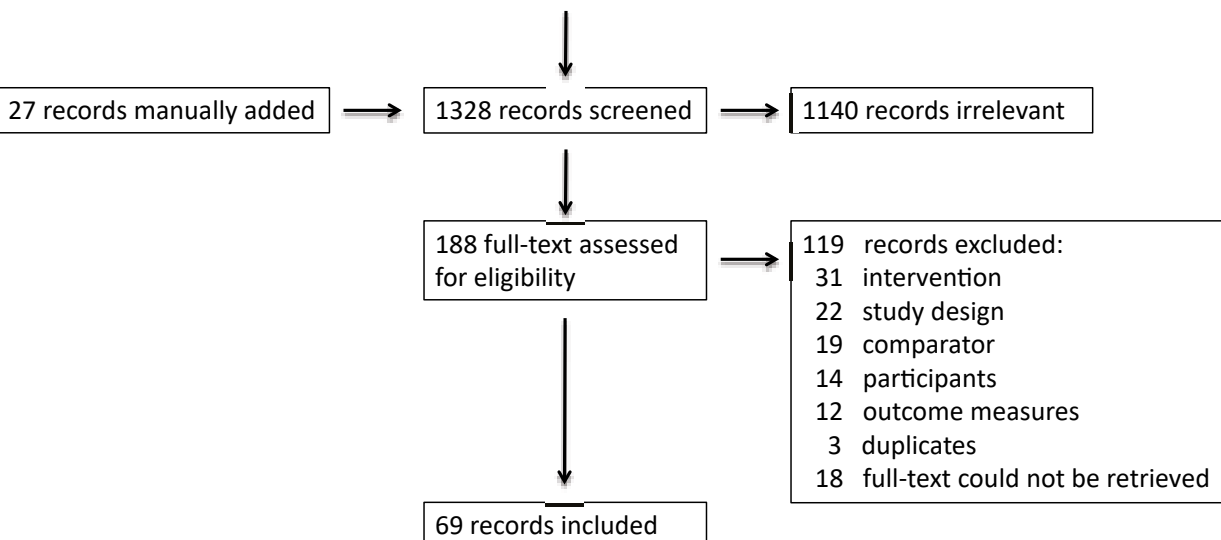

Figure 1 Flowchart 
Finally, 47 studies $^{12151623-66}$ were included in this systematic review, 22 feasibility studies ${ }^{12} 151623-41$ (46.8\%), 10 effectiveness studies ${ }^{57-66}(21.3 \%)$ and 15 studies $^{42-56}$ that reported on both feasibility and effectiveness (31.9\%). The remaining 22 records ${ }^{67-88}$ were conference abstracts. Seven initial studies described in these abstracts ${ }^{67-6976-79}$ developed into a full-text article (31.8\%).

\section{STUDY CHARACTERISTICS}

Of the studies that reported on effectiveness, two studies ${ }^{46} 65$ (8\%) were large RCTs (level of evi-

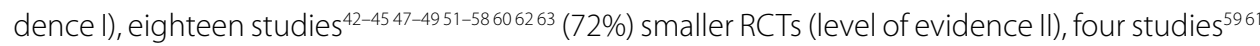
${ }^{6466}(16 \%)$ were single subject designs (one level of evidence III66 (no phases specified), the others ${ }^{59}$ ${ }^{61}{ }^{64}$ level of evidence IV) and one study ${ }^{50}$ (4\%) used a pretest-posttest cohort design, with the participants serving as their own controls.

The methodological quality of studies with a primary focus on intervention effectiveness, assessed by the Downs and Black checklist, is depicted in table 1. According to this scale, five studies $^{45-475556}$ (20\%) were rated as good, eleven studies ${ }^{4344484951-5357586265}$ (44\%) were fair and nine studies ${ }^{42505459-61636466}$ (36\%) were poor.

Table 1 Downs and Black checklist

\begin{tabular}{|c|c|c|c|c|c|}
\hline Authors & Yes & Partially & No & $\begin{array}{l}\text { Unable to } \\
\text { determine }\end{array}$ & $\begin{array}{l}\text { Not } \\
\text { applicable }\end{array}$ \\
\hline Wallen, Ziviani, Naylor, Evans, Novak, Herbert (2011) & 23 & & 1 & 2 & \\
\hline Kirkpatrick, Pearse, James \& Basu (2016) & 21 & 1 & 3 & 1 & \\
\hline $\begin{array}{l}\text { Gordon, Hung, Brandao, Ferre, Kuo, Friel, Petra, Chinnan, } \\
\text { Charles (2011) }\end{array}$ & 20 & 1 & & 5 & \\
\hline Hoare, Imms, Villanueva, Rawicki, Matyas \& Carey (2012) & 20 & 1 & 4 & 1 & \\
\hline James, Ziviani, Ware \& Boyd (2015) & 19 & 1 & 3 & 3 & \\
\hline $\begin{array}{l}\text { Facchin, Rosa-Rizzotto, Visonà Dalla Pozza, Turconi, Paglia- } \\
\text { no, Signorini, Tornetta, Trabacca, Fedrizzi (2011) }\end{array}$ & 18 & & 2 & 6 & \\
\hline Chiu, Ada \& Lee (2014) & 18 & 1 & 5 & 2 & \\
\hline $\begin{array}{l}\text { Sakzewski, Miller, Ziviani, Abbott, Rose, Macdonell \& Boyd } \\
\text { (2015) }\end{array}$ & 18 & 1 & 5 & 2 & \\
\hline Hsin, Chen, Lin, Kang, Chen \& Chen (2012) & 17 & 1 & 4 & 4 & \\
\hline Chen, Chen, Kang, Wu, Chen \& Hong (2014) & 17 & 1 & 5 & 3 & \\
\hline Novak, Cusick \& Lannin (2009) & 17 & 1 & 5 & 3 & \\
\hline $\begin{array}{l}\text { Eugster-Buesch, de Bruin, Boltshauser, Steinlin, Kuenzle, } \\
\text { Muller, Capone, Pfann \& Meyer-Heim (2012) }\end{array}$ & 17 & & 6 & 3 & \\
\hline $\begin{array}{l}\text { Klingels, Feys, Molenaers, Verbeke, Van Daele, Hoskens, } \\
\text { Desloovere \& De Cock (2013) }\end{array}$ & 16 & 1 & 6 & 2 & 1 \\
\hline
\end{tabular}




\begin{tabular}{|c|c|c|c|c|c|}
\hline Authors & Yes & Partially & No & $\begin{array}{l}\text { Unable to } \\
\text { determine }\end{array}$ & $\begin{array}{l}\text { Not } \\
\text { applicable }\end{array}$ \\
\hline Xu, He, Mai, Yan \& Chen (2015) & 16 & & 6 & 4 & \\
\hline Lin, Wang, Wu, Chen, Chang, Lin \& Chen (2011) & 15 & 1 & 5 & 4 & 1 \\
\hline $\begin{array}{l}\text { Preston, Weightman, Gallagher, Levesley, Mon-Williams, } \\
\text { Clarke \& O'Connor (2016) }\end{array}$ & 14 & 1 & 8 & 3 & \\
\hline Al-Oraibi \& Eliasson (2011) & 13 & 1 & 6 & 6 & \\
\hline Abd El-Kafy, Elshemy, Alghamdi (2014) & 13 & 1 & 7 & 5 & \\
\hline $\begin{array}{l}\text { Lowes, Mayhan, Orr, Batterson, Tonneman, Meyer, Alfano, } \\
\text { Wang, Whalen, Nelin, Lo \& Case-Smith (2014) }\end{array}$ & 12 & 1 & 7 & 3 & 3 \\
\hline Charles, Wolf, Schneider, Gordon (2006) & 12 & 1 & 8 & 5 & \\
\hline Naylor \& Bower (2005) & 11 & 1 & 5 & 6 & 3 \\
\hline Kim, Lee, Hwang, Lee, Kim, Park,You, Lee \& Lee (2012) & 9 & & 7 & 10 & \\
\hline Crocker, MacKay-Lyons \& McDonnell (1997) & 9 & & 8 & 4 & 5 \\
\hline Gross, Eudy, Drabman (1982) & 8 & & 5 & 6 & 7 \\
\hline Coker, Lebkicher, Harris, Snape (2009) & 4 & & 8 & 6 & 8 \\
\hline
\end{tabular}

The twelve qualitative studies ${ }^{12527-2931-34374042}$ found were scored with the JBI Critical Appraisal Checklist to determine risk of bias. A positive answer to the first five questions of this checklist is crucial for the assessment of risk of bias. The scores are given in table 2. In only four qualitative studies ${ }^{2932-34}$ (33\%), the first five questions of the JBI checklist could be answered. In other words, the risk of bias in these four studies was clear, whereas in eight studies 1225272831374042 (67\%) this risk could not be estimated from the data provided.

\section{Participant Characteristics}

Most studies targeted children with unilateral spastic CP, but there was a large variation in other child characteristics such as age, MACS, and GMFCS classification. The vast majority of studies did not report any parent characteristics. Only one study ${ }^{24}$ reported on age, gender and educational level of the parents. Nineteen percent of the studies only reported on gender characteristics. The number of study participants ranged from 1 to 147, with a maximum of 105 in an effectiveness study. All participant characteristics are shown in table 3. 
Table 2 JBI Critical Appraisal Checklist

\begin{tabular}{llll}
\hline Article & Yes & No & Unclear \\
\hline James, Ziviani, King \& Boyd (2016) & 9 & 1 & 1 \\
Peplow \& Carpenter (2013) & 8 & 1 & \\
Piggot, Paterson \& Hocking (2002) & 7 & 3 & 1 \\
Piggot, Hocking \& Paterson (2003) & 6 & 3 & 5 \\
Halvarsson, Asplund \& Fjellman-Wiklund (2010) & 4 & 1 & 5 \\
McBurney, Taylor, Dodd \& Graham (2003) & 4 & 1 & 5 \\
Novak (2011) & 4 & 1 & 5 \\
Sandlund, Dock, Hager \&Waterworth (2012) & 4 & 1 & 5 \\
Taylor, Dodd, McBurney \& Graham (2004) & 4 & 1 & 5 \\
Fergus, Buckler, Farrell, Isley, McFarland \& Riley (2008) & 3 & 2 & 5 \\
Hinojosa \& Anderson (1991) & 2 & 3 & 5 \\
Al-Oraibi \& Eliasson (2011) & 2 & 3 & 5 \\
\hline
\end{tabular}

\section{INTERVENTION CHARACTERISTICS}

The intervention characteristics of the studies included in this systematic review are shown in table 4. One should note that all characteristics described in the tables and results section below apply to the parent-delivered part of the intervention only. A more detailed description of the intervention is provided in the appendix.

The treatment approach used in the studies was predominantly (modified) CIMT $(40 \%)^{25}$ 35 42-45 48-50 54-57 59 61-65, also several studies ${ }^{30} 37-3941465258$ used computer-based rehabilitation (e.g. virtual reality, $17 \%)$. Very few studies used goal-directed $(n=2)^{2353}$ or bimanual training $(n=1)^{26}$. The comparators used were none (feasibility studies), other home-based training programs, care as usual, center-based occupational therapy or physiotherapy interventions. The objectives of the intervention were mostly unspecified, but when specified the focus was mainly on ICF activity level. The use of motor learning principles was often not mentioned; only 17 studies $252642-5053-5763$ (36\%) reported that their intervention was based on motor learning principles. The training duration of the home-based training programs varied from 2 weeks to 6 months (all parent-delivered), and the intensity ranged from 70 minutes to 21 hours a week (all parent-delivered). Therapy was mostly provided by parents (49\%), but there were also programs combining parent-delivered and therapist-delivered sessions (47\%). In the latter, the main part of the sessions was delivered by the parents. The coaching of the parents was often unspecified (57\%). Some studies mentioned different modes that were used by therapists to coach the parents, such as course/training, manual or other form of written instructions, DVD, reviewing of logbooks, e-mail, telephone or Skype calls, home visits, computer-feedback, and mutual discussion of goals and therapeutic activities. 


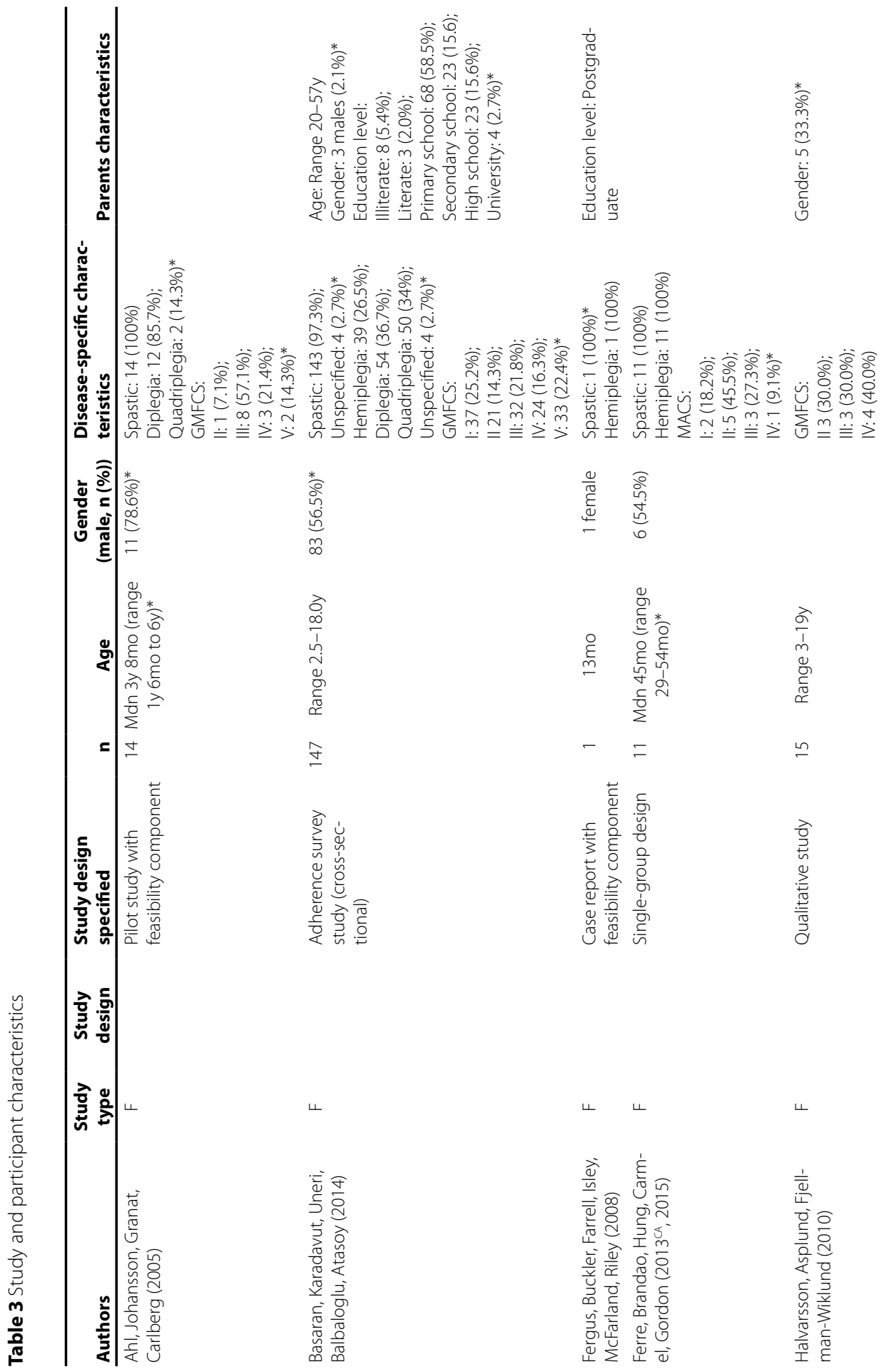



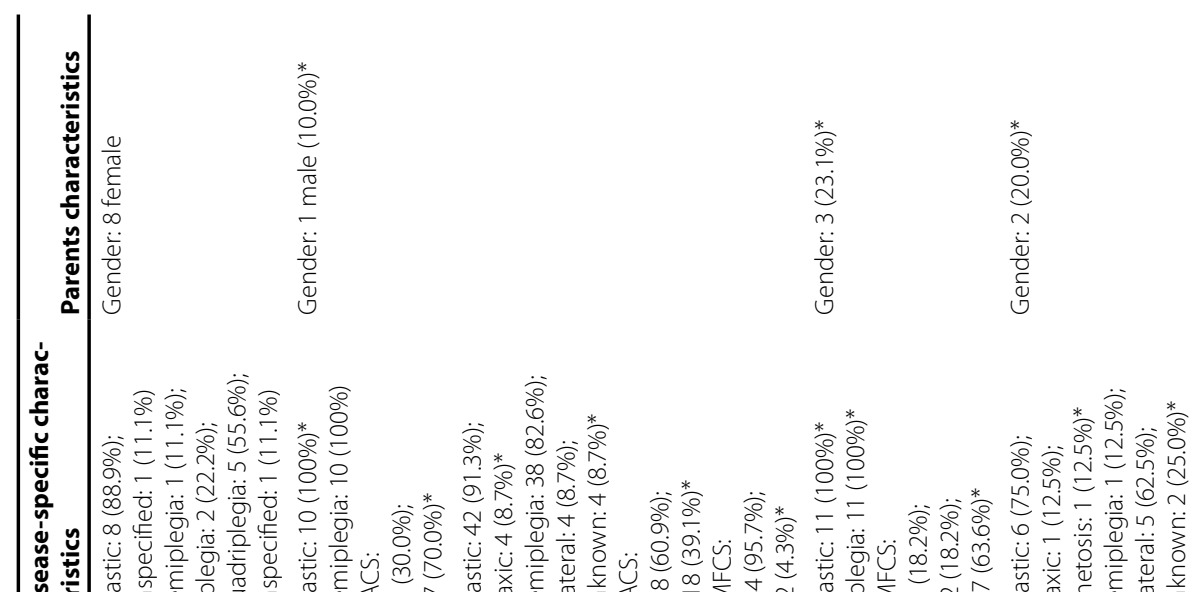

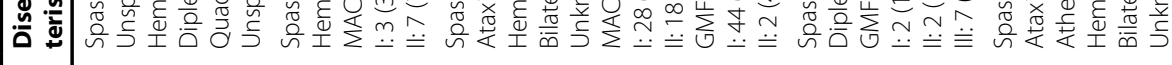
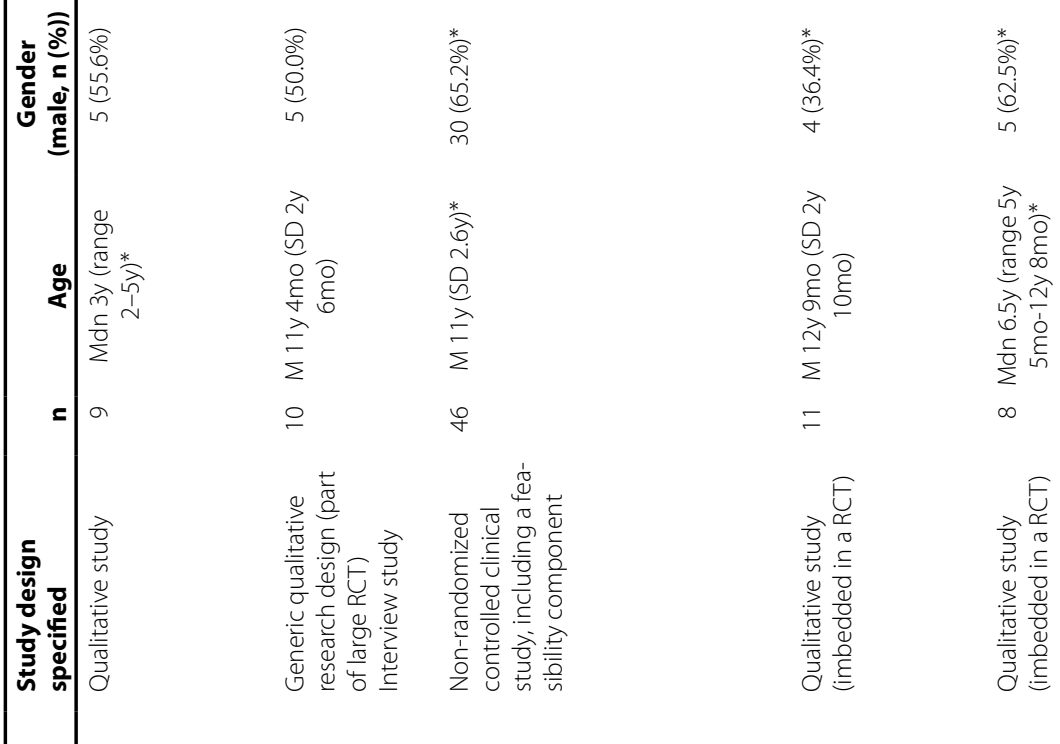

Е
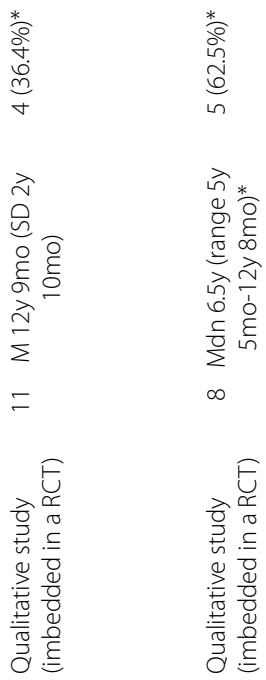

줄
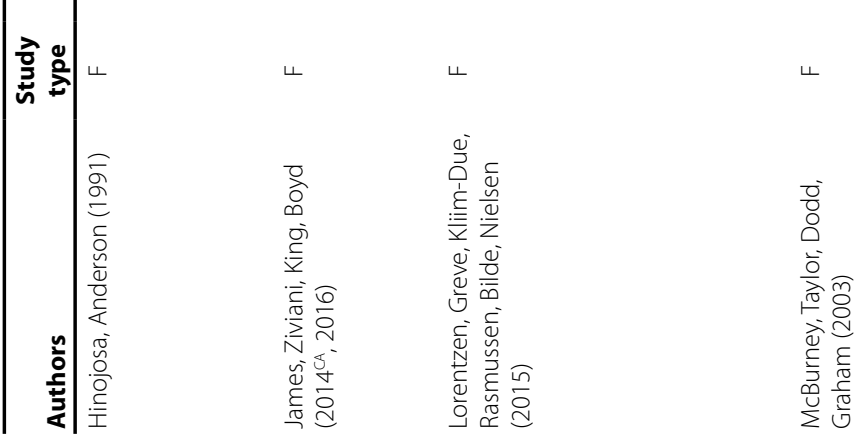

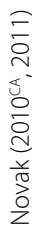


Systematic review

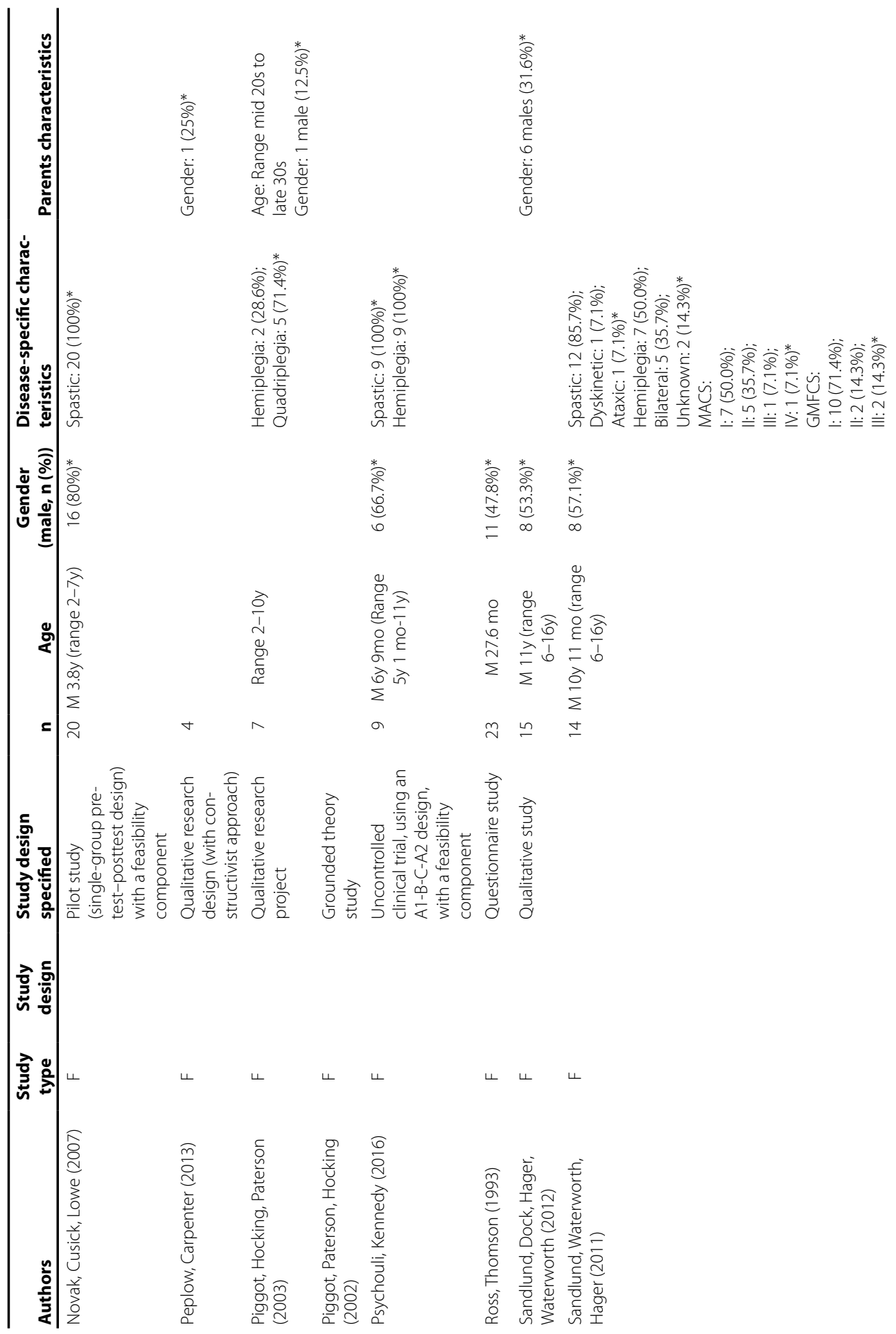

37 


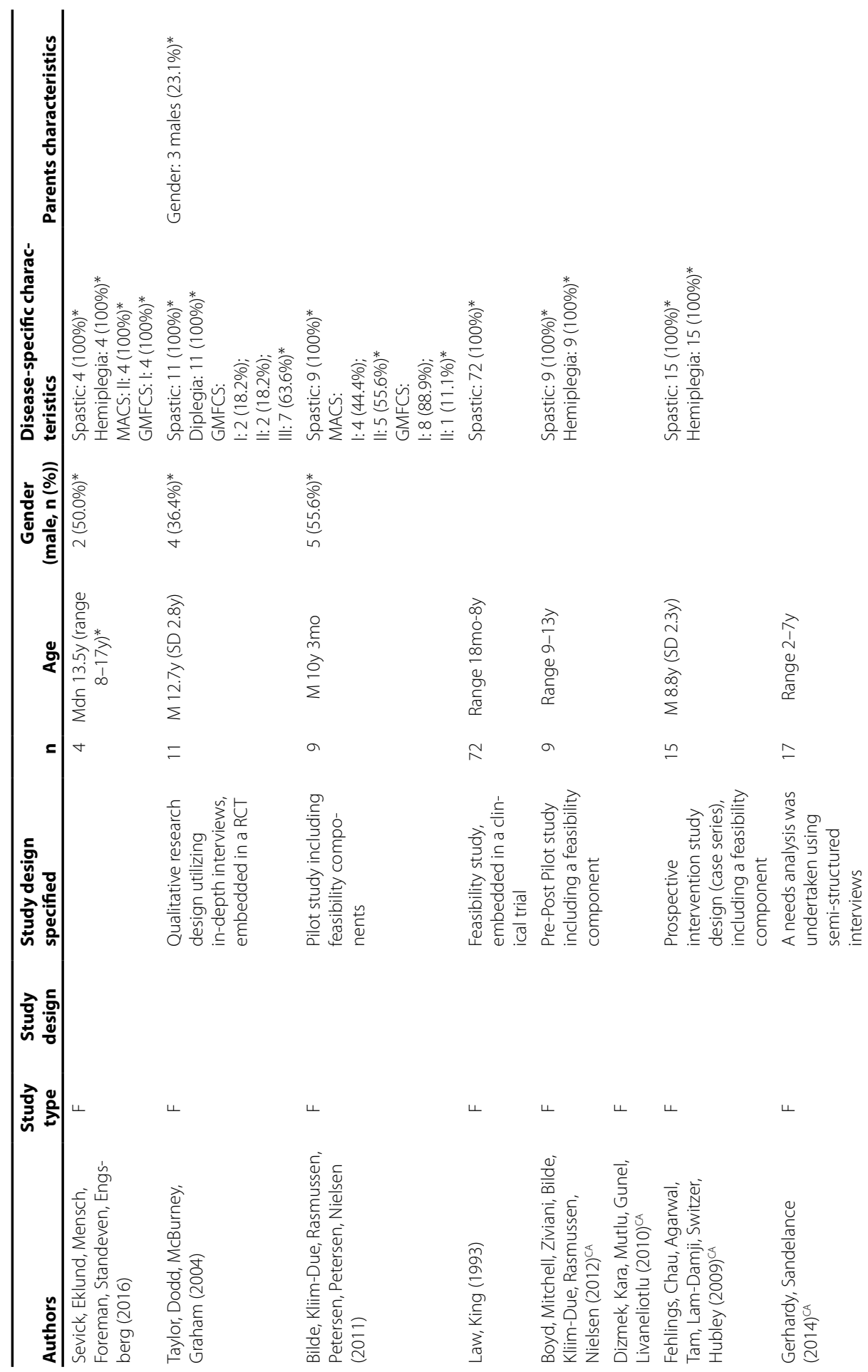




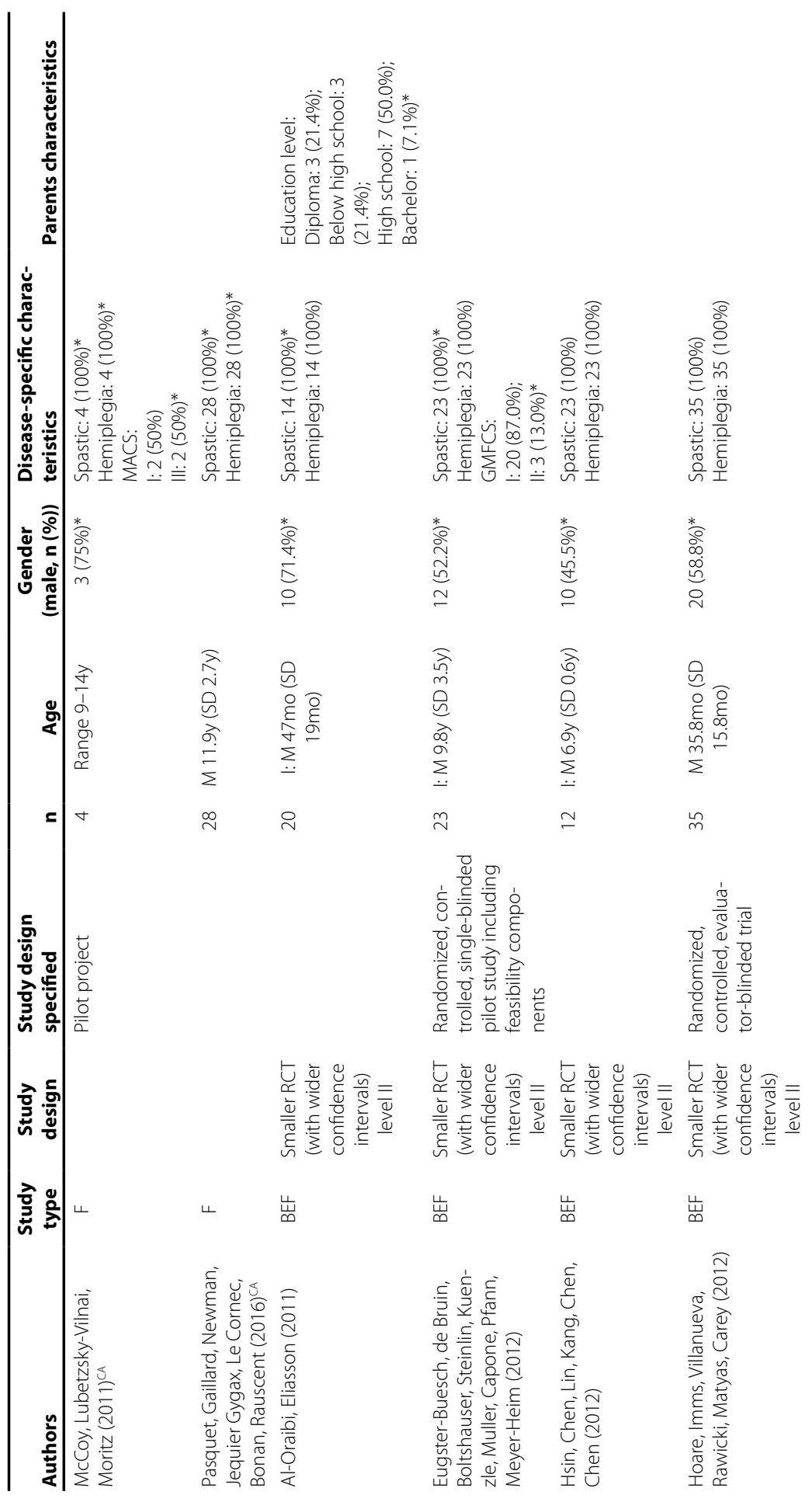




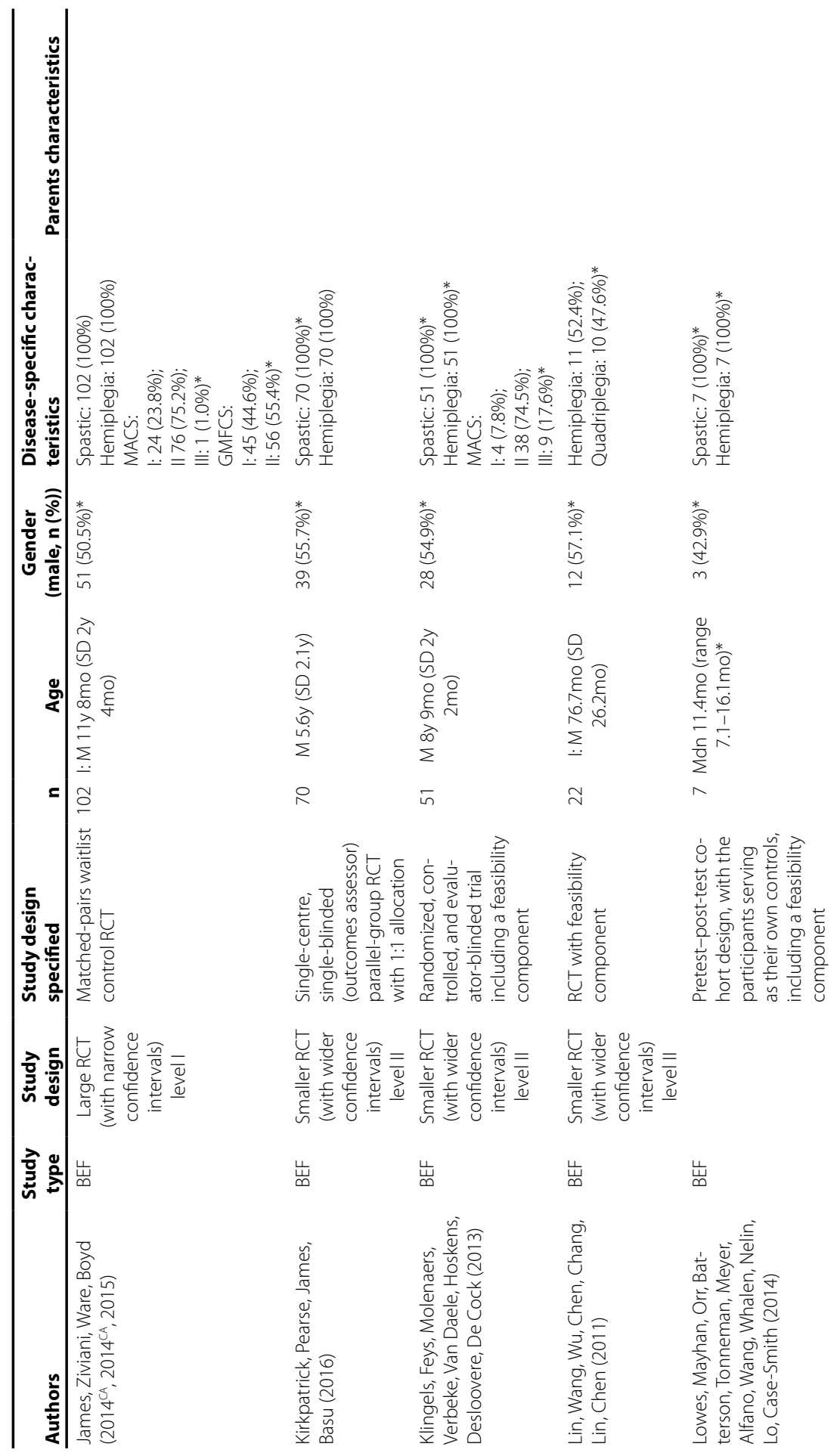




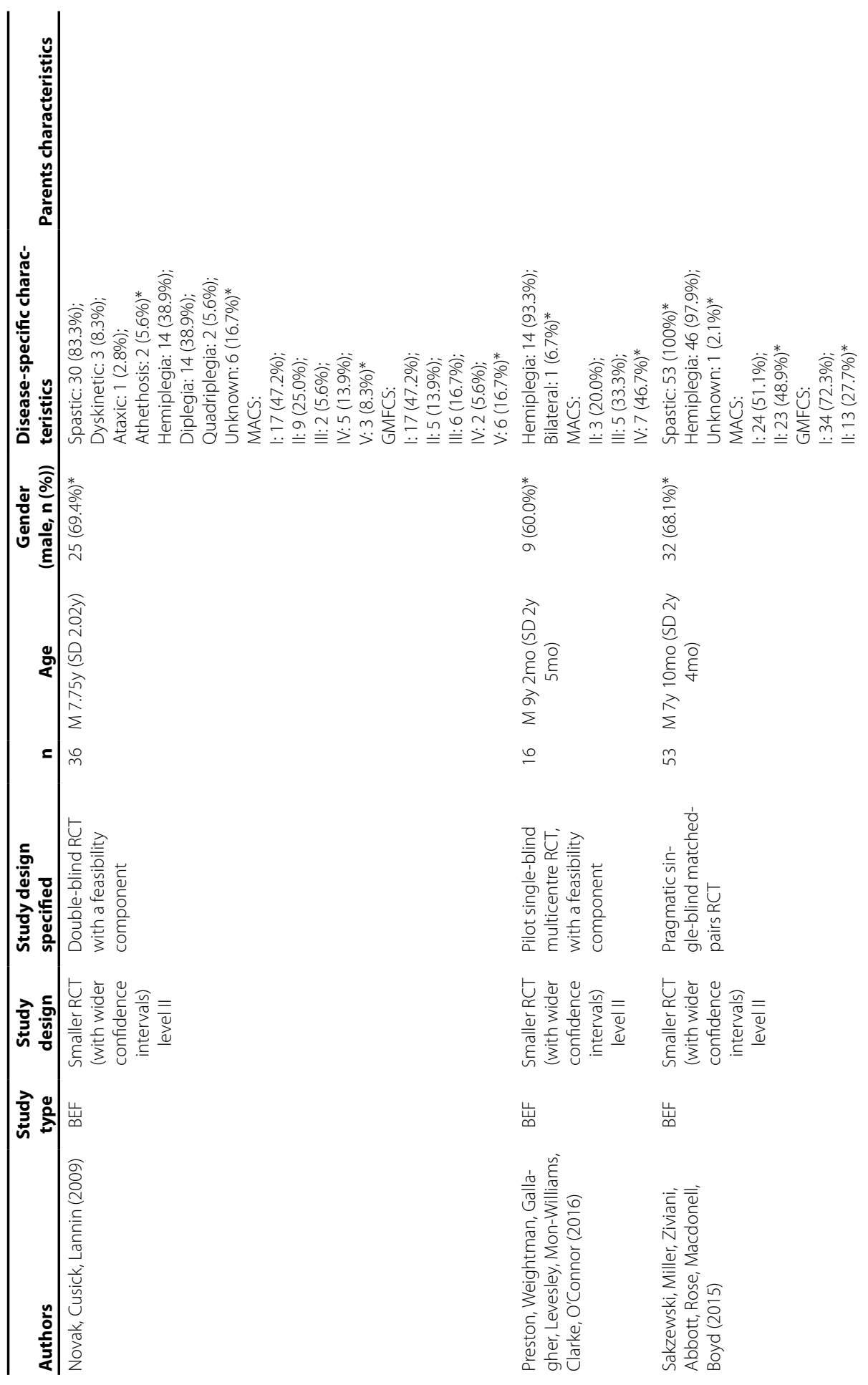




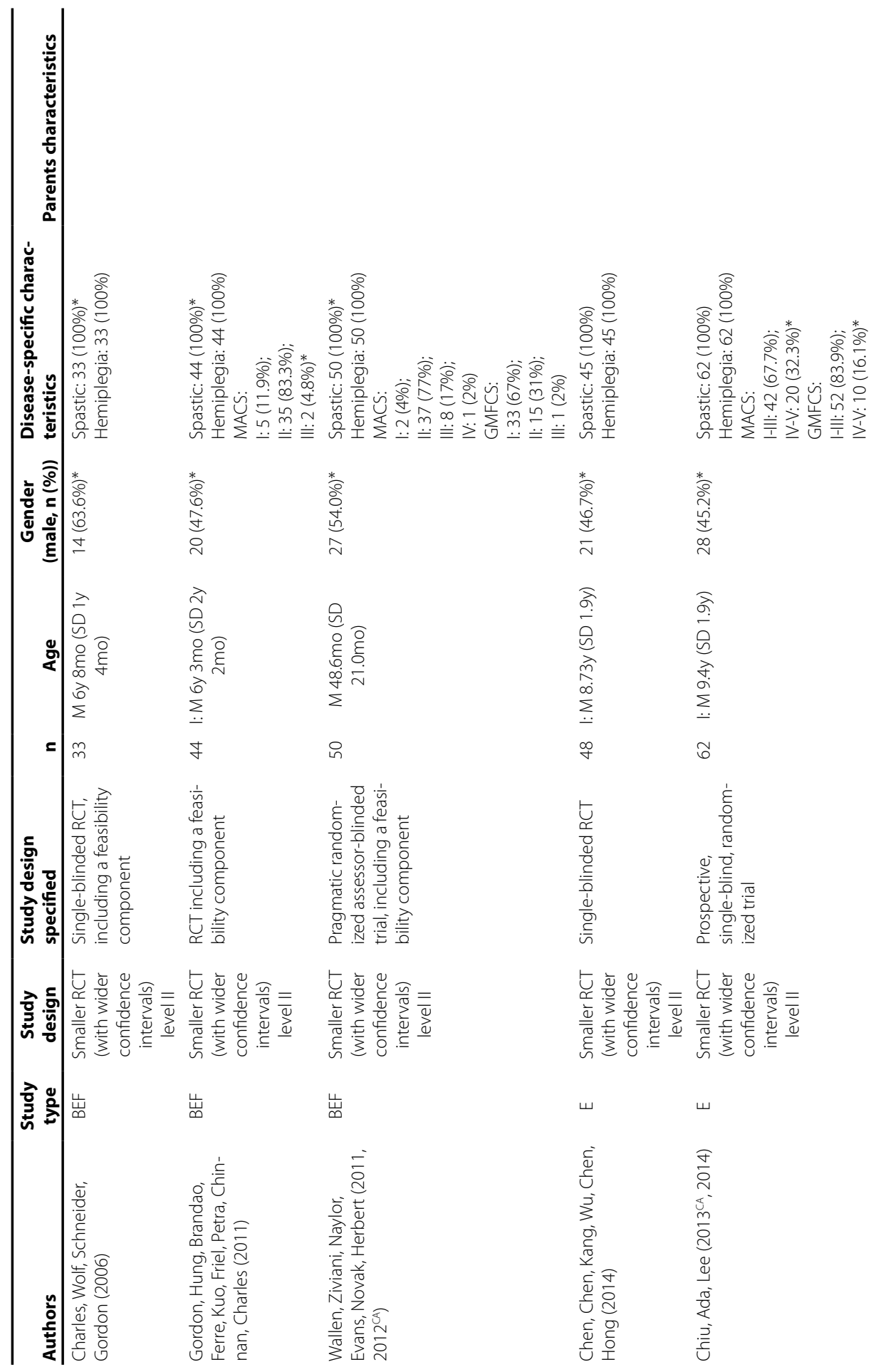




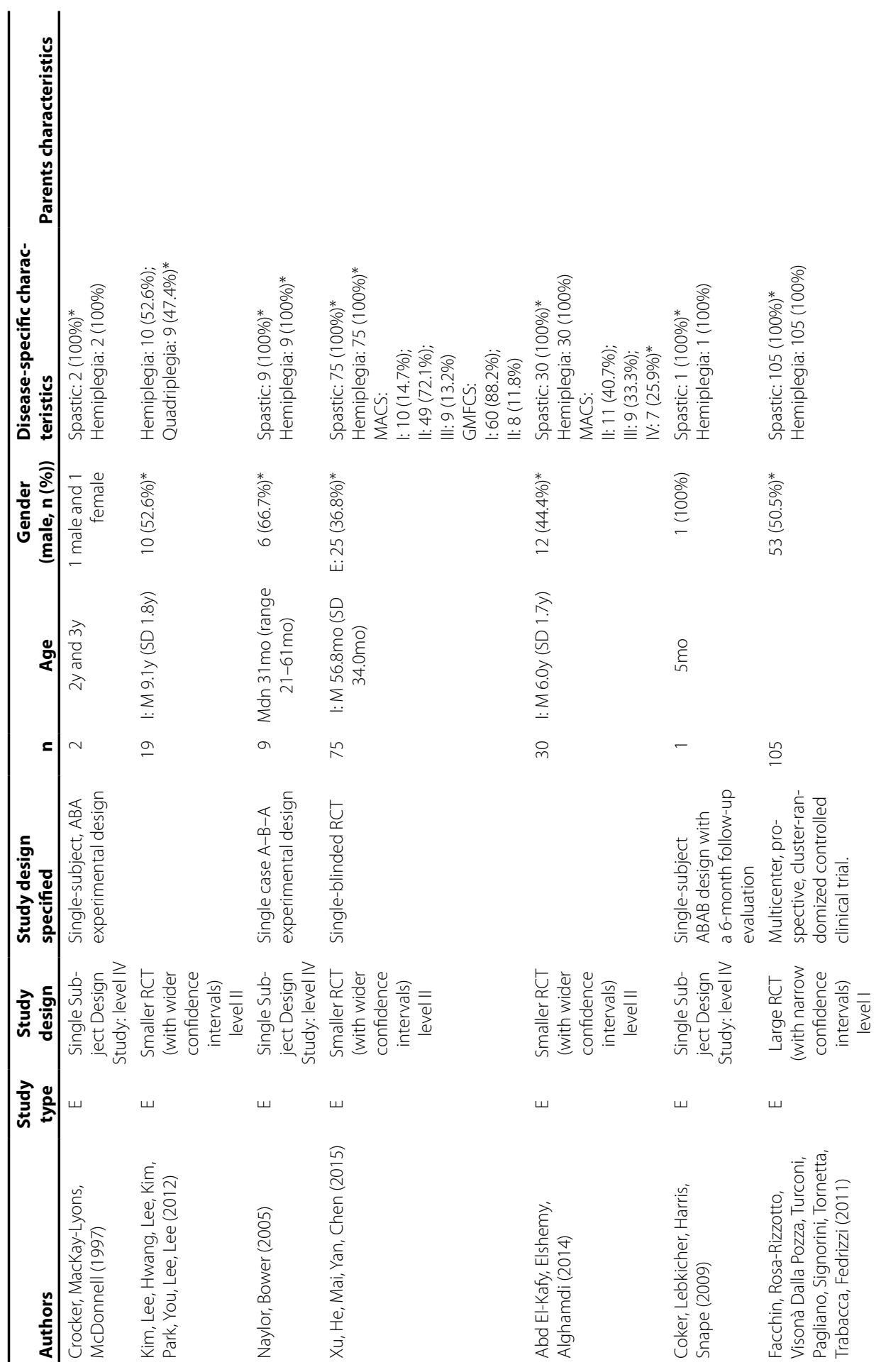




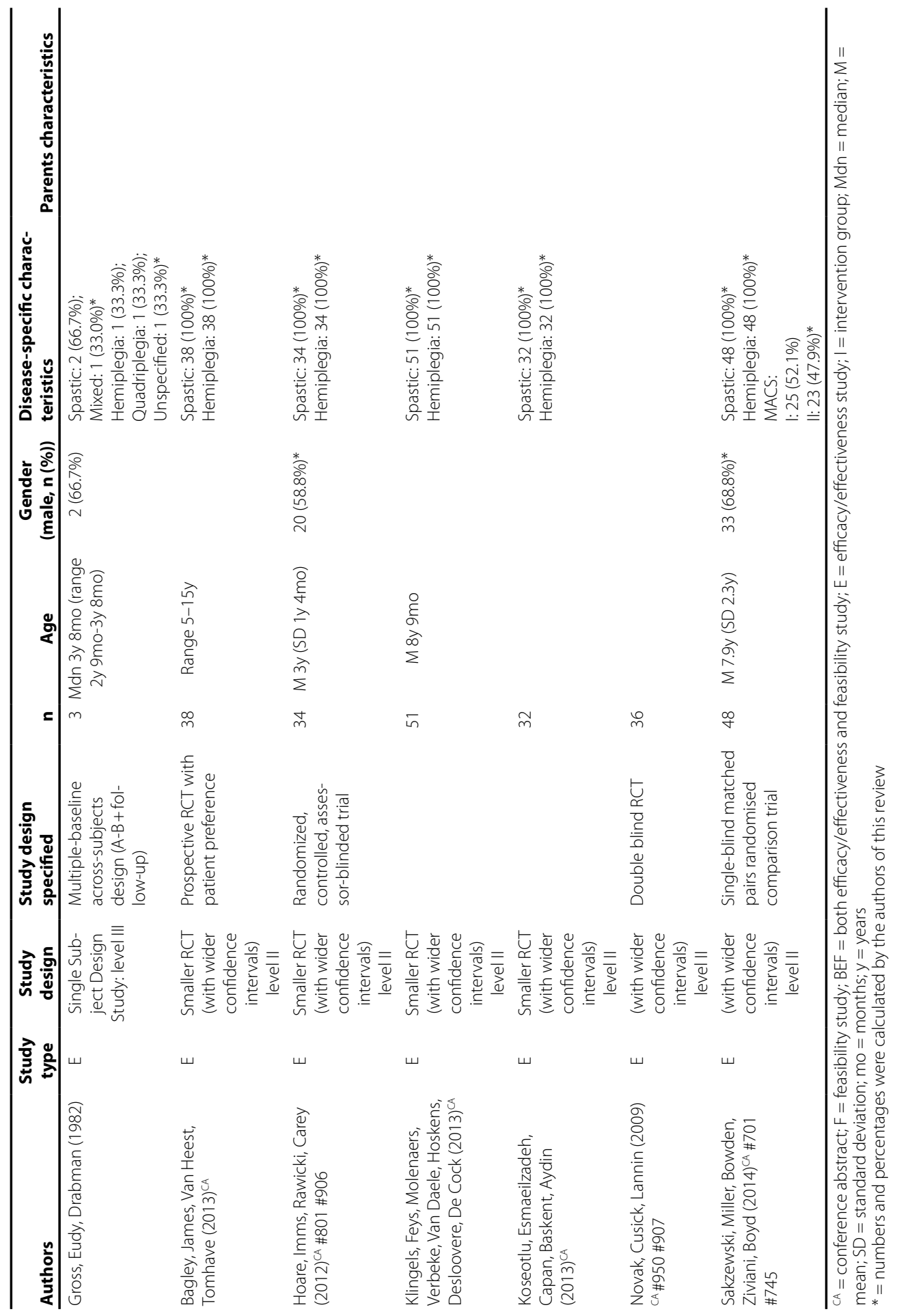




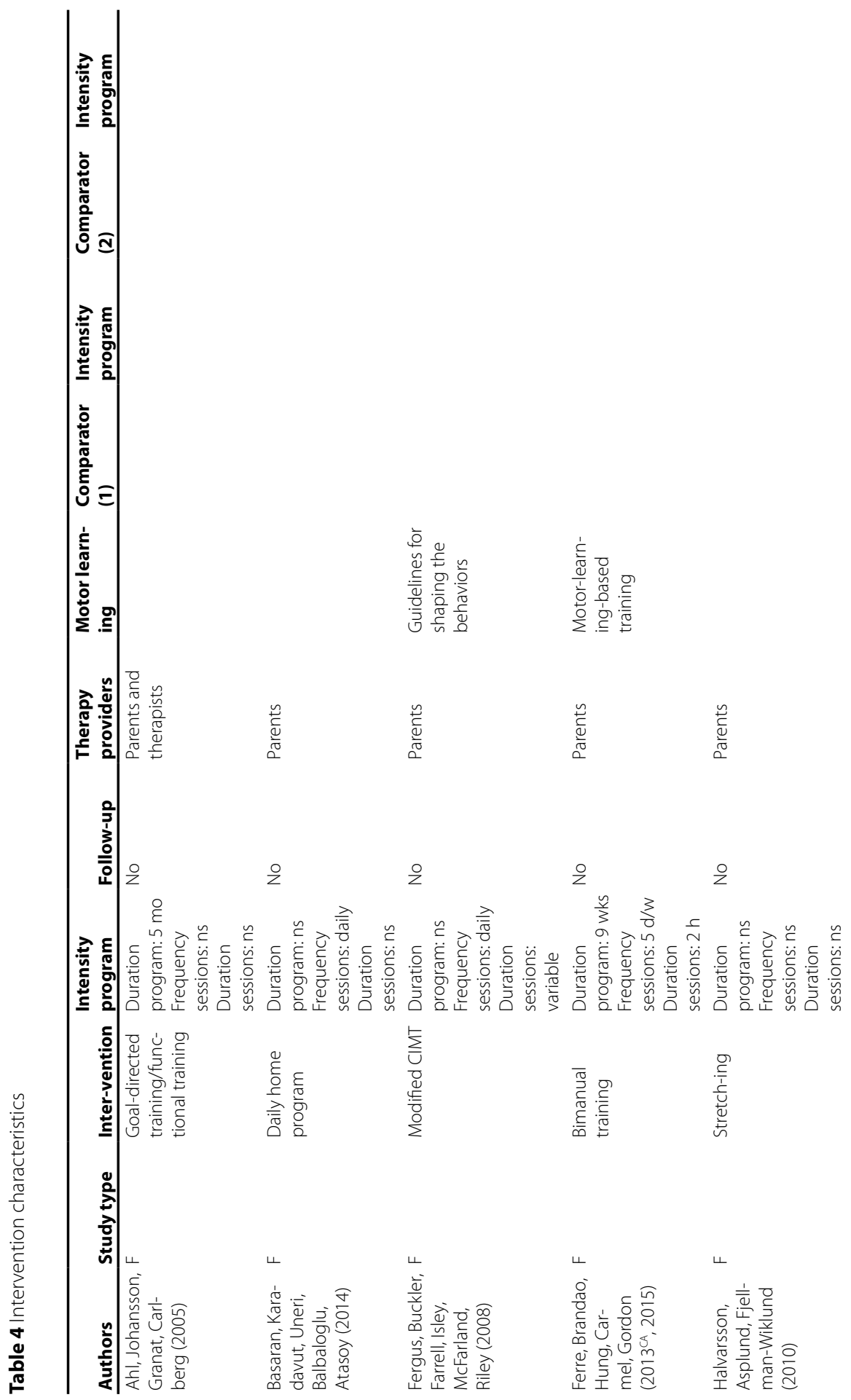




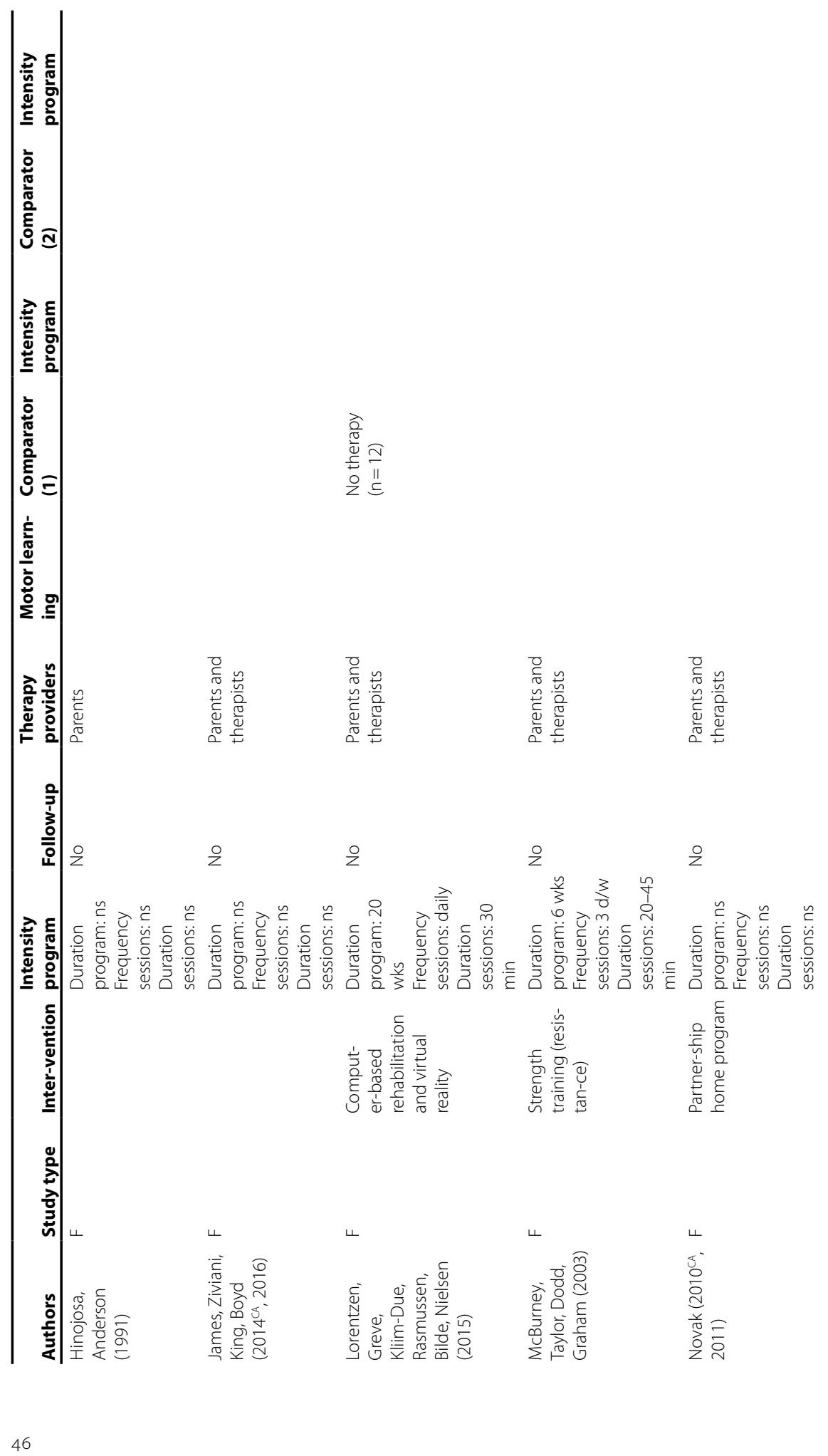




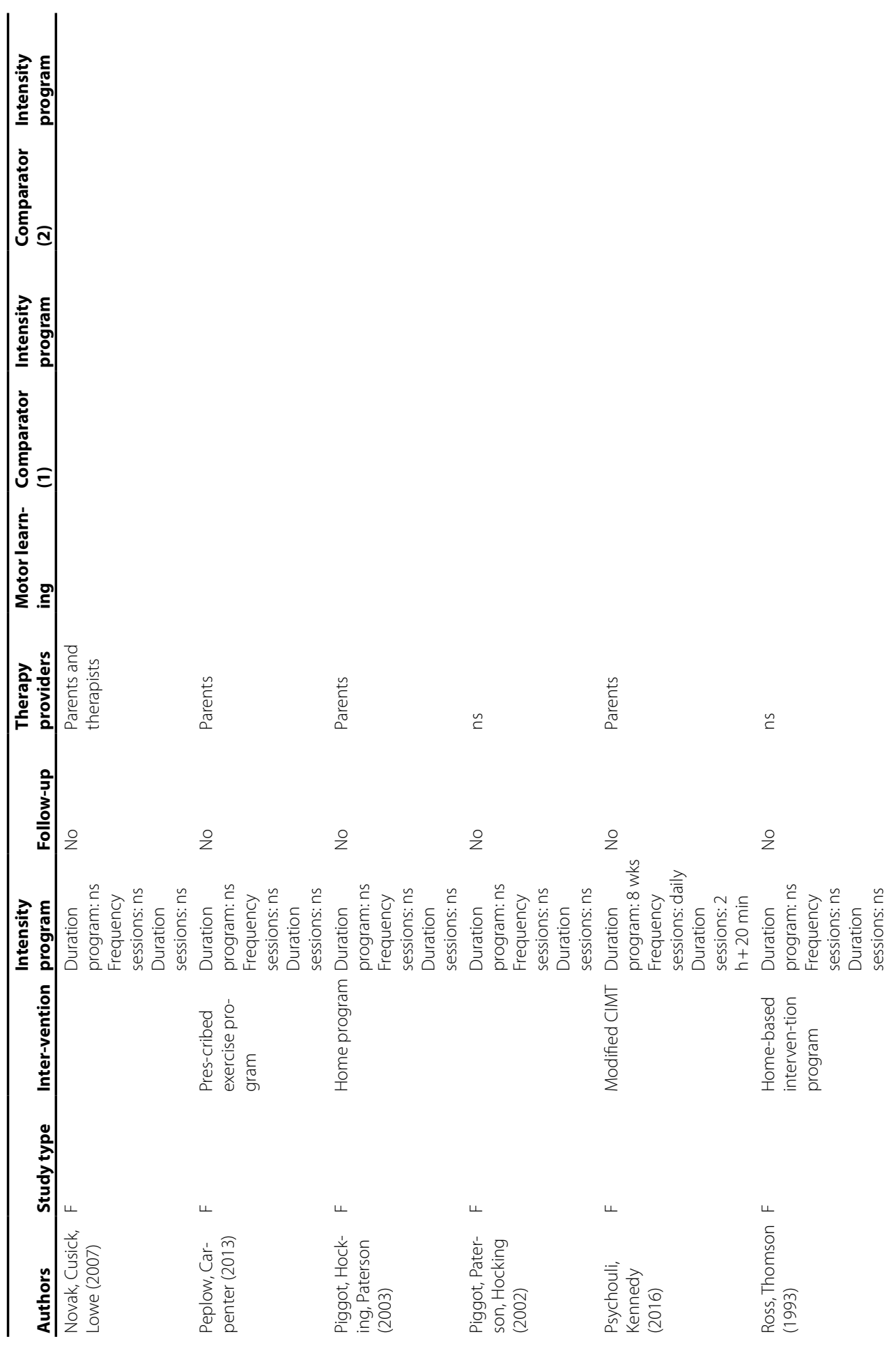




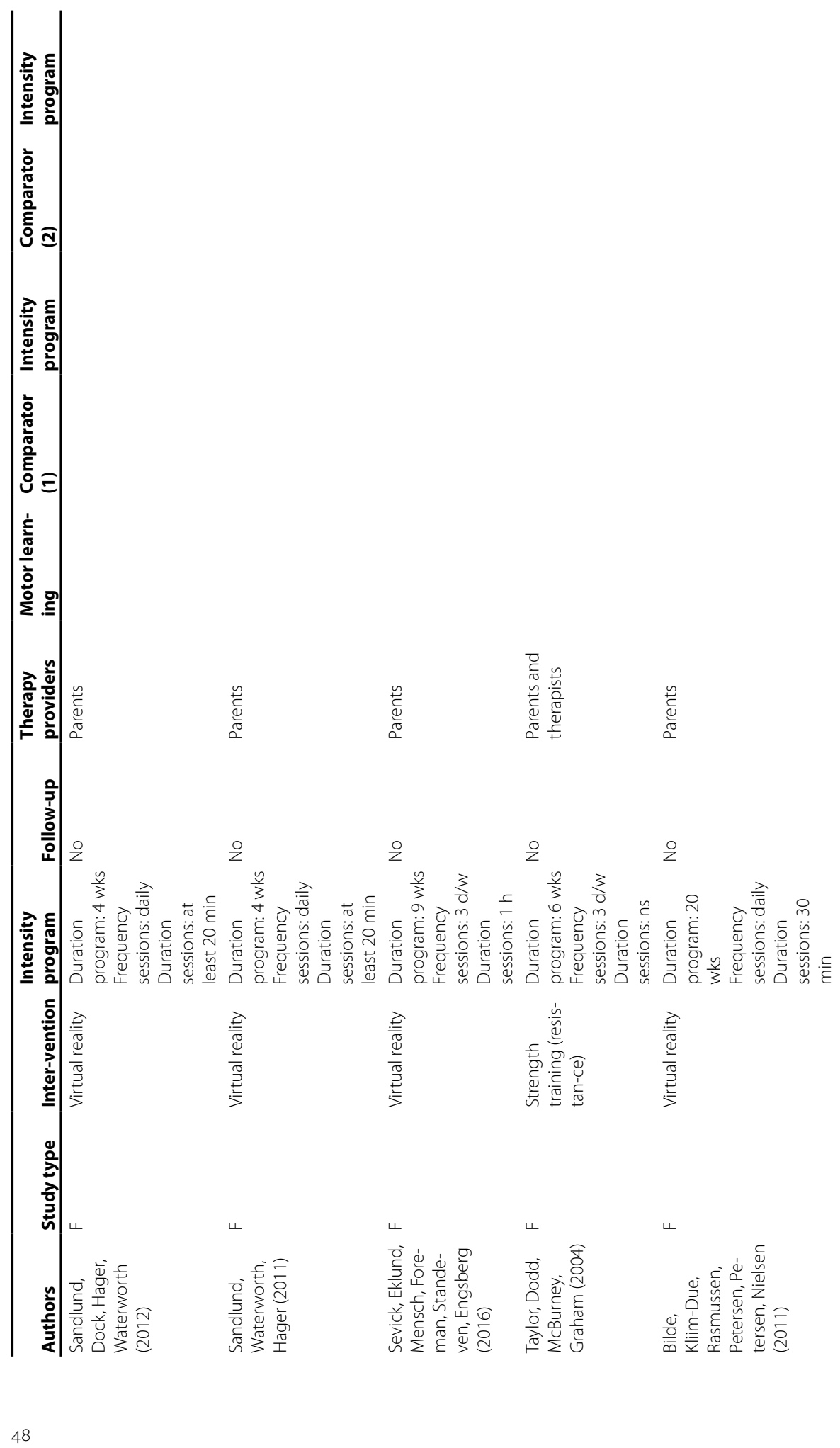




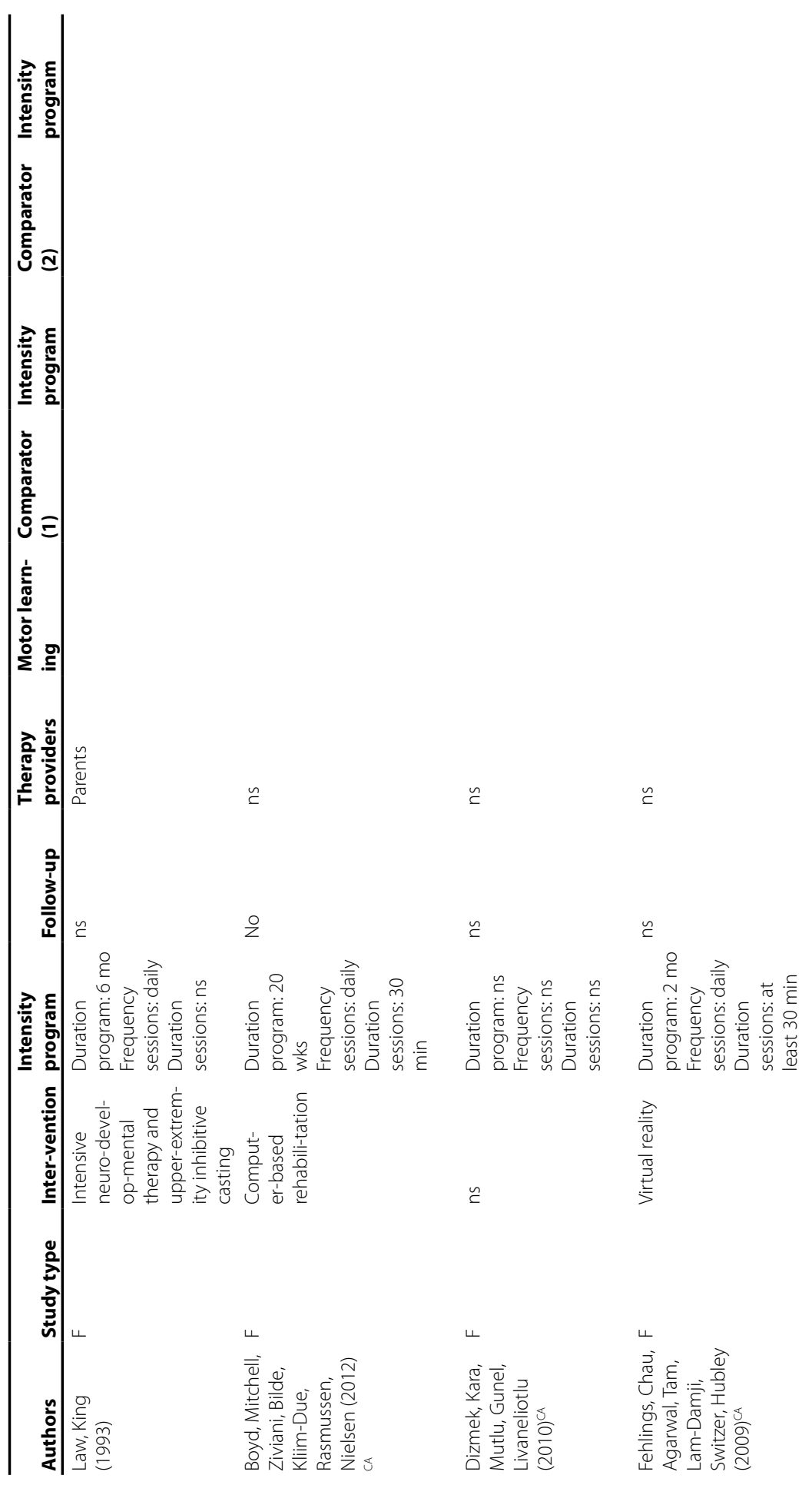



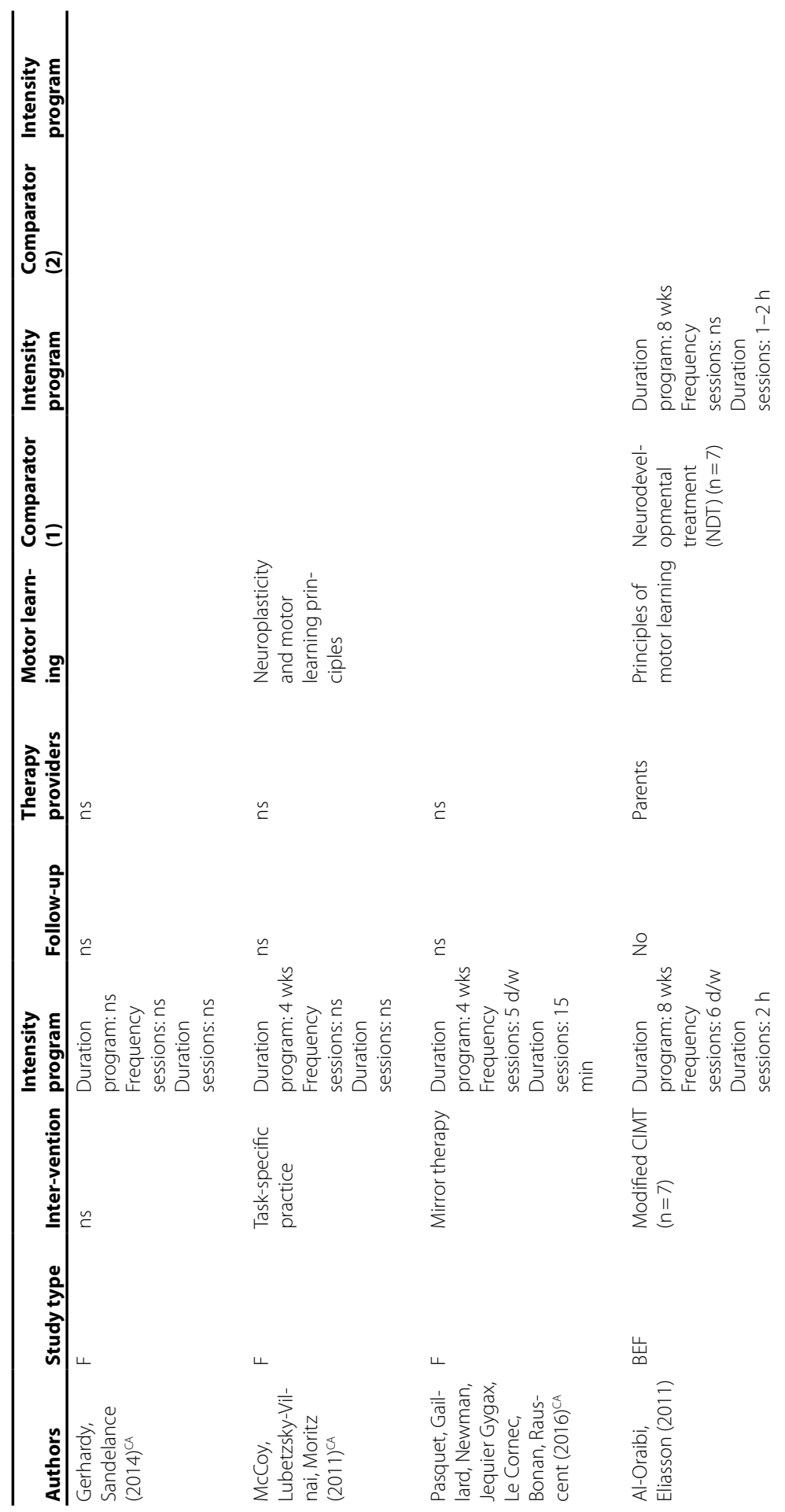

$\check{\complement}$

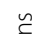

ํำ

竞

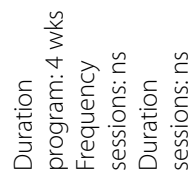

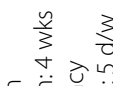

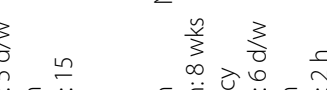

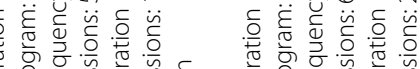
ด过吕领
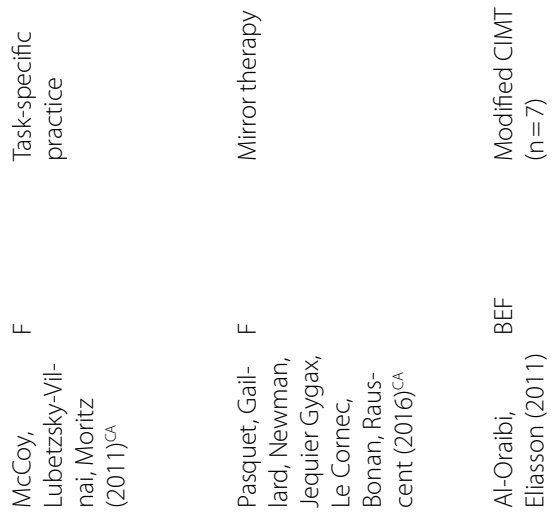


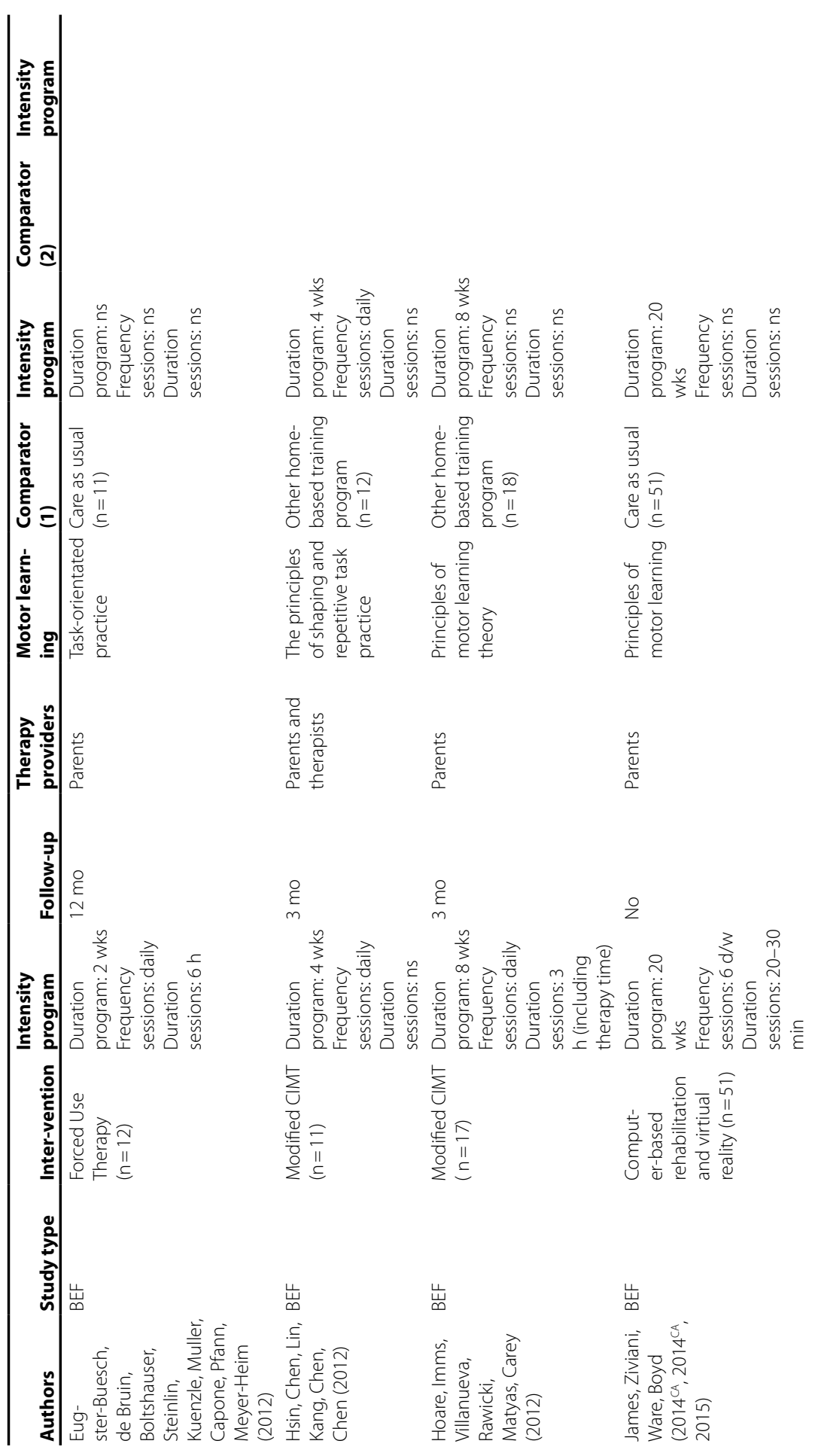




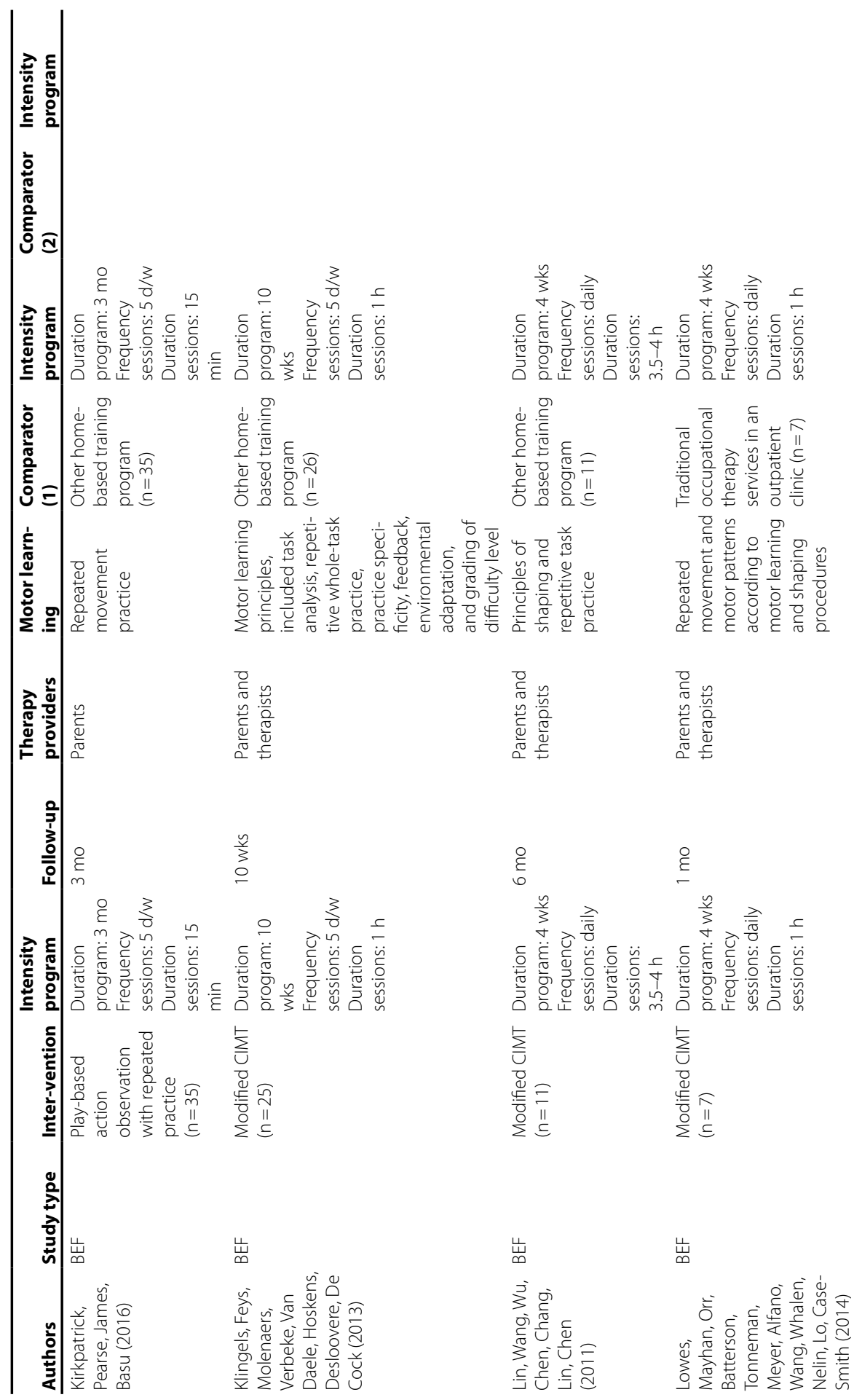




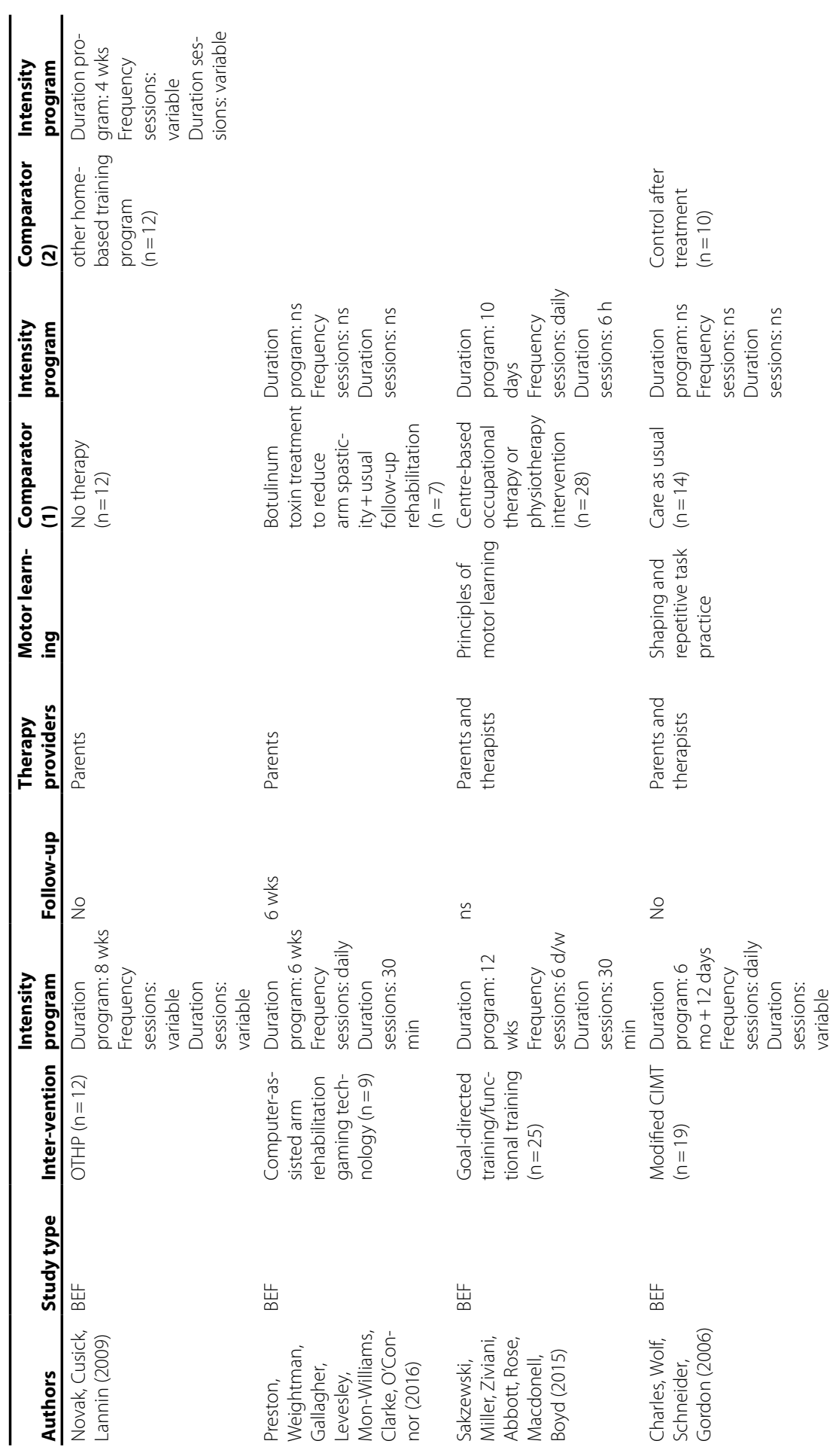




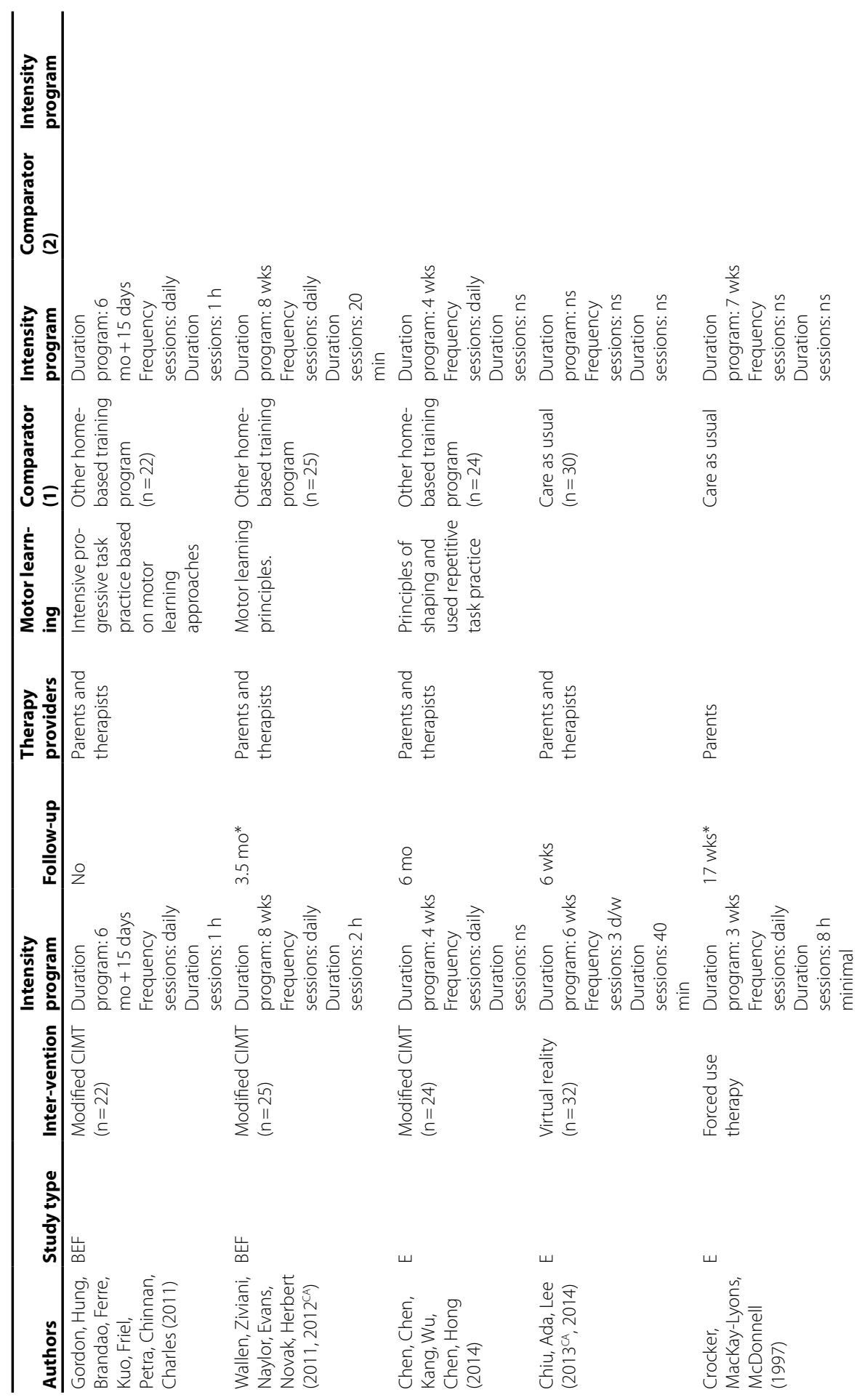




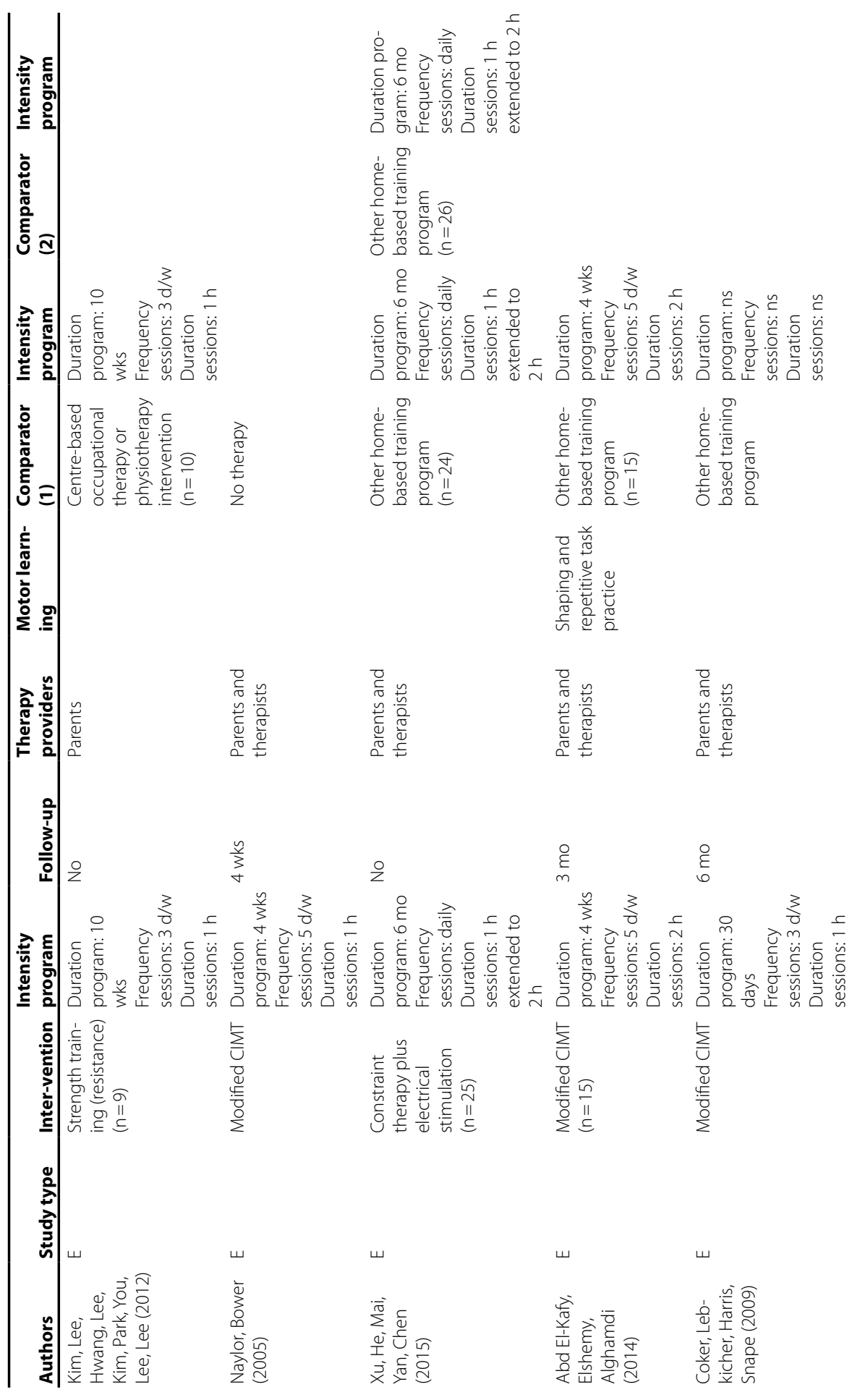




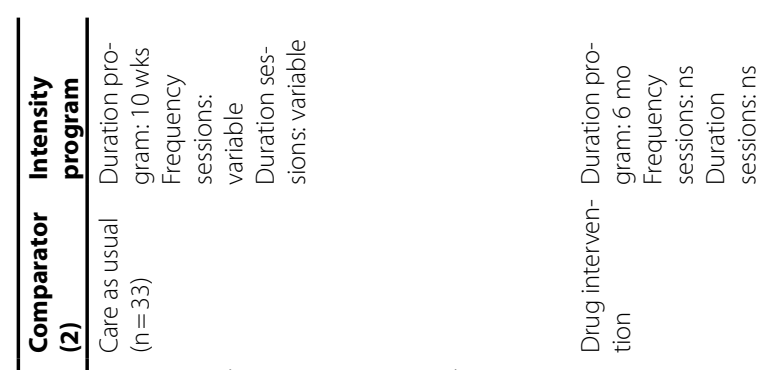

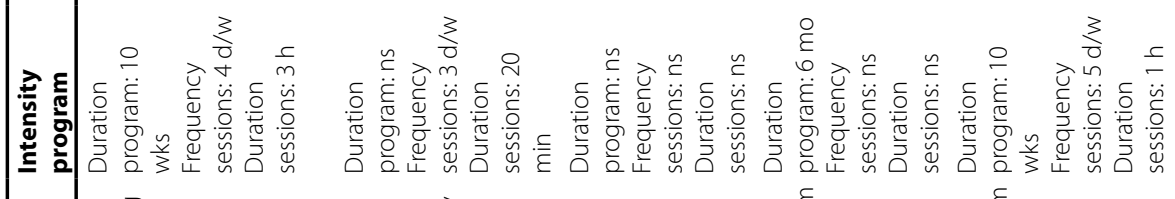
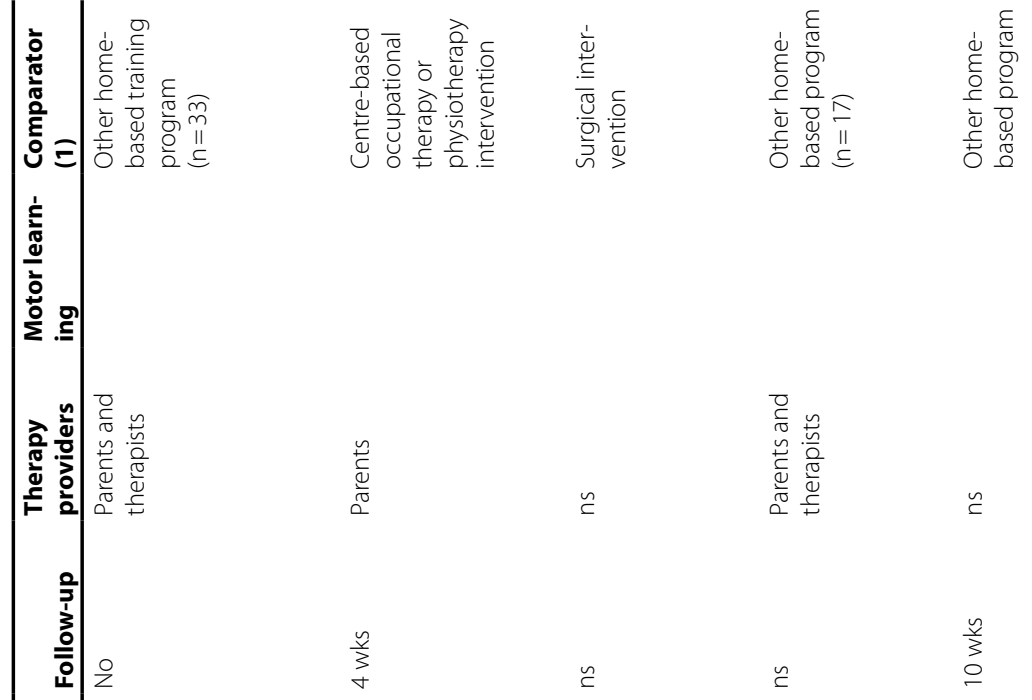

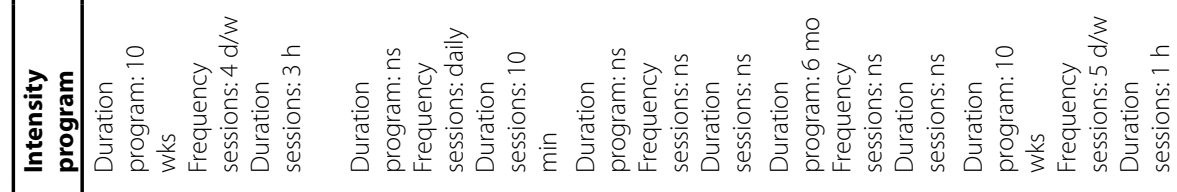

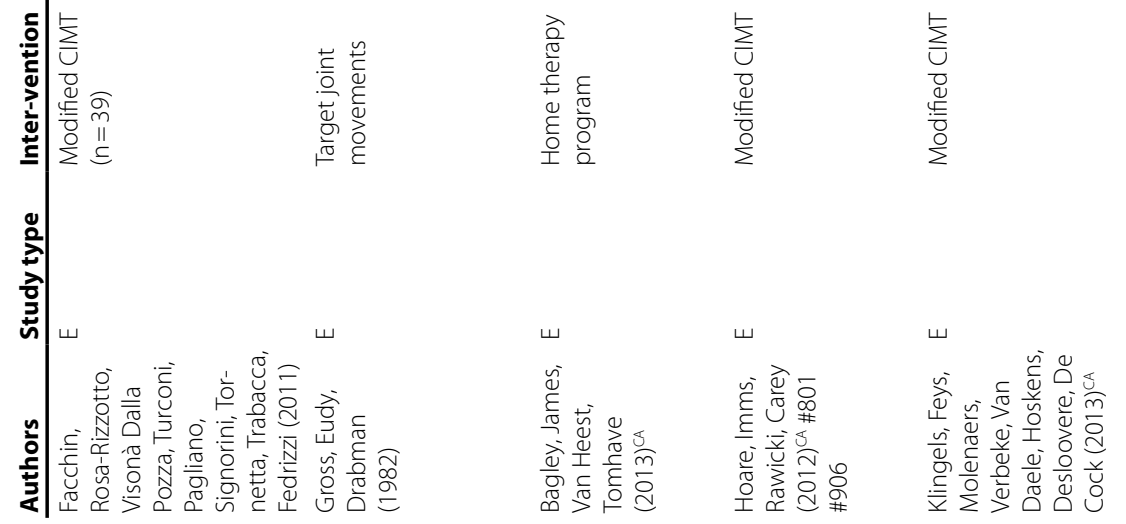




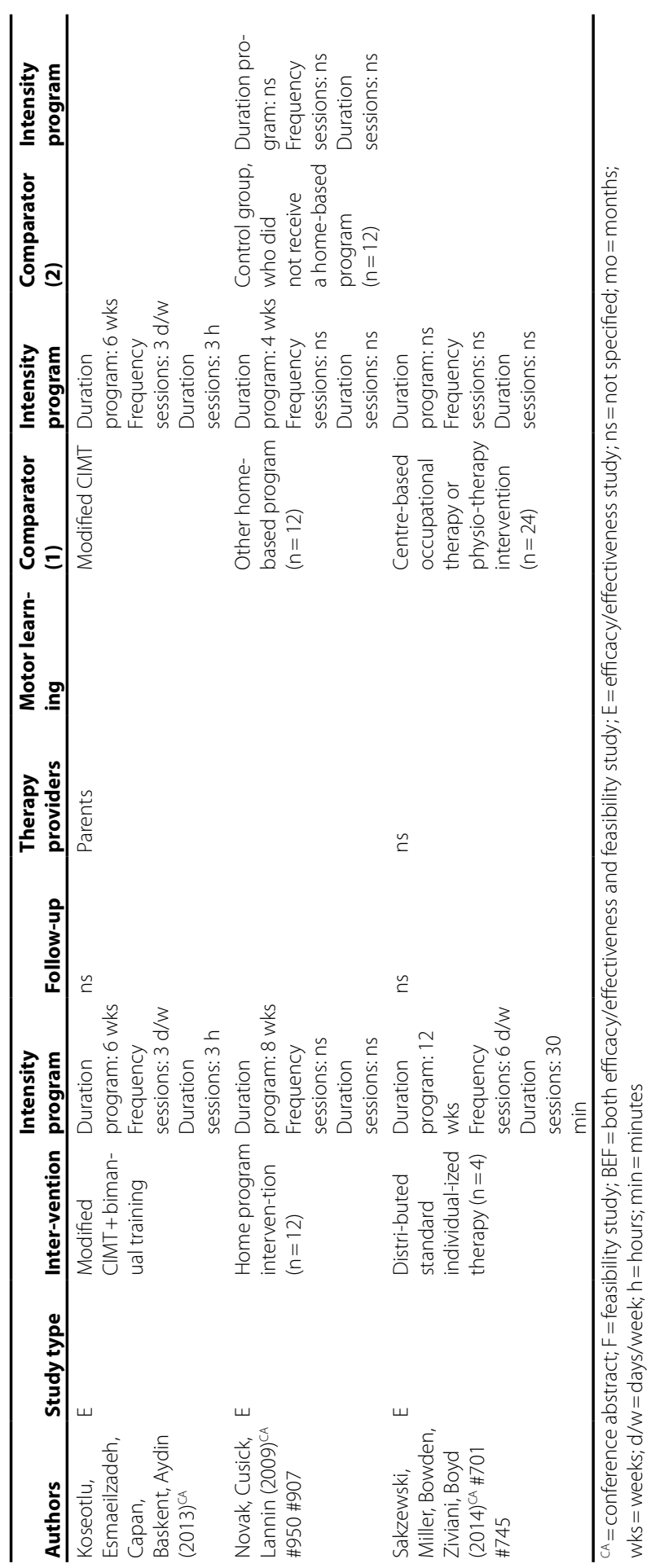




\section{Outcomes}

Feasibility studies only reported on the key areas acceptability and implementation. The overall compliance to home-based training programs (implementation) was moderate to high, ranging from $62 \%$ to $96.1 \%{ }^{15242630314041}$ The majority of the studies reported that parents found it easy to carry out the program and enjoyed seeing their children improve (acceptability).

In the effectiveness studies, more than 40 different child-related outcome measures were found. Child-related outcome measures on ICF-CY activity level were considered to be primary outcome measures in this review. Eleven different primary outcome measures were found, i.e. the Quality of Upper extremity Skills Test (QUEST 17x), Assisting Hand Assessment (AHA 12x), Canadian Occupational Performance Measure (COPM 10x), Melbourne Assessment of Unilateral Upper Limb Function (Melbourne 7x), Goal Attainment Scaling (GAS 4x), pediatric Motor Activity Log (pMAL 4x), Abilhand-kids (4x), video observation (3x), Shriners Hospital for Children Upper Extremity Evaluation (SHUEE 1x), Assessment of Motor and Process Skills (AMPS 1x) and the Children's Hand-use Experience Questionnaire (CHEQ 1X). The vast majority of these outcome measures showed an improvement in arm hand performance within-group, across time, i.e. before and after intervention. However, in case of effectiveness, this improvement (within-group) was not always sufficient to identify a difference between the interventions investigated (between-groups). Except for the study of Hsin et al. ${ }^{44}$ and Novak et al. ${ }^{51}$, who reported on the results of the Cerebral Palsy-specific Quality of Life (parent-proxy version) and Children's Assessment of Participation and Enjoyment (CAPE) respectively, none of the studies included outcome measures on ICF participation level. Both studies report gains in health-related quality of life. All other outcome measures were on ICF-CY function level. Again, the majority of the studies showed a positive change in hand function, within-group, before and after intervention, but a difference in effectiveness between interventions could not always be confirmed.

In contrast to the large amount of child-related outcome measures, only two studies ${ }^{26} 49$ reported on a parent-related outcome measure, i.e. the Parenting Stress Index-Short Form (PSISF). The study of Lin et al. ${ }^{49}$ as well as the study of Ferre et al. ${ }^{26}$ found no increase in parental stress during the intervention.

A detailed description of the results of the feasibility studies, efficacy/effectiveness studies and studies that reported on both feasibility and efficacy/effectiveness is given in table 5 to 7 . 


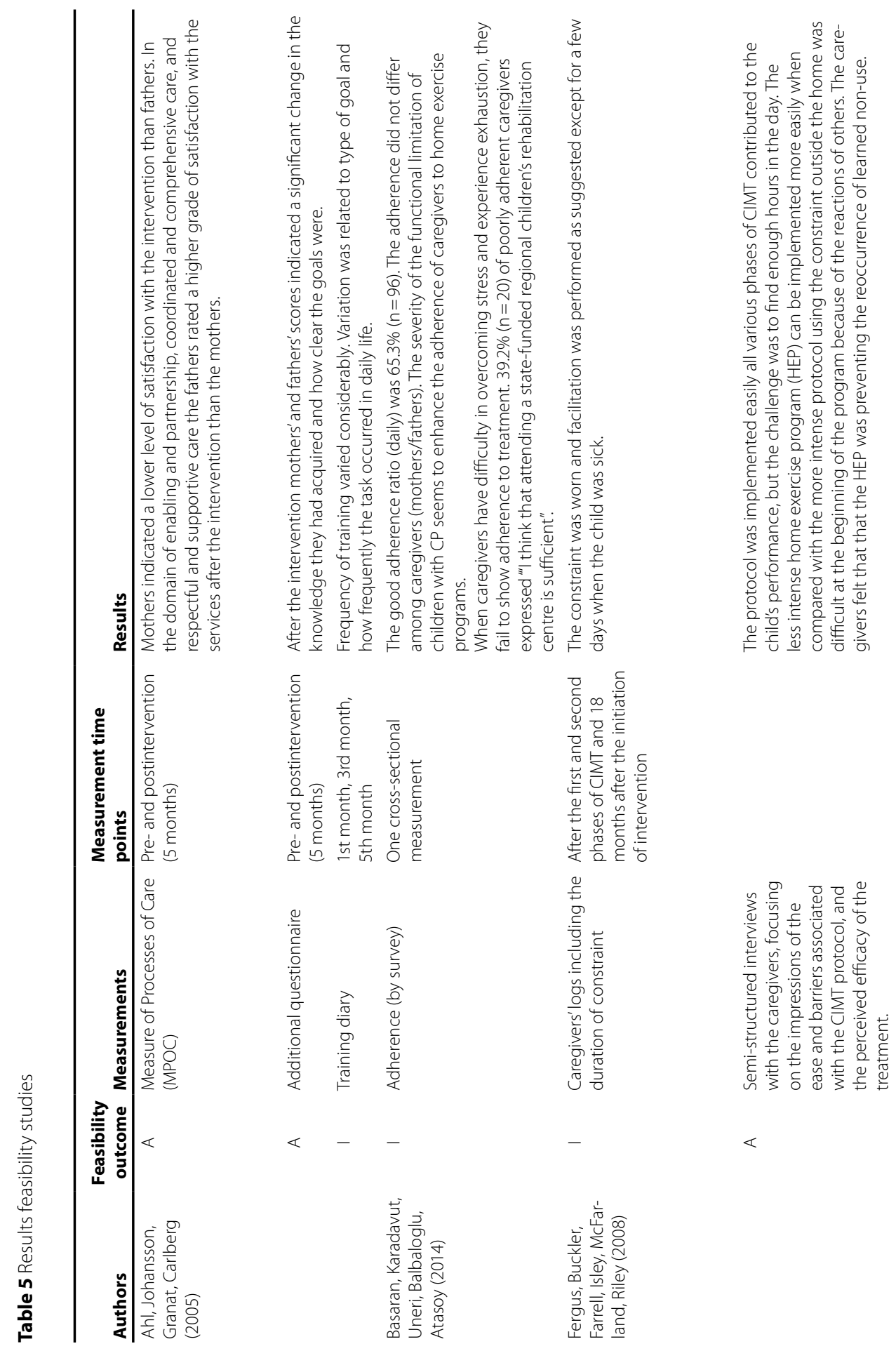




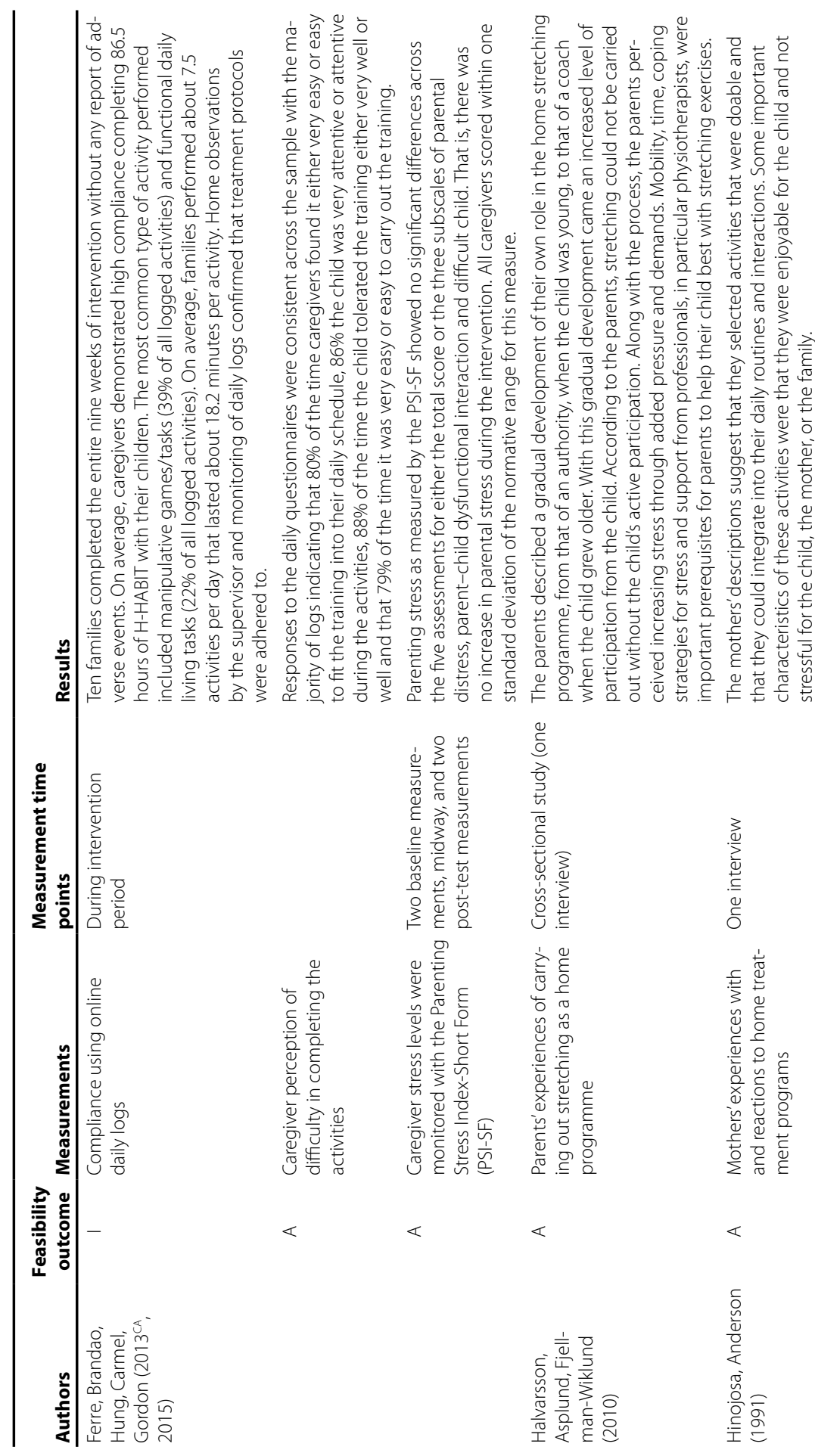




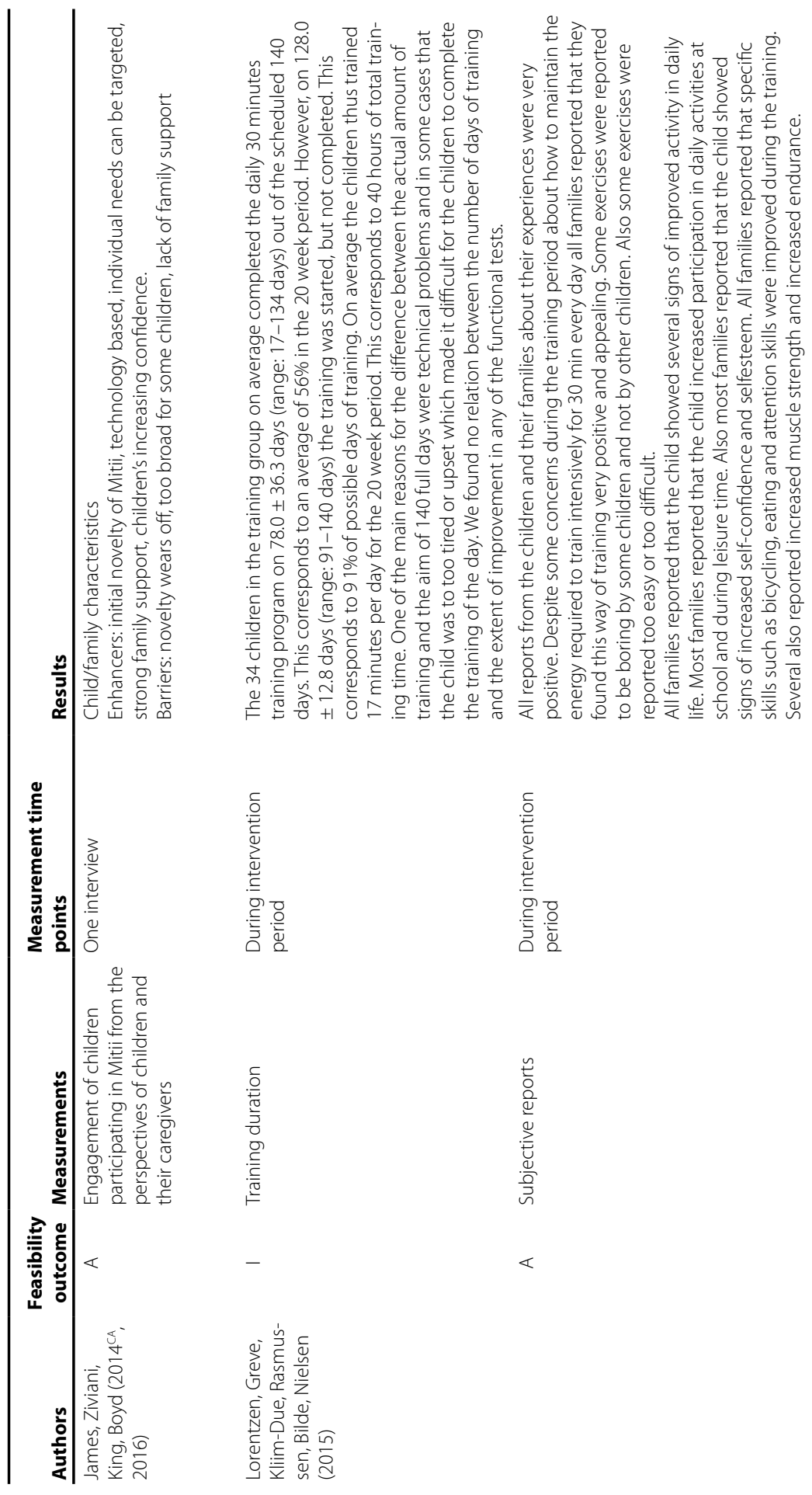




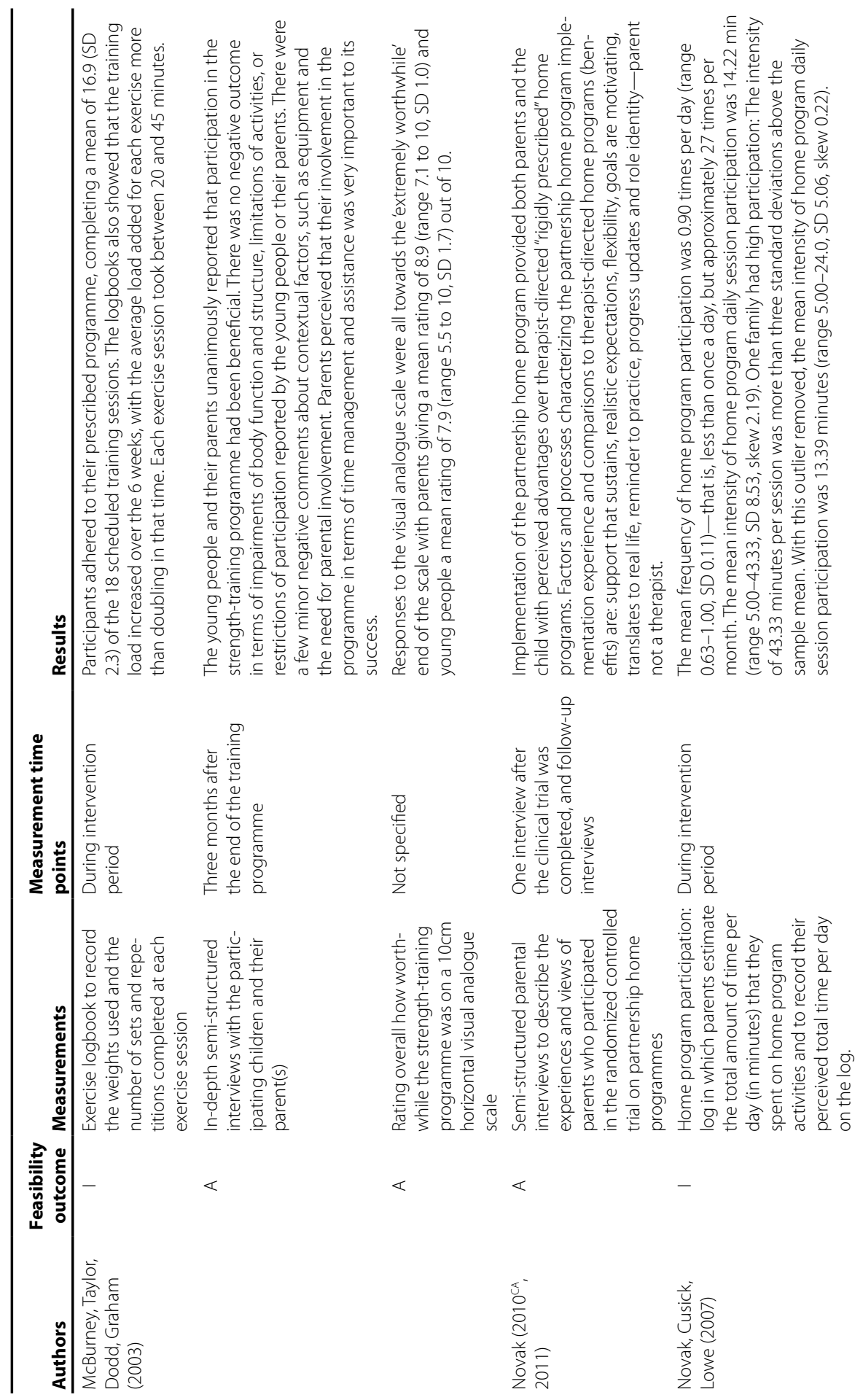




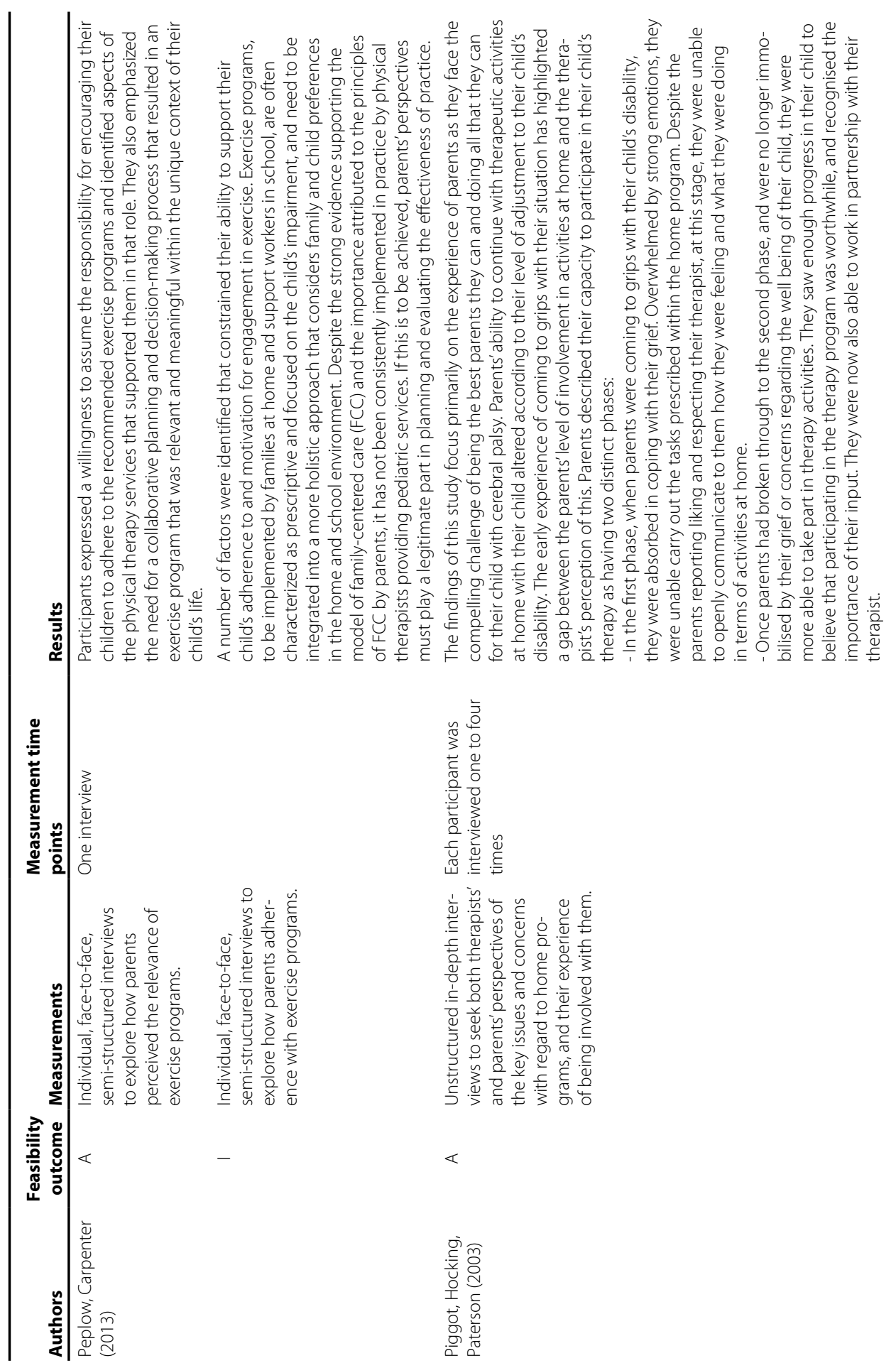




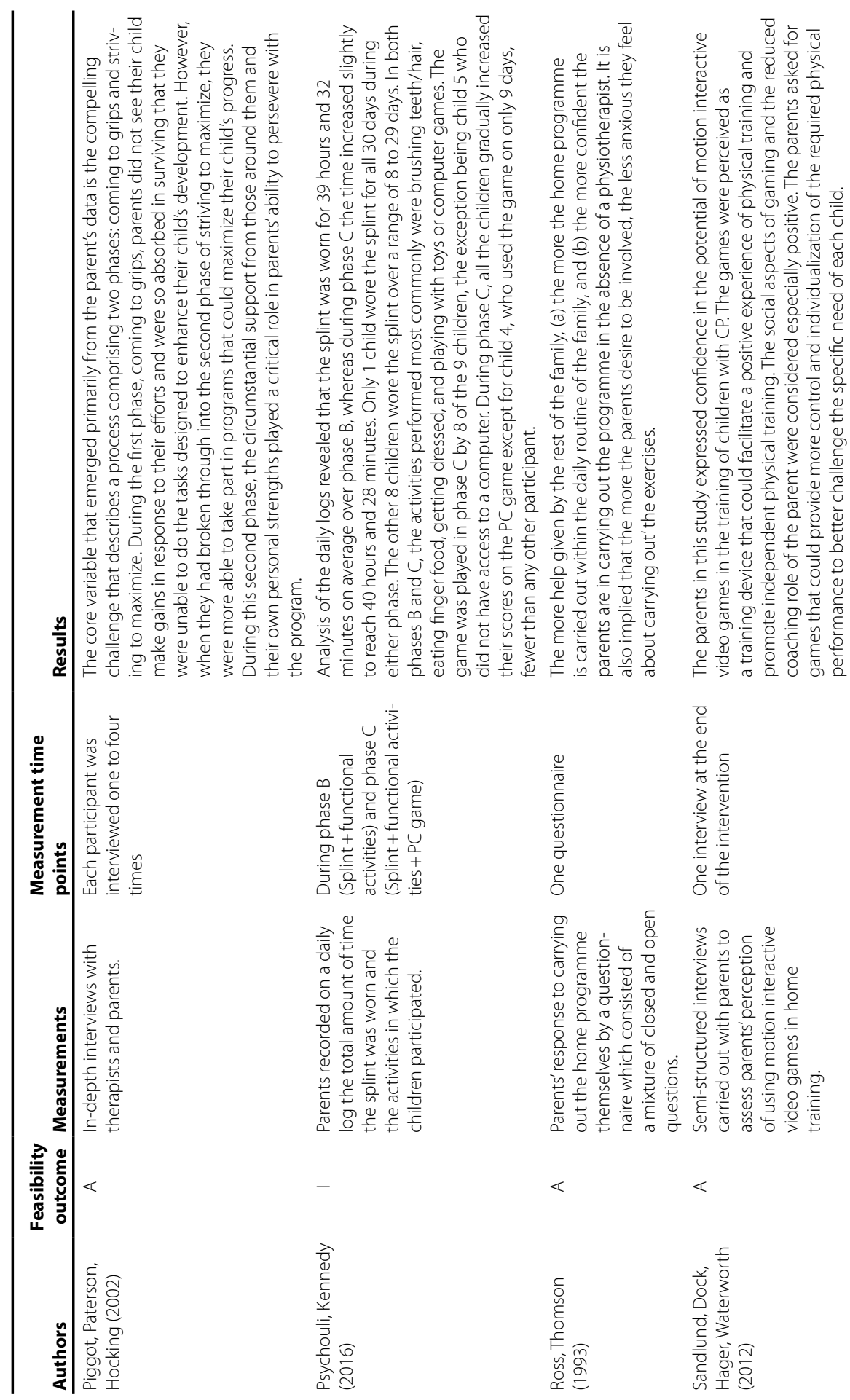




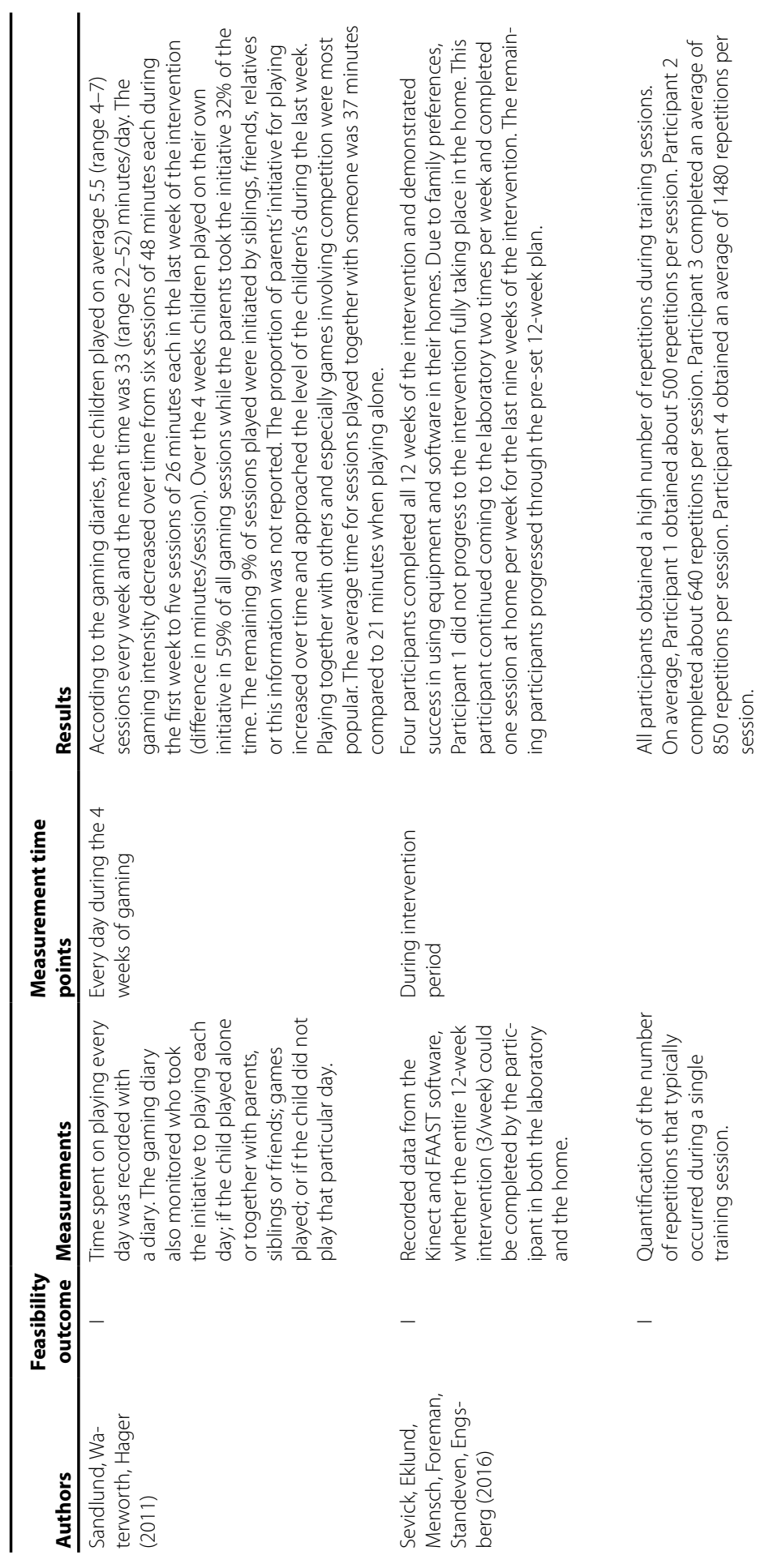




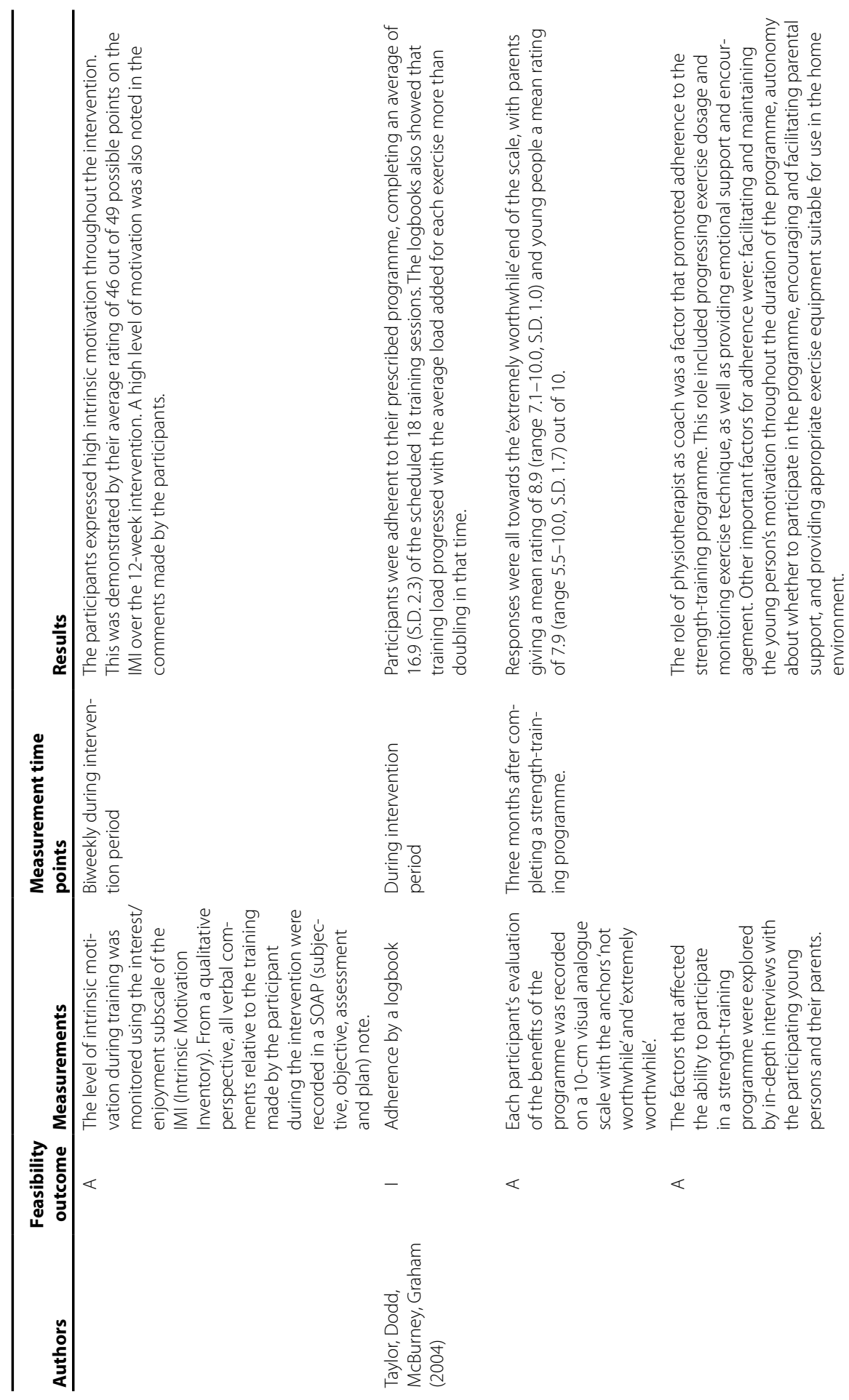



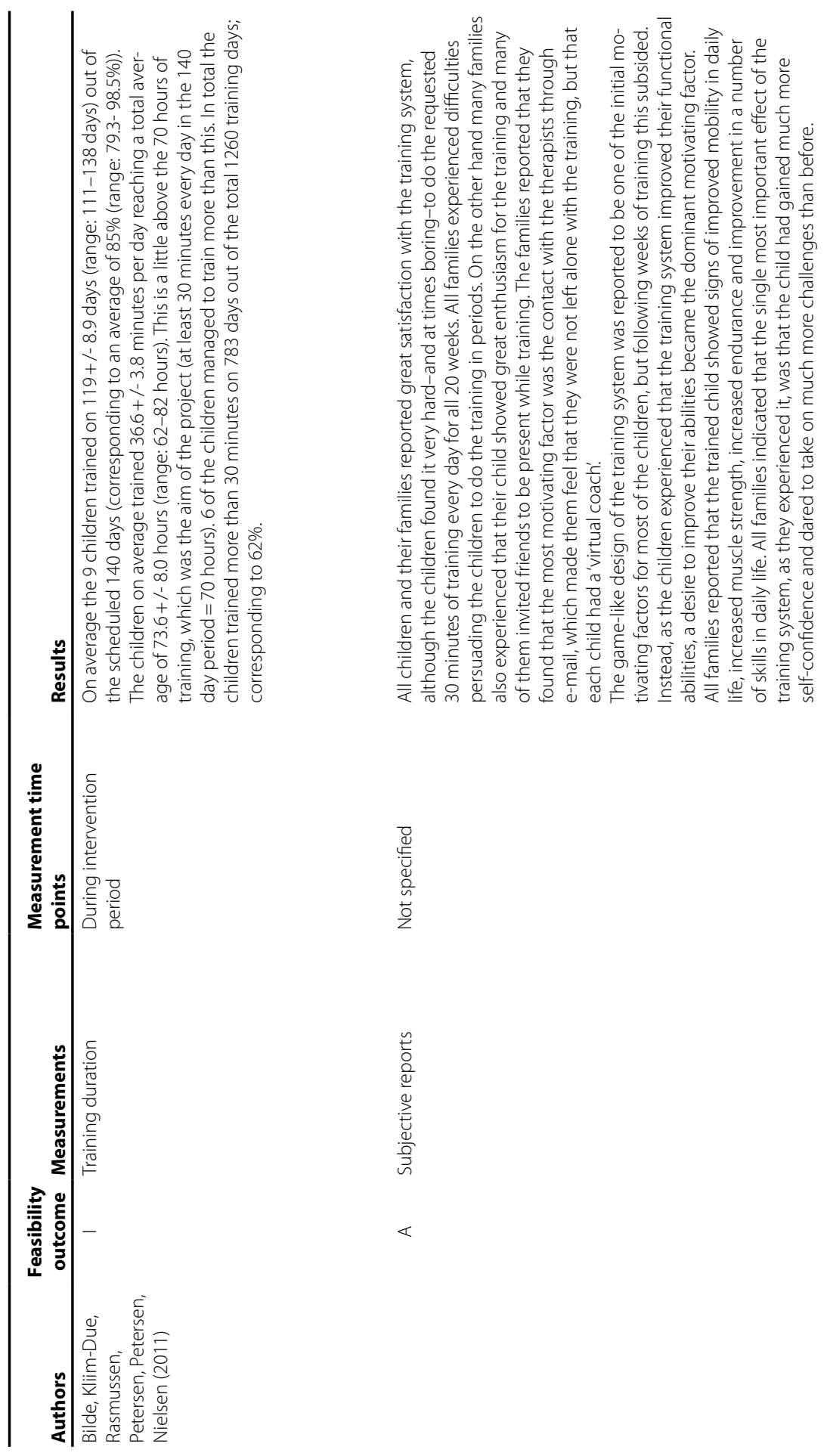

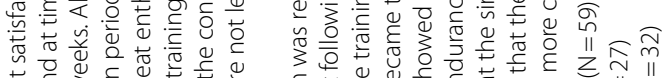

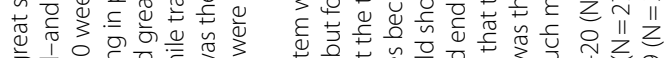

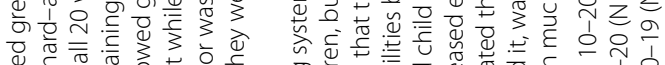

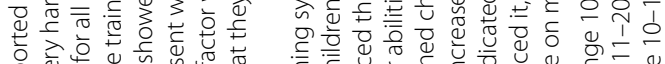

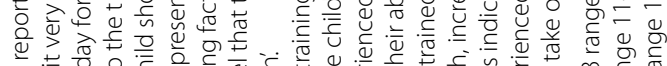

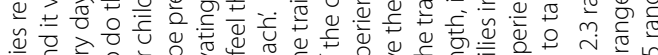

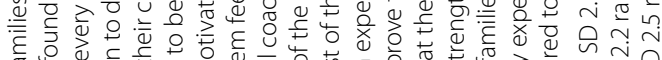

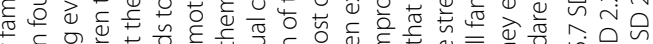

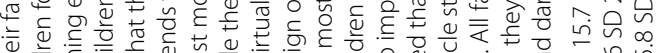

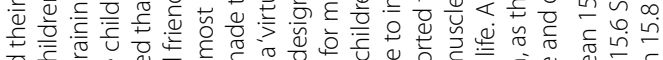

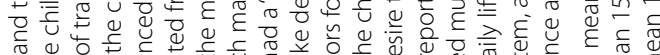

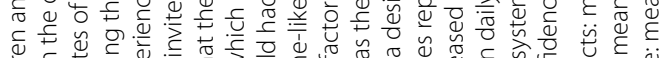

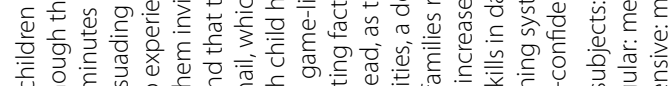

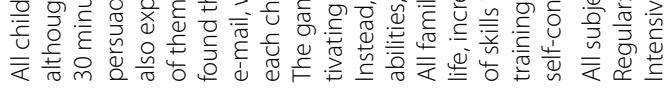

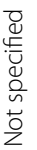

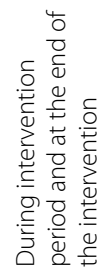

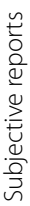

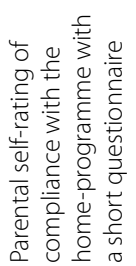

$\varangle$

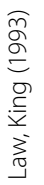




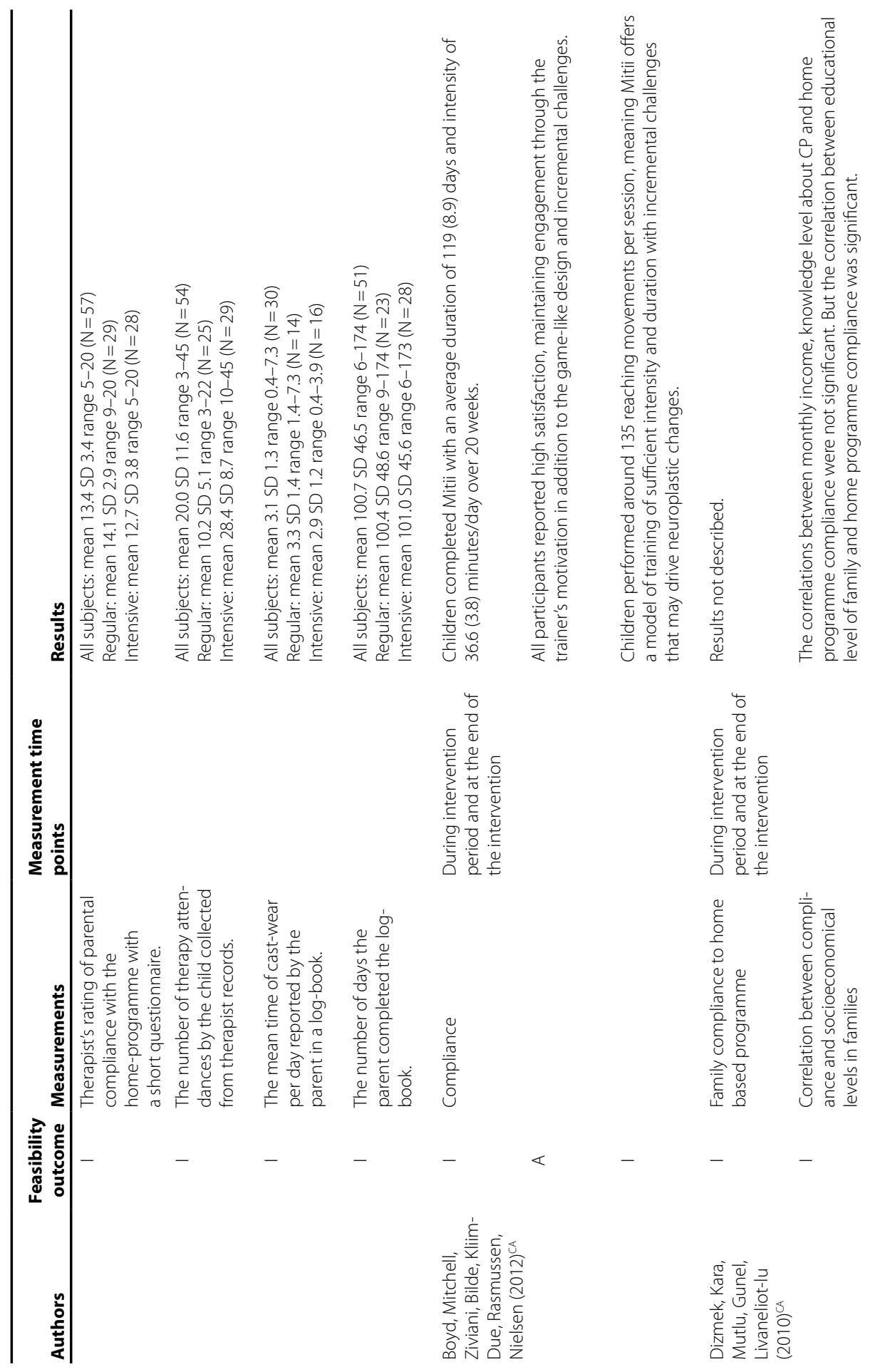




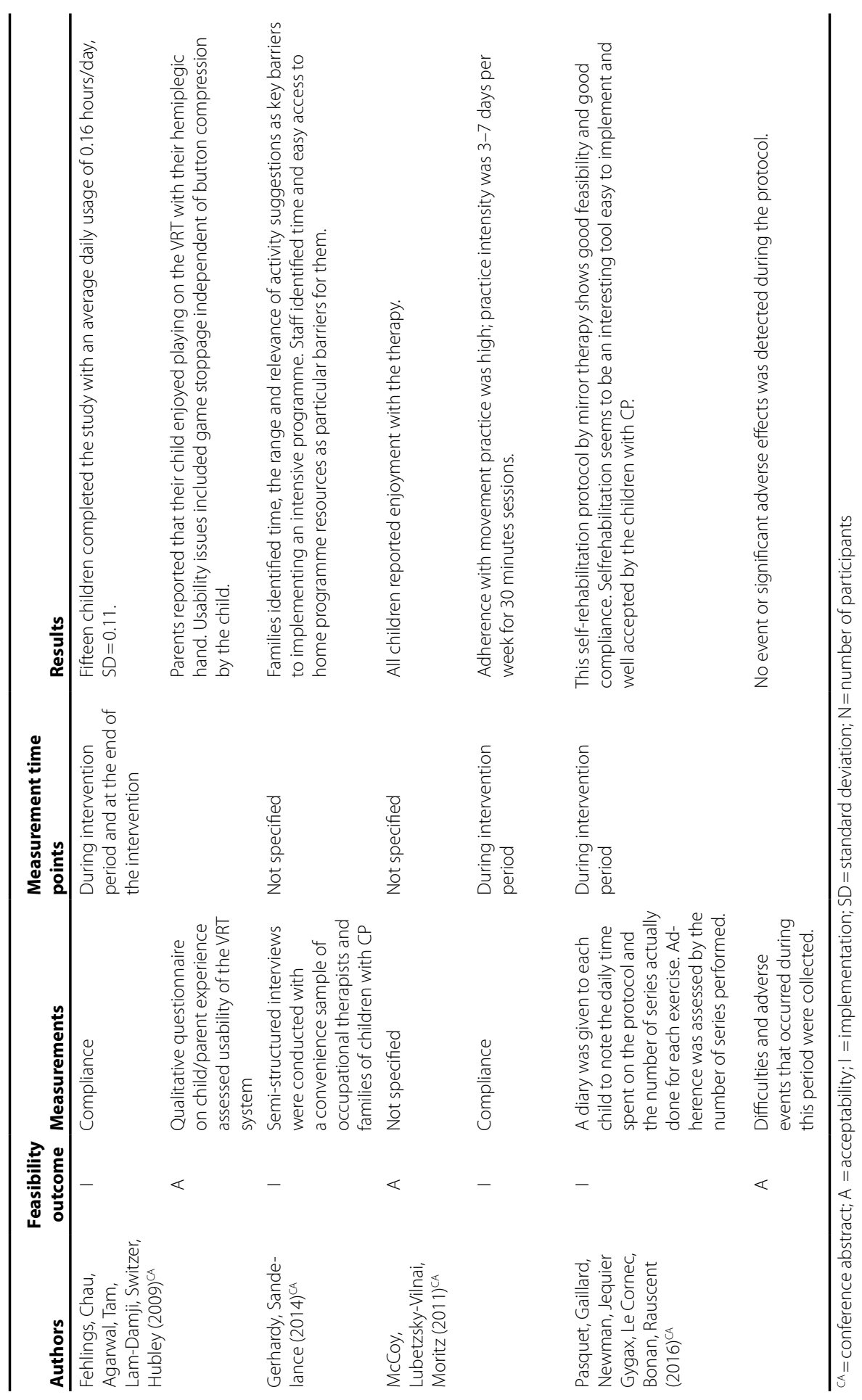




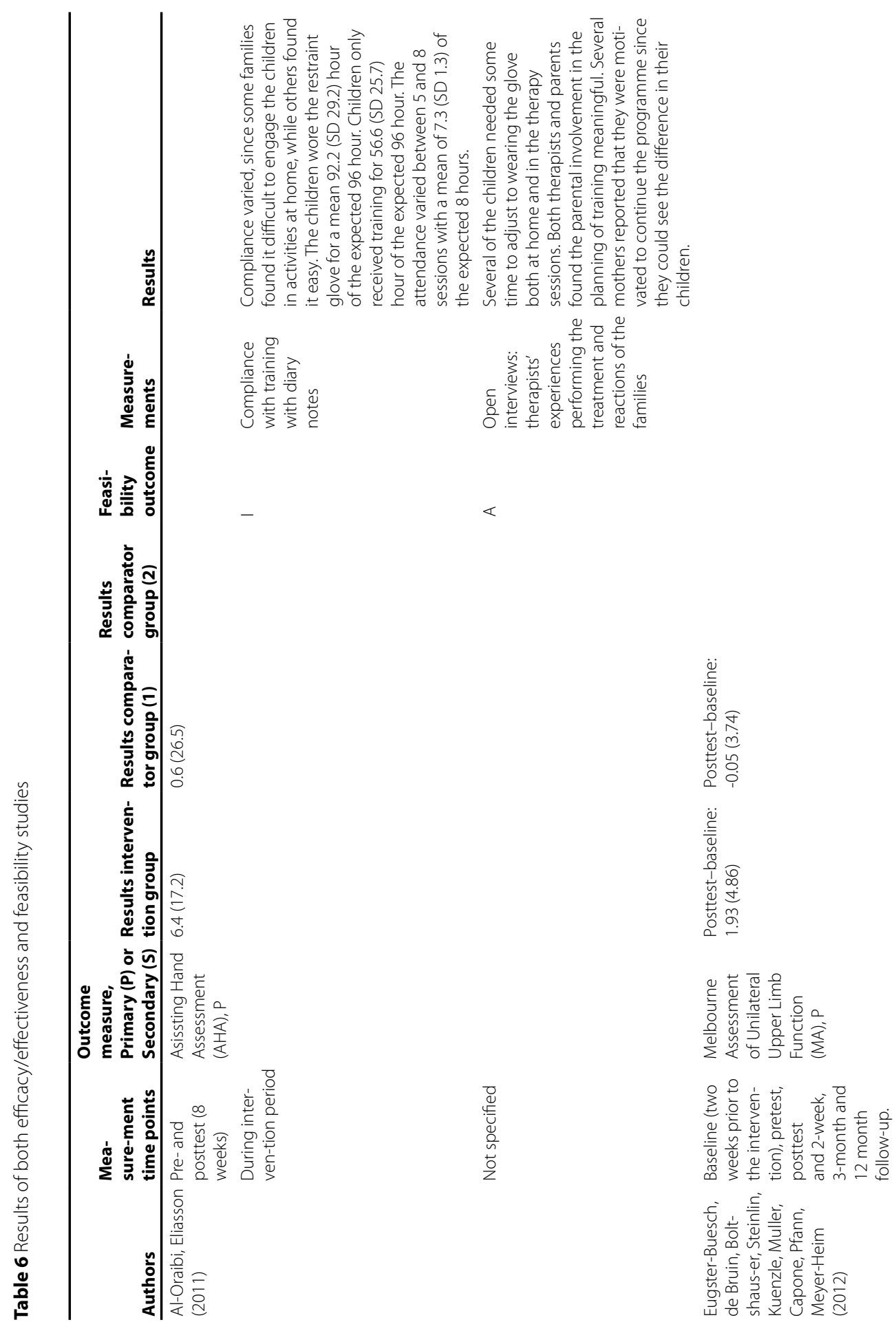




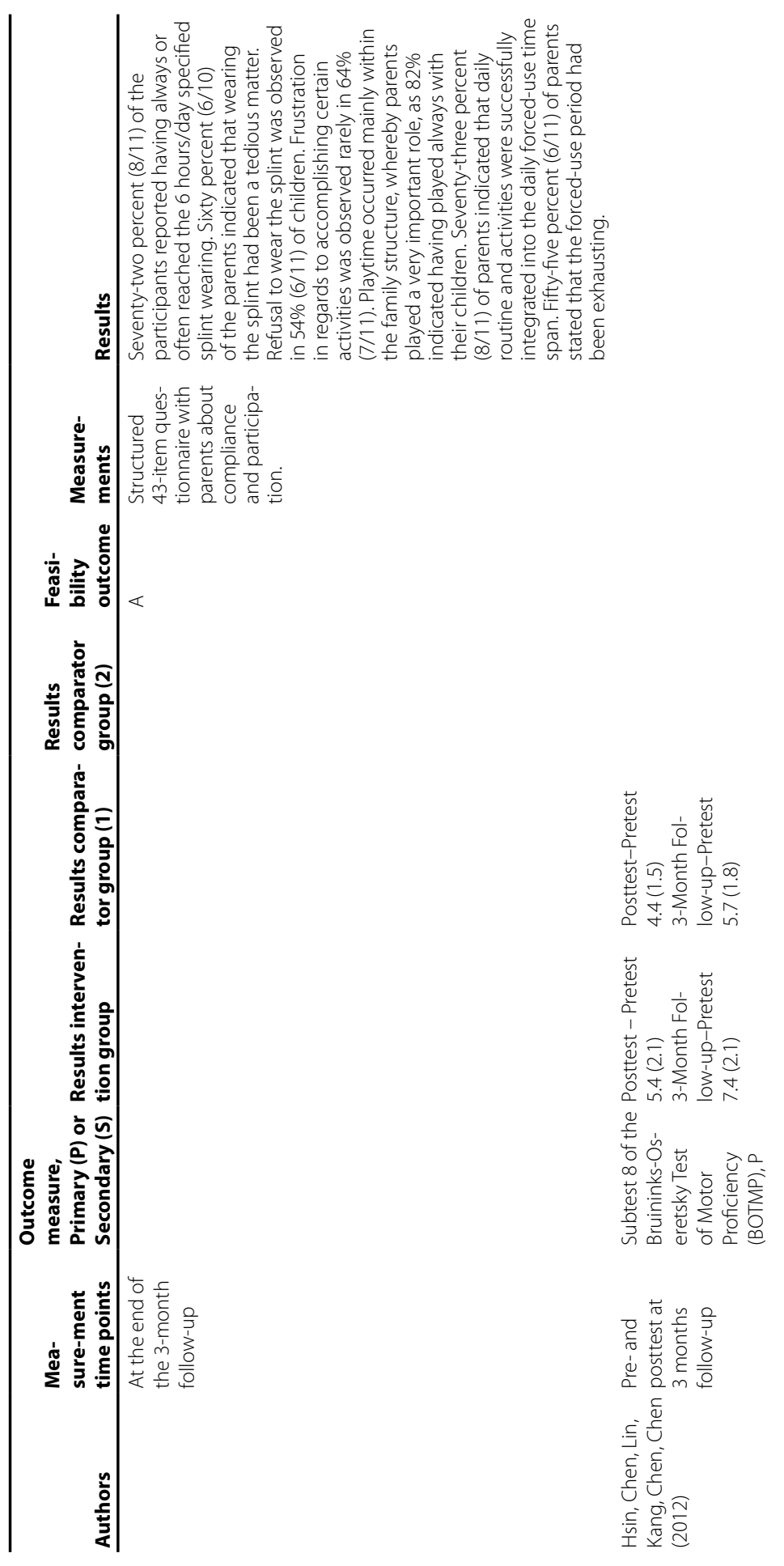




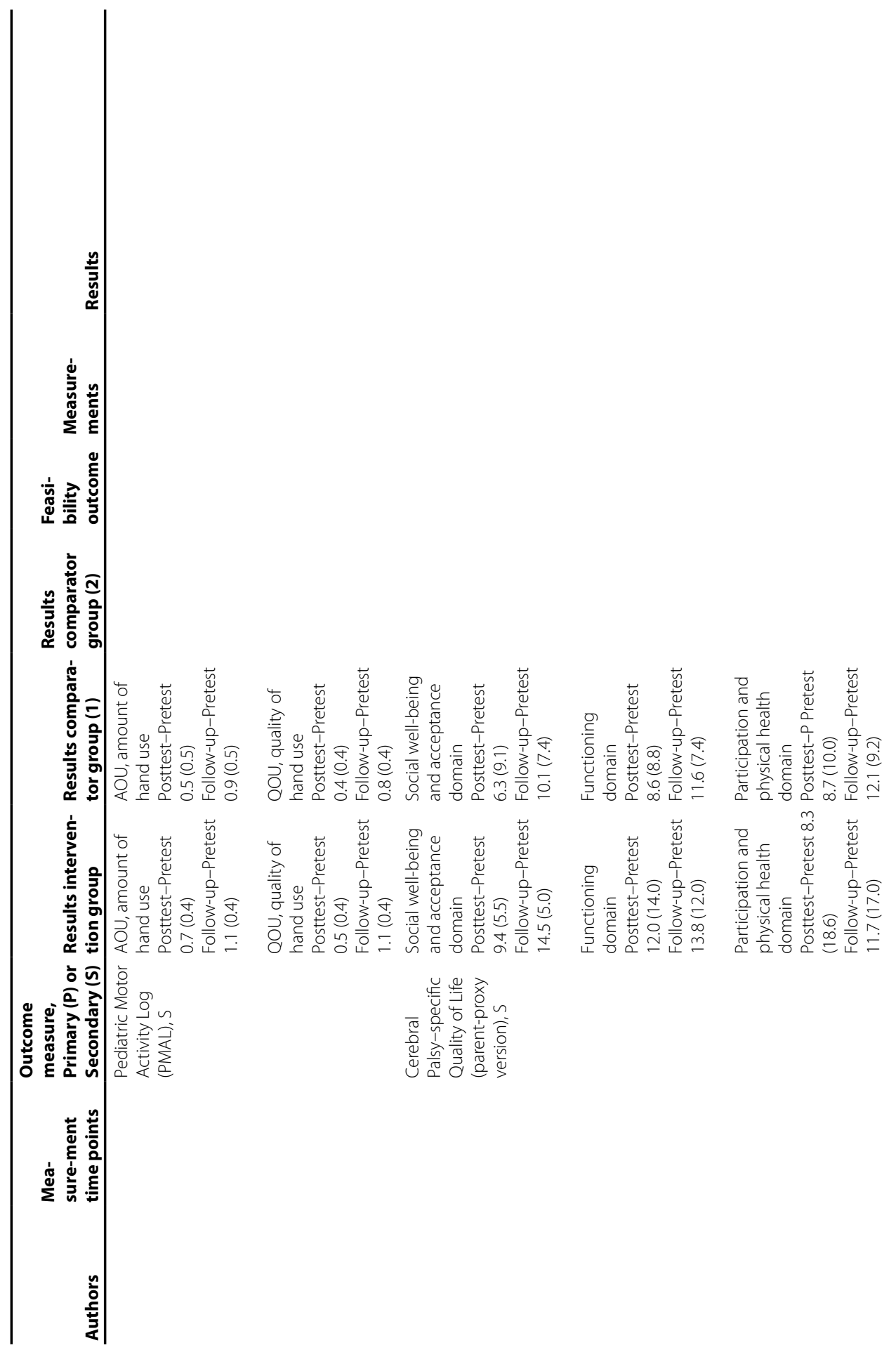




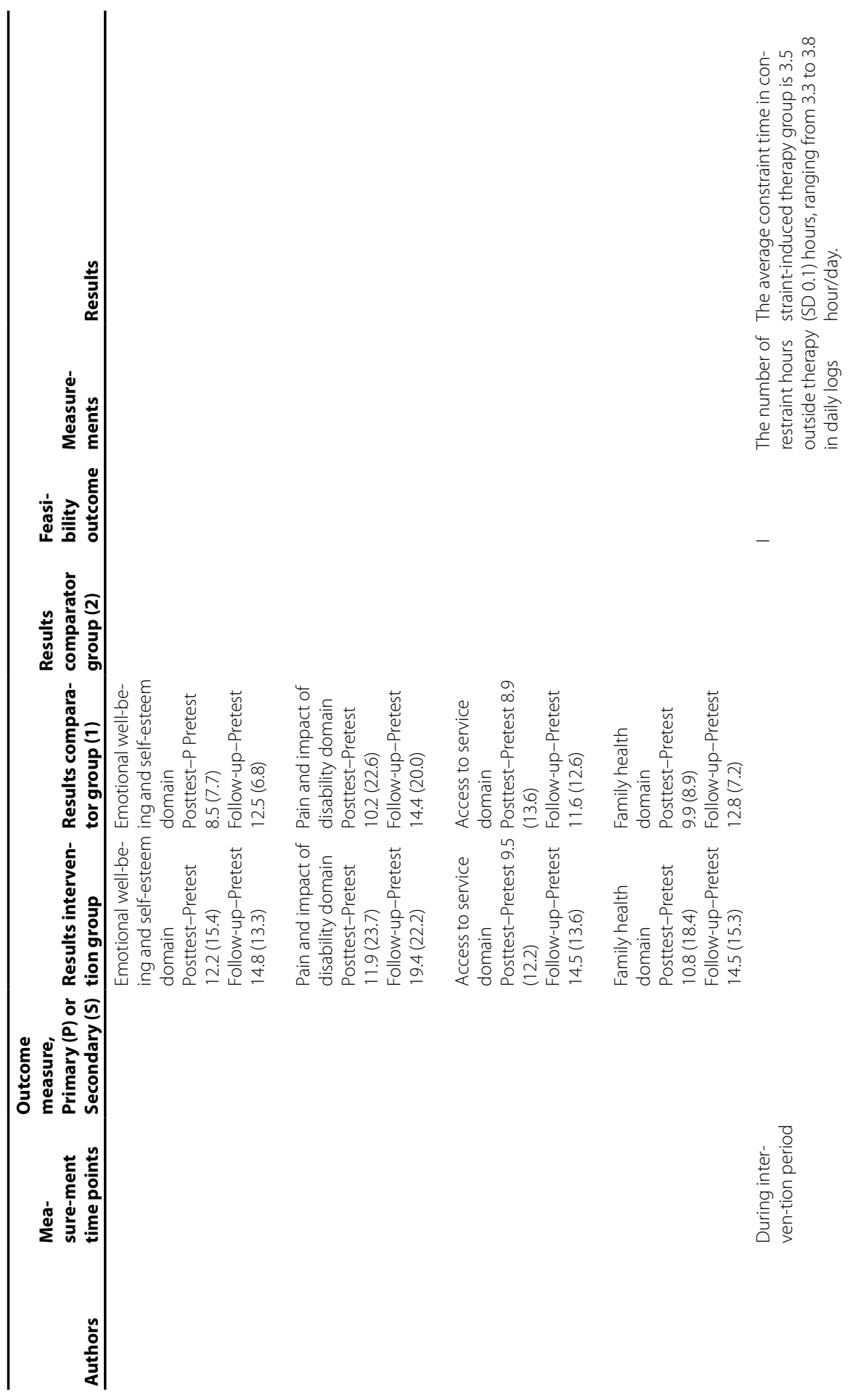




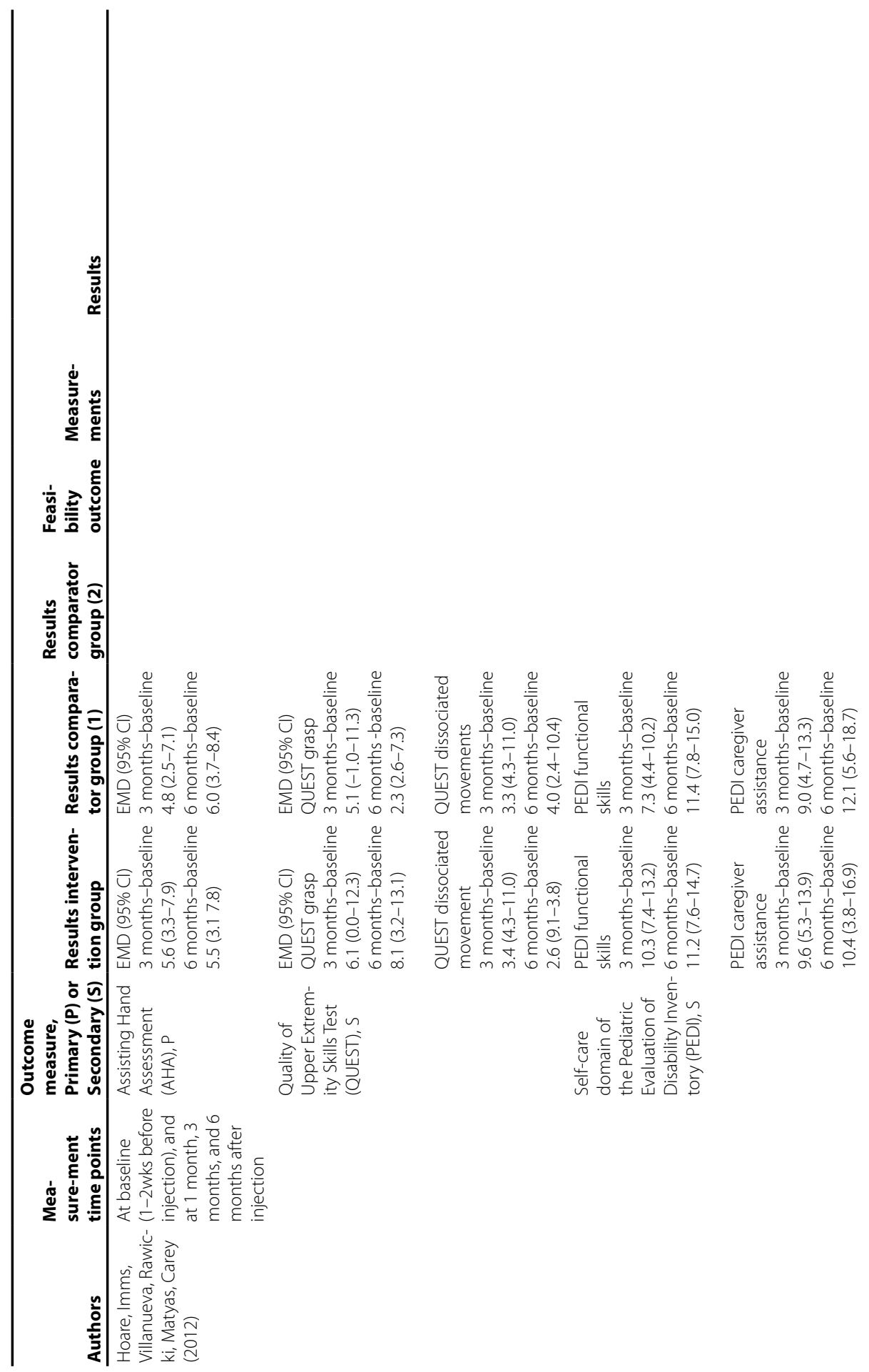



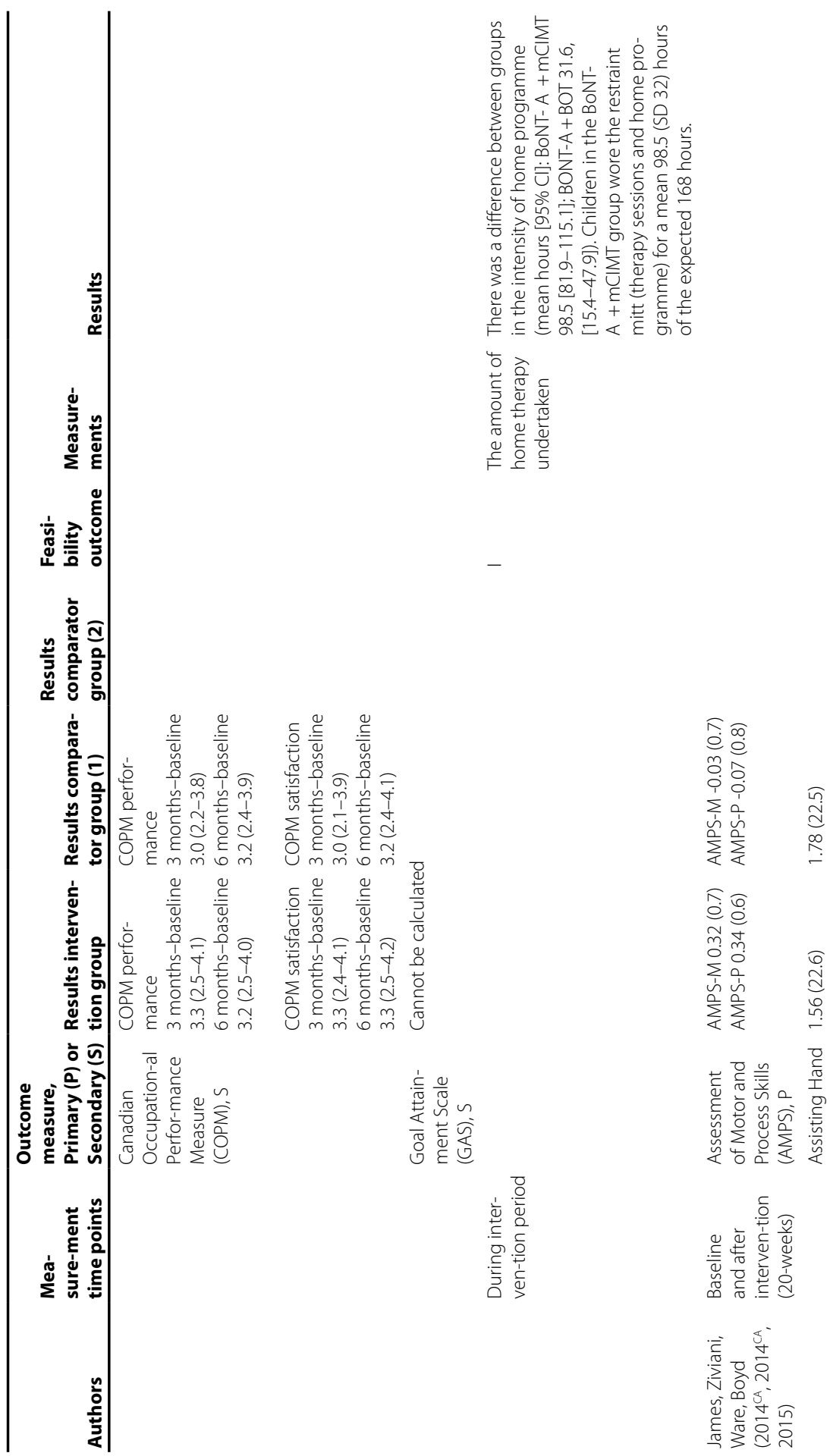

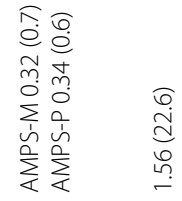

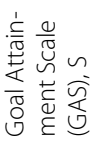

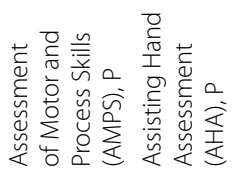

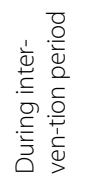

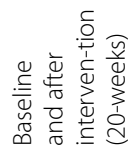

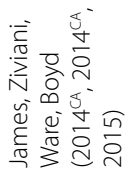




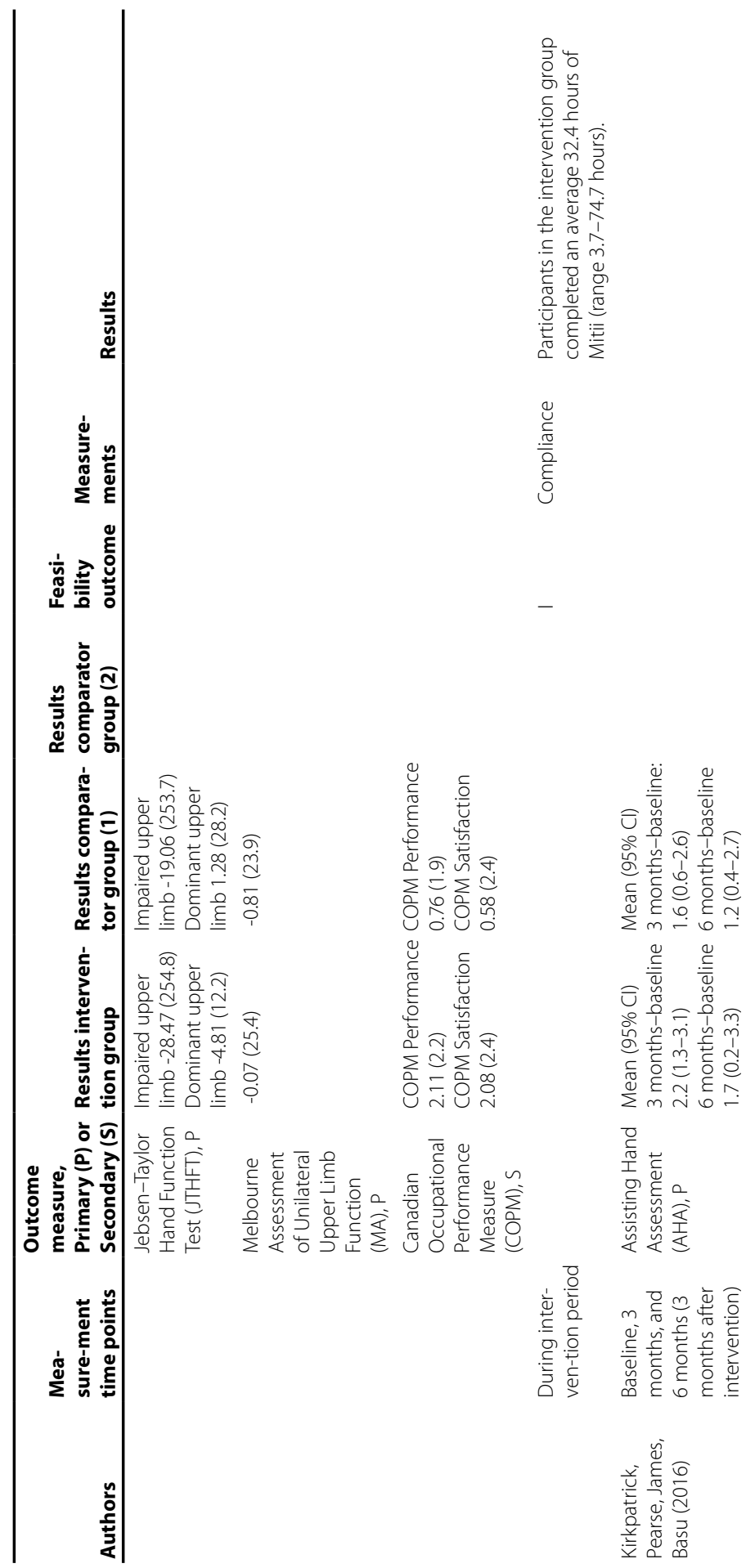




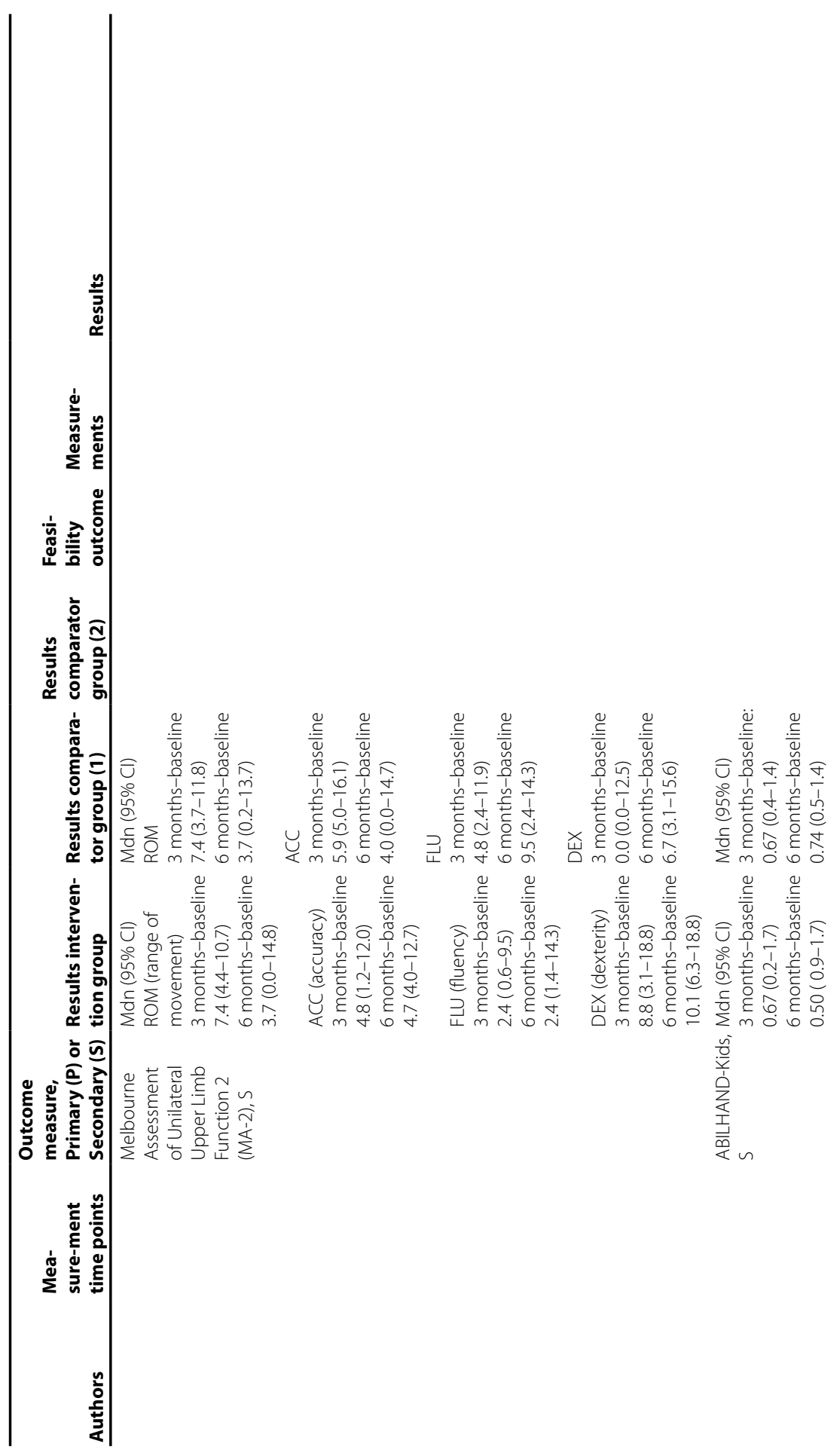




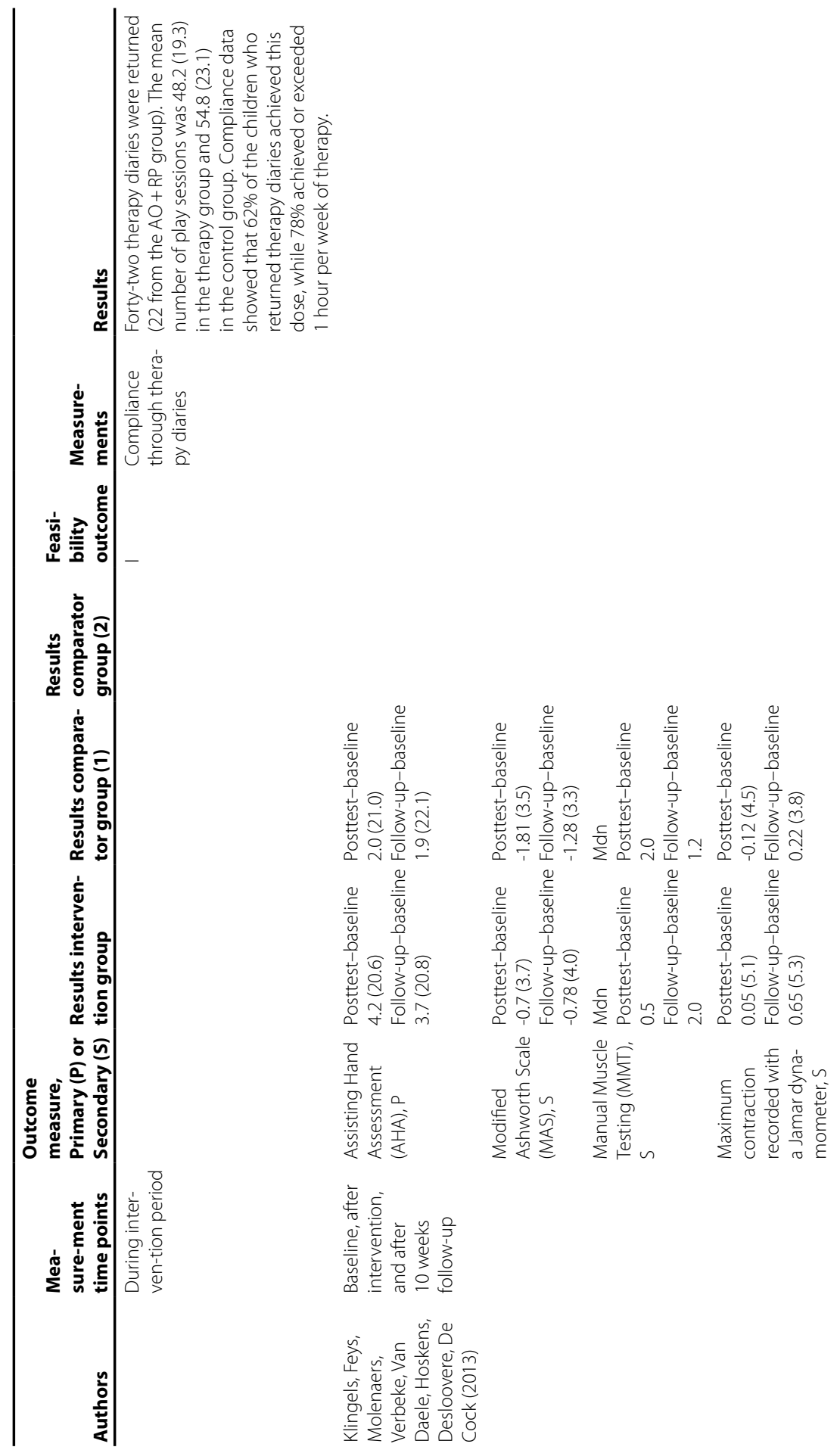




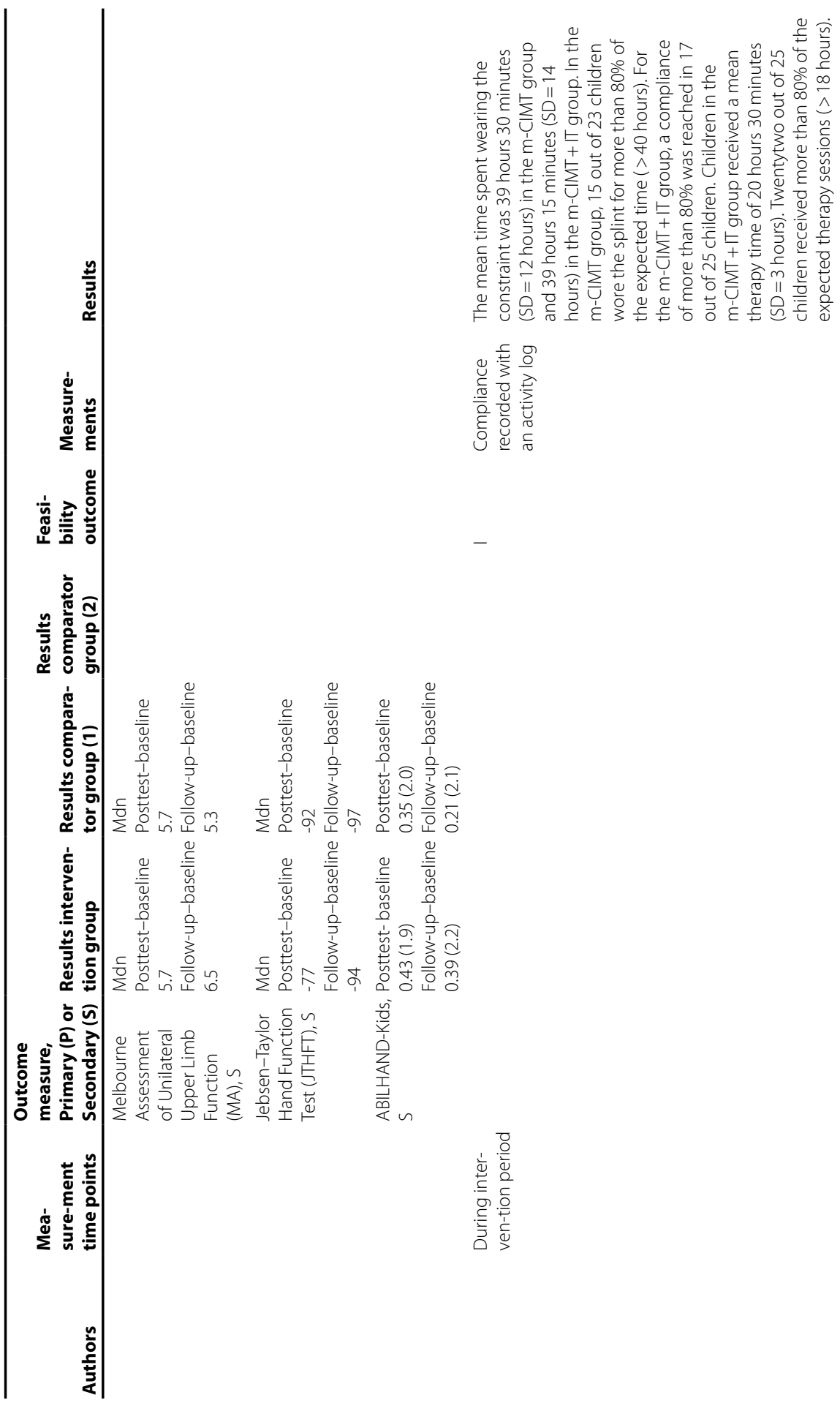




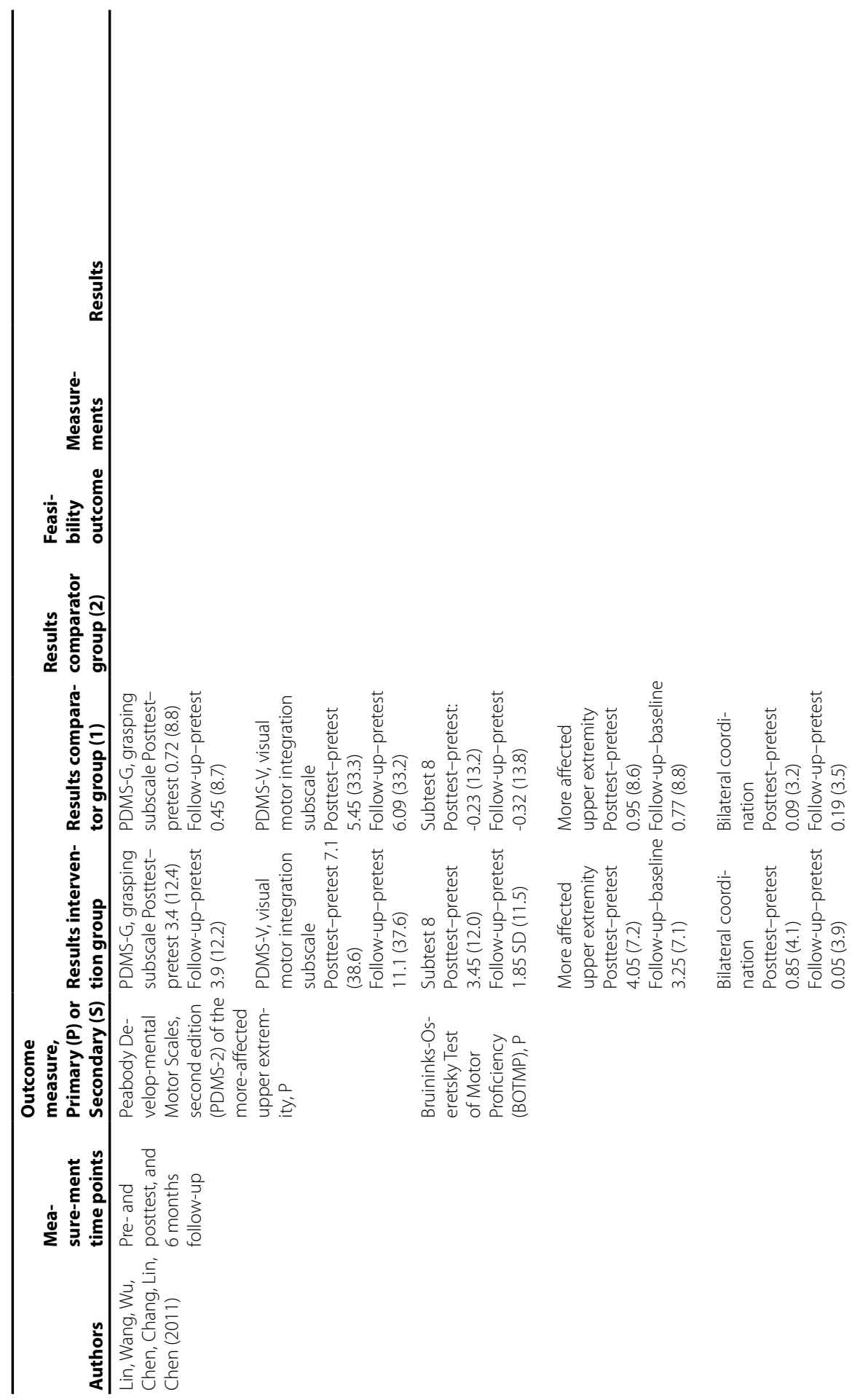




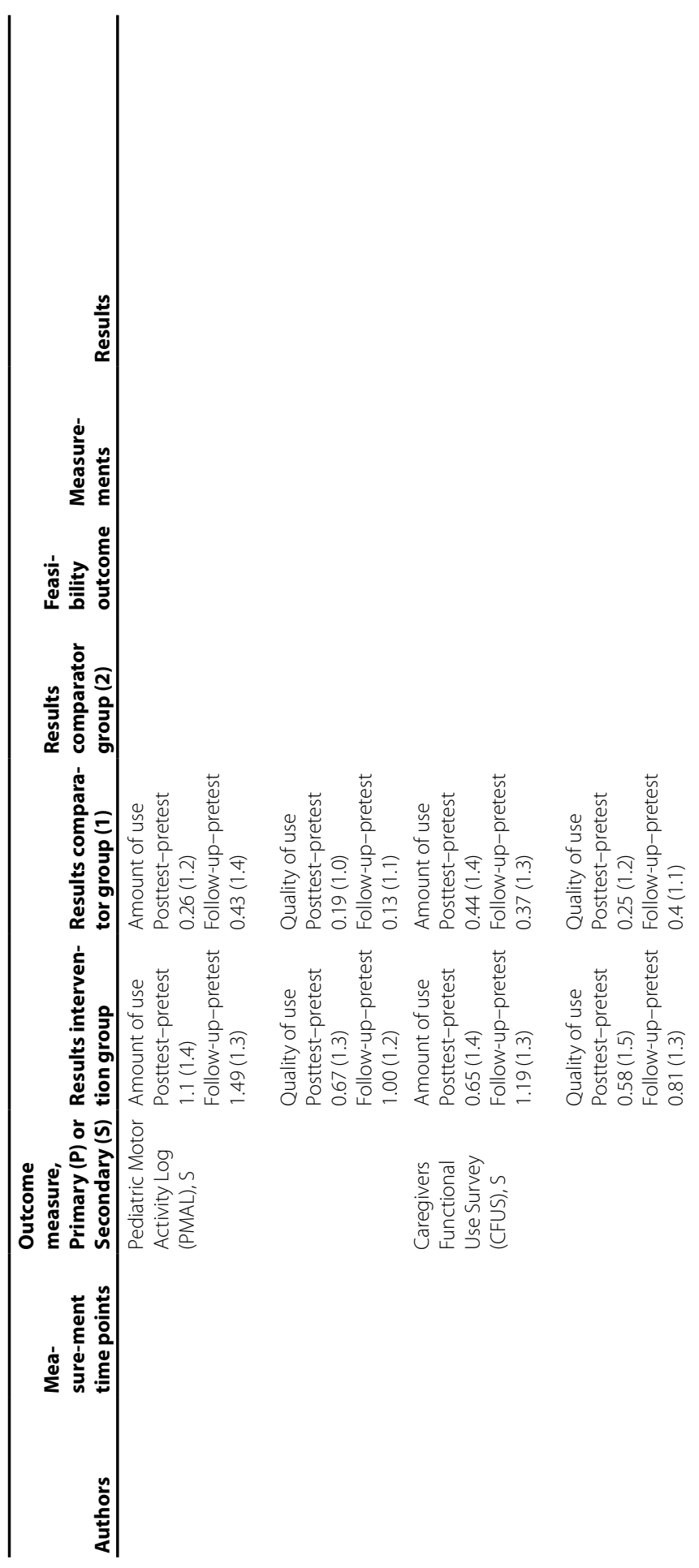




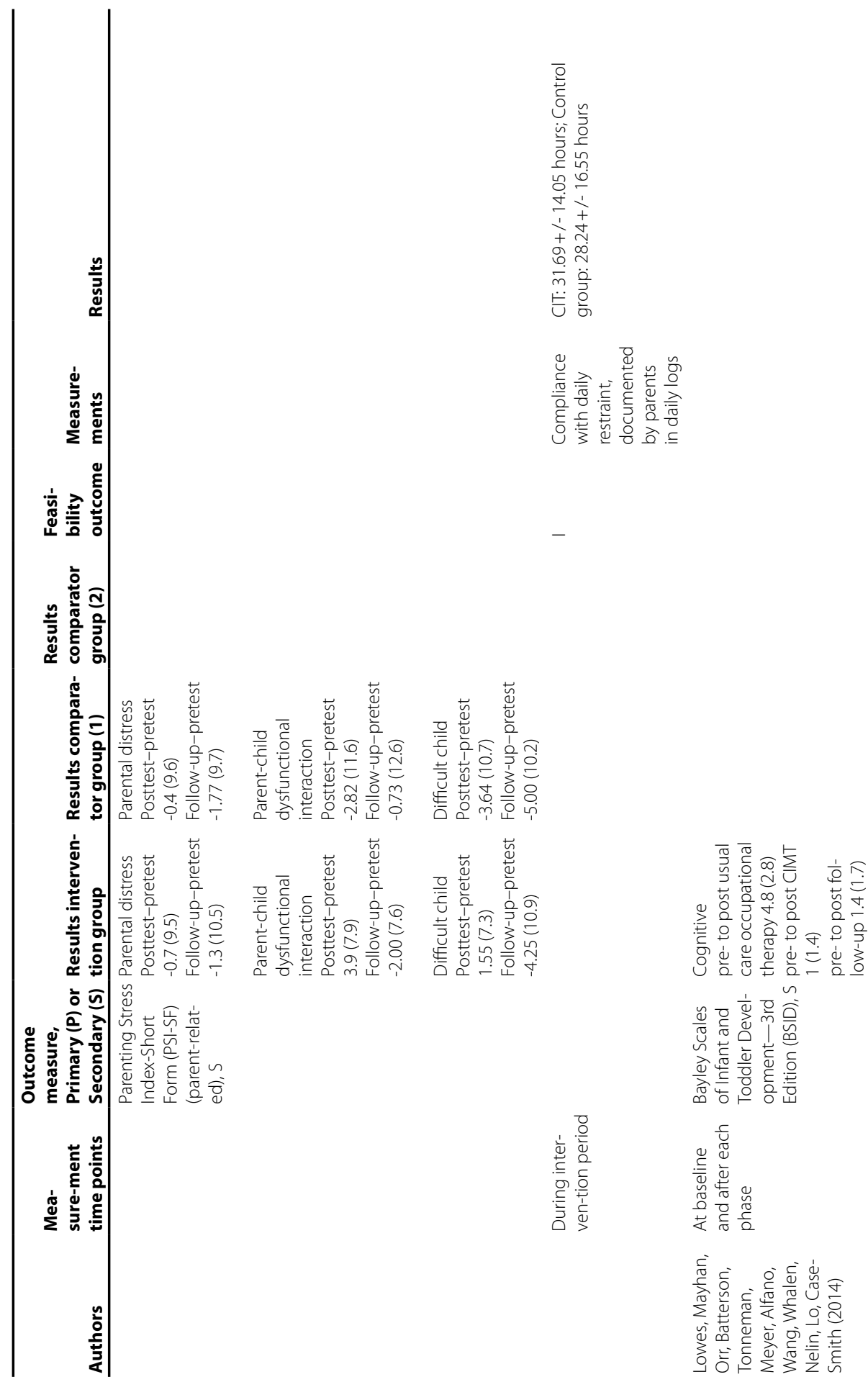




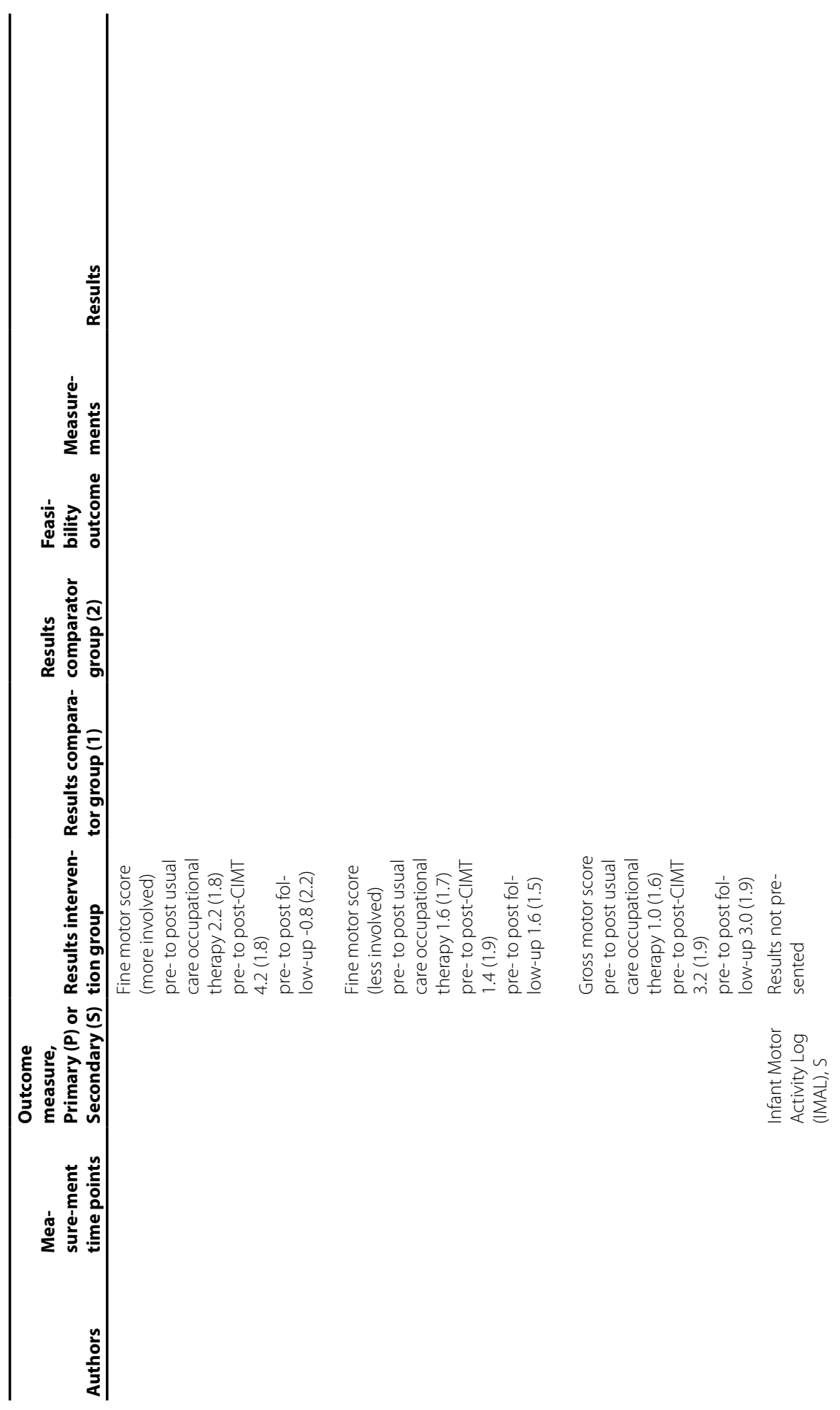




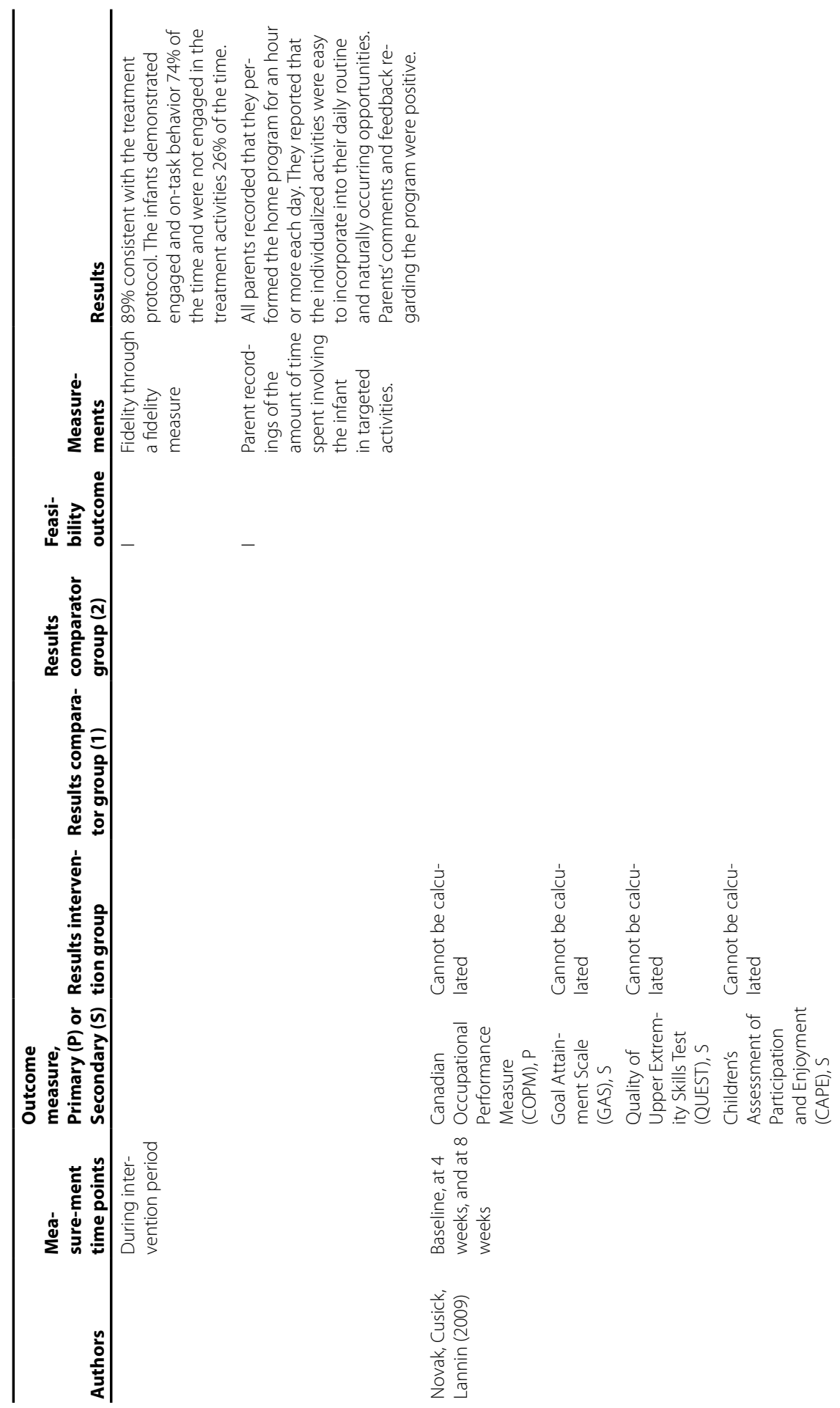




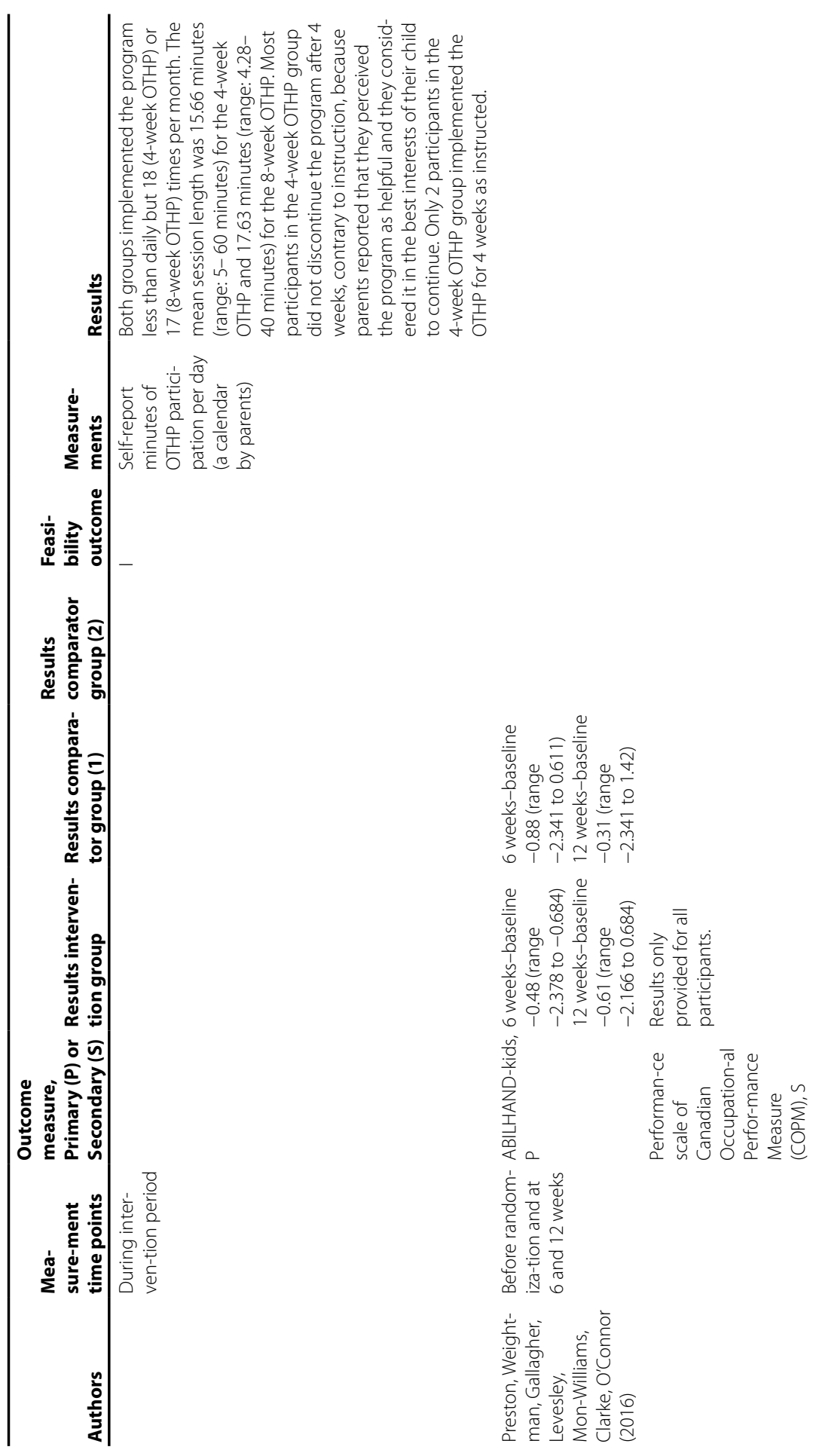




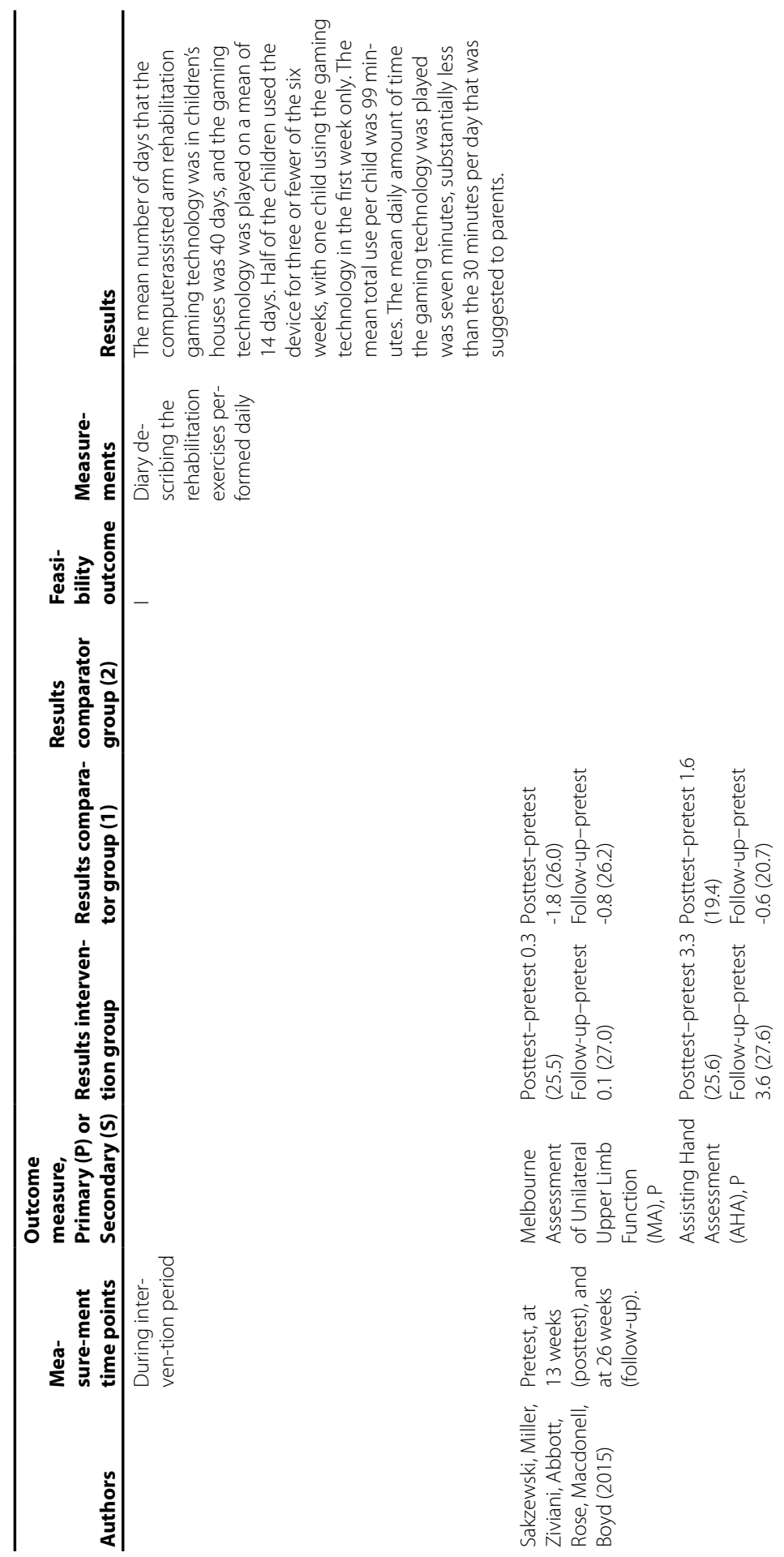




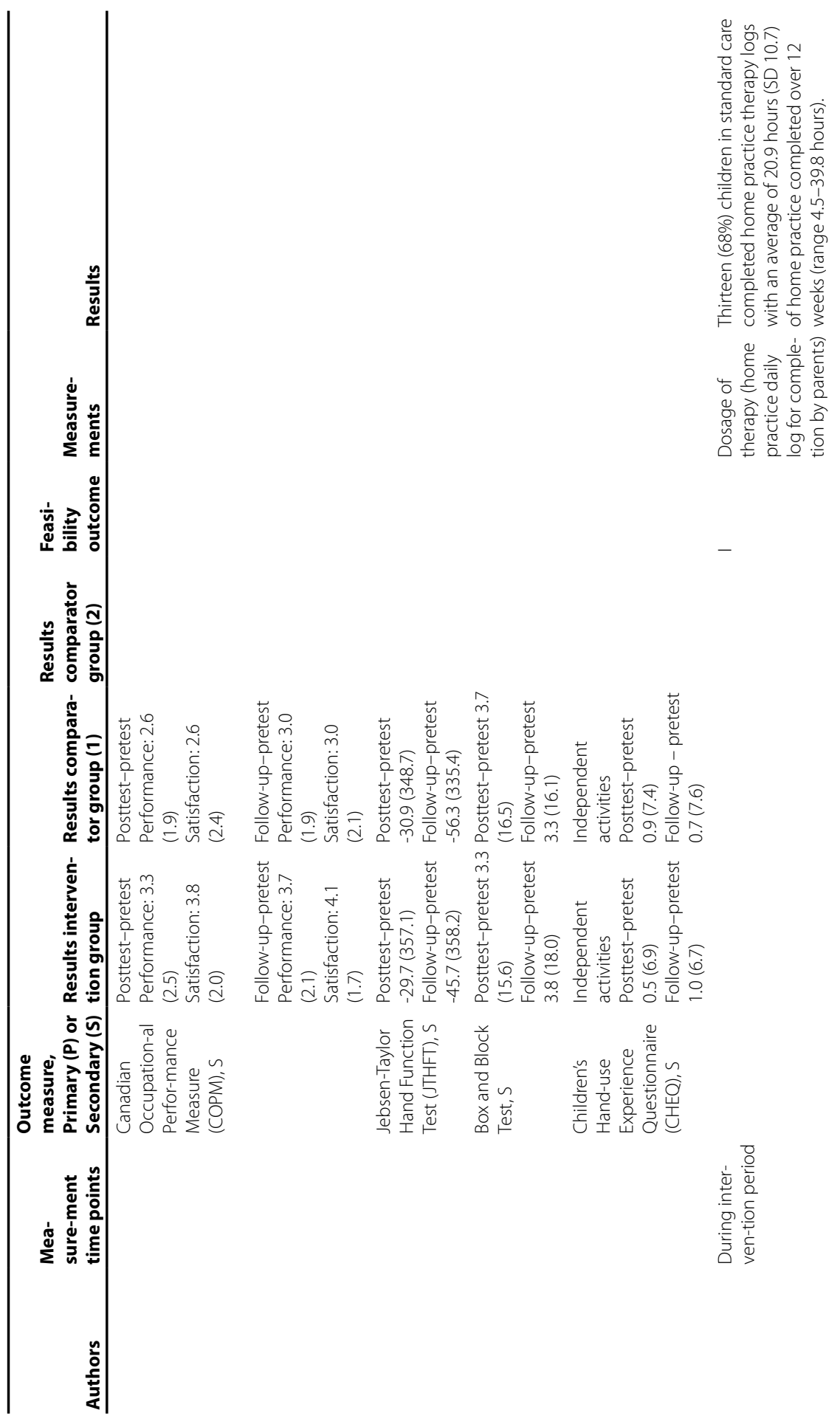




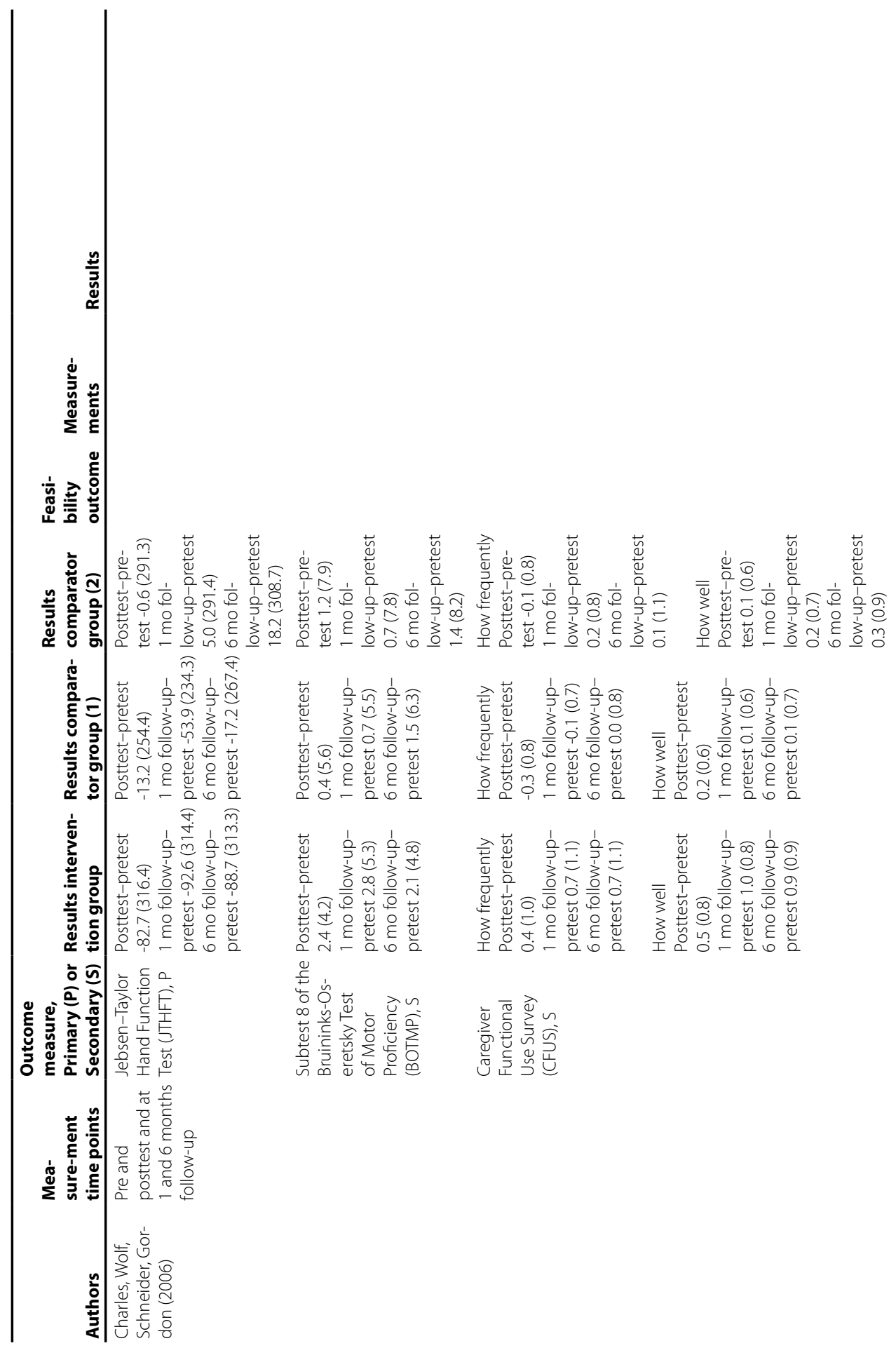




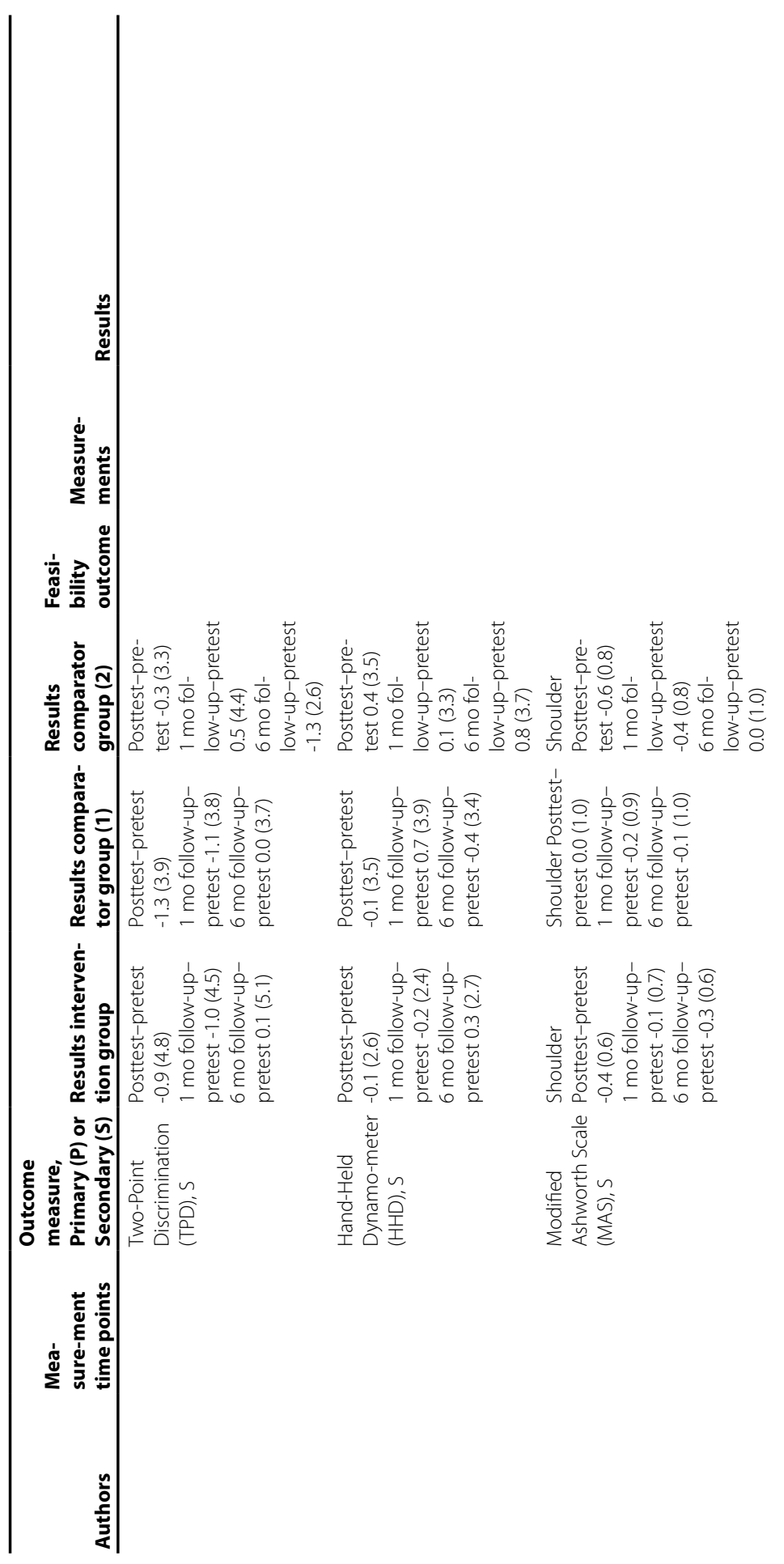




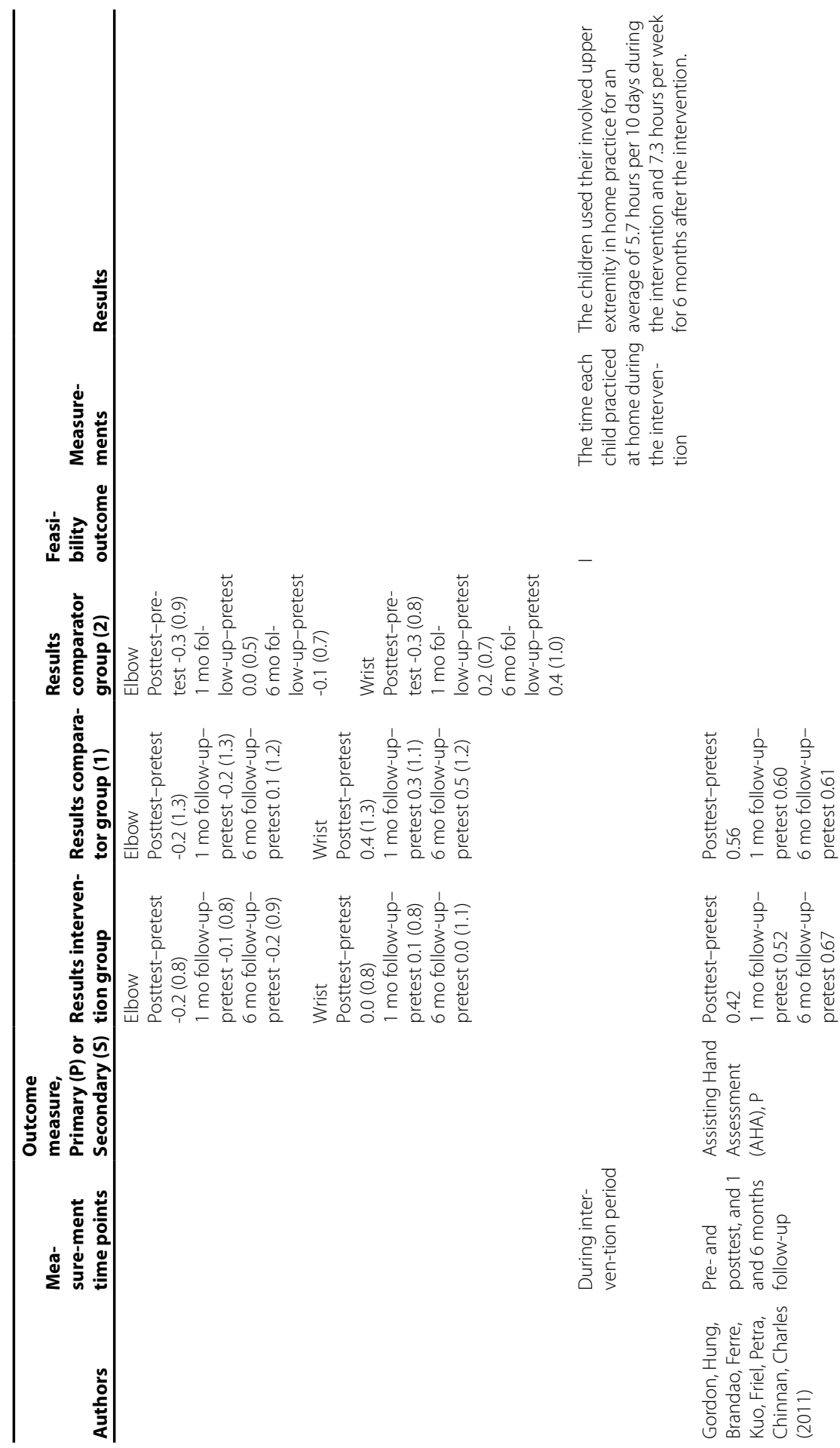




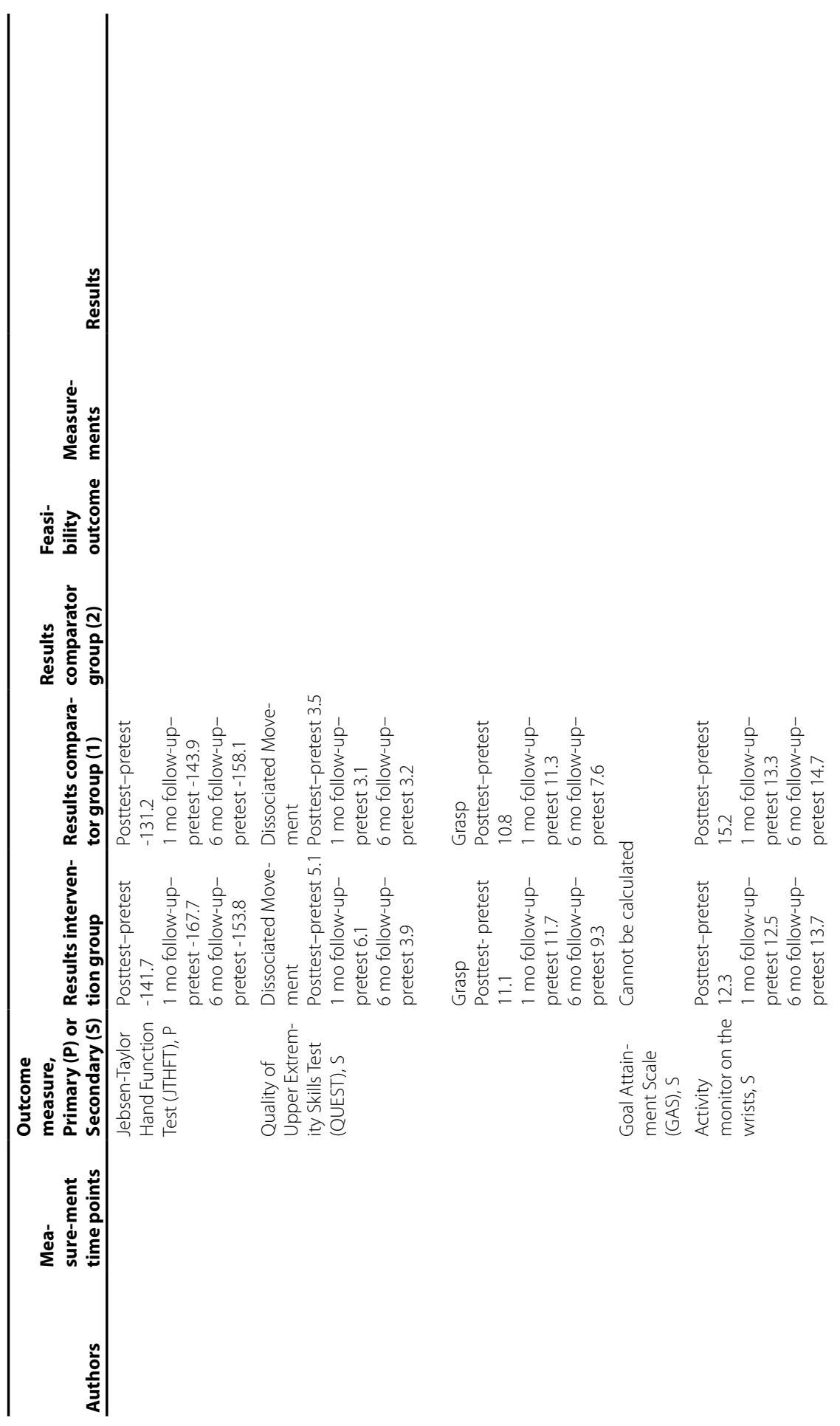




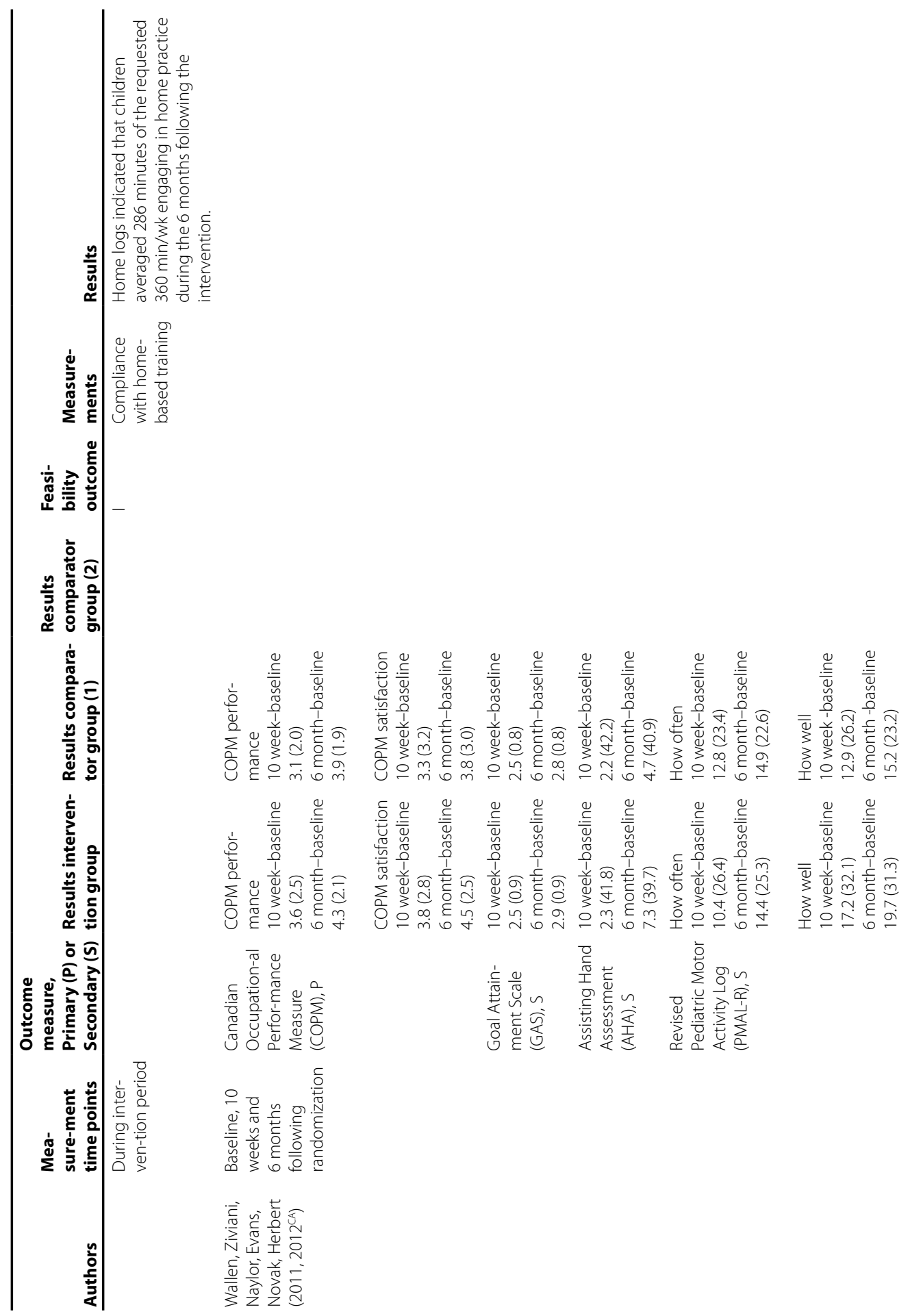




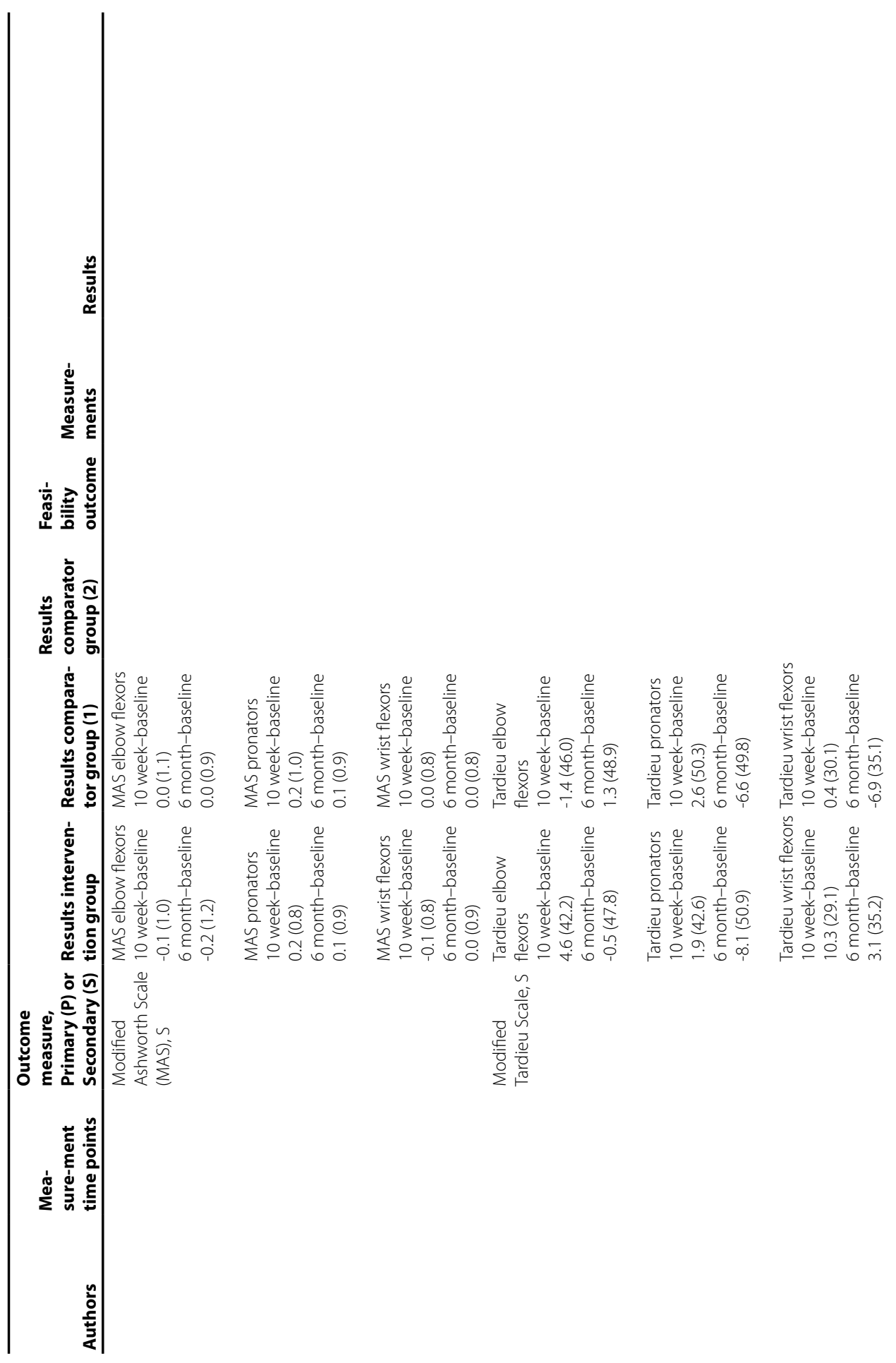




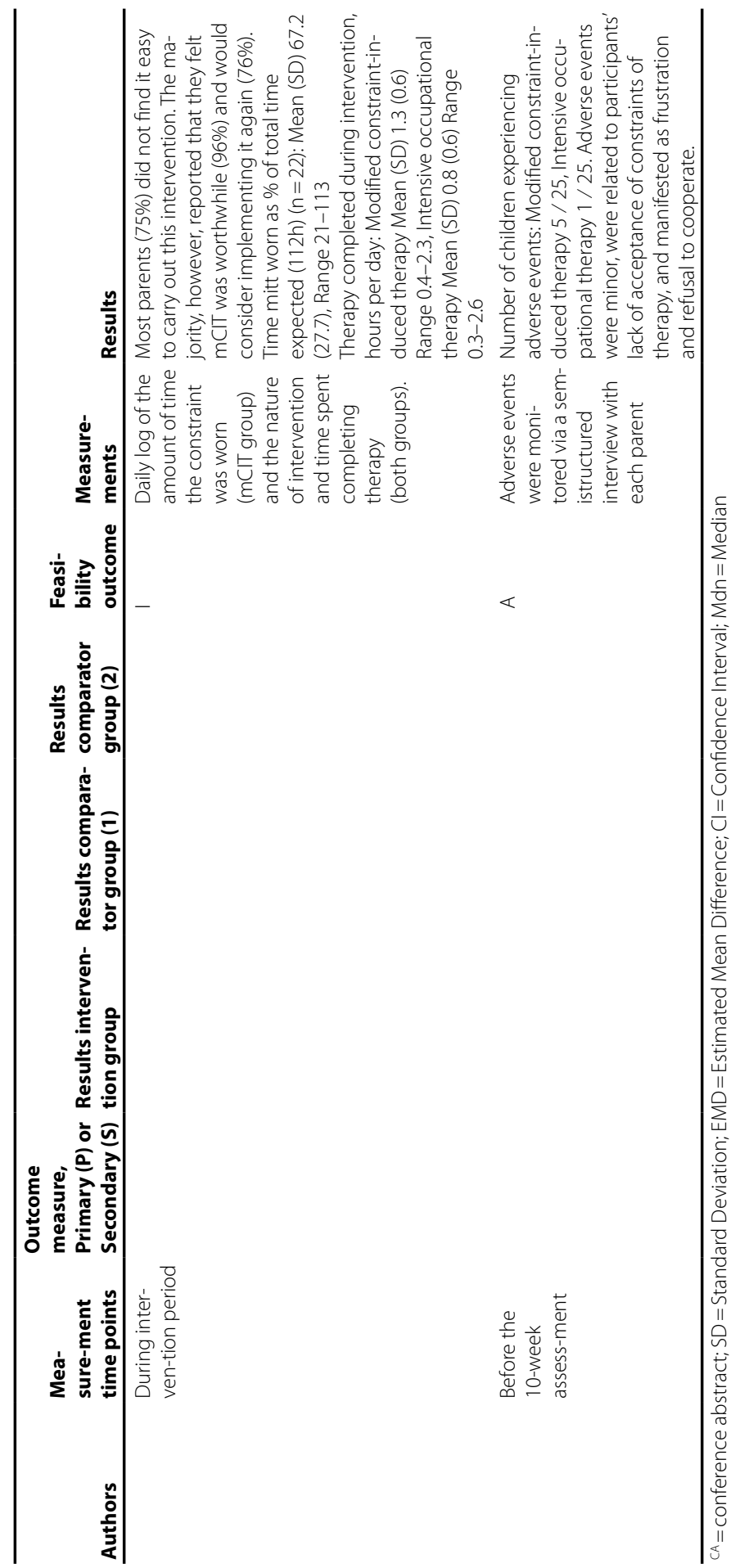




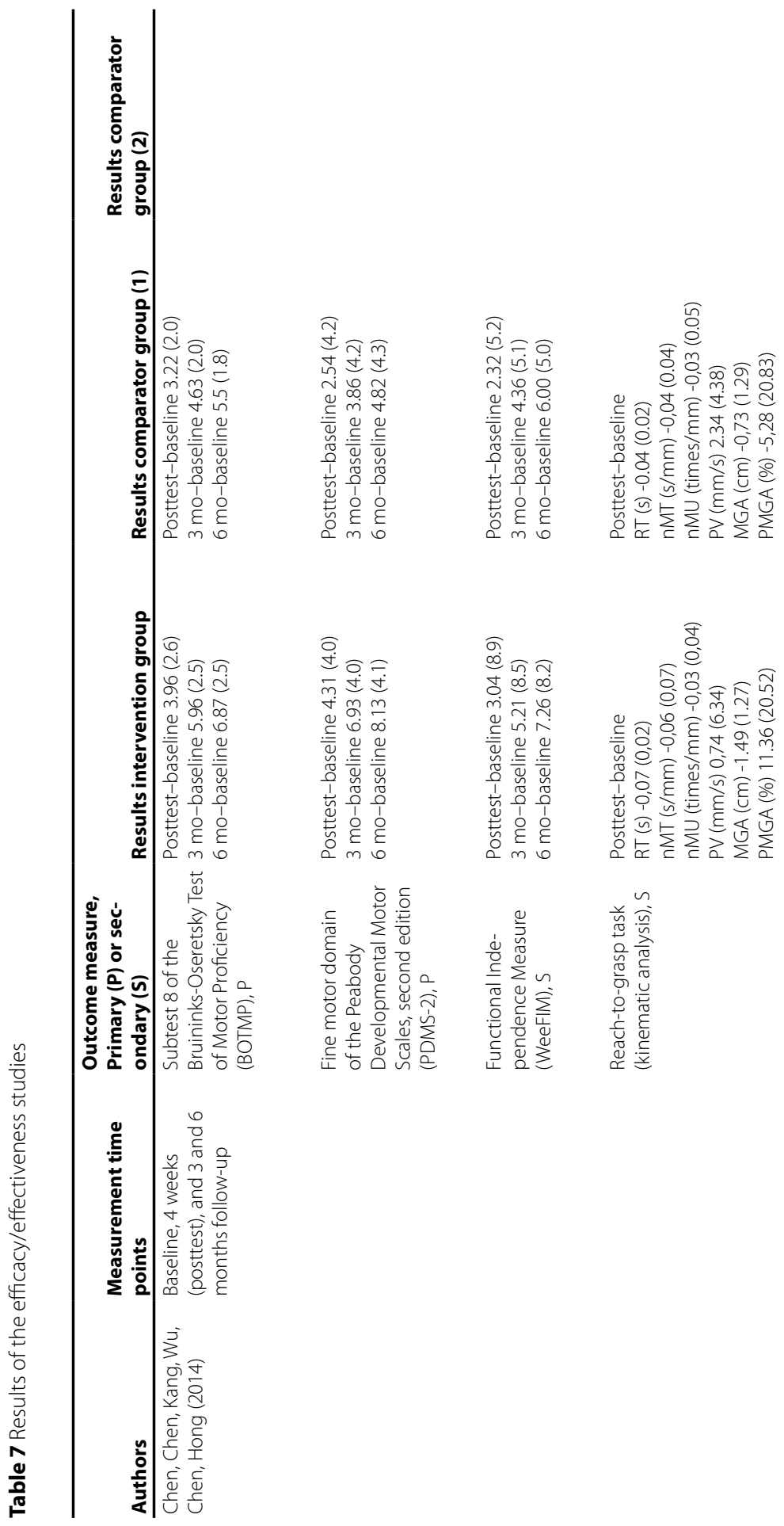




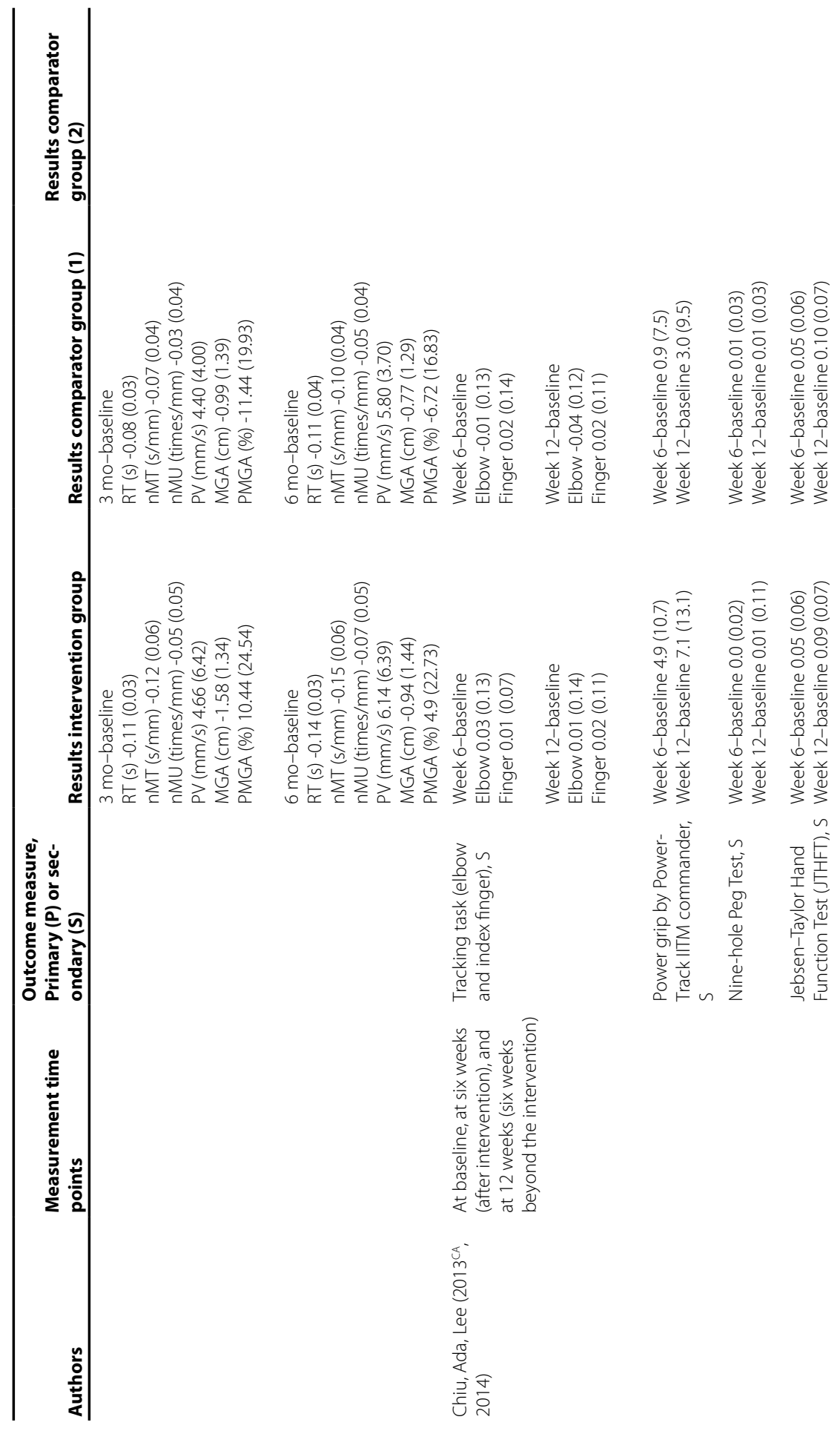




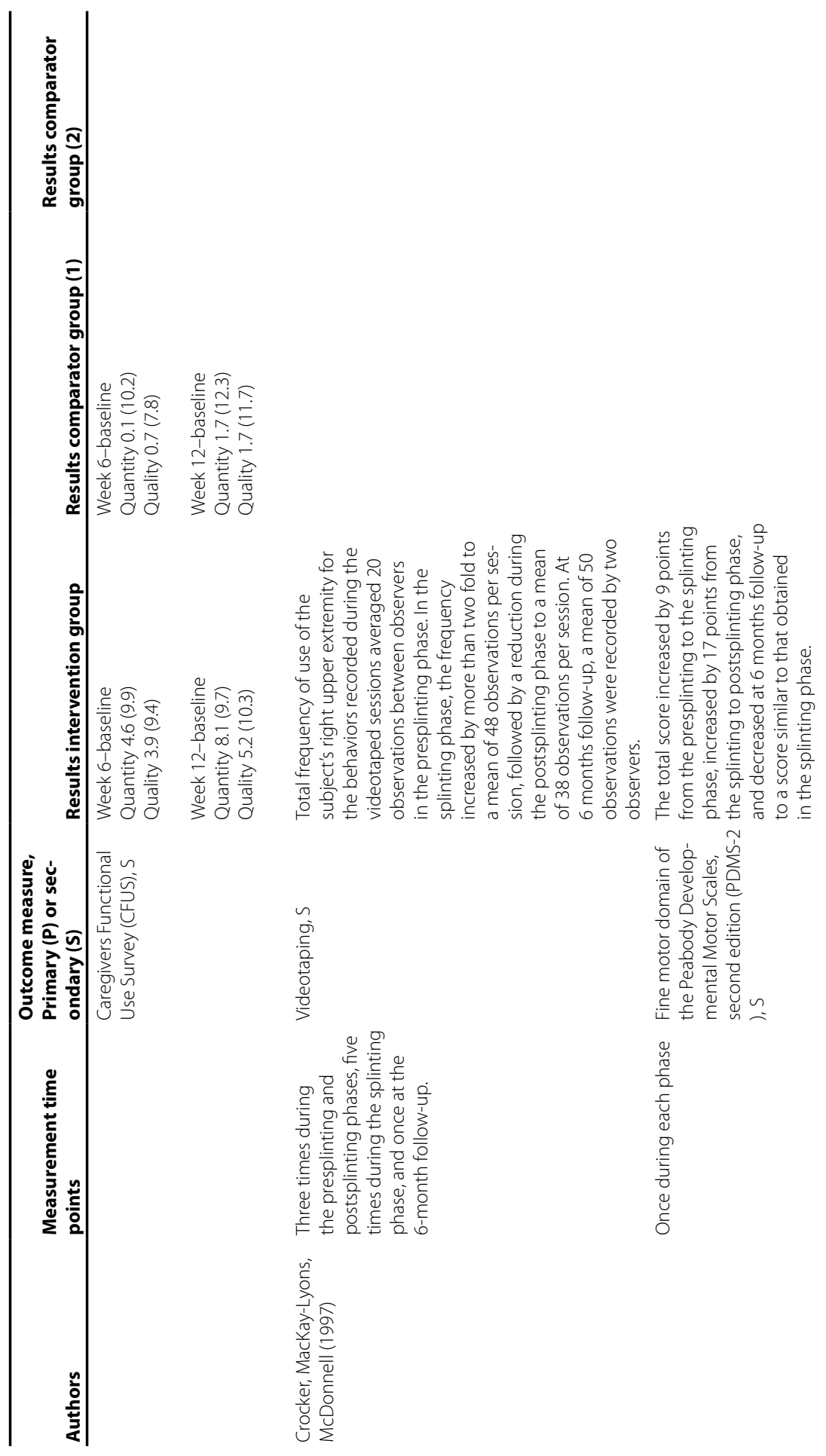



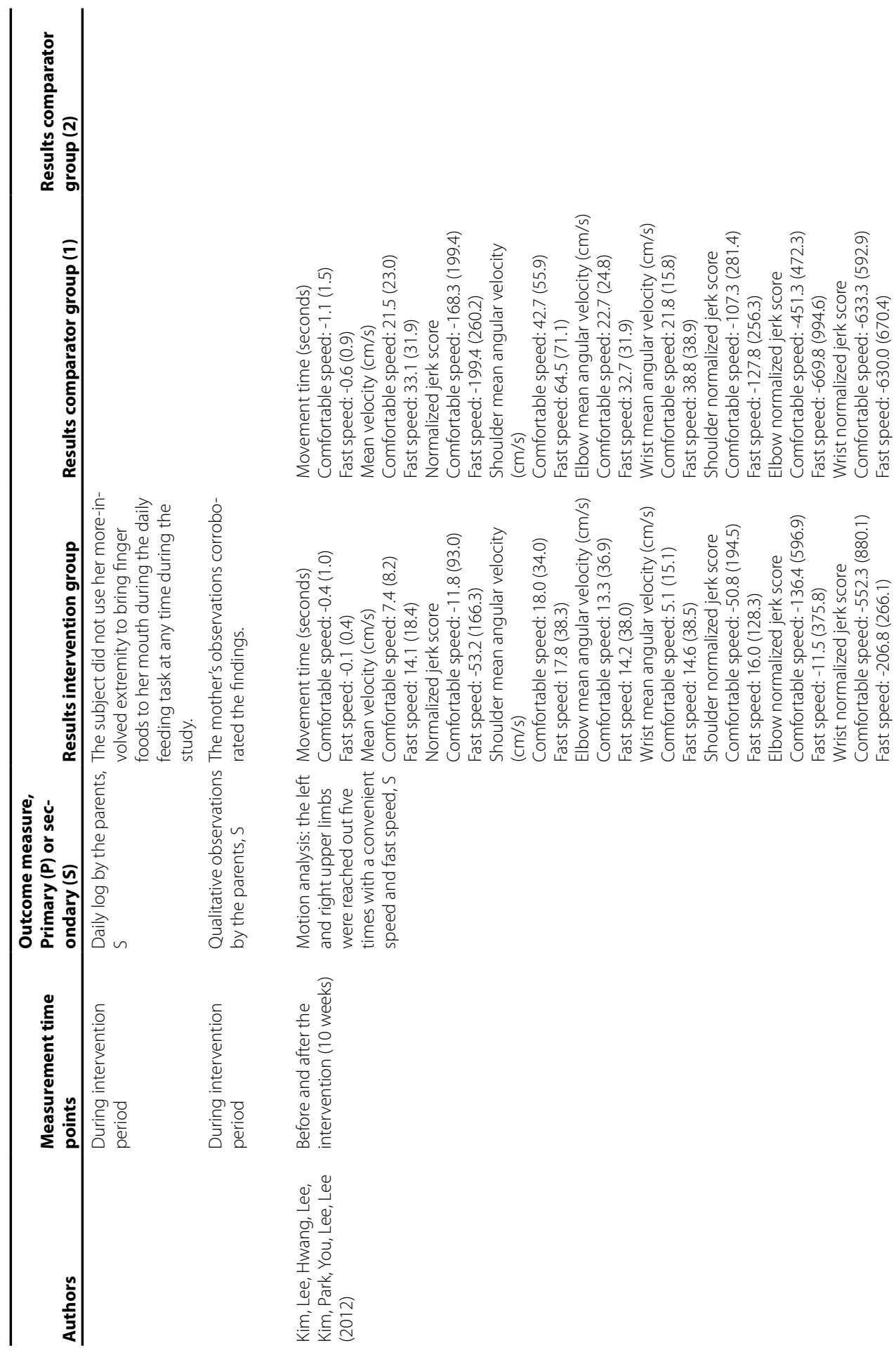

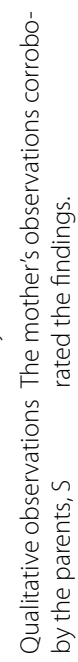

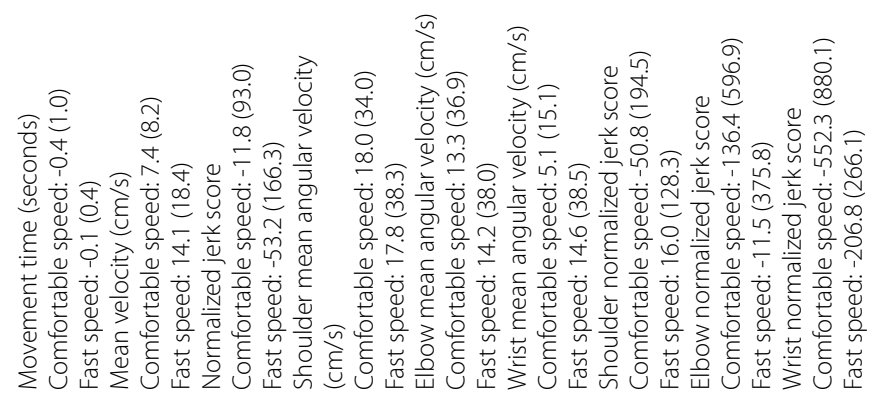

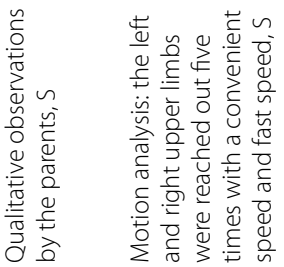

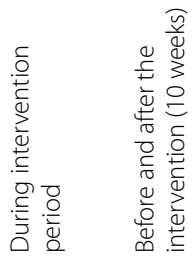

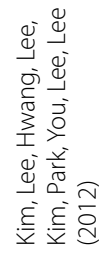



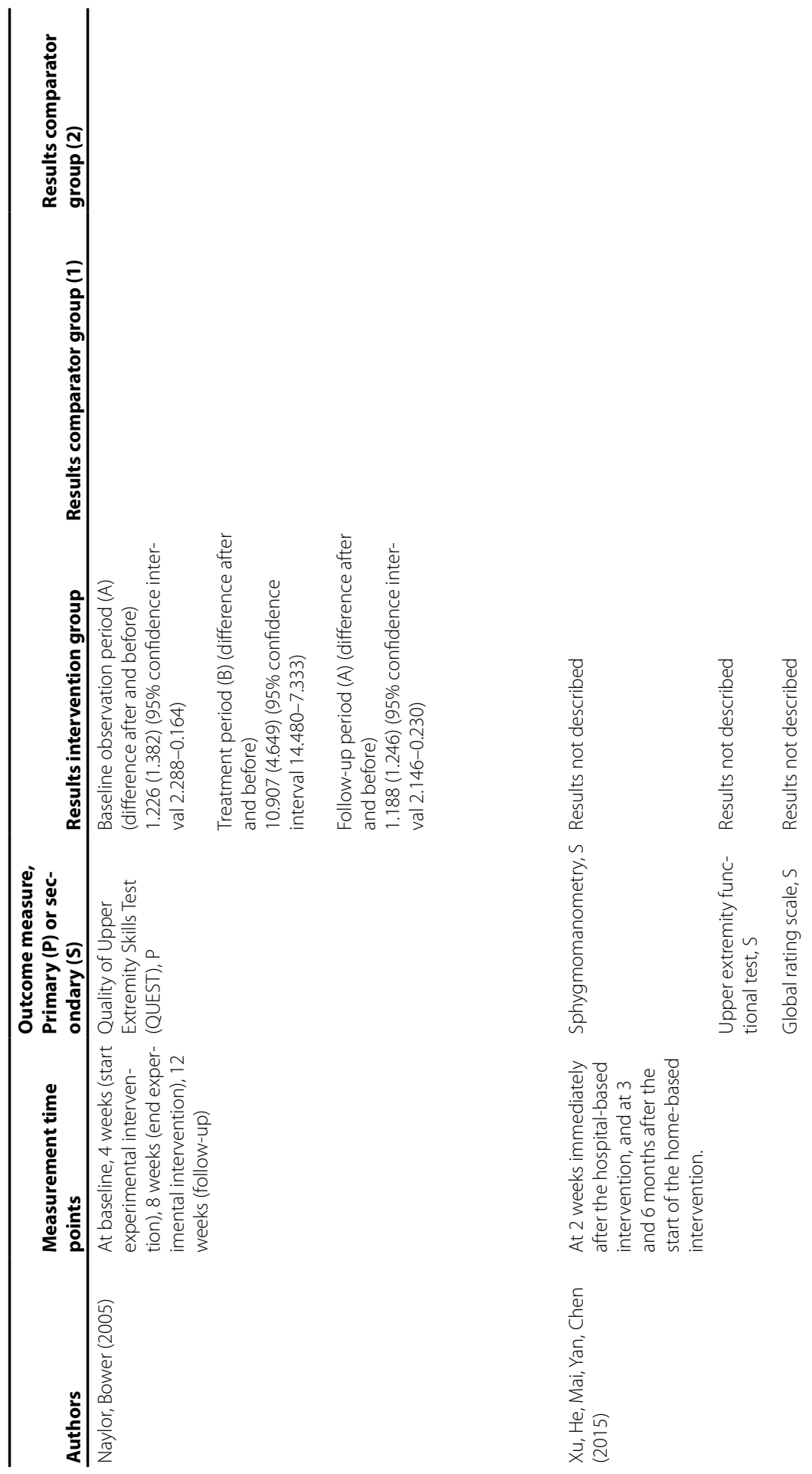


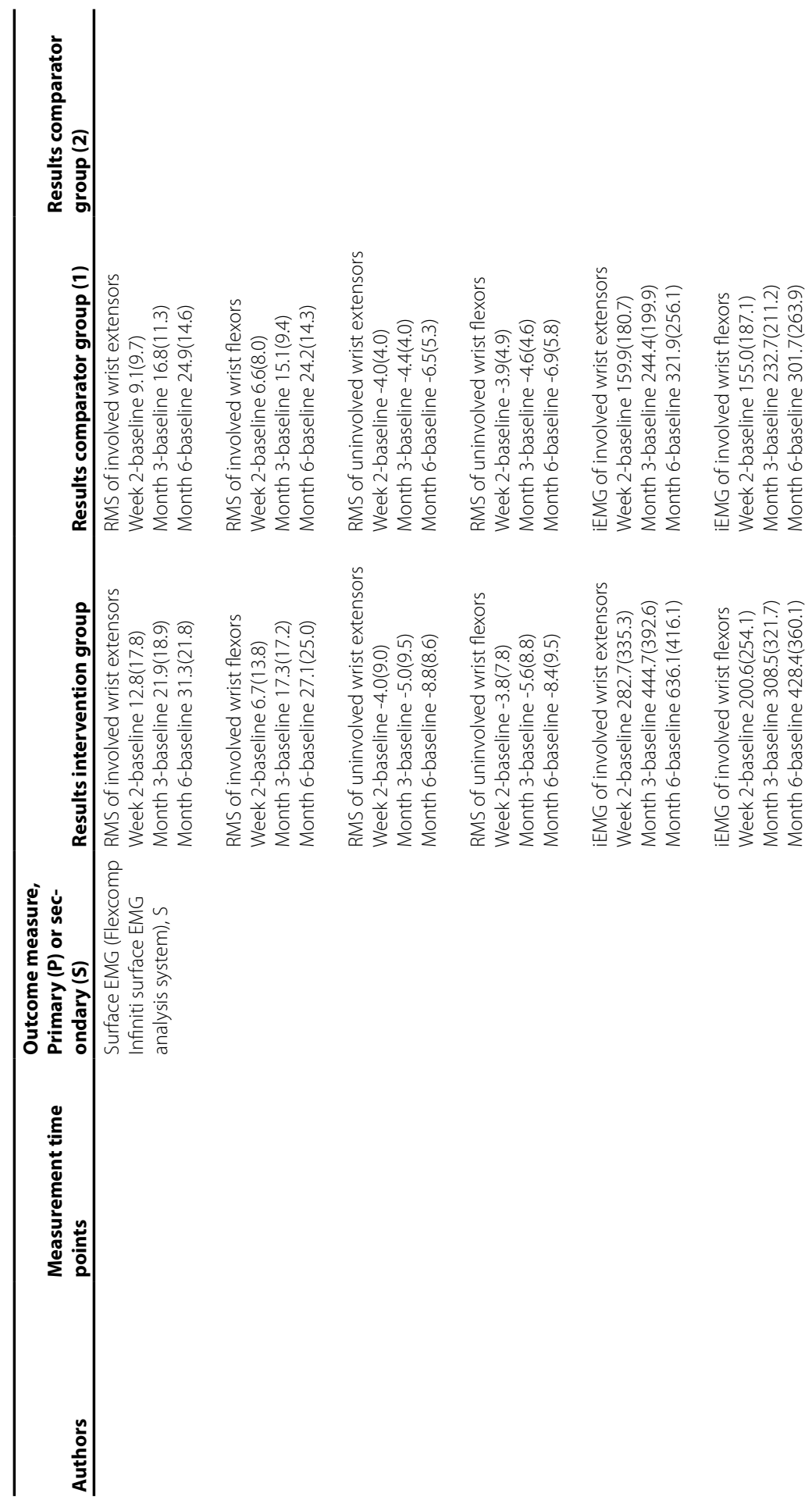




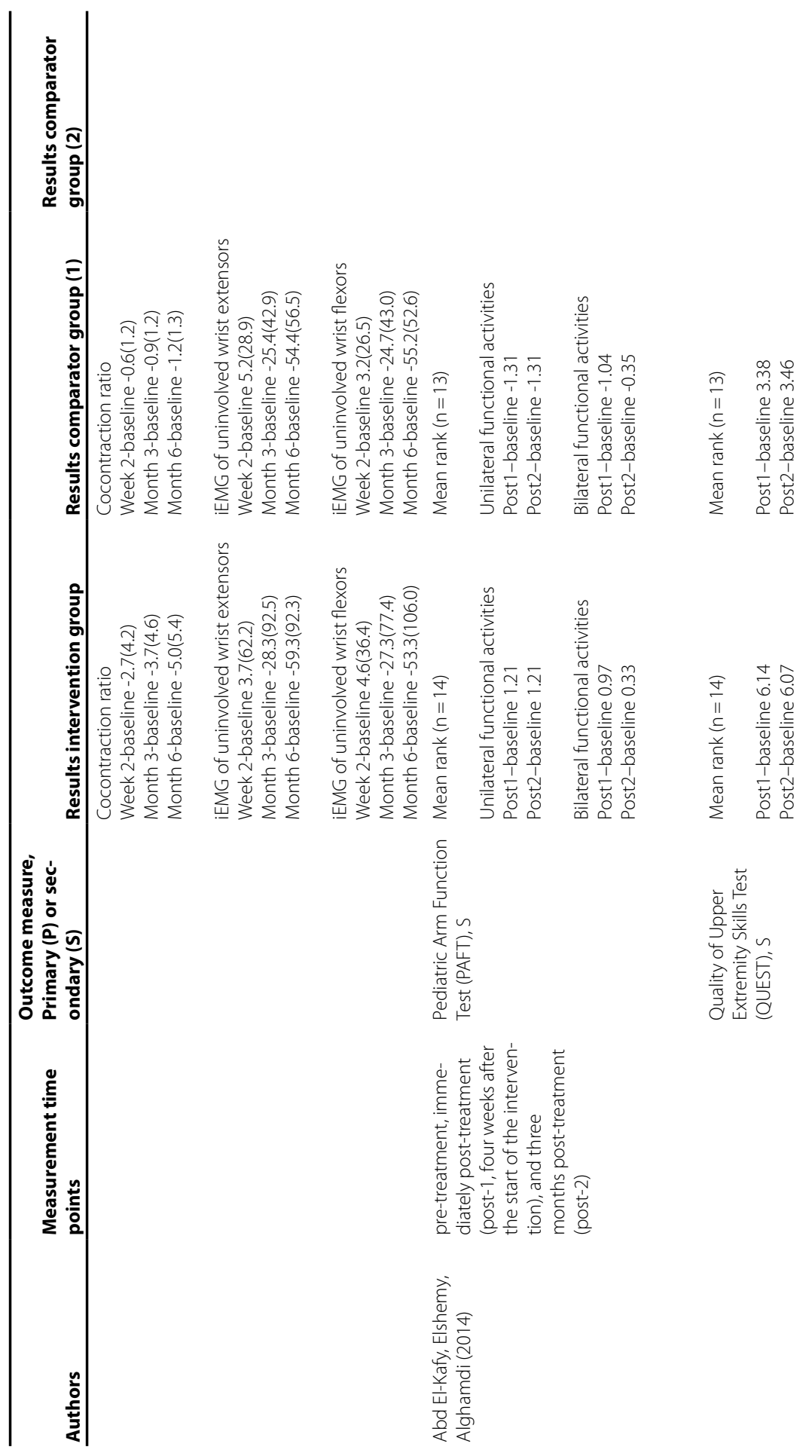




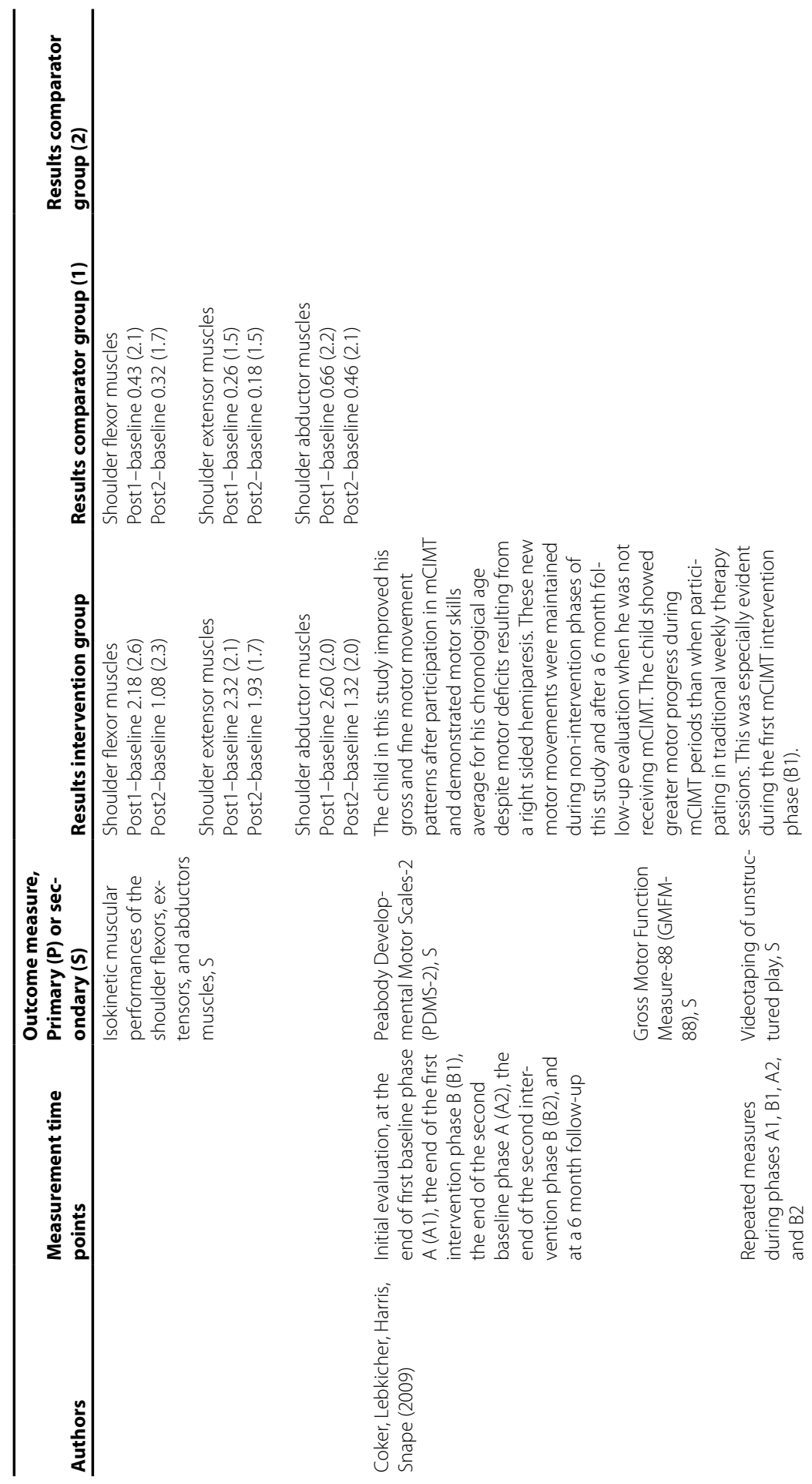




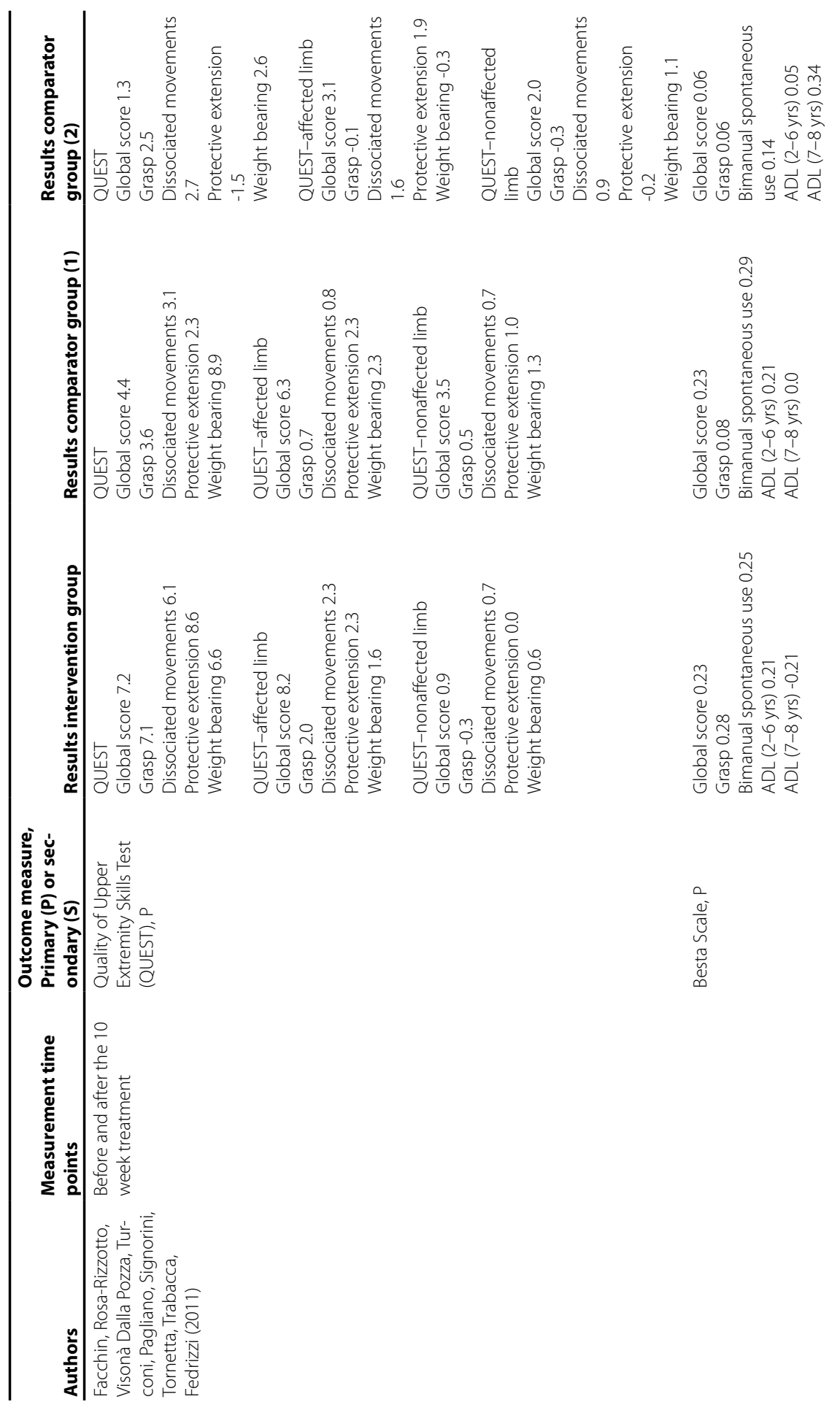




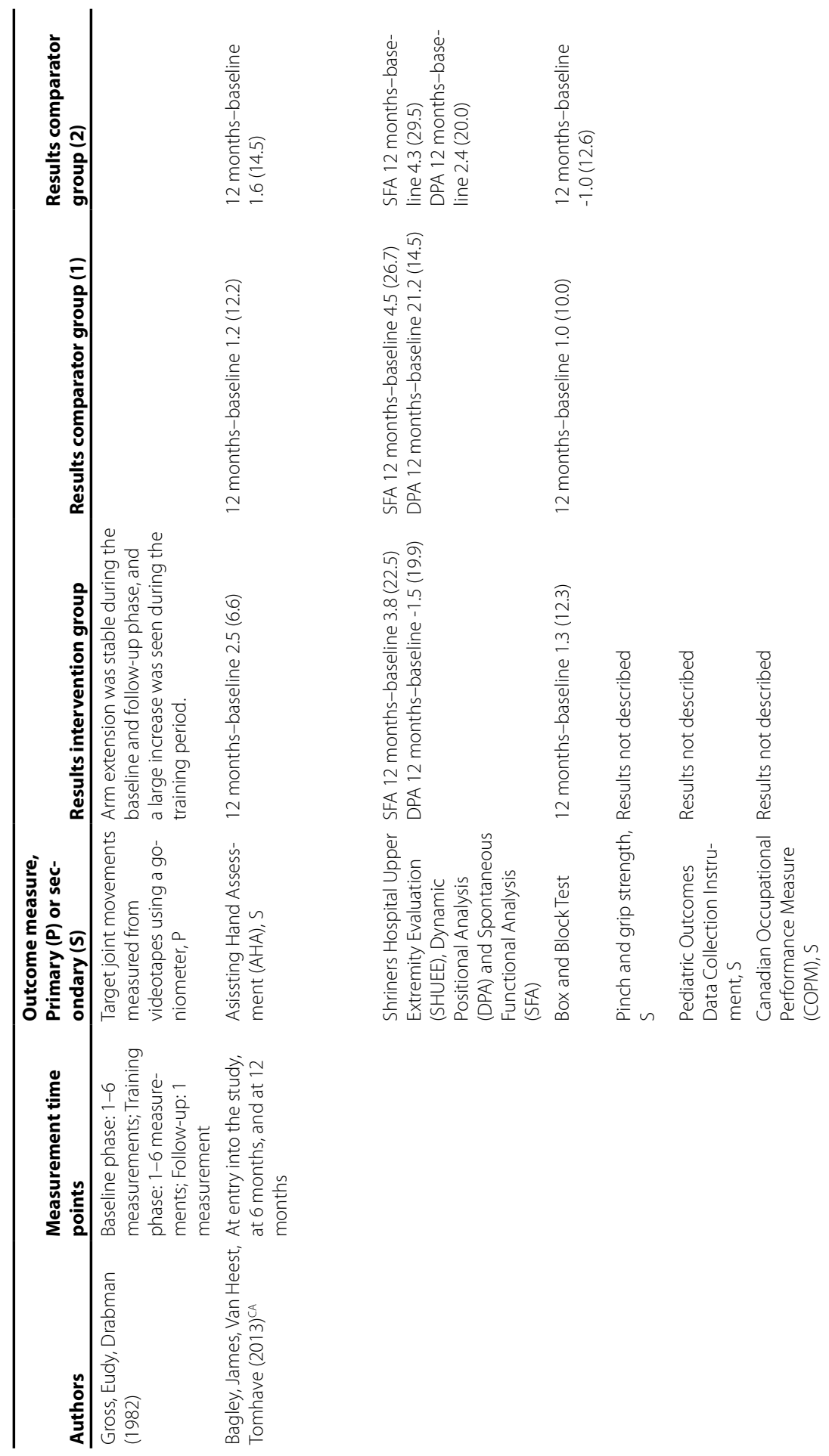



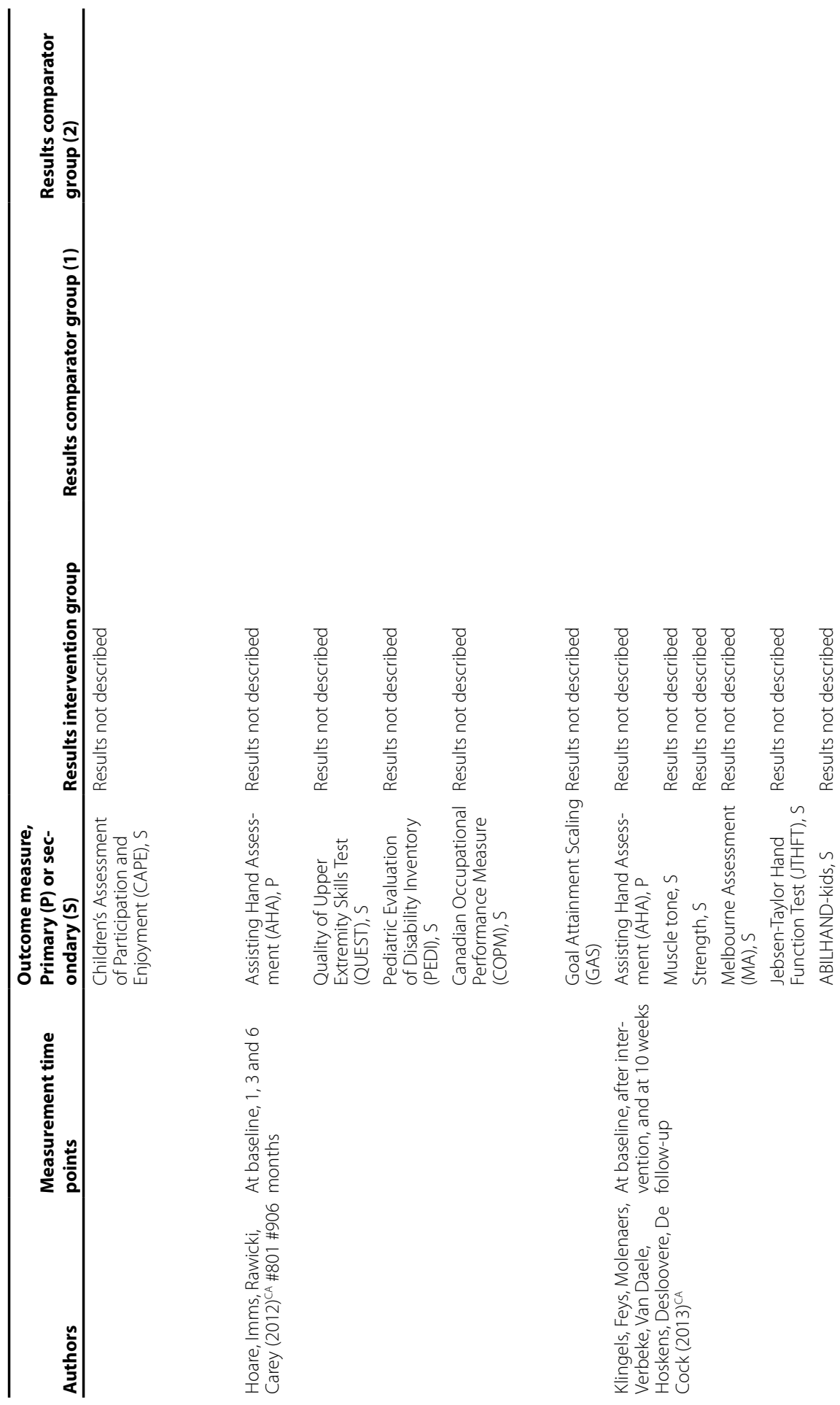


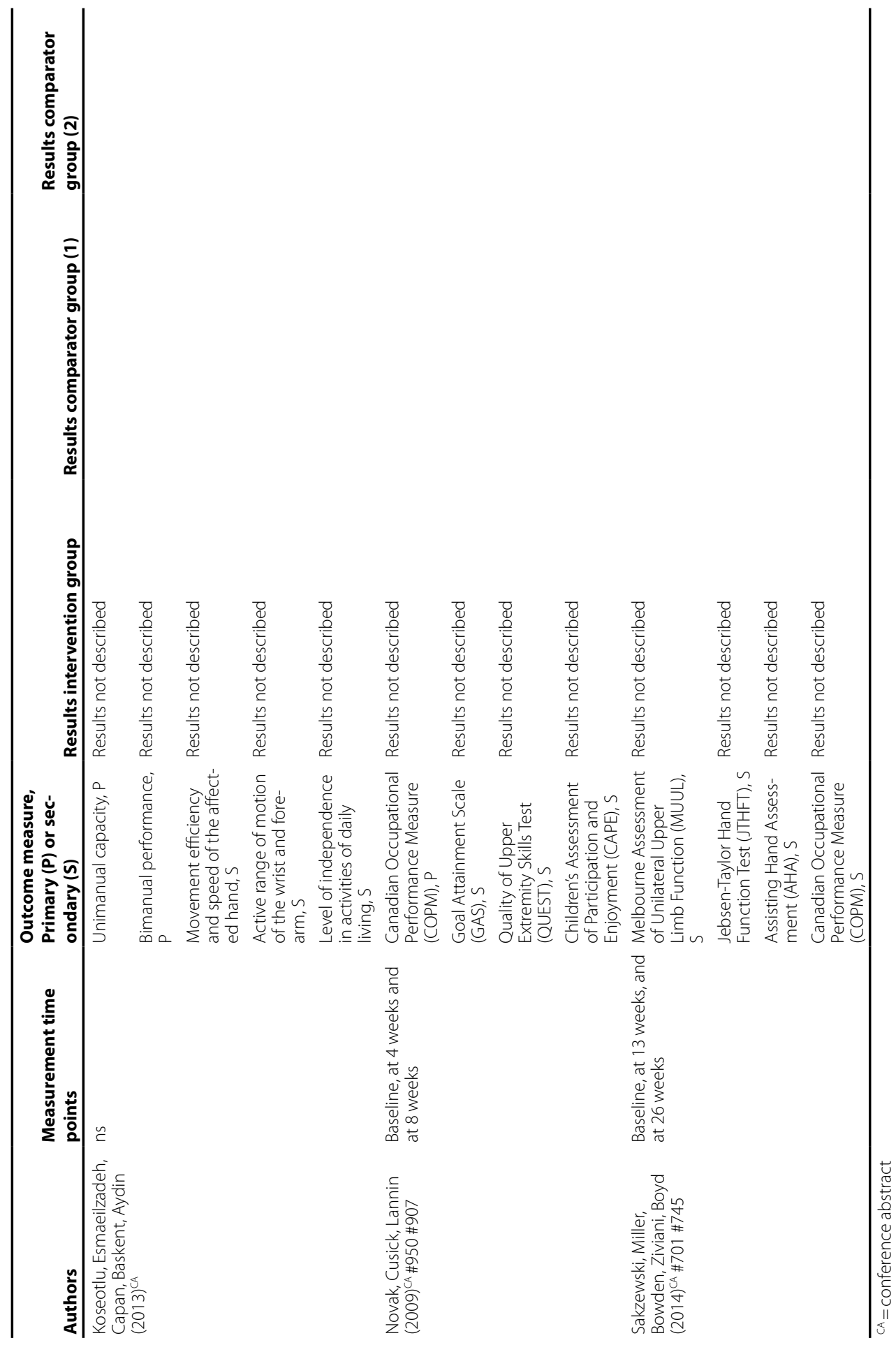




\section{Discussion}

This systematic review aimed to assess both feasibility and efficacy/effectiveness of home-based occupational therapy and physiotherapy programs in children with CP, specially focusing on the upper extremity. The objective was to investigate all relevant feasibility components according to Bowen et al. ${ }^{14}$, not only whether home programs were feasible in terms of compliance and adherence, as had been reported so far. However, the only feasibility outcomes mentioned to our opinion were implementation and acceptability. None of the included studies reported on aspects like satisfaction or practicality. Based on the implementation and acceptability results of the included studies, home-based training programs seem to be feasible. The overall compliance to home-based training programs was moderate to high, ranging from $62 \%$ to $96.1 \%$. Lorentzen and colleagues ${ }^{30}$, who found the lowest compliance, reported that technical problems and the fact that children were sometimes too tired or upset to complete the virtual reality training as main reasons for the difference between the actual amount and the intended amount of training. The high compliance reported by Ferre et al. ${ }^{26}$ may be due to the fact that they employed a strict selection of participants. Eleven parents and their child met the inclusion criteria and were willing to commit to the program requirements. One family dropped out after four weeks because the program was too demanding. Adjoining, they provided intensive coaching sessions to the parents. Overall the studies report that parents were positive about their experiences with the home programs. They found it easy to carry out the program and enjoyed seeing their children improve. However, there were also parents who found it difficult to incorporate the program in their daily life routine. Parents indicated that it was difficult to find enough hours in a day to perform the program next to their daily life activities. ${ }^{25}$ When the parent who delivered the program got support and help from other family members, it was easier for them to implement the training in their daily routine. ${ }^{36}$ Despite of these difficulties reported, general parental stress did not increase during the intervention. ${ }^{26} 28$

Conclusions about the effectiveness of home programs cannot be made because of the large variability in study, patient and intervention characteristics, comparators and outcome measures used in the included studies. Even within the same treatment approach, frequency and duration of the interventions varied. As training intensity is an important predictor for treatment success, an improvement in arm hand function and performance can therefore not be solely attributed to the intervention approach.

Many different treatment approaches were found in the included studies. The majority of the studies reported on the effectiveness of (modified) CIMT, whereas only one study ${ }^{26}$ investigated the effect of bimanual training. Both treatment approaches have shown to be effective in clinical rehabilitation. However, most activities in daily life require bimanual use of the hands. Therefore, an intervention focusing on the coordinated use of both hands in bimanual activities may have more impact on the child's daily life than a modified CIMT program focusing on improving the capacity of the affected hand.

According to Sakzewski et al. ${ }^{6}$, upper limb interventions in children with UCP should be goal-directed, adequately dosed, and based on motor learning approaches that use activity-based therapy. Most studies found in this review did not specify whether their intervention was based 
on motor learning principles. Some studies indicated that they used shaping and repetitive task practice, implying that the intervention was based on motor learning principles. The question which motor learning approach in the specific context of parent-delivered programs is the best suitable, remains, therefore, unanswered. Protocols from existing intramural programs may not always be feasible in a home setting, where parents are supervising the training of the child. They need to instruct their children and prompt the use of the affected upper extremity over and over again. Continuous prompting may pose an important stress factor on parents. ${ }^{89}$ Studies on basic motor learning in children with movement disorders have shown that implicit motor learning has positive effects on motivation and compliance and may therefore be better suited for a home setting. ${ }^{90-92}$ There is also evidence indicating that children with CP often have problems with working memory making it difficult for them to learn in an instruction-driven way. ${ }^{93}$ Moreover, implicit learning may lead to increased self-efficacy, which is important for motivation and compliance. Parents and clinicians rate motivation as the most influential personal characteristic, determining outcome and treatment adherence. ${ }^{94}$ An implicit motor learning approach seems very promising and should be explored in future studies.

Coaching of parents is a key element of home-based training programs. When parents are effectively coached by therapists and guided throughout the training period, parents become more confident in carrying out the home-based training program and find it easier to implement the program in their daily routine. ${ }^{1236}$ Surprisingly, information on how parents were coached to be therapy providers was lacking in most of the reported studies. Perhaps coaching received little attention during the interventions. Information on parent characteristics was also hardly given. Inferences about why some parents find it easy to carry out a home program while others struggle with finding ways to do so, for example, cannot be made. The fact that only two studies ${ }^{26}$

${ }^{49}$ reported on a parent-related outcome measure is also surprising given the major role of parents in the execution of a home-based program.

In conclusion one can state that a detailed description of home-based training protocols in most intervention studies is lacking. An extensive description of the interventions tested may take up a lot of words, but provides crucial information that increases our understanding on the working mechanism of an intervention. We therefore plea in favor of writing a protocol papers before publishing results.

\section{STUDY LIMITATIONS}

Due to the large variability in study, participants and intervention characteristics as well as child-related outcome measures found in the included studies, a meta-analysis on outcome measures was not possible. Although home-based training seems to be promising as most studies showed positive changes in child-related outcome measures, hard evidence on the effectiveness of these programs cannot be given. This also means that guidelines to improve the existing home-based programs or to develop new home programs are still to be awaited. As no synthesis of evidence was possible, the Grading of Recommendations Assessment, Development and Evaluation (GRADE) guidelines to judge the quality of evidence was not relevant and could not be used. ${ }^{95}$ With this, the review deviates from the protocol published by Beckers et al. ${ }^{17}$ 
Recommendations for future research would be to develop a core-set of outcome measures incorporating all levels of the ICF-CY to investigate the effects of interventions. Furthermore, parent-related characteristics, intervention elements and outcome measures should be part of and described in detail in studies investigating home-based training programs. Finally, future studies should focus on the comparison of two different home-based programs using a different motor learning approach while keeping aforementioned characteristics the same. 


\section{REFERENCES}

1. Oskoui M, Coutinho F, Dykeman J, et al. An update on the prevalence of cerebral palsy: a systematic review and meta-analysis. Developmental medicine and child neurology 2013;55(6):509-19.

2. Sellier E, Platt MJ, Andersen GL, et al. Decreasing prevalence in cerebral palsy: a multi-site European population-based study, 1980 to 2003. Developmental medicine and child neurology 2016;58(1):85-92.

3. Krigger KW. Cerebral Palsy: An Overview. American Family Physician 2006;73(1):91-100.

4. Arner M, Eliasson AC, Nicklasson S, et al. Hand function in cerebral palsy. Report of 367 children in a population-based longitudinal health care program. J Hand Surg Am 2008;33(8):1337-47.

5. van Eck M, Dallmeijer AJ, van Lith IS, et al. Manual ability and its relationship with daily activities in adolescents with cerebral palsy. J Rehabil Med 2010;42(5):493-8.

6. Sakzewski L, Ziviani J, Boyd RN. Efficacy of upper limb therapies for unilateral cerebral palsy: a meta-analysis. Pediatrics 2014;133(1):e175-204.

7. Milton Y, Roe S. Occupational therapy home programmes for children with unilateral cerebral palsy using bimanual and modified constraint induced movement therapies: A critical review. British Journal of Occupational Therapy 2016;80(6):337-49.

8. Novak I, Berry J. Home program intervention effectiveness evidence. Physical \& Occupational Therapy in Pediatrics 2014;34(4):384-9.

9. Fetters $L$, Kluzik J. The effects of neurodevelopmental treatment versus practice on the reaching of children with spastic cerebral palsy. Phys Ther 1996;76(4):346-58.

10. An M, Palisano RJ, Yi CH, et al. Effects of a Collaborative Intervention Process on Parent-Therapist Interaction: A Randomized Controlled Trial. Phys Occup Ther Pediatr 2018;20:1-17.

11. Novak I, Cusick A. Home programmes in paediatric occupational therapy for children with cerebral palsy: Where to start? Australian Occupational Therapy Journal 2006;53(4):251-64.

12. Novak I. Parent experience of implementing effective home programs. Physical \& Occupational Therapy in Pediatrics 2011;31(2):198-213.

13. Beckers LWME, Smeets RJEM, van der Burg JJW. Therapy-related Stress in Parents of Children with a Physical Disability: a Conceptual Analysis. submitted.

14. Bowen DJ, Kreuter M, Spring B, et al. How we design feasibility studies. Am J Prev Med 2009;36:452-7.

15. Novak I, Cusick A, Lowe K. A pilot study on the impact of occupational therapy home programming for young children with cerebral palsy. American Journal of Occupational Therapy 2007;61 (4):463-8.

16. Law M, King G. Parent compliance with therapeutic interventions for children with cerebral palsy. Developmental Medicine \& Child Neurology 1993;35(11):983-90.

17. Beckers LWME, Schnackers MLAP, Janssen-Potten YJ, et al. Feasibility and effect of home-based therapy programs for children with cerebral palsy: a protocol for a systematic review. BMJ Open 2017;7(e013687).

18. AACPDM. Methodology to Develop Systematic Reviews of Treatment Interventions (Revision 1.2. Secondary Methodology to Develop Systematic Reviews of Treatment Interventions (Revision 1.22008. https://http://www.aacpdm.org/UserFiles/file/systematic-review-methodology.pdf 
19. Organization WH. Towards a common language for functioning, disability and health. ICF: the international classification of functioning, disability and health. Secondary Towards a common language for functioning, disability and health. ICF: the international classification of functioning, disability and health. 2002. http://www.who.int/classifications/icf/icfbeginnersguide.pdf.

20. Institute TJB. The Joanna Briggs Institute Critical Appraisal tools for use in JBI systematic reviews. Checklist for qualitative research. Secondary The Joanna Briggs Institute Critical Appraisal tools for use in JBI systematic reviews. Checklist for qualitative research. 2016. http://joannabriggs.org/assets/docs/ critical-appraisal-tools/JBI_Critical_Appraisal-Checklist_for_Qualitative_Research.pdf

21. Downs SH, Black N. The feasibility of creating a checklist for the assessment of the methodological quality both of randomised and non-randomised studies of healthcare interventions. Epidemiol Community Health 1998;52:377-84.

22. O'Connor SR, Tully MA, Ryan B, et al. Failure of a numerical quality assessment scale to identify potential risk of bias in a systematic review: a comparison study. BMC research notes 2015;8:224.

23. Ahl LE, Johansson E, Granat T, et al. Functional therapy for children with cerebral palsy: an ecological approach. Developmental Medicine \& Child Neurology 2005;47(9):613-9.

24. Basaran A, Karadavut Kl, Uneri SO, et al. Adherence to home exercise program among caregivers of children with cerebral palsy. Turkiye Fiziksel Tip ve Rehabilitasyon Dergisi 2014;60(2):85-91.

25. Fergus A, Buckler J, Farrell J, et al. Constraint-induced movement therapy for a child with hemiparesis: a case report. Pediatric Physical Therapy 2008;20(3):271-83.

26. Ferre $C L$, Brandao MB, Hung YC, et al. Feasibility of caregiver-directed home-based hand-arm bimanual intensive training: a brief report. Developmental neurorehabilitation 2015;18(1):69-74.

27. Halvarsson S, Asplund R, Fjellman-Wiklund A. From authority to coach Parents' experiences of stretching as a home programme for children with cerebral palsy. Advances in Physiotherapy 2010;12(4):208-16.

28. Hinojosa J, Anderson J. Mothers' perceptions of home treatment programs for their preschool children with cerebral palsy. American Journal of Occupational Therapy 1991;45(3):273-9.

29. James S, Ziviani J, King G, et al. Understanding Engagement in Home-Based Interactive Computer Play: Perspectives of Children With Unilateral Cerebral Palsy and Their Caregivers. Physical \& Occupational Therapy in Pediatrics 2016;36(4):343-58.

30. Lorentzen J, Greve LZ, Kliim-Due M, et al. Twenty weeks of home-based interactive training of children with cerebral palsy improves functional abilities. BMC Neurology 2015;15:75.

31. McBurney H, Taylor NF, Dodd KJ, et al. A qualitative analysis of the benefits of strength training for young people with cerebral palsy. Developmental Medicine \& Child Neurology 2003;45(10):658-63.

32. Peplow UC, Carpenter C. Perceptions of parents of children with cerebral palsy about the relevance of, and adherence to, exercise programs: a qualitative study. Physical \& Occupational Therapy in Pediatrics 2013;33(3):285-99.

33. Piggot J, Hocking C, Paterson J. Parental adjustment to having a child with cerebral palsy and participation in home therapy programs. Physical \& Occupational Therapy in Pediatrics 2003;23(4):5-29.

34. Piggot J, Paterson J, Hocking C. Participation in home therapy programs for children with cerebral palsy: a compelling challenge. Qualitative Health Research 2002;12(8):1112-29.

35. Psychouli P, Kennedy CR. Modified Constraint-Induced Movement Therapy as a Home-Based Intervention for Children With Cerebral Palsy. Pediatric Physical Therapy 2016;28(2):154-60. 
36. Ross $\mathrm{K}$, Thomson D. An evaluation of parents' involvement in the management of their cerebral palsy children. Physiotherapy 1993;79(8):561-65.

37. Sandlund M, Dock K, Hager CK, et al. Motion interactive video games in home training for children with cerebral palsy: parents' perceptions. Disability \& Rehabilitation 2012;34(11):925-33.

38. Sandlund M, Waterworth EL, Hager C. Using motion interactive games to promote physical activity and enhance motor performance in children with cerebral palsy. Developmental neurorehabilitation 2011;14(1):15-21.

39. Sevick M, Eklund E, Mensch A, et al. Using Free Internet Videogames in Upper Extremity Motor Training for Children with Cerebral Palsy. Behav Sci (Basel) 2016;6(2).

40. Taylor NF, Dodd KJ, McBurney H, et al. Factors influencing adherence to a home-based strength-training programme for young people with cerebral palsy. Physiotherapy 2004;90(2):57-63.

41. Bilde PE, Kliim-Due M, Rasmussen B, et al. Individualized, home-based interactive training of cerebral palsy children delivered through the Internet. BMC Neurology 2011;11:32.

42. Al-Oraibi S, Eliasson AC. Implementation of constraint-induced movement therapy for young children with unilateral cerebral palsy in Jordan: a home-based model. Disability \& Rehabilitation 2011;33(2122):2006-12.

43. Eugster-Buesch F, de Bruin ED, Boltshauser E, et al. Forced-use therapy for children with cerebral palsy in the community setting: a single-blinded randomized controlled pilot trial. Journal of Pediatric Rehabilitation Medicine 2012;5(2):65-74.

44. Hsin YJ, Chen FC, Lin KC, et al. Efficacy of constraint-induced therapy on functional performance and health-related quality of life for children with cerebral palsy: a randomized controlled trial. Journal of Child Neurology 2012;27(8):992-9.

45. Hoare B, Imms C, Villanueva E, et al. Intensive therapy following upper limb botulinum toxin A injection in young children with unilateral cerebral palsy: a randomized trial. Developmental medicine and child neurology 2012;55(3):238-47.

46. James S, Ziviani J, Ware RS, et al. Randomized controlled trial of web-based multimodal therapy for unilateral cerebral palsy to improve occupational performance. Developmental Medicine \& Child Neurology 2015;57(6):530-8.

47. Kirkpatrick E, Pearse J, James P, et al. Effect of parent-delivered action observation therapy on upper limb function in unilateral cerebral palsy: a randomized controlled trial. Developmental Medicine \& Child Neurology 2016;58(10):1049-56.

48. Klingels K, Feys H, Molenaers $G$, et al. Randomized trial of modified constraint-induced movement therapy with and without an intensive therapy program in children with unilateral cerebral palsy. Neurorehabilitation \& Neural Repair 2013;27(9):799-807.

49. Lin KC, Wang TN, Wu CY, et al. Effects of home-based constraint-induced therapy versus dose-matched control intervention on functional outcomes and caregiver well-being in children with cerebral palsy. Research in Developmental Disabilities 2011;32(5):1483-91.

50. Lowes LP, Mayhan M, Orr T, et al. Pilot study of the efficacy of constraint-induced movement therapy for infants and toddlers with cerebral palsy. Physical \& Occupational Therapy in Pediatrics 2014;34(1):4-21.

51. Novak I, Cusick A, Lannin N. Occupational therapy home programs for cerebral palsy: double-blind, randomized, controlled trial. Pediatrics 2009;124(4):e606-14. 
52. Preston N, Weightman A, Gallagher J, et al. A pilot single-blind multicentre randomized controlled trial to evaluate the potential benefits of computer-assisted arm rehabilitation gaming technology on the arm function of children with spastic cerebral palsy. Clinical Rehabilitation 2016;30(10):1004-15.

53. Sakzewski L, Miller L, Ziviani J, et al. Randomized comparison trial of density and context of upper limb intensive group versus individualized occupational therapy for children with unilateral cerebral palsy. Developmental Medicine \& Child Neurology 2015;57(6):539-47.

54. Charles JR, Wolf SL, Schneider JA, et al. Efficacy of a child-friendly form of constraint-induced movement therapy in hemiplegic cerebral palsy: a randomized control trial. Developmental medicine and child neurology 2006;48(8):635-42.

55. Gordon AM, Hung YC, Brandao M, et al. Bimanual training and constraint-induced movement therapy in children with hemiplegic cerebral palsy: a randomized trial. Neurorehabilitation and neural repair 2011;25(8):692-702.

56. Wallen M, Ziviani J, Naylor O, et al. Modified constraint-induced therapy for children with hemiplegic cerebral palsy: a randomized trial. Developmental medicine and child neurology 2011;53(12):1091-9.

57. Chen HC, Chen CL, Kang $L$, et al. Improvement of upper extremity motor control and function after home-based constraint induced therapy in children with unilateral cerebral palsy: immediate and long-term effects. Archives of Physical Medicine \& Rehabilitation 2014;95(8):1423-32.

58. Chiu H-C, Ada L, Lee H-M. Upper limb training using wii sports resort ${ }^{\text {TM }}$ for children with hemiplegic cerebral palsy: A randomized, single-blind trial. Clinical Rehabilitation 2014;28(10):1015-24.

59. Crocker MD, MacKay-Lyons M, McDonnell E. Forced use of the upper extremity in cerebral palsy: a single-case design. American Journal of Occupational Therapy 1997;51(10):824-33.

60. Kim DA, Lee JA, Hwang PW, et al. The Effect of Comprehensive Hand Repetitive Intensive Strength Training (CHRIST) Using Motion Analysis in Children with Cerebral Palsy. Annals of Rehabilitation Medicine 2012;36(1):39-46.

61. Naylor CE, Bower E. Modified constraint-induced movement therapy for young children with hemiplegic cerebral palsy: a pilot study. Developmental Medicine \& Child Neurology 2005;47(6):365-9.

62. Xu K, He L, Mai J, et al. Muscle Recruitment and Coordination following Constraint-Induced Movement Therapy with Electrical Stimulation on Children with Hemiplegic Cerebral Palsy: A Randomized Controlled Trial. PLoS ONE [Electronic Resource] 2015;10(10):e0138608.

63. Abd El-Kafy EM, Elshemy SA, Alghamdi MS. Effect of constraint-induced therapy on upper limb functions: a randomized control trial. Scandinavian journal of occupational therapy 2014;21(1):11-23.

64. Coker P, Lebkicher C, Harris $L$, et al. The effects of constraint-induced movement therapy for a child less than one year of age. NeuroRehabilitation 2009;24(3):199-208.

65. Facchin P, Rosa-Rizzotto M, Visona Dalla Pozza L, et al. Multisite trial comparing the efficacy of constraint-induced movement therapy with that of bimanual intensive training in children with hemiplegic cerebral palsy: postintervention results. American journal of physical medicine \& rehabilitation 2011;90(7):539-53.

66. Gross AM, Eudy C, Drabman S. Training Parents to Be Physical Therapists with Their Physically Handicapped Child. Journal of Behavioral Medicine 1982;5(3):321-27.

67. Ferre CL, Hung Y, Brandao M, et al. Home-based bimanual training for young children with hemiplegia: Is it feasible to train caregivers as interventionists? Developmental medicine and child neurology 2013;Conference End: 20131019. Conference Publication: (var.pagings). 55:80. 
68. James S, Ziviani J, Boyd RN. Delivering online home-based rehabilitation: Insights from children with cerebral palsy and their caregivers. Developmental medicine and child neurology 2014;Conference End: 20140314. Conference Publication: (var.pagings). 56:15-16.

69. Novak I, Cusick A, Lannin N. Parent experience of implementing home programs: Semi-structured interviews. Developmental medicine and child neurology 2010;Conference End: 20100306. Conference Publication: (var.pagings). 52:51-52.

70. Boyd R, Mitchell L, Ziviani J, et al. Move it to improve it (Mitii)-Feasibility of a novel web-based therapy for children and adolescents with cerebral palsy. Developmental medicine and child neurology 2012; Conference End: 20120602. Conference Publication: (var.pagings). 54:76-77.

71. Dizmek P, Kara OK, Mutlu A, et al. Investigation of compliance to home based programme of families who have children with cerebral palsy, Serebral palsili cocuklarda ev programma aile uyumunun arastirilmasi. [Turkish, English]. Fizyoterapi Rehabilitasyon 2010;Conference End: 20101009. Conference Publication:(var.pagings). 21 (3):204.

72. Fehlings D, Chau T, Agarwal P, et al. A prospective case series evaluating the effectiveness of a virtual reality therapy home-based system to improve hand function in children with hemiplegia. Developmental medicine and child neurology 2009;Conference End: 20090926. Conference Publication: (var. pagings). 51:55.

73. Gerhardy A, Sandelance M. Demystifying home programmes: Resource development for families and clinicians to enhance upper limb intervention and outcomes in children. Developmental medicine and child neurology 2014;Conference End: 20140314. Conference Publication: (var.pagings). 56:73.

74. McCoy S, Lubetzsky-Vilnai A, Moritz C. Exploration of technology use for enjoyable task-specific practice to improve selective volitional muscle activation in children with cerebral palsy. Physiotherapy (United Kingdom) 2011;Conference End: 20110623. Conference Publication: (var.pagings). 97:eS777-eS78.

75. Pasquet T, Gaillard F, Newman CJ, et al. Feasibility of a self-rehabilitation program by mirror therapy in children with hemiplegic cerebral palsy. Annals of Physical \& Rehabilitation Medicine 2016;Conference Publication: (var.pagings). 595:e9.

76. Boyd R, Mitchell L, James S, et al. Multimodal training: Is the sum greater than the parts? Developmental medicine and child neurology 2014;Conference End: 20140705. Conference Publication: (var. pagings). 56:19.

77. James S, Ziviani J, Boyd RN. Efficacy of a web-based multimodal therapy program on occupational performance, upper limb function, and visual perception for children with unilateral cerebral palsy. Developmental medicine and child neurology 2014;Conference End: 20140913. Conference Publication: (var.pagings). 56:85-86.

78. Wallen M, Ziviani J, Evans R, et al. Modified constraint-induced therapy compared with intensive occupational therapy for children with hemiplegic cerebral palsy: Results of a randomised trial. Developmental medicine and child neurology 2012;Conference End: 20120602. Conference Publication: (var. pagings). 54:36-37.

79. Chiu H, Ada L, Lee H. Wii upper limb training in children with hemiplegic cerebral palsy (a randomized, singleblind trial). Developmental medicine and child neurology 2013;Conference End: 20131012. Conference Publication: (var.pagings). 55:31. 
80. Bagley A, James M, Van Heest A, et al. Is tendon transfer surgery in upper extremity cerebral palsy more effective than botulinum toxin injections or regular ongoing therapy? Developmental medicine and child neurology 2013;Conference End: 20131019. Conference Publication: (var.pagings). 55:82.

81. Hoare B, Imms C, Rawicki HB, et al. Bimanual occupational therapy is as effective as modified constraint-induced therapy following Botulinum toxin-A in young children with hemiplegic cerebral palsy. Developmental medicine and child neurology 2012;Conference End: 20120602. Conference Publication: (var.pagings). 54:37-38.

82. Hoare B, Imms C, Rawicki HB, et al. Intensive upper limb therapy following Botulinum toxin-A in young children with hemiplegic cerebral palsy: Results from a randomised controlled trial. Developmental medicine and child neurology 2010;Conference End: 20100306. Conference Publication: (var.pagings). 52:13-14.

83. Klingels K, Feys $\mathrm{H}$, Molenaers $\mathrm{G}$, et al. Randomized trial of modified constraint-induced movement therapy with and without an intensive therapy programme in children with unilateral cerebral palsy. Developmental medicine and child neurology 2013;Conference End: 20131012. Conference Publication: (var.pagings). 55:18.

84. Koseotlu PK, Esmaeilzadeh S, Capan N, et al. Comparison of the effects of modified constraint induced movement therapy combined with bimanual training, with modified constraint movement therapy approaches in the upper extremity functions in children with unilateral cerebral palsy, Unilateral Serebral Palsi'li Cocuklarda Modifiye Zorunlu Kullanim Terapisi ile Kombine Bimanuel Etitim ve Yalnizca Modifiye Zorunlu Kullanim Terapisi Yaklasimlarinin Ust Ekstremite Fonksiyonlari Uzerine Etkilerinin Karsilastirilmasi. [Turkish, English]. Turkiye Fiziksel Tip ve Rehabilitasyon Dergisi 2013;Conference End: 20130331. Conference Publication: (var.pagings). 59:232.

85. Novak I, Cusick A, Lannin N. Effectiveness of home program intervention for children with cerebral palsy: A double blinded randomised controlled trial. Developmental medicine and child neurology 2009;Conference End: 20090221. Sponsor: Allergan Australia . Conference Publication: (var.pagings). 51:13-14.

86. Novak M, Cusick A, Lannin N. Effectiveness of home program intervention for children with cerebral palsy: A double-blind randomised controlled trial. Developmental medicine and child neurology 2010;Conference End: 20100306. Conference Publication: (var.pagings). 52:12-13.

87. Sakzewski L, Miller L, Bowden J, et al. Randomised comparison trial of upper limb intensive group compared to individualised training for children with congenital hemiplegia. Developmental medicine and child neurology 2014;Conference End: 20140314. Conference Publication: (var.pagings). 56:55-56.

88. Boyd RN, Sakzewski L, Miller L, et al. Randomised trial of the density and context of upper limb intensive group compared to individualised training for children with congenital hemiplegia. Developmental medicine and child neurology 2013;Conference End: 20131019. Conference Publication: (var.pagings). 55:8.

89. van der Burg JJW, Aarts PBM, Steenbergen B. So you wanna be a popstar? Van'Handig2Handig'naar'Handig2HandigZelfstandig'! Nederlands Tijdschrift voor Revalidatiegeneeskunde 2013;35:125-26.

90. Dweck CS, Leggett EL. A social-cognitive approach to motivation and personality. Psychological review 1988;95(2):256.

91. Steenbergen B, van der Kamp J, Verneau M, et al. Implicit and explicit learning: applications from basic research to sports for individuals with impaired movement dynamics. Disabil Rehabil 2010;32(18):1509-16. 
92. Maxwell JP, Masters RSW, Kerr E, et al. The implicit benefit of learning without errors. Journal of Experimental Psychology Section A 2001;54(4):1049-68.

93. Jongbloed-Pereboom M, Janssen AJ, Steenbergen B, et al. Motor learning and working memory in children born preterm: a systematic review. Neurosci Biobehav Rev 2012;36(4):1314-30.

94. Majnemer A, Shevell M, Law M, et al. Level of motivation in mastering challenging tasks in children with cerebral palsy. Developmental Medicine \& Child Neurology 2010;52(12):1120-6.

95. Guyatt GH, Oxman AD, Schunemann HJ, et al. GRADE guidelines: a new series of articles in the Journal of Clinical Epidemiology. J Clin Epidemiol 2011;64(4):380-2. 
Chapter 4

\title{
Home-based bimanual training based on motor learning principles in children with unilateral cerebral palsy and their parents (the COAD-study): rationale and protocols
}

\author{
MLAP Schnackers*, LWME Beckers*, YJM Janssen-Potten, \\ PBM Aarts, EAA Rameckers, JJW van der Burg, IJM de Groot, \\ COAD Focus Group, RJEM Smeets, ACH Geurts, B Steenbergen \\ * contributed equally
}




\section{Abstract}

Background Home-based training is considered an important intervention in rehabilitation of children with unilateral cerebral palsy. Despite consensus on the value of home-based upper limb training, no evidence-based best practice exists. Promoting compliance of children to adhere to an intensive program while keeping parental stress levels low is an important challenge when designing home-based training programs. Incorporating implicit motor learning principles emerges to be a promising method to resolve this challenge.

Methods Here we describe two protocols for home-based bimanual training programs, one based on implicit motor learning principles and one based on explicit motor learning principles, for children with unilateral spastic cerebral palsy aged 2 through 7 years. Children receive goal-oriented, task-specific bimanual training in their home environment from their parents for $3.5 \mathrm{~h} /$ week for 12 weeks according to an individualized program. Parents will be intensively coached by a multidisciplinary team, consisting of a pediatric therapist and remedial educationalist. Both programs consist of a preparation phase (goal setting, introductory meetings with coaching professionals, design of individualized program, instruction of parents, home visit) and home-based training phase (training, video-recordings, registrations, and telecoaching and home visits by the coaching team). The programs contrast with respect to the teaching strategy, i.e. how the parents support their child during training. In both programs parents provide their child with instructions and feedback that focus on the activity (i.e. task-oriented) or the result of the activity (i.e. result-oriented). However, in the explicit program parents are in addition instructed to give exact instructions and feedback on the motor performance of the bimanual activities, whereas in the implicit program the use of both hands and the appropriate motor performance of the activity are elicited via manipulation of the organization of the activities.

Discussion With the protocols described here, we aim to take a next step in the development of much needed evidence-based home-based training programs for children with unilateral cerebral palsy.

Keywords Cerebral palsy, Rehabilitation, Upper extremity, Home program, Task-specific training, Implicit motor learning, Explicit motor learning, Bimanual performance, Parental stress 


\section{BACKGROUND}

Cerebral palsy (CP) is a group of neurodevelopmental disorders of movement and posture $[1,2]$. About one-third of children with CP experiences motor impairments predominantly affecting one side of the body, i.e. unilateral CP (UCP) [3], with impaired upper limb functioning as one of the most disabling symptoms [4]. Although the condition of CP is static, upper limb functioning is amendable to change, owing to the plasticity of the central nervous system [5]. Plasticity is the major entry point for the many rehabilitation programs that focus on improving upper limb functioning in these children [6]. This appears from studies on the effectiveness of centre-based rehabilitation programs for improving upper limb functioning such as goal-directed training (e.g. $[7,8])$, constraint-induced movement therapy (CIMT) (e.g. [9, 10]), and hand-arm bimanual intensive training (HABIT) (e.g. [11, 12]). Studies examining these programs have shown that the key ingredients for effective treatment constitute high training intensity combined with meaningful, task-specific, bimanual training [13]. Crucially, in order to reach this high intensity, training needs to be motivating for the child, and accommodated to the child's capabilities $[11,14,15]$.

An important next step in rehabilitation practice is (the continuation of) training in the child's daily life and home situation, such that empowerment of parents and independency from healthcare professionals of the parents and child are promoted. Furthermore, learning skills in the natural environment has been suggested to lead to better generalization of therapy effects [16]. In recent years, home-based training programs have been developed that have shown effectiveness [17]. However, at the same time these programs exemplified two important challenges that warrant further study in order for these programs to be feasible in the long term: 1) limit the therapy-related stress for the parents, and 2) promote compliance in children to adhere to an intensive program that involves repetitive practice $[18,19]$.

A critical remark on existing pediatric rehabilitation programs is their unspecified description and undifferentiated use of motor learning principles to train the children, i.e. explicit or implicit motor learning. In explicit motor learning conscious aspects of the motor learning process are targeted in particular, whereas in implicit motor learning especially non-conscious aspects of the motor learning process are targeted $[20,21]$. Generally, a combination of implicit and explicit motor learning is used in therapy programs in clinical practice, but the main focus is on explicit principles [22]. In home-based training programs based on explicit motor learning principles, parents need to prompt the use of the affected side over and over again to maintain a high training intensity. This continuous prompting may impose an important stress factor upon parents [23], possibly reducing the motivation of both the parents and the child. A more feasible method for home-based training may be implicitly eliciting the (proper) use of the affected hand. In this way, the burden on parents to continuously prompt their child may be reduced. In addition, studies on basic motor learning in children with movement disorders have shown that implicit motor learning has positive effects on motivation [24, 25] and self-efficacy [26]. As a consequence, the increased motivation of the child to keep practicing may reduce parental stress levels, because they are less involved in continuously prompting their child. 
Up until now, these promising advantages of implicit motor learning for home-based training have not been systematically studied. To enable this we have developed two home-based bimanual training programs. In this paper we present the protocols for two home-based training programs for young children with UCP, based on either implicit or explicit motor learning principles. A detailed description of the interventions is provided, in order to promote understanding of the content and to facilitate future research.

\section{METHODS/DESIGN}

The description of the protocols follows the Template for Intervention Description and Replication (TIDieR) guide [27].

\section{GENERAL DESCRIPTION}

The two interventions described in this protocol are:

- a home-based bimanual training program based on implicit motor learning principles;

- a home-based bimanual training program based on explicit motor learning principles.

The overall aim of both home-based training programs is to improve the bimanual skills of the child whilst minimizing the increase of therapy-related parental stress.

The target population of the home-based training programs is children with unilateral spastic CP aged 2 through 7 years with Manual Ability Classification System (MACS) level I-III [28] and Gross Motor Function Classification System (GMFCS) level I-III [29]. A physiatrist will determine whether the intervention is applicable for a child and the parents. One or two caregivers (either the parents or significant others, for example a grandparent) will participate in the training, which will be determined in consultation with members of the rehabilitation team involved, before the start of the program. For reason of readability we will use the word 'parents' in this paper.

The execution of the home-based training programs follows a triple action approach (Fig. 1). Since previous studies have indicated that support by professionals promotes the feasibility of home-based training [30,31], parents will be intensively coached by a multidisciplinary team, consisting of a pediatric therapist (occupational or physical therapist), and a remedial educationalist (or health care psychologist). As the focus of our home-based training programs is on the physical domain as well as the parent-child interaction and behavioral domain, parents will be coached by the therapist with regard to therapeutic content and implementation of the training in daily life, whereas the remedial educationalist will focus on the parent-child interaction and behavioral domain. In the programs, parents will teach the child new skills in the home environment. As a result, the child will learn new bimanual tasks in the needed context. 


\title{
Multidisciplinary team
}

\author{
】'soxhing \\ Parents \\ It teathing \\ Child 'learning'
}

Figure 1 Triple action approach in home-based training programs

The actual home-based training is focused on improving the child's bimanual performance of five personal rehabilitation goals (goal-oriented). To promote this process, a task-specific approach will be used, i.e. the activities will be consistent with the context of the particular goal. This task-specific approach is in agreement with the latest published version of the Dutch guidelines for treatment for children with spastic CP [32]. In these guidelines a task-specific intervention is defined as "the intervention is based on a task analysis aiming at practicing (sub-) activities that have been formulated in the goals" [32]. As proposed by Gordon [13] and based on the knowledge gained in centre-based programs, in both home-based training programs the same high intensity of bimanual training of meaningful task-specific activities is used.

Following the view on motor learning of Kleynen et al., the contrast between both programs is shaped by three elements: instructions, feedback and organization of the training [33].

Both home-based training programs consist of two phases, i.e. the preparation phase and the actual home-based training. Each phase comprises several intervention components (Table 1).

\section{Preparation of home-Based training}

The two-week preparation phase starts with setting goals for the home-based training. Next, introductory meetings between the parents and their child and the coaching professionals will take place. Thereafter the therapist will design the individualized program, while parents will be instructed concerning the home-based training. The last component of the preparation phase includes a home visit by the therapist. Each component will be elaborated on in the following paragraphs. 
Table 1 Overview of phases and intervention components

\begin{tabular}{ll}
\hline Phase & Intervention component \\
\hline Preparation of home-based training & Needs assessment and goal setting \\
& Introductory meetings with coaching professionals \\
& Design of individual program \\
& Instruction of parents \\
Home-based training & Home visit \\
& Training \\
& Video-recordings \\
& Registrations \\
& Telecoaching and home visits by the therapist \\
& Telecoaching by the remedial educationalist \\
\hline
\end{tabular}

\section{NeEdS ASSESSMENT AND GOAL SETTING}

The parents will prioritize five important needs on the domain of bimanual activities using the Canadian Occupational Performance Measure (COPM) child-adapted version [34, 35]. The COPM is a semi-structured interview for parents, in which they identify and rank their child's perceived problems in activities of self-care, play and/or leisure. The approach of this measure corresponds to the goal-oriented approach of the home-based training programs. The COPM has good psychometric properties [36, 37], which also apply to the child-adapted version [34]. The COPM will be administered over the phone by a therapist who is experienced in the COPM as well as in clinical practice in pediatric rehabilitation.

Later, the coaching therapist will transform the most important need into a SMART goal using video-recordings of the child's performance of the corresponding activity, and will use Goal Attainment Scaling (GAS) to formulate predetermined criteria for the progress towards the rehabilitation goal to be scored [38].

\section{INTRODUCTORY MEETINGS WITH COACHING PROFESSIONALS}

The parents and child will have a 60-min introductory meeting with the remedial educationalist and another 60-min meeting with the therapist at the rehabilitation centre to get acquainted and to discuss the professionals' role in the coaching team. Furthermore, the remedial educationalist will examine aspects of the interaction and behavioral domain that are of interest for the homebased training, for instance the parent-child interaction and organization of family life (e.g. weekly 
schedule and division of family responsibilities between parents). The therapist and parents will discuss the family situation and home environment, e.g. objects available that are related to the individual rehabilitation goals. In addition, the therapist will observe and make video-recordings of the child's performance of the activities corresponding to the needs.

\section{DESIGN OF INDIVIDUALIZED PROGRAM}

To enable a task-specific training program, several steps are followed. The treatment goals, based on the COPM and the video-recordings the therapist made of the child's performance of activities corresponding to the needs, as well as video-recordings of assessments, such as the Assisting Hand Assessment (AHA) [39], will provide the input. First, the coaching therapist will perform a task analysis based on stage 1 of the Perceive, Recall, Plan and Perform (PRPP) System of Task Analysis [40], by means of the video-recordings and an observation form. According to the PRPP system, each activity is separated into several logical successive steps of approximately equal size. As part of the task analysis, the therapist will evaluate the child's performance of each single activity step regarding four error types:

- errors of omission (such as omitting hooking the zipper before pulling it up);

- errors of repetition (such as grasping and releasing the zipper several times before pulling up the zipper);

- errors of accuracy (such as being unable to hook the zipper, by missing the hooker);

— errors of timing (such as the time needed to close the zipper being too long).

Consequently, the therapist will design an individualized program. The order in which the treatment goals will be addressed is jointly decided upon by therapist and parents. The therapy activities focus on the important steps with the accompanying errors that emerged from the task analysis. Progress is realized by increasing the complexity of performance in each relevant step emerged from the task analysis. This can be accomplished by adjustments to the initial posture of the child in which the activity is performed, the choice of objects (e.g. size of the zipper) or the environment in which the activity is carried out (e.g. alone or accompanied by others).

\section{INSTRUCTION OF PARENTS}

Parents will be instructed how to apply the home-based training according to the specific homebased training program through instruction videos and a manual. The instruction of the parents will address four topics, i.e. 1) the content of the home-based training program, 2) the teaching approach, 3) the support by the therapist and remedial educationalist during the home-based training program, and 4) the use of a digital communication tool that will be used for safe communication and exchange of documents and videos between parents and the therapist and remedial educationalist during the home-based training. 


\section{HOME VISIT}

The preparation phase ends by a home visit by the coaching therapist lasting approximately 90 min. During this visit, the therapist will discuss the general outline of the designed individualized program with the parents, the therapist will examine the particular home situation with the parents, and parents have the opportunity to ask questions.

\section{HOME-BASED TRAINING}

The second phase consists of concurrently the actual training, video-recordings and registrations created and shared by parents, and coaching of parents by the therapist and remedial educationalist through telecoaching and home visits. The components will be discussed consecutively.

\section{Training}

Children will receive $3.5 \mathrm{~h}$ per week (on average 30 min per day) of bimanual training, for 12 weeks in total. To support the task-specific approach, the home-based training will be performed in meaningful situations, embedded in family routines. To accommodate this, the hours of training can be divided across the week in training sessions with a minimum duration of $10 \mathrm{~min}$. The therapist and parents will select everyday objects or (therapeutic) toys that are appropriate for a specific rehabilitation goal. Use of objects and toys from the child's home situation is preferred. The therapist will strive for activities and objects that are varied and in line with the possibilities of the child, to encourage children and parents and avoid frustration or boredom.

Despite the fact that at the start of the program an estimation is being made whether the goals are realistic for 12 weeks of training, it is possible that goals have already been reached before the end of the program. Should this situation arise, the activities are being repeated in order to maintain and automate the achieved progress. However, for the training to remain challenging and motivating, parents and children may indicate one or two new bimanual rehabilitation goals. These goals will be trained during the remaining weeks of the program, in addition to the repetition of the five initial goals.

An interruption of the program for 1 week is allowed, for example due to holidays or illness. In the event of a one-week break, this week will be compensated for at the end of the home-based training program. If because of circumstances beyond one's control for more than 1 week cannot be trained, only 1 week will be made up for. The maximum duration of the home-based training program is therefore 13 weeks, namely 12 regular weeks and a maximum of one 'catch-up week'.

\section{VIDEO-RECORDINGS}

To enable the therapist and remedial educationalist to provide coaching that is tailored to the needs of the parents and child, parents will video-record a training session once a week. They will share the recordings with their coaching team through a digital communication tool. An extended and a short manual are available for both parents and the coaching team regarding the tool features needed for these interventions. 


\section{REGISTRATIONS}

Parents will register the training activities performed to obtain information about the actual intensity and content of the training. In both home-based training programs, parents register on a daily basis whether training activities were performed, and, if so, (1) how much time their child trained each rehabilitation goal, (2) the activities used to train each rehabilitation goal, and (3) particular details of that day (e.g. illness of the child). Daily registration time is expected to be approximately $5 \mathrm{~min}$. Moreover, on a weekly basis, parents register the experiences of both the child and the parent(s) with the training that week by means of emoticons. Parents are expected to need 5 additional minutes per weekly registration. The registrations will provide the coaching team with the information needed for remote coaching.

The training activities will be registered using a digital form (Excel-sheet) including written instructions. The registration form will be partially pre-filled by the therapist in order to 1) reduce the time burden and 2) to increase the chance on comprehensiveness of the data. Parents will share the registration forms with their coaching team by the digital communication tool.

\section{TELECOACHING AND HOME VISITS}

At the start of each week, scheduled contact moments with the parents and their coaching therapist will take place. During these contact moments, a small standard evaluation will take place, in which parents have the opportunity to ask questions, address problems regarding the support of the child during training, and indicate whether they need additional coaching by the remedial educationalist. During this evaluation, the registrations and video-recording of the training will be discussed. Furthermore, the content of the program for the upcoming week is formulated. This weekly schedule ensures that adaptations to the original plan are possible. These contacts will be mainly over the phone, lasting approximately $30 \mathrm{~min}$. Additionally, two times a home visit takes place, lasting approximately 60 min. The home visits will take place in week 5 and week 9 of the home-based training. During these home visits, the therapist will select objects and toys from the child's home situation for the training in the upcoming weeks and may provide additional objects if necessary. If desired by the therapist, it is allowed to schedule one extra home visit as a replacement for a telephone contact moment.

The remedial educationalist will contact the parents over the phone in the third week of the home-based training program. During this 30-min contact, the remedial educationalist discusses with the parents the process of home-based training, the parent-child interaction and, if applicable, the sources of stress and how to cope with these. Hereafter, the remedial educationalist exchanges the findings with and gives advice to the therapist. In case parents have a need for extra support by the remedial educationalist or if the coaching therapist indicates the necessity, an additional contact moment with the remedial educationalist can be planned during the 12week treatment period. 


\section{CONTRAST BETWEEN IMPLICIT PROGRAM AND EXPLICIT PROGRAM}

Both home-based training programs will contrast with respect to the teaching strategy, i.e. how the parents will support their child during training (Fig. 1). This is put into practice at the level of organization, instructions, and feedback [33] (Table 2).

Table 2 Contrast between implicit and explicit learning in home-based training programs

\begin{tabular}{lll}
\hline & Implicit & Explicit \\
\hline Instruction and feedback & - Task-oriented & - Focused on motor performance \\
& - Result-oriented & - Task-oriented \\
& & - Result-oriented \\
Organization & Eliciting & Prescribing \\
\hline
\end{tabular}

Parents participating in the implicit program will provide their child with instructions and feedback that solely focus on the activity itself (i.e. task-oriented) or the result of the activity (i.e. result-oriented) and that are aimed at motivating their child. A task-oriented instruction could for example be 'please focus on closing the zipper', whereas a result-oriented one would be 'now try to close the zipper within ten seconds'. No information on how the child actually performs or should perform the activity is provided. That is, no information is given related to the movements needed to accomplish the activity. In this home-based training program, the use of both hands and the appropriate motor performance of the activity are elicited via manipulation of the organization of the activities. The organization of the activities comprises for instance the type of objects used (e.g. size of the zipper), the position of the child (e.g. sitting on the floor), and the setting (e.g amount of distraction). Parents in this program will receive ideas from their coaching therapist, on activities and objects to elicit the proper bimanual performance by their child. Examples of corresponding instructions and feedback are also provided. During the home visits, the therapist will select a range of objects related to the rehabilitation goals that are available in the home environment and, if needed, additional objects.

Parents participating in the explicit program are instructed to give their child exact instructions and feedback on how to perform the bimanual activities, in addition to the instructions and feedback as described for the implicit program. In contrast to the implicit program, instructions in the explicit program are related to the movements needed to accomplish the activity, such as 'hold the bottom of the jacket with your left hand while pulling the zipper up with your right hand.' Parents will receive specific and elaborate exercises with the corresponding instructions from the coaching therapist. Only specific objects necessary for the execution of the exercises will be selected in the home environment, and, if needed, provided.

To enforce this contrast, the task analyses in the explicit program will be complemented by a movement analysis for each step in which a performance error is detected. This movement analysis will focus on the child's current performance (posture and movements) and the perfor- 
mance needed to complete the activity successfully. Based on this information, therapists operating in the explicit group can provide the parents with the instructions and feedback related to the appropriate motor performance.

\section{COURSE FOR THE THERAPISTS AND REMEDIAL EDUCATIONALISTS}

In order to coach the parents according to the specific home-based training program, therapists and remedial educationalists will be instructed by members of the research team. The course will take place prior to the start of inclusion of participants in the programs.

Therapists will be instructed during a one-day course by a physical therapist and an occupational therapist who have extensive experience with clinical practice, research and education in the field of pediatric rehabilitation. To prevent contamination, the instruction of the therapists will be provided for each home-based training program separately. During the course, therapists will be instructed how to perform a task analysis based on stage 1 of the PRPP System of Task Analysis [40], how to design the individualized training programs, and how to coach the parents during the intervention period. In addition, the use of a digital communication tool that can be used for the communication and exchange of documents and videos with parents will be addressed.

The course for remedial educationalists will last half a day. A remedial educationalist of the research team who has extensive experience with clinical practice, research and education in the field of pediatric rehabilitation will instruct the remedial educationalists operating in the home-based programs on how the parents should be coached during the intervention period, and on how to use the digital communication tool for the communication and exchange of documents with parents.

One year after the first instruction, a refresher course will be organized for all practitioners. For questions, therapists can contact the research team at any time.

The home-based training programs are elaborated in manuals. In these manuals, instructions and checklists are provided for all parts of the home-based training programs.

\section{STUDY ORGANIZATION}

The interventions described in this protocol are part of the 'Co-creation at hand: the road to independence' (COAD) study. The COAD-study is a collaboration between Maastricht University, Adelante, Radboud University, Sint Maartenskliniek, and Radboud University Medical Center. The home-based training programs and study were designed in consultation with a focus group consisting of the director of the Dutch association of people with physical disabilities, parents of children with CP, an adolescent with CP, as well as rehabilitation physicians, occupational and physical therapists, and a remedial educationalist experienced with rehabilitation of children with CP.

A process evaluation will be performed, with which we aim to systematically evaluate the processes and factors that influence implementation and effects of our home-based training programs. The methods of the process evaluation are described in a parallel paper (Beckers $L$, van der Burg J, Janssen-Potten Y, Rameckers E, Aarts P, Smeets R: Process evaluation of two home- 
based bimanual training programs in children with unilateral cerebral palsy (the COAD-study): protocol for a mixed methods study, submitted). In addition, a case series study will be executed to investigate the effects of the programs on the level of the child and the impact on the parents.

\section{Discussion}

In this paper we present two protocols for intensive home-based bimanual training in young children with UCP and their parents. Home-based training is considered an important intervention for rehabilitation care of children with CP, now and in the future. In home-based training, children learn new skills in their natural environment. This has been suggested to lead to better generalization of therapy effects [16]. For parents of children with CP, home-based training offers the possibility to become more engaged in the therapy of their child. This is in line with the framework of 'family-centred care' in which care is built on partnerships between parents and professionals [41]. This framework is regarded as the gold standard in therapy for children with CP $[41,42]$ and is claimed to enhance health outcomes [41]. Moreover, home-based training is relevant from a societal perspective, as it may lead to a reduction of healthcare costs [43].

Despite consensus on the importance of home-based upper limb training for all those involved [18], no evidence-based best practice exists yet. We aim to take the next step in the development of effective home-based upper limb training programs for children with uCP that are feasible in daily life situations. In order to provide intensive training in the home situation, it is essential that children are motivated and parents experience little stress. Previous studies, however, showed an increase in parental stress and a reduction of therapy compliance over time [18, 19, 44]. Incorporation of implicit motor learning principles seems to be a promising method to prevent or reduce these adverse effects. That is, implicit motor learning is expected to lead to improved motivation for training in children [24, 25], and to a reduced burden on parents due to less need for prompting their child to use the affected arm and hand properly. Moreover, we strive to resolve the challenges encountered in previous studies by coaching the parents not only with respect to bimanual performance of their child, but also with regard to parent-child interaction during practicing. Given the nature of the challenges observed in previous studies, this additional coaching by the remedial educationalists may be crucial. Because of their specific expertise on parent-child interaction and behavioral domain, the remedial educationalists are expected to be valuable in supporting the parents in their new role and in advising them on how to support and motivate their child. This will be important to empower the parents in their new role to facilitate an intensive training program.

Training intensity has been a crucial consideration regarding the feasibility of our homebased training programs. Studies on centre-based programs have shown that a high training intensity, i.e. 60-90 h in total, is essential for the improvement of upper limb functioning in children with CP [13]. However, a crucial difference between centre-based and home-based programs is the role of the parents in their child's therapy. In contrast to centre-based programs, parents have a pivotal role in home-based programs as the facilitator(s) of their child's training. However, their role has to be fulfilled in combination with their roles as parents and wage earners. A previous study on home-based training has shown that if training hours are not standardized, children with CP and their parents train, on average, 1-1.5 h per week [16]. Combining these findings, we 
have strived to adopt a middle course resulting in a training intensity of $3.5 \mathrm{~h}$ per week for 12 weeks (42 $\mathrm{h}$ in total). Through this training intensity, we aim for a level of parental involvement that is feasible for a heterogeneous group of parents to be able to implement the home-based training programs in a larger population.

\section{ACKNOWLEdgements}

The authors would like to acknowledge the members of the COAD Focus Group for their valuable input. The COAD Focus Group consists of (in alphabetical order) Nicole Brouwers, Anke Defesche, Yvonne Geerdink, Bregtje Janssen, Marjon Kissels, Martijn Klem, Denise Martens, Judith van Munster, Bianca Olive, Marleen Philippens, Lucianne Speth, and Ingrid van den Tillaar.

\section{FUNDING AND SPONSOR}

The COAD-project is part of the 3rd Program Rehabilitation Research of ZonMw, the Netherlands Organization for Health Research and Development (grant number 630000001). Under this program, the COAD-project has been funded by Revalidatiefonds and Johanna Kinderfonds / Stichting Rotterdams Kinderrevalidatie Fonds Adriaanstichting. Additional grants were provided by Revalidatiefonds (project number R2016006) and Stichting Vooruit (signed 18-05/YvH/NS).

The funding bodies have had no role in the design of the study and in writing the manuscript, nor will they have a role in collection, management, analysis, and interpretation of the data, and in the decision to submit manuscripts for publication. These are the responsibilities of the COAD steering committee.

\section{Authors' contributions}

MS and LB drafted the initial manuscript. BS and YJ-P advanced and critically appraised the manuscript. All authors contributed to designing the study. MS, LB, YJ-P, PA, ER, JB, BS developed the homebased training programs. The home-based training programs have been constructed in consultation with members of the COAD Focus Group. All authors read and approved the final manuscript.

\section{ETHICS APPROVAL AND CONSENT TO PARTICIPATE}

Ethical approval for the COAD-study has been granted by the Committee on Research Involving Human Subjects of the region Arnhem-Nijmegen in the Netherlands (protocol number NL53670.091.15). No potential risks for participants in the interventions described here have been identified. Written informed consent will be obtained for the participation of both the parents and the children in the home-based training programs.

\section{COMPETING INTERESTS}

The authors declare that they have no competing interests. 


\section{REFERENCES}

1. Rosenbaum P, Paneth N, Leviton A, Goldstein M, Bax M, Damiano D, et al. A report: the definition and classification of cerebral palsy April 2006. Dev Med Child Neurol Suppl. 2007;109:8-14.PubMedGoogle Scholar

2. Graham HK, Rosenbaum P, Paneth N, Dan B, Lin JP, Damiano DL, et al. Cerebral palsy. Nat Rev Dis Primers. 2016; https://doi.org/10.1038/nrdp.2015.82.

3. Cans C. Surveillance of cerebral palsy in Europe: a collaboration of cerebral palsy surveys and registers. Surveillance of cerebral palsy in Europe (SCPE). Dev Med Child Neurol. 2000;42:816-24.View ArticleGoogle Scholar

4. Beckung E, Hagberg G. Neuroimpairments, activity limitations, and participation restrictions in children with cerebral palsy. Dev Med Child Neurol. 2002;44:309-16.View ArticlePubMedGoogle Scholar

5. Eyre JA. Development and plasticity of the corticospinal system in man. Neural Plast. 2003; https://doi. org/10.1155/np.2003.93.

6. Aisen ML, Kerkovich D, Mast J, Mulroy S, Wren TA, Kay RM, et al. Cerebral palsy: clinical care and neurological rehabilitation. Lancet Neurol. 2011; https://doi.org/10.1016/s1474-4422(11)70176-4.

7. Ketelaar M, Vermeer A, Hart H, van Petegem-van Beek E, Helders PJ. Effects of a functional therapy program on motor abilities of children with cerebral palsy. Phys Ther. 2001;81:1534-45.View ArticlePubMedGoogle Scholar

8. Lowing K, Bexelius A, Brogren Carlberg E. Activity focused and goal directed therapy for children with cerebral palsy_do goals make a difference? Disabil Rehabil. 2009; https://doi. org/10.1080/09638280902822278.

9. Eliasson AC, Shaw K, Berg E, Krumlinde-Sundholm L. An ecological approach of constraint induced movement therapy for 2-3-year-old children: a randomized control trial. Res Dev Disabil. 2011; https:// doi.org/10.1016/j.ridd.2011.05.024.

10. Wallen M, Ziviani J, Naylor O, Evans R, Novak I, Herbert RD. Modified constraint-induced therapy for children with hemiplegic cerebral palsy: a randomized trial. Dev Med Child Neurol. 2011; https://doi. org/10.1111/j.1469-8749.2011.04086.x.

11. Gordon AM, Schneider JA, Chinnan A, Charles JR. Efficacy of a hand-arm bimanual intensive therapy (HABIT) in children with hemiplegic cerebral palsy: a randomized control trial. Dev Med Child Neurol. 2007; https://doi.org/10.1111/j.1469-8749.2007.00830.x.

12. Sakzewski L, Ziviani J, Abbott DF, Macdonell RA, Jackson GD, Boyd RN. Randomized trial of constraint-induced movement therapy and bimanual training on activity outcomes for children with congenital hemiplegia. Dev Med Child Neurol. 2011; https://doi.org/10.1111/j.1469-8749.2010.03859.x.

13. Gordon AM. To constrain or not to constrain, and other stories of intensive upper extremity training for children with unilateral cerebral palsy. Dev Med Child Neurol. 2011; https://doi.org/10.1111/j.14698749.2011.04066.x.

14. Gordon AM, Charles J, Wolf SL. Methods of constraint-induced movement therapy for children with hemiplegic cerebral palsy: development of a child-friendly intervention for improving upper-extremity function. Arch Phys Med Rehabil. 2005;86:837-44.View ArticlePubMedGoogle Scholar 
15. Majnemer A, Shevell M, Law M, Poulin C, Rosenbaum P. Level of motivation in mastering challenging tasks in children with cerebral palsy. Dev Med Child Neurol. 2010; https://doi.org/10.1111/j.14698749.2010.03732.x.

16. Novak I, Cusick A, Lannin N. Occupational therapy home programs for cerebral palsy: double-blind, randomized, controlled trial. Pediatrics. 2009; https://doi.org/10.1542/peds.2009-0288.

17. Sakzewski L, Ziviani J, Boyd RN. Efficacy of upper limb therapies for unilateral cerebral palsy: a meta-analysis. Pediatrics. 2014; https://doi.org/10.1542/peds.2013-0675.

18. Novak I, Cusick A. Home programmes in paediatric occupational therapy for children with cerebral palsy: where to start? Aust Occup Ther J. 2006;53:251-64.View ArticleGoogle Scholar

19. Scales LH, McEwen IR, Murray C. Parents' perceived benefits of physical therapists' direct intervention compared with parental instruction in early intervention. Pediatr Phys Ther. 2007; https://doi. org/10.1097/PEP.0b013e3180cabc57.

20. Masters RS. Knowledge, knerves and know-how: the role of explicit versus implicit knowledge in the breakdown of a complex motor skill under pressure. Br J Psychol. 1992;83:343-58.View ArticleGoogle Scholar

21. Masters RS, Poolton J. Advances in implicit motor learning. In: Hodges N, Williams A, editors. Skill acquisition in sport: research, theory and practice. 2nd ed. London: Routledge; 2012. p. 59-75.Google Scholar

22. Steenbergen B, van der Kamp J, Verneau M, Jongbloed-Pereboom M, Masters RS. Implicit and explicit learning: applications from basic research to sports for individuals with impaired movement dynamics. Disabil Rehabil. 2010; https://doi.org/10.3109/09638288.2010.497035.

23. van der Burg J, Aarts P, Steenbergen B. So you wanna be a popstar: van Handig2Handig naar Handig2. Nederlands Tijdschrift voor Revalidatiegeneeskunde 2013;35:125-126.Google Scholar

24. Dweck CS, Leggett EL. A social-cognitive approach to motivation and personality. Psychol Rev. 1988;95:256.View ArticleGoogle Scholar

25. Maxwell JP, Masters RS, Kerr E, Weedon E. The implicit benefit of learning without errors. Q J Exp Psychol A. 2001; https://doi.org/10.1080/713756014.

26. Bandura A. Self-efficacy: toward a unifying theory of behavioral change. Psychol Rev. 1977;84:191-215. View ArticlePubMedGoogle Scholar

27. Hoffmann TC, Glasziou PP, Boutron I, Milne R, Perera R, et al. Better reporting of interventions: template for intervention description and replication (TIDieR) checklist and guide. BMJ. 2014;7(348):g1687.View ArticleGoogle Scholar

28. Eliasson AC, Krumlinde-Sundholm L, Rosblad B, Beckung E, Arner M, Ohrvall AM, et al. The manual ability classification system (MACS) for children with cerebral palsy: scale development and evidence of validity and reliability. Dev Med Child Neurol. 2006; https://doi.org/10.1017/s0012162206001162.

29. Palisano R, Rosenbaum P, Walter S, Russell D, Wood E, Development GB. Reliability of a system to classify gross motor function in children with cerebral palsy. Dev Med Child Neurol. 1997;39:214-23. View ArticlePubMedGoogle Scholar

30. Ferre CL, Brandão MB, Hung Y-C, Carmel JB, Gordon AM. Feasibility of caregiver-directed home-based hand-arm bimanual intensive training: a brief report. Dev Neurorehabil. 2015; https://doi.org/10.3109/ 17518423.2014 .948641$. 
31. Novak I. Parent experience of implementing effective home programs. Phys Occup Ther Pediatr. 2011; https://doi.org/10.3109/01942638.2010.533746.

32. De Nederlandse Vereniging van Revalidatieartsen. Richtlijn Spastische cerebrale parese bij kinderen. Utrecht:VRA; 2015.Google Scholar

33. Kleynen M, Braun SM, Rasquin SM, Bleijlevens MH, Lexis MA, Halfens J, et al. Multidisciplinary views on applying explicit and implicit motor learning in practice: an international survey. PLoS One. 2015; https://doi.org/10.1371/journal.pone.0135522.

34. Cusick A, Lannin NA, Lowe K. Adapting the Canadian occupational performance measure for use in a paediatric clinical trial. Disabil Rehabil. 2007; https://doi.org/10.1080/09638280600929201.

35. Law M, Baptiste S, McColl M, Opzoomer A, Polatajko H, Pollock N. The Canadian occupational performance measure: an outcome measure for occupational therapy. Can J Occup Ther. 1990;57:82-7.View ArticlePubMedGoogle Scholar

36. Cup EH, Scholte op Reimer WJ, Thijssen MC, van Kuyk-Minis MA. Reliability and validity of the Canadian occupational performance measure in stroke patients. Clin Rehabil. 2003;17:402-9.View ArticlePubMedGoogle Scholar

37. McColl MA, Paterson M, Davies D, Doubt L, Law M. Validity and community utility of the Canadian occupational performance measure. Can J Occup Ther. 2000;67:22-30.View ArticlePubMedGoogle Scholar

38. Steenbeek D, Ketelaar M, Galama K, Gorter JW. Goal attainment scaling in paediatric rehabilitation: a critical review of the literature. Dev Med Child Neurol. 2007; https://doi.org/10.1111/j.14698749.2007.00550.x.

39. Krumlinde-Sundholm L, Holmefur M, Kottorp A, Eliasson AC. The assisting hand assessment: current evidence of validity, reliability, and responsiveness to change. Dev Med Child Neurol. 2007; https://doi. org/10.1111/j.1469-8749.2007.00259.x.

40. Nott MT, Chapparo C, Heard R. Reliability of the perceive, recall, plan and perform system of task analysis: a criterion-referenced assessment. Aust Occup Ther J. 2009; https://doi.org/10.1111/j.14401630.2008.00763.x.

41. Rosenbaum P, King S, Law M, King G, Evans J. Family-centred service: A conceptual framework and research review. Phys Occup Ther Pediatr. 1998;18:1-20.Google Scholar

42. Bailey DB, Buysse V, Edmondson R, Smith TM. Creating family-centered services in early intervention: perceptions of professionals in four states. Except Child. 2016;58(4):298-309.View ArticleGoogle Scholar

43. Hillier S, Inglis-Jassiem G. Rehabilitation for community-dwelling people with stroke: home or centre based? A systematic review. Int J Stroke. 2010; https://doi.org/10.1111/j.1747-4949.2010.00427.x.

44. Peplow UC, Carpenter C. Perceptions of parents of children with cerebral palsy about the relevance of, and adherence to, exercise programs: a qualitative study. Phys Occup Ther Pediatr. 2013; https://doi.or g/10.3109/01942638.2013.773954. 
Chapter 5

\section{Therapy-related stress in parents of children with a physical disability: a conceptual analysis}

LWME Beckers, RJEM Smeets, JJW van der Burg 


\section{Abstract}

Purpose The aim of this paper is to conceptualize the phenomenon of therapy-related parental stress (TRPS) in parents of children with a physical disability.

Methods Three models related to parental stress were reviewed, i.e. general parental stress, burden of caregiving in parents of children with disabilities, and experiences of these parents with their child's therapy.

Results We define TRPS as the subjective stress and subsequent changes of functioning and health experienced by a parent of a child with a physical disability in response to paramedical therapies (i.e. physical, occupational, and/or speech and language therapy). A theoretical model is proposed to describe the process of TRPS. Available questionnaires will most likely not be valid and responsive to capture the (changes in) stress parents experience related to therapy their child receives.

Conclusion This article provides a first definition of TRPS and a theoretical model to visualize the processes with regard to this topic. Empirical testing of the presented components and their coherence is needed to confirm or improve the model. A questionnaire that specifically measures the construct of TRPS is needed, along with evaluating TRPS in clinical practice and research.

Keywords parental stress; family-professional collaboration; adverse effects of therapy; paediatric rehabilitation; theoretical model. 


\section{INTRODUCTION}

Family-professional collaboration and shared planning and implementation of an intervention are recognized as crucial steps in the rehabilitation of children with physical disabilities. To accomplish this collaborative service delivery, parental engagement is essential [1]. The parents' engagement can vary in type and degree, with parents being coached by therapists to be the primary interventionists as the most extreme.

Various papers have reported that family-professional collaboration is effective in improving child functioning. Examples include caregiver-provided, home-based bimanual training in children with cerebral palsy (CP) [2], and family-supported rehabilitation for

children with traumatic brain injury [3]. On the other hand, literature recognizes drawbacks of such therapy programs. Often, protocols require high doses, and thus many hours of training, to be successful [4]. It has been found that therapy adherence is limited and parents sacrifice employment-related activities, leisure time, and family activities to enable commitment to therapy [5]. A negative correlation between such parental sacrifices and family well-being has also been confirmed [5]. Similarly, data from previous research suggest that parents feel pressure to adhere to therapeutic activities, and the required efforts to do so negatively impact their family relationships [6,7]. In addition, parents may encounter a conflict between their attitude as a parent and their approach as a therapy provider. The parenting role is relationship-oriented and characterized by, for instance, sensitivity and responsiveness to the child's feelings and needs, in order to establish or sustain a supportive parent-child relationship. The therapy, however, focuses on improving performance of the child and requires a task-oriented approach. Hence, implementation of therapeutic strategies can interfere with the parent-child emotional availability and interaction [6,8]. These potential disadvantageous consequences of collaborative service delivery can lead to an increase of perceived parental stress as a secondary, adverse effect of paramedical therapies [7]. This can be considered therapy-related parental stress (TRPS).

So far, little attention has been paid to the phenomenon of TRPS in either paediatric rehabilitation treatment, intervention research, or stress-oriented research in parents of children with disabilities. However, TRPS may affect the well-being of parents as well as the child and siblings, because it adds to the more general stress of parenting a child with physical disabilities. Parental stress in general has been associated with less nurturing, supportive, patient, and involved parenting. Instead, parents can be irritable, negative or punitive [9]. This in turn puts the parent-child interaction and relation even further under pressure. TRPS may also negatively impact the quality of the execution of therapy, hereby reducing its effect on child functioning. Consequently, it is imperative to recognize paramedical therapies as a potential stressor for parents. To allow evaluation of TRPS, its concept should be defined.

\section{Purpose Statement}

The purpose of this paper is to conceptualize the phenomenon of therapy-related stress in parents of children with a physical disability. A definition and theoretical model are proposed. Furthermore, this paper reflects on how to assess TRPS in clinical practice and research. We aim to provide a starting point for the debate about TRPS. 


\section{Defining Therapy-Related parental Stress}

Stress is difficult to define and conceptualize. In threatening situations, stress can be useful as it supports physiological, cognitive, and behavioural responses. In this paper, however, we focus on the negative variant, also referred to as psychological distress, which is "the unique discomforting, emotional state experienced by an individual in response to a specific stressor or demand that results in harm, either temporary or permanent, to the person" [10]. For parents of children with a physical disability we distinguish three related and accumulating scopes of parental stress. The first scope is general parental stress, which can be experienced by anyone raising a child. The second scope represents stress that is unique for parenting and caring for a child with a physical disability, whereas the third scope includes TRPS.

\section{General parental Stress}

Parental stress arises from attempts to adapt to the demands of parenthood. De Brock [11] describes a theoretical stress process model, considering parental stress as an interactive chain of factors. A stress-provoking condition (stressor) acts as a stimulus. Stressors can vary from minor everyday annoyances to life changing events. The primary response to a stressor is defined as the subjective stress experience, which can be physical and/or psychological. Moderating variables of the subjective stress experience are social resources (e.g. social support) and psychological resources (i.e. personality characteristics such as sense of competence). Depending on one's degree of psychological resilience, a subjective stress experience may lead to a secondary stress response, which is a change of physical and/or psychological functioning (depression, for instance) and tends to be more enduring. This secondary stress response will again influence parental behaviour and, indirectly, the development of the child. These pedagogical and developmental consequences can then in itself become stressors for the parent [11].

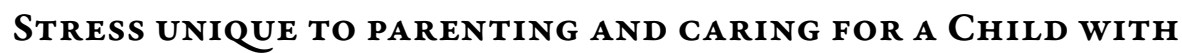 a physical Disability}

Parents of children with a physical disability face particular stressors [12]. Often, there is uncertainty during the search for a diagnosis and prognosis. Once the diagnosis is confirmed, parents have to adjust their expectations regarding the child's and their own future. Depending on the severity of the disability, more effort of the child and family is required for acquisition of skills, performance of daily activities, and participation $[13,14]$.

Often, the functional prognosis is predicted to be dependent on stimulation of the child's development. This may impose a feeling of pressure and responsibility on the parents. Moreover, due to the complex care needs, parents have to fulfil the role of 'case manager'. These additional demands tend to result in elevated stress levels [15]. 


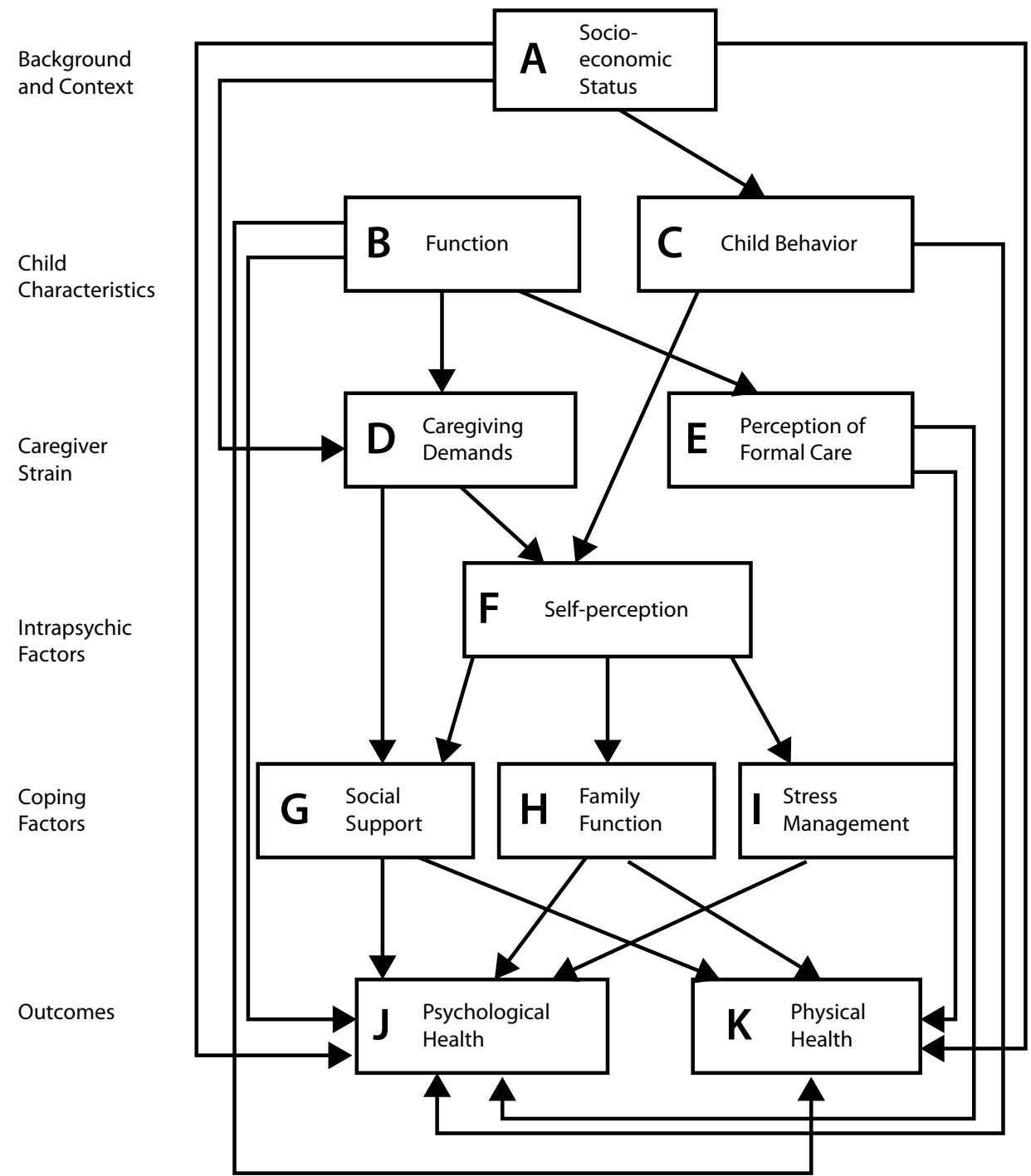

Figure 1 Process and burden of caregiving in parents of children with disabilities [17]. Reproduced with permission from Journal Pediatrics, Vol. 115, Page e628, Copyright $\odot 2005$ by the AAP. 
Raina et al. [16,17] developed a multidimensional model to describe the process and burden of caregiving in parents of children with CP and other disabilities (figure 1).

The model was developed based on literature review and has thereafter been empirically tested. Psychological health and physical health are included in the model as outcomes. Stress is the main indicator of psychological health. Caregiving demands and perception offormal care constitute the caregiver strain. The former are daily demands as well as conflicts between the caregiving and occupational roles. Perception of formal care is considered the perceived family-centeredness of services. Caregiving strain is affected by the child's impairment (motor severity and cognitive functioning, dependency in daily life, and medical problems), and leads to stress through several mediating coping factors: social support from others, family function, and stress management $[16,17]$.

Despite their similarities, this model and the stress process model by De Brock differ on important fundamental aspects. First, the stress process model deservedly acknowledges parental stress as a cyclic process, whereas the model of Raina et al. is a linear model. Second, in Raina et al.'s model functioning of the child is only incorporated as an initiator of caregiver strain. De Brock, on the other hand, describes development of the child as both a consequence of parental stress and a stressor. Third, a strength of the model of Raina et al. is the incorporation of perception of formal care. This factor is relevant in parenting and caring for a child with a disability, making the model appropriate for this population.

\section{Perception of formal Care}

Raina et al. $[16,17]$ hypothesize that increased family-centeredness of services is associated with less parental stress. We, however, acknowledge stress as a possible adverse effect of certain components of collaborative service delivery, as it may tax family resources [5]. Paramedical therapies, i.e. physical, occupational, and speech and language therapy, are important components in these services. Kruijsen-Terpstra et al. [18] elaborate on the experiences of parents of children with CP with these kinds of therapies. Based on their mixed studies review, they describe a model of context- and process-related factors that may influence outcomes of physical and occupational therapy (figure 2).

Context-related factors are clustered within the subdomains culture, community, and family. The family factor contains support from the extended family as well as shared responsibility and roles within the family. Another context-related factor is balancing the demands of daily life with the child's therapy. The process domain in this model can be perceived as a more detailed elaboration of the perception of formal care domain in Raina et al's model. Within the process domain, several factors are clustered within two subdomains: family-professional partnership and intervention. Family-professional partnership includes factors such as shared decision-making and open communication. The intervention subdomain includes implementation of the intervention at home, therapist-child relationship, and design and content of the intervention. Additional process factors are coordinated care, comprehensive care, continuity of care, dedicated time, and receiving information [18]. We recognize the aforementioned process-related factors as contributors to therapy being a possible stressor, affecting whether and to what extent parents experience TRPS as an outcome. 


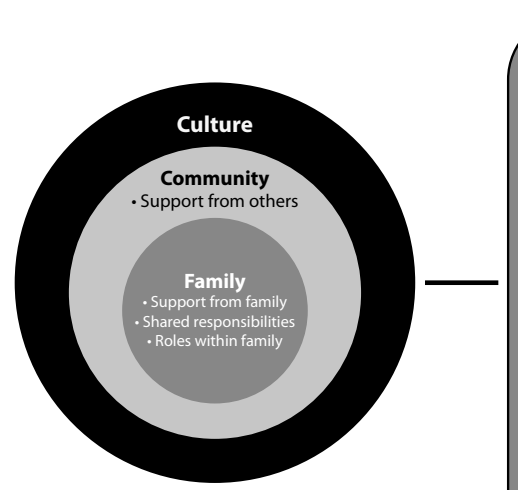

- Demands of everyday life
- Co-ordinated care

- Comprehensive care

- Continuity of care

- Dedicated time

- Information

\section{Context}

\section{Process}

\section{Outcomes}

Child health and well-being

- Improvements

Parental outcomes

- Positive

- Negative

Increased access

Increased satisfaction

with care

Figure 2 Experiences of parents of children with CP with their child's physical and occupational therapy [18]. Reproduced with permission from Journal Child: Care, Health and Development, Vol. 40, Page 793, Copyright (c) 2013 by John Wiley and Sons.

\section{Therapy-RELATED Parental STRESS}

TRPS is a distinctive component of parental stress, with therapy being the particular stressor of interest. Thus, we define TRPS as the subjective stress and subsequent changes of functioning and health experienced by a parent of a child with a physical disability in response to paramedical therapies (i.e. physical, occupational, and/or speech and language therapy). Therapies vary in content and design parameters and differ with regard to parental engagement, ranging from parents getting advice to performing an intensive home-based program with their child themselves. The concept of TRPS applies to all kinds of therapies, although its relevance may be positively correlated with parental engagement in therapy.

In accordance with this definition, we propose a theoretical model for the process of TRPS (figure 3). Preceding its development, a focus group discussion with mothers of children with CP confirmed the relevance of the TRPS construct and revealed its main contributors. These parents have had experience with various therapeutic interventions for their child and were involved, in a 'co-thinker' role, in a related research project [19,20]. In addition to the results of the focus group discussion, the three abovementioned models of De Brock, Raina et al., and Kruijsen-Terpstra et al. were reviewed and taken into account. 

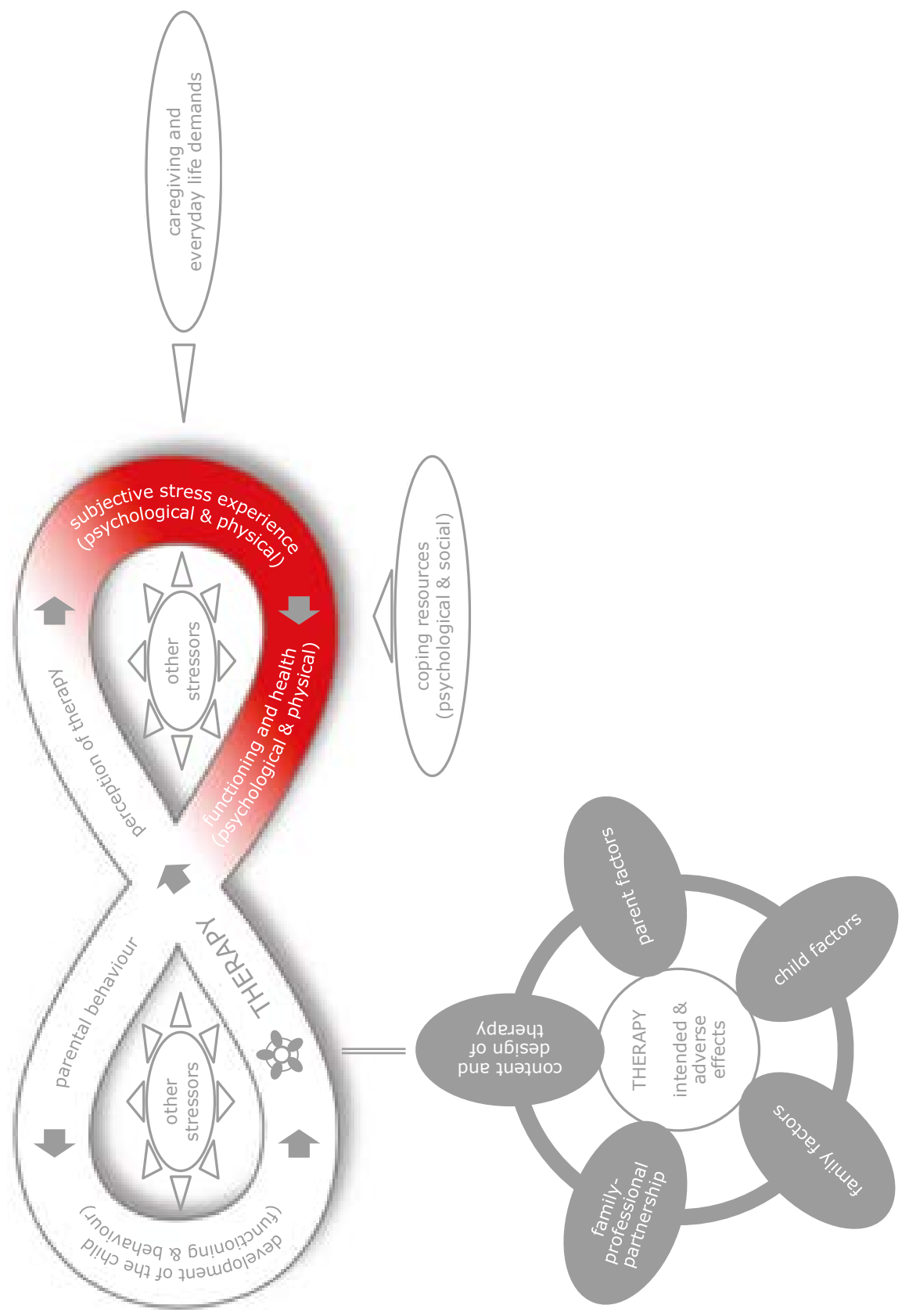

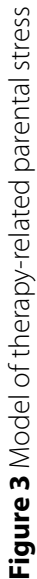


In our model, the process of TRPS is visualized by a lemniscate (i.e. infinity symbol). This represents the constant influence of the perception and impact of therapy on the implementation of subsequent interventions during the many years of rehabilitation of a child. The phases are continuous rather than intermittent.

Therapy is the focus of attention as the potential stressor. The enlarged steering wheel shaped figure visualizes five distinctive contributors to the course of therapy: content and design of the therapy, family-professional partnership, family factors, child factors, and parent factors. The former two are represented in the process domain of Kruijsen-Terpstra et al's model [18]. In our view, the content and design of the therapy contain the treatment modality, frequency of appointments, implementation of therapy and related exercises at home (including adaptations to daily routines and parent-child interaction), etcetera. Family-professional partnership comprises therapist-child and therapist-parent(s) relationship. Elements considered important in family-centred care, like shared decision-making and open communication between professional and family, which are also incorporated in Kruijsen-Terpstra et al's model [18], are of interest for this contributor. Although Kruijsen-Terpstra et al. consider the family subdomain as part of the context, in our model we acknowledge family factors as a central element in the course of therapies. This contributor mainly includes roles and responsibilities within the family, family planning, and interactions between the child with a disability, parents, and siblings. The previously described contributors and examples were confirmed to contribute to the course of therapy by the parents in our focus group discussion.

Furthermore, child factors and parent factors were derived from the focus group as separate contributors. The child's character (e.g. being stubborn), feelings and emotions (e.g. frustration), behaviour (e.g. rebellion), mental and physical condition (e.g. fatigue), and motivation are recognized as child factors affecting the course of therapy. Similarly, the parent's feelings (e.g. disappointment), mental and physical condition (e.g. fatigue), and motivation constitute the parent factors. All five contributors are assumed to interact with each other and together result in intended and adverse effects of the therapy.

Intended effects are for instance improved performance of activities. Adverse effects can vary from mild reactions to major events, like frustration and increase of spasticity, respectively.

All aforementioned contributors, together with the intended and adverse effects, determine the perception of therapy by the parent. This is in line with Raina et al. [16,17], who acknowledge perception of formal care in relation to caregiver strain. In accordance with De Brock [11], the subsequent components of the process of TRPS are subjective stress experience by the parent, functioning and health of the parent, parental behaviour, and development of the child, respectively. Since the development of the child may influence the course of therapy, the process is regarded cyclic. The perception of therapy results in the subjective stress experience, which can be psychological and/or physical, as described by De Brock [11]. The subjective stress experience, i.e. primary stress response, influences the secondary stress response, which includes the functioning and health of the parent (again psychological and/or physical). We have included health on the basis of Raina et al.'s model $[16,17]$. Together, the subjective stress experience and the change of functioning and health of the parent constitute the TRPS. This successively affects parental behaviour, and development of the child (functioning and behaviour). 
Aspects other than the therapy can influence the subjectively experienced stress. Raina et al's model $[16,17]$ indicates that caregiving demands have consequences for the experienced burden of parents of children with a disability. In addition, Kruijsen-Terpstra et al. [18] imply that the demands of everyday life impact parents' experiences with therapy. Hence, caregiving and everyday life demands are incorporated as interacting factors for the subjective stress experience. Coping resources can also act as a mediator between the primary and secondary stress response, as stated through the models of De Brock, Raina et al, and Kruijsen-Terpstra et al. [11,16-18]. In conformity with these models, we differentiate psychological and social resources as moderating factors for subjective stress experience affecting functioning and health of the parent. Psychological coping resources are, for example, self-esteem and stress management. Social resources include family function and social support (e.g. from extended family). Coping resources cohere with the previously described parent factors as contributor of the therapy. Notwithstanding, the parent factors are variable over time and directly related to the course of therapy, whereas coping resources are more stable personality characteristics. The same applies to other related elements, for example the contributor family factors and family function as part of social resources. Other stressors, both related and unrelated to caregiving, can intervene with the TRPS process at any stage. Although contextual factors like culture and socio-economic status are expected to influence the TRPS [16-18], these are not included to warrant comprehensibility of the model.

\section{Evaluating Therapy-Related Parental Stress}

Several questionnaires are available to measure the amount of general stress parents may perceive, of which the Parenting Stress Index (PSI) is probably the most used [21]. It focuses on stressors that are unique to the parent-child relationship. Such generic parental stress questionnaires, however, do not focus particularly on parents of children with a disability. As a consequence, they may not be able to differentiate stressors significant to parenting and caring for a child with a disability. In spite of that, they are frequently used in this population to investigate the occurrence of stress and the effect of interventions [6,22].

For these purposes instruments like the Questionnaire on Resources and Stress for Families with Chronically III or Handicapped Members (QRS), and Distress Thermometer for Parents (DT-P) are likely more appropriate, since they include domains such as overprotection or dependency $[23,24]$. Also, the Measure of Processes of Care (MPOC) evaluates the perception of formal care, i.e. parents' perceptions of the extent to which the health services their child receives are family-centred [25]. These measures are more population specific than generic parental stress questionnaires and an association may be expected between their constructs and TRPS. Still, the items of such questionnaires will most likely not be valid to capture the stress parents experience related to therapy their child receives, nor sufficiently responsive to pick up relevant changes in parental stress during an intervention program. This will result in an inadequate evaluation of the degree of TRPS and thus a probable underestimation of the adverse effect of therapies, both in clinical practice and intervention research. Likewise, information regarding particular sources of stress should be assessed to guide efforts or interventions to reduce TRPS. Therefore, a questionnaire that specifically measures the construct of TRPS is required. 
We hypothesize that the perceived TRPS may fluctuate during the course of a therapeutic intervention. For example, while experiencing a high stress level during the start-up of a new therapy, this may diminish if parents feel more empowered to deliver the therapy, or increase if the child does not benefit from the therapy as expected. These kinds of processes require repeated measurements of TRPS before, during, and after the therapy instead of a single retrospective assessment. Also, in our opinion, both effectiveness and adverse effects of therapeutic interventions should always be assessed, comparable to the standard use of measures like number needed to treat and number needed to harm in drug interventions [26].

\section{Discussion}

This article provides a first definition of TRPS and a theoretical model to visualize the processes with regard to its construct. Furthermore, the need for measures to evaluate this particular type of parental stress is emphasized.

The conceptual analysis of TRPS draws attention to a highly important, but under-considered, topic and clarifies the current state of knowledge. The proposed model can facilitate health professionals to comprehend this phenomenon in relation to the potential influence of their therapy programs, both positive and negative. Nevertheless, the proposed model is purely theoretical and, as most models, is a simplified representation of reality. Empirical testing of the presented components and their mutual associations is needed to confirm or improve the model. In addition to child-related measures, we advocate that TRPS should be an outcome of interest in both formal care and research projects in order to evaluate the effect of therapies on the family level. This will avoid overvaluation of the effectiveness of a therapy, by not solely taking a beneficial effect on the functioning of the child into account. After all, even a therapy that effectively increases, for instance, motor performance of the child, can also have TRPS as an adverse effect. To allow evaluation of TRPS, psychometrically sound instruments must be developed.

Family-centred approaches are considered best practice for formal care [1]. One of the six main concepts of family-centred care is "encouraging the development of true collaborative relationships between families and health-care providers, and partnership" [27]. Notwithstanding, a study comparing families and health professionals' perspectives on family-centred care has identified that the collaborative processes are often not sufficiently implemented when family-centred care is operationalized [27]. Particularly the decision of the parents' role in the treatment of their child is frequently not made collaboratively, considering the wishes and abilities of the parents at the particular point in time. Instead, health-care providers delegate responsibilities to the parents, irrespectively of their needs and resources $[27,28]$. This reverses the potential of familycentred care to reduce the risk of TRPS. The field of tension between the parents' perspective and evidence based requirements of training principles and doses may account for this paradox.

Future studies are required to establish to what extent TRPS is an issue for families and to unravel its complexity, leading to well-founded recommendations and guidelines for clinical practice. Preventing TRPS, evaluating both the experienced level of stress and supportive/ hindering factors throughout interventions, and incorporating remediating methods in therapy 
programs to reduce the effect of stress provoking factors should become standard procedures in the clinical treatment. To accomplish this, interdisciplinary collaboration among therapists, social workers, remedial educationalists, and parents will be necessary.

The issue of TRPS requires further attention in order to advance paediatric rehabilitation and its evidence base. This is supported by a recent editorial of Zebracki [29], advocating to treat paediatric patients within the context of the family system, and to consider the well-being of the caregiver. One of the aims of the present paper is to stimulate awareness of the phenomenon of TRPS. We invite the field to debate about an accurate definition, to determine the position of TRPS in relation to other scopes of parental stress, and advance our model, to guide paediatric rehabilitation practice and research.

\section{Acknowledgements}

This work was supported by Revalidatiefonds and Johanna Kinderfonds / Stichting Rotterdams Kinderrevalidatie Fonds Adriaanstichting through ZonMw; Revalidatiefonds; and Stichting Vooruit.

\section{DECLARATION OF INTEREST}

The authors report no conflicts of interest. 


\section{REFERENCES}

[1] An M, Palisano RJ. Family-professional collaboration in pediatric rehabilitation: a practice model. Disabil Rehabil. 2014;36(5):434-40.

[2] Ferre $C L$, Brandao M, Surana B, et al. Caregiver-directed home-based intensive bimanual training in young children with unilateral spastic cerebral palsy: a randomized trial. Dev Med Child Neurol. 2017;59(5):497-504.

[3] Braga LW, Da Paz AC, Ylvisaker M. Direct clinician-delivered versus indirect family-supported rehabilitation of children with traumatic brain injury: a randomized controlled trial. Brain Inj. 2005;19(10):819-31.

[4] Gordon AM. To constrain or not to constrain, and other stories of intensive upper extremity training for children with unilateral cerebral palsy. Dev Med Child Neurol. 2011;53Suppl4:56-61.

[5] McConnell D, Parakkal M, Savage A, et al. Parent-mediated intervention: adherence and adverse effects. Disabil Rehabil. 2015;37(10):864-72.

[6] Lin KC, Wang TN, Wu CY, et al. Effects of home-based constraint-induced therapy versus dose-matched control intervention on functional outcomes and caregiver well-being in children with cerebral palsy. Res Dev Disabil. 2011;32(5):1483-91.

[7] Peplow UC, Carpenter C. Perceptions of parents of children with cerebral palsy about the relevance of, and adherence to, exercise programs: a qualitative study. Phys Occup Ther Pediatr. 2013;33(3):285-99.

[8] Barfoot J, Meredith P, Ziviani J, et al. Parent-child interactions and children with cerebral palsy: An exploratory study investigating emotional availability, functional ability, and parent distress. Child Care Health Dev. 2017;43(6):812-822.

[9] Holden GW. Parenting. A Dynamic Perspective. London (UK): Sage Publications Inc; 2010.

[10] Ridner SH. Psychological distress: concept analysis. J Adv Nurs. 2004;45(5):536-45.

[11] De Brock, AJLL. Ouderlijk opvoedkundig handelen: de invloed van ouder-, kinden contextuele kenmerken [dissertation]. Nijmegen (Netherlands): Universiteitsdrukkerij Nijmegen; 1994.

[12] Butcher PR, WindT, Bouma A. Parenting stress in mothers and fathers of a child with a hemiparesis: sources of stress, intervening factors and long-term expressions of stress. Child Care Health Dev. 2008;34(4):530-41

[13] Piškur B, Beurskens AJ, Jongmans MJ, et al. Parents' actions, challenges, and needs while enabling participation of children with a physical disability: a scoping review. BMC Pediatr. 2012;12:177.

[14] Piškur B, Beurskens AJ, Ketelaar M, et al. Daily actions, challenges, and needs among Dutch parents while supporting the participation of their child with a physical disability at home, at school, and in the community: a qualitative diary study. BMC Pediatr. 2017;17(1):12.

[15] Pinquart M. Parenting stress in caregivers of children with chronic physical condition. A meta-analysis. Stress Health. 2017;34(2):197-207.

[16] Raina P, O'Donnell M, Schwellnus H, et al. Caregiving process and caregiver burden: conceptual models to guide research and practice. BMC Pediatr. 2004;4:1

[17] Raina P, O'Donnell M, Rosenbaum P, et al. The Health and Well-Being of Caregivers of Children With Cerebral Palsy. Pediatrics 2005;115(6):e626-36.

[18] Kruijsen-Terpstra AJ, Ketelaar M, Boeije H, et al. Parents' experiences with physical and occupational therapy for their young child with cerebral palsy: a mixed studies review. Child Care Health Dev. 2014;40(6):787-96. 
[19] Schnackers M, Beckers L, Janssen-Potten Y, et al. Home-based bimanual training based on motor learning principles in children with unilateral cerebral palsy and their parents (the COAD-study): rationale and protocols. BMC Pediatr. 2018;18(1):139.

[20] Beckers L, van der Burg J, Janssen-Potten Y, et al. Process evaluation of two home-based bimanual training programs in children with unilateral cerebral palsy (the COAD-study): protocol for a mixed methods study. BMC Pediatr. 2018;18(1):141.

[21] Johnson AO. Test review: Parenting Stress Index, Fourth Edition (PSI-4). J Psychoeduc Assess. 2015;33(7):698-702.

[22] Ketelaar M, Volman MJM, Gorter JW, et al. Stress in parents of children with cerebral palsy: what sources of stress are we talking about? Child Care Health Dev. 2008;34(6):825-9.

[23] Holroyd J. A review of criterion validation research on the Questionnaire on Resources and Stress for Families with Chronically III or Handicapped Members. J Clin Psychol. 1988;44(3):335-54.

[24] Haverman L, van Oers HA, Limperg PF, et al. Development and Validation of the Distress Thermometer fr Parents of a Chronically III Child. J Pediatr. 2013;163(4):1140-6.

[25] Cunningham BJ, Rosenbaum PL. Measure of processes of care: a review of 20 years of research. Dev Med Child Neurol. 2014;56(5):445-52.

[26] Andrade C. The numbers needed to treat and harm (NNT, NNH) statistics: what they tell us and what they do not. J Clin Psychiatry. 2015;76(3):e330-3.

[27] Mackean GL, Thurston WE, Scott CM. Bridging the divide between families and health professionals' perspectives on family-centred care. Health Expect. 2005;8(1):74-85.

[28] Leiter V. Dilemmas in sharing care: maternal provision of professionally driven therapy for children with disabilities. Soc Sci Med. 2004;58(4):837-49.

[29] Zebracki K. Perspectives on caring for the child and the caregiver. Dev Med Child Neurol. 2018;60(2):112. 
Chapter 6

\section{Cross-cultural Translation and Adaptation of the Lifestyle Assessment Questionnaire (LAQ-CP) Into Dutch: A Brief Report}

LWME Beckers, LAWM Speth, EAA Rameckers, YJM Janssen-Potten

Pediatric Physical Therapy (2017) DOI: 10.1097/PEP.0000000000000428 


\section{Abstract}

Purpose To produce a Dutch translation of the Lifestyle Assessment Questionnaire for children with cerebral palsy (LAQ-CP), adapted for cross-cultural differences.

Methods The translation process consisted of 6 stages, following a guideline for cross-cultural adaptations including duplicate forward- and back-translations, expert group review, pilot-testing, and a process audit.

Results Several adaptations to the questionnaire were required due to cross-cultural differences As a result of the pilot-test, the layout was adapted to the desires of the users. The process auditor stated that the process had been comprehensive and valued the quality of the work.

Conclusion The project resulted in a Dutch translation of the LAQ-CP, adapted for cross-cultural differences. Validation of the translated questionnaire is required before use in clinical practice and research is recommended (Dutch abstract, Supplemental Digital Content 1, available at: http://links.lww.com/PPT/A164).

Keywords cerebral palsy; clinimetrics; cross-cultural translation; family; LAQ-CP; measurement; participation; questionnaire 


\section{INTRODUCTION AND PURPOSE}

Children with cerebral palsy (CP) may experience restricted participation as a result of their impaired motor function and activity limitations. Within the International Classification of Functioning, Disability and Health, participation restrictions have been defined as "problems an individual may experience in involvement in life situations."1 In children with CP, this may include participation with peers and family, at school and in leisure activities. ${ }^{2}$ In rehabilitation of children with CP, goal setting aims at retention or improvement of participation at home, at school, and in society. As a result, defining the level of participation is warranted to identify potential participation problems, to support goal setting, to evaluate changes in participation over time, or to monitor therapy. ${ }^{3}$ Despite this need, the number of available instruments to measure participation in children with CP is limited. In the Netherlands, four questionnaires are recommended in a guideline of the Netherlands Society of Rehabilitation Specialists on diagnosis and treatment of children with spastic CP, that is, the APCP (Assessment of Preschool Children's Participation); the CAPE (Children's Assessment of Participation and Enjoyment); the LAQ-CP (Lifestyle Assessment Questionnaire); and the LifeH (Assessment of Life Habits). In contrast to the APCP, CAPE, and LifeH, the LAQ-CP is not available in Dutch. The LAQ-CP is a condition-specific parent/carer-completed questionnaire, which measures the effect of disability on participation of children with CP ages 3 to 10 years as well as on their families' participation. ${ }^{4}$ The latter distinguishes the LAQ-CP from the other recommended questionnaires, which are restricted to participation of the child. ${ }^{5}$ The LAQ-CP consists of 46 items with 6 domains: physical independence, clinical burden, mobility, schooling, economic burden, and social integration. ${ }^{4,6}$ The required time to complete the questionnaire is 20 minutes. ${ }^{6}$ The item scores of each dimension can be summed and scaled, to derive a dimensional score on a quasicontinuous scale from 0 to 100. The 6 dimension scores may be used to describe the child by a descriptive profile. In addition, the Lifestyle Assessment Score can be calculated. Dimensional weightings are integrated in this total impact score, which is expressed as a percentage. A higher Lifestyle Assessment Score indicates a more severe effect on the child's life. ${ }^{4,6}$ Mackie et $\mathrm{l}^{4}$ evaluated reliability and validity of the LAQ-CP in children aged 3 to 10 years. Test-retest reliability was found to be high $(r=0.97, P<.0001)$ and differences between individuals remained large and stable ( $x 2=0.29$, not significant). Convergent validity has been investigated by comparing LAQ-CP scores with functional limitation scores as measured by the Central Motor Deficit Form.? A significant correlation ( $r=0.76, \mathrm{P}<.0001)$ was reported, indicating good construct validity. Internal consistency and responsiveness have not been assessed. ${ }^{4-6}$ Thus far, the LAQ-CP is only validated for discriminative purposes. Additional research is needed to determine whether it can be used as an evaluative or predictive measure. ${ }^{6}$

Morris et $\mathrm{al}^{5}$ have recommended the LAQ-CP in their review on measures of participation for children with CP. It has a broad coverage of most domains of the International Classification of Functioning, Disability and Health and is distinctive from other questionnaires because of the focus on the effect on participation of the family unit, in addition to participation of the child. ${ }^{5}$ Because the LAQ-CP measures a particular construct with regard to participation, rehabilitation of children with CP may benefit from a Dutch translation. Hence, the aim of this project was to produce a high-quality Dutch translation of the LAQ-CP, adapted for cross-cultural differences. 


\section{Methods}

A review of guidelines for cross-cultural translation and adaptation of questionnaires by Epstein et $\mathrm{l}^{8} \mathrm{did}$ not lead to a consensus in methods. Nonetheless, they recommend using a validated approach that preferably involves more than one translator, reconciliation of the multiple translations into 1 , and an expert committee. ${ }^{8}$ The guideline provided by Beaton et a $l^{9}$ includes all these stages. In addition, it contains a back-translation. ${ }^{8,9}$ We consider this is as an advantage, because it can facilitate communication with the developers of the original questionnaire. ${ }^{8} \mathrm{~A}$ drawback of the guideline is that involvement of the target population is limited to the pilot-test at the end of the process.

The translation process consisted of 6 stages, following the guideline for cross-cultural adaptations by Beaton et $\mathrm{al}^{9,10}$ (Figure 1). The project was coordinated by a project leader with a background in physical therapy and experience on 2 projects concerning clinimetrics. The project was conducted by the Department of Rehabilitation Medicine at Maastricht University and Adelante Centre of Expertise in Rehabilitation and Audiology from December 2015 to July 2016. Before stage 1, a preparation stage was performed to facilitate the translation process. During the preparation stage, translators and members of the expert group were recruited, cooperation agreements were made, report forms were created, and the pilot-test prepared.

\section{Stage 1}

The LAQ-CP was independently translated from English into Dutch by 2 translators. Both translators were bilingual, with Dutch as their primary language. One translator translated the questionnaire from a clinical viewpoint, whereas a professional translator translated the questionnaire without medical background and without knowledge of the concept the questionnaire aims to measure. Both translators provided a written report of the translation process, including challenging phrases, uncertainties, and reasoning for their decisions. Stage 1 resulted in 2 Dutch translations: $\mathrm{T} 1$ and $\mathrm{T} 2$.

\section{StAge 2}

Both translators from stage 1 and the project leader combined the 2 translations of the LAQ-CP into 1 version of the questionnaire: T12. A report clarified the consensus process.

\section{StAge 3}

The translation of the LAQ-CP (T12) was independently back-translated from Dutch into English by 2 translators. Both professional translators were bilingual, with English as their primary language. The translators had no knowledge of the concept the questionnaire aims to measure and were masked to the original English version of the questionnaire. Both translators provided a written report of the translation process, including challenging phrases, uncertainties, and reasoning for their decisions. Stage 3 resulted in 2 English translations: BT1 and BT2. 


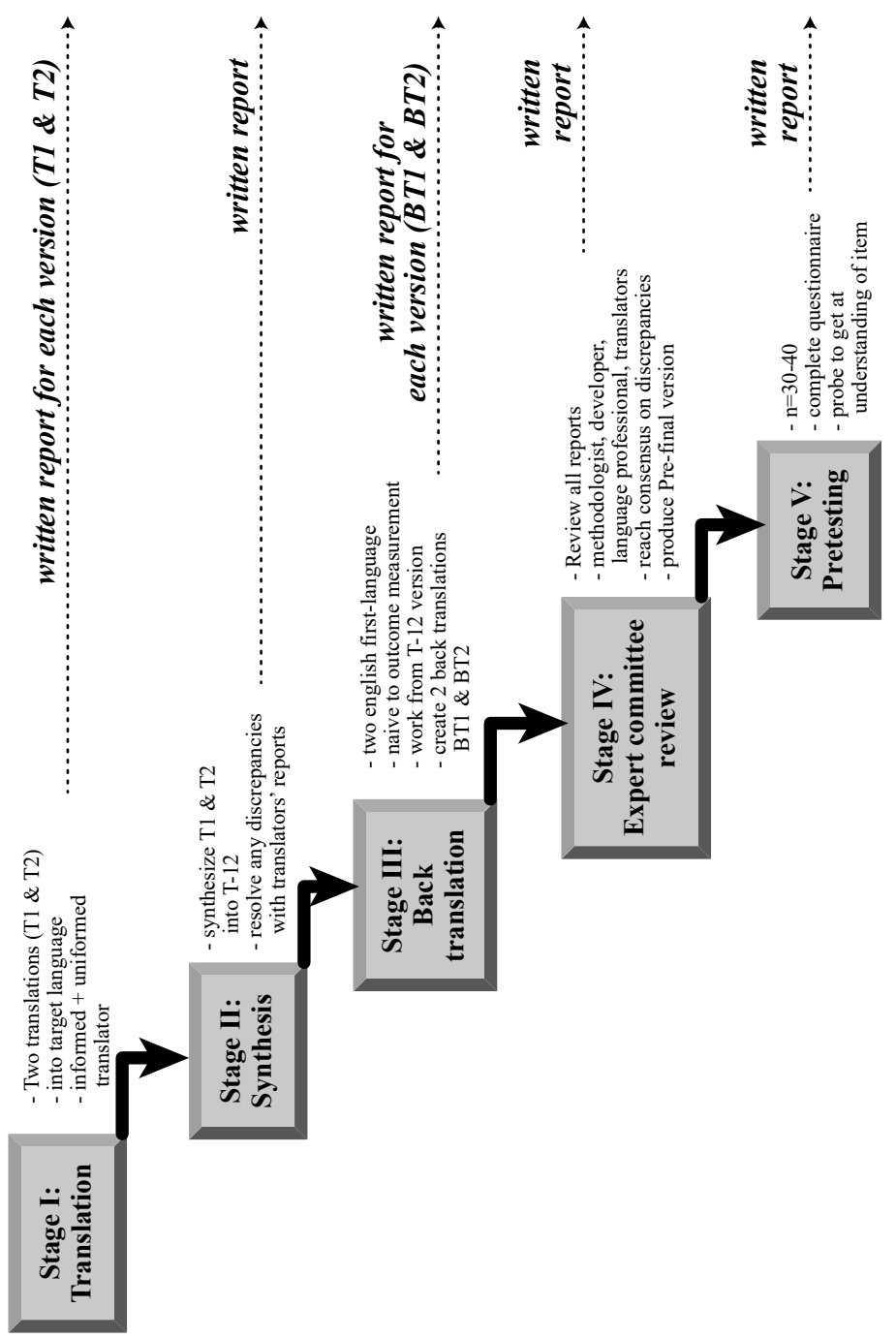




\section{STAGE 4}

An expert group composed a prefinal version of the LAQ-CP, on the basis of the translations and reports of the previous stages. The expert group consisted of 7 people: the project leader, the 4 translators, a pediatric physical therapist, and a pediatric rehabilitation physician. The last 2 both have approximately 30 years of clinical experience in pediatric rehabilitation. They participate in CP-Net, which is a Dutch network of health care professionals, researchers, patients with CP and their parents. The expert group made a report of the process during stage 4 . One of the developers of the original English version of the LAQ-CP was consulted on cross-cultural issues during this as well as previous stages.

\section{STAGE 5}

The prefinal translation of the LAQ-CP was pilot-tested in a convenience sample of parents of children with CP ages 3 to 10 years. Direct recruitment of potential participants known within the pediatric rehabilitation network of the experts was through an invitation letter. Parents were asked for their opinions on the questionnaire by a digital survey. Questions were primarily with regard to comprehensibility and applicability. No personal or medical data were collected. Furthermore, this study was considered not to lead to infringement of the physical or psychological integrity of our participants. Hence, ethical approval was not required, following the "Wet medisch-wetenschappelijk onderzoek met mensen" (WMO; Medical Research Involving Human Subjects Act). ${ }^{11}$

\section{STAGE 6}

The translation process was assessed during a process audit by means of review of the translations and reports of stages 1 through 5 by one of the developers of the original English version of the LAQ-CP. She judged the process and quality of the translation and created a report including comments to be considered by the expert group.

\section{RESULTS}

The project resulted in the Dutch translation of the LAQ-CP, entitled "Vragenlijst ter beoordeling van de leefstijl van kinderen." The final version of the questionnaire is provided as Supplemental Digital Content 2 (available at: http://links.Iww.com/PPT/A165).

\section{STAGES 1 AND 2 (Forward TRANSLATION)}

Throughout the translation process, several arguments were taken into consideration. Simplicity and terms generally used by clinical practitioners in their communication with parents were preferred, in order that the questionnaire be comprehensible for parents across socioeconomic groups. Moreover, consistency of terms and syntax was strived for throughout the questionnaire. 
The translators experienced translation of response options of items 8,11 , and 27 as challenging.

Several adaptations to the questionnaire were required because of cross-cultural differences. The types of services (item 11) and school types (item 26) that are accessible in the Netherlands are not the same as the types of services and school types that are provided in the United Kingdom. Subsequently, the response options were adapted to the situation in the Netherlands. The translation regarding types of services was formulated in a way that it remains applicable after future changes of regulations.

Amounts in pounds (items 13 and 14) were converted into amounts in euros. The value in euros for each response option is equal to the value in pounds, to maintain generalization between the questionnaires. However, the amounts were rounded. Distances in miles (item 23) were converted to kilometers, using the same approach for conversion.

Because cereal (item 17) is not as common to eat for breakfast in the Netherlands as it is in the United Kingdom, the activity "eating a bowl of muesli" was replaced by "eating a bowl of soup."

\section{Stages 3 ANd 4 (BACK-Translation ANd Expert Group)}

The expert group addressed several fundamental remarks.

The abbreviation of the English name of the questionnaire (ie, LAQ-CP) was preferred over a new Dutch abbreviation. For clarification of the abbreviation, besides the Dutch name of the questionnaire the English name was included.

In the introduction text, the translation "families" was replaced by the more specific "gezinnen" (meaning immediate families).

The expert group questioned why "leg support" (item 5) is mentioned separately, whereas support to the upper limb is not. Therefore, one of the designers of the original English questionnaire was consulted. The designer answered that ever since the questionnaire was designed, arm/ wrist splints and lycra suits have been used frequently. Therefore, in the Dutch translation "body support" was used. Examples were included to clarify for parents.

The expert group felt that eating a bowl of cereal and eating a bowl of soup are not equally difficult (item 17). Therefore, the Dutch T12 translation "eating a bowl of soup" was replaced by "eating a bowl of yoghurt."

The expert team did not agree as to what is meant by the original item "getting out of bed" (item 17): the activity getting out of bed (ie, coming from supine position to sitting position to standing) or the activity of getting up in the morning (ie, the morning routine, including grooming, getting dressed, etc). Consultation with one of the designers of the original English questionnaire clarified that the former was intended. The Dutch translation was amended accordingly.

The expert team did not agree as to what is meant by the original term "areas" (item 20). Hence, one of designers of the original English questionnaire was consulted. The designer answered that it concerns other daily activities and it is not about other physical locations. The Dutch translation was changed correspondingly.

Moreover, the response options of items 8, 11, and 27 were amended after expert group discussion. 


\section{Stage 5 (Pilot-Test)}

Seven parents were recruited for the pilot-test. Respondents commented on multiple layout issues. They felt the layout was disorderly, disliked circling answers, preferred more space to write down remarks, and disliked the childish pictures. Accordingly, the layout of the English version was adapted to the desires of the users, for example by including checking boxes, adding line breaks, and removing the pictures. Moreover, several minor changes in wording were made. In addition, respondents made content-related comments, for example regarding questions'lack of specificity. To retain comparability between the Dutch translation and the original English questionnaire, these did not lead to changes to the construct to be measured.

\section{Stage 6 (Process Audit)}

The process auditor stated that the process had been comprehensive and valued the quality of the work. Based on comments of the process auditor, some additional changes were made.

The response options of item 11 were reconsidered. It was ensured that the number of response options in the Dutch translation is equal to the English questionnaire, to make generalization of the score calculation possible.

During the translation process, the activity "eating a bowl of cereal" (item 17) had been adapted to "eating a bowl of yoghurt," because this is a more common activity in the Netherlands and was considered equally difficult to perform by the expert group. As a result of the process audit, however, eating yoghurt was changed back to eating soup, which in the end was estimated most similar to eating cereal regarding difficulty to perform the activity.

\section{Discussion}

The project resulted in a Dutch translation of the LAQ-CP, adapted for cross-cultural differences. The key strength of the project is the extensive procedure that was used for development of the translated questionnaire, consisting of duplicate forward- as well as back-translations, criticism by an expert group, pilot-testing, and a process audit. We feel that this approach was successful in capturing the relevant cross-cultural differences and making valid adaptations. Specifically, the expert group discussions revealed remarkable insights. The number of participants of the pilot-test, however, was small and its scope relatively narrow, which resulted in limited information on perception of the target population regarding the questionnaire.

The Dutch LAQ-CP is promising to contribute to clinical practice and as an outcome for research purposes, because it provides a valuable addition to measure participation in children with CP. Despite its promising benefits, the Dutch LAQ-CP should not be used in clinical practice and research until it has been validated. This is an important issue for future research. Psychometric properties of the instrument should be established with Dutch children. It is recommended to 
investigate for which subpopulations (eg, regarding age) reliability, validity, and responsiveness are satisfying. The amended layout of the Dutch LAQ-CP is expected to result in a slightly higher usability and reliability than obtained from the original English version. Validity and responsiveness, however, are expected to be comparable.

\section{ACknowledgment}

We are grateful to Professor Helen McConachie for performing the process audit and the valuable discussion on cross-cultural differences. 


\section{REFERENCES}

1. World Health Organization. Towards a Common Language for Functioning, Disability and Health. ICF: The International Classification of Functioning, Disability and Health. Geneva, Switzerland: World Health Organization; 2002. http://http://www.who.int/classifications/icf/icfbeginnersguide.pdf. Accessed October 6, 2016.

2. Imms C. Children with cerebral palsy participate: a review of the literature. Disabil Rehabil. 2008;30(24):1867-1884.

3. Nederlandse Vereniging van Revalidatieartsen. Richtlijn Spastische Cerebrale Parese Bij Kinderen. Utrecht, the Netherlands: De Nederlandse Vereniging van Revalidatieartsen; 2015. https://revalidatiegeneeskunde.nl/sites/default/files/attachments/Kwaliteit/Richtlijnen/richtlijn_cerebrale_parese_revisie_2015_-_def.pdf. Accessed Februar 20, 2017.

4. Mackie PC, Jessen EC, Jarvis SN. The Lifestyle Assessment Questionnaire: an instrument to measure the impact of disability on the lives of children with cerebral palsy and their families. Child Care Health Dev. 1998;24(6):473-486.

5. Morris C, Kurinczuk JJ, Fitzpatrick R. Child or family assessed measures of activity performance and participation for children with cerebral palsy: a structured review. Child Care Health Dev. 2005;31(4):397407.

6. Mackie PC, Jessen EC, Jarvis SN. The Lifestyle Assessment Questionnaire. LAQ-CP. North of England Collaborative Cerebral Palsy Survey; 2003. http://research.ncl.ac.uk/cargo-ne/LAQ-CP\%20Manual\%20 2003.pdf. Accessed February 20, 2017.

7. Stewart A. Standard recording of central motor deficit. Dev Med Child Neurol. 1989;31(1):120-127.

8. Epstein J, Santo RM, Guillemin F. A review of guidelines for cross-cultural adaptation of questionnaires could not bring out a consensus. J Clin Epidemiol. 2015;68(4):435-441.

9. Beaton DE, Bombardier C, Guillemin F, Ferraz MB. Guidelines for the process of cross-cultural adaptation of self-report measures. Spine. 2000;25(24):3186-3891.

10. de Vet HCW, Terwee CB, Mokkink LB, Knol DL. Measurement in Medicine. New York, NY: Cambridge University Press; 2011.

11. Central Committee on Research Involving Human Subjects. Medical/Scientific Research and the WMO. http://http://www.ccmo.nl/en/medical-scientific-research-and-the-wmo. Accessed March 20, 2017. 


\section{ChaPter 7}

\section{Barriers to recruitment of children with cerebral palsy in a trial on home-based training}

LWME Beckers, EAA Rameckers, RJEM Smeets, JJW van der Burg, PBM Aarts, MLAP Schnackers, YJM Janssen-Potten 


\section{Abstract}

Many trials fail to include the targeted number of participants, causing scientific and ethical problems. The COAD trial of home-based training programs for children with unilateral cerebral palsy (CP) encountered recruitment problems, even though the parent-delivered home-based approach complies with recent health-care developments in the Netherlands. The current project aimed to identify the barriers to recruitment in the COAD trial. This summative, multidimensional evaluation comprised informal conversational interviews in which stakeholders who had been involved reflected on the factors that impeded successful recruitment of participants into the COAD trial. Barriers to implementation and recruitment were clustered according to the constructs of the Consolidated Framework for Implementation Research (CFIR). Member checking validated the findings. A total of 41 stakeholders contributed to the evaluation. Barriers to the implementation of the HBTPs were identified within every domain of the CFIR (intervention characteristics, outer setting, inner setting, characteristics of individuals, and process). Parent-delivered home-based training was perceived as highly complex and in conflict with the pressures on and the needs of parents. Many parents preferred the alternative center-based group interventions. The involvement of a resonance group was highly valued, and opportunities for further enhancements emerged. Additionally, the importance of research consortia was emphasized. The appropriateness of the RCT as the study design was criticized. The findings of this study are summarized in a tool which provides a dozen directions for the successful recruitment of participants in pediatric rehabilitation research. 


\section{INTRODUCTION}

Successful recruitment of the requisite number of research participants is known to be challenging. A Dutch study showed that $38 \%$ of completed trials failed to include the targeted number of participants. ${ }^{1}$ Similar and even lower rates have been reported elsewhere.,3 The scientific validity of underpowered studies is questionable, while participants are unnecessarily exposed to burdens and risks. ${ }^{1}$ Additionally, it is a waste of already limited resources.

Numerous factors that influence recruitment have been reported for medical trials. ${ }^{4}$ In rehabilitation research, however, motives for taking part are likely to be different. Firstly, interventions focus on activities, participation, and quality of life rather than body functions and structures, or extending life. Secondly, rehabilitation interventions often require more active and time-consuming involvement from participants and their families than do medical interventions. ${ }^{5}$ Pediatric rehabilitation is even more distinctive, because children are vulnerable and parents advocate for their child's needs.

The COAD trial (CO-creation At hanD: the road to independence) is the most recent example of a study in Dutch pediatric rehabilitation that has encountered recruitment problems. The trial (NTR5743) aimed to include 78 children with unilateral cerebral palsy (CP) and their parents during a two-year period. After enrollment of only 12 participants in one year, the trial was terminated early. The experimental interventions were two home-based training programs (HBTPs), using either implicit or explicit motor learning principles. The HBTPs comprised a task-specific, bimanual approach. Parents provided intensive training in their home setting for 12 consecutive weeks. An interdisciplinary team consisting of an occupational or physical therapist and a remedial educationalist coached the parents. The protocols of the HBTPs have been reported elsewhere. ${ }^{6}$ A three-armed, multicenter randomized controlled trial (RCT) of a pragmatic nature was planned to evaluate the effectiveness of the HBTPs (Figure 1). The research team collaborated with a 'resonance group' consisting of parents of children with $C P$, a youngster with $C P$, the director of the BOSK (Dutch association for people with a physical disability and their parents), therapists, physiatrists, and a remedial educationalist. This group was consulted repeatedly during the planning and execution of the trial. The BOSK was also a co-applicant on the grant proposal.

The parent-delivered home-based approach of the COAD trial complies with recent developments within health care towards self-reliance of families, professionals becoming coaches, and the use of e-health technologies.' A basic needs assessment among parents, including focus group discussions and surveys, confirmed the potential of home-based training. Despite these preconditions, enrollment in the COAD trial was disappointing. This justifies exploring whether and how the concept of home-based training contributed to the troublesome recruitment process. Many previous Dutch trials (i.e. BoBiVa, BOLIEN, LEARN 2 MOVE, POPEYE, and SPACE BOP), investigating other types of interventions, have also struggled to meet recruitment goals. Thus, the COAD trial also provided an excellent opportunity to advance the understanding of additional factors that impede successful recruitment in pediatric rehabilitation research. Hence, the aim of this project was to identify the barriers associated with the recruitment during the COAD trial, using a multidimensional perspective. 


\section{Study population}

Children with unilateral spastic CP aged 2.5 through 7 years (MACS level I-III and GMFCS level I-III) and their parents

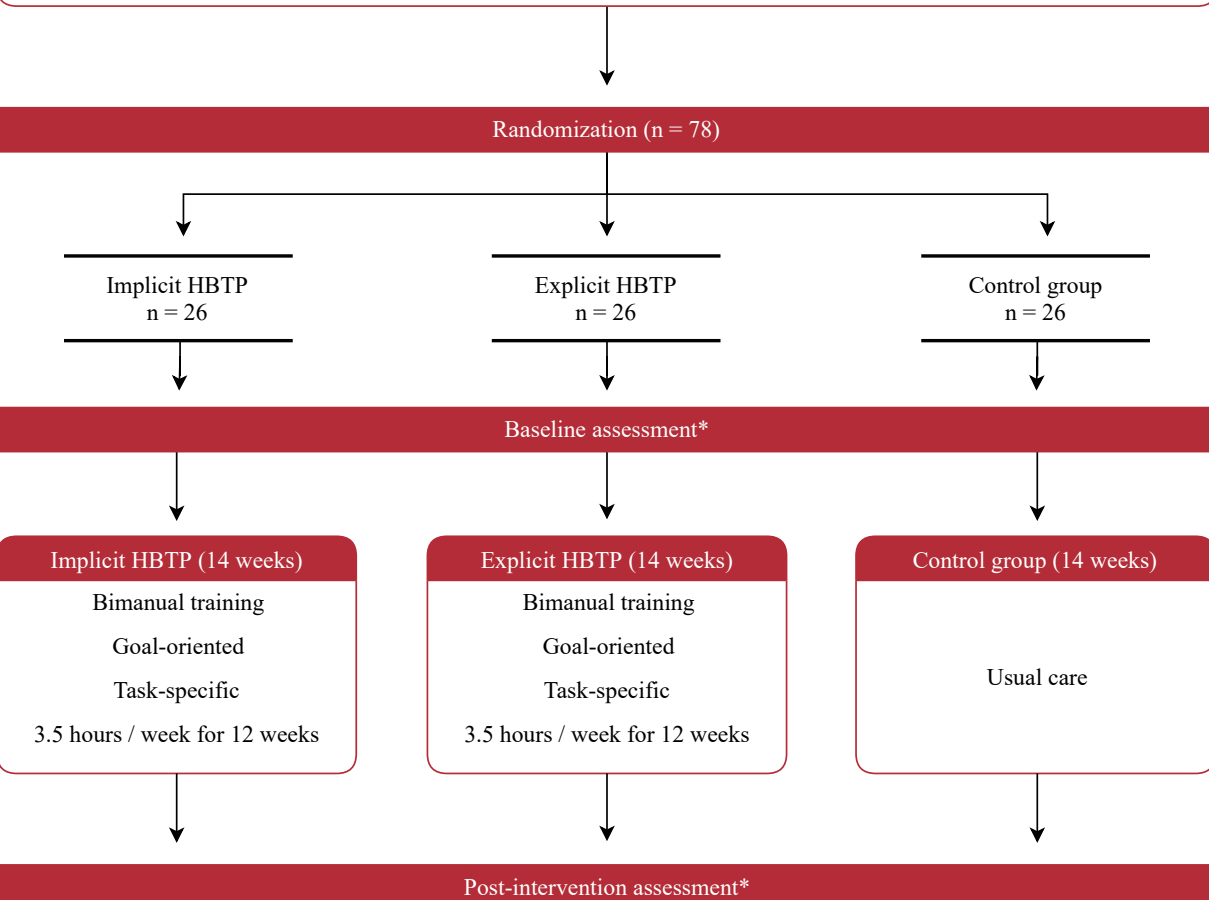

\section{Follow-up (12 weeks)}

Usual care

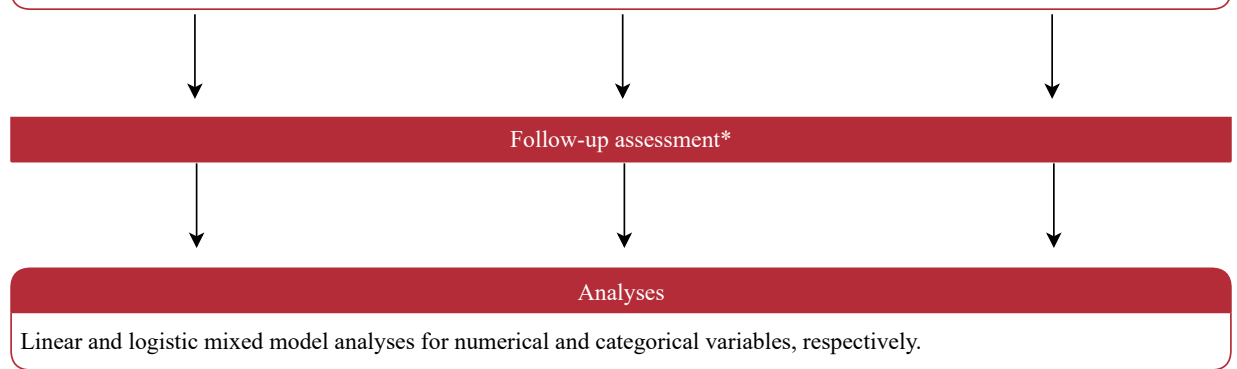

* Primary outcome measures: performance of bimanual activities (Canadian Occupational Performance Measure - COPM performance scale); therapy-related parental stress (in-depth parental interviews)

Secondary outcome measures: bimanual capacity and performance (Assisting Hand Assessment - AHA; Canadian Occupational Performance Measure - COPM satisfaction scale; Goal Attainment Scale - GAS; Hand-Use-at-Home questionnaire - HUH; Observational Skills Assessment Score - OSAS ); participation (Lifestyle Assessment Questionnaire LAQ-CP); parental stress (Opvoedingsbelasting vragenlijst - OBVL; Vragenlijst Ouderlijke Stress als gevolg van Therapie van het kind - VOST); parental empowerment (Empowerment Vragenlijst - EMPO)

Figure 1 Design of the COAD trial 


\section{METHODS}

A summative evaluation of the COAD trial was made after its closing. To enable a multidimensional evaluation, six relevant stakeholder groups that had been involved in the COAD trial were invited to participate: collaborating rehabilitation centers, grant provider (i.e. manager of the subsidy program) and funding bodies, the approving Medical Research Ethics Committee (MREC), non-collaborating rehabilitation centers, parents of children with $\mathrm{CP}$, and the research team (Figure 2).

All stakeholders willing to contribute reflected on the trial from their perspectives during informal conversational interviews with the principal investigator (LB). The conversations were either in person or over the phone and mostly individually. The interviews revolved around the question 'What factors impeded successful recruitment of participants into the COAD trial'? The recruitment of participants and the implementation of the HBTPs and of the trial occurred within the collaborating rehabilitation centers. Consequently, recruitment issues were interrelated with and indistinguishable from implementation issues. Therefore, LB extracted barriers to recruitment as well as to implementation from the interview notes and clustered them according to the Consolidated Framework for Implementation Research (CFIR). ${ }^{8}$ The CFIR provides a framework of constructs that have been associated with effective implementation and is considered particularly useful for complex interventions. In this project, it was applied to evaluate the recruitment of participants and implementation of the HBTPs into clinical practice in the context of research. The definitions of the constructs as utilized are described in Supplemental File 1. Member checking of the preliminary report by the stakeholders was used to validate the findings.

\section{RESULTS}

A total of 41 stakeholders, covering all six stakeholder groups, contributed to the evaluation (Figure 2). The rehabilitation centers were represented by occupational and physical therapists, physiatrists, local ethical committee members, and a researcher. Thirty-two of the stakeholders completed the member checking. Many stakeholders stressed the importance of home-based training and still expected this to be the therapy of the future. Barriers to the implementation of the HBTPs in the COAD trial were identified within every domain, but not all constructs, of the CFIR (Supplemental File 1). The results are presented following the five CFIR domains: intervention characteristics, outer setting, inner setting, characteristics of individuals, and process. The stakeholders' subjective commentaries are represented as faithfully as possible, allowing contradictory opinions and speculations. In the discussion section, the considerations will be put into perspective and recommendations will be provided. 

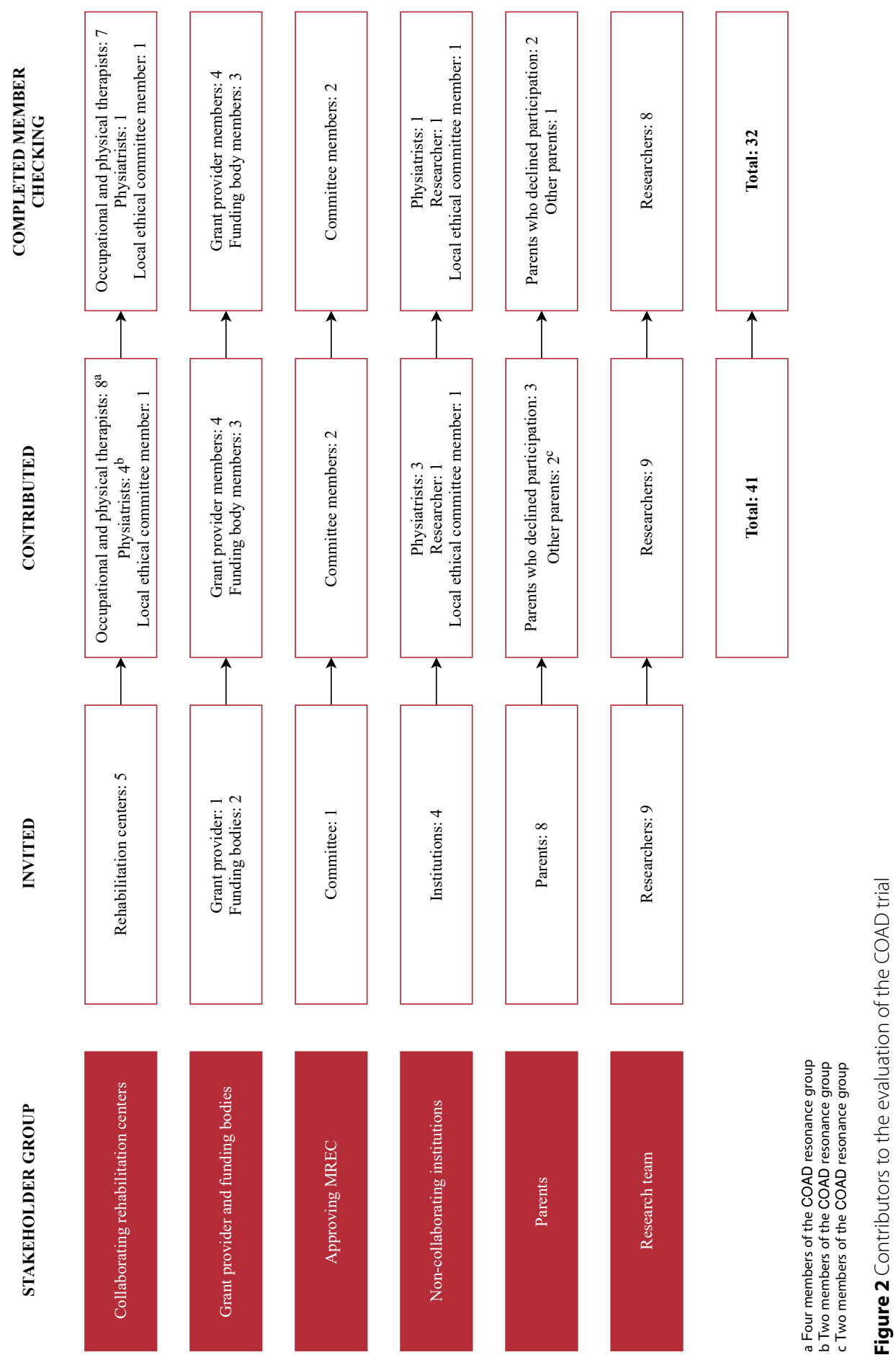


\section{INTERVENTION CHARACTERISTICS}

\section{INTERVENTION SOURCE}

The involvement of the resonance group was valued by many stakeholders, especially the participation of the parents, the youngster with CP, and the patient association BOSK. One parent stated that the research team had sufficiently listened to the opinions of the resonance group. Nevertheless, several other stakeholders felt that some suggestions, for example reducing the training intensity, were overruled by the research team, based on scientific arguments. According to the involvement matrix, an instrument to define the level of involvement of patients and parents in research, ${ }^{9}$ the resonance group participated in the role of co-thinker. It was suggested that this involvement matrix might have been used to discuss the roles parents wanted to fulfill at each stage of the project, enabling their involvement as advisors or partners. Also, parents, as well as children and youngsters with CP, could have been more explicitly involved in the earliest stages of the project to jointly establish the topic, research question, research design, and intervention content and structure. However, the parents in the resonance group were well informed, highly engaged, and hence perhaps not fully representative of the average parent of a young child with CP. The research team might have attempted to involve a larger and more heterogeneous group of parents, using low threshold strategies such as social media, social events, or consultation with fellow parents by dedicated parents. Likewise, the resources and preferences of the collaborating rehabilitation centers might have been taken into account more at the beginning of the project. Such an impact analysis might have resulted in more convenient procedures. A different balance between conflicting scientific, family, and practical perspectives would probably have increased feasibility.

\section{Relative ADVANTAge}

While the trial was running, many alternative clinical treatment options were available to families. Intensive upper-extremity interventions using a playgroup setting around one theme, e.g. pirates, ${ }^{10}$ were very common. During the inclusion period, most parents had to decide whether their child would participate in either the COAD trial, including the unconventional concept of parent-delivered home-based training and remote coaching, or in a therapist-delivered group intervention of proven effectiveness at the center. Parents declared that these group interventions can be logistically challenging, having to travel to the center several times a week, arranging childcare for siblings, and requiring the child to be absent from school. Still, many parents seemed to favor these group interventions because they expected the burden for their family to be lower and felt it easier to relinquish control. Furthermore, clinicians observed that parents preferred the social contact with fellow parents and the motivational effect of the group process. Additionally, one parent expressed appreciation for the child's not having to compete with typically-developing peers. Children presumably liked the playful setting of the group interventions better than a task-specific approach at home. HBTPs, on the other hand, offer parents the opportunity to adjust both training moments and content to their family routine and interests. If families succeed in performing relevant activities in a meaningful setting and at a convenient time, practice may 
not feel like therapy. However, it appeared that parents' positive experiences of attending group interventions outweighed the potential benefits of the new HBTPs. For clinicians, a difficult home situation and the benefits of a group-based setting were important reasons to prefer group interventions over HBTPs for some potential participants. Since a sufficient number of participants is also needed for the center-based group interventions to be profitable, these and the COAD trial turned out to be competitors.

Finally, parents were frequently invited to participate in studies, which made them selective. Numerous outcome measures in this trial resulted in a relatively high research-related burden, also disadvantageous. The centers reported a similar 'research fatigue'.

\section{Adaptability}

The centers perceived a lack of flexibility in the trial protocol with regard to two of the intervention components: the home visits, and coaching by pre-specified therapists. Both conflicted with the practical organization of other treatments provided by the rehabilitation teams. This led to the non-participation of some centers. Similarly, although the content of the HBTPs was individualized, the process was standardized, which provided limited possibilities to tailor it fully to the family needs.

\section{Trialability}

An RCT was used to test the newly developed HBTPs. In retrospect, many stakeholders suggested that this was premature, since developing and testing of interventions should be an iterative process. A priori focus group discussions with parents showed a positive attitude towards comparable interventions. A similar needs assessment in the specific target population of the HBTPs might have added useful information regarding parents' perceptions of the concept and approach of home-based training and its clinical meaningfulness. Afterwards, feasibility and pilot studies by early adopters might have been beneficial in optimizing the interventions. Concerning effectiveness, other experimental designs (e.g. (stepped-wedge) cluster randomization) might have been less demanding for centers. A consecutive execution of several smaller projects can result in greater success in terms of sample size, coordination within dynamic clinical settings, costs, and early detection of difficulties. Hence, in retrospect, it seems that an RCT was not the most suitable approach for such complex interventions at this early stage.

Conversely, several stakeholders referred to the view of many funders, journals, referees, and others of the RCT as the 'gold standard' in intervention research, valuing other designs, especially qualitative studies, less. Similarly, the grant call for this project explicitly demanded the use of an RCT. The grant provider and funders mentioned in the interviews that their organizations recently initiated transitions to match appropriate designs to research questions. ${ }^{11}$ Hence, a trend is beginning towards supporting small-scale, attainable projects. Positive results can then encourage funders to financially support more complex follow-up studies. 


\section{COMPLEXITY}

The interventions were innovative in two ways, resulting in great complexity. The first was the home-based setting. The transition from conventional center-based to home-based interventions posed great demands on therapists and parents. Changing their habits and committing themselves to an unfamiliar treatment provoked resistance. Within centers, it was difficult and time-consuming for managers to facilitate deployment and for planners to arrange unusual appointments (e.g. home visits) alongside regular clinical care. Even though the research team provided an instructional course, extensive manual, and time-task matrix, therapists struggled to comprehend the expected duties and to complete them within the anticipated time. For families, the intensive training, including making video-recordings for the remote coaching, required an exceptional effort. Twelve weeks of repetitive, daily practice of the specific tasks was regarded as lengthy. Because remote coaching was unfamiliar to parents and practitioners, the research team provided a digital tool to facilitate secured data sharing. However, practical limitations of this system increased complexity.

Secondly, the incorporation of implicit and explicit motor learning approaches was innovative. Operationalization of these theoretical concepts led to complex intervention protocols. Parents, as well as therapists, indicated that the programs required background knowledge and a relatively high cognitive level in parents. The programs were also outside the 'comfort zone' of therapists, which made the programs even more challenging and time-consuming to grasp and apply. Furthermore, the research focused on both an implicit and an explicit program simultaneously and no crossover of therapists was allowed (to restrict contamination). This demanded the dedication of two therapists per intervention for each center and resulted in additional inconvenience in management and planning.

Regarding the research design, the comparison of three treatments was identified as a complicating factor. Especially with young children, parents advocate for the best care possible. Hence, potential randomization to a control group of usual care was mentioned as a reason for non-participation. Parents might also have been uncomfortable being randomized to an implicit or explicit teaching style. It was suggested that a wait-list control group and preference-based randomization might have resolved these delicate issues. ${ }^{12}$

The complexity of both the interventions and the study limited comprehensibility of their benefits, which increased the threshold for centers and parents to participate.

\section{DESIGN QUALITY AND PACKAGING}

Due to parental involvement during its development, some parents as well as other stakeholders greatly esteemed the participant information, whereas others criticized the presentation of the study as being too research-oriented. To complement the parents' needs, it was suggested that the information provided to parents could have given more emphasis to the relevance of the HBTPs for families, revolving around the question 'What's in it for me?' 


\section{Cost}

As previously described, the research team developed the interventions according to theoretical and scientific standards. Consequently, the content was prioritized over the cost. Dependent of other care provided to individual children, the financial return sometimes made it unprofitable to provide the HBTPs. The HBTPs being merely cost-covering was a drawback for centers to participate. It emerged that centers and the research team had conflicting opinions about the trade-off between financial sustainability and desired functional outcomes.

Due to changes in the health care regulations (see the construct 'External policies and incentives'), insurance companies would not pay for travel time and expenses for the home visits and this was not accounted for in the research budget, resulting in a major cost to centers and a reason to resist the intervention. An option might have been for the research team to consult with health insurance companies to find a solution to compensate for this.

\section{OUTER SETTING}

\section{Patient NeEds AND Resources}

Numerous concerns regarding the needs and resources of the families arose from parents themselves, as well as from clinicians' interactions with parents. Most frequently-mentioned was the pressure perceived by families. Parents of children with CP generally have a busy life, combining the care of their child(ren) with work, sports, and social events. In addition, some parents encounter more complex circumstances, such as moving house, divorce, or a sibling also requiring special care. On top of this, CP is a complex disorder, involving a range of problems with, for instance, mobility, communication, or behavior, aside from the arm-hand impairments. Hence, rehabilitation is multifaceted, which results in many time-consuming activities and arm-hand treatments sometimes not being prioritized. Children attend either special education, involving time-consuming door-to-door transportation, or mainstream education, which can be demanding, cause burden, and leave little time for practice. Parents balance the time and energy investment of their family with the expected benefits. Both parents and clinicians emphasized that non-participating parents did not use these points as excuses, implying that they were unable, rather than unwilling, to perform a HBTP. If they could not give it their best, they would decide against participation.

Additionally, clinicians reflected that, in present Dutch society, health professionals tend to patronize families and that many parents accept this, with restriction of their autonomy as a consequence. A reason why families accept this dependency on health professionals may be parents' lack of self-confidence. From clinicians' impressions, many parents have more confidence in therapists than in their own competence and therefore feel unable to be highly engaged in a HBTP, making it difficult to achieve self-management. Several stakeholder groups suggested that, as the rehabilitation setting was not yet ready for parent-delivered interventions, the COAD trial was probably ahead of its time. 
Some parents preferred to limit themselves to their parenting role, instead of being a co-therapist. They considered the home to be a safe environment for the child to unwind, wanting to be there for their child and to provide stimulation, but without forcing the child to practice activities. Parents who are employed in the health sector themselves either valued HBTPs more or, conversely, wanted to separate their occupation from their role at home. In certain cases, parents might have been discouraged because their social network was incomprehending of the effort parents needed to put into the treatment. Last, the intention of parents to avoid emphasizing the disability at home, in order to normalize the child's condition, was mentioned as a reason to decline home-based training.

It was also suggested that the parents' views regarding HBTPs may differ with time. Children in the project's target population make the transition from a (therapeutic) playgroup to kindergarten, and from kindergarten to school. During these transition periods, most parents do not want to start a new treatment on top of the child's getting acquainted with the new situation. In the youngest children with CP, HBTPs may overcome developmental disregard and fit in well with usual care (i.e. screening and providing advice). On the other hand, HBTPs may be less feasible for these young children from the perspective of parental coping. Clinicians indicated that, following the diagnosis, some parents appear to be looking for guidance and prefer the therapist to be involved continuously. Later on, parents may have come to terms with the child's disability and the prognosis may be clearer, decreasing the emotional pressure on parents. They may also have become more able to express their children's treatment needs.

Parents declared a need for guidance in stimulating and guiding their child in a playful way at home. In that context, the HBTPs may have created an impression more of therapy than play. In addition, the interventions may have lacked a'wow factor'to motivate children and parents. Alternating therapy periods at the center and at home, following a looser protocol, might have been preferred. However, in one parent's opinion, parents should simply make time for home training.

\section{EXTERNAL POLICIES AND INCENTIVES}

First, although a multi-institutional research team carried out the COAD trial, stakeholders identified a lack of extensive collaboration between institutions before and during this and other projects, resulting in fragmentation of research across the country. The rehabilitation sector should agree on a research agenda reflecting the mutual interests of stakeholders, such as research teams, patient associations, and grant providers. Next, research should be performed by large consortia, alternating the coordinating role among the institutions while other centers facilitate, either in partnership or by providing potential participants. This strategy would be expected to be less sensitive to competition over acquiring funding or participants, and might positively affect mutual commitment. Collaboration is considered insurmountable for studies within pediatric rehabilitation in the Netherlands to succeed. To achieve this, transparency and willingness to share are regarded as highly important. Readiness to make steps in this direction has been perceived. The Dutch rehabilitation association (Revalidatie Nederland) and the Netherlands Society of Rehabilitation Medicine (NSRM/VRA) are currently initiating these transitions. 
Second, despite providing a statement of intent, which is needed for the MREC to assess the attainability of recruitment targets, centers withdrew before the start of the study, as a result of reviews by their local research committees. The research team agreed that this was undesirable and that more binding agreements were needed. Proposed solutions were legal penalty clauses and a track-record of misconduct. Moreover, concerns were expressed about the procedure of local review. Incongruity was observed between experts on the content who were aware of what was going on in the workplace (e.g. therapists), those providing the statement of intent (i.e. the head of department), the local review committees, and the boards of directors who needed to approve the execution of the study. Reciprocal communication about interests, consequences, and deployability should be more effective to get and keep collective agreement about study involvement.

Third, as of 2015, due to decentralization of social policy, the Dutch financial incentives of the health care system have been amended..$^{13}$ This development has put pressure on the rehabilitation setting and restricted the possibilities of financial investments in innovation and research by centers (see the available resources described in the construct'Readiness for implementation').

\section{INNER SETTING}

\section{STRUCTURAL CHARACTERISTICS}

Since the HBTPs impacted the primary processes to a large extent, the infrastructure within centers had to be efficient for the implementation to succeed.

\section{IMPLEMENTATION CLIMATE}

Compatibility: the interventions under study did not fit with the existing workflow of the usual center-based therapies and, as previously mentioned, their adaptability was limited. Because the organizational systems varied, the perceived impracticality differed from one center to another. Some centers were, due to privacy concerns, reluctant to use video-recordings.

Relative priority: because of the regular workload and the urgency to achieve production levels, recruitment for the trial was only a side issue. For managers and clinicians to commit to the project, the expected gains had to outweigh the time and energy spent on implementation, for instance regarding the instructional course for the practitioners involved in the HBTPS.

As a result of the complex and time-consuming development of the intervention protocols, the research team was compelled to postpone recruitment, which affected the implementation climate.

\section{READINESS FOR IMPLEMENTATION}

Leadership engagement: in addition to the financial considerations, two criteria were decisive for centers to participate, namely the ability to deliver the programs according to the protocols, and inclusion of the requisite number of subjects for both the center itself and the study as a whole being feasible. 
Available resources: given the health care system changes, the available resources in the centers did not allow for additional financial investment. Moreover, the limited availability of therapists and remedial educationalists was a restraint on centers' ability to include sufficient participants.

\section{ChARACTERISTICS OF INDIVIDUALS}

\section{INDIVIDUAL STAGE OF CHANGE}

Overall, the clinicians declared that they could not have done better in recruiting families, taking the aforementioned matters into account. The enthusiasm and focus of attention on recruitment of the professionals within the centers varied. Some clinicians stayed focused, while the alertness of others decreased during the study. Several circumstances reduced the clinicians' motivation: 1) a relatively long period between training and start of the interventions; 2 ) interruption of recruitment during holidays; 3 ) randomization of the first participant to the usual care control group; 4) the actual training being home-based and therefore not visible to the department; 5) problems with the digital data sharing tool; and 6) perceived insufficiency in active leadership by the research team to facilitate the therapists.

\section{OTHER PERSONAL ATtRIBUTES}

Certain clinicians were selective in their recruitment, for example, based on knowledge of the families' home situations. They put more effort into the recruitment of parents that they expected to participate successfully and omitted to approach others.

\section{Process}

\section{PlanNing}

A new research team from multiple institutions carried out the COAD trial. Despite its dedication, the group dynamics in combination with the challenging operationalization of the interventions resulted in a too-gradual evolution of the project. Consequently, when the research team was ready to start implementation, the centers' eagerness had declined. Furthermore, this delayed recruitment, hindering proceeding by centers and shortening the inclusion period.

The three-armed design required a relatively large sample size. The estimation of the number of potential participants was exaggerated for several reasons. First, a survey was conducted prior to the study in order to assess willingness to participate in HBTPs in a group of parents whose children were engaged in an intensive treatment program. This was likely a non-representative sample. Also, the characteristics and time-investments of the therapy and research-related activities were not known at that point. Second, the specified inclusion and exclusion criteria reduced the number of eligible participants. Third, expectations of recruitment rates were too optimistic. Therefore, centers were initially invited to collaborate based on their geographical location as 
a practical argument. Taking into account 'Lasagna's law' on patient recruitment might have reduced the overestimation. ${ }^{14}$ The MREC members emphasized the need for them to be more critical in assessing whether the estimation of expected participants in future study proposals is adequate.

The grant provider believed that the research team, like most scientists, lacked strategic thinking skills. A priori barrier analysis, for instance including developments in the health care sector and competitive interventions, might have prevented some of the problems.

\section{ENGAGING}

External change agents: announcing the COAD trial and involving the centers from the beginning, as described earlier, might have had a positive influence on the centers' enthusiasm and commitment. Clear communication of the benefits for the center and primarily focusing on the content experts were suggested as key factors in generating goodwill. The research team reflected that it was not proactive enough in the enrollment and follow-up of centers, and did not sufficiently take the dynamics of each individual organization into account. Moreover, the importance of PR was underestimated and options to better promote the study were missed. Likewise, the project lacked a clear figurehead. Consequently, the pediatric rehabilitation sector was not nationally attentive to the COAD trial.

\section{EXECUTING}

In most centers, it was challenging to identify potential participants. Local or national registers could have eased recruitment for clinicians and researchers.

\section{REFLECTING AND EVALUATING}

Some stakeholders associated with the centers declared that the research team did not sufficiently inform them about the state of affairs, in particular when the project did not go well, while others appreciated the thoughtful communication by the research team. The research team felt that it had taken sufficient initiative and had been very transparent about the recruitment struggles. The grant provider, in contrast, stated that the researchers should have alerted them in a timelier manner. Their self-reflection revealed that they could have been more critical of the progress reports. Additionally, miscommunication between the grant provider and the funding bodies responsible for the grant was said to be partly accountable for the early termination of the trial.

\section{Discussion}

This project identified many interacting factors across all domains of the CFIR (i.e. intervention characteristics, outer setting, inner setting, characteristics of individuals, and process) that impeded implementation of the HBTPs and the COAD trial. Within the complex combination of barriers, three levels can be distinguished: barriers that directly affected the recruitment of participants; barriers related to implementation that indirectly influenced recruitment; and barriers related to the study design that indirectly influenced the recruitment conditions. 


\section{RECRUITMENT}

Most factors that directly impeded recruitment were related to the home-based training. The parent-delivered HBTPs' being frequently perceived to be incompatible with the pressures and the needs of parents is likely to be associated with the elevated levels of parental stress in this population. ${ }^{15}$ An imbalance between personal patient burden and benefit, which has previously been reported as a barrier, ${ }^{16}$ is therefore particularly relevant in families of children with CP. An unanticipated finding was that, despite the unique potential of HBTPs to integrate training moments and activities into the family routine, many parents preferred the alternative center-based group interventions. There are similarities between the present results and a recent review which shows that parents'feelings of capability is a determinant of parent-delivered interventions. Some parents do not see their parenting role as including delivery of therapy and tend to feel overwhelmed. ${ }^{17}$ Consistent with the literature, the high complexity of the HBTPs also appeared to be an underlying factor. ${ }^{4}$ This was to some extent an inevitable consequence of the operationalization of the innovative home-based training and the fundamental theoretical principles of motor learning that were used. These substantial barriers unique to the home-based training conflicted with the intended shift towards reinforcement of self-reliance of families.

Aarts et al's RCT is an example of a study in the Netherlands that successfully enrolled the targeted number of children with CP and reported not a single drop-out in the experimental group. The authors noticed the treatment's being attractive to children as a major strength. Based on the results of the current evaluation, procedural simplicity may have been a facilitating factor from the therapists' viewpoint. ${ }^{18}$

Considering the methodology of the study, the randomization aspect was assumed to be a disincentive for parents to participate. This is in agreement with the BoBiVa, as well as the SPACE BOP study, in which the intended recruitment procedures had to be adapted because many parents had a strong preference for or aversion to the experimental treatment (i.e. botulinum toxin A) and therefore did not want their child with CP to participate in a randomized trial. ${ }^{19,20}$

\section{IMPLEMENTATION}

The two essential barriers of implementation identified were both associated with collaboration. While identifying facilitators was not the aim of the project, the engagement of the resonance group clearly emerged as being highly valued. This is consistent with the literature reporting that an internally-developed intervention, with transparency and participation in the decision-making process, is an effective engagement strategy for stakeholders to feel ownership of the innovation, and this contributes to implementation success. ${ }^{21}$ In this context, the current study pointed out specific opportunities for further enhancements in pediatric rehabilitation: empowering parents to be involved as an advisor or partner, involving stakeholders from the very beginning of the project, and including a representative sample of targeted users. Our results support the general recommendation to engage all stakeholders as equal partners in the process. ${ }^{4}$ It is important to realize that, besides being an opportunity, participation in research teams can be perceived as an additional pressure by parents. Therefore the level of parent and patient participation should cor- 
respond with the family wishes. A leading example of successful partnership between researchers, families, and health care professionals is the Peninsula Cerebra Research Unit for Childhood Disability Research (PenCRU). ${ }^{22}$

This evaluation emphasized the importance of research consortia in strengthening recruitment capacity. A research consortium has previously been defined as "a group of individuals and their organizations working together to address specific research needs and attract funding. Consortia design and implement research over one or several countries and sites."23 El Ansari et al. determined benefits and challenges of research consortia and provides recommendations for effective collaboration. 23,24

\section{STUDY DESIGN}

The appropriateness of an RCT as the study design and the estimated ability to recruit the necessary number of study participants were criticized. The former criticism is in agreement with, amongst others, the new Medical Research Council guidance, which recommends an iterative development and evaluation process for complex interventions. ${ }^{25}$ Although Dutch grant providers and funders have recently started to encourage smaller-scale research projects, opportunities to gradually develop and investigate interventions are still limited, given the highly competitive process of acquiring research funding and associated demands. At the same time, the understanding and appreciation of alternative designs that require smaller sample sizes need to be advanced. Given the heterogeneity of the population of children with $\mathrm{CP}$, single-case experimental design (SCED) can be a powerful design to evaluate intervention effectiveness.

In interpreting this evaluation, it must be recognized that the results may point to contradictory conclusions. For instance, it was argued that there should be more intensive involvement of parents as stakeholders in the research project, while on the other hand the burden on parents was mentioned as a limiting factor to their participation in the trial. Similarly, other recommendations would have had drawbacks, such as a significant increase in time and costs necessary to develop and execute the study. In some instances, the research team decided from the outset that theoretical and scientific requirements of the HBTPs and the trial outweighed other interests. An example concerns the home visits, which were adhered to because the content of the HBTPs and their standardization within the trial were prioritized over the centers' concerns about the associated costs. Overall, it is a challenge to make a trade-off between the many sometimes incompatible interests, including family needs, centers' perspectives in terms of feasibility and costs, scientific rigor, formal policies, rules and regulations, and time and costs to prepare and perform the study. Effective collaboration approaches are assumed to constructively advocate each stakeholder's viewpoint.

In addition to the many aspects related to the COAD trial that could have been influenced, the amendment of the health care policy was a circumstance beyond the control of the research team and other stakeholders involved. This, as well as competitive group-based interventions, applies specifically in the Netherlands. The other results are expected to be generalizable to other Western societies and health care systems. 
The number and variety of stakeholders involved was the major strength of this evaluation. However, due to legal and ethical constraints about approaching non-participants, the proportion of parents was relatively low. Another limitation was that, given the aim of the project, the focus was on barriers to participation only. Facilitators of recruitment were not systematically explored and their role in the process may therefore have been underestimated. Last, this evaluation produced a narrative of all opinions, sometimes opposed, rather than consensus-based outcomes.

Barriers directly related to the home-based training imply that those concerned were not yet receptive to the major transition to parent-delivered interventions. Efforts are needed to ensure that pediatric rehabilitation care is compatible with societal developments as well as family needs. A hybrid combination of home-based and center-based features may yield maximum benefits. The findings of this evaluation may improve the planning and conduct of studies and other implementation projects in pediatric rehabilitation. Figure 3 provides a dozen directions for successful recruitment of participants in pediatric rehabilitation research. Further work is required to establish facilitators of both participant recruitment and implementation of interventions, and to create evidence-based strategies to facilitate these processes. Contrary to expectations, many of the barriers addressed are shared with other health care fields and populations. It is therefore essential that lessons learned from previous projects are also recognized.

\section{Conclusion}

This evaluation has shown that barriers to recruitment of participants during the COAD trial were mostly related to the home-based training approach. The findings can be used to develop a targeted transition towards parent-delivered interventions in pediatric rehabilitation. Furthermore, it has been confirmed that comprehensive collaboration between all stakeholders involved is fundamental in order to balance the many different interests. Last, the results favor the facilitation of an iterative process, including small-scale studies, of intervention development and evaluation.

\section{Competing InTERESTS}

The authors have no competing interests to declare.

\section{FUNDING}

This work was supported by Revalidatiefonds, Johanna Kinderfonds, and Stichting Rotterdams Kinderrevalidatie Fonds Adriaanstichting in the 3rd Program Rehabilitation Research of ZonMw (the Netherlands Organization for Health Research and Development), [grant number 630000001]; Revalidatiefonds [grant number R2016006]; and Stichting Vooruit [grant number 18-05/YvH/NS]. Representatives of the grant provider and funding bodies contributed as stakeholders to the data collection and member checking. They had no other role in study design, in the analysis and interpretation of data, in the writing of the report, and in the decision to submit the article for publication. 


\section{A Dozen Directions}

\section{for successful recruitment of participants in pediatric rehabilitation research}

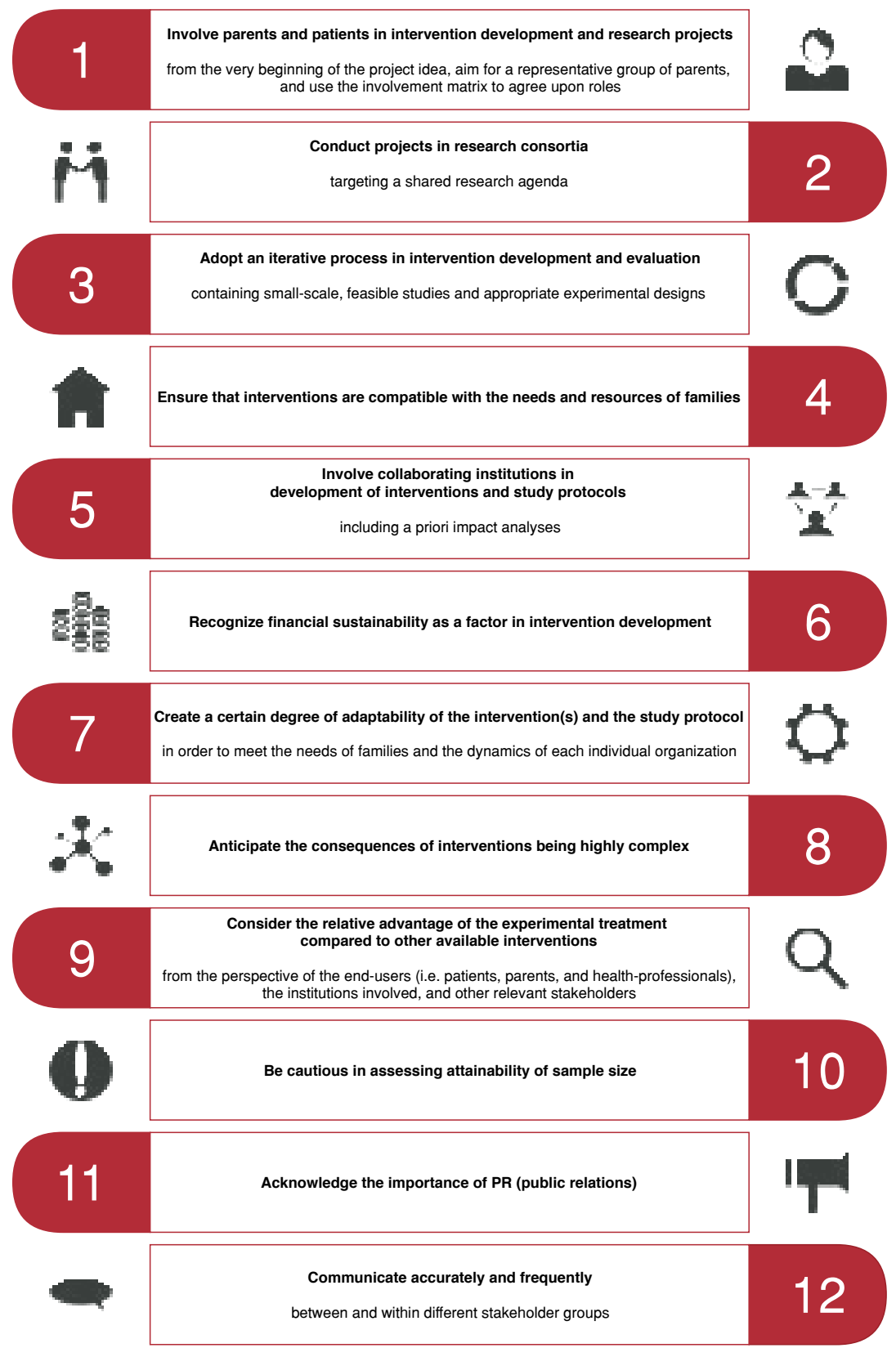

Figure 3 A dozen directions for successful recruitment of participants in pediatric rehabilitation research 


\section{Acknowledgements}

The authors thank all stakeholders for their contribution to this evaluation: Dr. Mattijs Alsem (Amsterdam UMC), Dr. Hans Arendzen (ZonMw), Monique Arts (Libra), Joop Beelen (HandicapNL, former Revalidatiefonds), Nienke Bosboom (parent), Nicole Brouwers (parent, COAD resonance group), Mechteld van den Beld (HandicapNL, former Revalidatiefonds), Dr. Annemieke Buizer (Amsterdam UMC), Martijn da Costa (ZonMw), Lianne Damen (Commissie Mensgebonden Onderzoek regio Arnhem - Nijmegen), Anke Defesche (Adelante, COAD resonance group), Hanneke Denissen (Adelante), Dr. Yvonne Geerdink (Sint Maartenskliniek, COAD resonance group), Marianne Geerts (Libra), Prof. dr. Sander Geurts (Radboudumc, COAD research team), Dr. Imelda de Groot (Radboudumc, COAD research team), Ellen Hanselaar (JKF Kinderfonds), Sarina Ishak (ZonMw), Anne Jensen (Rijndam), Marjon Kissels (Adelante, COAD resonance group), Martijn Klem (parent, BOSK, COAD resonance group), Joyce van de Laar (parent), Judith van Munster (Sint Maartenskliniek, COAD resonance group), Dr. Ingrid van de Port (Revant), Liset de Reus (ZonMw), Dr. Jolt Roukema (Commissie Mensgebonden Onderzoek regio Arnhem - Nijmegen), Caroline Scheijmans (Libra), Dr. Lucianne Speth (Adelante, COAD resonance group), Prof. dr. Bert Steenbergen (Radboud University, COAD research team), Ingrid van den Tillaar (Sint Maartenskliniek, COAD resonance group), Sandra Titulaer (Rijndam), Dr. Cecile Utens (Libra), Eef Vennix (Libra), Jan Weurding (parent), and Anne Wind (Treant). Thanks to Les Hearn for proofreading the manuscript. 


\section{REFERENCES}

1. Damen L, van Agt F, de Boo T, Huysmans F. Terminating clinical trials without sufficient subjects. Journal of medical ethics. Jul 2012;38(7):413-416.

2. McDonald AM, Knight RC, Campbell MK, et al. What influences recruitment to randomised controlled trials? A review of trials funded by two UK funding agencies. Trials. Apr 7 2006;7:9.

3. Carlisle B, Kimmelman J, Ramsay T, MacKinnon N. Unsuccessful trial accrual and human subjects protections: an empirical analysis of recently closed trials. Clinical trials (London, England). Feb 2015;12(1):77-83.

4. Huang GD, Bull J, Johnston McKee K, Mahon E, Harper B, Roberts JN. Clinical trials recruitment planning: A proposed framework from the Clinical Trials Transformation Initiative. Contemporary clinical trials. Mar 2018;66:74-79.

5. Patterson KK. Rehabilitation research: who is participating? Physiotherapy Canada. Physiotherapie Canada. Summer 2013;65(3):201-203.

6. Schnackers M, Beckers L, Janssen-Potten Y, et al. Home-based bimanual training based on motor learning principles in children with unilateral cerebral palsy and their parents (the COAD-study): rationale and protocols. BMC pediatrics. Apr 18 2018;18(1):139.

7. Dutch Association of Medical Specialists. Vision document: Medical Specialist 2025 - Ambition, trust, cooperation2017.

8. Damschroder LJ, Aron DC, Keith RE, Kirsh SR, Alexander JA, Lowery JC. Fostering implementation of health services research findings into practice: a consolidated framework for advancing implementation science. Implementation science: IS. Aug 7 2009;4:50.

9. Alsem MW. Family needs and the role of information in paediatric rehabilitation care Utrecht (Netherlands), Utrecht University; 2018.

10. Aarts PB, van Hartingsveldt M, Anderson PG, van den Tillaar I, van der Burg J, Geurts AC. The Pirate group intervention protocol: description and a case report of a modified constraint-induced movement therapy combined with bimanual training for young children with unilateral spastic cerebral palsy. Occupational therapy international. Jun 2012;19(2):76-87.

11. May A, Mathijssen J. "Alternatieven voor RCT bij de evaluatie van effectiviteit van interventies!?" Eindrapportage2015.

12. Torgerson DJ, Sibbald B. Understanding controlled trials. What is a patient preference trial? BMJ (Clinical research ed.). Jan 31 1998;316(7128):360.

13. Vermeulen W. Decentralization of social policy in the Netherlands. CPB Netherlands Bureau for Economic Policy Analysis.

14. Bachenheimer JF, Brescia BA. Reinventing Patient Recruitment: Revolutionary Ideas for Clinical Trial Success. New York, NY: Routledge; 2016.

15. Pinquart M. Parenting stress in caregivers of children with chronic physical condition-A meta-analysis. Stress and health : journal of the International Society for the Investigation of Stress. Apr 2018;34(2):197207.

16. Adams M, Caffrey L, McKevitt C. Barriers and opportunities for enhancing patient recruitment and retention in clinical research: findings from an interview study in an NHS academic health science centre. Health research policy and systems. Mar 12 2015;13:8. 
17. Lord C, Rapley T, Marcroft C, Pearse J, Basu A. Determinants of parent-delivered therapy interventions in children with cerebral palsy: A qualitative synthesis and checklist. Child: care, health and development. Sep 2018;44(5):659-669.

18. Aarts PB, Jongerius PH, Geerdink YA, van Limbeek J, Geurts AC. Effectiveness of modified constraint-induced movement therapy in children with unilateral spastic cerebral palsy: a randomized controlled trial. Neurorehabilitation and neural repair. Jul-Aug 2010;24(6):509-518.

19. Speth L, Janssen-Potten Y, Rameckers E, et al. Effects of botulinum toxin A and/or bimanual task-oriented therapy on upper extremity activities in unilateral Cerebral Palsy: a clinical trial. BMC neurology. Aug 19 2015;15:143.

20. Schasfoort F, Dallmeijer A, Pangalila R, et al. Value of botulinum toxin injections preceding a comprehensive rehabilitation period for children with spastic cerebral palsy: A cost-effectiveness study. Journal of rehabilitation medicine. Jan 10 2018;50(1):22-29.

21. CFIR Research Team-Center for Clinical Management Research. Intervention Source. https://cfirguide. org/constructs/intervention-source/. Accessed 11 November, 2018.

22. Peninsula Cerebra Research Unit for Childhood Disability Research (PenCRU). http://www.pencru.org/. Accessed 10 November, 2018.

23. El Ansari W, Maxwell AE, Mikolajczyk RT, Stock C, Naydenova V, Kramer A. Promoting public health: benefits and challenges of a Europeanwide research consortium on student health. Central European journal of public health. Jun 2007;15(2):58-65.

24. El Ansari W, Maxwell AE, Stock C, Mikolajczyk R, Naydenova V, Kramer A. Nurses'involvement in international research collaborations. Nursing standard (Royal College of Nursing (Great Britain) : 1987). Mar 7-13 2007;21(26):35-40.

25. Craig P, Dieppe P, Macintyre S, Michie S, Nazareth I, Petticrew M. Developing and evaluating complex interventions: the new Medical Research Council guidance. International journal of nursing studies. May 2013;50(5):587-592. 

Chapter 8

\section{Effect of home-based bimanual training in children with unilateral cerebral palsy (the COAD-study): a case series}

LWME Beckers, EAA Rameckers, PBM Aarts, JJW van der Burg,

RJEM Smeets, COAD Author Group, MLAP Schnackers,

B Steenbergen, IJM de Groot, ACH Geurts, YJM Janssen-Potten 


\section{Abstract}

Aims To explore the child- and parent-related effects of home-based bimanual training, adopting implicit or explicit motor learning, in children with unilateral cerebral palsy.

Methods Case series of 14 children (2-7 years) who completed the training. Five children performed the implicit and nine the explicit programs. Both included goal-oriented task-specific training for 3.5 hours/week for 12 weeks. A therapist and remedial educationalist coached parents in providing the training. Progression on bimanual goals and therapy-related parental stress were of primary interest, measured with the Canadian Occupational Performance Measure (COPM) and by parental interviews. Data were collected at baseline (T0), halfway through the training (T1), at the end of training (T2), and after 12 weeks (T3).

Results On the COPM performance scale, a clinically-relevant change was seen in 50\% (7/14), $86 \%(12 / 14)$, and $85 \%(11 / 13)$, and, on the satisfaction scale, in $43 \%(6 / 14), 64 \%(9 / 14)$, and $54 \%$ (7/14) of the children, at $\mathrm{T} 1, \mathrm{~T} 2$, and $\mathrm{T} 3$, respectively. During the interviews, some parents indicated that they had experienced stress because of the intensity of the training. The secondary outcomes showed a tendency toward reduction of therapy-related and generic parental stress.

Conclusions The programs seem to positively affect children's bimanual performance and parental stress. 
Children with unilateral spastic cerebral palsy (UCP) often encounter problems in the development of their arm-hand function and skill performance. The ultimate aim of rehabilitation is to increase independence and participation. To achieve this, effective therapies are required to improve the performance of bimanual activities in everyday life. Bimanual training in the children's daily home environment seems to be the best approach to realize this.

Previous research has established the effectiveness of bimanual training (Novak et al., 2013), and evidence for home-based programs is growing (Sakzewski, Ziviani, \& Boyd, 2014). However, to our knowledge, only one effectiveness study has so far focused on parent-delivered bimanual training at home. In that randomized controlled trial (RCT), children performed home-based activities for 2 hours a day, 5 days a week, for 9 weeks. This intensive training was more successful in making progress on functional goals than was lower extremity home-based training (Ferre et al., 2017).

According to Sakzewski et al., upper limb interventions in children with UCP should be goal-directed, adequately dosed, and based on motor-learning approaches that use activity-based therapy (Sakzewski et al., 2014). These guidelines also apply to home-based training. A remaining question to be addressed is the suitability of different motor-learning approaches in the specific context of parent-delivered programs. Explicit motor learning generates consciously accessible information about how to produce appropriate movements (Eliasson \& Burtner, 2008; van der Kamp, Steenbergen, \& Masters, 2018). In the implicit approach, procedural learning about how a movement is performed occurs as a result of repeated practice, without active accumulation of declarative knowledge (Eliasson \& Burtner, 2008; van der Kamp et al., 2018). In order to evaluate the potential of these contrasting motor-learning principles in home-based training, two programs were developed: a home-based bimanual training program adopting implicit motor-learning principles, hereafter called 'implicit program,' and a home-based bimanual training program adopting explicit motor-learning principles, hereafter called 'explicit program.'

There are indications that home-based programs can induce parental stress (Lin et al., 2011; Peplow \& Carpenter, 2013). It is imperative to take this into account in the development and evaluation of such interventions. Consequently, coaching by a remedial educationalist was incorporated in the home-based training programs under study, and therapy-related parental stress was monitored throughout.

The main objective of the programs was to improve bimanual performance of targeted activities by the child, while preventing an increase of (therapy-related) parental stress. Secondary effects were expected on manual capacity, general bimanual performance, and participation of the child, and on empowerment of the parents. The objective of this case series was to explore the child- and parent-related effects of the implicit and explicit home-based bimanual training programs in children with UCP. 


\section{CASE DESCRIPTION}

\section{Patient information}

Figure 1 illustrates the participant flow. Initially, 12 participants were enrolled in an RCT ("COAD: home-based training programs for young children with cerebral palsy,"), of whom seven were allocated to either the implicit or explicit training approach, and five to the control group. After early closure of the RCT (Stage 1) due to recruitment problems (Beckers, Rameckers, et al., 2018), 11 additional participants were recruited for the purpose of this case series (Stage 2). These participants received either the implicit or explicit program, according to the parents' preference. Of the total of 18 children who started a home-based training program, 14 completed the training and are reported on in this case series. Five families performed the implicit program (two randomized and three self-chosen) and nine families the explicit program (three randomized and six self-chosen).

Participants were recruited from five rehabilitation centers in the Netherlands between April 2016 and March 2018. Children were eligible if they had a clinically-confirmed diagnosis of unilateral spastic CP, were aged 2 through 7 years, and had Manual Ability Classification System (MACS), as well as Gross Motor Function Classification System (GMFCS), Levels I to III (Eliasson et al., 2006; Palisano et al., 1997). Potential participants were excluded if 1) the child had a co-morbidity affecting arm-hand function; 2) the child had received surgery or other medical intervention within the nine months prior to or during the study that might affect motor function; 3) the child was to participate in an intensive therapy program focusing on the upper limbs during the study; 4) parents were unable to respond to interviews and questionnaires in Dutch; 5) the rehabilitation team expected parents to be unable to adhere to the programs; or 6) there were any other medical or psychosocial contraindications.

Ethical approval was granted for both study stages by the medical ethical committee Commissie Mensgebonden Onderzoek regio Arnhem - Nijmegen (Protocol Number NL53670.091.15). Informed consent was obtained for each participant.

\section{DEMOGRAPHIC INFORMATION}

Table 1 provides characteristics of participants and baseline findings. Eleven boys and three girls participated. Their median age was 4.0 years (IQR 1.5). The majority of children had MACS Level II, and GMFCS Level I. Supplemental File 1 presents the children's motor impairments (range of motion and strength).

All children had problems in performance of bimanual activities. The rehabilitation needs defined by parents at the start of the program included five categories of activities: (un)dressing (26 needs), eating and drinking (18 needs), crafts (10 needs), play (7 needs), and gross motor (outdoor) activities (9 needs). The most common needs were related to putting on, taking off and zipping a jacket ( 9 children), pulling lower garments down and up (6 children), using cutlery while eating ( 6 children), and holding paper while cutting, drawing, coloring, or erasing ( 9 children). 


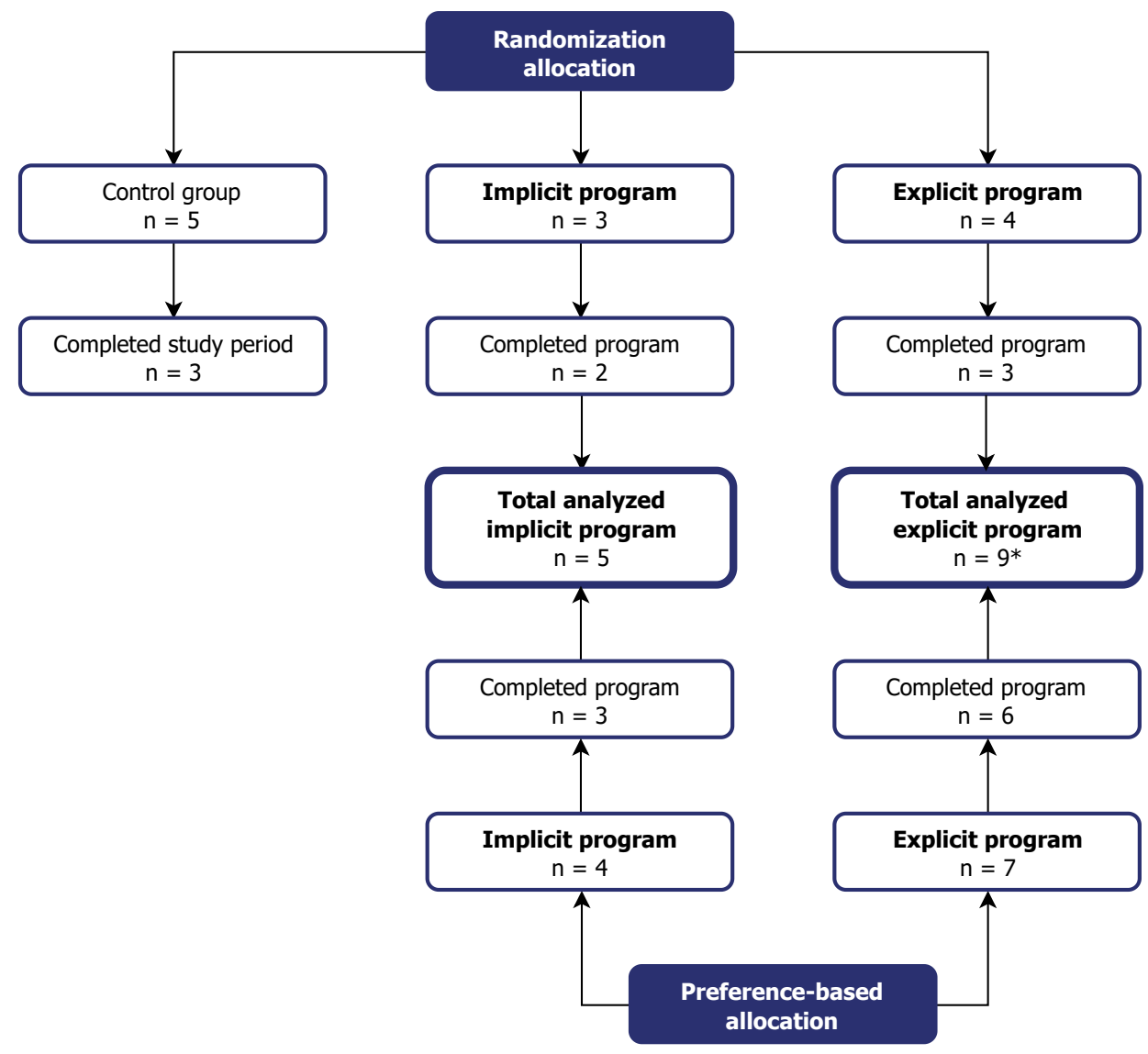

* One participant that was included in the analyses dropped out at follow-up (T3)

Figure 1 Participant flow 


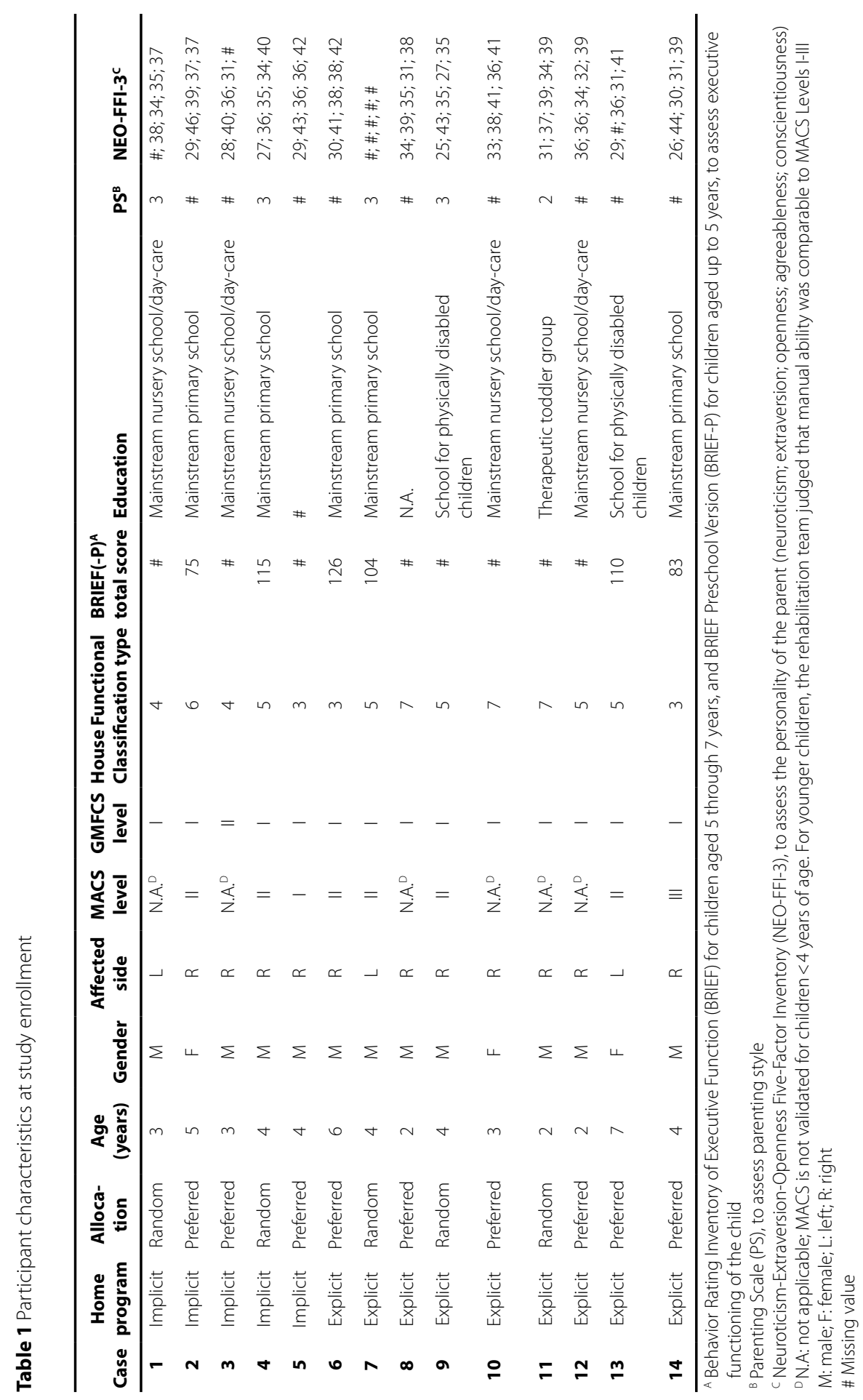




\section{THERAPEUTIC INTERVENTIONS}

The implicit and explicit programs both incorporated goal-oriented and task-specific training principles. For each child, five bimanual needs were identified collaboratively with parents, providing the input for the intervention goals. Therapists analyzed the children's performance for each identified activity and prepared individual training programs. Exclusively, in the explicit program, a movement analysis by the therapist informed the content of the training. Parents were provided with instructional videos and a manual before the start of the training. Intended training intensity was 3.5 hours per week for 12 weeks. Parents in the implicit program elicited the desired bimanual performance by organization of the training activities, complemented with task-oriented instructions and feedback; parents in the explicit program gave instructions and feedback focused on motor performance, in addition to task-oriented instructions and feedback. A therapist coached parents in applying the training, and a remedial educationalist concentrated on parent-child interaction and child behavior. The therapist had a face-to-face meeting with parents at the start of the program and had weekly contact with them throughout the training, primarily by telephone and (three times) through home visits. The remedial educationalist met parents before the start of the training and phoned them two weeks afterwards. Support during the remaining weeks of the program was available on request of either the parents themselves or the therapist, if training was impeded by parent-child interaction or child behavior. The coaching was provided by professionals at the child's rehabilitation center. The professionals had attended an instructional course and received a manual. A detailed protocol of the programs is published elsewhere (Schnackers et al., 2018).

After finishing the home-based training, children received usual care for 12 weeks before the follow-up.

\section{OUTCOME MEASURES}

In selecting the outcome measures, four aspects were taken into account: 1) the robustness of psychometric properties (e.g. sensitivity to change); 2) the ability to compare the results with existing and future studies; 3 ) the intention to capture potential changes across the activity and participation domains of the International Classification of Functioning, Disability, and Health (ICF); and 4) the importance of assessing constructs of the motor functioning of the child and of parental stress that are of specific interest in the implementation of home-based bimanual training.

\section{Parent-Rated outcomes AT CHILd LeVEl}

\section{CANadian OCCupational PeRformance Measure (COPM)}

Parents identified and ranked five needs involving bimanual activities of self-care, play and/or leisure, using the child-adapted version of the COPM. For each activity, parents rated the performance and their satisfaction with this performance, generating an averaged performance and satisfaction 
score. As the COPM performance scale corresponds to the goal-oriented and family-centered approach of the interventions, this was the primary outcome. The COPM has good psychometric properties, which also hold in the child-adapted version (Cusick, Lannin, \& Lowe, 2007).

\section{HAND-USE-AT-HOME QUESTIONNAIRE (HUH)}

The amount of spontaneous use of the child's affected hand at home was assessed using the $\mathrm{HUH}$, which consists of 18 age-related play and self-care activities for which bimanual execution is required or preferred. Parents rated the frequency of spontaneous use of the affected hand in these activities on a 5-point scale. Test-retest reliability and construct validity of this Dutch questionnaire in children with CP are very good and good, respectively (Geerdink et al., 2017; van der Holst et al., 2018).

\section{LIFESTYLE ASSESSMENT QUESTIONNAIRE FOR CP (LAQ-CP)}

The child's participation was assessed through the LAQ-CP, a parent-reported questionnaire. The LAQ-CP consists of 46 items and examines the impact of the disabilities on six dimensions. Weighted aggregation of the domain scores is used to calculate a total Lifestyle Assessment Score (LAS) (Mackie, Jessen, \& Jarvis, 1998). The Dutch version has not yet been validated.

\section{OBSERVER-RATED OUTCOMES AT CHILD LEVEL}

\section{GOAL ATTAINMENT SCALING (GAS)}

The highest prioritized rehabilitation goal was scaled using GAS. The progress towards the rehabilitation goal was scored in relation to predetermined criteria as formulated in the GAS scale. As knowledge about the capacities of the child was needed, the personal GAS scale was formulated by the coaching therapist. GAS is responsive to measurements of individual change over time in children with CP and has good inter-rater reliability and acceptable validity (Steenbeek, Ketelaar, Galama, \& Gorter, 2007; Steenbeek, Ketelaar, Lindeman, Galama, \& Gorter, 2010).

\section{Assisting Hand ASSESSMENT (AHA)}

Bimanual performance of the child was assessed using the AHA version 5.0 (either Small Kids or School Kids version). The AHA measures the typical application of a child's assisting hand in a range of standardized bimanual activities. The AHA has been reported to have good validity, reliability and responsiveness to change in children with UCP (Krumlinde-Sundholm \& Eliasson, 2003; Krumlinde-Sundholm, Holmefur, Kottorp, \& Eliasson, 2007). 


\section{ObSERVATIONAL SKILLS ASSESSMENT SCORE (OSAS)}

The OSAS was used to measure the quality of use of the affected hand in age-appropriate, standardized bimanual motor tasks in which the use of both hands is required. The OSAS tasks involve many repetitions of actions to reliably assess quality of use. Quality of use is scored every second in order to make the OSAS sensitive to subtle differences. There is evidence that OSAS has satisfactory reliability (Speth et al., 2013).

\section{Parent-Related outcomes}

\section{PARENTAL INTERVIEWS}

We explored parental stress experienced throughout the programs with a qualitative approach. Parents participated in interviews during and after the intervention as part of a mixed-methods process evaluation that was embedded in the effect study. The specific procedures have been reported elsewhere (Beckers, van der Burg, et al., 2018).

\section{THERAPY-RELATED PARENTAL STRESS QUESTIONNAIRE (IN DUTCH: VRAGENLIJST OUdERLIJKE StREsS ALS GEVOLG VAN THERAPIE VAN HET KIND (VOST))}

The project team has developed the VOST to measure therapy-related parental stress and the factors that contribute to this stress. The questionnaire consists of three parts. In Part I, parents rate their perceived overall parental stress due to therapy of their child on a Visual Analogue Scale (VAS) and describe in their own words what caused the stress. In Part II, 34 potential stress-inducing factors are presented, covering six domains. For each domain, parents select those factors which have caused them stress and have the opportunity to define additional factors. Moreover, parents rate the amount of stress for each domain separately on a VAS. In Part III of the questionnaire, parents identify the three factors selected in Part II which led to most stress. The psychometric properties of VASs are considered to be satisfactory (Lesage, Berjot, \& Deschamps, 2012), while those of the VOST are still under investigation.

\section{PARENTING STREsS QUeSTIONNAIRE (IN DUtCh: OPVOEDINGSBELASTINGVRAGENLIJST (OBVL))}

The OBVL was used to assess generic stress related to parenting. This Dutch questionnaire consists of five scales (total of 34 items). The reliability and validity of the OBVL are good (Vermulst, Kroes, De Meyer, Nguyen, \& Veerman, 2015). 


\section{EMPOWERMENT QUESTIONNAIRE (EMPO)}

The EMPO Version 3.1 was used to assess empowerment of parents. This is a 27-item questionnaire divided into three subscales. Reliability and validity of the EMPO have been confirmed to be sufficient to good (Damen et al., 2017).

\section{DAta COLlection}

Data were collected at four time points: at baseline (T0), halfway through the training period (T1, after 6 weeks of training), at the end of the program (T2, after 12 weeks of training), and at follow-up (T3, 12 weeks after the end of the program). The COPM and therapy-related parental stress evaluated by parental interviews were of primary interest. To limit the burden on families during the training period, secondary outcome measures were not assessed at T1.

The parent most involved in the training rated the parent-reported outcome measures. COPM was thrice assessed face-to-face and once by telephone (T1). All questionnaires were internet-administered by Castor Electronic Data Capture.

Assessments were completed by one of two research assistants who were trained and experienced in the use of the outcome measures and were blinded as to intervention. The GAS (i.e. performance of the activity set by the rehabilitation goal), AHA, and OSAS were video recorded and scored on subsequent viewing by trained assessors blinded as to treatment and time point. The AHA evaluator was qualified to administer this measure.

\section{Findings}

\section{Parent-Rated outcomes at Child LeVel}

\section{Canadian Occupational Performance Measure (COPM)}

On the COPM performance scale, $86 \%(12 / 14)$ of the children exceeded the clinically-relevant change of two points after the program. The scores between T0 and T1 already showed an improvement of at least two points in half of the children (7/14). At follow-up, 85\% (11/13) of the children showed a clinically-relevant increase compared to baseline. The COPM satisfaction scores improved by clinically-relevant amounts during the first half of the program in $43 \%(6 / 14)$ and during the total program in 64\% (9/14) of the children. In 88\% (7/8) of the improved cases, the T3 score was still > 2 points higher than T0 (Table 2).

As can be seen from Figure 2, clinically-relevant change scores on the COPM performance and satisfaction scales were achieved with both the implicit and explicit programs. 


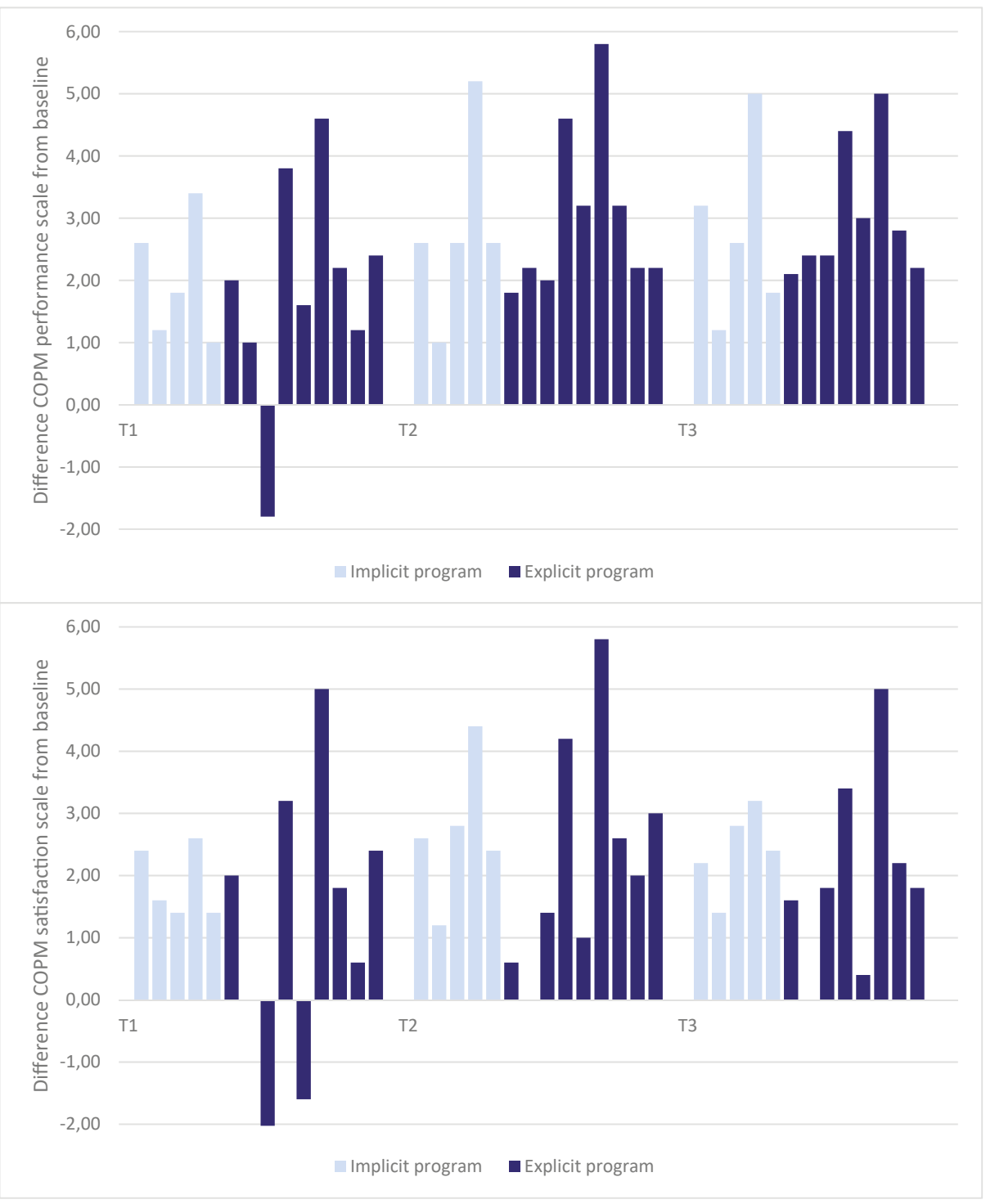

Figure $\mathbf{2}$ COPM change scores 


\section{HAND-USE-AT-Home QUESTIONNAIRE (HUH)}

For individual children, the minimal detectable change of the $\mathrm{HUH}$ is 1.66 logits (van der Holst et al., 2018). For none of the children was an effect of this magnitude found (Table 2).

\section{Lifestyle Assessment Questionnaire for CP (LAQ-CP)}

No values for statistically-significant or clinically-relevant change scores for LAQ-CP have been reported in the literature, so we chose an arbitrary difference of 20 points to be regarded as relevant. During the program, one child (8\%) improved and one child (8\%) deteriorated on the physical independence domain. The physical independence of three other children (27\%) was better at follow-up than at baseline. An unintended change was an increase of economic burden in two families (15\%) at T2 and in three other families at T3 (27\%). The other domain scores and the LAS indicated no changes in participation (Table 2).

\section{OBSERVER-RATED OUTCOMES AT THE CHILD LEVEL}

\section{Goal Attainment Scaling (GAS)}

At T2, two children (17\%) scored -3 (deterioration), four children (33\%) scored -2 (equal to starting level), three children (25\%) scored 0 (expected goal), one child (8\%) scored 1 (somewhat more than expected), and two children (17\%) scored 2 (much more than expected). Thus, after the program, $50 \%$ of the children (6/12) reached the expected goal or more. At T3, two children (18\%) scored -2 , four children (36\%) scored -1 (less than expected), two children (18\%) scored 0, and three children (27\%) scored 1 , meaning that $45 \%$ of the children $(5 / 11)$ reached or maintained the expected goal or more at follow-up (Table 3).

\section{Assisting Hand Assessment (AHA)}

In two children, the AHA score showed a clinically-relevant (at least 5 units) increase at T2 (14\%), as well as T3 (15\%), compared to baseline. In two other children (14\%), this change was only found at T2 and, in another two children (15\%), this change only occurred at T3. The scores of one child (7\%) showed a clinically-relevant decrease at both T2 and T3 (Table 3).

\section{Observational Skills Assessment Score (OSAS)}

When compared to the smallest detectable difference as reported by Speth et al. (Speth et al., 2013), the individual data showed no significant effects (Table 3). 


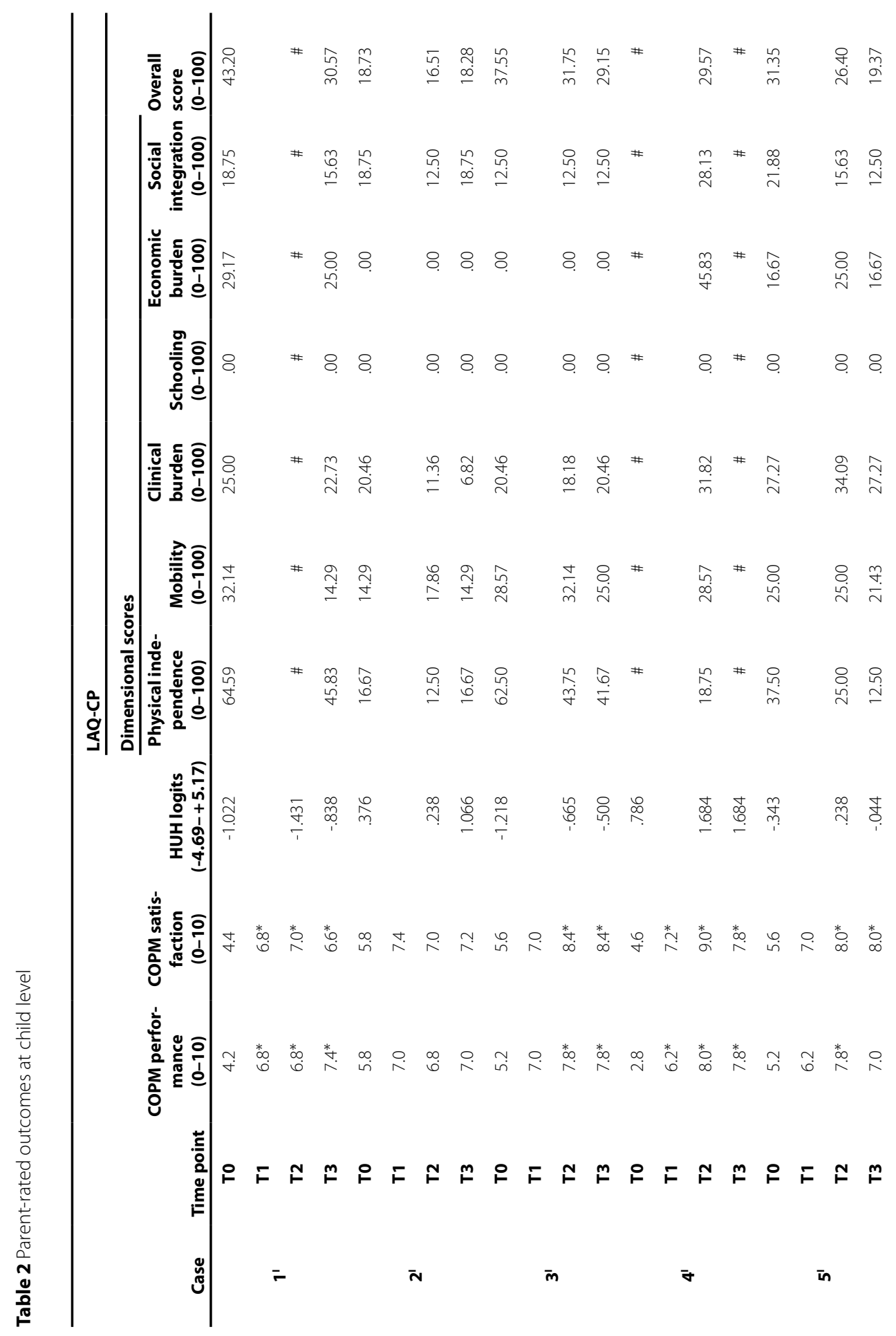




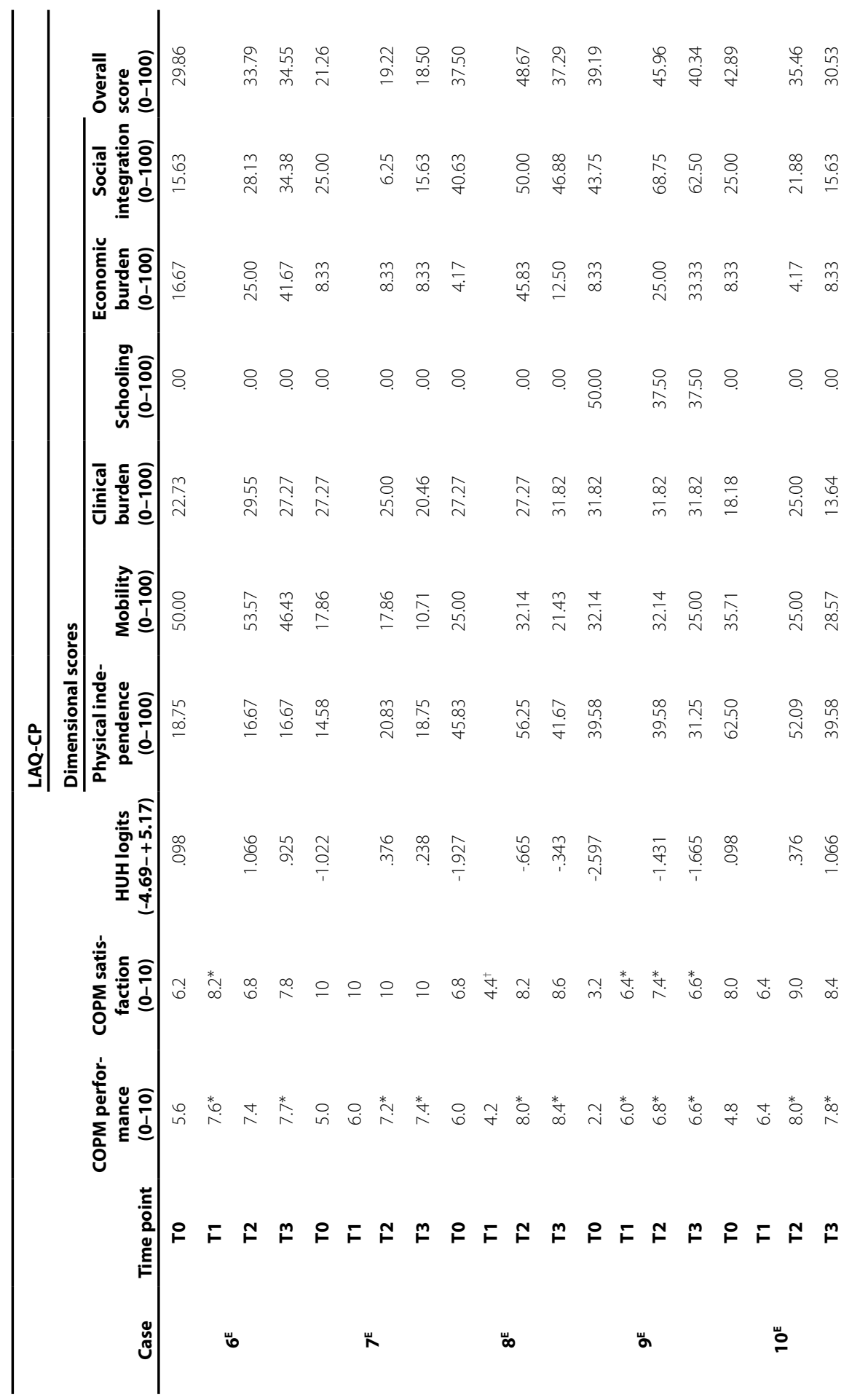




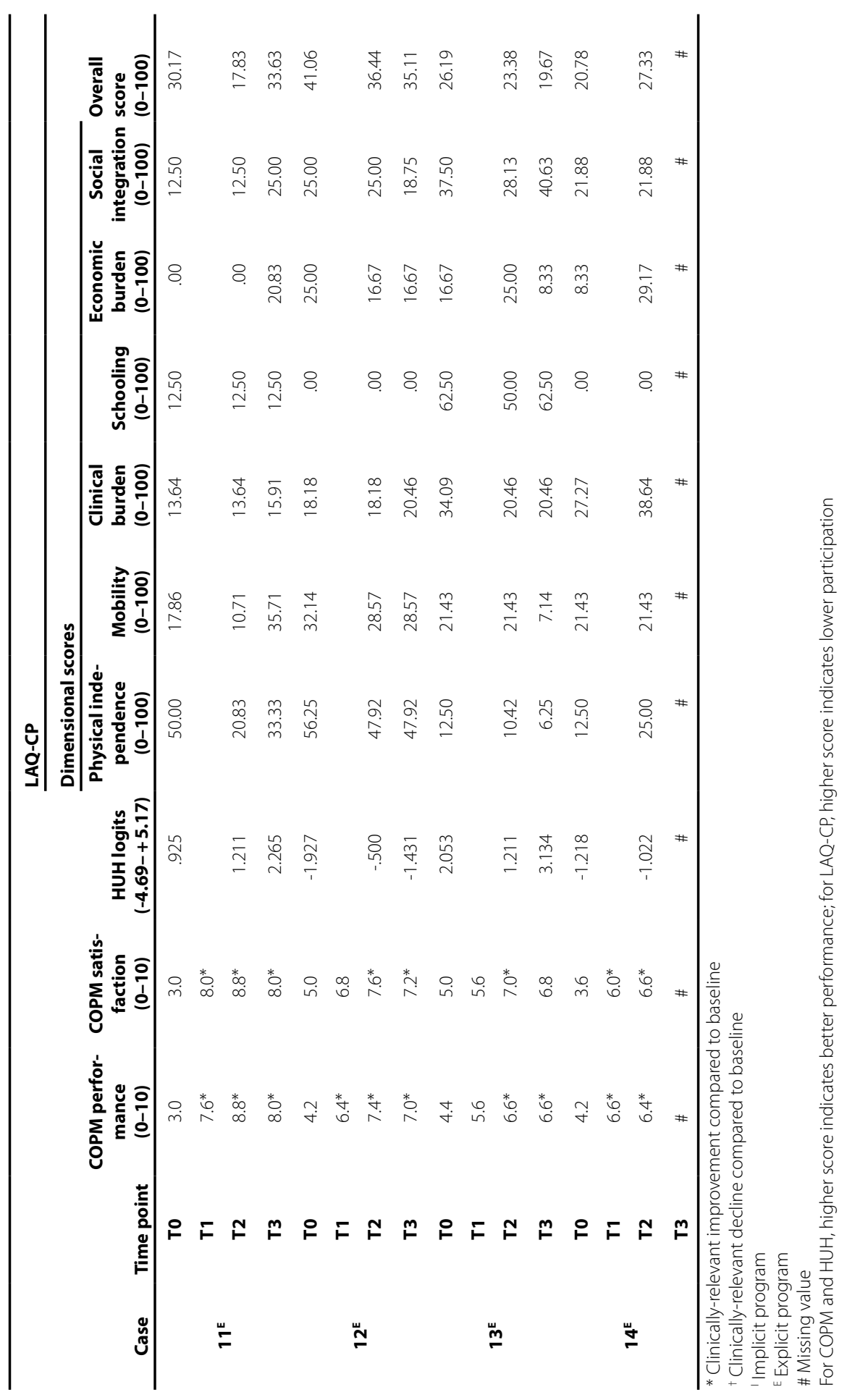


Table 3 Observer-rated outcomes at child level

\begin{tabular}{|c|c|c|c|c|c|c|c|c|c|}
\hline \multirow[b]{2}{*}{ Case } & \multirow[b]{2}{*}{$\begin{array}{l}\text { Time } \\
\text { point }\end{array}$} & \multirow[b]{2}{*}{$\begin{array}{c}\text { GAS } \\
(-3-+2)\end{array}$} & \multicolumn{4}{|c|}{ OSAS ${ }^{A}$} & \multirow[b]{2}{*}{$\begin{array}{c}\text { Hold } \\
\text { fingers } \\
(1-4)\end{array}$} & \multirow[b]{2}{*}{$\begin{array}{c}\text { Hold wrist } \\
\qquad(1-5)\end{array}$} & \multirow[b]{2}{*}{$\begin{array}{c}\text { Release } \\
(1-4)\end{array}$} \\
\hline & & & $\begin{array}{c}\text { AHA- } \\
\text { units } \\
(0-100) \\
\end{array}$ & $\begin{array}{c}\text { Reach } \\
(0 / 1)\end{array}$ & $\begin{array}{c}\text { Grasp } \\
\text { fingers } \\
(1-3) \\
\end{array}$ & $\begin{array}{l}\text { Grasp } \\
\text { wrist } \\
(1-5) \\
\end{array}$ & & & \\
\hline \multirow[t]{6}{*}{$1^{\prime}$} & TO & -2 & 50 & 0 & 2 & 1 & 2 & 1 & 2.5 \\
\hline & & & & 0 & 2 & 1 & 1 & 1 & 1 \\
\hline & T2 & -2 & 50 & 0 & 2 & 1 & 2 & 1 & $3^{*}$ \\
\hline & & & & 0 & 2 & 1 & $2^{*}$ & 1 & $2.0^{*}$ \\
\hline & T3 & -2 & 49 & 1 & 2 & 1 & 2 & 1 & $2.0^{+}$ \\
\hline & & & & 0 & $1^{+}$ & 1 & 1 & 1 & 1 \\
\hline \multirow[t]{6}{*}{$2^{\prime}$} & TO & -2 & 55 & 0 & 2 & 1 & 2 & 1 & 3.0 \\
\hline & & & & 0 & 2 & 1 & 2 & 1 & 3 \\
\hline & T2 & -2 & 57 & 0 & 2 & 1 & 2 & 1 & 3 \\
\hline & & & & 0 & 2 & 1 & 2 & 1 & $2.0^{+}$ \\
\hline & T3 & 1 & 57 & 0 & 2 & 1 & 2 & 1 & 3.0 \\
\hline & & & & 0 & 2 & $3^{*}$ & 2 & $3^{*}$ & 3 \\
\hline \multirow[t]{6}{*}{$3^{\prime}$} & TO & -2 & 48 & 0 & 2 & 1 & 2 & 1 & 2.0 \\
\hline & & & & 0 & 2 & 1 & 2 & 1 & 2 \\
\hline & T2 & $\#$ & $62^{*}$ & 0 & 2 & 1 & 2 & 1 & $3^{*}$ \\
\hline & & & & 0 & 2 & 1 & 2 & 1 & 2.0 \\
\hline & T3 & \# & 52 & 0 & 2 & 1 & 2 & 1 & 2.0 \\
\hline & & & & 0 & 2 & 1 & 2 & 1 & 2 \\
\hline \multirow[t]{6}{*}{$4^{\prime}$} & TO & -2 & 63 & 0 & 2 & 3 & 2 & 1 & 2.5 \\
\hline & & & & 0 & 2 & 1 & 3 & 1 & 2 \\
\hline & T2 & -2 & 59 & 0 & 2 & $1^{+}$ & 2 & 1 & $3^{*}$ \\
\hline & & & & 1 & 2 & 1 & 3 & 1 & $3.0^{*}$ \\
\hline & T3 & 1 & 61 & 0 & $1^{+}$ & $2^{+}$ & $3^{*}$ & $2^{*}$ & $1.0^{\dagger}$ \\
\hline & & & & 1 & 2 & 1 & $2^{+}$ & 1 & $3^{*}$ \\
\hline \multirow[t]{6}{*}{$5^{\prime}$} & TO & -2 & 52 & 0 & 2 & 3 & 2 & 3 & 3.0 \\
\hline & & & & 0 & 2 & 3 & 3 & 3 & 2 \\
\hline & T2 & 0 & $63^{*}$ & 0 & 2 & 3 & 2 & 3 & 3 \\
\hline & & & & 0 & 2 & $1^{+}$ & 3 & 3 & 2.0 \\
\hline & T3 & 0 & $58^{*}$ & 0 & 2 & 3 & 2 & 3 & $2.0^{+}$ \\
\hline & & & & 0 & 2 & $1^{+}$ & $2^{+}$ & $1^{+}$ & 2 \\
\hline \multirow[t]{6}{*}{$6^{\mathrm{E}}$} & TO & -2 & 50 & 0 & 2 & 1 & 2 & 1 & 2.0 \\
\hline & & & & 0 & 2 & 1 & 2 & 1 & 2 \\
\hline & T2 & 0 & 49 & 0 & 2 & 1 & 2 & 1 & 2 \\
\hline & & & & 0 & 2 & 1 & 2 & 1 & 2.0 \\
\hline & T3 & 0 & $58^{*}$ & 0 & 2 & 1 & 2 & 1 & 2.0 \\
\hline & & & & 0 & 2 & 1 & 2 & 1 & 2 \\
\hline \multirow[t]{6}{*}{$7^{\mathrm{E}}$} & TO & -2 & 63 & 1 & 2 & 1 & 2 & 1 & 2.0 \\
\hline & & & & 0 & 2 & 1 & 2 & 1 & 1 \\
\hline & T2 & 2 & 64 & 0 & 2 & 1 & 2 & 1 & $3^{*}$ \\
\hline & & & & 0 & 2 & 1 & 2 & 1 & $2.5^{*}$ \\
\hline & T3 & -1 & 65 & 0 & 2 & 1 & 2 & 1 & $3.0^{*}$ \\
\hline & & & & 0 & 2 & 1 & 2 & 1 & $3^{*}$ \\
\hline \multirow[t]{6}{*}{$8^{\mathrm{E}}$} & TO & -2 & 71 & 0 & 2 & 1 & 2 & 1 & 3.0 \\
\hline & & & & 0 & 2 & 1 & 3 & 1 & 2 \\
\hline & T2 & 0 & 73 & 0 & 2 & 1 & 2 & 1 & 3 \\
\hline & & & & 0 & 2 & $3^{*}$ & 3 & 1 & $3.0^{*}$ \\
\hline & T3 & -2 & $79^{*}$ & 0 & 2 & 1 & 2 & 1 & 3.0 \\
\hline & & & & 0 & 2 & 1 & 3 & 1 & $3^{*}$ \\
\hline
\end{tabular}




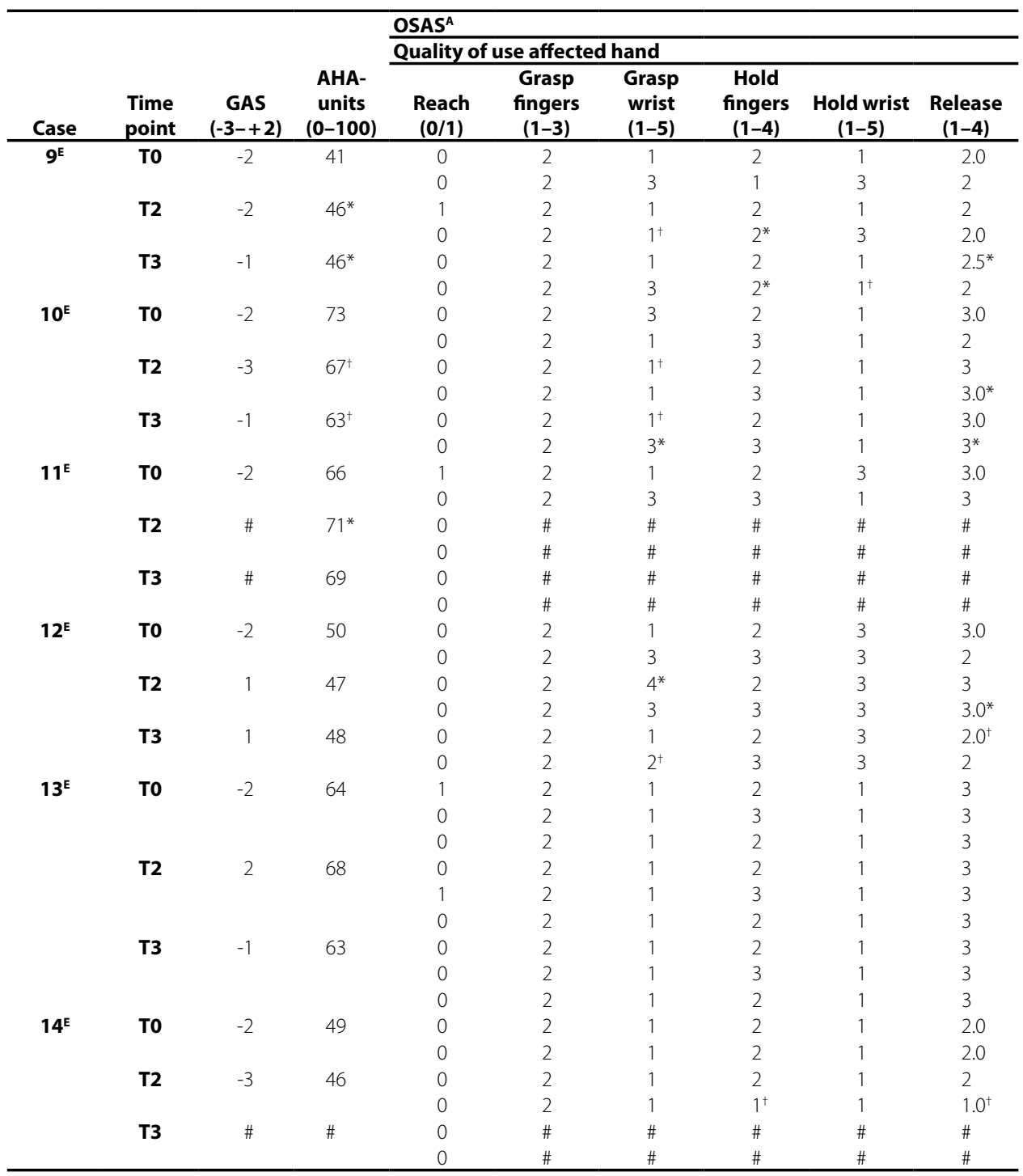

* Clinically-relevant/statistically-significant improvement compared to baseline

+ Clinically-relevant/statistically-significant decline compared to baseline

A OSAS tasks are (from top to bottom) threading beads, and building with 'Pop-Onz' (Fisher Price') for children aged 2.5 to 6 years, and buttering and cutting bread, small screw and nut construction, and large screw and nut construction for children from 7 years of age

' Implicit program

Explicit program

\# Missing value

For GAS, AHA, and OSAS, higher score indicates higher ability 


\section{Parent-Related outcomes}

\section{Parental interviews}

During the interviews, some parents indicated that they felt no stress at all, but for others the home-based training led to varying degrees of stress. Generally, parents mentioned feelings like frustration, irritation, and disquiet rather than stress, which in their opinion was too strong a word. The most stress-inducing factor was the prescribed intensity of the training, i.e. to find a time for the training as well as the need to meet the required training hours. As one parent explained:

"... I think that if we, for example, could determine ourselves how much I practice, that that would give meless stress." [parent]

Parents experienced most stress at the beginning of the program, when they were still figuring out how to implement the training.

\section{Therapy-related Parental Stress Questionnaire (VOST)}

In their answers to the open-ended question at baseline, three parents expressed feelings of stress during preparation for the home-based training program. This indicates that the reported stress related to the programs was already affecting the baseline VOST score. Five parents did not consider the parent-delivered training as therapy and therefore did not fill out the questionnaire at T2. In addition, there were many missing data, particularly for the VASs for the six domains. Consequently, only six cases had sufficient data to evaluate therapy-related parental stress throughout the study by means of this questionnaire. Five of these six cases (83\%) showed a decline of overall parental stress during the program. The results of the stress levels for the separate domains were inconsistent, although the data revealed a slight decreasing tendency on the child, parent, and family domains, and a minor increase of stress on the domain 'characteristics of the therapy', at T2 and T3 (Table 4). At baseline, parents indicated 'incorporating the therapy into the planning and/or organization of my family' and 'my personal condition' most frequently as the factors most contributing to their stress (both 83\%, 5/6), while at the end of the home-based training most frequently given was 'the time needed to exercise at home' $(83 \%, 5 / 6)$.

\section{Parenting Stress Questionnaire (OBVL)}

At baseline, nine parents (64\%) had a score outside of the normal range regarding one or more subscores (T-score $\geq 60$ ) and/or the total score (T-score $\geq 65$ ). The change scores of the OBVL were analyzed in light of the smallest detectable difference given in the questionnaire manual (Vermulst et al., 2015). Change scores from baseline to the end of the program (T2) showed a reduction of parental stress on one or more scales in eight cases (57\%), an increase in three parents (21\%), and no change in three cases (21\%) (Table 4). 


\section{EMPOWERMENT QUESTIONNAIRE (EMPO)}

At baseline, three parents (21\%) had a score outside of the 'non-concerned range' for the total score (T-score $>40$ ) and one or more subscores (T-score $>35$ ). Based on the reliable change index (Zahra \& Hedge, 2010), the scores on the intrapersonal subscale significantly increased between T0 and T2 in nine parents (69\%) and declined in one (8\%). At T3, the scores of five parents (42\%) were improved and of four parents (33\%) worsened. For the interactional subscale, an approximately equal number of parents improved and worsened at T2 (5/13 (38\%) and 7/13 (54\%), respectively), as well as at T3 (3/12 (25\%) and 4/12 (33\%), respectively). The same applies to the behavioral control subscale at T2 (four (31\%) improved and five (38\%) worsened), while at follow-up the score of eight parents (67\%) had increased and of only two (17\%) decreased. The results of the total score indicate that the empowerment of four parents (31\%) had increased at T2 and of one (8\%) decreased. At T3, five parents (42\%) had a higher empowerment than baseline, and two (17\%) a lower one (Table 4).

\section{INTERVENTION ADHERENCE AND TOLERABILITY}

Intervention adherence and tolerability were assessed in a process evaluation, to be published separately. The median of the average hours each child trained weekly is 2.8 (IQR 1.1). No adverse events were reported, except for one hospital admission due to an epileptic seizure, considered unrelated to the home-based training. 


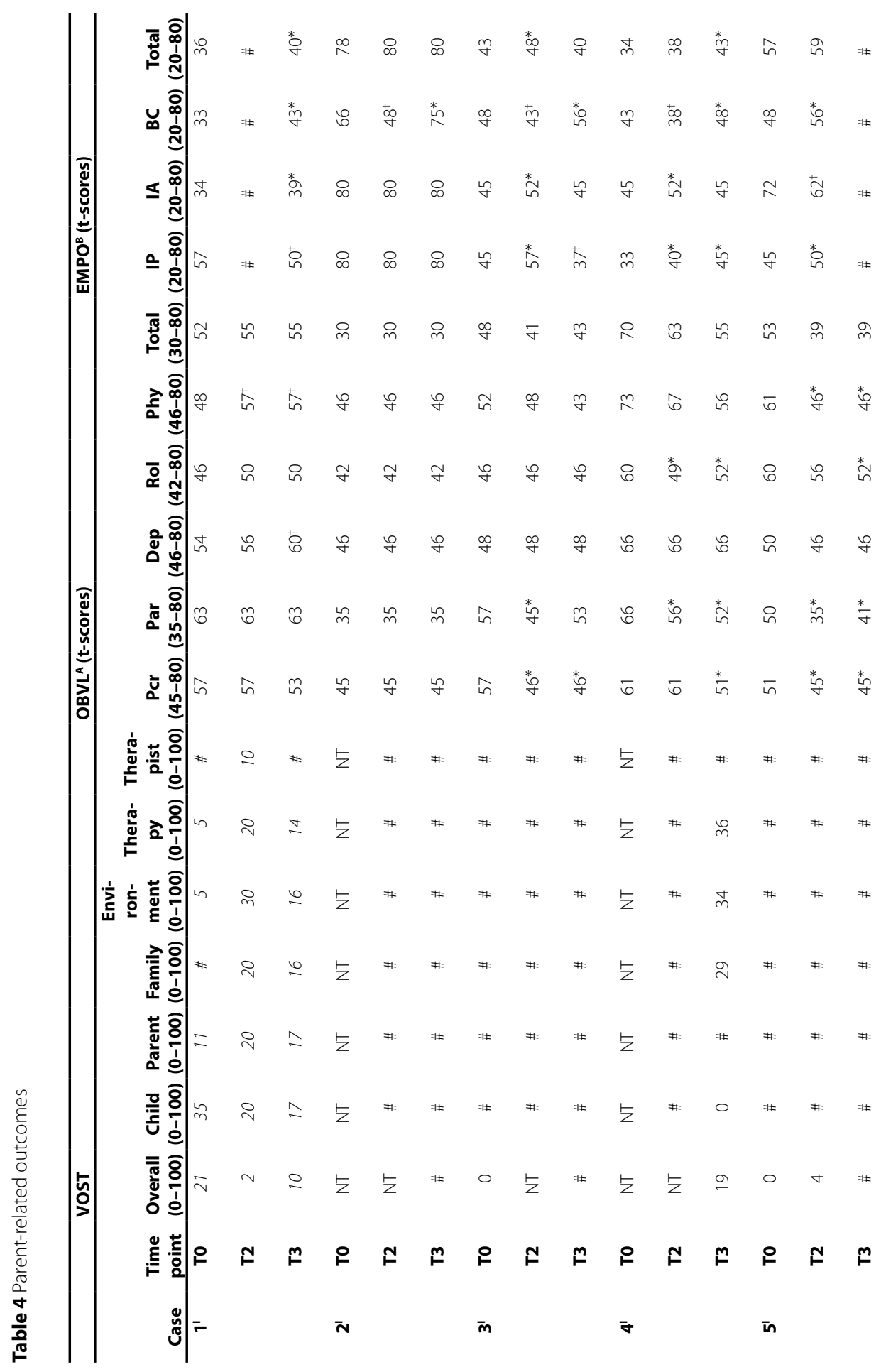




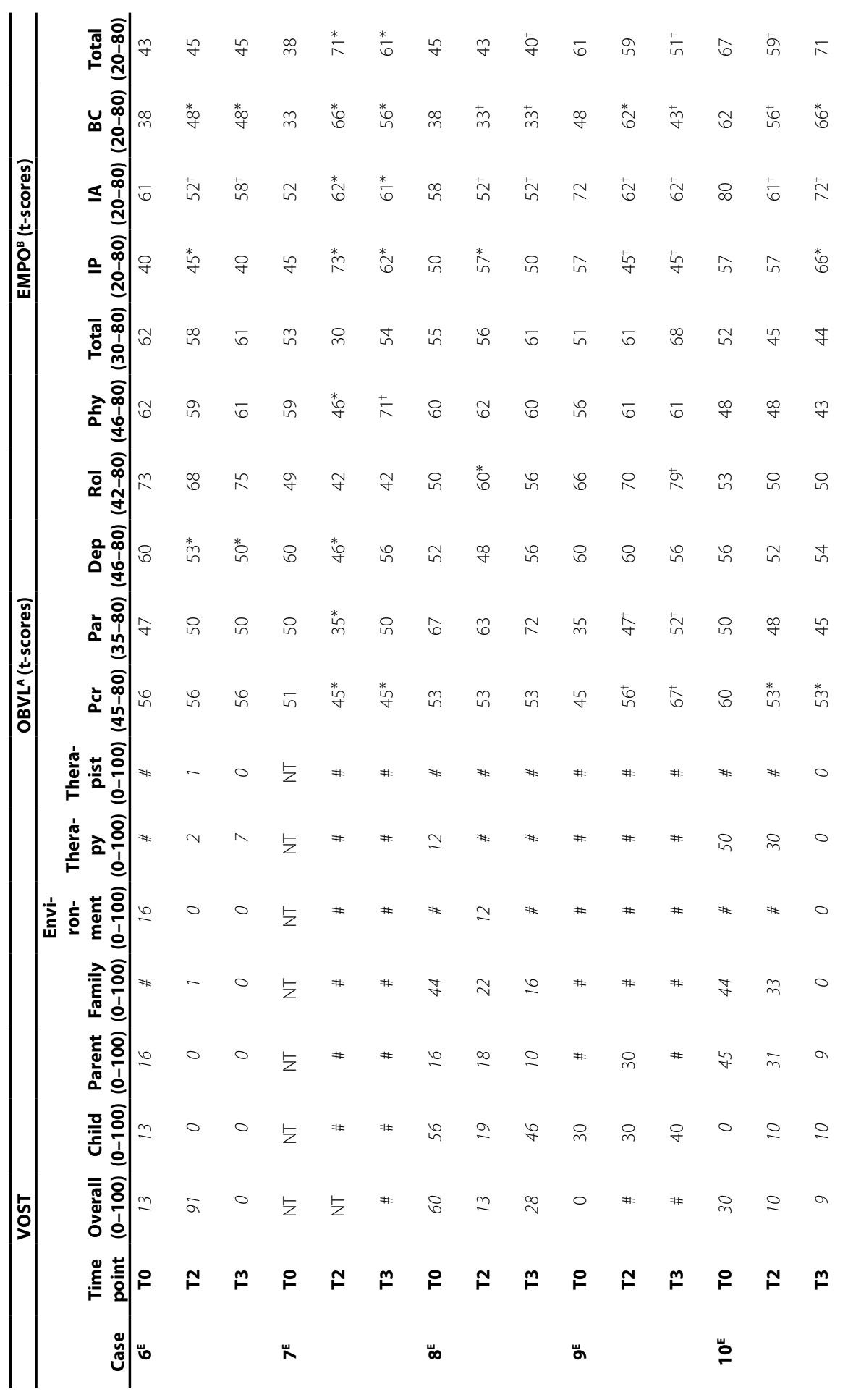




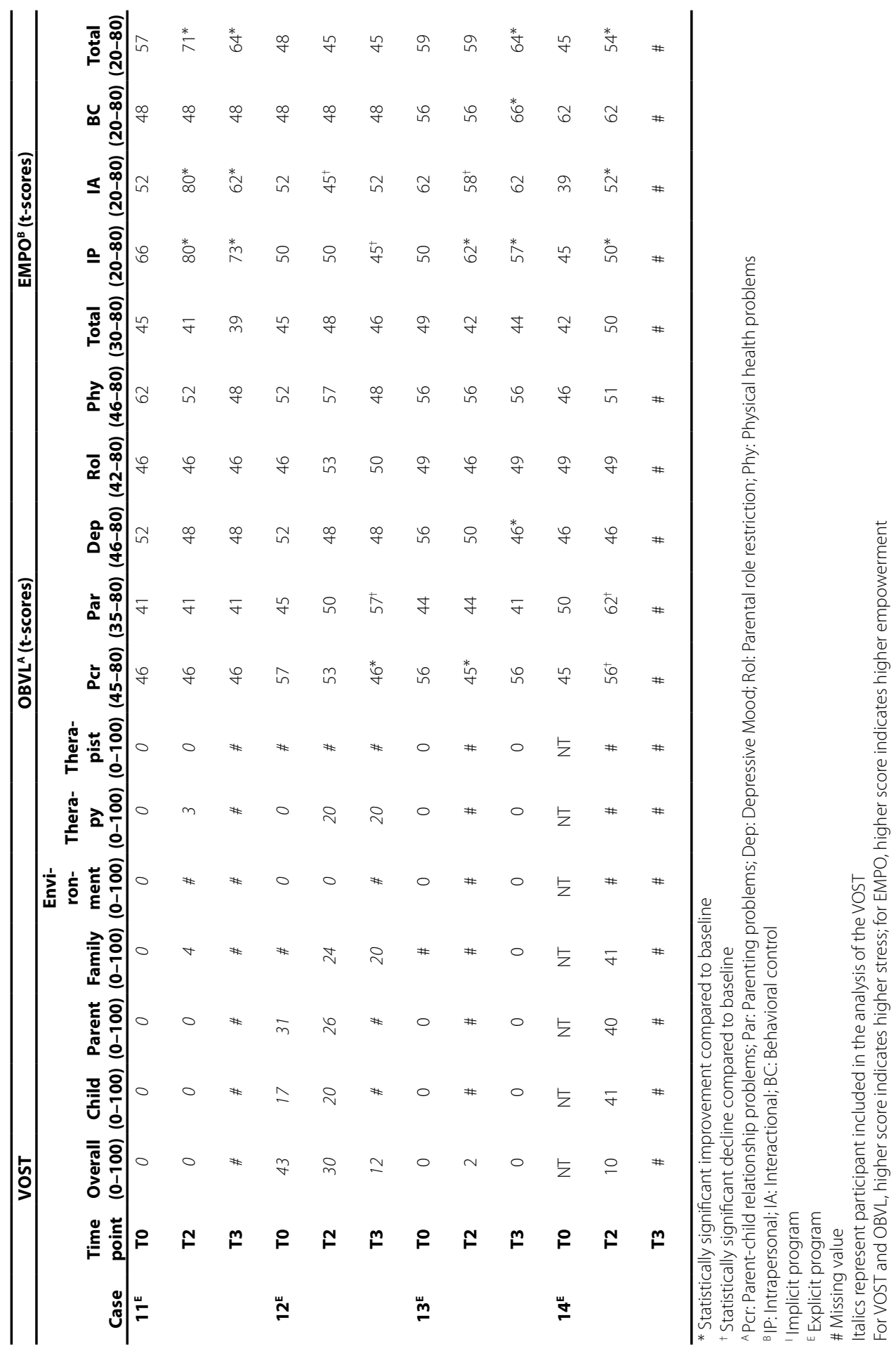




\section{Discussion}

This case series study aimed to investigate the effect of implicit and explicit home-based bimanual training programs on children with UCP and on their parents. The results suggest that improvements in performance of targeted bimanual activities can be achieved with both approaches when delivered by parents, and suggest that parental stress may decrease during these programs.

The most important clinically-relevant finding was improvement of performance of, and satisfaction with, targeted activities. A retention of these effects 12 weeks after the end of the program was observed with the COPM. These findings are consistent with Ferre et al. (Ferre et al., 2017), who found a similar effect of home-based intensive bimanual training with the COPM, although their total training dose was twice as high as in our study. Although a few children in our case series improved on several other outcome measures, there is no apparent evidence for transfer of treatment effect to general manual capacity and/or performance of non-practiced bimanual tasks. Parents experienced some stress because of the training intensity. However, earlier findings of stable or increased stress levels were not confirmed (Ferre, Brandao, Hung, Carmel, \& Gordon, 2015; Lin et al., 2011). Rather, there was a tendency toward reduction of both therapy-related and generic parental stress during home-based training. This may be attributed to the novel role of the remedial educationalist in the programs, who coached the parents in addressing difficult behavior and problems in emotion regulation, motivation, and (sustained) attention of the child. The increased empowerment of some parents, reflected in their higher scores on the intrapersonal subscale of the EMPO, implies that their feelings of competence and self-efficacy grew (Damen et al., 2017).

\section{STUDY LIMITATIONS}

The original research questions, particularly concerning the comparison of implicit and explicit motor learning, could not be answered as intended, due to recruitment problems during the RCT (Beckers, Rameckers, et al., 2018). As causality cannot be assumed and improvements may have been affected by maturation, the Hawthorne effect or other mediating or moderating factors, the results should be interpreted with caution. Moreover, participants who discontinued the homebased training also withdrew from the assessments and could therefore not be analyzed. This fact should be considered in order to avoid an overestimation of the presented findings. Last, the lack of psychometrically-sound instruments to assess some constructs of specific interest to home-based training left no other option but to use new measures that have not yet, or only to a limited extent, been validated.

\section{FUTURE RESEARCH}

The combination of findings for child- and parent-related outcomes, though preliminary, provides support for the potential effectiveness of goal-directed task-specific bimanual home-based training programs. Interdisciplinary coaching, involving a remedial educationalist or health-care 
psychologist, is recommended to prevent therapy-related parental stress and may even produce reduction of generic parental stress. High-quality effect studies are warranted to confirm the hypotheses that can be generated from this study.

\section{Conclusion}

This study has been the first attempt to examine bimanual home-based training programs adopting interdisciplinary coaching for children with uCP. The programs seem to positively affect children's bimanual performance. A decrease of therapy-related and generic parental stress levels occurred more often than did an increase. These encouraging results require continued efforts to establish the effectiveness of interdisciplinary home-based training programs, both on child- and parent-related variables, in children with UCP.

\section{Acknowledgements}

We sincerely thank the children and their parents who generously gave their time and effort to participate in this study. We also greatly appreciate the dedicated commitment of the therapists, remedial educationalists, and their colleagues. We would like to acknowledge the research assistants who conducted and scored the assessments. We highly esteem the contribution of the COAD Author Group: Nicole Brouwers, Anke Defesche, Hanneke Denissen, Rosemarie Derickx, Yvonne Geerdink, Ruth Heijsen-van den Heuvel, Bregtje Janssen, Anne Jensen, Marjon Kissels, Martijn Klem, Nanne Land, Denise Martens, Judith van Munster, Bianca Olive, Marleen Philippens, Caroline Scheijmans, Lucianne Speth, Ingrid van den Tillaar, and Eef Vennix. Finally, we thank Les Hearn for proofreading the manuscript.

\section{FUNDING}

This research was supported by a ZonMw (the Netherlands Organization for Health Research and Development) grant within the context of the 3rd Program Rehabilitation Research (630000001), funded by HandicapNL (former Revalidatiefonds), Johanna Kinderfonds, and Stichting Rotterdams Kinderrevalidatie Fonds Adriaanstichting. HandicapNL (former Revalidatiefonds, R2016006) and Stichting Vooruit (18-05/YvH/NS) provided additional funding. 


\section{REFERENCES}

Beckers, L., Rameckers, E., Smeets, R., Van der Burg, J., Aarts, P., Schnackers, M., \& Janssen-Potten, Y. (2018). Barriers to recruitment of children with cerebral palsy in a trial of home-based training Manuscript submitted for publication.

Beckers, L., van der Burg, J., Janssen-Potten, Y., Rameckers, E., Aarts, P., \& Smeets, R. (2018). Process evaluation of two home-based bimanual training programs in children with unilateral cerebral palsy (the COAD-study): protocol for a mixed methods study. BMC Pediatr, 18(1), 141. doi:10.1186/s12887-018$1111-1$

COAD: home-based training programs for young children with cerebral palsy. Nederlands Trial Register Retrieved 9 January 2019, from Academic Medical Center http://www.trialregister.nl/trialreg/admin/ rctview.asp?TC $=5743$

Cusick, A., Lannin, N. A., \& Lowe, K. (2007). Adapting the Canadian Occupational Performance Measure for use in a paediatric clinical trial. Disabil Rehabil, 29(10), 761-766. doi:10.1080/09638280600929201

Damen, H., Veerman, J. W., Vermulst, A. A., Nieuwhoff, R., de Meyer, R. E., Scholte, R. H. J. J. J. o. C., \& Studies, F. (2017). Parental Empowerment: Construct Validity and Reliability of a Dutch Empowerment Questionnaire (EMPO). 26(2), 424-436. doi:10.1007/s10826-016-0568-5

Eliasson, A. C., \& Burtner, P. (2008). Neuropsychology of movement sequence learning. In Improving Hand Function in Children with Cerebral Palsy: Wiley.

Eliasson, A. C., Krumlinde-Sundholm, L., Rosblad, B., Beckung, E., Arner, M., Ohrvall, A. M., \& Rosenbaum, P. (2006). The Manual Ability Classification System (MACS) for children with cerebral palsy: scale development and evidence of validity and reliability. Dev Med Child Neurol, 48(7), 549-554. doi:10.1017/ s0012162206001162

Ferre, C. L., Brandao, M., Surana, B., Dew, A. P., Moreau, N. G., \& Gordon, A. M. (2017). Caregiver-directed home-based intensive bimanual training in young children with unilateral spastic cerebral palsy: a randomized trial. Dev Med Child Neurol, 59(5), 497-504. doi:10.1111/dmcn.13330

Ferre, C. L., Brandao, M. B., Hung, Y. C., Carmel, J. B., \& Gordon, A. M. (2015). Feasibility of caregiver-directed home-based hand-arm bimanual intensive training: a brief report. Dev Neurorehabil, 18(1), 69-74. doi: 10.3109/17518423.2014.948641

Geerdink, Y., Aarts, P., van der Holst, M., Lindeboom, R., Van Der Burg, J., Steenbergen, B., \& Geurts, A. C. (2017). Development and psychometric properties of the Hand-Use-at-Home questionnaire to assess amount of affected hand-use in children with unilateral paresis. Dev Med Child Neurol, 59(9), 919-925. doi:10.1111/dmcn.13449

Krumlinde-Sundholm, L., \& Eliasson, A. C. (2003). Development of the Assisting Hand Assessment: A Raschbuilt Measure intended for Children with Unilateral Upper Limb Impairments. Scandinavian Journal of Occupational Therapy, 10(1), 16-26. doi:10.1080/11038120310004529

Krumlinde-Sundholm, L., Holmefur, M., Kottorp, A., \& Eliasson, A. C. (2007). The Assisting Hand Assessment: current evidence of validity, reliability, and responsiveness to change. Dev Med Child Neurol, 49(4), 259-264. doi:10.1111/j.1469-8749.2007.00259.x

Lesage, F. X., Berjot, S., \& Deschamps, F. (2012). Clinical stress assessment using a visual analogue scale. Occup Med (Lond), 62(8), 600-605. doi:10.1093/occmed/kqs140 
Lin, K. C., Wang, T. N., Wu, C. Y., Chen, C. L., Chang, K. C., Lin, Y. C., \& Chen, Y. J. (2011). Effects of home-based constraint-induced therapy versus dose-matched control intervention on functional outcomes and caregiver well-being in children with cerebral palsy. Res Dev Disabil, 32(5), 1483-1491. doi:10.1016/j. ridd.2011.01.023

Mackie, P. C., Jessen, E. C., \& Jarvis, S. N. (1998). The lifestyle assessment questionnaire: an instrument to measure the impact of disability on the lives of children with cerebral palsy and their families. Child Care Health Dev, 24(6), 473-486.

Novak, I., Mclntyre, S., Morgan, C., Campbell, L., Dark, L., Morton, N., .. Goldsmith, S. (2013). A systematic review of interventions for children with cerebral palsy: state of the evidence. Dev Med Child Neurol, 55(10), 885-910. doi:10.1111/dmcn.12246

Palisano, R., Rosenbaum, P., Walter, S., Russell, D., Wood, E., \& Galuppi, B. (1997). Development and reliability of a system to classify gross motor function in children with cerebral palsy. Dev Med Child Neurol, 39(4), 214-223.

Peplow, U. C., \& Carpenter, C. (2013). Perceptions of parents of children with cerebral palsy about the relevance of, and adherence to, exercise programs: a qualitative study. Phys Occup Ther Pediatr, 33(3), 285-299. doi:10.3109/01942638.2013.773954

Sakzewski, L., Ziviani, J., \& Boyd, R. N. (2014). Efficacy of upper limb therapies for unilateral cerebral palsy: a meta-analysis. Pediatrics, 133(1), e175-204. doi:10.1542/peds.2013-0675

Schnackers, M., Beckers, L., Janssen-Potten, Y., Aarts, P., Rameckers, E., van der Burg, J., . . Steenbergen, B. (2018). Home-based bimanual training based on motor learning principles in children with unilateral cerebral palsy and their parents (the COAD-study): rationale and protocols. BMC Pediatr, 18(1), 139. doi:10.1186/s12887-018-1110-2

Speth, L., Janssen-Potten, Y., Leffers, P., Rameckers, E., Defesche, A., Geers, R., .. Vles, H. (2013). Observational skills assessment score: reliability in measuring amount and quality of use of the affected hand in unilateral cerebral palsy. BMC Neurol, 13, 152. doi:10.1186/1471-2377-13-152

Steenbeek, D., Ketelaar, M., Galama, K., \& Gorter, J. W. (2007). Goal attainment scaling in paediatric rehabilitation: a critical review of the literature. Dev Med Child Neurol, 49(7), 550-556. doi:10.1111/j.14698749.2007.00550.x

Steenbeek, D., Ketelaar, M., Lindeman, E., Galama, K., \& Gorter, J. W. (2010). Interrater reliability of goal attainment scaling in rehabilitation of children with cerebral palsy. Arch Phys Med Rehabil, 91(3), 429-435. doi:10.1016/j.apmr.2009.10.013

van der Holst, M., Geerdink, Y., Aarts, P., Steenbeek, D., Pondaag, W., Nelissen, R. G., ... Vliet Vlieland, T. P. (2018). Hand-Use-at-Home Questionnaire: validity and reliability in children with neonatal brachial plexus palsy or unilateral cerebral palsy. Clin Rehabil, 32(10), 1363-1373. doi:10.1177/0269215518775156

van der Kamp, J., Steenbergen, B., \& Masters, R. S. W. (2018). Explicit and implicit motor learning in children with unilateral cerebral palsy. Disabil Rehabil, 40(23), 2790-2797. doi:10.1080/09638288.2017.1360403

Vermulst, A., Kroes, G., De Meyer, R., Nguyen, L., \& Veerman, J. (2015). Handleiding OBVL. In. Retrieved from http://www.praktikon.nl/wp-content/uploads/2016/03/handleiding_obvl_2015_1_.pdf

Zahra, D., \& Hedge, C. (2010). The Reliable Change Index: Why isn't it more popular in academic psychology? PsyPag Quarterly(76), 14-19. 


\section{Chapter 9}

\section{Process evaluation of two home-based bimanual training programs in children with unilateral cerebral palsy (the COAD-study): protocol for a mixed methods study}

LWME Beckers, JJW van der Burg, YJM Janssen-Potten,

EAA Rameckers, PBM Aarts, RJEM Smeets 


\section{Abstract}

Background As part of the COAD-study two home-based bimanual training programs for young children with unilateral Cerebral Palsy (UCP) have been developed, both consisting of a preparation phase and a home-based training phase. Parents are coached to use either an explicit or implicit motor learning approach while teaching bimanual activities to their child. A process evaluation of these complex interventions is crucial in order to draw accurate conclusions and provide recommendations for implementation in clinical practice and further research. The aim of the process evaluation is to systematically assess fidelity of the home-based training programs, to examine the mechanisms that contribute to their effects on child-related and parent-related outcomes, and to explore the influence of contextual factors.

Methods A mixed methods embedded design is used that emerges from a pragmatism paradigm. The qualitative strand involves a generic qualitative approach. The process evaluation components fidelity (quality), dose delivered (completeness), dose received (exposure and satisfaction), recruitment and context will be investigated. Data collection includes registration of attendance of therapists and remedial educationalists to a course regarding the home-based training programs; a questionnaire to evaluate this course by the instructor; a report form concerning the preparation phase to be completed by the therapist; registration and video analyses of the home-based training; interviews with parents and questionnaires to be filled out by the therapist and remedial educationalist regarding the process of training; and focus groups with therapists and remedial educationalists as well as registration of drop-out rates and reasons, to evaluate the overall home-based training programs. Inductive thematic analysis will be used to analyse qualitative data. Qualitative and quantitative findings are merged through meta-inference.

Discussion So far, effects of home-based training programs in paediatric rehabilitation have been studied without an extensive process evaluation. The findings of this process evaluation will have implications for clinical practice and further research regarding development and application of home-based bimanual training programs, executed by parents and aimed at improving activity performance and participation of children with UCP.

Keywords Cerebral palsy, Process evaluation, Mixed methods, Complex intervention, Home program, Bimanual training, Upper extremity, Explicit motor learning, Implicit motor learning, Parental stress 


\section{BACKGROUND}

Cerebral Palsy (CP) is the most common cause of motor disability in children [1]. The restricted motor function of one upper extremity in children with unilateral CP (UCP) mainly leads to perceived difficulty in performing bimanual activities of daily living [2]. These activity limitations often restrict the children's level of participation with their peers and family, at school and in leisure activities [3]. Most of these children are enrolled in different kinds of interventions during childhood to improve performance of bimanual activities and to promote participation.

There seems to be consensus among clinicians and researchers on the importance of home-based training programs for children with CP [4]. To allow implementation of these programs within the context of family life, collaborative service delivery is required, meaning families collaborate with professionals in the delivery of treatment. An et al. have defined three main principles of importance to collaborative service delivery in paediatric rehabilitation: family identified needs, shared responsibility, and family empowerment [5]. Taking into account the unique needs of the family promotes parents' perceptions of shared planning. This shared responsibility is crucial for successful and effective implementation of interventions. Since families and professionals have expertise on different areas, various essential perspectives on the child can be taken into consideration. Because of the parents' engagement in collaborate service delivery, the empowerment of the family is supposed to be supported [5]. As a result, parents may become less dependent on health care professionals in the treatment of their child's disability. Moreover, home-based training programs enable children to practice bimanual activities within the specific context of their daily lives. As a result, the neural processing demands during training are equal to the demands while performing the tasks in daily life. Therefore, no transfer of training to a new environment is required [6]. Hence, home-based training programs are expected to be highly effective in improving bimanual performance of activities and maintenance of training effects is more likely to occur.

Although there is evidence that home-based bimanual training programs are effective in improving bimanual performance [7, 8], data from several studies suggest that these programs can have adverse consequences too. In a qualitative study, Peplow and Carpenter showed that parents feel a lot of pressure to adhere to the training, eliciting perceived stress. In addition, ensuring that the child performed the prescribed training activities required time and effort from parents and impacted family relationships [9]. Likewise, Lin et al. reported that home-based training led to dysfunctional parent-child interaction and an increase of parental stress [10]. Since parental stress seems to have a negative impact on adherence, this may also limit the effectiveness of home-based programs [11].

Our research group currently performs the COAD-study (co-creation at hand: the road to independence). For this study, two home-based bimanual training programs for young children with UCP have been developed. Both programs aim to increase bimanual activity performance of the child, without increasing parental stress [12]. To pursue this, the programs differ from existing home-based bimanual training programs in two ways. First, a remedial educationalist or health care psychologist (referred to as 'remedial educationalist') collaborates with a paediatric occupational or physical therapist (referred to as 'therapist') in the coaching of parents in order 
to specifically focus on the parent-child interaction. The main aim is to establish a functional parent-child interaction, resulting in optimal adherence to the training with as minimal as possible increase of therapy-related parental stress. Second, as it is not clear what type of learning is most effective, parents in one program are coached to use an explicit motor learning approach while teaching motor activities to their child, whereas parents in the other program are coached to use an implicit motor learning approach. Within both programs, the therapist coaches the parents to provide particular instructions and feedback to their child and to organize the training activities in a specific way [13]. The latter includes for instance the type of object to be used (e.g. shape), the position of the child (e.g. sitting at a table) and the setting (e.g. slipperiness of the working surface). In both programs, parents provide task-oriented and result-oriented instructions and feedback to the child. An example of a task-oriented instruction is to tell the child to grab the jar of peanut butter and open it. Result-oriented feedback is for instance to compliment the child on opening the jar himself. Using the explicit approach, parents give additional instructions and feedback to their child regarding specific motor execution of the task. An example of an explicit instruction is asking the child to grab the jar of peanut butter with the affected hand whilst doing 'the trick of the thumb' (i.e. abducting the thumb), and to subsequently squeeze the jar with the affected hand while turning the lid with the non-affected hand. By contrast, using the implicit approach, parents do not give any instructions or feedback regarding motor execution of the task. Instead, parents provoke specific motor execution by the organization of the task, for example by positioning the jar of peanut butter on the affected side to elicit the child to grab it with the affected hand, and by using a sufficiently small-sized jar that can easily be squeezed with the affected hand while opening the lid with the non-affected hand. We hypothesize that by using an explicit approach, parents have to prompt their child frequently to attain the specified way of motor execution of the task, which may result in frustration and conflicts between the child and the parent. This may lead to an increase of perceived parental stress and limited adherence to the training. Moreover, the explicit instruction with regard to specific motor execution of the task has to be remembered by the child during task performance. Hence, working memory demands in the explicit approach are expected to be higher than in the implicit approach. As children with CP often have limited working memory abilities [14], this may cause complications during the training. The implicit approach is expected to have less adverse consequences than the explicit approach, as the parents do not need to prompt their child regarding motor execution of the task and working memory demands are lower.

As part of the COAD-study, the home-based training programs are tested for their effects and compared with each other, using a comparative case series design. Effects are measured on child-related outcomes regarding bimanual activity and participation. Additionally, parent-related effects are investigated, concerning parental stress and empowerment.

In addition to the effect evaluation, a process evaluation is needed, in particular because we consider these home-based training programs to be complex interventions [15]. This assumption is based on four reasons. First, each program comprises multiple components that interact with each other. The components are for example instructional videos to train the parents, a task analysis performed by the therapist, performance of the home-based training by the child and parents, and a phone call from the remedial educationalist to coach the parents. Second, the 
actions required by both the health care professionals and the parents are numerous as well as difficult. Third, the programs aim to produce change on a range of child-related and parent-related outcomes. Fourth, tailoring of the programs to the individual child and parents is permitted to a relatively large extent.

Results regarding effects of our home-based training programs alone are not sufficient to come to accurate conclusions and recommendations for implementation in clinical practice and further research. For example, in case one or both home-based training programs are found to be successful, it is valuable to know why they are effective as well as whether and how they can be optimized. Likewise, if a program is unsuccessful, it is important to know why it is ineffective or has unanticipated effects. Because of the complexity of the programs, we consider that three aspects are important to investigate specifically. First, evaluation of effects must be related to the evaluation of fidelity, indicating whether the programs were performed (i.e. implemented) as intended within the comparative case series. Second, causal mechanisms should be clarified as far as possible, by exploring which components of the programs did and did not contribute to the effects of the programs. Third, it is relevant to identify what contextual factors with regard to children, parents and health care professionals are associated with possible variation in implementation and outcomes between child-parents triads and corresponding health care professionals [15]. In conclusion, a process evaluation of the home-based training programs is a crucial addition to the evaluation of their effects.

Consequently, this study aims to systematically evaluate the processes and factors which influence implementation and effects of the programs. For this purpose, a process evaluation of the programs will be performed using mixed methods embedded in the case series. Tashakkori \& Creswell describe mixed-methods research as "... research in which the investigator collects and analyses data, integrates the findings, and draws inferences using both qualitative and quantitative approaches or methods in a single study or program of inquiry" [16]. Quantitative data collection will be used for factual information, for instance the number of minutes spent per day on each treatment goal, as well as basic opinions such as the degree of confidence of parents in the cooperation with the therapist. Qualitative data collection will be used to gain understanding of in-depth experiences, for example regarding the experiences of parents with the program. In the process evaluation, the quantitative and qualitative data will be combined to draw conclusions regarding the processes of the home-based training programs.

\section{Methods}

\section{COAD-STUDY SUMMARY}

The COAD-study consists of a comparative case series and a process evaluation. It is a multicentre study with a pragmatic nature, which will be performed in the Netherlands from April 2017 until October 2018. It is expected that participants will be enrolled from five rehabilitation centres on nine locations situated in both urban and rural areas. The study population consists of children aged 2 through 7 years with a clinically confirmed unilateral spastic CP and Manual Ability Classification System (MACS) level I-III [17], and their parents. A total of approximately 18 children and 
their parents will participate in the COAD-study. Children will be allocated to either the implicit home-based training program or the explicit home-based training program based on the preference of the parents. Parents receive an information leaflet regarding the difference between the programs and can discuss their decision with a health professional. We have described the protocols of the interventions in detail elsewhere [12].

The child-related primary outcome of the case series is performance of predetermined, individual rehabilitation goals, focused on bimanual daily life activities, as measured with the performance scale of the Canadian Occupational Performance Measure (COPM) [18]. With respect to the parents, therapy-related parental stress, explored with in-depth parental interviews, is of primary interest. Secondary outcomes focus on bimanual activity and participation of the child as well as (general) parental stress and empowerment.

\section{DESIGN AND PARADIGM}

We will employ a mixed methods embedded design, that is a mixed methods approach in which quantitative as well as qualitative data collection and analyses are combined within a traditional quantitative or qualitative research design [19]. This study follows the embedded variant. The qualitative strand is implemented during the case series, thus a concurrent timing occurs. We consider the qualitative and quantitative parts of the case series and process evaluation equally important. The quantitative and qualitative strand will be interactive: mixing of methods occurred at the level of design and will continue during data collection (i.e. quantitative data will support selection of participants for elements of the qualitative strand) and during interpretation.

This study emerges from the pragmatism paradigm, which focuses on the consequences of actions, is problem centred, pluralistic and real-world practice oriented [19]. The qualitative strand involves a generic qualitative approach. Merriam describes generic qualitative research as an approach that cannot be specified as a particular type of qualitative study, such as grounded theory [20]. The aim of generic qualitative research studies is to understand the way people make sense of their lives and their experiences. However, it does not have an additional dimension that other designs have, such as understanding of a certain phenomenon in a phenomenological design [20].

\section{HOME-BASED TRAININg PROgRAMS}

A multidisciplinary team of certified care providers, consisting of a paediatric physical or occupational therapist and a remedial educationalist or healthcare psychologist will deliver the programs. Each therapist will operate within only one home-based training program to prevent contamination. Allocation of a therapist to a program is based on the preference of the therapist. Remedial educationalists will operate across both programs. No contamination is expected, because remedial educationalists are instructed to avoid coaching with regard to the therapeutic content of the programs. 


\section{COURSE FOR THERAPISTS AND REMEDIAL EDUCATIONALISTS}

Each therapist will complete a one-day course regarding the home-based training program. The course targets either the implicit approach or the explicit approach and mainly focuses on performing task analyses and designing an individualized home-based training plan in accordance with the specific learning approach. During a half-day course, the remedial educationalists will be informed on the content of both home-based training programs and instructed how to coach parents. Members of the research team who are experienced clinicians and educators will provide the courses. Since inclusion of participants within the centres will start consecutively, the courses are repeatedly delivered during the study. Refresher courses will be organized for therapists as well as remedial educationalists approximately one year after the initial training.

The home-based training programs consist of two phases: a preparation phase and the home-based training phase.

\section{PREPARATION PHASE}

The 2-week preparation phase involves four aspects. First, a blinded therapist who is not involved in the home-based training program of the child will determine five individual rehabilitation goals of importance to the parents and child, using the COPM [18]. Second, the coaching team gets acquainted with the parents and the child in an introductory meeting between the parents and the remedial educationalist and another meeting between the parents, child and coaching therapist, at the rehabilitation centre. During the latter meeting, the therapist will observe the manual abilities of the child and will videotape the child performing the activities that the rehabilitation goals comprise. Third, based on these videos the therapist will perform a task analysis. The task analysis approach is based on principles of stage 1 of the Perceive, Recall, Plan and Perform (PRPP) System of Task Analysis [21]. According to this task analysis, each task will be divided in steps. Thereafter, the error types in performance of each step, i.e. errors of omission, repetition, accuracy or timing, are registered. For participants in the explicit program, therapists additionally perform a movement analysis for each step in which errors occur. This movement analysis contains the posture and movement related actions the child does, as well as should perform in order to successfully complete the step. Subsequently, for all children therapists design an individualized training plan including instruction, feedback and organization of the task. Videos of the child during administration of the Assisting Hand Assessment (AHA) and the Observational Skills Assessment Score (OSAS) can facilitate training design [22, 23]. Fourth, the parents will be trained, which is twofold. Parents will receive instructional videos and a manual to study at home during the preparation phase. These materials address the content of the program, the coaching and teaching approach, and the use of the communication tool Quli (i.e. a Dutch online system for safe transfer of data such as documents, messages and videos between health care providers and health care consumers) [24]. Subsequently, at the end of the preparation phase, the therapist will visit the child and parents at home. This home visit allows the therapist to clarify the instruc- 
tional videos, discuss the training plan, assess the physical home situation and available objects related to the rehabilitation goals, and answer parents' questions. In case parents or the therapist have questions in the behavioural and social interaction domain, the remedial educationalist will be consulted. The manuals for therapists and remedial educationalists include various checklists, for example to guide the home visit.

\section{HOME-BASED TRAINING PHASE}

The aim of the home-based training is improvement of the child's performance of individual rehabilitation goals through training that is congruent with the context of the particular goal (i.e. task-specific therapy). This is in accordance with the latest version of the recommendations for care of children with spastic CP in the Netherlands, i.e. the 'Richtlijn Spastische cerebrale parese bij kinderen' [25]. During this phase, parents will provide training to their child in their home environment. The parent or caregiver most involved in the training will, as 'primary trainer', also have an active role in the data collection for the study. To foster implementation, a second parent or caregiver may also be involved in the training.

During the 12-week home-based training phase, parents and children will train for $3.5 \mathrm{~h} \mathrm{per}$ week, preferably in meaningful situations. Parents can subdivide these training hours across the week in sessions with a minimum duration of $10 \mathrm{~min}$.

In both programs, parents provide task-oriented and result-oriented instructions and feedback to the child. In the explicit approach, parents additionally give instructions and feedback to their child regarding specific motor execution of the tasks, whereas in the implicit approach parents provoke specific motor execution by the organization of the tasks.

Throughout the home-based training, parents will be coached by a therapist and a remedial educationalist. The parents and therapist will have a 30-min appointment over the phone weekly. In week 5 and week 9 the therapist will visit the parents at home for 60 min. If necessary, the therapist may schedule one additional home visit during the home-based training phase. Furthermore, parents will be contacted by phone by the remedial educationalist after the second week of home-based training. If requested by parents or therapist, basically one additional contact with the remedial educationalist can be planned.

To facilitate remote coaching, parents will register the amount and content of training they have performed with their child. Moreover, once a week they will record a training session on video. Parents will send the training registration form and the videos to the therapist and remedial educationalist via the communication tool Quli.

\section{FOLLOW-UP PERIOD}

A follow-up period succeeds the home-based training program, during which children will receive usual care. At the end of this 12-week period the final data will be collected to investigate the retention of training. 


\section{Process evaluation methods}

The approach described by Saunders et al. is used as the framework for the process evaluation. Saunders et al. describe five components of process evaluation: fidelity, dose, reach, recruitment, and context [26].

- Fidelity (quality) is defined as "the extent to which an intervention was implemented as planned".

- Dose consists of dose delivered (completeness) and dose received (exposure and satisfaction). Dose delivered includes "the amount or number of intended units of each intervention or component delivered or provided by interventionists". The exposure aspect of dose received is defined as "the extent to which participants actively engage with, interact with, are receptive to, and/or use materials or recommended resources". The satisfaction aspect of dose received comprises "participant satisfaction with program, interactions with staff and/or investigators".

- Reach (participation rate) is defined as "the proportion of the intended priority audience that participates in the intervention".

- Recruitment comprises "procedures used to approach and attract participants at individual or organizational levels; includes maintenance of participant involvement in the intervention and measurement components of the study".

- Context contains "aspects of the environment that may influence intervention implementation or study outcomes; includes contamination or the extent to which the control group was exposed to the program" [26].

Four of these five components of process evaluation will be investigated in our study. Reach of the home-based training programs in the context of the COAD-study is expected to be highly influenced by study related factors and will therefore not be assessed. Please note that throughout this paper implementation within the scope of the study is meant, not implementation within clinical practice.

\section{Data COLlection}

Table 1 shows the data collection methods, which will be used to assess the components fidelity, dose, recruitment and context of the process evaluation. The methods contain questionnaires, report and registration forms, videos, interviews and focus groups. In case of non-response to questionnaires and forms a maximum of three reminders will be send two, four and six days after the initial invitation. For the parent-related methods, the parent who is primary trainer will be asked to provide the requested input.

The following paragraphs will elaborate on the data collection. 
Table 1 Overview of data collection methods and respondents used to assess each process evaluation component per phase

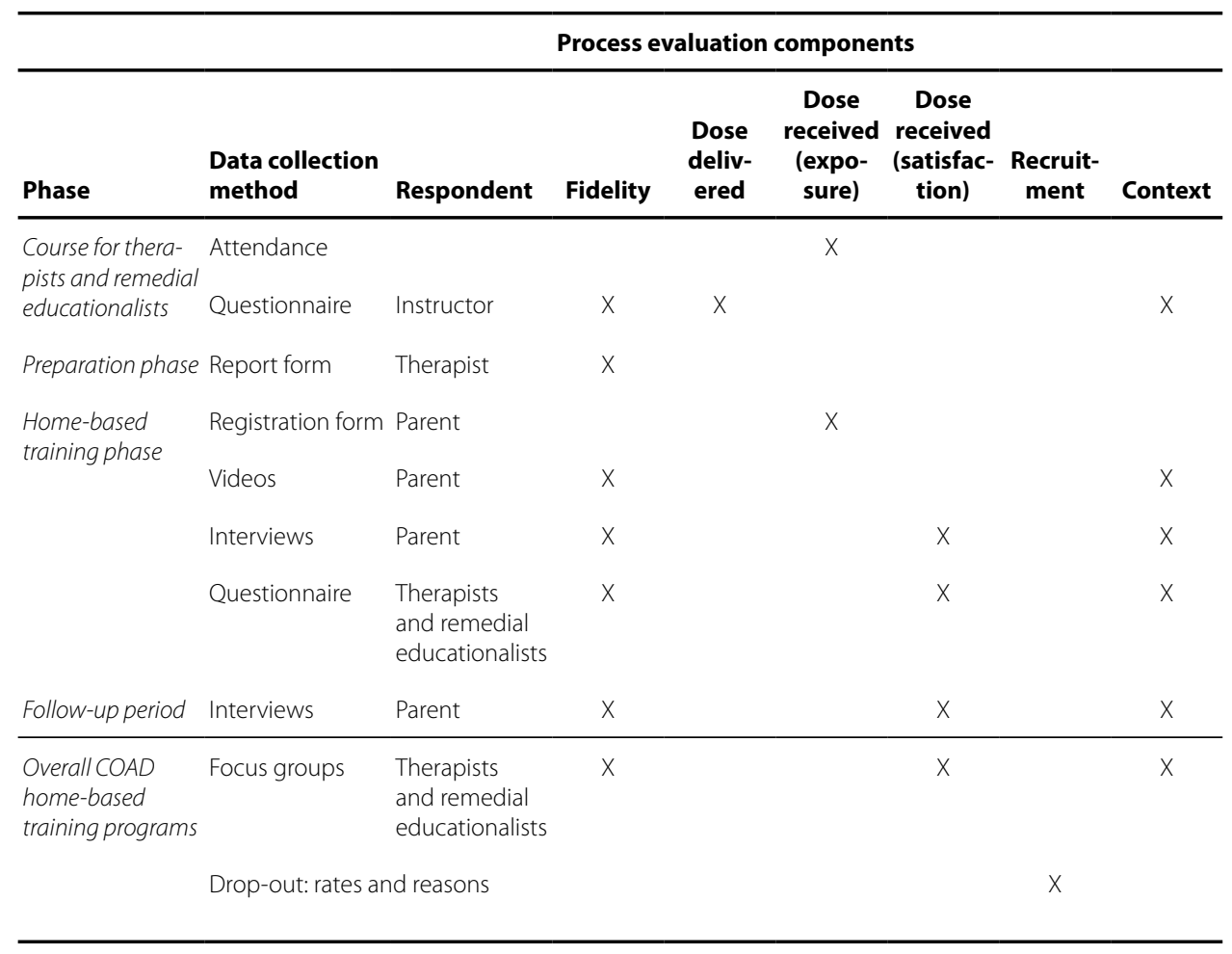

\section{COURSE FOR THERAPISTS AND REMEDIAL EDUCATIONALISTS}

For each course, attendance of therapists and remedial educationalists will be registered.

The instructors of the therapists and remedial educationalists will evaluate the course by a digital questionnaire. The questionnaire includes questions regarding duration, location, content, positive and negative experiences, competence of the participants after the course, received feedback of the participants, and suggestions for improvement. All instructors are invited to fill out the questionnaire as soon as possible after the course and return it by e-mail.

\section{PREPARATION PHASE}

The first home visit will be evaluated by the therapist using a digital report form. This form includes for each point of the checklist for the first home visit provided by the protocol (as described in the paragraph 'home-based training programs, preparation phase') items regarding duration, understanding by parents, and particulars; other topics that were discussed; therapist's impression of 
parents' competence to execute the home-based training; and whether the remedial educationalist was going to be consulted instantly. The report form will be administered directly after the home visit by the online data collection application Castor EDC.

\section{HOME-BASED TRAINING PHASE AND FOLLOW-UP PERIOD}

During the home-based training, all parents will register the daily amount of training and the kind of activities that were performed regarding each treatment goal (for example, closing the button of cotton trousers, while seated with the trousers on a table in front of the child); if applicable particular details per day, such as illness of the child; and perceived emotions of the parent as well as parent-rated emotions of the child related to the training via the use of emoticons. The digital registration form (Microsoft ${ }^{\circledR}$ Excel format) will be uploaded in the online data collection application Castor EDC by the parents at the end of the training program.

Besides, parents will be asked to make one video recording of a training session every week. From these twelve videos, six videos will be selected randomly. The video material will be scored by blinded assessors making use of a rating tool. The purpose of the tool is to define the degree of implicit and explicit approaches that parents use in training their child. This will be established by the proportion of task-oriented and result-oriented instruction/feedback versus instruction/ feedback regarding motor execution of the task. A random selection of $20 \%$ of the videos will be rated in duplicate in order to calculate the inter-rater reliability of the tool.

Moreover, three in-depth parental interviews will be conducted: halfway the home-based training phase, after the home-based training has ended and after the follow-up period of 12 weeks. The interviews will be performed either with one parent or a parent-couple, including at least one parent actively engaged in the home-based training. The interviews will be semi-structured, following a pre-defined interview protocol. The duration of each interview will be approximately $60 \mathrm{~min}$. To facilitate transferability of the different aims of the process evaluation, for the interviews a specific sub research question has been formulated: How do parents experience the home-based training program and how do they integrate the program in the context of family life? This question directs the interview process as well as data-analysis. Topics that will be covered during the first two interviews are: overall experience with the home-based training program; integrating the home-based training in the daily life of the child, the parent and the family; influence of the training on the parent and on the child; experiences of the parent as a co-therapist and perceived reactions of the child; the coaching by the health professional(s); and suggestions for improvement of the home-based training program. The third interview will cover retrospective experience with the home-based training program; (appreciation of) influence of the home-based training on the parent and the child after the intervention had ended; suggestions for improvement; retrospective considerations regarding participation in the home-based training program; and recommendation of the program to fellow parents. Additionally, therapy-related parental stress will be investigated by means of the interviews in the context of the case series of the COAD-study. The first interview will take place in person at the parents'home, the second and third by video call. The interviews will be audiotaped. Trained interviewers will perform the interviews. 
To evaluate the particular course of the home-based training program for each child, the therapist and remedial educationalist will each fill out a questionnaire. The questionnaires include questions regarding the execution and timing of program elements; content-related and procedural particularities; and their opinion on the application of the program by the parents. The questionnaires will be administered by the online data collection application Castor EDC, after the program of an individual child has ended.

\section{OVERALL HOME-BASED TRAINING PROGRAMS}

Focus groups will be held with therapists and remedial educationalists involved in the study to explore their experiences with the home-based training programs. The health professionals will attend separate focus groups, based on their occupation and the home-based program they provided. Participation of all health professionals is desired, while practical difficulties to accomplish this are expected to result in a convenience sample. The duration of the focus groups will be 90-120 min. A topic list will guide the focus group discussions. This list will be designed based on the results of the other elements of the process evaluation. If a meeting in person is logistic not feasible, a simultaneous online focus group will take place. The focus groups will be videotaped. A trained researcher and assistant moderator will moderate the focus groups.

Dropout rates will be assessed for each home-based training program and, if available, reasons for drop-out will be recorded.

\section{DATA ANALYSIS}

A graphical presentation of the data collection methods and the analysis process is given in Fig. 1.

Thematic analysis will be used for qualitative data, following the method described by Braun and Clarke $[27,28]$. The analysis will be inductive (i.e. the identified themes will derive from the data) and on a latent/interpretative level. The latter indicates that ideas, assumptions, conceptualisations and ideologies will be determined that are theorized as underlying to the semantic content of the data. The first phase of the thematic analysis involves familiarization with the data. Verbatim transcripts will be created, the data will be read repeatedly and initial ideas will be noted. Phase 2 comprises systematic generation of initial codes. During phase 3 themes will be searched for by arranging codes into potential themes. Next, applicability of these themes to the coded extracts as well as the entire data set will be reviewed in phase 4 . Phase 5 involves creating clear definitions and names for the themes, in order to refine their specifics. Phase 6 will offer the final opportunity for analysis. After selecting decent extract examples, final analysis and comparison of the analysis to the research aim and literature, the report will be produced [27, 28]. The qualitative data analysis software NVivo will be used throughout the data analysis.

Descriptive statistics include mean (standard deviation) or median (range) and number (\%) for continuous and categorical data, respectively.

Initially the qualitative and quantitative data will be analysed concurrently. Thereafter, a side-byside comparison will be performed using a summary table in which the qualitative and quantitative findings are merged. This meta-inference will be followed by interpretation of the combined results. 


\section{VALIDITY AND RELIABILITY OF THE QUALITATIVE STRAND}

The process evaluation applies both between- and across-method triangulation by combining several quantitative and qualitative data-collection procedures, such as questionnaires, registration forms, in-depth interviews and focus groups. Data sources triangulation is performed by variance in respondents, namely trainers, parents, therapists and remedial educationalists. Two researchers will perform the data analysis. Hence, investigator triangulation occurs.

The results of the focus groups will be validated with the participating health professionals. No other member checking will be performed.

A researcher with expertise in qualitative research and with no other involvement in the project will peer review the process evaluation by verification of the analysis of $20 \%$ of the interviews and focus groups. In addition, she will critically analyse whether the conclusions are founded. By the peer review process, it is strived for sufficient independence in conducting the process evaluation and interpretation of its results.

\section{RESEARCHER BIAS AND ASSUMPTION}

The interviews will be executed by $L B$, the data analysis will be performed by LB and MM. $L B$ is a physical therapist by origin, currently she is a PhD candidate on the COAD-study. MM is a research assistant with a background as medical analyst. Because of the pragmatic nature of the study, the researchers involved in the process evaluation will remain passive observers during the study by avoiding interference with the home-based training programs and its delivery. Since the process evaluation will be executed by the project team, which is also responsible for development and delivery of the programs and for the evaluation of outcomes of the case series, peer review will be performed as described in the previous paragraph.

The study, including data analysis, will be performed in Dutch. The findings and supporting evidence will be translated into English. Back translation of $20 \%$ of this material will be executed to increase credibility.

\section{Discussion}

This protocol outlines the background and design of the process evaluation of two home-based bimanual training programs for children with UCP. Evaluating two programs that differ regarding the approach by which parents teach motor activities to their child is innovative. Comparing an implicit with an explicit approach originates from the demand to increase bimanual activity and participation of the child whilst minimizing parental stress as a result of home-based training. Furthermore, to our knowledge, this is the first study to perform an extensive process evaluation parallel to an effect evaluation regarding home-based training programs in paediatric rehabilitation. The aim of the process evaluation is to assess fidelity of the home-based training programs, to examine the mechanisms that cause the relation between the programs and their effects, and to determine the influence of contextual factors. 


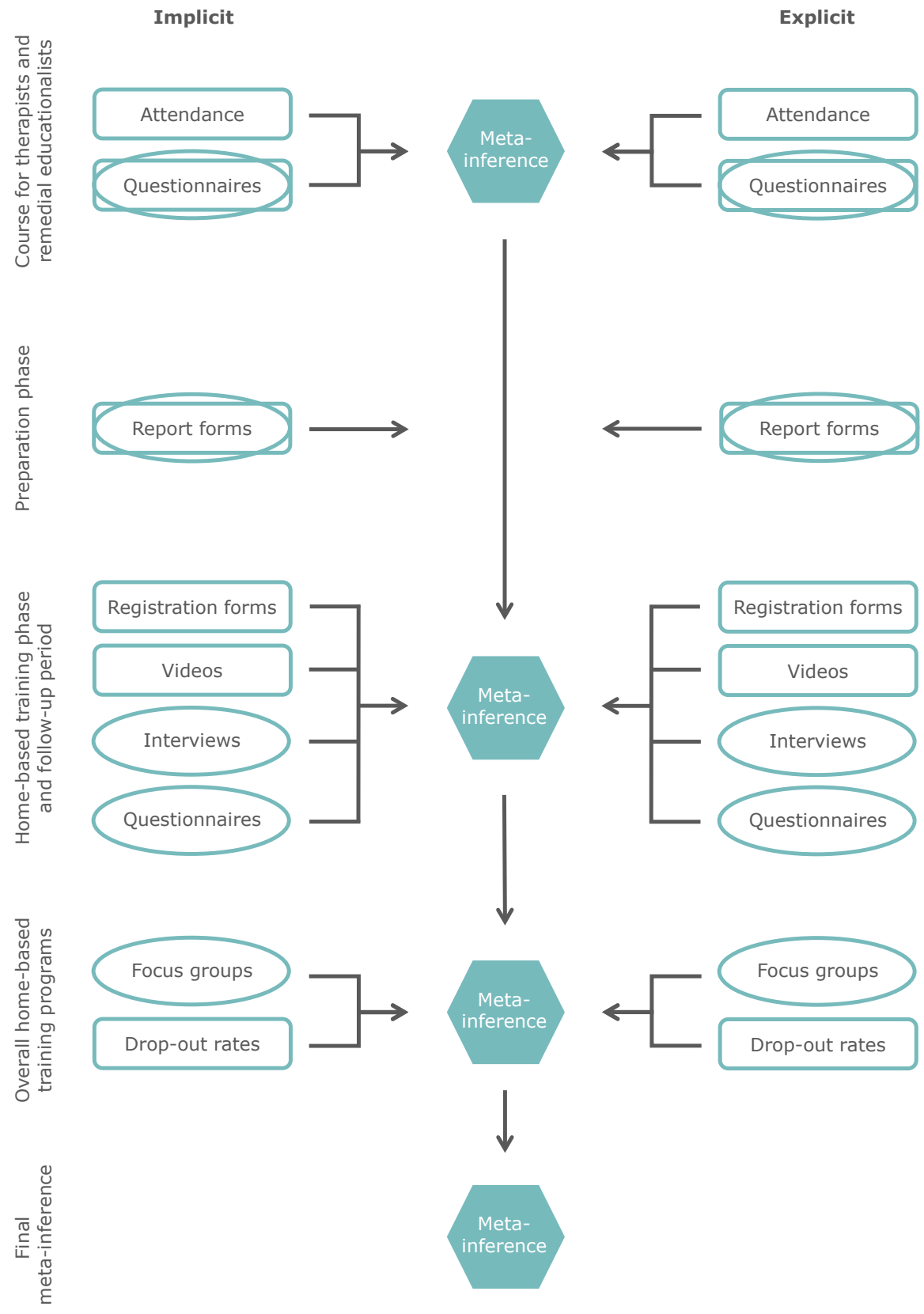

Figure 1 Overview data collection methods and the analysis process. Ovals represent primarily qualitative parts, rectangles primarily quantitative parts and hexagons meta-inferences 
A key strength of the process evaluation is the use of mixed methods. In general, it is assumed that triangulation of quantitative and qualitative methods leads to greater validity than either single one alone, and that combining them offsets the weaknesses of each individual method [19]. For this specific study, the mixed methods design is chosen to get a more complete understanding of processes occurring with regard to the home-based training programs and experiences of parents with the programs. Moreover, the qualitative data are expected to facilitate the explanation of the quantitative findings. The use of different types of triangulation as well as data collection during all phases of the home-based training programs will enhance the credibility (i.e. internal validity) of the study.

Member checking is limited to the focus groups at the end of the study. Member checking throughout the study would be expected to influence behaviour of parents, therapists and remedial educationalists during the home-based training programs, possibly affecting the results of the process and/or effect evaluation. Another limitation includes execution of the process evaluation by the project team itself, which may introduce researcher bias. However, acknowledging this possibility as well as peer review by an independent researcher is assumed to reduce this risk.

The findings of this process evaluation will have implications for clinical practice and further research regarding development and application of home-based bimanual training programs, executed by parents and aimed at improving activity performance and participation of children with UCP.

\section{Acknowledgements}

We would like to thank our focus group for their contribution to the COAD-study.

\section{FUNDING}

This work was supported by Revalidatiefonds and Johanna Kinderfonds / Stichting Rotterdams Kinderrevalidatie Fonds Adriaanstichting through the 3rd Program Rehabilitation Research of ZonMw (the Netherlands Organization for Health Research and Development), grant number 630000001. Revalidatiefonds and Stichting Vooruit provided additional grants, project number R2016006 and 18-05/YvH/NS, respectively. The funding bodies had no role in the design of the study or writing the manuscript.

\section{Authors' Contributions}

YJ-P had a predominant role in the application for funding of the COAD-study, in which JB, ER, PA, and RS gave their input. LB conceptualized and designed the process evaluation described in this protocol, under supervision of JB and RS. LB drafted the initial manuscript, where after LB, JB and RS contributed to its enhancement providing intellectual input. All authors commented on the manuscript and approved its final version. 


\section{ETHICS APPROVAL AND CONSENT TO PARTICIPATE}

The medical ethical committee 'Commissie Mensgebonden Onderzoek regio Arnhem - Nijmegen' approved this study (protocol number NL53670.091.15). Participating parents and the legal guardians of the participating children must provide written informed consent before enrolment in the COAD-study. Additional written permission will be gained for audio recording the in-depth interviews and video recording the focus groups.

\section{Competing interests}

The authors declare that they have no competing interests. 


\section{REFERENCES}

1. Rosenbaum P. Cerebral palsy: what parents and doctors want to know. BMJ. 2003;326(7396):970-974.

2. Lemmens RJ, Janssen-Potten YJ, Timmermans AA, Defesche A, Smeets RJ, Seelen HA. Arm hand skilled performance in cerebral palsy: activity preferences and their movement components. BMC Neurol. 2014;14:52.

3. Imms C. Children with cerebral palsy participate: a review of the literature. Disabil Rehabil. 2008;30(24):1867-1884.

4. Novak I, Cusick A. Home programmes in paediatric occupational therapy for children with cerebral palsy: where to start? Aust Occup Ther J. 2006;53(4):251-264.

5. An M, Palisano RJ. Family-professional collaboration in pediatric rehabilitation: a practice model. Disabil Rehabil. 2014;36(5):434-440.

6. Shumway-Cook A, Woollacott MH. Motor learning and recovery of function. In: Shumway-Cook A, Woollacott MH, editors. Motor control: translating research into clinical practice. Philadelphia: Lippincott Williams \& Wilkins; 2007.

7. Ferre CL, Brandão M, Surana B, Dew AP, Moreau NG, Gordon AM. Caregiver-directed home-based intensive bimanual training in young children with unilateral spastic cerebral palsy: a randomized trial. Dev Med Child Neurol. 2016;59(5):497-504.

8. Novak I, Cusick A, Lannin N. Occupational therapy home programs for cerebral palsy: double-blind, randomized, controlled trial. Pediatrics. 2009;124(4):e606-e614.

9. Peplow UC, Carpenter C. Perceptions of parents of children with cerebral palsy about the relevance of, and adherence to, exercise programs: a qualitative study. Phys Occup Ther Pediatr. 2013;33(3):285-299.

10. Lin KC, Wang TN, Wu CY, Chen CL, Chang KC, Lin YC, Chen YJ. Effects of home-based constraint-induced therapy versus dose-matched control intervention on functional outcomes and caregiver well-being in children with cerebral palsy. Res Dev Disabil. 2011;32(5):1483-1491.

11. Başaran A, Karadavut KI, Üneri SO, Balbaloğlu O, Atasoy N. Adherence to home exercise program among caregivers of children with cerebral palsy. Turk J Phys Med Rehab. 2014;60:85-91.

12. Schnackers M, Beckers L, Janssen-Potten Y, Aarts P, Rameckers E, van der Burg J, et al. Home-based bimanual training based on motor learning principles in children with unilateral cerebral palsy and their parents (the COAD-study): rationale and protocols. Submitted to BMC Pediatr. 2018;18:139.

13. Kleynen M, Braun SM, Rasquin SM, Bleijlevens MH, Lexis MA, Halfens J, Wilson MR, Masters RS, Beurskens AJ. Multidisciplinary views on applying explicit and implicit motor learning in practice: an international survey. PLoS One. 2015;10(8):e0135522.

14. Jenks KM, de Moor J, van Lieshout EC. Arithmetic difficulties in children with cerebral palsy are related to executive function and working memory. J Child Psychol Psychiatry. 2009;50(7):824-833.

15. Medical Research Council . Developing and evaluating complex interventions: new guidance. 2006.

16. Tashakkori A, Creswell JW. Editorial: exploring the nature of research questions in mixed methods research. J Mix Methods Res. 2007;1 (3):207-211.

17. Eliasson AC, Krumlinde-Sundholm L, Rosblad B, Beckung E, Arner M, Ohrvall AM, Rosenbaum P. The manual ability classification system (MACS) for children with cerebral palsy: scale development and evidence of validity and reliability. Dev Med Child Neurol. 2006;48(7):549-554. 
18. Law M, Baptiste S, McColl M, Opzoomer A, Polatajko H, Pollock N. The Canadian occupational performance measure: an outcome measure for occupational therapy. Can J Occup Ther. 1990;57(2):82-87.

19. Creswell JW, Plano Clark VL. Designing and Conducting mixed methods research. Thousand Oaks: SAGE Publications; 2011.

20. Merriam SB, Tisdell EJ. Six common qualitative research designs. In: Merriam SB, Tisdell EJ, editors. Qualitative research: a guide to design and implementation. San Francisco: Jossey-Bass; 2015. pp. 22-42.

21. Nott MT, Chapparo C, Heard R. Reliability of the perceive, recall, plan and perform system of task analysis: a criterion-referenced assessment. Aust Occup Ther J. 2009;56(5):307-314.

22. Krumlinde-sundholm L, Eliasson A-c. Development of the assisting hand assessment: a Raschbuilt measure intended for children with unilateral upper limb impairments. Scand J Occup Ther. 2003;10(1):16-26.

23. Speth L, Janssen-Potten Y, Leffers P, Rameckers E, Defesche A, Geers R, Smeets R, Vles H. Observational skills assessment score: reliability in measuring amount and quality of use of the affected hand in unilateral cerebral palsy. BMC Neurol. 2013;13:152.

24. Quli. Wat is Quli? 2017. https://www.quli.nl/wat-is-quli/. Accesssed 8 Feb 2018.

25. De Nederlandse Vereniging van Revalidatieartsen. Richtlijn Spastische cerebrale parese bij kinderen. 2015. https://revalidatiegeneeskunde.nl/sites/default/files/attachments/Kwaliteit/Richtlijnen/richtlijn_cerebrale_parese_revisie_2015_-_def.pdf. Accessed 22 May 2017.

26. Saunders RP, Evans MH, Joshi P. Developing a process-evaluation plan for assessing health promotion program implementation: a how-to guide. Health Promot Pract. 2005;6(2):134-147.

27. Braun V, Clarke V. Using thematic analysis in psychology. Qual Res Psychol. 2006;3(2):77-101.

28. Braun V, Clarke V. What can "thematic analysis" offer health and wellbeing researchers? Int J Qual Stud Health Well-being. 2014;9:26152. 
Chapter io

\section{Process evaluation of two home- based bimanual training programs in children with unilateral cerebral palsy (the COAD-study): a mixed methods study}

LWME Beckers, RJEM Smeets, MAC de Mooij, B Piškur, JJW van der Burg, EAA Rameckers, PBM Aarts, COAD Author Group, YJM Janssen-Potten 



\section{INTRODUCTION}

Unilateral cerebral palsy (UCP) is characterized by impairments in bimanual performance [1]. There is robust evidence that bimanual intensive movement therapy results in meaningful improvements of bimanual coordination, bilateral spontaneous hand use, and activities that are relevant for the child [2-4]. However, it is of great importance that learning is generalized to the natural environment where the child eventually has to perform bimanual activities, become independent, and participate [5]. Practice in the home setting meets this requirement of practice specificity and optimizes transfer of learning, which is a great advantage compared to center-based treatment. Parent-delivered training at home also complies with principles of family-centered care [6], and may increase self-efficacy, empowerment, and self-reliance of parents.

Home-based programs can be effective in improving clinical outcomes [2,7]. However, studies are mostly limited to constraint-induced movement therapy (CIMT) as the treatment approach, and are incomplete in describing the intervention protocol, especially in terms of coaching approaches and motor learning principles [8]. A common distinction is made between implicit and explicit techniques, of which various definitions exist. Kleynen et al. [9] define implicit motor learning as "learning which progresses with no or minimal increase in verbal knowledge of movement performance (e.g., facts and rules) and without awareness". Explicit motor learning on the other hand is considered to be "learning which generates verbal knowledge of movement performance (e.g., facts and rules), involves cognitive stages within the learning process and is dependent on working memory involvement" [9]. Implicit motor learning is less demanding for the working memory [10], which is relatively often impaired in children with CP [11]. Moreover, it is believed to put less strain on the parent-child interaction when applied in a training program [12]. However, the applicability of both principles for home-based training has not been systematically studied. In conclusion, more research is needed on bimanual training in the home environment and on the multiplicity of components in home programs.

Within the COAD-study (CO-creation At hanD: the road to independence), two home-based bimanual training programs have been developed, targeting young children with UCP [12]. The aim of both programs is to increase bimanual activity performance of the child, without increasing parental stress. The training is parent-delivered, aided by multidisciplinary coaching by an occupational/physical therapist and a remedial educationalist or health-care psychologist. The latter is involved as it is known that home-based programs can negatively impact parent-child interaction and wellbeing of the family, impeding therapy compliance. The motor learning approach in one program is at the implicit end of the motor learning spectrum, while in the other program parents are instructed to use a predominantly explicit motor learning approach.

Rehabilitation interventions are complex, involving a process that includes numerous actions, and are carried out in complex environments [13]. A major challenge in rehabilitation research is to evaluate the interacting intervention components as a whole. For this reason, Wade et al. recommend a process evaluation of interventions delivered in studies [13]. The delivery of training by parents and the collaboration between parents and health-care professionals further complicate home-based training programs. Nonetheless, studies thus far have not performed a comprehensive process evaluation, but only examined compliance with and acceptability of 
the prescribed home-based training. These evaluations were related to the overall programs, but did not provide an assessment of specific aspects of the home programs [8]. It is intended that the current research will contribute to a deeper understanding of parent-delivered home-based bimanual training programs.

The aims of the COAD-study were to: (1) evaluate the effects of the home-based training programs; and (2) evaluate the processes and factors that influenced implementation and effects of the programs. This paper reports on the outcomes of the process evaluation. The effect analysis will be published separately [14]. The COAD-study adopted a mixed methods methodology. In the process evaluation, the quantitative strand included factual and elementary information. Indepth experiences were appraised by qualitative data collection. Conclusions were drawn from the meta-inference of the quantitative and qualitative data. Four main research questions guided the process evaluation:

1. To what extent were the home-based training programs implemented as intended?

2. How did parents experience the home-based training program and how did they integrate the program into family life?

3. Which components of the home-based training programs contributed to the effects of the programs?

4. What contextual factors with regard to children, parents and health-care professionals were associated with possible variation in implementation and outcomes between child:parents:health-care-professionals triads?

\section{MATERIALS AND METHODS}

\section{DESIGN AND PARADIGM}

A mixed methods study, which can be defined as "... research in which the investigator collects and analyses data, integrates the findings, and draws inferences using both qualitative and quantitative approaches or methods in a single study or program of inquiry" [15], was conducted. Specifically, a concurrent embedded experimental design was utilized: the mainly qualitative process evaluation was embedded in the quantitative experimental design (i.e. RCT and thereafter case series). Quantitative and qualitative data had equal priority and were gathered longitudinally throughout the same period. The quantitative and qualitative strands had an interactive relationship and were primarily mixed at the level of design and interpretation. A pragmatic worldview guided this study. This paradigm addresses the consequences of research, is problem-centered, pluralistic, and real-world-practice oriented [16]. A generic qualitative approach was applied to the qualitative strand [17]. The full protocol of the process evaluation has been described elsewhere [18].

\section{Participants}

A total population sample of the effect study participants was included in the process evaluation. Participants were recruited from five rehabilitation centers on seven locations across urban as well as rural areas in the Netherlands between April 2016 and March 2018. This comprised children 
with UCP, aged 2 through 7 years with Manual Ability Classification System (MACS) Levels I-III [19], together with their parents. Allocation to the implicit or explicit program followed either randomization ( $\mathrm{RCT}$ ) or the parents' preference (case series).

In addition to the families, the health-care professionals involved in the interventions participated in the process evaluation. Apart from being employed as a physical or occupational therapist, remedial educationalist, or health-care psychologist in one of the collaborating rehabilitation centers, no eligibility criteria were applied. Each therapist was engaged with one of the programs, whereas the remedial educationalists and health psychologists were involved in both.

Ethical approval for the study was granted by the medical ethical committee 'Commissie Mensgebonden Onderzoek regio Arnhem - Nijmegen' (NL53670.091.15). Written informed consent was obtained from all participating parents and the legal guardians of participating children. Participants gave permission for audio (and video) recordings. Confidentiality was ensured by the removal of all identifying information.

\section{INTERVENTIONS}

The interventions under study were two home-based bimanual training programs, of which the detailed protocols have previously been published [12]. The home-based training programs encompassed bimanual task-specific training delivered by the parents, intending to improve the child's performance of bimanual activities, without inducing therapy-related parental stress. A pediatric physical or occupational therapist (referred to as therapist) and a remedial educationalist or health-care psychologist (referred to as remedial educationalist) from the child's rehabilitation center jointly coached parents. The professionals attended an instructional course and received a manual so that they might become acquainted with the home-based training protocols before they started coaching. The entire home-based training programs lasted 14 weeks, comprising a two-week preparation phase followed by a 12-week home-based training phase.

\section{Preparation PHASE}

Parents identified five rehabilitation needs for their child (i.e. bimanual activities) during the administration of the COPM by an independent therapist [20]. Thereafter, an introductory meeting between the parents and the coaching therapist took place at the rehabilitation center, including observation of the child's bimanual functioning, as well as a meeting between the parents and the remedial educationalist to explain the involvement of the remedial educationalist and to examine the child, parent, and family conditions. Next, the therapist conducted a task analysis for the five target bimanual activities and developed an individualized training plan. In the explicit program, the task analysis was complemented by a movement analysis. Further, in both programs parents prepared themselves by means of watching instructional videos and reading a manual. A home visit by the therapist to assess and discuss the specific home and family situation concluded the preparation phase. 


\section{HOME-BASED TRAINING PHASE}

The aim of the home-based training was for the child to accomplish personal goals. Hence, the programs adopted a goal-oriented, task-specific approach of bimanual training, preferably in meaningful situations for the child. The training was parent-delivered in the home environment and scheduled for 3.5 hours per week.

Parents gave task-oriented and result-oriented instructions and feedback to the child in both programs. The explicit program was characterized by additional instructions and feedback focused on the motor performance, while in the implicit program the organization of the activities elicited the targeted motor performance [12].

Parents registered the dose and specifics of training, and video-recorded a training moment each week. Initially, the communication tool 'Quli' was provided to parents to share these data with the health professionals. When practical issues emerged that impeded the process, the tool'arQive' was implemented instead. The interdisciplinary remote coaching comprised weekly telephone appointments with the therapist, two home visits by the therapist (at four and eight weeks after the start of the training), one standard telephone appointment with the remedial educationalist (after two weeks), and additional consultation with the remedial educationalist if required.

\section{FOLLOW-UP}

Children received 12 weeks of usual care following the home-based training programs.

\section{Process evaluation methods}

The components fidelity, dose, recruitment, and context were appraised, as represented in the guide for process evaluations by Saunders et al. [21].

\section{DATA COLLECTION}

The data collection approaches are presented in Table 1 and included attendance monitoring, questionnaires, a registration form, video analyses, interviews, and focus group discussions. The parent most involved in the training (i.e.' primary trainer') participated in the parent-related procedures.

\section{COURSE ATTENDANCE MONITORING}

Attendance at the instructional course for therapists and remedial educationalists was monitored by the research team.

\section{QUESTIONNAIRES}

The evaluation contained three different semi-structured questionnaires. First, the instructors (i.e. research team members) completed a questionnaire regarding the content and procedures 
applied during the instructional course for therapists and remedial educationalists. Second, the therapist described the conduct of the first home visit for each participant by a questionnaire. Third, both the therapist and remedial educationalist completed a questionnaire at the end of each individual home-based training program to evaluate its execution.

\section{REGISTRATION FORM}

In a registration form, parents logged the amount of time spent on training each treatment goal, including any relevant details, as well as the emotions experienced by themselves and observed in their child due to the training.

\section{VIDEO ANALYSIS}

Blinded raters ( $\mathrm{JB}$ and $\mathrm{YJ}$ ) scored the weekly video recordings of the training for the type of instruction and feedback used by parents (i.e. either task-oriented and result-oriented and/or focused on motor performance). The rating tool was applied using a 10-second partial interval time sampling procedure. It was intended to analyze six randomly selected videos per child. However, since the complications with the data sharing tool resulted in a large number of missing videos, all available videos were scored. Inter-rater reliability was assessed for $20 \%$ of the videos.

\section{INTERVIEWS}

Following the longitudinal methodology, parents were interviewed repeatedly: halfway through the home-based training phase; at the end of the home-based training; and at the end of the 12-week follow-up. The aim of the interviews was to evaluate the parents' experiences with the home-based training programs and the integration of the programs in the context of their family life, including a descriptive inventory of perceived therapy-related parental stress. A protocol was used to guide the semi-structured interviews. A trained interviewer (LB) conducted and audiotaped the interviews, the first time at the parents' home and twice by phone or video call.

\section{FOCUS GROUP DISCUSSION}

After the closure of inclusion, a convenience sample of therapists and remedial educationalists reflected on the home-based training programs during focus group discussions. The remedial educationalists formed one and the therapists two focus groups, independent of the home-based program they were involved in. The latter was a minor deviation from the protocol, which occurred due to planning issues. A trained researcher (LB) and assistant moderator (MM) guided the focus group discussions, which contained a combination of in-person and simultaneous online/telephone sessions. The focus group discussions followed a protocol and were audio- (and video-)taped. 


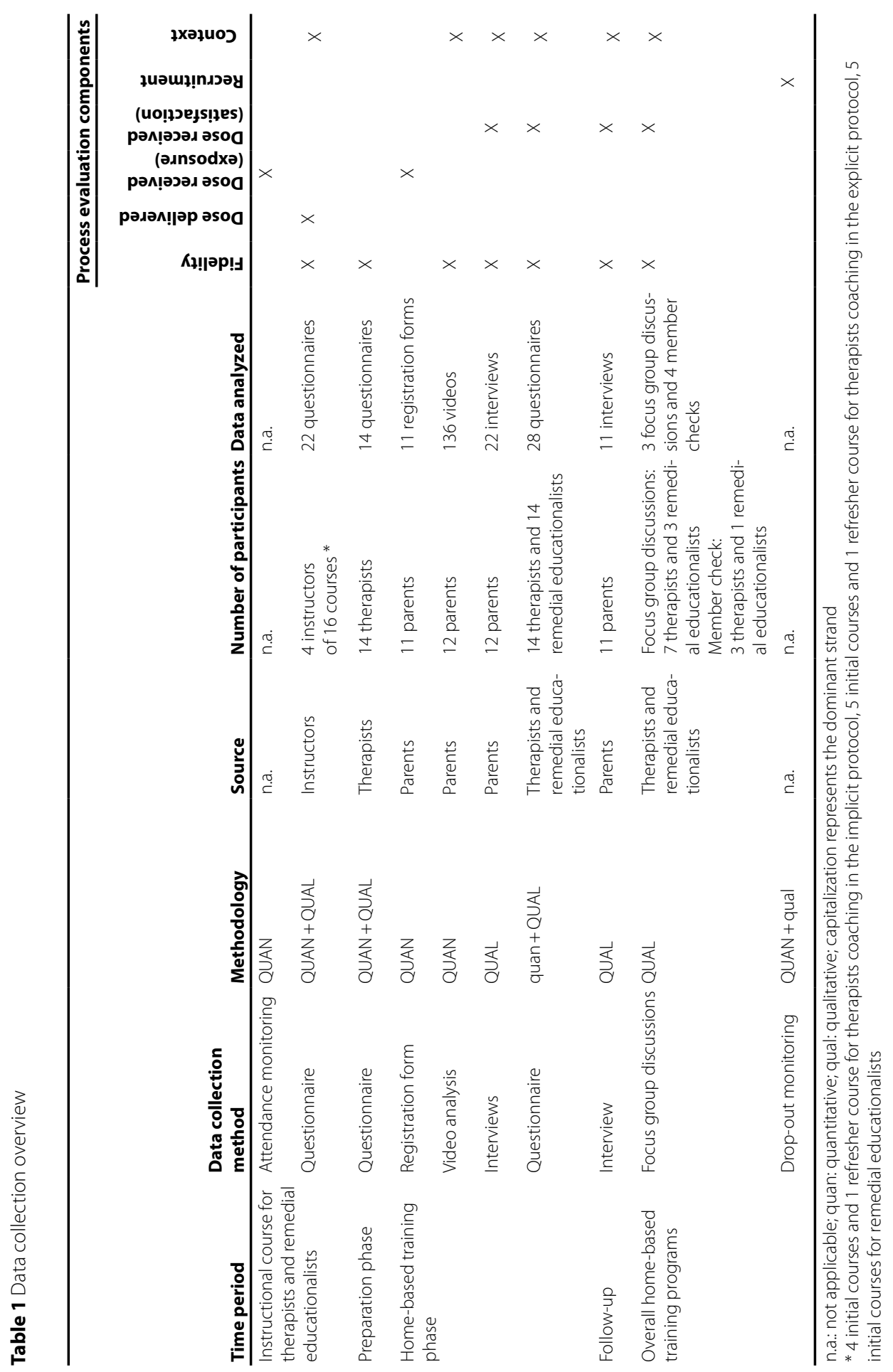




\section{DROPOUT MONITORING}

Dropout rates and reasons were registered for each home-based training program.

\section{Data ANALYSis}

The quantitative data were analyzed using descriptive statistics. Analysis of the qualitative data was carried out by thematic analysis as reported by Braun and Clarke [22,23], adopting inductive and latent (i.e. interpretative) approaches. Two researchers (LB and MM) analyzed the interview transcripts and focus group material, followed by debriefing sessions. The quantitative and qualitative data were analyzed simultaneously, followed by a side-by-side comparison of the qualitative and quantitative findings. Finally, meta-inferences were drawn.

\section{TRUSTWORTHINESS}

Several procedures were applied in order to enhance credibility. The evaluation involved between- and across-method triangulation, data sources triangulation, site triangulation, and investigator triangulation. Second, the accuracy of the data was checked throughout and at the end of interviews and focus group discussions by paraphrasing and summarizing. In addition, member checking of the preliminary report was carried out by the health professionals who were not able to attend the focus group discussions.

The first author (LB) is a PhD candidate with a background in physical therapy, whereas MM is an experienced research assistant, originally educated as a medical analyst. The researchers attempted to prevent the process evaluation's affecting the delivery of the home-based training programs, and to view the evaluation with detachment. Considering the nature of self-evaluation, an independent expert (BP) performed peer review in several stages in order to minimize researcher bias. She is a qualified occupational therapist with a doctoral degree and extensive experience in pediatric rehabilitation and qualitative research. During debriefing sessions, the researchers ( $(\mathrm{B}$ and $\mathrm{MM})$ and the peer reviewer (BP) discussed their interpretations and perceptions.

\section{RESULTS}

\section{SAMPLE CHARACTERISTICS}

Fourteen families participated in the process evaluation. Nine performed the explicit program and five the implicit program. The characteristics of the families are provided in Table 2.

Eleven therapists and seven remedial educationalists coached parents. They all participated in the process evaluation. The median time they had been working in pediatric rehabilitation was 15 years (range $5-31$ years) and 14 years (range 2-22 years) for therapists and remedial educationalists, respectively. Ten of the therapists (91\%) reported previous experience with the coaching role. 
Table 2 Participant characteristics

\begin{tabular}{|c|c|c|c|}
\hline \multicolumn{2}{|l|}{ Child characteristics $(n=14)$} & \multirow{2}{*}{$\begin{array}{c}\mathbf{n} \\
5 \\
9\end{array}$} & \multirow{2}{*}{$\begin{array}{l}\% \\
36 \\
64\end{array}$} \\
\hline Home-based program & $\begin{array}{l}\text { Implicit } \\
\text { Explicit }\end{array}$ & & \\
\hline Gender & $\begin{array}{l}\text { Male } \\
\text { Female }\end{array}$ & $\begin{array}{r}11 \\
3\end{array}$ & $\begin{array}{l}79 \\
21\end{array}$ \\
\hline & $\begin{array}{l}2 \\
3\end{array}$ & $\begin{array}{l}3 \\
3\end{array}$ & $\begin{array}{l}21 \\
21\end{array}$ \\
\hline Age (years) & $\begin{array}{l}4 \\
5 \\
6 \\
7\end{array}$ & $\begin{array}{l}5 \\
1 \\
1 \\
1\end{array}$ & $\begin{array}{r}36 \\
7 \\
7 \\
7\end{array}$ \\
\hline MACS level & $\begin{array}{l}\text { I } \\
\text { III } \\
\text { Not applicable* }\end{array}$ & $\begin{array}{l}1 \\
6 \\
1 \\
6\end{array}$ & $\begin{array}{r}7 \\
43 \\
7 \\
43\end{array}$ \\
\hline GMFCS level & $\begin{array}{l}\text { I } \\
\text { II }\end{array}$ & $\begin{array}{r}13 \\
1\end{array}$ & $\begin{array}{r}93 \\
7\end{array}$ \\
\hline Type of education & $\begin{array}{l}\text { Mainstream nursery school or day-care } \\
\text { Therapeutic toddler group } \\
\text { Mainstream primary school } \\
\text { School for physically disabled children } \\
\text { Not applicable } \\
\text { Missing value }\end{array}$ & $\begin{array}{l}4 \\
1 \\
5 \\
2 \\
1 \\
1\end{array}$ & $\begin{array}{r}29 \\
7 \\
36 \\
14 \\
7 \\
7\end{array}$ \\
\hline Siblings & $\begin{array}{l}1 \\
2 \\
\text { None } \\
\end{array}$ & $\begin{array}{l}9 \\
2 \\
3\end{array}$ & $\begin{array}{l}64 \\
14 \\
21\end{array}$ \\
\hline Caregiver characteristics of the & primary trainer $(n=14)$ & $\mathbf{n}$ & $\%$ \\
\hline Gender & $\begin{array}{l}\text { Male } \\
\text { Female }\end{array}$ & $\begin{array}{r}1 \\
13\end{array}$ & $\begin{array}{r}7 \\
93\end{array}$ \\
\hline Relationship to the child & $\begin{array}{l}\text { Natural (or adoptive) parent } \\
\text { Foster parent }\end{array}$ & $\begin{array}{r}12 \\
2\end{array}$ & $\begin{array}{l}86 \\
14\end{array}$ \\
\hline Family structure & $\begin{array}{l}\text { Traditional family } \\
\text { Reconstituted family }\end{array}$ & $\begin{array}{r}13 \\
1\end{array}$ & $\begin{array}{r}93 \\
7\end{array}$ \\
\hline Highest education completed & $\begin{array}{l}\text { Secondary vocational education } \\
\text { Senior general secondary education/Pre-university } \\
\text { education } \\
\text { Higher professional education } \\
\text { University education }\end{array}$ & $\begin{array}{l}4 \\
1 \\
7 \\
2\end{array}$ & $\begin{array}{r}29 \\
7 \\
50 \\
14\end{array}$ \\
\hline Working hours per week & $\begin{array}{l}0 \\
10-20 \\
20-30 \\
30-40\end{array}$ & $\begin{array}{l}2 \\
2 \\
7 \\
3\end{array}$ & $\begin{array}{l}14 \\
14 \\
50 \\
21\end{array}$ \\
\hline $\begin{array}{l}\text { Another family member requiring } \\
\text { special care or attention }\end{array}$ & $\begin{array}{l}\text { Yes } \\
\text { No }\end{array}$ & $\begin{array}{r}2 \\
12\end{array}$ & $\begin{array}{l}14 \\
86\end{array}$ \\
\hline
\end{tabular}




\begin{tabular}{|c|c|c|c|}
\hline \multicolumn{2}{|c|}{ Caregiver characteristics of the secondary trainer $(n=13)$} & \multirow{2}{*}{$\begin{array}{r}\mathbf{n} \\
10 \\
3\end{array}$} & \multirow{2}{*}{$\begin{array}{l}\% \\
77 \\
23\end{array}$} \\
\hline Gender & $\begin{array}{l}\text { Male } \\
\text { Female }\end{array}$ & & \\
\hline Relationship to the child & $\begin{array}{l}\text { Natural (or adoptive) parent } \\
\text { Foster parent } \\
\text { Grandparent }\end{array}$ & $\begin{array}{r}11 \\
1 \\
1\end{array}$ & $\begin{array}{r}85 \\
8 \\
8\end{array}$ \\
\hline Family structure & $\begin{array}{l}\text { Traditional family } \\
\text { Single-parent family }\end{array}$ & $\begin{array}{r}12 \\
1\end{array}$ & $\begin{array}{r}92 \\
8\end{array}$ \\
\hline Highest education completed & $\begin{array}{l}\text { Pre-vocational secondary education } \\
\text { Secondary vocational education } \\
\text { Higher professional education } \\
\text { University education }\end{array}$ & $\begin{array}{l}1 \\
6 \\
5 \\
1\end{array}$ & $\begin{array}{r}8 \\
46 \\
38 \\
8\end{array}$ \\
\hline $\begin{array}{l}\text { Number of working hours per } \\
\text { week }\end{array}$ & $\begin{array}{l}10-20 \\
20-30 \\
30-40 \\
>40\end{array}$ & $\begin{array}{l}1 \\
3 \\
6 \\
3\end{array}$ & $\begin{array}{r}8 \\
21 \\
46 \\
21\end{array}$ \\
\hline
\end{tabular}

MACS: Manual Ability Classification System; GMFCS: Gross Motor Function Classification System; * MACS is not validated for children $<4$ years of age. For younger included children, the rehabilitation team judged that manual ability was comparable to MACS Level I-III.

The results are clustered by twelve main themes that derived from the analysis: instructional course, goal setting, instructional videos, training, registration form, video recording, sharing of data, coaching by therapist, telephone appointments by therapist, home visits by therapist, remedial educationalist coaching, and impact of the program.

\section{THEME 1: INSTRUCTIONAL COURSE}

All therapists and remedial educationalists (100\%) attended the instructional course. Each therapist who had coached a family for more than one year after the initial course additionally joined a refresher session. The remedial educationalists deemed a refresher session unnecessary and did not attend it.

The total duration of the initial course for therapists ranged from 2 to 7.5 hours. Most of this time (100-280 minutes) included discussion of either the implicit or explicit content. The refresher courses lasted 70-110 minutes. The total course duration for the remedial educationalists ranged from 1 to 3.75 hours, including 60-135 minutes of instruction for the remedial educationalists' role. The number, prior knowledge, and preparation of attendants and their capability to apprehend the content caused the variation in durations. For the vast majority of the courses (94\%, 15/16) the duration and content of the total course and the different elements were considered adequate. For all courses, the mode of delivery (in-person, video call, and once by telephone) was deemed adequate $(100 \%, 16 / 16)$.

After most courses $(63 \%, 10 / 16)$, the instructors judged that the course attendants were sufficiently competent in all relevant topics. In three initial courses for therapists, the instructors predicted that the therapists would be insufficiently prepared to develop a GAS, which was part of the study procedures. In two initial courses for the implicit therapists, the instructor(s) doubted whether all course attendants had sufficient insight to work out both task analysis and treatment 
program. In the opinion of the instructors, one participant had little insight in the task analysis and program content, and seemed not to improve during the course. Lastly, after one course, the instructors judged that the professionals were not yet sufficiently skilled to use Quli.

\section{SUBTHEME 1: GROUP COURSES ENABLE DYNAMIC AND SUPPORTIVE LEARNING}

The course instructors commented that, although individual instruction was goal-oriented, fast, and enabled anticipation of prior knowledge and reasoning of the attendant, multiple attendants resulted in an interactive and lively course. The group dynamics provided opportunity for exchange and learning from each other's questions and remarks. The instructors expressed appreciation of the attitude of the health-care professionals: enthusiastic, motivated, open-minded, and actively participating by providing critical questions and suggestions, which led to substantive discussions. However, during one group course, a different level and active participation of attendants was disadvantageous. The instructors recommended that courses should be given in a group setting.

\section{SUBTHEME 2: COURSE CONTENT CAN BE OPTIMIZED}

Regarding the content, participants described three different aspects. First, for some initial courses of therapists providing the implicit training, the instructor(s) and attending therapists reported that they would have appreciated more time to discuss and practice the content of the implicit program, and elaborate several examples. Second, the instruction for Quli was acknowledged to need attention, although suggestions for improvement varied widely, from 'being a more prominent part of the course' to 'no integral part of the course anymore'. Third, some videos presenting examples of cases were not suitable, because they were not recorded in a home situation.

\section{SUbThEME 3: COURSE INSTRUCTORS' AND ATTENDANTS' EXPERIENCES WERE POSITIVE}

The intensive practice-oriented approach, including directly applying the learning to examples of cases, provided better understanding. The attending therapists also appreciated this practical part, indicating to the instructors that they were satisfied with the course and thought it worthwhile. They were enthusiastic regarding the content of the program, which they thought was interesting, well set up, and comprehensive. Therapists felt that the implicit program required a different mindset and was therefore challenging. They desired consultation with fellow therapists about the program. For the remedial educationalists, the course content was clear, and they valued the written information for later reference. 


\section{THEME 2: GOAL SETTING}

\section{SUbThEME 4: GOAL SETTING PROCESS SHOULD BE GUIDED BY THE COACHING THERAPIST}

Therapists commented that they would have preferred to administer the Canadian Occupational Performance Measure (COPM) themselves, instead of this being done by an independent therapist. They explained that the COPM assessment also would have provided input for the treatment plan, which they therefore lacked.

\section{SUbThEME 5: GOALS NEED TO BE MEANINGFUL}

Therapists questioned whether some goals reflected the child's needs, as the parents defined them. The child's interest and frustration varied per goal, sometimes against the therapist's and/ or parents' expectations, as one of the therapists explained:

"We had four goals that were really based on daily activities and one goal [...] that was about dressing and undressing a doll. And mother thought: that is very nice for her, because that is something, a play activity. And that turned out to be something that did totally not work, because she really did not want to go along. She wanted mother to do it. And it was indeed difficult, but she really did not want that, while, for example, putting on the jacket, taking off shoes, spreading a sandwich... Those were, among others, goals, those went very automatic." (therapist)

Moreover, parents described that, for the goal setting process, it was beneficial when targeted activities were part of daily routine. Those goals were the most convenient ones for families to practice. In particular, for the child practicing these recurring activities, it felt least like doing therapy. Parents also observed that children made most progress when goals were meaningful to the child. Therapists noticed that they did not have to provide any additional training materials for such goals. On the other hand, parents also experienced that it was beneficial if a goal was a play activity or if a goal provided variation of the activity to be practiced. Additionally, it was considered important for defined goals to be realistic and age-appropriate. Lastly, both parents and therapists indicated that it was important that goals be suitable to the specific home situation and season. Activities related to coats or shoes were, for example, difficult to practice in good weather and, conversely, bad weather negatively affected practice of outdoor activities.

\section{SubThEME 6: ADAPTing goals WAS BENEFicial}

Therapists reported that, for $23 \%$ (3/13) of the children, one or two goals were added to the five predefined goals, while goals were sometimes no longer pursued. Reasons for these changes included the child's success with multiple goals, the arising of new needs, or the child's frustration with an activity. Parents appreciated the opportunity to establish new goals during the program. However, some others would have preferred fewer goals to practice, because they found it challenging to divide their attention between five goals in limited training time. 


\section{THEME 3: INSTRUCTIONAL VIDEOS}

\section{SUBTHEME 7: INSTRUCTIONAL VIDEOS WERE USEFUL}

Therapists reported that the parents of some children did not watch the instructional videos, or did so only partially. Parents were positive about the instructional videos, although many had little or no recall of their opinion of them during the interviews. One parent stated:

"Those instructional films, those work very well, because it really gives a good picture of [...] what you can do yourself in the training." (parent)

\section{THEME 4: TRAINING}

The median training duration was 12.6 weeks (range 11.0-14.7). With 36\% (5/14) of the participants, an interruption, and therefore extension, of the program occurred. Most parents did not comply with the 3.5 hours (210 minutes) of training per week. As can be seen from Table 3, the mean weekly training duration ranged from 106 to 211 minutes (median 165, IQR 67,5). The median training duration amounted to $79 \%$ of the intended intensity.

Table 3 Results registration form $(n=11)$

\begin{tabular}{lcccc}
\hline Child (pseudonym) & $\begin{array}{c}\text { Minimum weekly } \\
\text { training duration } \\
\text { (minutes) }\end{array}$ & $\begin{array}{c}\text { Maximum weekly } \\
\text { training duration } \\
\text { (minutes) }\end{array}$ & $\begin{array}{c}\text { Mean weekly } \\
\text { training duration } \\
\text { (minutes) }\end{array}$ & $\begin{array}{c}\text { Total training } \\
\text { duration } \\
\text { (hours) }\end{array}$ \\
\hline Julia & 40 & 165 & 106 & 21.2 \\
Thomas & 80 & 160 & 120 & 24.1 \\
Emma & 0 & 172 & 129 & 25.8 \\
David & 0 & 260 & 134 & 26.8 \\
Ryan & 0 & 310 & 160 & 31.9 \\
Noah & 0 & 265 & 165 & 33.0 \\
Kevin & 105 & 207 & 178 & 35.6 \\
Mike & 2 & 340 & 195 & 39.0 \\
Robert & 185 & 216 & 203 & 40.5 \\
Oliver & 178 & 277 & 209 & 41.9 \\
Alexander & 160 & 255 & 211 & 42.3 \\
\hline
\end{tabular}

From analysis of available video-recorded training sessions, it was calculated that the average percentage of intervals that included task-oriented and/or result-oriented instruction or feedback was 85.2 (SD 2.9) in the implicit program, and 87.4 (SD 10.8) in the explicit program. The 
percentage of intervals that included instruction or feedback with regard to motor performance was 20.9 (SD 3.7) in the implicit program. In the explicit program, this percentage was 42.0 (SD 13.7), twice as high (Table 4). Kappa was 0.54. According to the interpretation guidelines of Landis and Koch [24], this represents a moderate agreement between the raters.

Table 4 Results video analysis $(n=12)$

\begin{tabular}{lcccccc}
\hline $\begin{array}{l}\text { Child } \\
\text { (pseud- } \\
\text { onym) }\end{array}$ & $\begin{array}{l}\text { Training } \\
\text { program }\end{array}$ & Videos & Intervals & $\begin{array}{c}\text { Scorable } \\
\text { intervals }\end{array}$ & $\begin{array}{c}\text { Intervals that } \\
\text { included task-ori- } \\
\text { ented and/or } \\
\text { result-oriented } \\
\text { instruction or } \\
\text { feedback }\end{array}$ & $\begin{array}{c}\text { ortervals that in- } \\
\text { Iluded instruction } \\
\text { or feedback with } \\
\text { regard to motor } \\
\text { performance }\end{array}$ \\
\hline Mike & Implicit & 7 & 100 & 71 & $87.3 \%$ & $18.3 \%$ \\
Samantha & Implicit & 8 & 52 & 21 & $81.0 \%$ & $19.0 \%$ \\
Noah & Implicit & 3 & 27 & 15 & $86.7 \%$ & $20.0 \%$ \\
Robert & Implicit & 13 & 75 & 57 & $86.0 \%$ & $26.3 \%$ \\
Julia & Explicit & 40 & 585 & 556 & $98.2 \%$ & $19.6 \%$ \\
Emma & Explicit & 9 & 126 & 123 & $91.9 \%$ & $29.3 \%$ \\
Kevin & Explicit & 3 & 53 & 40 & $97.5 \%$ & $37.5 \%$ \\
Ryan & Explicit & 2 & 34 & 28 & $71.4 \%$ & $39.3 \%$ \\
Oliver & Explicit & 21 & 145 & 130 & $81.5 \%$ & $45.4 \%$ \\
Thomas & Explicit & 20 & 75 & 57 & $78.9 \%$ & $47.4 \%$ \\
Alexander & Explicit & 1 & 9 & 9 & $100 \%$ & $55.6 \%$ \\
Christian & Explicit & 9 & 70 & 34 & $79.4 \%$ & $61.8 \%$ \\
\hline & & & & & & \\
\hline
\end{tabular}

\section{SUbTheme 8: HOME-BASED TRAINING IS VALUABLE}

Parents, therapists, and remedial educationalists agreed that the strength of the program was the training in the home situation. They considered it a valuable addition to the center-based therapy programs already available, as the comment below illustrates:

"I think particularly by considering how you can practice certain actions in the home situation, just brings so much peace, and without the feeling that you are practicing all of the time." (parent)

Parents regarded the overall program highly positive, because the results achieved outweighed the effort they put in. Additionally, therapists appreciated the experience of how to coach parents from a distance and how to stimulate them to train in the home situation. 


\section{SUBTHEME 9: TRAINING OF ACTIVITIES OF DAILY LIVING WERE EASILY IMPLEMENTED}

Parents implied that they successfully managed to integrate training of activities of daily living in the family routine. Talking about this topic a parent said:

"The things that we proposed are of course practical things that our son encounters in daily life, in his daily routine. So if that came along, like pulling up the pants, closing the zipper, eating from a bowl: in fact, you encounter that every day and then you can train that every day. And then it also makes sense for him to train it and [...] that way it just comes back daily and you can just train at times when it is necessary, and that it occurs. And that actually works well." (parent)

Practicing such tasks, therefore, became a habit: tasks were practiced automatically, without having to think about them. Moreover, the child did not experience these training moments as therapy. As a result, these training activities became routine, being practiced using the same techniques following the program, and often continuing to improve after the program had ended. However, after the program parents no longer created additional practice moments, as they had during the program.

\section{SUbTHEME 10: TRAINING WAS TIME-CONSUMING}

Parents stated that they perceived the programs to be very time-consuming. They felt that it was an extra focus of attention that was difficult to fit into already busy family life, including work, school, health-care-related tasks, and leisure activities. As one parent put it:

"Perhaps it is just a feeling. You have to, there is just an extra point of attention during the week. [...] I experience family life as quite busy and hectic next to a busy working life. So you also have to think: oh yeah, we still have to practice or train with him. We have to fill in the form. We have to make a video-recording. And that is also a repetitive thing. And I think that is quite time-consuming." (parent)

The treatment goals that did not involve daily recurring activities required parents to find time to offer training. On school days, there was little time left for training, and children were often tired after school. During holidays and days off, it was harder to comply with the training program as the days were less structured. Some parents chose at times to sacrifice leisure activities for the purpose of training, whereas others did not. Parents also had to choose to what extent they put their and their child's time and energy in either the home-based training of bimanual activities or in other developmental aspects that required attention, such as gross motor activities or cognitive learning skills.

In most families, both parents naturally alternated supervision of training. For parents, this was convenient when they were not in the mood or had lost their patience. The child also benefited from being approached in different ways. Siblings were generally not involved in nor affected by the training. However, for parents it was sometimes challenging to divide their attention between their children because of the needs of the training. The home-based training enabled parents to flexibly arrange their schedule themselves, though, and did not require leave from school or travelling to the rehabilitation center. However, distractions in the home situation could be a drawback. One parent explained: 
"At the moment that you just plan certain things, like: well, we are going to do that and that and that, and the doorbell rings and there is a friend at the door to ask if she comes to play outside, then it is made very difficult for her to say: no, I have to do therapy now and I cannot play outside. Well, then you very easily say: then we do it [practice] another time." (parent)

As a result, families did not always manage to comply with the training intensity. For some parents, being occupied with the training and seeing progression was more relevant than the practice time, while others were eager to adhere to the prescribed training hours. A therapist wrote:

"I had to put the rules of the research in perspective as the parents felt a lot of pressure. If they continue to give so much weight to this, they will not hold on and it will be very burdensome. I spent time on putting these demands into perspective. They should benefit from it and it must be feasible within the situation." (therapist)

Additionally, for some young children it was not always doable to practice for at least ten consecutive minutes, as prescribed in the protocol.

Prior to the start, parents underestimated how time-intensive the program would be. They were relieved when the program was finished, as the need to exercise stopped.

Therapists mentioned problems with behavior, concentration, and motivation as restricting factors while, for other children, willingness to learn, motivation, and perseverance positively attributed to training success. Parents found that dedication, willpower, perseverance, and patience as parental personality traits were beneficial to the training. Perfectionism was helpful in achieving the required training time, but was also stress-inducing.

Health-care professionals reported that children and parents worked hard. They noticed that the home situation had to be strong and the child had to feel well for the program to be a success. The timing of the program was mentioned as another important factor. Implementation of the program when parents and/or the child were already experiencing strain, for instance because of work or school, was difficult.

Therapists also observed that, when goals were achieved or a ceiling level reached, parents started searching for how to continue the practice. In this 'generalization phase,' they applied the learned skill in a variety of situations, rather than practicing the activity in the original situation for which the training was initially provided.

\section{SubThEME 11: THE TRAINING SHOULD BE APPEALING TO THE CHILD}

Parents stressed that it was beneficial to make training appealing and stimulating to the child. For example, one parent explained:

"[...] that it [training] will not become a punishment, but that it just remains fun. And that it is not an obligation, but that it remains fun. Because I think that that is the most important thing, that it is important that you train, but I think the most important thing is that the fun in the training, for us as a parent and for the child, must remain the highest priority. Like: gosh, that is fun, we are going to do this now and not: oh gosh, we need to again." (parent) 
Strategies that parents used to accomplish this varied with the child. They generally involved not emphasizing activities as being therapy nor imposing practice, but rather practicing when the child showed that it felt like performing the activity, and using a playful, enthusiastic, and varied approach. This required a certain amount of creativity of parents in the provision of training, which they became better at during the program. In addition, parents were challenged in selecting suitable training materials and offering subsequent steps of increasing difficulty. A parent suggested creating a database of exercises, games, and ideas for parents in order to offer variations of certain bimanual activities.

\section{SUBTHEME 12: PARENTS WERE WELL ABLE TO PROVIDE TRAINING TO THEIR CHILD}

Parents said that they, as well as their child, were not aware of the parents' role as 'therapist'. As one parent said:

"For him, it is actually just playing with his daddy or with his mummy. And that we have a purpose in that, he is not aware of." (parent)

Parents did not experience separate roles, but explained that providing the therapy naturally mixed with their usual parenting role. Additionally, parents liked to remain in the role of parent to their child and did not want to impose training. Sometimes parents struggled because their children were more resistant in their trusted home situation than they were with therapists. For instance, one parent told:

"I also notice, he does things better with, so to speak, with someone else than he does with us. Because then he will sooner get recalcitrant: "I do not feel like it", and he will act silly. If (therapist) would be here, he would, for example, do it. But that is someone else, with a different position." (parent)

\section{SUBTHEME 13: THE INVOLVEMENT OF THE EXTENDED FAMILY OR SOCIAL ENVIRONMENT WAS LIMITED}

Parents said that the involvement of grandparents in the training varied. In some families, grandparents, particularly those who provided structural child care, were actively involved in the training. The degree to which they were instructed by parents and how much time and effort this required also varied. Other parents chose only to practice with their child themselves, to keep control. Parents often informed other persons involved in the child's life, like teachers, about the family's participation in the home-based training program, the goals of the training, and sometimes more detailed instructions, although they had no or a limited role in the training. Parents thought that training activities performed by grandparents or at (pre)school not within their sight was disadvantageous, because others practiced in a way that the parents were not satisfied with.

Therapists suggested identifying who has an important role in the daily life of the child, and to involve these people in the coaching from the beginning. Involving, for instance, a grandparent, primary care physiotherapist, or day-carer would lead to coaching in the whole life of the child, which would be expected to benefit generalization after the program has ended. It was recognized that this would require a bigger investment for parents and therapists. 


\section{SubThEME 14: PROgRAM DURATION TOO INTENSIVE}

Parents as well as therapists felt that 12 weeks working on the same goals was too long for the parents and the child to continue being motivated. They suggested reducing the duration to eight weeks. They also suggested approaches combining home-based and center-based training. A suggested example of such a hybrid model was that the child would be seen at the center once every three weeks, and the parents would provide training at home for the other two weeks. They also argued that it would be too intensive to perform home-based training continuously during the child's rehabilitation trajectory.

\section{SUBTHEME 15: PROTOCOL DEVIATIONS OCCURRED WITH TASK-SPECIFICITY}

The answers of parents and therapists revealed that for some goals they adopted a looser interpretation. Although the activities practiced were in some way related to the targeted goals, not all training was task-specific. This included, for instance, a range of activities that required the same actions, for instance in the way of holding an object as in the originally targeted activity (i.e. pudding cup, sandbox toys, or Yahtzee). Another example was given by a parent:

"At one point I had that video where she was braiding her hair. And that was just a very cute video, so I forwarded it to the therapist, like: yes, it does not match the goals, but I think it is such a nice movie. And then she said: [...] of course it belongs to your goals, because that is again, on the basis of tying shoelaces, that is a particular act." (parent)

These varying activities were chosen in order for parents to attain the practice time and were considered as training by parents as well as therapists.

\section{SUBTHEME 16: MOTOR LEARNING STRATEGIES SHOULD EVOLVE AROUND THE CHILD'S AND PARENTS' NEEDS}

Parents and therapists stated that they did not always comply with the implicit program, since instructions included motor performance elements. Similarly, although the focus was on explicit motor learning, implicit elements were also integrated to some extent in the explicit program.

Parents for which the implicit or explicit training approach corresponded with their natural teaching style and their child's natural learning style were doing well. Parents who were used to the opposite teaching style struggled and needed quite some time and coaching to get accustomed with the new approach. It could also happen that the child was not comfortable with the implicit approach, as a therapist explained:

"By doing, mother actually said: oh, I find out that he actually has much more need for explicit explanation and instruction. Because the child then literally said: but mama, say what I have to do! So how do I hold that scissors now?" (therapist)

Therapists suggested not differentiating anymore between the implicit and explicit approaches. Rather, aspects of implicit and explicit motor learning should be used in accordance with the child (what does he or she respond to), parents (what types are they), and the phase of the therapy. 


\section{THEME 5: REGISTRATION FORM}

Two parents (14\%) did not complete the digital registration form. Additionally, one parent (7\%) provided a hand-written narrative description of the training and one (7\%) filled out a different but comparable logbook on paper.

\section{SUBTHEME 17: COMPLETING THE REGISTRATION FORM WAS BURDENSOME}

Parents complained that completing the registration form every week was a burden for them for several reasons. Filling in and sending it to the therapist was time-consuming. In addition, it was troublesome having to keep it in mind at all times and to retrospectively quantify the time practiced, especially for goals that were incorporated into the daily routine. Some parents felt that the format of the registration form was not very useful, had trouble completing it on their device, or preferred keeping track on paper. The therapists observed differences between parents: some actively filled in the form, whereas it was not part of the routine of others. Likewise, the form provided some parents with something to hold on to, but others did not attach importance to the time practiced and experienced it as a lot of paperwork. For example, one parent noted:

"Well, the only thing that I liked less [...] filling out that schedule. If I did not have to do that, just only practice, and that then an occupational therapist visited our home, discussing things, etcetera, I think that is absolutely fine. But indeed the keeping track of lists and things, and that documentation, that is something that, yes, that I do not like." The parent continued: "those registration forms add nothing for us, has no added value. It only generates unnecessary irritation" (parent)

From the therapists' view, this led to parents writing down just anything, which was not relevant. Therapists did not put much emphasis on the use of this registration form because this would have been counter-productive.

\section{SUBTHEME 18: THE REGISTRATION FORM HAD LIMITED ADDED VALUE}

Therapists recognized an added value of the form in that it made parents aware of and motivated to practice. They considered parents applying therapy regularly as more important than the specific training duration. Some parents generalized the training time (i.e. not only time-on-task, but time spent within a broader activity), which prevented parental stress. The registration form provided the therapists with insight into the days and total time practiced, to be used as a subject of discussion with questions like 'why did you not succeed?', what can we do differently?' or 'what will help you?'. However, for the therapists, the registration form had lower priority than the video recordings. One therapist did not use the form, as it felt as if she was checking up on parents. The remedial educationalists did not utilize the registration form. 


\section{THEME 6: VIDEO RECORDING}

\section{SUBTHEME 19: VIDEO RECORDINGS WERE VALUABLE}

The therapists stated that the videos were valuable for coaching parents. They could retrieve a lot of valuable information from the videos: how parents instructed the child, the interaction between child and parents, how well the training could be applied by parent and child, ambient factors (e.g. the presence of siblings or disruptive radio sounds), the impact of the training, and achievements of the training. This enabled therapists to follow the process and, in subsequent coaching, they could respond to what had happened at home: knowing the conditions involved made it easier for them to think things through. It enabled them to give feedback to parents, and to provide more focused coaching and more specific advice. It also helped them to take parents along in the process, which helped parents to understand how goals were composed and how activities and training were related to the goals. Therapists emphasized the importance of filming in particular those activities that were difficult or that parents had questions about, since there the therapist could really make a difference. If videos were of poor quality or rotated, coaching based on the video recordings was felt to be difficult.

Parents were also positive about the video recordings, although it appeared challenging to provide the training while filming. They benefited from the tips they received from their coaching therapist in response to the videos. For some children, the filming was an extra motivation to show to the therapist what they learned, whereas others did not like to be filmed.

For the remedial educationalists, it was enriching to observe behavior in the videos. They appreciated being able to view the footage themselves, from a different viewpoint than the therapist. They referred to the video recordings in conversations with parents and the therapist.

\section{THEME 7: SHARING OF DATA}

Only 29\% of parents (4/14) and therapists (4/14) used the data sharing system provided to send videos and treatment information, respectively.

\section{SUBTHEME 20: THE DATA SHARING SYSTEM DID NOT WORK}

Many parents and therapists reported that the data sharing tool Quli did not function as expected. Problems experienced included logging in as well as making, uploading, sending, and watching videos. Quli was also described as non-user-friendly, because parents needed a laptop either to film or upload videos. It would have been more convenient for parents to both film and send the video recordings with their smartphone with the push of a button. The problems encountered cost parents and therapists much time and caused a lot of frustration and irritation. Many parents found a more satisfying way of sending video recordings, mostly using WeTransfer or WhatsApp instead. Other parents decided, in consultation with their coaching therapist, not to share video recordings at all. The registration form was mostly sent by e-mail. 
In the opinion of participants who used arQive, this was a pleasant system. They perceived only occasional problems with logging in, uploading long videos, and viewing videos. However, the registration form could not be transferred via arQive.

\section{THEME 8: COACHING BY THERAPIST}

\section{SUBTHEME 21: THERAPISTS HAD CONFIDENCE IN PARENTS' CAPABILITIES}

Therapists had confidence in parents' capability of delivering the training prior to the program. Familiarity with home-based training, motivation, enthusiasm, and knowing how to stimulate their child were seen as beneficial to this. Therapists were concerned about parents being busy or not having completed the preparations, and one expected that a lot of support and positive reinforcement of the parents would be needed to carry out the training. One parent-couple specifically told the therapist that they needed very concrete written information about what and how they had to do the training. Their therapist expected that they would become more independent over time.

\section{SUBTHEME 22: TASK-ANALYSIS WAS VALUABLE}

Therapists reasoned that the task-analysis helped them in defining the training plan and in coaching parents, particularly at the beginning of the trajectory. Normally, they only did an analysis mentally and gave treatment according to what they felt was right to do. The structured task-analysis made them consciously think about how to work towards a goal within a certain number of weeks. They shared and/or discussed the task-analysis with parents. Using the task-analysis, therapists took parents through the process and the therapists' way of thinking: what steps to take in the development of the child's learning of a task? What makes a task easier or more difficult? How to carry out the training? What are points of attention for each goal? Both therapists and parents thought that this information was supportive for parents and enabled them to teach their child other activities themselves.

\section{SUBTHEME 23: COACHING WAS CHALLENGING FOR THERAPISTS}

Therapists revealed that they sometimes experienced the coaching as challenging. They used different ways of coaching: for a parent who needed a lot of positive reinforcement, the therapist used solution-oriented coaching by asking questions (e.g. what do you see?). A second example is the main focus on practicing being fun for the child, which gives the child energy. A third therapist opted for video recordings of herself where she personally addressed the child and gave a message to the parents. The video messages included instructions, for which the therapist imitated the hand function of the child. It was beneficial if the therapist and the family already knew each other, since they would already have built rapport and would need less time to reach 
agreement. Parents thinking along and bringing ideas in was also regarded beneficial, which mostly gradually developed during the program. In a few cases, the therapist also (indirectly) instructed the primary care physiotherapist or day-carer.

\section{SUBTHEME 24: COACHING BY THE THERAPIST WAS VALUABLE}

Parents stated that they highly appreciated the coaching by the therapist. They thought that therapists were enthusiastic, positive, committed, and available when they needed them. In addition to the telephone appointments and home visits, parents and therapists were also in touch with each other by e-mail and WhatsApp. Parents received tools, tips, and advice that were very helpful to them. This included suggestions about dividing a task into sub-tasks (e.g. to start practicing closing the zipper, instead of hooking it in), motor performance (e.g. to hold the cutlery differently), organization of activities (e.g. practicing zipper use while the jacket lies on the table in front of the child), materials to be used (e.g. using a large zipper instead of a small one), innovative variation in offering activities (e.g. creating a contest), and dealing with child behavior (e.g. introducing a sticker reward chart). Parents also made purchases on advice of the therapist, for instance a more suitable chair or left-handed scissors.

Although one parent would have preferred a more critical approach, the positive feedback and confirmation by the therapist that they were doing well generally reinforced parents and children. The following comment illustrates this:

"As a parent, you just do something. I mean, I did not obtain a diploma for it. You have an idea about how to handle your child well, but if someone else sees that on a video and then gives you feedback and that is generally positive, yes, it is nice to hear from an outsider that you are doing quite well. That gives you confidence as a parent." (parent)

In one case, the child was sensitive to the feedback provided by the therapist:

"At a certain point, he [the child] said: oh, because she thinks I am not doing it right anyway. So I emphasized right away, like: yes, she actually thinks it is very good. So then I thought like: oh yeah, apparently he realizes it somehow, that he gets the feeling that he is judged or that he indeed gets the feeling he is not doing it right. And that has actually been one time and after that he did not say it again. And I am also aware of myself, the way I tell those tips and tricks to him, that I should bring that somewhat different. So also because of that he may not have said it again." (parent)

\section{SUBTHEME 25: PROGRAMS WERE COMPLEX}

Therapists mentioned that the planning and preparations, such as the task-analysis, as well as the coaching (watching video-recordings, adjusting the treatment plan, telephone appointments, and home visits) were time-consuming for them. The peak load at the beginning required flexibility and investment of their own time. They also indicated that the program was complex and it took a while for them to get accustomed to it, although this was a prerequisite for coaching parents. The manual was clear and useful as a reference. For implementation purposes, they suggested defining core elements of the program that could be adapted to the requirements of each individual process. 


\section{THEME 9: TELEPHONE APPOINTMENTS BY THERAPIST}

Telephone appointments with the therapist took place every week according to protocol in 57\% (8/14) of the participants. Therapists commented that telephone appointments were sometimes cancelled because it was not a good time for parents or they had forgotten, or for holidays or illness.

\section{SubTheme 26: TELEPHONE APPOINTMENTS WERE VALUABLE}

Therapists thought the telephone appointments were important for various reasons: to have regular contact with parents, to check how things are going, to be on the same page with parents, to indicate points for attention after seeing the videos, to evaluate last week's agreements and difficulties parents encountered, and to confirm that parents were on the right track. In a few cases, therapists called both parents alternately, to enable them both to receive feedback and ask questions. Although this was sometimes difficult to plan, it was beneficial to speak to both parents. Sometimes therapists e-mailed instead of telephone appointments, or wrote down and e-mailed the advice and agreements for goals following the phone call. This worked particularly well for parents who had trouble recalling information discussed verbally.

\section{SUBTHEME 27: NUMBER OF TELEPHONE APPOINTMENTS CAN BE REDUCED AND CUSTOMIZED}

Some therapists indicated that the duration of the phone calls fluctuated throughout the program from five to thirty minutes, depending on the topics to be discussed and questions of parents. Others observed that the frequency and duration declined as the program went on. Parents considered the planned frequency and duration of telephone appointments too much at the end of the program. A decreasing and customized coaching intensity as the program developed was suggested.

\section{THEME 10: HOME VISITS BY THERAPIST}

All three home visits with the therapist took place according to protocol in 43\% (6/14) of participants. However, therapists might visit families at home at a later time in the program than scheduled (Table 5).

During the first home visit, most time was spent on discussing the teaching approach, the program overview, and the plan for the first training week. Except for the registration of the home-based training, therapists had the impression that parents sufficiently understood the topics discussed (Table 6). 
Table 5 Quantitative results questionnaire evaluation individual programs ( $n=14)$

\begin{tabular}{|c|c|c|}
\hline \multicolumn{2}{|c|}{ Topic therapist questionnaire } & \multirow[t]{2}{*}{ Result } \\
\hline \multicolumn{2}{|c|}{ Home visits } & \\
\hline 1. & Participants for which all three home visits took place & $43 \%(6 / 14)$ \\
\hline 2. & Period between introduction meeting and first home visit (weeks) & Median 2.0 (range 1.0-3.0) \\
\hline 3. & Period between start of the training and second home visit (weeks) & Median 5.0 (range 4.0-8.0) \\
\hline 4. & Period between start of the training and third home visit (weeks) & Median 11.0 (range 10.0-12.0) \\
\hline 5. & Participants for which an extra home visit took place & $0 \%(0 / 14)$ \\
\hline
\end{tabular}

Note: Some participants returned incomplete questionnaires, resulting in missing values.

\section{SUBTHEME 28: HOME VISITS WERE VALUABLE}

Therapists defined that the first home visit was very valuable, especially if they did not know the child yet. It was particularly beneficial to see the home and family situation: the house and the environment where the family lived, the conditions for the training (e.g. the child's seating position at the table), and the attributes and toys that were available and that might be used during training. For example, one therapist wrote:

"The home visit was very enlightening to get an overview of everything that is going on in this specific family." (therapist)

Additional aspects that were discussed during the first home visit included the personal goals, the approach for training the targeted activities and points of attention, training moments that would fit into the daily routine, care of siblings, and questions of parents. In one case, the therapist observed one of the targeted activities at the playground, as this had not been possible in the rehabilitation center before. Sometimes, the second home visit was also useful, for instance to discuss topics in more detail, to adjust the program and give advice on how to approach the training, or because of goals that were changed. The third home visit had little added value for therapists. In addition, the travel time for therapists to visit families was significant.

As with the therapists, parents indicated that the home visits had added value to them in showing performance of activities in person. In one case, the parent explained the effect of modeling by the therapist:

"If she writes it down, she puts it exactly the same. But in my case that just works, I remember it better, it does work better if I just see it at such a moment and someone doing it, than that I have it written down on paper." (parent) 
Table 6 Quantitative results report form first home visit $(n=14)$

\begin{tabular}{|c|c|c|}
\hline Question & $\begin{array}{l}\text { Minutes the topic was } \\
\text { discussed } \\
\text { (median (range)) }\end{array}$ & $\begin{array}{l}\text { Number of parents who } \\
\text { sufficiently understood } \\
\text { the topic discussed }\end{array}$ \\
\hline \multicolumn{3}{|l|}{ Instructional videos } \\
\hline 1. Questions of parents & $3.5(0-10)$ & n.a. \\
\hline 2. Teaching approach & $10.0(0-75)$ & $100 \%(12 / 12)$ \\
\hline \multicolumn{3}{|l|}{ Home-based training program } \\
\hline 3. Overview of entire 12-week program & $10.0(0-45)$ & $100 \%(11 / 11)$ \\
\hline 4. Coaching approach & $7.5(1-10)$ & $100 \%(11 / 11)$ \\
\hline 5. Plan and teaching approach for the first training week & $15.0(2-60)$ & $100 \%(10 / 10)$ \\
\hline 6. Registration & $5.0(1-10)$ & $80 \%(8 / 10)$ \\
\hline 7. Video recordings & $5.0(0-10)$ & $100 \%(10 / 10)$ \\
\hline \multicolumn{3}{|l|}{ Setting of home-based training } \\
\hline 8. Available training objects & $5.0(3-10)$ & \\
\hline 9. Training objects brought by the therapist & $5.0(0-15)$ & \\
\hline 10. Location of training & $5.0(0-10)$ & \\
\hline \multicolumn{3}{|l|}{ Data sharing system } \\
\hline 11. Setting up data sharing system & $5.0(0-10)$ & \\
\hline \multicolumn{3}{|l|}{ Registration form } \\
\hline 12. Providing the digital registration form to parents & $1.0(0-10)$ & \\
\hline
\end{tabular}

Note: Some participants returned incomplete questionnaires, resulting in missing values. n.a.: not applicable

The home visits helped the therapist to think through and provide suggestions relevant to the specific home situation. For a child whose goals involved largely school-related activities, the parent appreciated one home visit's taking place at school.

\section{SUBTHEME 29: NUMBER OF HOME VISITS NEED TO BE REDUCED OR CUSTOMIZED}

Many therapists remarked that home visits did not take place because of parents being busy, their own planning, travel time, illness, or because it was combined with an appointment in the rehabilitation center. Moreover, therapists and parents often agreed that the third home visit did not have an additional benefit over contact by telephone. In general, therapists and parents indicated that three home visits in the training period was a lot given the pressure on parents as well as therapists visiting families in the evening. They suggested that the number of home visits could be either reduced or more customized. 


\section{THEME 11: REMEDIAL EDUCATIONALIST COACHING}

The telephone appointment with the remedial educationalist took place with $77 \%$ of parents. Some remedial educationists said they were unable to reach the parents by telephone. For 29\% of the children, the therapist consulted the remedial educationalist at the start of the program for a specific question. Throughout the program, with $23 \%$ of the participants the therapist consulted with the remedial educationalist (Table 7).

Table 7 Quantitative results questionnaire evaluation individual programs ( $n=14$ )

\begin{tabular}{|c|c|c|}
\hline \multicolumn{2}{|c|}{ Topic remedial educationalist questionnaire } & \multirow{2}{*}{$\begin{array}{c}\text { Result } \\
77 \%(10 / 13)\end{array}$} \\
\hline 1. & Participants for which the telephone appointment took place & \\
\hline 2. & Period between start of the training and telephone appointment (weeks) & Median 2.6 (range 1.3-4.0) \\
\hline 3. & Participants for which additional appointments took place & $0 \%(0 / 14)$ \\
\hline 4. & Participants for which the therapist consulted the remedial educationalist & $23 \%(3 / 13)$ \\
\hline
\end{tabular}

Note: Some participants returned incomplete questionnaires, resulting in missing values.

\section{SUbTHEME 30: DEGREE OF INVOLVEMENT AND ADDED VALUE OF REMEDIAL EDUCATIONALIST DIFFERED}

The remedial educationalists explained that, for most children, their role was limited to appointments according to the protocol. In these situations, where the relationship between parents and therapist was good and parents had no problems or questions, a more active role of the remedial educationalist was not needed. Hence, the remedial educationalist and therapist had no contact or only occasional contact during informal meetings at the rehabilitation center: to verify that the program was going well; or to discuss concerns of the therapist regarding emotion regulation, behavior, attention, or motivation of the child, or the parents' capability to provide the training. The health-care professionals agreed that the therapist would inform the remedial educationalist if difficulties arose in the course of the program. Remedial educationalists were confident that therapists knew when to approach them and therefore made the therapist more responsible for keeping an eye on the situation than the parents. In a few cases, the remedial educationalist was more involved because of behavioral difficulties. This involved in-depth discussions of the video recordings between remedial educationalist and therapist, whereafter the therapist communicated the advice of the remedial educationalist to the parents. In another family, the remedial educationalist accompanied the therapist during two home visits, and supported the parents by answering their questions.

Because the remedial educationalist looks at the child and family from a different perspective than the therapist, they felt it was beneficial to follow families. For the families in which the remedial educationalist had no active role, the remedial educationalists felt they could not make a difference. However, the remedial educationalists acknowledged that, should parents experi- 
ence high stress levels, need guidance, or have questions about their child's behavior, a remedial educationalist could be of great value. The therapists appreciated the remedial educationalists' involvement in more complicated cases. Overall, the remedial educationalists felt they had less impact than therapists who were in contact with parents week in and week out. They felt that they were a superfluous part of the program.

In agreement with the before-mentioned, most parents said that coaching by the remedial educationalist was not necessary for them and they saw no advantage in their individual situation. However, they did not mind that it was part of the training program and they appreciated the possibility of consulting the remedial educationalist. They also thought that fellow parents might have a need for it:

"I am glad that it is included, because I can imagine that others may have a great need for that, and I think it is also an essential part of it. But for us it does not apply." (parent)

The remedial educationalists suggested an alternative approach: to arrange a group meeting with a remedial educationalist when multiple parents are performing a home-based program. They expected that parents might benefit from each other's experiences and the exchange of tips and tricks, perhaps even more than from the advice of the remedial educationalist. At the same time, they doubted whether this format would work in practice, since inviting parents to a center might impose an additional burden on them.

\section{SUBTHEME 31: TELEPHONE APPOINTMENTS HAVE LIMITATIONS}

The remedial educationalists reflected that the telephone appointment did not bring the opening to parents they would have liked, since it is distant. A second conversation in person would have been better to recognize the needs of parents and to provide guidance to them. However, they also recognized that opportunities for an appointment in the rehabilitation center are restricted, because the program is executed at a distance.

\section{THEME 12: IMPACT OF THE PROGRAM}

\section{SUbTHEME 32: THE PROGRAM WAS DEMANDING FOR THE CHILD}

Parents described that part of the time their child liked to perform the training activities and enjoyed the attention of the parent that went along with it. At other times, the child got frustrated or angry and showed resistant behavior. This occurred mainly if the child felt that it had to practice, if the activity was difficult for the child, and/or if the child was tired or moody. Sometimes parents related this to the child's toddler age:

"That is also a bit, his age is of course also, if I want it too much then he thinks himself: uh-uh. That is a bit the disadvantage of two and a half: no is no. And he also knows that he can say no" (parent)

Some parents felt that the training did not impose stress on their child, while others thought that the training was demanding. This expressed itself in tiredness, in a few cases negatively affecting school results or generating the need of an afternoon nap. 


\section{SUbTHEME 33: BIMANUAL PERFORMANCE IMPROVED}

Parents indicated that their child's performance of the activities targeted by the treatment goals improved substantially. Some children accomplished certain tasks even subconsciously, without focused attention on the performance. The performance of some activities improved more than others. Parents attributed this to the extent to which an activity was trained. The performance of activities that were integrated in daily life improved most. The parents of most children also reported an increase of spontaneous use of the affected hand in bimanual activities that were not related to the goals set, and improved performance of these activities: as one parent put it:

"So there are more and more activities that he first said of: I cannot, you must do it. He now wants to do that himself. So he also becomes more independent in that two-handed activity. He just uses the right [hand] very well. And that is just great to see." (parent)

Another parent spoke about the affected hand:

"He simply has it in the picture again. And he just uses it again, he tries to use it more. Simply during play with Duplo ${ }^{\circledR}$, that kind of things. He remembers it easier again. And he still does not use it at all times where he could use it, but yes, you know that probably is not going to happen. But you see that very clearly, that it is indeed just more in the picture." (parent)

In addition to the parents and therapists, other persons who were involved in the child's life, like teachers, complimented the child's improvements. On the personal level, parents observed that their child was proud if it succeeded in completing a task, that the child's self-confidence grew, that it got more eager to perform activities by itself, and that independence increased.

After the program ended, progression in most treatment goals continued. However, when children did not keep practicing activities or did not integrate these in daily life, parents noted that the performance declined.

\section{SUbTHEME 34: THE PROGRAM BOTH POSITIVELY AND NEGATIVELY AFFECTED PARENTS}

Parents declared that the home-based training program was a stimulus for them to consciously put effort into practicing with their child. It enhanced their awareness that as parents they were able to get good results when practicing at home. Moreover, it made them understand better how to teach their child, as they learned a certain way of thinking with regard to skill acquisition. Sometimes parents also got a better insight into the personality of their child. This made that parents felt more competent and confident at training with their child. The progress of the child motivated and stimulated parents to keep going. The results gave them positive feelings of satisfaction and pride, which meant that they enjoyed performing the training. Parents also mentioned that they appreciated that the program gave them a more active role, more control, and more responsibility in guiding their child and the rehabilitation process. As one parent said:

"I am actually very positive in the sense that I find it very nice that as parents we have literally something to do. Because you, it sometimes feels so helpless that you cannot really help him, and this is really just very concrete." (parent)

Additionally, a therapist observed that parents were more able to put their expectations of their child's abilities into perspective. 
One parent felt very responsible for teaching the child but felt that she lacked knowledge in occupational therapy. Moreover, she missed the therapists at the rehabilitation center keeping an eye on the child and its general development. This made the home-based training feel like a lonely process for her.

For some parents, the home-based training was not stressful at all, while others felt varying degrees of stress. Parents generally felt that the term 'stress' was too strong to describe their feelings: they used words like 'frustration,' 'irritation,' and 'unrest.' The prescribed intensity of the training was the most stress-inducing factor. This included making time for the training and the urge to meet the required training hours. Parents experienced most stress at the beginning of the program, when they were still figuring out how to implement the training.

\section{DROPOUT MONITORING}

Overall, four out of 18 participants (22\%) withdrew from the study. When the RCT was terminated early and continued as a case series, the drop-out rate declined from 29\% (2/7) in the RCT to $18 \%(2 / 11)$ in the case series. All four families discontinued participation because of personal circumstances (e.g. house renovation) that interfered with the home-based training. In two cases, the time demand of the training combined with the home situation appeared to be a limiting factor. In one of these families, an additional reason was that it was a struggle to make the weekly video recordings. In the two other cases, no reasons were mentioned that were related to the intervention.

\section{META-INFERENCE}

Table 8 summarizes the qualitative and quantitative findings for each process evaluation component. The findings of the different components support each other. 


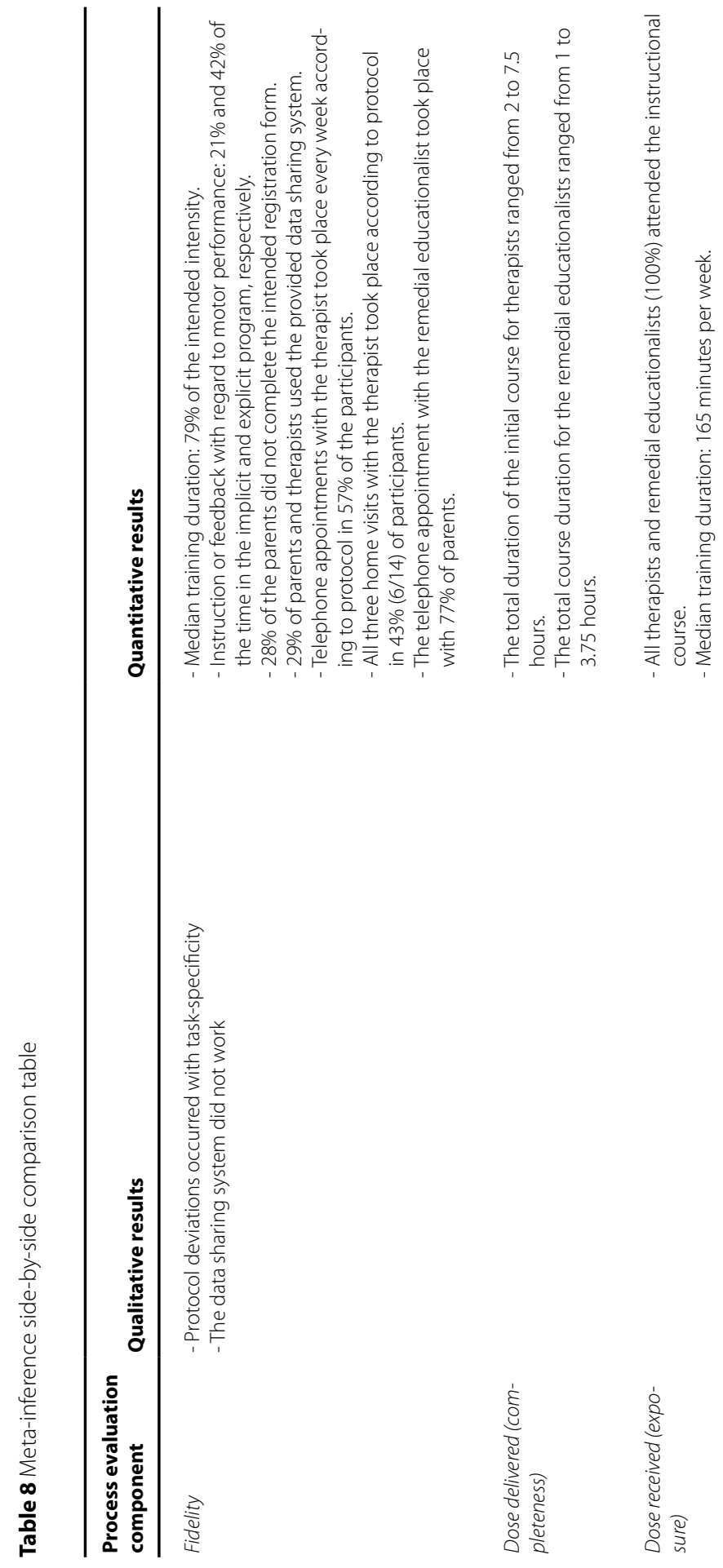




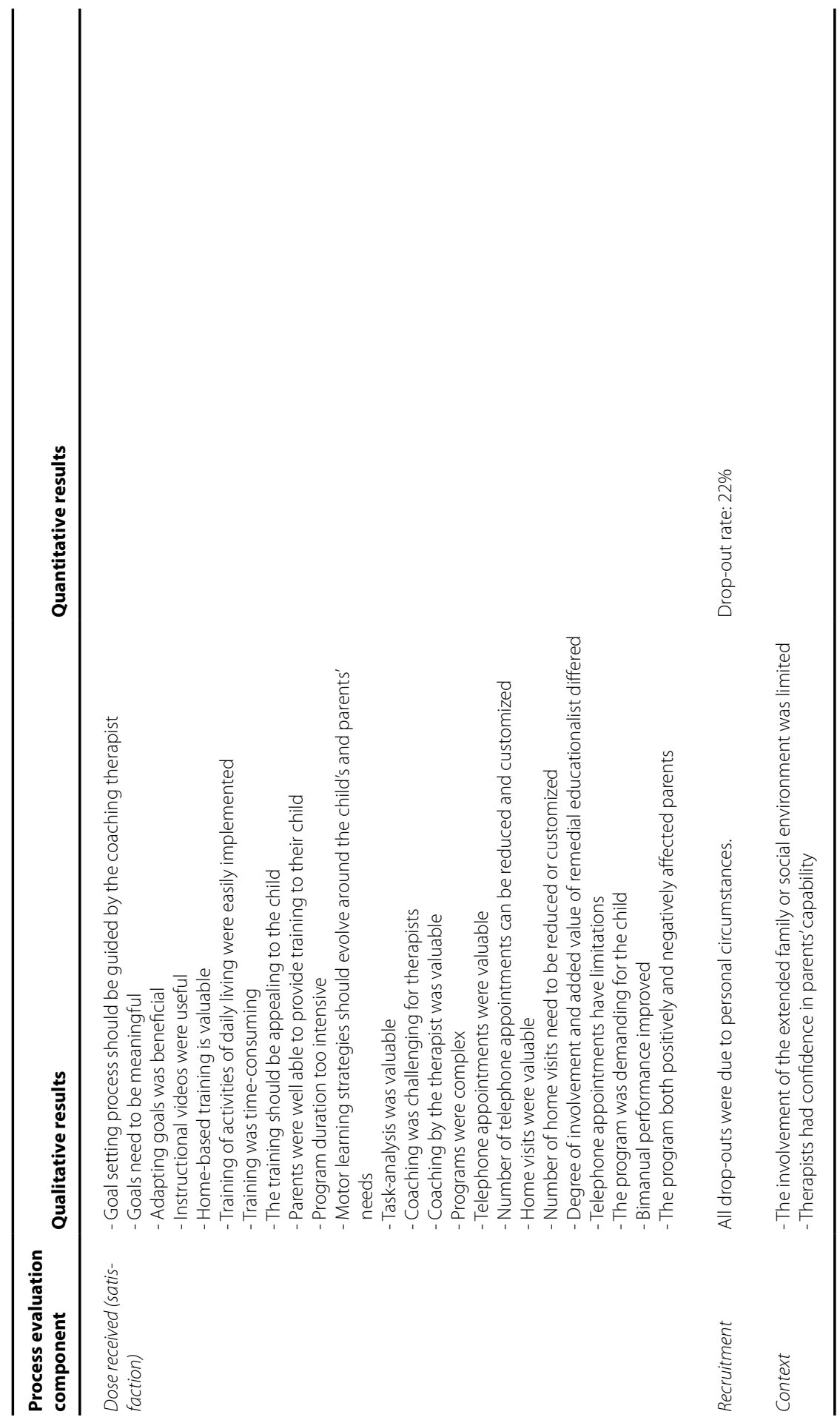




\section{Discussion}

This study aimed to evaluate the processes and factors that influenced implementation and effects of an implicit and explicit home-based training program for children with uCP. We addressed (1) the extent to which the programs were implemented as intended, (2) parental experiences and the integration of the programs in their family life, (3) contributing program components, and (4) contextual factors.

The programs were not fully implemented as intended. The results show a good compliance of $79 \%$ of the intended training intensity, consistent with the average compliance (62\% to $96.1 \%$ ) of other home-based training programs in children with CP [8]. However, only about a third of parents and therapists used the provided data sharing system, likely due to the shortcomings of the system for the specific intervention purposes. Telephone appointments with the therapist took place each week in 57\%, and all three home visits in $43 \%$ of participants. These moderate rates can be explained by the qualitative comments of participants that the scheduled frequency of appointments was too high at the end of the program and therefore not adhered to. Instruction or feedback with regard to motor performance was observed $20.9 \%$ and $42.0 \%$ of the time in the implicit and explicit program, respectively. The doubled amount in the explicit program substantiates the difference between the implemented motor learning strategies by the parents in both programs. In a previous study, 36\% of motor-related instruction or feedback was found for an intensive center-based program. The rate for our implicit program is considerably lower than this previously reported rate [25]. However, as neither interpretation guidelines nor a priori hypotheses exist, caution must be applied to this finding.

Overall, parents experienced the home-based training as positive. They were well able to provide the therapy, while paying particular attention to making the training appealing to the child. Activities of daily living were most easily practiced, which confirms earlier findings [26,27]. For the children, the program was demanding. Still, parents thought the program was worthwhile as the bimanual performance of their child improved. Altogether, parents found the program time-consuming. This is consistent with results of others, who identified time as a key barrier to implementing an intensive program [28-30]. The added pressure and time demands resulted in stress in a few parents. This agrees with a previous study of Halvarsson et al. [31]. Parents also experienced the 12-week program duration as too long. Parents perceived both intensity and duration as too much. At the same time, dosage is known to be one of the most crucial factors in attaining treatment effects [2]. A hybrid model of treatment, merging home-based and center-based elements, was proposed by parents and therapists. This solution may ensure training intensity and at the same time increase feasibility for families.

The combined coaching by the therapist and remedial educationalist was a highly appreciated component of the program. Likewise, previous studies reported that a therapist as coach provides emotional support, encouragement, and motivation, thus promoting adherence [32,33]. The instructional course for health-care professionals, the instructional videos for parents, the task-analysis, the telephone appointments, and the home visits all positively contributed to the program. Nevertheless, the frequency of appointments was considered too high as the program progressed. This could be attributed to a selective sample of motivated and capable parents par- 
ticipating in the study and as such needing less coaching. Other parents likely did not opt for the study or were even not invited by their child's rehabilitation team who may have thought the parents unable to provide such home-based training [34]. If the programs are to be implemented in clinical practice and a more diverse group of parents to participate, a more intensive coaching process may be necessary for some. Differentiation has to be made in coaching parents, based on their circumstances (e.g. skills, motivation, and context). Moreover, conversations by telephone had limitations for coaching by remedial educationalists. They would prefer in-person meetings, but this would put additional burden on parents. Video-conversations may be a satisfactory resolution. Last, everyone involved was annoyed by the problems with the data sharing system and parents disliked filling out the registration form. Thus, these components did not contribute to the success of the program.

The involvement of the extended family or the family's social network in the training was limited, because parents preferred to be in control of the training. However, therapists advocated involving significant persons in the child's life from the beginning, and expected this to facilitate generalization of treatment effects. In our opinion, however, there may be a risk that this would make the organization of the training and the coaching more complex and time-consuming for parents and therapists.

The results of this study are subject to certain limitations. The focus group discussions with the health professionals were conducted when the last participant had ended the program. As the study period encompassed several years, therapists and remedial educationalists acknowledged that they had a hard time remembering the details of their coaching in the home-based program. Hence, recall bias may have affected the results. Likewise, during the interviews, parents could not fully recall their opinions on the instructional videos they had watched at the very beginning of the program. Moreover, fidelity of the task analysis was not assessed. The COAD-study encountered recruitment problems and consequently potential selection bias [34]. Therefore, the conclusions of this study may not generalize to the entire population of young children with UCP and their parents. Last, during the intervention, some research activities had to take place. Parents as well as the health-care professionals may not have been able to completely disregard this when describing their experiences. This may have influenced the process evaluation.

The findings of this process evaluation, together with the results of the parallel effect evaluation [14], imply that home-based bimanual training forms a sound therapeutic approach. In clinical practice, we advocate integrating several core components in home-based training programs: setting meaningful goals, a priori task-analysis, instructional videos, and coaching by a therapist and remedial educationalist by means of video recordings, telephone appointments, and home visits. For the remedial educationalist's coaching, video-call appointments may be more effective than telephone appointments. The frequency and duration of appointments need to be customized to the family circumstances. Implications of other outcomes of this evaluation should be respected for each individual process. Until the optimal training duration is established, a length between eight and twelve weeks is advised.

A natural progression of this study is to confirm the appropriateness of the proposed modifications, by investigating either an amended version of the current program or a new program using a user-centered design in which the recommendations are incorporated. In particular, the 
consequences of a reduced duration to eight weeks on the motor performance improvements should be examined. Given the importance of the video-recordings in the coaching process, a well-operating data sharing system is imperative. The arQive system seems promising. However, as this was implemented later on in the study, the credibility of this finding is limited. More studies need to be done to establish whether arQive does indeed suffice, or to assess the feasibility of alternatives. A better understanding of interacting variables in implicit and explicit motor learning is an essential next step in defining their eligibility for particular children, parents, and phases of learning. Ideally, a model will be developed to predict the optimal proportion of implicit and explicit motor learning techniques for each individual family and situation. Following the suggestion of parents and therapists, further work is recommended to explore and test the optimal design of a hybrid model, integrating home-based and center-based training.

\section{Acknowledgements}

We are grateful to the children, parents, and health-care professionals who participated in this study. We thank the COAD Author Group for their contribution: Nicole Brouwers, Anke Defesche, Hanneke Denissen, Rosemarie Derickx, Yvonne Geerdink, Kiki van Heel, Ruth Heijsen-van den Heuvel, Bregtje Janssen, Anne Jensen, Marjon Kissels, Martijn Klem, Denise Martens, Judith van Munster, Bianca Olive, Marleen Philippens, Caroline Scheijmans, Lucianne Speth, Ingrid van den Tillaar, and Eef Vennix. We acknowledge Les Hearn for proofreading the manuscript. 


\section{REFERENCES}

[1] Gordon AM, Bleyenheuft Y, Steenbergen B. Pathophysiology of impaired hand function in children with unilateral cerebral palsy. Dev Med Child Neurol 2013;55 Suppl 4:32-7.

[2] Sakzewski L, Ziviani J, Boyd RN. Efficacy of upper limb therapies for unilateral cerebral palsy: a meta-analysis. Pediatrics 2014;133:e175-204.

[3] Novak I, McIntyre S, Morgan C, Campbell L, Dark L, Morton N, Stumbles E, Wilson SA, Goldsmith S. A systematic review of interventions for children with cerebral palsy: state of the evidence. Dev Med Child Neurol 2013;55:885-910.

[4] Andersen JC, Majnemer A, O'Grady K, Gordon AM. Intensive upper extremity training for children with hemiplegia: from science to practice. Semin Pediatr Neurol 2013;20:100-5.

[5] Palisano RJ, Campbell SK, Orlin M. Physical Therapy for Children-E-Book. Elsevier Health Sciences; 2014.

[6] Mackean GL, Thurston WE, Scott CM. Bridging the divide between families and health professionals' perspectives on family-centred care. Health Expect 2005;8:74-85.

[7] Novak I, Berry J. Home program intervention effectiveness evidence. Phys Occup Ther Pediatr 2014;34:384-9.

[8] Beckers LWME, Geijen MME, Kleijnen J, Rameckers EAA, Schnackers MLAP, Smeets RJEM, Janssen-Potten YJM. Feasibility and effectiveness of home-based therapy programs for children with cerebral palsy: a systematic review. (In preparation).

[9] Kleynen M, Braun SM, Bleijlevens MH, Lexis MA, Rasquin SM, Halfens J, Wilson MR, Beurskens AJ, Masters RS. Using a Delphi technique to seek consensus regarding definitions, descriptions and classification of terms related to implicit and explicit forms of motor learning. PLoS One 2014;9:e100227.

[10] Steenbergen B, van der Kamp J, Verneau M, Jongbloed-Pereboom M, Masters RS. Implicit and explicit learning: applications from basic research to sports for individuals with impaired movement dynamics. Disabil Rehabil 2010;32:1509-16.

[11] Jenks KM, de Moor J, van Lieshout EC. Arithmetic difficulties in children with cerebral palsy are related to executive function and working memory. J Child Psychol Psychiatry 2009;50:824-33.

[12] Schnackers M, Beckers L, Janssen-Potten Y, Aarts P, Rameckers E, van der Burg J, de Groot I, Smeets R, Geurts S, Steenbergen B. Home-based bimanual training based on motor learning principles in children with unilateral cerebral palsy and their parents (the COAD-study): rationale and protocols. BMC Pediatr 2018;18:139.

[13] Wade DT, Smeets RJ, Verbunt JA. Research in rehabilitation medicine: methodological challenges. J Clin Epidemiol 2010;63:699-704.

[14] Beckers LWME, Rameckers EAA, Aarts PBM, van der Burg JJW, Smeets RJEM, COAD Author Group, Schnackers MLAP, Steenbergen B, de Groot IJM, Geurts ACH and others. Effect of home-based bimanual training in children with unilateral cerebral palsy (the COAD-study): a case series. (In preparation).

[15] Tashakkori A, Creswell JW. Editorial: Exploring the Nature of Research Questions in Mixed Methods Research. 2007;1:207-11.

[16] Creswell JW, Clark VLP. Designing and Conducting Mixed Methods Research. SAGE Publications; 2011.

[17] Merriam SB, Tisdell EJ. Qualitative Research: A Guide to Design and Implementation. San Francisco (CA): Wiley; 2015. 
[18] Beckers L, van der Burg J, Janssen-Potten Y, Rameckers E, Aarts P, Smeets RJBP. Process evaluation of two home-based bimanual training programs in children with unilateral cerebral palsy (the COAD-study): protocol for a mixed methods study. 2018;18:141.

[19] Eliasson AC, Krumlinde-Sundholm L, Rosblad B, Beckung E, Arner M, Ohrvall AM, Rosenbaum P. The Manual Ability Classification System (MACS) for children with cerebral palsy: scale development and evidence of validity and reliability. Dev Med Child Neurol 2006;48:549-54.

[20] Law M, Baptiste S, McColl M, Opzoomer A, Polatajko H, Pollock N. The Canadian occupational performance measure: an outcome measure for occupational therapy. Can J Occup Ther 1990;57:82-7.

[21] Saunders RP, Evans MH, Joshi P. Developing a process-evaluation plan for assessing health promotion program implementation: a how-to guide. Health Promot Pract 2005;6:134-47.

[22] Braun V, Clarke V. Using thematic analysis in psychology. Qualitative Research in Psychology 2006;3:77-101.

[23] Braun V, Clarke V. What can "thematic analysis" offer health and wellbeing researchers? International Journal of Qualitative Studies on Health and Well-being 2014;9:26152.

[24] de Vet HCW, Terwee CB, Mokkink LB, Knol DL. Measurement in Medicine: A Practical Guide. Cambridge University Press; 2011.

[25] Leijzer S. De Betrouwbaarheid van een Observatiesysteem voor Impliciete en Expliciete Aanleerstrategieën tijdens Bimanuele Therapie bij Kinderen met unilaterale Cerebrale Parese: Radboud University; 2017.

[26] Ahl LE, Johansson E, Granat T, Carlberg EB. Functional therapy for children with cerebral palsy: an ecological approach. Developmental Medicine \& Child Neurology 2005;47:613-9.

[27] Hinojosa J, Anderson J. Mothers' perceptions of home treatment programs for their preschool children with cerebral palsy. American Journal of Occupational Therapy 1991;45:273-9.

[28] Fergus A, Buckler J, Farrell J, Isley M, McFarland M, Riley B. Constraint-induced movement therapy for a child with hemiparesis: a case report. Pediatric Physical Therapy 2008;20:271-83.

[29] Gerhardy A, Sandelance M. Demystifying home programmes: Resource development for families and clinicians to enhance upper limb intervention and outcomes in children. Developmental Medicine and Child Neurology 2014;Conference End: 20140314. Conference Publication: (var.pagings). 56:73.

[30] Lorentzen J, Greve LZ, Kliim-Due M, Rasmussen B, Bilde PE, Nielsen JB. Twenty weeks of home-based interactive training of children with cerebral palsy improves functional abilities. BMC Neurology 2015;15:75.

[31] Halvarsson S, Asplund R, Fjellman-Wiklund A. From authority to coach Parents' experiences of stretching as a home programme for children with cerebral palsy. Advances in Physiotherapy 2010;12:208-16.

[32] Bilde PE, Kliim-Due M, Rasmussen B, Petersen LZ, Petersen TH, Nielsen JB. Individualized, homebased interactive training of cerebral palsy children delivered through the Internet. BMC Neurology 2011;11:32.

[33] Taylor NF, Dodd KJ, McBurney H, Graham HK. Factors influencing adherence to a home-based strength-training programme for young people with cerebral palsy. Physiotherapy 2004;90:57-63.

[34] Beckers LWME, Rameckers EAA, Smeets RJEM, Van der Burg JJW, Aarts PBM, Schnackers MLAP, Janssen-Potten YJM. Barriers to recruitment of children with cerebral palsy in a trial of home-based training. (In preparation). 



\section{Chapter iI}

\section{Single-case experimental design studies in children with cerebral palsy: a scoping review}

LWME Beckers, RA Stal, RJEM Smeets, P Onghena, CHG Bastiaenen 


\section{Abstract}

Background Cerebral palsy (CP) research is dominated by group-based designs and the randomized controlled trial (RCT) has long been considered superior. Single-case experimental design (SCED) provides a rigorous alternative, respecting the heterogeneity of the population. This study aims to critically evaluate SCED studies performed within the population of children and adolescents with $C P$.

Methods A scoping review was performed. Studies were identified by searching electronic databases and included if they reported on a SCED study of children and/or adolescents up to 21 years with CP. Demographic, methodological, and statistical data were extracted. Articles were graded using the Risk of Bias in N-of-1 Trials (RoBiNT) Scale and evaluated for quality of reporting using the Consolidated Standards of Reporting Trials (CONSORT) extension for N-of-1 trials (CENT 2015). Comments regarding strengths and limitations were extracted and analysed.

Results Studies investigated the effects of a wide range of interventions on various outcomes. Most SCED types were adopted in multiple studies. All studies used visual inspection rather than visual analysis, often complemented with basic statistical descriptives. Risk of bias was high, particularly concerning internal validity. Many items of the CENT were insufficiently reported. Although the number of studies had increased over time, their risk of bias had not decreased. Several benefits and limitations of SCED were identified.

Conclusions SCED has potential within the context of personalized evidence-based medicine in children and adolescents with CP, provided that the quality of evidence from results is increased through reduction of risk of bias. 


\section{BACKGROUND}

Cerebral palsy (CP) is a group of disorders with heterogeneous aetiologies and clinical presentations. Consequently, rehabilitation of children and adolescents with CP requires an individualized approach. In contrast, research within this population is dominated by group-based designs, and the randomized controlled trial (RCT) has for a long time been considered superior in intervention research. Because children may respond differently or even oppositely to the same intervention, the application of this group design leads to several problems in conducting and interpreting, such as very large confidence intervals for mean changes. In addition, RCTs generally include a narrow selection of the wide diversity in characteristics of children with CP and often end up with rather small study samples, limiting the generalizability of results to an even larger extent. Because the variability in treatment effects is masked, the applicability of RCT findings to clinical decision-making in individual children is very limited.' The single-case experimental design (SCED) provides an alternative to the traditional group-based research to establish intervention effectiveness in this diverse and relatively small population.

The SCED is a within-subject design, performed in one or a small number of cases. The participants serve as their own controls by comparing distinct experimental phases for the outcome of interest. This ordinarily includes a baseline phase to determine a representative stable state or trend, with which subsequent phases can be compared. ${ }^{2}$ For a single-case design to be considered experimental, the time-points at which the interventions are implemented have to be manipulated and, preferably, randomized. Additionally, the main outcomes are to be measured systematically and frequently within and across all phases, in order to assess individual change across different conditions. 'There is a variety of SCEDs: the A-B design, the withdrawal/reversal (A-B-A) design, the multiple baseline design, the alternating treatments design, and changing-criterion design. The design characteristics have been described in detail elsewhere., 3

The specific design used and the study's methodology determine the confidence in any causal relationship found. Various means can be used to increase the study's validity, such as multiple baselines, randomization, and replication within or between participants., ${ }^{1,2}$ On the other hand, some specific threats to internal validity make the SCED methodologically challenging. These include the issue of autocorrelation due to repeated measures within cases and the possibility of carry-over effects from interventions. ${ }^{2}$ In developing children return to baseline is not necessarily expected and favourable carry-over effects are desirable: retention of improvements after the treatment has ended is what is aimed for. Equally important, it would be unethical to withdraw an effective intervention in a subsequent phase, especially from a developing child. Likewise, lengthy baseline periods can be undesirable, and provoke resistance from families, who advocate the best care at all times. ${ }^{1}$ These matters are to be carefully considered regarding SCED studies in children with CP.

SCED studies can provide a rigorous alternative to $\mathrm{RCTs},{ }^{2}$ respecting the heterogeneity regarding clinical performance and prognosis that characterize the population of children with CP. Replication of results among cases increases generalization of findings, whereas dissimilar results enable identification of factors that interfere with the children's response to the intervention. Hence, SCED studies inform treatment providers whether an intervention is expected to benefit 
their individual patient, facilitating translation of research findings into clinical practice.' Similarly, clinicians can employ a pragmatic SCED as a clinical decision tool to identify the best option for care for their patient.

The level of evidence from SCED studies is a subject of debate. Recently, there has been a shift towards acceptance of the SCED as a methodology equivalent to the RCT. Some even consider the level of evidence from SCED studies to be the highest for clinical decision-making."

${ }^{4}$ In our opinion, the appropriateness of the study design to answer the research question and its methodological quality determine the strength of the evidence, rather than the study type itself.

Although SCED seems to be underused', there are no data on the extent to which this design has been adopted in CP-related research, the methodological and statistical approaches applied, or the validity and the quality of reporting of these studies. A better understanding of the (dis)advantages of specific methodological aspects would contribute to epidemiological advancements in terms of design and analysis of SCED studies. This study, therefore, aims to critically evaluate SCED studies performed within the population of children and adolescents with CP.

\section{METHODS}

The current study is explorative in nature, intending to systematically map the available literature relevant to the topic of interest. Because of the broadness and complexity of this aim, a scoping review was deemed most appropriate.

The methodological framework of Arksey and O'Malley, ${ }^{5}$ and enhancements to this approach suggested by Levac et al., ${ }^{6}$ as well as the Joanna Briggs Institute Reviewers' Manual 2015 (Methodology for JBI Scoping Reviews), ${ }^{7}$ were adopted for designing this scoping review. Its objectives, inclusion criteria, and methods were specified in advance in a protocol.

\section{IDENTIFICATION OF RESEARCH QUESTIONS}

The research questions that guided this review were:

1. How many SCED studies have been performed in the population of children and adolescents with CP and what were their aims?

2. What methodological and statistical approaches were used in the available SCED studies?

3. What are the validity of these SCED studies and the quality of their reporting?

4. What are strengths and limitations of these SCED studies according to their authors?

\section{IDENTIFICATION OF RELEVANT STUDIES}

\section{SEARCH STRATEgy}

The iterative process started with an initial limited search in the electronic databases EMBASE (Ovid interface) and MEDLINE (Ovid interface). The query included terms related to the population and to SCED. The text words of titles and abstracts along with the index words of retrieved 
reports were analyzed. Thereafter, the identified keywords and index words were used to perform a second amended search in the electronic databases CINAHL (EBSCO interface), EMBASE (Ovid interface), MEDLINE (Ovid interface) and PsycINFO (EBSCO interface), without restriction on the year of publication. The complete search query for all databases is presented in Supporting Information 1. The first author developed the search strategies, in consultation with a research librarian, and performed the searches.

In order to identify grey literature, the members of the European Academy of Childhood Disability (EACD) and the Australasian Academy for Cerebral Palsy and Developmental Medicine (AusACPDM) were requested by a newsletter to inform the project group of any published or unpublished SCED studies, executed either by their research group or associates.

\section{INCLUSION CRITERIA}

\section{TYPES OF PARTICIPANTS}

Studies with children and/or adolescents aged up to 21 years with CP were included. Studies of which $>50 \%$ of the participants did not meet these criteria were excluded. At the outset of the study, the population of interest also consisted of children and adolescents with acquired brain injury. However, based on the more numerous results of the initial search than anticipated and to keep the study feasible, a protocol amendment was made to restrict the scoping review to CP only.

\section{CONCEPT}

Studies reporting on any SCED were evaluated. This included, but was not limited to, multiple crossover N-of-1 trials. SCED studies were defined as having the following characteristics, in accordance with the single case designs technical documentation from What Works Clearinghouse: ${ }^{8}$

- "An individual 'case' is the unit of intervention and unit of data analysis. A case may be a single participant or a cluster of participants."

- "Within the design, the case provides its own control for purposes of comparison."

- "The outcome variable is measured repeatedly within and across different conditions or levels of the independent variable."

An element of experimental manipulation by the researcher(s) was required to meet the definition of SCED.

\section{CONTEXT}

Laboratory studies were excluded. No other limits were used for the setting, type of interventions, or outcomes. 


\section{TYPES OF SOURCES}

Completed or ongoing empirical research studies or protocols were included. Studies published in English, Dutch, or German were considered for inclusion.

\section{STUDY SELECTION}

The inclusion criteria were applied during two selection phases: (1) title and abstract screening, where in case of doubt the study remained included, and (2) assessing full texts for eligibility. The first and second author independently performed both phases of the selection process. One German article was evaluated by the first and last author instead. All cases of discrepancy were discussed until consensus was reached. The online Covidence systematic review software (Veritas Health Innovation, Melbourne, Australia) was used for management of the study.

\section{Charting The data}

\section{DATA COLLECTION PROCESS}

Demographic, methodological, and statistical data were extracted. Two independent reviewers (first and second author) carried out the data collection by using a customized digital charting form. Any differences were resolved via consensus. A third reviewer (last author) mediated if required.

\section{RISK OF BIAS APPRAISAL AND REPORTING APPRAISAL}

The selected articles were graded for risk of bias using the Risk of Bias in N-of-1 Trials (RoBiNT) Scale. The RoBiNT Scale has been developed to critically appraise SCED studies, primarily in the field of neurorehabilitation. It contains a 7-item Internal Validity subscale and an 8-item External Validity and Interpretation subscale. For each item, 0, 1, or 2 points can be awarded, which enables a total score from 0 to 30 to be calculated. The higher the score, the lower the risk of bias. Inter-rater reliability and construct validity of the RoBiNT Scale are satisfactory and an extensive manual is available, providing scoring procedures and rating guidelines. ${ }^{3,9}$

The Consolidated Standards of Reporting Trials (CONSORT) extension for N-of-1 trials (CENT 2015) was used to evaluate the quality of reporting of the full-text publications. This standard provides guidance to facilitate preparation and appraisal of, specifically, multiple crossover N-of-1 trials. ${ }^{10}$ This is in contrast to the RoBiNT scale, which has a wider application. In this review, all 25 items and 44 sub-items of the CENT were rated as sufficiently reported, insufficiently reported, or not applicable. 
To increase consistency between the reviewers, the first, second, and last author independently assessed five studies. They reflected on the results and aligned their conceptualizations of the appraisal tools. Thereafter, the first and second author appraised the risk of bias and reporting quality for all studies independently. Disagreements between the reviewers' assessments were debated and a final decision agreed upon.

\section{Content analysis}

The first author searched the introduction, methods, and discussion sections of all included studies for comments regarding the strengths and limitations of studies, including rationales for using SCED approaches. Relevant sentences or paragraphs were tracked and coded per study. These coded extracts were analysed for recurrent issues across studies and overarching themes were sought.

\section{Consultation}

A consultation element was embedded in the scoping review to enhance the validity of the study results. The stakeholder consulted (PO) was a professor of educational and behavioural statistics and methodology, with expertise in SCED research. A report describing the preliminary findings was shared with PO to inform the consultation, followed by a discussion of his perspectives. The provided insights were incorporated into this paper.

\section{RESULTS}

Figure 1 displays the scoping review flow diagram. After removal of duplicates, the database search (last updated 13 September 2018) retrieved 417 citations. The newsletter item, which was sent to 1261 EACD and AusACPDM members, obtained no responses. Eighty records were included in the review, reporting on 74 unique studies. One of the full text publications reported on two separate studies and another described methods only.

\section{STUDY CHARACTERISTICS}

Supporting Information 2 contains the aims and characteristics of the included studies. The studies focused on a wide range of interventions and outcomes. Besides establishing effects, a few studies aimed to answer feasibility questions, for instance concerning satisfaction of users with the treatment(s). The sample sizes varied from one to 14 children and adolescents. The demographics of participants were diverse with respect to types of $C P$, age, and gender. 


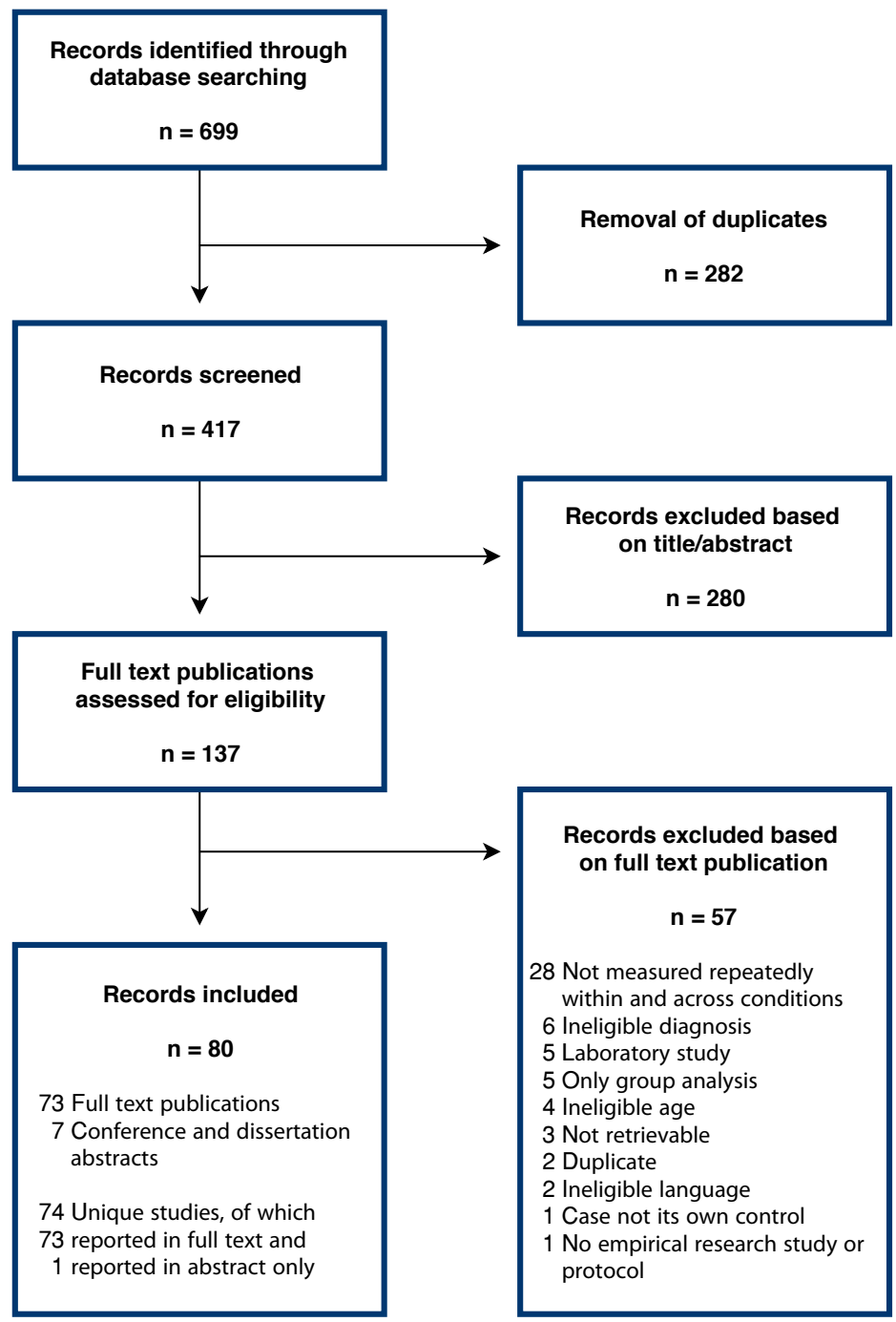

Figure 1 Flowchart of the study selection process 


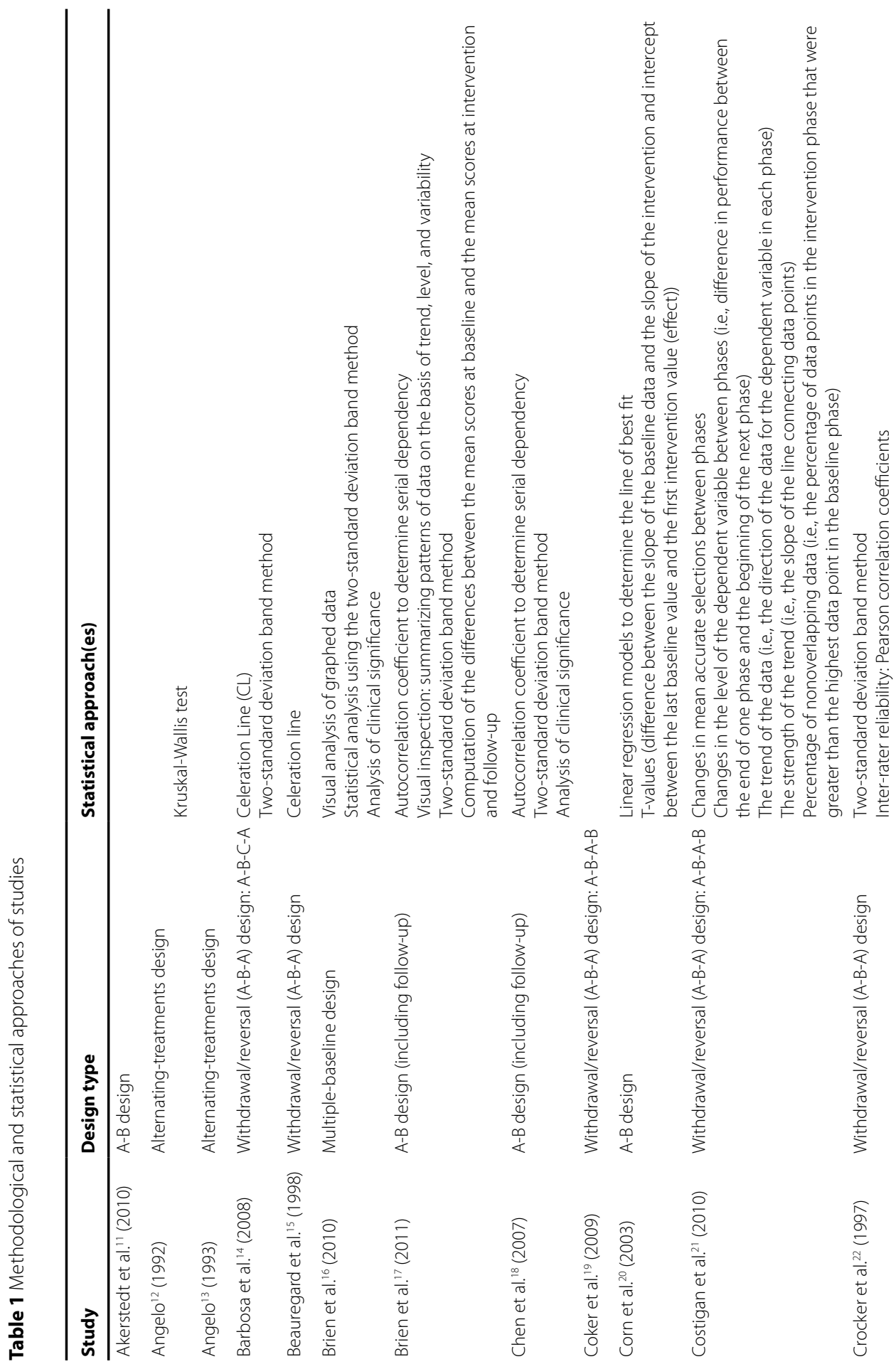




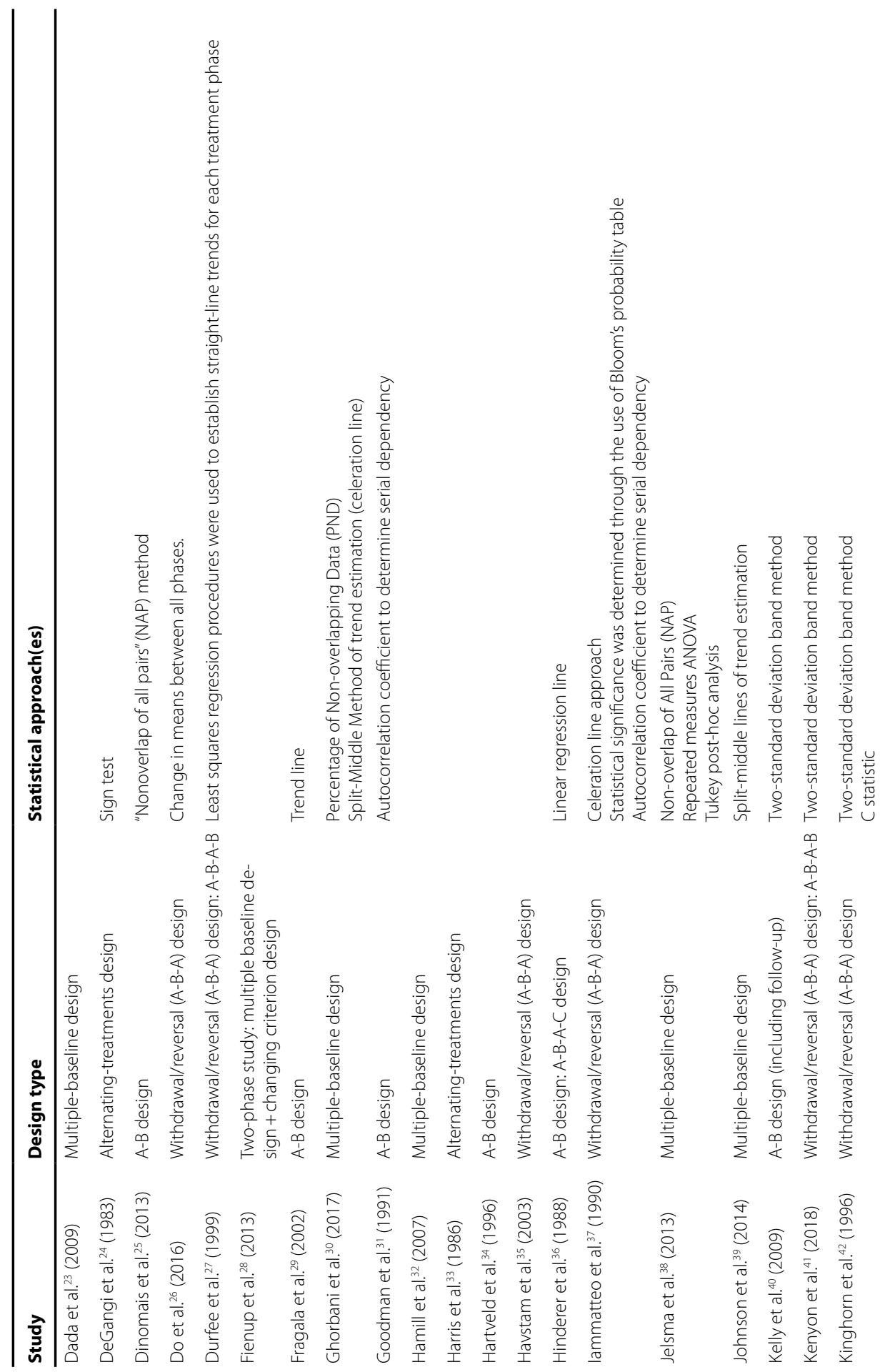




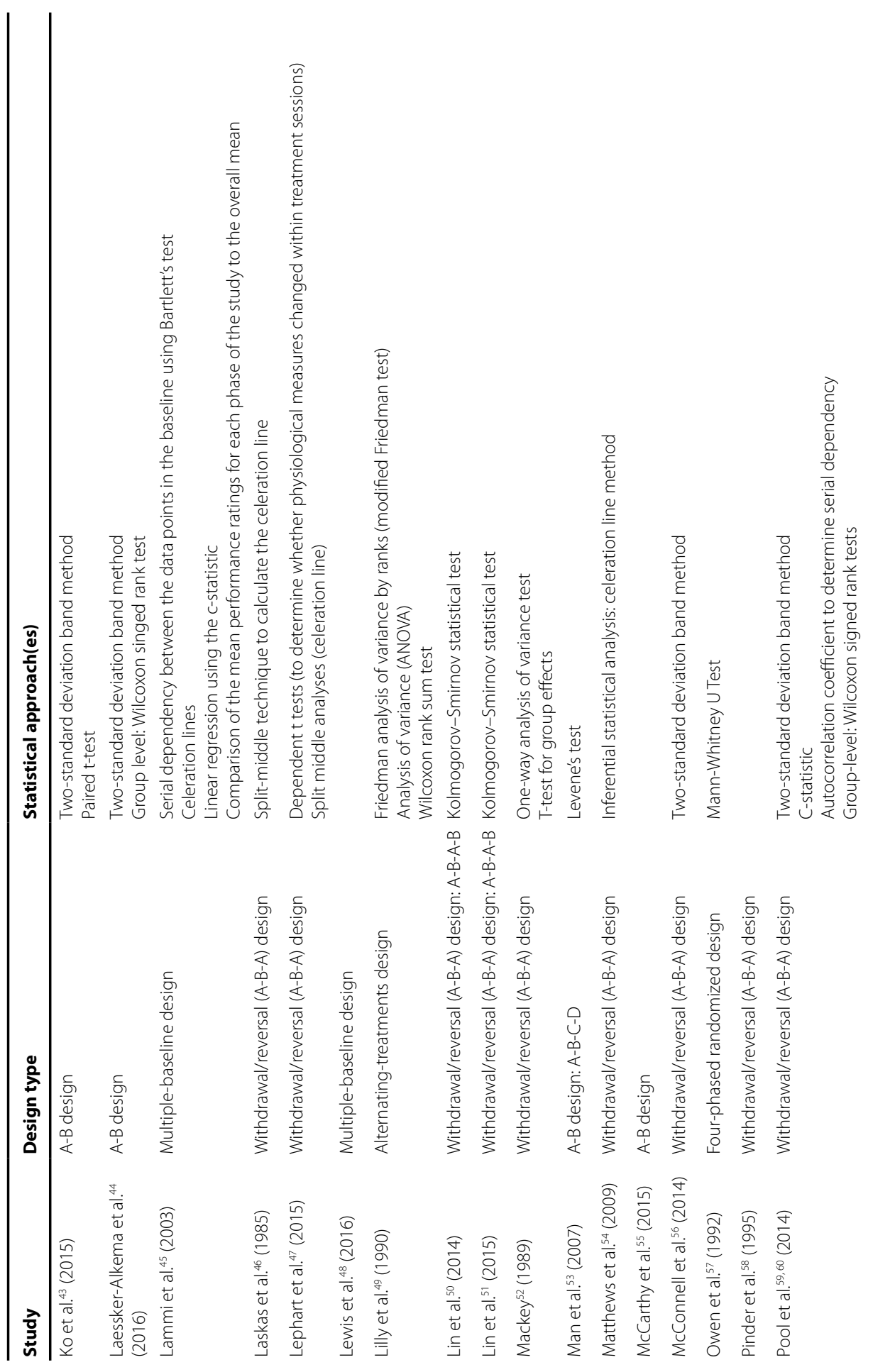




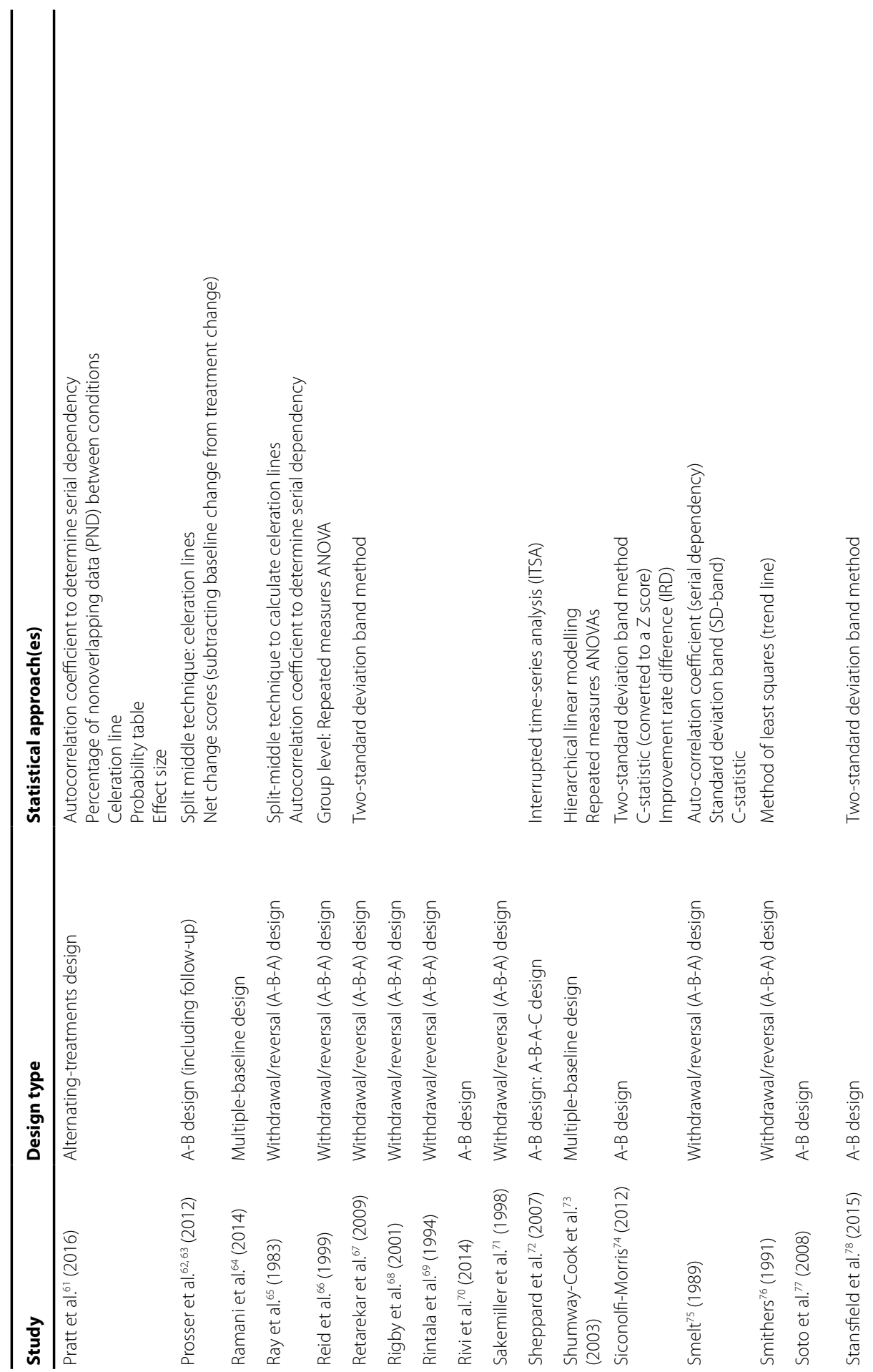




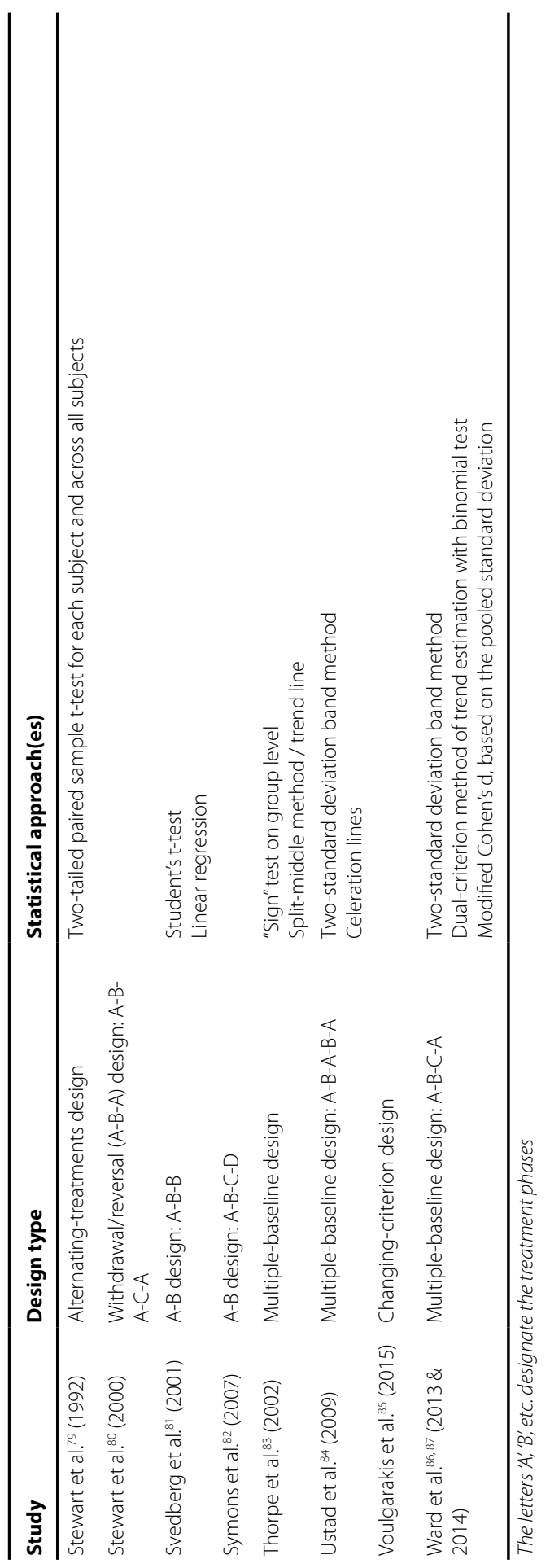


Table 2 Risk of bias per item of the RoBiNT Scale, and inter-rater agreement of RoBiNT scale scores $(\mathrm{N}=73)$

\begin{tabular}{|c|c|c|c|c|c|c|c|}
\hline & \multicolumn{6}{|c|}{ Mean (range) } & $\%$ of agreement \\
\hline Interval Validity Subscale & \multicolumn{6}{|c|}{$3.6(1-10)$} & 79 \\
\hline $\begin{array}{l}\text { External Validity and Interpretation } \\
\text { Subscale }\end{array}$ & \multicolumn{6}{|c|}{$7.6(2-13)$} & 72 \\
\hline Total score & \multicolumn{6}{|c|}{$11.2(4-22)$} & 75 \\
\hline \multirow[t]{3}{*}{ Item } & \multicolumn{6}{|l|}{ Score } & \\
\hline & \multicolumn{2}{|c|}{0 points } & \multicolumn{2}{|c|}{1 point } & \multicolumn{2}{|c|}{2 points } & \\
\hline & Absolute & $\%$ & Absolute & $\%$ & Absolute & $\%$ & \\
\hline \multicolumn{8}{|l|}{ Interval Validity Subscale } \\
\hline 1. Design with control & 27 & 37.0 & 29 & 39.7 & 17 & 23.3 & 74 \\
\hline 2. Randomization & 63 & 86.3 & 0 & 0.0 & 10 & 13.7 & 93 \\
\hline 3. Sampling of behaviour & 8 & 11.0 & 30 & 41.1 & 35 & 47.9 & 78 \\
\hline $\begin{array}{l}\text { 4. Blinding of people involved in the } \\
\text { intervention }\end{array}$ & 70 & 95.9 & 3 & 4.1 & 0 & 0.0 & 93 \\
\hline 5. Blinding of assessor(s) & 56 & 76.7 & 3 & 4.1 & 14 & 19.2 & 77 \\
\hline 6. Inter-rater agreement & 47 & 64.4 & 15 & 20.5 & 11 & 15.1 & 53 \\
\hline 7. Treatment adherence & 67 & 91.8 & 3 & 4.1 & 3 & 4.1 & 84 \\
\hline
\end{tabular}

\section{External Validity and Interpretation Subscale}

\begin{tabular}{lccccccc} 
8. Baseline characteristics & 37 & 50.7 & 36 & 49.3 & 0 & 0.0 & 58 \\
$\begin{array}{l}\text { 9. Setting } \\
\begin{array}{l}\text { 10. Dependent variable } \\
\text { (target behaviour) }\end{array}\end{array}$ & 27 & 37.0 & 29 & 39.7 & 17 & 23.3 & 59 \\
$\begin{array}{l}\text { 11. Independent behaviour (therapy/ } \\
\text { intervention) }\end{array}$ & 0 & 0.0 & 13 & 17.8 & 60 & 82.2 & 78 \\
$\begin{array}{l}\text { 12. Raw data record } \\
\text { 13. Data analysis }\end{array}$ & 12 & 16.4 & 43 & 58.9 & 18 & 24.7 & 67 \\
$\begin{array}{l}\text { 14. Replication } \\
\text { 15. Generalization }\end{array}$ & 34 & 46.6 & 24 & 32.9 & 15 & 20.5 & 58 \\
\hline
\end{tabular}




\section{Methodological and Statistical APPROACHeS}

As can be seen from Table 1, the withdrawal/reversal design was most frequently used, followed by the A-B design, the multiple baseline design, and the alternating treatments design. The changing-criterion design was seldom used. The data analysis of all studies comprised visual inspection to a greater or lesser degree. Nevertheless, none of the studies systematically evaluated all six components (i.e. level, trend, variability, immediacy of the effect, overlap, and consistency of data patterns across similar phases) that are considered fundamental to demonstrate whether a causal relation exists and which would have been regarded as a visual analysis. ${ }^{8}$ The descriptive two-standard deviation band method and the split-middle technique (celeration line) were the most frequently-mentioned statistical approaches.

\section{RISK OF BIAS}

Table 2 presents the results obtained from the risk of bias appraisal. The included studies scored on average 11.2 points on the RoBiNT scale. Total scores ranged from 4 to 22 points. The Interval Validity Subscale mean score was 3.6 points. The mean score on the External Validity and Interpretation Subscale was 7.6 points, more than twice as high.

\section{INTERNAL VALIDITY SUbSCALE}

For the item 'design with control', 37.0\% of the articles scored 0 points, 39.7\% scored 1 point and $23.3 \%$ scored 2 points. Many multiple baseline designs were rewarded 0 points since concurrency was not demonstrated. In as many as $86.3 \%$ of the studies, no randomization was applied. With regard to item 3, it was found that $41.1 \%$ and $47.9 \%$ of the studies used at least 3 or at least 5 data points or sets of alternating sequences, respectively. Blinding of either the participant or the practitioner involved in the intervention was not applied in $95.9 \%$ of the studies. The majority of studies (80.8\%) also did not use a blinded assessor. As to inter-rater agreement, 0 points, 1 point, and 2 points were given to $64.4 \%, 20.5 \%$, and $15.1 \%$ of the studies, respectively. Finally, it appeared that $91.8 \%$ of the studies did not (adequately) confirm treatment adherence.

\section{EXTERNAL VALIDity and INTERPRETATION SUbSCALE}

Although half of the studies did sufficiently report on relevant biomedical variables, none provided an analysis of the baseline conditions or behaviours using a functional analysis, case formulation, or other systematic investigation. Only $23.3 \%$ of the studies described both the general location and the specific environment in which the intervention was conducted. A large proportion of the studies (82.2\%) sufficiently defined the dependent variable (outcome), including the measuring method. Similarly, $61.6 \%$ of the studies described the content of the intervention and the procedure of delivery in detail. For the item 'raw data record' a score of 1 point was awarded to the largest group of studies. Many studies were penalized because the figures were of such poor quality that the data points could not be read. The data analysis of studies was rated 0 
points in 46.6\%, 1 point in 32.9\%, and 2 points in 20.5\% of the studies. Many studies did conduct statistical analyses, but justification was missing, which is a requirement for a maximum score of 2 points. A total of $34.2 \%$ of studies did not contain any replication. The study was replicated (across participants, settings, behaviours, or practitioners) once or twice in $27.4 \%$ of the studies, and three or more times in $38.4 \%$ of the studies. Just 2 studies indicated a generalization measure to determine transfer of intervention effects to other variables or settings.

\section{INTER-RATER AGREEMENT}

The inter-rater agreement between the two assessors per item of the RoBiNT scale ranged from 53\% to 99\% (Table 2). The agreement for the Internal Validity Subscale, External Validity and Interpretation Subscale, and total score were $79 \%, 72 \%$, and $75 \%$, respectively.

\section{REPORTING OF QUALITY}

Table 3 compares the proportion of studies that sufficiently and insufficiently reported the items of the CENT.

\section{Title AND ABSTRACT}

Merely a quarter of the publications identified their study as a SCED in the title. According to the specific CENT guidance, the abstract should describe such items as the trial design, participant(s), interventions, objective, outcome, randomization, blinding, results, and conclusion. None of the included studies fully met these criteria. Particularly, randomization and blinding procedures, and harms were often missing.

\section{INTRODUCTION}

The majority of the studies reported the scientific background, explained the rationale, and specified the objectives or hypotheses. However, only $29.2 \%$ of the authors reported their rationale for using a SCED approach. 


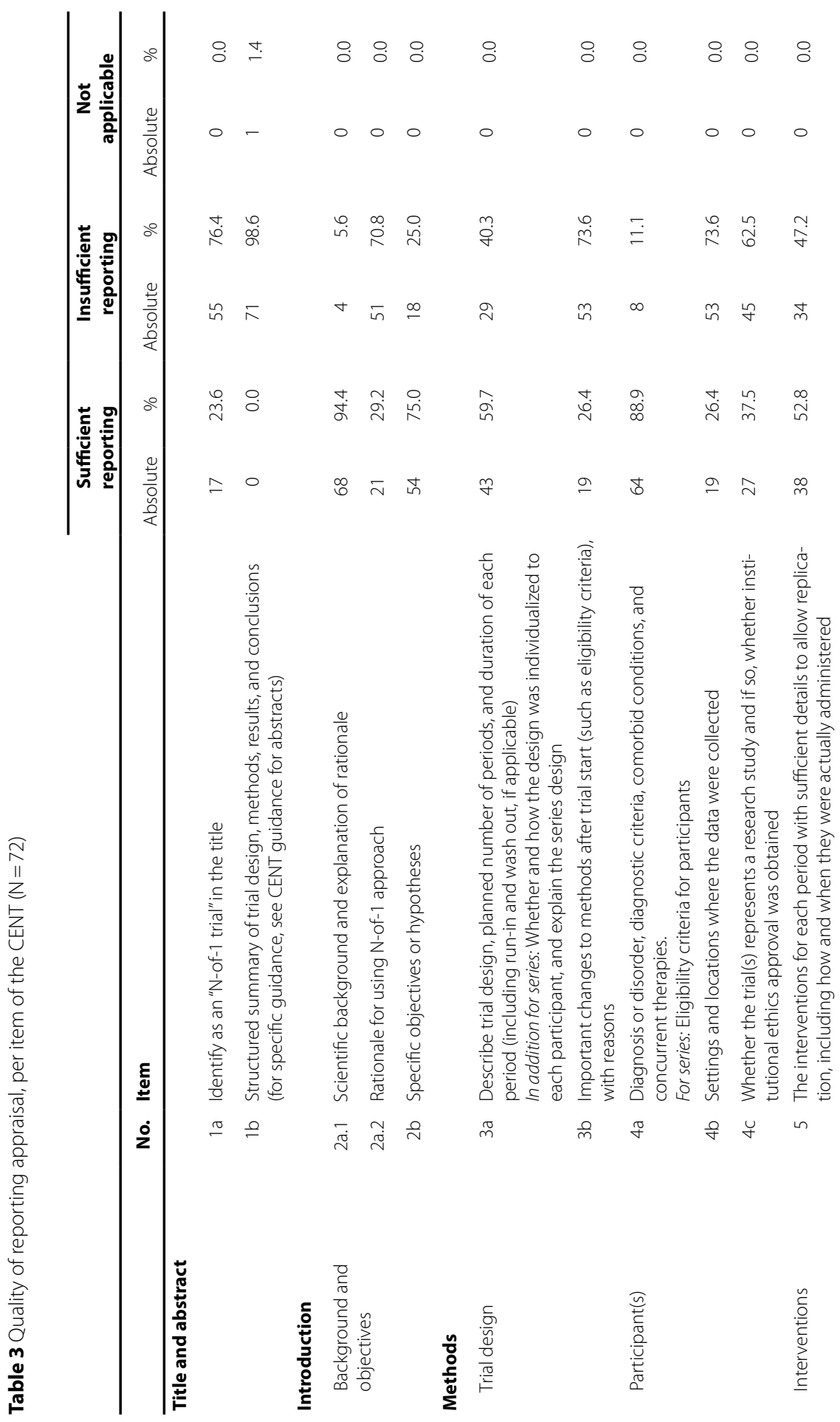




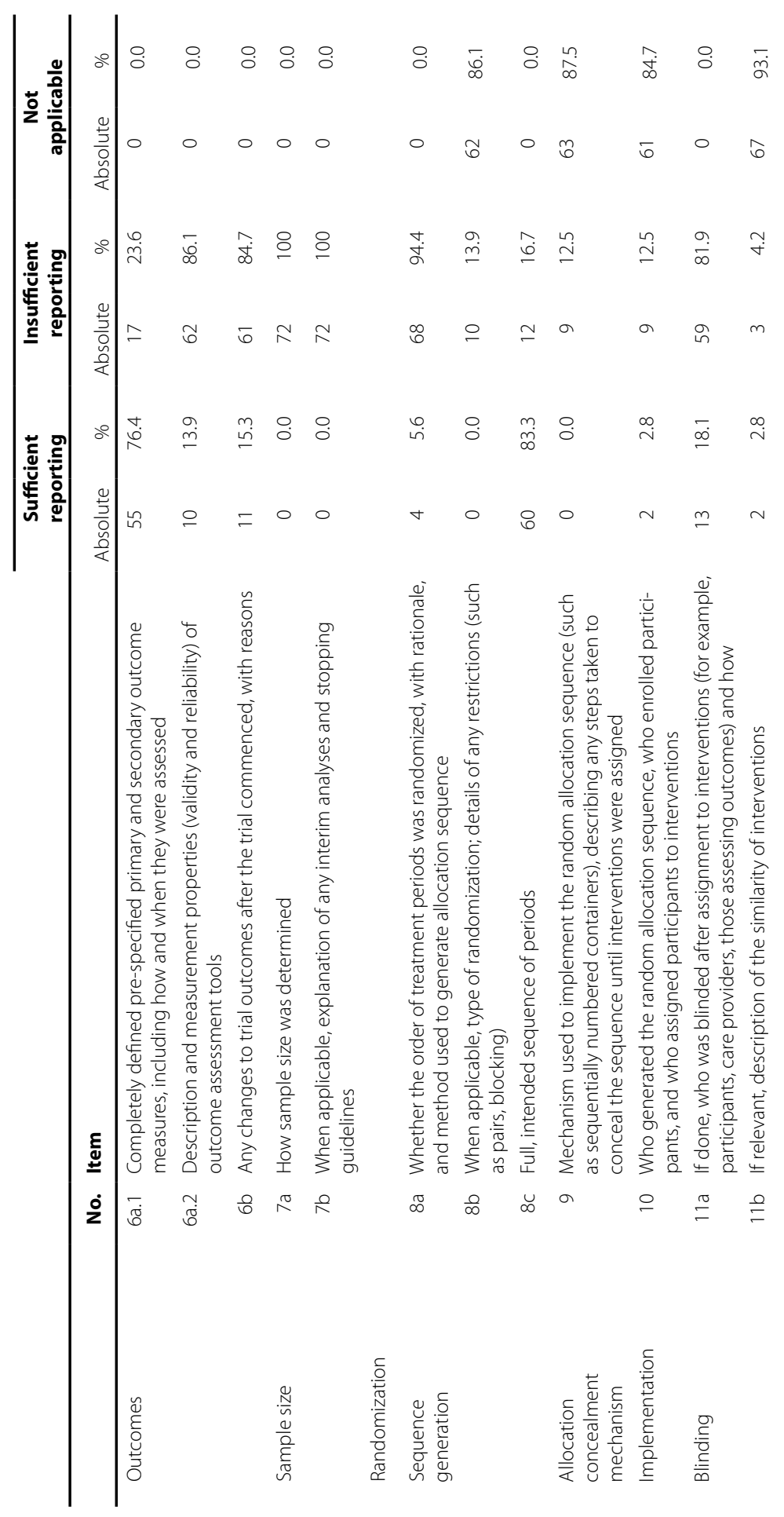




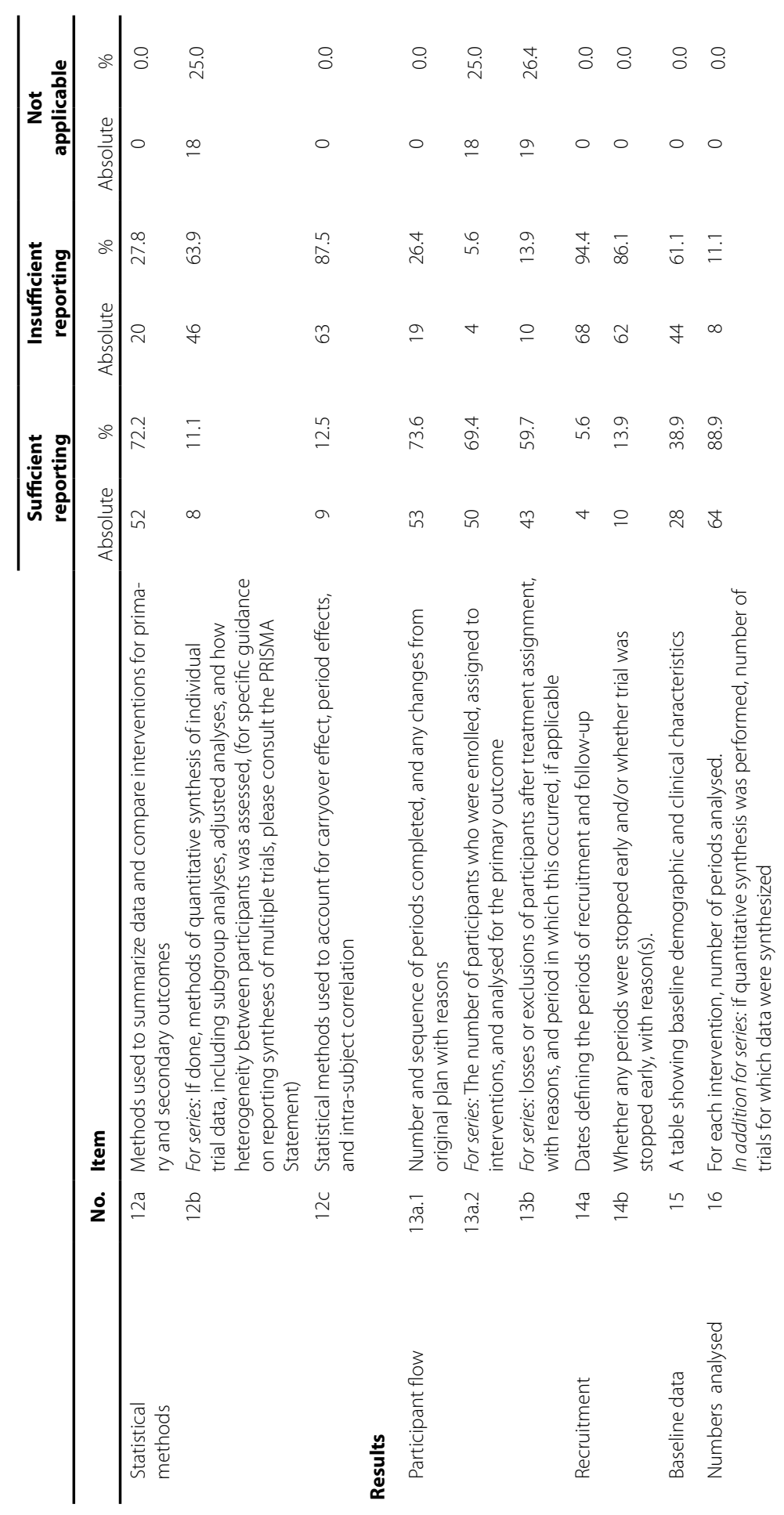




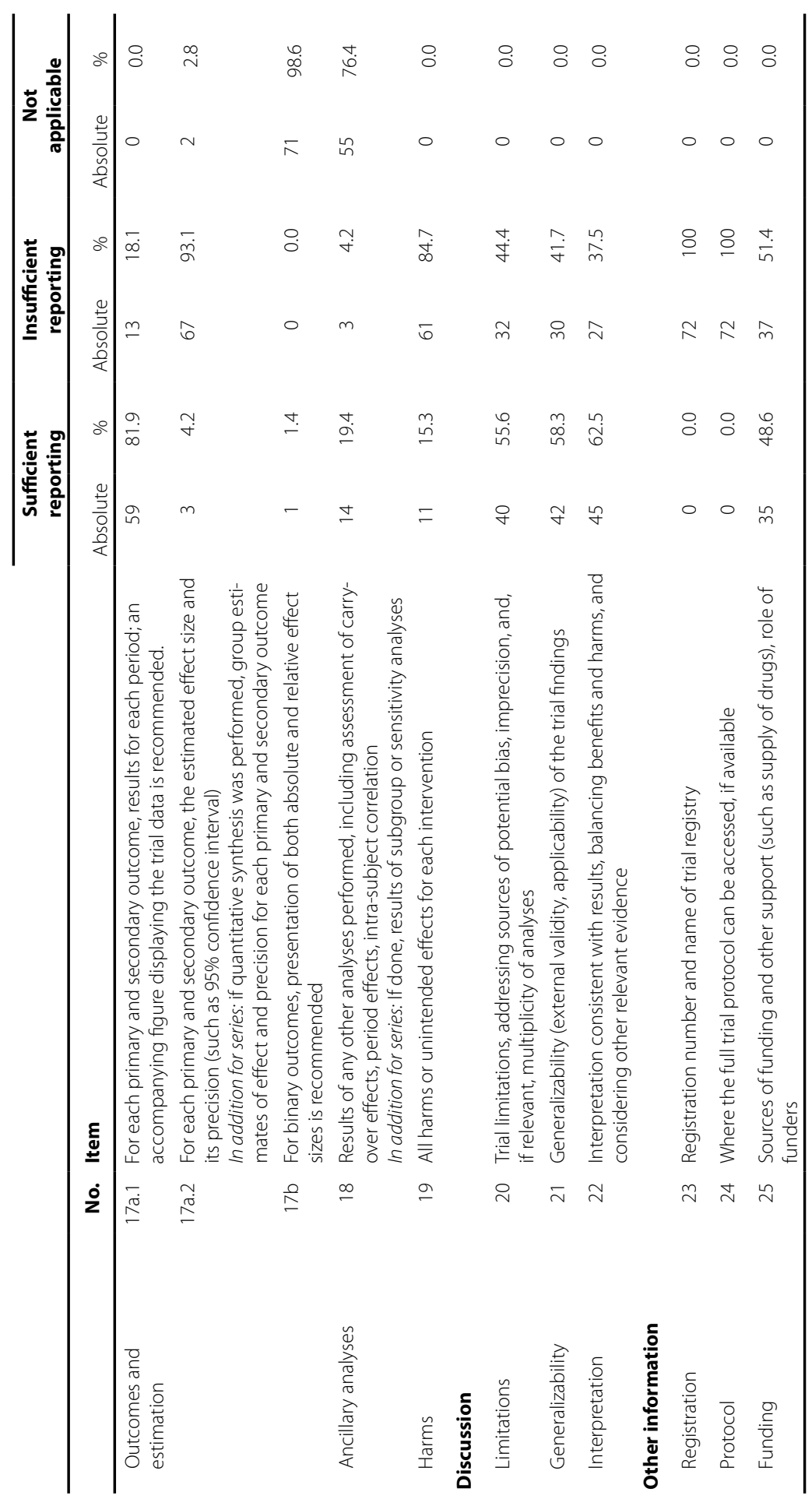




\section{Methods}

Although $59.7 \%$ of the studies sufficiently described the trial design, only $26.4 \%$ described whether and how methods changed after the start of the study. Patient characteristics or eligibility criteria were well reported. Still, in most studies information on settings and locations were lacking, and it was not addressed whether both a health research ethics board reviewed and approved the research study and parent and/or patient consent or assent was obtained. About half of the studies reported the interventions for each period insufficiently. Baseline intervention procedures were frequently not reported. While $76.4 \%$ of the studies completely defined pre-specified primary and secondary outcomes, the minority adequately described the measurement properties of outcome assessment tools and changes to outcomes after the trial commenced. None of the studies defined how sample size was determined nor explained interim analysis and stopping guidelines. Only four studies reported both whether and how randomization was applied. Consequently, item numbers 8b, 9, and 10 were often not applicable. Studies that did use randomization generally did not describe the type of randomization, allocation concealment mechanism, and implementation. Blinding was not mentioned in $81.9 \%$ of the studies. A total of $72.2 \%$ of the studies satisfactorily reported the statistical methods that were used to summarize data and compare interventions. On the other hand, methods of quantitative synthesis of individual trial data and assessing heterogeneity between participants (for series), and methods to account for carryover effect, period effects, and intra-subject correlation were reported in only $11.1 \%$ and $12.5 \%$ of the studies, respectively.

\section{RESULTS}

Overall, the participant flow was well reported. Dates of the recruitment and follow-up period were not defined, with the exception of four studies. Further, just 13.9\% of the studies reported whether and why any periods or the study itself were stopped early. Baseline demographic and clinical characteristics were too limited or not provided at all in $61.1 \%$ of the studies. Most studies sufficiently reported the numbers analyzed. The assessment of outcomes and estimation revealed opposing results: $81.9 \%$ of studies presented results for each outcome and each period, while no more than 3 studies estimated an effect size and its precision. The items concerning binary outcomes and ancillary analysis were mainly irrelevant. Few studies (15.3\%) reported any harms or unintended effects of interventions.

\section{Discussion}

Limitations, generalizability, and interpretation of the results were discussed satisfactorily in comparable proportions of studies: $55.6 \%, 58.3 \%$, and $62.5 \%$, respectively. 


\section{OTHER INFORMATION}

A registration or protocol of the study was not referred to in any of the publications. Sources of funding were named in about half of the studies.

\section{DEVELOPMENT OVER TIME}

From 1983 to 2018, the number of published SCED studies fluctuated between 0 and 7 per year, with an increase over time (Figure 2). The mean RoBiNT Scale total score of studies published each year remained stable over the last decades (Figure 2).

\section{CONTENT ANALYSIS}

The analysis of the authors' commentaries on the utilization of SCEDs resulted in a synthesis of the findings in two themes: applicability of the SCED and methodological considerations.

\section{APPlicability of THE SCED}

The most mentioned argument for applying a SCED was the appropriateness of this design for the heterogeneous population of children with CP and related variability in responses to interventions. Authors specified that the SCED approach met the demands of individualized (evaluation of) treatments, thereby being highly relevant for clinical practice. A secondary reason for using a SCED was limited availability of potential participants. Finally, some expressed the SCED to be a rigorous design with a high level of internal validity.

Authors frequently discussed limited generalizability of their findings, either to patients comparable to the children under research or to a wider population, as a major limitation of their study. Likewise, a small sample size was mentioned as a limitation. Replication or a follow-up study in a larger and/or more heterogeneous sample and using a longer follow-up period were often recommended. Several studies explicitly planned or recommended an RCT following the SCED study. In agreement with this, many authors referred to their work as preliminary, exploratory, or a pilot study.

\section{Methodological CONSIDERATIONS}

Practical concerns and constrains limited the possibilities of the research design. These were often inherent to the clinical nature of the study. Additionally, in many cases illness, holidays, or other events interrupted or otherwise disturbed the study procedures. Another recurrent example was the lack of a blinded assessor, due to insufficient resources. 


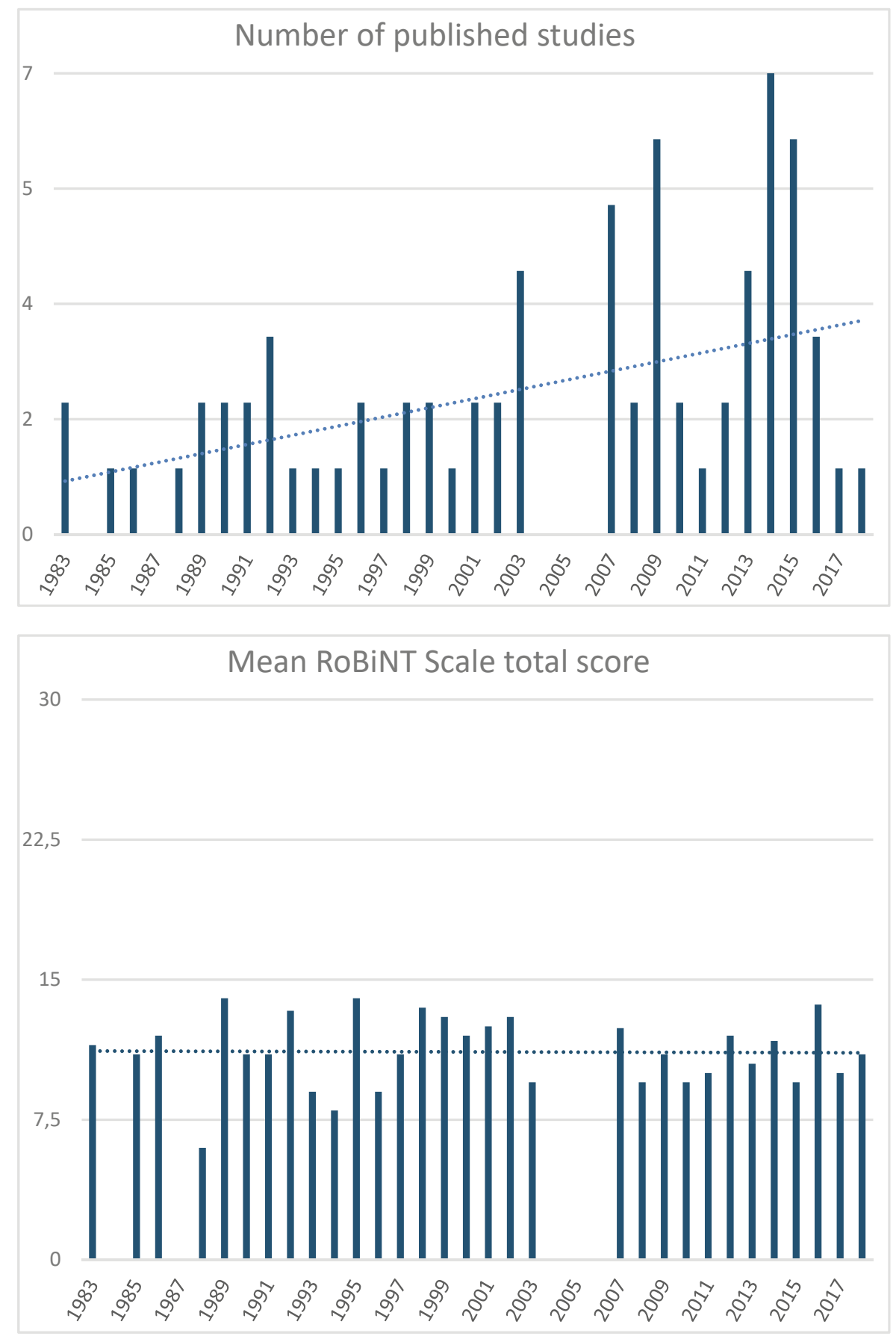

Figure 2 Development of SCED studies over time (including linear least squares trend lines) 
Concerning the study population, authors noticed that motivation, compliance, rapport with the assessor, and other mood and behavioural aspects of the child affected the assessment process. Second, confounding effects of growth, maturity and the ongoing process of motor development were recognized in the interpretation of findings. Researchers explained that they carefully considered the time and effort required of participants in order to ease the burden on children and their families, sometimes at the expense of study rigour.

The relevance as well as the challenge of standardized measurement conditions (e.g. same time each day) were acknowledged. Moreover, authors balanced the importance of outcome measures being sensitive to change with clinical relevance and ease of administration. A few specifically stated that the Gross Motor Function Measure (GMFM) was not responsive to changes in the case of short intervals between measurements.

In several publications, it was argued that withdrawal of treatment was inappropriate, either ethically or because permanent change of the child's functioning was intended. In some other studies, where researchers did withdraw an intervention, a carry-over effect was demonstrated. Furthermore, some authors identified other limitations of their studies related to the SCED: the absence of a control group, too few data points collected, the variability of data points within phases and thus no stable baseline, and practice effects.

\section{Discussion}

With respect to the first research question of this study, 74 SCED studies have been identified. These studies aimed to investigate the effects of a range of interventions on a variety of outcomes. The second question focused on the methodological and statistical approaches of the included studies. Except for the changing-criterion design, all SCED types were adopted in multiple studies. All studies used visual inspection rather than visual analysis, often complemented with inconsistent, basic statistical approaches. The third question sought to determine the risk of bias and quality of reporting. The overall risk of bias was relatively high, particularly in terms of internal validity. Additionally, many items of the CENT were insufficiently reported in the included full text publications. Although the annual number of studies increased, their risk of bias did not decrease over time. The fourth and last question was designed to identify the strengths and limitations of SCED studies. Benefits and limitations of the applicability of the SCED in the population of interest were determined and several methodological considerations discussed.

The A-B design and the non-concurrent multiple baseline design, which can be seen as a series of $A-B$ designs, ${ }^{3}$ were included in this scoping review because they encompass single-case methodology. It should be emphasized, though, that generally causal inferences cannot be derived from these designs. ${ }^{3}$ A recent article argued that A-B designs in which the start point of the treatment phase is randomized can be valid experimental designs under certain conditions, ${ }^{88}$ but the numerous studies included in this review that used this design did not comply with these criteria. The analysis of SCED is controversial in terms of the applicability of visual and (advanced) statistical analysis available. Beyond this debate, there is abundant room for improvement in the reporting of the analyses used, their rationales, and the techniques used. Due to the nature of 
the interventions in paediatric rehabilitation, in most studies neither families nor professionals involved in delivery of the intervention could have been blinded. Except for corresponding Item 4 of the RoBiNT scale, studies missed opportunities to comply with quality criteria for SCEDs. Contrary to expectations, this review did not find an increase of the total score of the RoBiNT scale over time. This may be explained by the fact that guidelines for SCED design and reporting were only published some 10 years ago., 89 One of the findings of the content analysis was that the clinical setting of SCED studies negatively affects the capability to perform the experiment in conformity with recommended procedures. This is a possible explanation for the high risk of bias of studies. Similarly, acceptable measurement burdens for participating families may have been prioritized over measurement intensity or phase lengths.

Next to the systematic and thorough search and data charting process, the consultation phase in which an expert in SCED statistics and methodology reviewed the results strengthened the validity of this scoping review. Unfortunately, the lack of response to the newsletter items did not allow for the inclusion of grey literature. It is possible, therefore, that unpublished studies were missed. The CENT reporting standard was chosen along with the RoBiNT Scale to cover both the policies of medical and behavioural sciences. Unlike the alternative Single-Case Reporting Guideline In BEhavioural Interventions (SCRIBE), the CENT has been specifically designed for the medical N-of-1 trial, i.e. a multiple crossover design. As a consequence, not all items were equally relevant for each type of SCED. Hence, results of the quality or reporting assessment need to be interpreted with caution to avoid overestimating the inadequacy of reporting. Last, the inter-rater agreement of the risk of bias appraisal was slightly lower than the agreement of $81 \%$ between trained novice raters in an inter-rater reliability study of the RoBiNT scale. ${ }^{3}$

We are convinced that adopting SCED methodology is the way forward for clinically-meaningful intervention research in the heterogeneous population of children and adolescents with CP. Many authors of included studies confirmed this potential of the SCED. Epidemiological principles and design standards are available to guide the planning of a SCED study and increase the credibility of results. General tools include the RoBiNT Scale, the SCRIBE, the CENT, and the single-case design (SCD) standards by What Works Clearinghouse., 8-10,90 So far only one CP-specific guideline is available, which is rather concise, with limited testing for validity. ${ }^{91}$ Hence, standards should be advanced for the complex rehabilitation setting to fit the specific needs of paediatric rehabilitation. To initiate a trend towards improvement of validity, it is imperative that researchers follow available recommendations for SCEDs. In addition, the importance of generic principles of intervention research should not be underestimated, such as clinical relevance of treatments and reporting of harms.

A number of issues is still under debate among experts and requires consensus, for instance consistent SCED terminology and appropriate statistical approaches per design. Best practices need to be agreed upon for CP-related research, supplementing earlier work.' Some specific concerns need particular attention. On the one hand, the non-reversal multiple baseline design seems most convenient, since many CP-treatments are intended to produce a permanent change. On the other hand, if no immediate effect is expected, it will reduce the suitability of the multiple baseline design. Hence, directions for the applicability of SCED designs to certain re- 
search situations are desired. Furthermore, the frequently-repeated measures require instruments with specific psychometric properties that are at the same time clinically relevant and convenient to administer often. A core set of suitable measurement instruments could aid in the selection from the many options available in paediatric rehabilitation.

The SCED has potential in the context of personalized evidence-based medicine in children and adolescents with CP, provided that the level of evidence of results is increased by minimizing risk of bias. Therefore, it is imperative that researchers comply with recommendations and guidelines. We invite the field to fill the gaps identified by us by building the evidence base for a SCED methodology that is highly valid and relevant to clinical practice. 


\section{REFERENCES}

1. Romeiser-Logan L, Slaughter R, Hickman R. Single-subject research designs in pediatric rehabilitation: a valuable step towards knowledge translation. Dev Med Child Neurol. 2017; 59:574-580.

2. Grossman J, Mackenzie FJ. The randomized controlled trial: gold standard, or merely standard? Perspect Biol Med. 2005; 48:516-534.

3. Tate RL, Rosenkoetter U, Wakim D, Sigmundsdottir L, Doubledaty J, Togher L, et al. The Risk of Bias in N-of-1 Trials (RoBiNT) Scale: An expanded manual for the critical appraisal of single-case reports. Sydney, Australia2015.

4. OCEBM Levels of Evidence Working Group. The Oxford 2011 Levels of Evidence. Oxford Centre for Evidence-Based Medicine.

5. Arksey H, O'Malley L. Scoping studies: towards a methodological framework. International Journal of Social Research Methodology. 2005; 8:19-32.

6. Levac D, Colquhoun H, O'Brien KK. Scoping studies: advancing the methodology. Implementation Science. 2010; 5:69.

7. The Joanna Briggs Institute. Joanna Briggs Institute Reviewers' Manual: 2015 edition / Supplement: The Joanna Briggs Institute; 2015. Available from: http://joannabriggs.org/assets/docs/sumari/Reviewers-Manual_Methodology-for-JBI-Scoping-Reviews_2015_v2.pdf.8. Kratochwill TR, Hitchcock J, Horner RH, Levin JR, Odom SL, Rindskopf DM, et al. Single-case designs technical documentation. What Works Clearinghouse; 2010.

9. Tate RL, Perdices M, Rosenkoetter U, Wakim D, Godbee K, Togher L, et al. Revision of a method quality rating scale for single-case experimental designs and n-of-1 trials: the 15-item Risk of Bias in N-of-1 Trials (RoBiNT) Scale. Neuropsychol Rehabil. 2013; 23:619-638.

10. Shamseer L, Sampson M, Bukutu C, Schmid CH, Nikles J, Tate R, et al. CONSORT extension for reporting N-of-1 trials (CENT) 2015: Explanation and elaboration. Bmj. 2015; 350:h1793.

11. Akerstedt A, Risto O, Odman P, Oberg B. Evaluation of single event multilevel surgery and rehabilitation in children and youth with cerebral palsy-A 2-year follow-up study. Disability and rehabilitation. 2010; 32:530-539.

12. Angelo J. Comparison of three computer scanning modes as an interface method for persons with cerebral palsy. The American journal of occupational therapy. 1992; : official publication of the American Occupational Therapy Association. 46:217-222.

13. Angelo J. Using single-subject design in clinical decision making: the effects of tilt-in-space on head control for a child with cerebral palsy. Assistive technology: the official journal of RESNA. 1993; 5:46-49.

14. Barbosa AP, Vaz DV, Benseman Gontijo Ana Paula AP, Fonseca ST, Mancini MC. Therapeutic effects of electrical stimulation on manual function of children with cerebral palsy: Evaluation of two cases. Disability and Rehabilitation. 2008; 30:723-728.

15. Beauregard R, Thomas JJ, Nelson DL. Quality of reach during a game and during a rote movement in children with cerebral palsy. Physical and Occupational Therapy in Pediatrics. 1998; 18:67-84.

16. Brien M, Sveistrup H. An intensive virtual reality program impoves balance and functional mobility of adolescents with cerebral palsy. Archives of Physical Medicine and Rehabilitation. 2010; 91 (10):e45. 
17. Brien $M$, Sveistrup $H$. An intensive virtual reality program improves functional balance and mobility of adolescents with cerebral palsy. Pediatric physical therapy: the official publication of the Section on Pediatrics of the American Physical Therapy Association. 2011; 23:258-266.

18. Chen Y, Kang L, Chuang T, Doong J, Lee S, Tsai M, et al. Use of virtual reality to improve upper-extremity control in children with cerebral palsy: a single-subject design. Physical Therapy. 2007; 87:1441-1457.

19. Coker P, Lebkicher C, Harris L, Snape J. The effects of constraint-induced movement therapy for a child less than one year of age. NeuroRehabilitation. 2009; 24:199-208.

20. Corn K, Imms C, Timewell G, Carter C, Collins L, Dubbeld S, et al. Impact of second skin lycra splinting on the quality of upper limb movement in children. British Journal of Occupational Therapy. 2003; 66:464-472.

21. Costigan FA, Light J. Effect of seated position on upper-extremity access to augmentative communication for children with cerebral palsy: Preliminary investigation. American Journal of Occupational Therapy. 2010; 64:596-604.

22. Crocker MD, MacKay-Lyons M, McDonnell E. Forced use of the upper extremity in cerebral palsy: a single-case design. The American journal of occupational therapy. 1997; : official publication of the American Occupational Therapy Association. 51:824-833.

23. Dada $\mathrm{S}$, Alant E. The effect of aided language stimulation on vocabulary acquisition in children with little or no functional speech. American Journal of Speech-Language Pathology. 2009; 18:50-64.

24. DeGangi GA, Hurley L, Linscheid TR. Toward a methodology of the short-term effects of neurodevelopmental treatment. American Journal of Occupational Therapy. 1983; 37:479-484.

25. Dinomais M, Veaux F, Yamaguchi T, Richard P, Richard I, Nguyen S. A new virtual reality tool for unilateral cerebral palsy rehabilitation: Two single-case studies. Developmental Neurorehabilitation. 2013; 16:418-422.

26. Do JH, Yoo EY, Jung MY, Park HY. The effects of virtual reality-based bilateral arm training on hemiplegic children's upper limb motor skills. NeuroRehabilitation. 2016; 38:115-127.

27. Durfee $J$, Billingsley FF. A comparison of two computer input devices for uppercase letter matching. The American journal of occupational therapy. 1999; : official publication of the American Occupational Therapy Association. 53:214-220.

28. Fienup DM, Mudgal D, Pace G. Increasing money-counting skills with a student with brain injury: skill and performance deficits. Brain Inj. 2013; 27:366-376.

29. Fragala MA, O'Neil ME, Russo KJ, Dumas HM. Impairment, disability, and satisfaction outcomes after lower-extremity botulinum toxin a injections for children with cerebral palsy. Pediatric Physical Therapy. 2002; 14:132-144.

30. Ghorbani N, Rassafiani M, Izadi-Najafabadi S, Yazdani F, Akbarfahimi N, Havaei N, et al. Effectiveness of cognitive orientation to (daily) occupational performance (CO-OP) on children with cerebral palsy: A mixed design. Research in Developmental Disabilities. 2017; 71:24-34.

31. Goodman G, Bazyk S. The effects of a short thumb opponens splint on hand function in cerebral palsy: a single-subject study. The American journal of occupational therapy. 1991; : official publication of the American Occupational Therapy Association. 45:726-731.

32. Hamill D, Washington $K$, White OR. The effect of hippotherapy on postural control in sitting for children with cerebral palsy. Physical \& Occupational Therapy in Pediatrics. 2007; 27:23-42. 
33. Harris SR, Riffle K. Effects of inhibitive ankle-foot orthoses on standing balance in a child with cerebral palsy: A single-subject design. Physical Therapy. 1986; 66:663-667.

34. Hartveld A, Hegarty J. Frequent weightshift practice with Computerised feedback by cerebral palsied children-Four single-case experiments. Physiotherapy. 1996; 82:573-580.

35. Havstam C, Buchholz M, Hartelius L. Speech recognition and dysarthria: A single subject study of two individuals with profound impairment of speech and motor control. Logopedics Phoniatrics Vocology. 2003; 28:81-90.

36. Hinderer KA, Harris SR, Purdy AH, Chew DE, Staheli LT, McLaughlin JF, et al. Effects of 'tone-reducing' vs. standard plaster-casts on gait improvement of children with cerebral palsy. Dev Med Child Neurol. 1988; 30:370-377.

37. Iammatteo PA, Trombly C, Luecke L. The effect of mouth closure on drooling and speech. The American journal of occupational therapy. 1990; : official publication of the American Occupational Therapy Association. 44:686-691.

38. Jelsma J, Pronk M, Ferguson G, Jelsma-Smit D. The effect of the Nintendo Wii Fit on balance control and gross motor function of children with spastic hemiplegic cerebral palsy. Developmental Neurorehabilitation. 2013; 16:27-37.

39. Johnson BA, Salzberg C, MacWilliams BA, Shuckra AL, D'Astous JL. Plyometric training: effectiveness and optimal duration for children with unilateral cerebral palsy. Pediatric physical therapy: the official publication of the Section on Pediatrics of the American Physical Therapy Association. 2014; 26:169-179.

40. Kelly ME, Darrah J, Sobsey R, Haykowsky M, Legg D. Effects of a community-based aquatic exercise program for children with cerebral palsy: a single subject design. Journal of Aquatic Physical Therapy. 2009; 17:1-11.

41. Kenyon LK, Farris JP, Aldrich NJ, Rhodes S. Does power mobility training impact a child's mastery motivation and spectrum of EEG activity? An exploratory project. Disability \& Rehabilitation: Assistive Technology. 2018; 13:665-673.

42. Kinghorn J, Roberts $G$. The effect of an inhibitive weight-bearing splint on tone and function: a single-case study. The American journal of occupational therapy. 1996; : official publication of the American Occupational Therapy Association. 50:807-815.

43. Ko MS, Lee JA, Kang SY, Jeon HS. Effect of Adeli suit treatment on gait in a child with cerebral palsy: a single-subject report. Physiotherapy theory and practice. 2015; 31:275-282.

44. Laessker-Alkema K, Eek MN. Effect of Knee Orthoses on Hamstring Contracture in Children With Cerebral Palsy: Multiple Single-Subject Study. Pediatric physical therapy : the official publication of the Section on Pediatrics of the American Physical Therapy Association. 2016; 28:347-353.

45. Lammi BM, Law M. The effects of family-centred functional therapy on the occupational performance of children with cerebral palsy. Can J Occup Ther. 2003; 70:285-297.

46. Laskas CA, Mullen SL, Nelson DL, Willson-Broyles M. Enhancement of two motor functions of the lower extremity in a child with spastic quadriplegia. Physical Therapy. 1985; 65:11-16.

47. Lephart K, Kaplan SL. Two Seating Systems' Effects on an Adolescent With Cerebral Palsy and Severe Scoliosis. Pediatric physical therapy : the official publication of the Section on Pediatrics of the American Physical Therapy Association. 2015; 27:258-266.

48. Lewis J, Pin T. Dynamic elastomeric fabric orthosis in managing shoulder subluxation in children with severe cerebral palsy: A case series. Dev Med Child Neurol. 2016; 58:61. 
49. Lilly LA, Powell NJ. Measuring the effects of neurodevelopmental treatment on the daily living skills of 2 children with cerebral palsy. The American journal of occupational therapy. 1990; : official publication of the American Occupational Therapy Association. 44:139-145.

50. Lin CY, Chang YM. Increase in physical activities in kindergarten children with cerebral palsy by employing MaKey-MaKey-based task systems. Research in Developmental Disabilities. 2014; 35:1963-1969.

51. Lin CY, Chang YM. Interactive augmented reality using Scratch 2.0 to improve physical activities for children with developmental disabilities. Research in Developmental Disabilities. 2015; 37:1-8.

52. Mackey $\mathrm{S}$. The use of computer-assisted feedback in a motor control task for cerebral palsied children. Physiotherapy. 1989; 75:143-148.

53. Man DW, Wong MS. Evaluation of computer-access solutions for students with quadriplegic athetoid cerebral palsy. The American journal of occupational therapy. 2007; : official publication of the American Occupational Therapy Association. 61:355-364.

54. Matthews MJ, Watson M, Richardson B. Effects of dynamic elastomeric fabric orthoses on children with cerebral palsy. Prosthetics and Orthotics International. 2009; 33:339-347.

55. McCarthy JH, Hogan TP, Beukelman DR, Schwarz IE. Influence of computerized sounding out on spelling performance for children who do and do not rely on AAC. Disability and rehabilitation. 2015; Assistive technology. 10:221-230.

56. McConnell K, Johnston L, Kerr C. Efficacy and acceptability of reduced intensity constraint-induced movement therapy for children aged 9-11 years with hemiplegic cerebral palsy: a pilot study. Physical \& occupational therapy in pediatrics. 2014; 34:245-259.

57. Owen SE, Stern LM. Management of drooling in cerebral palsy: three single case studies. International Journal of Rehabilitation Research. 1992; 15:166-169.

58. Pinder GL, Olswang LB. Development of communicative intent in young children with cerebral palsy: a treatment efficacy study. Infant-Toddler Intervention. 1995; 5:51-69.

59. Pool D, Blackmore AM, Bear N, Valentine J. Effects of functional electrical stimulation in children with spastic hemiplegia. Dev Med Child Neurol. 2014; 56:9-10.

60. Pool D, Blackmore AM, Bear N, Valentine J. Effects of short-term daily community walk aide use on children with unilateral spastic cerebral palsy. Pediatric physical therapy: the official publication of the Section on Pediatrics of the American Physical Therapy Association. 2014; 26:308-317.

61. Pratt B, Hartshorne NS, Mullens P, Schilling ML, Fuller S, Pisani E. Effect of Playground Environments on the Physical Activity of Children With Ambulatory Cerebral Palsy. Pediatric physical therapy: the official publication of the Section on Pediatrics of the American Physical Therapy Association. 2016; 28:475-482.

62. Prosser LA, Ohlrich LB, Curatalo LA, Alter KE, Damiano DL. Feasibility and preliminary effectiveness of a novel mobility training intervention in infants and toddlers with cerebral palsy. Developmental Neurorehabilitation. 2012; 15:259-266.

63. Prosser LA, Ohlrich L, Curatalo L, Alter K, Damiano D. Novel mobility training in prewalking toddlers with cerebral palsy. Dev Med Child Neurol. 2012; 54:23.

64. Ramani KK, Police SR, Jacob N. Impact of low vision care on reading performance in children with multiple disabilities and visual impairment. Indian journal of ophthalmology. 2014; 62:111-115.

65. Ray SA, Bundy AC, Nelson DL. Decreasing drooling through techniques to facilitate mouth closure. Am J Occup Ther. 1983; 37:749-753. 
66. Reid D, Rigby P, Ryan S. Functional impact of a rigid pelvic stabilizer on children with cerebral palsy who use wheelchairs: Users' and caregivers' perceptions. Pediatric Rehabilitation. 1999; 3:101-118.

67. Retarekar R, Fragala-Pinkham MA, Townsend EL. Effects of aquatic aerobic exercise for a child with cerebral palsy: single-subject design. Pediatric physical therapy : the official publication of the Section on Pediatrics of the American Physical Therapy Association. 2009; 21:336-344.

68. Rigby P, Reid D, Schoger S, Ryan S. Effects of a Wheelchair-Mounted Rigid Pelvic Stabilizer on Caregiver Assistance for Children with Cerebral Palsy. Assistive Technology. 2001; 13:2-11.

69. Rintala P, Era P. Posture control in children with cerebral palsy; a pilot study. Journal of Rehabilitation Sciences. 1994; 7:9-14.

70. Rivi E, Filippi M, Fornasari E, Mascia MT, Ferrari A, Costi S. Effectiveness of standing frame on constipation in children with cerebral palsy: a single-subject study. Occupational therapy international. 2014; 21:115-123.

71. Sakemiller LM, Nelson DL. Eliciting functional extension in prone through the use of a game. The American journal of occupational therapy. 1998; : official publication of the American Occupational Therapy Association. 52:150-157.

72. Sheppard L, Mudie H, Froude E. An investigation of bilateral isokinematic training and neurodevelopmental therapy in improving use of the affected hand in children with hemiplegia. Physical and Occupational Therapy in Pediatrics. 2007; 27:5-25.

73. Shumway-Cook A, Hutchinson S, Kartin D, Price R, Woollacott M. Effect of balance training on recovery of stability in children with cerebral palsy. Dev Med Child Neurol. 2003; 45:591-602.

74. Siconolfi-Morris GC. Use of a video game based balance training intervention on the balance and function of children with developmental disabilities: University of Kentucky; 2012.

75. Smelt HR. Effect of an inhibitive weight-bearing mitt on tone reduction and functional performance in a child with cerebral palsy. Physical and Occupational Therapy in Pediatrics. 1989; 9:53-80.

76. Smithers JA. Facilitation of rolling in a child with athetoid cerebral palsy-A single-subject design. Physiotherapy. 1991; 77:243-248.

77. Soto G, Yu B, Kelso J. Effectiveness of multifaceted narrative intervention on the stories told by a 12-yearold girl who uses AAC. Augmentative and alternative communication (Baltimore, Md. 2008; : 1985). 24:76-87.

78. Stansfield S, Dennis C, Larin H, Gallagher C. Movement-Based VR gameplay therapy for a child with cerebral palsy. Annual Review of CyberTherapy and Telemedicine. 2015; 13:153-157.

79. Stewart H, Noble G, Seeger BR. Isometric joystick: A study of control by adolescents and young adults with cerebral palsy. Australian Occupational Therapy Journal. 1992; 39:33-39.

80. Stewart H, Wilcock A. Improving the communication rate for symbol based, scanning voice output device users. Technology \& Disability. 2000; 13:141-150.

81. Svedberg LE, NordahI UE, Lundeberg TC. Effects of acupuncture on skin temperature in children with neurological disorders and cold feet: an exploratory study. Complement Ther Med. 2001; 9:89-97.

82. Symons FJ, Tervo RC, Kim O, Hoch J. The effects of methylphenidate on the classroom behavior of elementary school-age children with cerebral palsy: A preliminary observational analysis. Journal of Child Neurology. 2007; 22:89-94.

83. Thorpe DE, Valvano J. The effects of knowledge of performance and cognitive strategies on motor skill learning in children with cerebral palsy. Pediatric Physical Therapy. 2002; 14:2-15. 
84. Ustad T, Sorsdahl AB, Ljunggren AE. Effects of intensive physiotherapy in infants newly diagnosed with cerebral palsy. Pediatric Physical Therapy. 2009; 21:140-149.

85. Voulgarakis H, Forte S. Escape extinction and negative reinforcement in the treatment of pediatric feeding disorders: A single case analysis. Behavior Analysis in Practice. 2015; 8:212-214.

86. Ward R, Leitao S, Strauss G. An evaluation of the effectiveness of PROMPT therapy in improving speech production accuracy in six children with cerebral palsy. International journal of speech-language pathology. 2014; 16:355-371.

87. Ward R, Strauss G, Leitao S. Kinematic changes in jaw and lip control of children with cerebral palsy following participation in a motor-speech (PROMPT) intervention. International journal of speech-language pathology. 2013; 15:136-155.

88. Michiels B, Onghena P. Randomized single-case AB phase designs: Prospects and pitfalls. Behav Res Methods. 2018.

89. Tate RL, McDonald S, Perdices M, Togher L, Schultz R, Savage S. Rating the methodological quality of single-subject designs and n-of-1 trials: introducing the Single-Case Experimental Design (SCED) Scale. Neuropsychol Rehabil. 2008; 18:385-401.

90. Tate RL, Perdices M, Rosenkoetter U, Shadish W, Vohra S, Barlow DH, et al. The Single-Case Reporting Guideline In BEhavioural Interventions (SCRIBE) 2016 Statement. Neuropsychol Rehabil. 2017; 27:1-15.

91. Romeiser Logan L, Hickman RR, Harris SR, Heriza CB. Single-subject research design: recommendations for levels of evidence and quality rating. Dev Med Child Neurol. 2008; 50:99-103. 


\section{Chapter I2}

General discussion 

The overall aim of this dissertation was to generate scientific knowledge on the chances and challenges of home-based bimanual training in children with UCP. The previous chapters reported the results of the research project COAD ("CO-creation At hanD: the road to independence"). In this chapter, first the main findings of these studies are discussed, followed by an overview of methodological considerations, implications for clinical practice, and directions for future research.

\section{INTERPRETATION OF MAIN FINDINGS}

\section{HOME-BASED BIMANUAL TRAINING}

This section presents an overview of the conclusions from the COAD-project regarding homebased bimanual training. To describe the coherence between the main findings of the different studies of this dissertation, a logic model was developed. A logic model is a graphical representation of a specific program that links outcomes with program activities and processes as well as the theoretical assumptions. ${ }^{1}$ The current logic model proposes an improved version of the home-based training program. The amendments are based on the conclusions of this dissertation. The input for the logic model was derived from Chapter 7, 8, and 10. The study on barriers to recruitment provided the main input for the identification of resources. Resources (or inputs) include the human, financial, and organizational resources available to the program. The expected outcomes of the program are established from the hypotheses generated from the case series and process evaluation. I would like to emphasize that the logic model, presented in Figure 1, is an instant overview at this point in time. As the training program and evidence base develops, the model should be refined.

The assumptions on which the original training program was based have been formed by a literature search, described in the general introduction, and are also applicable to the improved program. Enabling and limiting resources are distinguished in the model for the improved program. Enabling resources are financial reimbursement by health insurance companies; an efficient infrastructure within centers; and an effective data sharing system. Limiting resources, on the other hand, are decentralization of social policy, restricting the possibilities of financial investments by centers; travel time and uncovered expenses; limited availability of therapists and remedial educationalists; a culture in which health-care providers patronize families; pressure perceived by families, restricting their time and energy investment in training; and parents being hesitant to provide therapy. These resources all influence the implementation of the program activities. These activities are the instructional course for health-care professionals; the task-analysis; the exchange of video-recorded training sessions; coaching by a therapist, including telephone appointments and home visits; and coaching by a remedial educationalist, including video call appointments. The instructional course, task-analysis, and the exchange of video-recorded training sessions affect the coaching by the therapist. The former and latter also affect the coaching by the remedial educationalist. The program activities are expected to result in varying doses of delivered home-based training. From the findings of varying training intensity and parents experiencing no or limited parental stress, it can be hypothesized that parents, either consciously 
or unconsciously, self-regulate training intensity in order to avoid overburdening. In addition, the low inclusion numbers may reflect parental consideration of their ability to accomplish the home-based training program under study with an acceptable level of stress.

The outcomes for the child are hypothesized to show an increased quality and quantity of performance of the activities indicated as personal goals, increase of spontaneous use of the affected hand in bimanual activities that were not related to the personal goals, and improved performance of these activities, increased self-confidence, and increased independency. The parent-related outcome measures revealed that parents' knowledge on skill acquisition increased, as well as their self-confidence. The findings of the case series and process evaluation were not completely consistent with each other with regard to the performance of non-trained activities. In the case series no effects on performance tests was found, whereas parents indicated in the process evaluation that their child more often and better performed bimanual activities that were not trained. Three possible explanations for these differences can be given. First, a previous study showed that after intensive rehabilitation treatment, parents' and experts' perception of activities of daily living status is incongruent: parents estimated their child's performance as better. ${ }^{2}$ It is likely that this effect is even stronger after parent-delivered therapy, because of the effort of parents. Second, standardized tests may not be sensitive enough to detect subtle changes in a wide variety of activities that can only be observed in daily context and shared by parents. Third, parents also explained that their child's self-confidence grew, resulting in increased initiative to perform activities. Hence, these changes instead of improved bimanual capacity may lead to increased bimanual performance in daily life, not detectable by the tests. Only short-term outcomes are incorporated in the logic model, as the research that led to this dissertation was not focused on long-term outcomes. In addition, the impact of the program, i.e. intended or unintended change occurring in rehabilitation organizations, the CP community, or family systems as a result of program activities, was not gauged. This impact is still a black box and therefore presented as such in the logic model.

\section{THERAPY-RELATED PARENTAL STRESS}

Therapy-related parental stress has not been included as an outcome in the logic model, since this is an unintended adverse effect of the program. From the results of the systematic review and our own findings, an interesting phenomenon has been observed. Qualitative interview studies showed that parents who delivered home-based training experienced stress to a greater or lesser extent. ${ }^{3}$ However, quantitative measurement with the Parental Stress Index (PSI), or the comparable Dutch Opvoedingsbelastingvragenlijst (OBVL) in the present study, did not detect increased stress levels. ${ }^{4,5}$ This can be attributed to the fact that these questionnaires measure more generic parental stress and are therefore not responsive to changes in therapy-related parental stress. Therefore, in Chapter 5 we proposed that therapy-related parental stress needs more attention, and that an instrument to measure this specific construct for clinical and research objectives is needed. For this reason, we initiated a satellite project on the development of such an instrument. We aimed to assess both stress intensity and contributing stressors. The latter requires a formative framework, meaning that the items (i.e. stressors) constitute the construct (i.e. therapy-related 


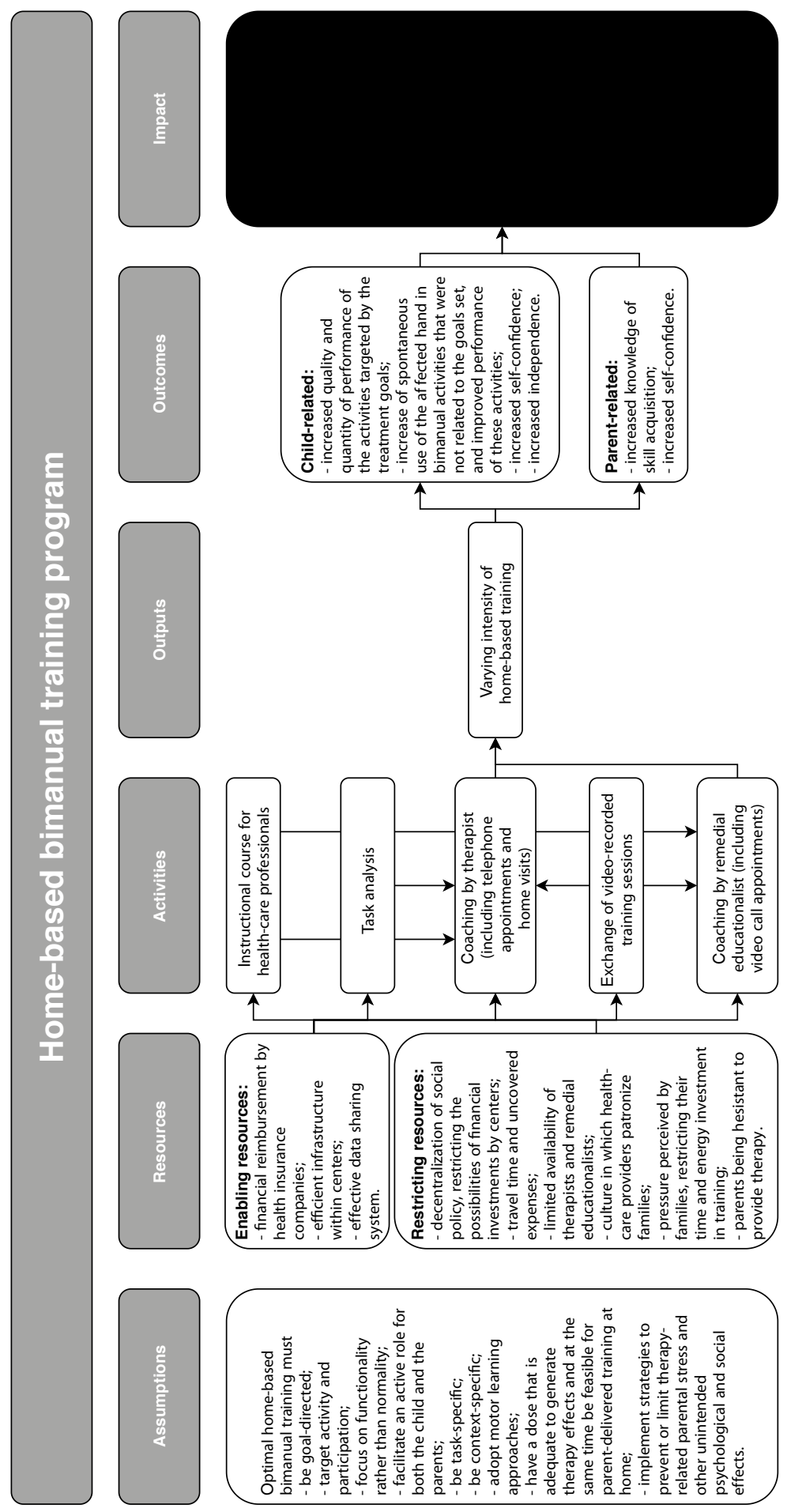

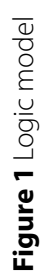


parental stress). ${ }^{6}$ During the development process, we first organized a focus group with parents of children with physical disabilities and a remedial educationalist to explore the construct of therapy-related parental stress. The results of the focus group were used to identify stressors whereby parents may experience therapy-related parental stress. Using this information, we constructed a first draft of a questionnaire. Thereafter, consultation with two experts on questionnaire development and psychometric evaluation led to adaptions and a provisional version of the questionnaire, entitled the Therapy-related Parental Stress Questionnaire (in Dutch:Vragenlijst Ouderlijke Stress als gevolg van Therapie van het kind (VOST)). The content of this provisional questionnaire has been described in Chapter 8. An extensive pilot test among parents of children with a disability has been performed to investigate parents' experiences filling out the questionnaire and to evaluate applicability, completeness, terminology, and lay-out. Currently a field test of the amended questionnaire is running to reduce the number of items and to develop a scoring system. Assessment of the psychometric properties is a necessary next step.

A significant point of attention emerged during the interviews of the process evaluation as well as the field test. Parents experience impact of attending and/or providing therapy on their well-being, but they often do not describe these feelings with the term stress. This finding raises the fundamental concern that conceptualization of the constructs of caregiver strain and therapy-related parental stress need to be improved.

\section{RESEARCH DESIGNS}

The appropriateness of different research designs for intervention research in pediatric rehabilitation was not a topic of interest at the beginning of the project, but emerged as the project progressed. Chapter 7 showed that the RCT design partly explained the recruitment problems of the effect study. RCTs are generally accompanied with high costs, demanding coordination within multiple dynamic clinical settings, and large sample sizes. Recruiting large numbers of participants is a well-known challenge within the field of (pediatric) rehabilitation research. Many parents are invited to participate, which may attribute to the research fatigue and selectivity that has been reported by parents and health-care providers, in turn negatively affecting the recruitment of future studies. Additionally, the randomization aspect is a reason for non-participation of families, since parents can have strong opinions towards an intervention or do not want to withhold their child a possible effective intervention. We argue that an iterative process in intervention development and evaluation is better achievable. It enables early detection of difficulties, particularly given the complexity of rehabilitation interventions.? Stakeholders suggested wait-list control group and preference-based randomization, as well as (stepped-wedge) cluster randomization as potential alternatives. Since these designs still require many participants, to my opinion the single-case experimental design (SCED) is the way forward. In our scoping review (Chapter 11) we discussed that the SCED enables clinically-meaningful intervention research in the heterogeneous population of children and adolescents with CP. To make this design even more powerful, standards for the complex pediatric rehabilitation setting should be advanced and carefully followed by researchers. In addition to these methodological issues, there is another major concern. Funders and their referees still regard the RCT as superior in intervention research. 
I challenge them to move forward and to prioritize the suitability of the design to answer the research question. For calls where proposals of different fields compete, one should consider the unique features of rehabilitation and their consequences for the methodological choices. Researchers who share our opinion, for their part, should attempt to educate others. Sharing exemplary high-quality studies in the (pediatric) rehabilitation area may help to convince criticists.

\section{METHODOLOGICAL CONSIDERATIONS}

The individual chapters include an overview of limitations and methodological reflections. Particularly Chapter 7 provides a profound analysis of lessons learned from the COAD-study. This general discussion is therefore limited to some overarching and additional considerations.

\section{MIXED METHODS RESEARCH}

The COAD-study adopted a mixed methods methodology, because neither a quantitative nor a qualitative approach would have been sufficient to answer our research questions. An additional benefit of this methodology was that the strengths of each approach compensated for the weaknesses of the other. ${ }^{8}$ Creswell and Clark recognize that researchers need to possess knowledge and skills of quantitative as well as qualitative procedures in order to conduct a proper mixed methods study. ${ }^{8}$ Since the composition of the research team in terms of expertise was merely quantitative, an expert in qualitative research and mixed methods methodology contributed to the project. We are confident that this was appropriate to achieve a rigorous research process. A second challenge of mixed methods research is the demand of research time, study duration, and associated costs. ${ }^{8}$ Because of the embedded design of our study, the participants were involved in both the quantitative and qualitative data collection. This created an issue of high research burden for the participants, particularly the parents.

\section{Children's involvement}

Given the young age of the participants (2-7 years), parent proxies identified the needs of the children, which was the starting point for goal setting and the home-based training. One of the findings of the process evaluation was that the goals should match the child's interest, and that this was not always the case. In other studies, children of the same age as our participants identified and scored goals themselves, using the Canadian Occupational Performance Measure (COPM), Goal Attainment Scaling (GAS), or Perceived Efficacy and Goal Setting System (PEGS). ${ }^{9}$ The latter measure uses illustrated and blank picture cards to indicate the activities the child wants to improve on. Thus, in our study it may have been feasible to take the opinion of the children into account during the goal setting process as well as evaluation. Likewise, the children's voices were not directly heard in the process evaluation. Young children can be interviewed, although this requires time, effort, and specific techniques. ${ }^{10,11}$ While this was not feasible in terms of research burden of families, interviewing the participating children would have likely added an additional level of information gathered during our process evaluation. 


\section{OUTCOME MEASURES}

Our study aimed to cover all relevant constructs of the activity and participation components of the Children \& Youth Version of the International Classification of Functioning, Disability, and Health (ICF-CY). This resulted in a set of relatively many relevant measures. Even though this was in itself a strength of the study, some problems arose by doing so. To begin with, parents indicated that they felt uncomfortable to complete the questionnaires that targeted their feelings and behavior (parenting style, personality of the parent, and parental stress), resulting in a high number of missing data. They had difficulties recognizing the relevance for the research and did not identify themselves with the questions asked. This highlights the importance of a continued dialogue between participants and researchers from the start of planning until the closure of the study. I think that participants need to be better educated about the rationale of standardized data collection, outcome measures, and repeated measurements. Researchers, on the other hand, are responsible for hearing and taking into account the participants' concerns. Another issue was that the measurements were obviously time-consuming for parents, particularly at baseline. A possible solution may be computer-adaptive tests (CATs). Using item-response theory (IRT) models and developments in technology, the presented questions are based on the respondent's answers on previous ones. This targeting to the individual, results in more relevant items. It is also more efficient, requires fewer items and thus a shorter response time and less burden for the participant. ${ }^{12}$ The Patient Reported Outcomes Measurement Information System (PROMIS $\left.{ }^{\circ}\right)$ network generates domain-specific measures operationalized by large item bank, which can be administered by CATs. ${ }^{13}$ For the pediatric population, Dutch-Flemish translations are available for nine self-report item banks, including physical function-upper extremity, physical function mobility, peer relationships, and depressive symptoms. ${ }^{14}$ CATs and the PROMIS are continuously enhanced. Validating CATs and implementing them in intervention studies is a promising future research priority.

Goal Attainment Scaling (GAS), an individualized outcome measure of our effect study, involves a comprehensive procedure. Krasny-Pacini et al. propose GAS quality appraisal criteria, that were not available prior to our study, though. ${ }^{15}$ They acknowledge that it is challenging to meet all criteria. Our study applied collaborative goal setting; GAS corresponding to the functional ICF-CY activity domains; adaptation to the specific time frame of the study; precise description of GAS levels preintervention, and verification of GAS scales by an external judge to check if the scale represented relevant goals, specificity to the aim of home-based training, clinically meaningful change, equal distance of levels, attainability/difficulty, measurability, and unidimensionality. In conclusion, our study met 13 of the 17 criteria. A recent review systematically appraised the scientific rigor of studies in the field of pediatric rehabilitation using GAS, and found that on average 4.71 criteria were met. ${ }^{16}$ Comparison with this result indicates that our GAS procedure was overall accurate. Still, four criteria were not met. First, not all therapists who were supposed to set the GAS had completed a formal GAS training before the start of our COAD-study. Therefore, a practical GAS training, which is in accordance with recommendations, was incorporated in the instructional course of the COAD-study. ${ }^{15,16}$ However, this training was less extensive and included limited 
practicing of GAS writing. Previous research suggested that months of experience are needed before the GAS can be used in a valid and reliable wayt. ${ }^{17,18}$ Second, context of performance measurement should be clearly defined and controlled for to warrant scoring reproducibility. ${ }^{15}$ Retrospectively, this was inadequately done, particularly for instructions and amount of verbal or physical help provided. Third, preintervention performance should be verified and correspond to the initial level described in the scale $(-2) .{ }^{15}$ This was not employed in our study. Performance in general and specifically in children can be variable, for instance due to motivation and before mentioned contextual aspects. Due to this, not having preintervention performance verified may have led to an inaccurate reflection of the true level of initial performance during formulation of the GAS scales. Fourth and last, interrater reliability was not calculated for the GAS scales of our study. The potentially insufficient training, limited controlled context, lack of verification of preintervention performance, and absence of interrater reliability calculation could all have negatively affected the validity of the findings.

\section{STATISTICAL APPROACH}

As in almost all studies in pediatric rehabilitation, for our RCT we planned statistical methods based on the frequentist (classical) paradigm. An alternative approach is Bayesian statistics. The Bayesian paradigm offers a different view: "progress in science generally comes about by learning from previous research findings and incorporating information from these research findings into our present studies.19 ${ }^{\prime 19}$ Cumulative knowledge is generated by compromising the prior distribution (based on all knowledge available) and the likelihood function (obtained from the data) into the posterior distribution. Advantages of this method include a reduction of the required sample size to obtain statistically significant results, more accurate results for non-normally distributed data, and more intuitive interpretation of results. ${ }^{19}$ Because at the time, effectiveness of homebased bimanual training had not been addressed in previous studies, there was not sufficient prior information available (i.e. a noninformative prior distribution) to determine posterior distributions. This made us decide against Bayesian statistical methods. However, the data from the COAD-study can be a source of information for prior probability of future studies. In addition, I plea for more recognition of Bayesian approaches, and strongly encourage fellow researchers to consider applying them. Particularly in view of the recruitment problems encountered by us and many others.

\section{IMPLICATIONS FOR CLINICAL PRACTICE}

The findings of this dissertation have a number of practical implications. Taken together, the results of the case series and process evaluation do support the use of home-based bimanual training in clinical practice. Home-based bimanual training should be considered as part of a care pathway interspersed with center-based training. Ensuring appropriate home-based bimanual training of children with $\mathrm{UCP}$, programs should include (1) a priori instruction of the health-care professionals, (2) a task-analysis performed by the therapist, (3) exchange of video-recorded 
training sessions, and (4) coaching of the parents by a therapist and remedial educationalist. It is important to individualize the frequency and mode of coaching contact (e.g. telephone appointment or home visit) to the needs and preferences of the parents.

In both home-based training and other interventions, health-care professionals should acknowledge the potential of therapy-related parental stress. Awaiting validated questionnaires, I recommend professionals to discuss parental well-being, caregiver strain and therapy-related parental stress repeatedly with parents during their child's rehabilitation process.

\section{DIRECTIONS FOR FUTURE RESEARCH}

Given the case series design, our study provides inconclusive evidence of the effectiveness of the home-based bimanual training programs. More work is required to confirm our findings. Likewise, an effect study is needed to demonstrate causality of some outcomes that were derived from the process evaluation, such as increased self-confidence. Future research should target an amended version of our home-based training program, using the suggestions of this dissertation as incorporated in the logic model. In addition, there is abundant room for progress in determining alternative home-based bimanual training programs, for instance a combined home- and center-based program. Special attention should be paid to the coaching process, well-being of parents during the home-based training, and the way children perceive the training. The programs under research should either be described in detail in the final report, or published in separate protocols. Research on implicit and explicit motor learning needs to focus on the suitability of approaches for individual children, parents, and phases of learning, rather than testing the dominance of one strategy compared to the other.

There are still many unanswered questions about therapy-related parental stress. To develop a complete picture of this construct and to find the best way to measure it, additional studies will be needed. Moreover, the area of therapy-related parental stress, caregiver strain, and parent well-being should be embedded in intervention research in pediatric rehabilitation.

Despite the promising benefits of SCEDs, future studies on the methodological and statistical procedures of this design within pediatric rehabilitation are advocated. In the meantime, I strongly encourage fellow researchers to consider this design for intervention studies and transparently share detailed protocols a priori and their experiences with the design afterwards. 


\section{REFERENCES}

1. W.K. Kellogg Foundation. Using Logic Models to Bring Together Planning, Evaluation, and Action. Logic Model Development Guide. 2004.

2. Paradis J, Dispa D, De Montpellier A, et al. Reliability of questionnaires after an intensive motor intervention for children with cerebral palsy (CP): a randomized trial. In: European Academy of Childhood Disability Conference Stockholm (Sweden), 2016.

3. Peplow UC and Carpenter C. Perceptions of parents of children with cerebral palsy about the relevance of, and adherence to, exercise programs: a qualitative study. Physical \& occupational therapy in pediatrics 2013; 33: 285-299. 2013/03/13. DOI: 10.3109/01942638.2013.773954.

4. Ferre $C L$, Brandao $M B$, Hung $Y C$, et al. Feasibility of caregiver-directed home-based hand-arm bimanual intensive training: a brief report. Developmental neurorehabilitation 2015; 18: 69-74. 2014/09/03. DOl: 10.3109/17518423.2014.948641.

5. Lin KC, Wang TN, Wu CY, et al. Effects of home-based constraint-induced therapy versus dose-matched control intervention on functional outcomes and caregiver well-being in children with cerebral palsy. Research in developmental disabilities 2011; 32: 1483-1491. 2011/03/25. DOI: 10.1016/j.ridd.2011.01.023.

6. de Vet HCW, Terwee CB, Mokkink LB, et al. Measurement in Medicine: A Practical Guide. Cambridge University Press, 2011.

7. Wade DT, Smeets RJ and Verbunt JA. Research in rehabilitation medicine: methodological challenges. Journal of clinical epidemiology 2010; 63: 699-704. 2009/10/01. DOI: 10.1016/j.jclinepi.2009.07.010.

8. Creswell JW and Clark VLP. Designing and Conducting Mixed Methods Research. SAGE Publications, 2011.

9. Tam C, Teachman G and Wright V. Paediatric Application of Individualised Client-Centred Outcome Measures: A Literature Review. 2008; 71: 286-296. DOI: 10.1177/030802260807100706.

10. O'Reilly M and Dogra N. Interviewing Children and Young People for Research. SAGE Publications, 2016.

11. Morse JM. Qualitative Nursing Research. SAGE Publications, 1991.

12. Smith $A B$, Hanbury A and Retzler J. Item banking and computer-adaptive testing in clinical trials: Standing in sight of the PROMISed land. Contemporary clinical trials communications 2019; 13: 005-005. 2018/12/26. DOI: 10.1016/j.conctc.2018.11.005.

13. Alonso J, Bartlett SJ, Rose M, et al. The case for an international patient-reported outcomes measurement information system (PROMIS(R)) initiative. Health and quality of life outcomes 2013; 11: 210. 2013/12/24. DOl: 10.1186/1477-7525-11-210.

14. Haverman $L$, Grootenhuis MA, Raat $H$, et al. Dutch-Flemish translation of nine pediatric item banks from the Patient-Reported Outcomes Measurement Information System (PROMIS)(R). Quality of life research : an international journal of quality of life aspects of treatment, care and rehabilitation 2016; 25: 761-765. 2015/03/31. DOI: 10.1007/s11136-015-0966-y.

15. Krasny-Pacini A, Evans J, Sohlberg MM, et al. Proposed Criteria for Appraising Goal Attainment Scales Used as Outcome Measures in Rehabilitation Research. Archives of physical medicine and rehabilitation 2016; 97: 157-170. 2015/09/08. DOI: 10.1016/j.apmr.2015.08.424.

16. Harpster K, Sheehan A, Foster EA, et al. The methodological application of goal attainment scaling in pediatric rehabilitation research: a systematic review. Disability and rehabilitation 2018: 1-10. 2018/06/30. DOI: 10.1080/09638288.2018.1474952. 
17. KiresukTJ, Smith A and Cardillo JE. Goal Attainment Scaling: Applications, Theory, and Measurement. Taylor \& Francis, 2014.

18. Steenbeek $D$, Ketelaar M, Lindeman E, et al. Interrater reliability of goal attainment scaling in rehabilitation of children with cerebral palsy. Archives of physical medicine and rehabilitation 2010; 91: 429-435. 2010/03/20. DOI: 10.1016/j.apmr.2009.10.013.

19. van de Schoot R, Kaplan D, Denissen J, et al. A gentle introduction to bayesian analysis: applications to developmental research. Child development 2014; 85: 842-860. 2013/10/15. DOI: 10.1111/cdev.12169. 


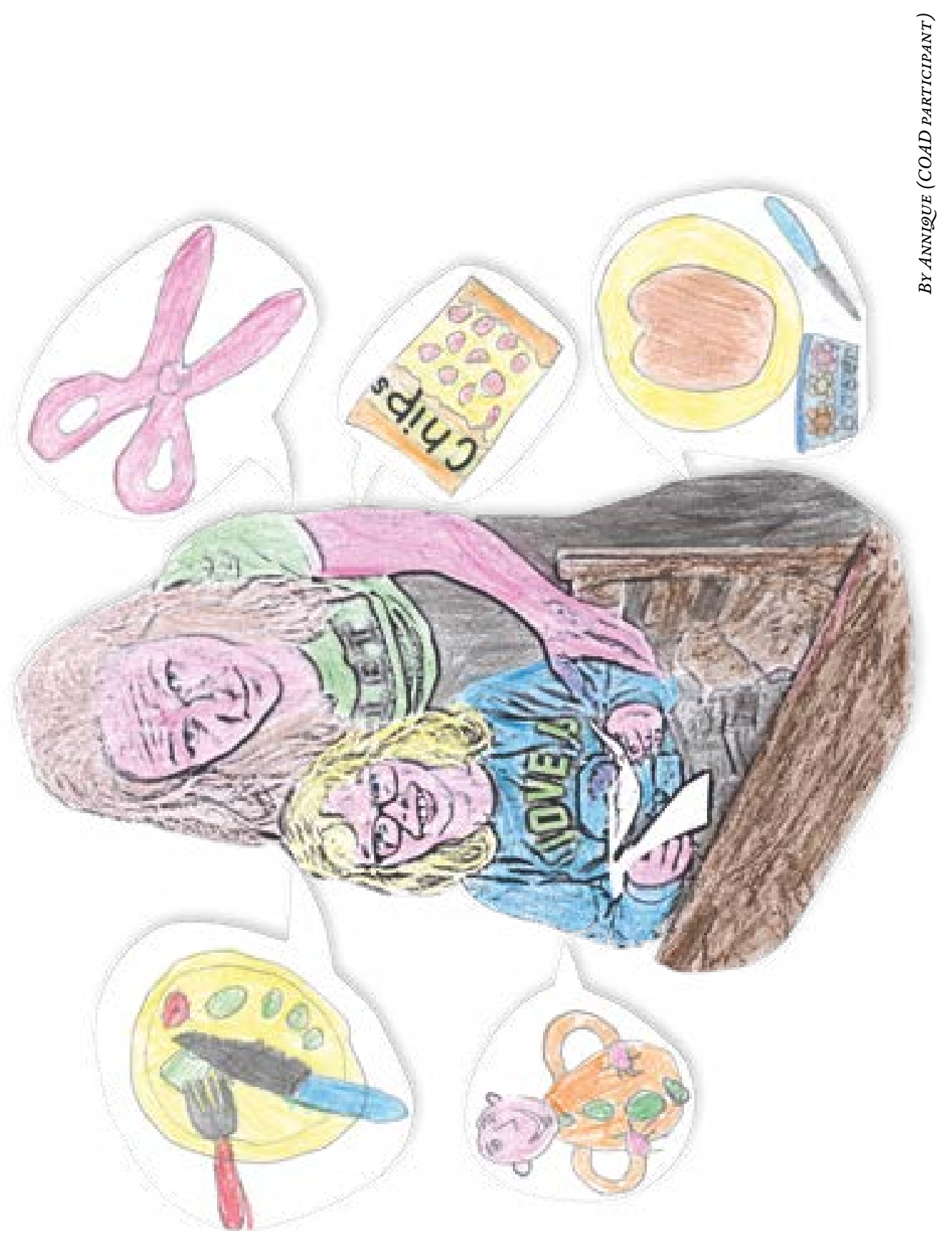



VALORIZATION ADDENDUM 

With the work presented in this dissertation we aimed to generate- scientific knowledge on the chances and challenges of home-based bimanual training in children with unilateral cerebral palsy (UCP). The theoretical and practical implications of our results were described in the various chapters throughout this dissertation. Below, our findings are discussed in a broader societal context. According to the 'Regulations for obtaining a PhD at UM', in this addendum I consider valorization as "the process of creating value from knowledge, by making knowledge suitable and/or available for social (and/or economic) use and by making it suitable for translation into competing products, services, processes and new activities" (definition taken from the report of the National Valorisation Committee, Waardevol: Indicatoren voor Valorisatie [Valuable: Indicators for valorisation] (2011) The Hague: Rathenau Institute, p. 8). The addendum will address 1) the social relevance of the research results; 2 ) the target groups to whom the research results are of interest; 3 ) the products and activities in which the research results will be applied and given shape; 4) the extent to which the research results can be called innovative; and 5) how the valorization plan will be implemented.

\section{RELEVANCE}

$\mathrm{CP}$ is the most common cause of motor disability in children. The impaired functioning of the arm and hand in children with UCP negatively affects independence, participation, and quality of life. In addition to the child itself, these consequences also impact the parents and siblings. Optimizing the care for children with UCP, to which the results of this dissertation contribute, is therefore valuable for the many families involved. Because no cost-effectiveness study has been performed, no conclusions can be drawn yet regarding the economic relevance of our findings.

\section{TARGET GROUP}

We intended to generate research evidence for the benefit of children with $\mathrm{CCP}$ and their parents. Hence, in addition to researchers, grant providers, and others within the academic community, the results of this dissertation are primarily of interest to these children and their parents. The research is also highly relevant for the rehabilitation teams that are involved in the care of these families. Pediatric occupational and physical therapists as well as remedial educationalists are important target groups, because they are the parents' coaches during the home-based training. Moreover, physiatrists can use the findings when discussing treatment plans with parents. It should be recognized that at this point no generalization is possible to children other than the population of interest in the study. To a lesser extent, policy makers can adopt our conclusions in the development or update of clinical guidelines, although the limited evidential value of the case series should be considered appropriately. Last, awaiting future research on the cost-effectiveness of home-based training, our results are of interest to health insurance companies who fund the program. 


\section{Products and activities}

Our work has already been shaped into several products and activities. First, the paper as presented in chapter 4 provides a detailed description of the intervention protocol, in order for clinicians and fellow researchers to use a comparable home-based training program. Second, the translation and adaptation of the Lifestyle Assessment Questionnaire for children with cerebral palsy (LAQ-CP) resulted in a Dutch version of the questionnaire, which is freely available at: http:// links.Iww.com/PPT/A165. Last, dissemination activities included presentations and mini-symposia at national and international conferences. For the occasion of the defense of this dissertation, a one-day symposium is organized by the Centre of Expertise in Rehabilitation and Audiology of Adelante in collaboration with the Department of Rehabilitation Medicine (Care and Public Health Research Institute (CAPHRI)) of Maastricht University. The symposium targets researchers, clinicians, as well as parents. During the symposium both our results and work of others will be discussed.

There are various other opportunities to translate the insights of this dissertation into products and activities, apart from the scientific output. Given the complexity of the home-based training program, during the research we developed and provided a manual and instructional course to the healthcare professionals, and instructional videos to parents. These can be shaped into products to be implemented within the Netherlands or, after translation, internationally. The products should be amended using the results of this dissertation. The process evaluation pointed out the importance of a user-friendly tool to share videos and other data between parents and healthcare professionals. The existing system arQive was used in an inventive way to suit the needs within our intervention. It was concluded that arQive is promising to facilitate homebased training. A next step is to optimize its functionalities and implement it thereafter. In order to inform parents and the general population about the research results, lay information will be disseminated where possible, for instance through social media, and opportunities for media attention will be seized.

\section{INNOVATION}

As the systematic review pointed out, many home-based interventions have been investigated, although not in the Netherlands. Furthermore, our study was one of the first to apply bimanual training in the home-based setting. Another important innovative aspect was the interdisciplinary coaching by a therapist and remedial educationalist, which emerged to be a highly important component of the program. The distinction between an implicit and explicit motor learning approach, though not further recommended based on our results, was another novel facet. In addition to the intervention itself, the extensive process evaluation was an original research method in respect to home-based interventions. 


\section{Planning and implementation}

Part of the research team recently started a new study: the \#Eurekah project (Effectieve data-Uitwisseling tijdens de REvalidatie van Kinderen met Arm-/handproblematiek: een Haalbaarheidsstudie). In this feasibility study it will be investigated whether arQive is indeed suitable to exchange data effectively during rehabilitation of children with arm/hand problems.

More work is needed to confirm the effectiveness of the home-based training program, before valorization of the intervention-related products should occur. Based on the experiences during this project, as described in chapter 7, I would recommend a well-designed single-case experimental design study of the optimized home-based training program. Pending these developments, no planning to implement the program can be provided yet. 

SUMMARY 

This dissertation presents the results of the research project COAD ("CO-creation At hanD: the road to independence"). The overall aim of this dissertation was to generate scientific knowledge on the chances and challenges of home-based bimanual training in children with unilateral cerebral palsy (uCP).

Given the promising advantages of upper extremity home-based programs in children with cerebral palsy (CP), a systematic review of the available literature on this topic was warranted. Chapter $\mathbf{2}$ describes the protocol of a systematic review, of which the purpose was to investigate currently available home-based occupational therapy and physiotherapy programs regarding both their feasibility and effect. The systematic review was developed in accordance with the Preferred Reporting Items for Systematic Review and Meta-Analysis Protocols (PRISMA-P) 2015. Studies were included in which primary data were collected, participants were children aged $<18$ years with any type of CP and the intervention of interest was a home-based occupational therapy or physiotherapy intervention. Comparators of interest were: no therapy, care as usual, center-based occupational therapy or physiotherapy, an alternative home-based program and a medical intervention. Studies were included that report either on feasibility (i.e., acceptability, demand, implementation, practicality, adaptation, expansion or integration) or on efficacy/ effectiveness (i.e., child-related upper extremity outcomes within all International Classification of Functioning, Disability and Health levels or parent-related/caregiver-related outcomes on the psychological and social domain). Relevant studies were identified by searching the databases MEDLINE, EMBASE, CINAHL, PsycINFO, PEDro, OTSeeker and CPCI-S as well as the trial registers ICTRP and CENTRAL, the reference lists of included records and by circulating a bibliography of the included records to authors of included studies. There were no restrictions on language or year of publication. The search strategy consisted of terms related to the population and intervention. Data were extracted in duplicate using a digital data extraction form.

Chapter 3 describes the results of this systematic review. A total of 47 studies were included, 22 feasibility studies (46.8\%), 10 effectiveness studies (21.3\%) and 15 studies that reported on both (31.9\%). There was a large variation in child characteristics, whereas the vast majority of studies did not report any parent characteristics. The treatment approach was predominantly (modified) Constraint-Induced Movement Therapy (CIMT) (40\%). The objectives of the intervention, the use of motor learning principles, and the coaching of parents were mostly unspecified. The training duration of the parent-delivered home-based training varied from 2 weeks to 6 months, and intensity from 70 minutes to 21 hours per week. The overall compliance to training (implementation) was moderate to high (62\% to 96.1\%). The majority of feasibility studies reported that parents found it easy to carry out the program and enjoyed seeing their children improve (acceptability). Conclusions about the effectiveness of home programs could not be made because of the large variability in study, patient and intervention characteristics, comparators and outcome measures used in the included studies.

Despite consensus on the value of home-based upper limb training in rehabilitation of children with UCP, no evidence-based best practice exists. Promoting compliance of children to adhere to an intensive program while keeping parental stress levels low is an important challenge when designing home-based training programs. Incorporating implicit motor learning principles emerged to be a promising method to resolve this challenge. In chapter $\mathbf{4}$ we describe two 
protocols for home-based bimanual training programs, one based on implicit motor learning principles and one based on explicit motor learning principles, for children with unilateral spastic CP aged 2 through 7 years. Children receive goal-oriented, task-specific bimanual training in their home environment from their parents for $3.5 \mathrm{~h} /$ week for 12 weeks according to an individualized program. Parents are intensively coached by a multidisciplinary team, consisting of a pediatric therapist and remedial educationalist. Both programs consist of a preparation phase (goal setting, introductory meetings with coaching professionals, design of individualized program, instruction of parents, home visit) and home-based training phase (training, video-recordings, registrations, and tele-coaching and home visits by the coaching team). The programs contrast with respect to the teaching strategy, i.e. how the parents support their child during training. In both programs parents provide their child with instructions and feedback that focus on the activity (i.e. task-oriented) or the result of the activity (i.e. result-oriented). However, in the explicit program parents are in addition instructed to give exact instructions and feedback on the motor performance of the bimanual activities, whereas in the implicit program the use of both hands and the appropriate motor performance of the activity are elicited via manipulation of the organization of the activities. With the protocols described, we aim to take a next step in the development of much needed evidence-based home-based training programs for children with UCP.

Chapter 5 aims to conceptualize the phenomenon of therapy-related parental stress (TRPS) in parents of children with a physical disability. Three models related to parental stress were reviewed, i.e. general parental stress, burden of caregiving in parents of children with disabilities, and experiences of these parents with their child's therapy. We define TRPS as the subjective stress and subsequent changes of functioning and health experienced by a parent of a child with a physical disability in response to paramedical therapies (i.e. physical, occupational, and/or speech and language therapy). A theoretical model is proposed to describe the process of TRPS. Available questionnaires will most likely not be valid and responsive to capture the (changes in) stress parents experience related to therapy their child receives. This chapter provides a first definition of TRPS and a theoretical model to visualize the processes with regard to this topic. Empirical testing of the presented components and their coherence is needed to confirm or improve the model. A questionnaire that specifically measures the construct of TRPS is needed, along with evaluating TRPS in clinical practice and research.

Chapter 6 provides the development of a Dutch translation of the Lifestyle Assessment Questionnaire for children with cerebral palsy (LAQ-CP), adapted for cross-cultural differences. The translation process consisted of 6 stages, following a guideline for cross-cultural adaptations including duplicate forward- and back-translations, expert group review, pilot-testing, and a process audit. Several adaptations to the questionnaire were required due to cross-cultural differences. As a result of the pilot-test, the layout was adapted to the desires of the users. The process auditor stated that the process had been comprehensive and valued the quality of the work. The project resulted in a Dutch translation of the LAQ-CP, adapted for cross-cultural differences. Validation of the translated questionnaire is required before use in clinical practice and research is recommended. 
Many trials fail to include the targeted number of participants, causing scientific and ethical problems. The COAD trial of home-based training programs for children with UCP encountered recruitment problems, even though the parent-delivered home-based approach complies with recent health-care developments in the Netherlands. The project presented in chapter 7 aimed to identify the barriers to recruitment in the COAD trial. This summative, multidimensional evaluation comprised informal conversational interviews in which stakeholders who had been involved reflected on the factors that impeded successful recruitment of participants into the COAD trial. Barriers to implementation and recruitment were clustered according to the constructs of the Consolidated Framework for Implementation Research (CFIR). Member checking validated the findings. A total of 41 stakeholders contributed to this evaluation. Barriers to the implementation of the HBTPs were identified within every domain of the CFIR (intervention characteristics, outer setting, inner setting, characteristics of individuals, and process). Parent-delivered home-based training was perceived as highly complex and in conflict with the pressures on and the needs of parents. Many parents preferred the alternative center-based group interventions. The involvement of a resonance group was highly valued, and opportunities for further enhancements emerged. Additionally, the importance of research consortia was emphasized. The appropriateness of the randomized controlled trial (RCT) as the study design was criticized. The findings of this study are summarized in a tool which provides a dozen directions for the successful recruitment of participants in pediatric rehabilitation research.

The aim of chapter $\mathbf{8}$ is to explore the child- and parent-related effects of home-based bimanual training, adopting implicit or explicit motor learning, in children with uCP. We present a case series of 14 children (2-7 years) who completed the training. Five children performed the implicit and nine the explicit programs. Both included goal-oriented task-specific training for 3.5 hours/week for 12 weeks. A therapist and remedial educationalist coached parents in providing the training. Progression on bimanual goals and TRPS were of primary interest, measured with the Canadian Occupational Performance Measure (COPM) and by parental interviews. Data were collected at baseline (T0), halfway through the training (T1), at the end of training (T2), and after 12 weeks (T3). On the COPM performance scale, a clinically relevant change was seen in 50\% (7/14), 86\% (12/14), and 85\% (11/13), and, on the satisfaction scale, in 43\% (6/14), $64 \%$ (9/14), and $54 \%(7 / 14)$ of the children, at T1, T2, and T3, respectively. During the interviews, some parents indicated that they had experienced stress because of the intensity of the training. The secondary outcomes showed a tendency toward reduction of therapy-related and generic parental stress. It was concluded that the programs seem to positively affect children's bimanual performance and parental stress.

A process evaluation of the complex home-based bimanual training programs is crucial in order to draw accurate conclusions and provide recommendations for implementation in clinical practice and further research. Chapter $\mathbf{9}$ describes the protocol of the process evaluation, which aimed to systematically assess fidelity of the home-based training programs, to examine the mechanisms that contributed to their effects on child-related and parent-related outcomes, and to explore the influence of contextual factors. A mixed methods embedded design was used 
that emerged from a pragmatism paradigm. The qualitative strand involved a generic qualitative approach. The process evaluation components fidelity (quality), dose delivered (completeness), dose received (exposure and satisfaction), recruitment and context were investigated. Data collection included registration of attendance of therapists and remedial educationalists of a course regarding the home-based training programs; a questionnaire for the instructor to evaluate this course; a report form concerning the preparation phase to be completed by the therapist; registration and video analyses of the home-based training; interviews with parents and questionnaires filled out by the therapist and remedial educationalist regarding the process of training; and focus groups with therapists and remedial educationalists as well as registration of drop-out rates and reasons, to evaluate the overall home-based training programs. Inductive thematic analysis was used to analyze qualitative data. Qualitative and quantitative findings were merged through meta-inference.

Chapter 10 provides the results of the process evaluation. The programs were not fully implemented as intended, but showed a good compliance of $79 \%$ of the intended training intensity. Overall, parents experienced the home-based training as positive. They were well able to provide the therapy. Activities of daily living were most easily practiced. For the children the program was demanding. Still, parents thought the program was worthwhile, as the bimanual performance of their child improved. Furthermore, altogether parents perceived the program as time-consuming. The extra pressure and time demands resulted in stress in few parents. Parents also experienced the 12-week program duration as too long. The combined coaching by the therapist and remedial educationalist was a highly appreciated component of the program. The instructional course for health care professionals, the instructional videos for parents, the task-analysis, the telephone appointments, and the home visits all positively contributed to the program. Nevertheless, the frequency of appointments was considered too high when the program progressed, conversations by telephone induced limitations for the coaching by the remedial educationalist, everyone involved was annoyed by the problems with the data sharing system, and parents disliked filing out the registration form.

$\mathrm{CP}$ research is dominated by group-based designs and the RCT has long been considered superior. Single-case experimental design (SCED) provides a rigorous alternative, respecting the heterogeneity of the population. The study described in chapter $\mathbf{1 1}$ aimed to critically evaluate SCED studies performed within the population of children and adolescents with CP. A scoping review was performed. Studies were identified by searching electronic databases and included if they reported on a SCED study of children and/or adolescents up to 21 years with CP. Demographic, methodological, and statistical data were extracted. Articles were graded using the Risk of Bias in N-of-1 Trials (RoBiNT) Scale and evaluated for quality of reporting using the Consolidated Standards of Reporting Trials (CONSORT) extension for N-of-1 trials (CENT 2015). Comments regarding strengths and limitations were extracted and analyzed. Studies investigated the effects of a wide range of interventions on various outcomes. Most SCED types were adopted in multiple studies. All studies used visual inspection rather than visual analysis, often complemented with basic statistical descriptives. Risk of bias was high, particularly concerning internal validity. Many items of the CENT were insufficiently reported. Although the number of studies had increased over time, their risk of bias had not decreased. Several benefits and limitations of SCED were 
identified. The conclusions were that SCED has potential within the context of personalized evidence-based medicine in children and adolescents with CP, provided that the quality of evidence from results is increased through reduction of risk of bias.

In chapter 12 a general discussion of the main findings of the studies is provided. First, to describe the coherence between the results of the different studies, a logic model is presented, including assumptions, enabling and limiting resources, program activities, output, and outcomes. Second, we discuss a satellite project on the development of the Therapy-related Parental Stress Questionnaire (in Dutch:Vragenlijst Ouderlijke Stress als gevolg van Therapie van het kind (VOST)). Third, we argue for an iterative process in intervention development and evaluation in the complex pediatric rehabilitation setting. The SCED enables clinically-meaningful intervention research. Thereafter, we discuss some overarching methodological considerations: the mixed methods research approach, the children's involvement in the project, the outcome measures used, and an alternative statistical approach. In conclusion, our results do support the use of home-based bimanual training in clinical practice. Programs for children with UCP should include (1) a priori instruction of the healthcare professionals, (2) a task-analysis performed by the therapist, (3) exchange of video-recorded training sessions, and (4) individualized coaching of the parents by a therapist and remedial educationalist. More work is required to confirm our findings and to determine alternative home-based bimanual training programs. Special attention should be paid to the coaching process, the well-being of parents during the home-based training, the way children perceive the training, and the suitability of motor learning approaches for individual children, parents, and phases of learning. Additional studies will be needed to develop a complete picture of the construct of TRPS and to find the best way to measure it. Lastly, future studies on the methodological and statistical procedures of the SCED within pediatric rehabilitation are advocated. 

Nederlandse SAmenvatting (DUtCh SUmmary) 
Dit proefschrift beschrijft de resultaten van het COAD onderzoeksproject ("CO-creation At hanD: the road to independence "). Het algehele doel van dit proefschrift was het genereren van wetenschappelijke kennis over de mogelijkheden en uitdagingen van bimanuele thuistraining voor kinderen met een unilaterale Cerebrale Parese (uCP).

Gezien de veelbelovende voordelen van thuisinterventies voor de bovenste extremiteit voor kinderen met Cerebrale Parese (CP), is een systematische review van de beschikbare literatuur over dit onderwerp noodzakelijk. Hoofdstuk 2 beschrijft het protocol van een systematische review, met als doel om de haalbaarheid en het effect van de momenteel beschikbare ergo- en fysiotherapeutische thuisinterventies te onderzoeken. De systematische review werd ontwikkeld in overeenstemming met de 'Preferred Reporting Items for Systematic Review and Meta-Analysis Protocols (PRISMA-P) 2015'. Studies werden geïncludeerd indien primaire data werd verzameld, de deelnemers kinderen in de leeftijd $<18$ jaar met om het even welk type van CP waren, en de interventie een ergo- of fysiotherapeutische thuisinterventie was. Ter vergelijking werden de volgende behandelingen meegenomen: geen therapie, gebruikelijke zorg, ergo- of fysiotherapie in het centrum, een alternatieve thuisinterventie en een medische interventie. Studies werden geïncludeerd indien gerapporteerd werd over haalbaarheid (dat wil zeggen aanvaardbaarheid, behoefte, implementatie, bruikbaarheid, aanpassing, uitbreiding of integratie) of over effectiviteit (dat wil zeggen kind-gerelateerde uitkomstmaten voor de bovenste extremiteit binnen alle levels van de'International Classification of Functioning, Disability and Health' of ouder-/verzorger gerelateerde uitkomstmaten binnen het psychologische en sociale domein). Relevante studies werden geïdentificeerd door het doorzoeken van de databases MEDLINE, EMBASE, CINAHL, PsycINFO, Pedro, OTseeker en CPCI-S, evenals de trial registraties ICTRP en CENTRAL, de referentie-lijsten van opgenomen studies en door de verspreiding van een bibliografie van de geïncludeerde studies naar auteurs van opgenomen studies. Er waren geen beperkingen voor taal of publicatiejaar. De zoekstrategie bestond uit termen gerelateerd aan de populatie en interventie. Gegevens werden in tweevoud geëxtraheerd met behulp van een formulier voor digitale gegevensextractie.

Hoofdstuk 3 beschrijft de resultaten van deze systematische review. In totaal werden 47 studies geïncludeerd: 22 haalbaarheidsstudies (46,8\%), 10 effectiviteitsstudies (21,3\%) en 15 studies die beide rapporteerden (31,9\%). Er was een grote variatie in kind karakteristieken, terwijl de overgrote meerderheid van de onderzoeken geen karakteristieken van de ouders rapporteerde. De behandelvorm was overwegend (gemodificeerde) 'Constraint-Induced Movement Therapy' (CIMT) (40\%). De doelstellingen van de interventie, het gebruik van motorische leerprincipes en de coaching van ouders waren meestal niet gespecificeerd. De trainingsduur van de door ouders geleverde thuistraining varieerde van 2 weken tot 6 maanden en de intensiteit van 70 minuten tot 21 uur per week. De algehele therapietrouw van de training (implementatie) was matig tot hoog (62\% tot 96,1\%). De meeste haalbaarheidsstudies rapporteerden dat ouders het gemakkelijk vonden om het programma uit te voeren en het leuk vonden om hun kinderen vooruit te zien gaan (aanvaardbaarheid). Er konden geen conclusies over de effectiviteit van thuisprogramma's worden getrokken vanwege de grote variabiliteit in studie-, patiënt- en interventiekarakteristieken, interventies waarmee vergeleken werd en uitkomstmaten die werden gebruikt in de geselecteerde studies. 
Ondanks consensus over de waarde van thuisinterventies voor de bovenste extremiteit in de revalidatie van kinderen met een uCP, bestaat er geen 'evidence-based best practice'. Het bevorderen van de therapietrouw van kinderen om zich aan een intensief programma te houden, terwijl het niveau van ouderlijke stress laag blijft, is een belangrijke uitdaging bij het ontwerpen van thuistrainingsprogramma's. Het integreren van impliciete motorische leerprincipes leek een veelbelovende methode om deze uitdaging op te lossen. In hoofdstuk 4 beschrijven we twee protocollen voor bimanuele thuistrainingsprogramma's, één gebaseerd op impliciete motorische leerprincipes en één gebaseerd op expliciete motorische leerprincipes, voor kinderen met een unilaterale spastische CP van 2 tot 7 jaar. Kinderen krijgen doelgerichte, taak-specifieke, bimanuele training in hun thuisomgeving van hun ouders gedurende 3,5 uur per week gedurende 12 weken volgens een geïndividualiseerd programma. Ouders worden intensief gecoacht door een multidisciplinair team, bestaande uit een kindertherapeut en orthopedagoog. Beide programma's bestaan uit een voorbereidingsfase (doelen stellen, kennismakingsgesprekken met coaching professionals, ontwerpen van een geïndividualiseerd programma, instructie van ouders, huisbezoek) en een trainingsfase (training, video-opnamen, registraties als ook tele-coaching en huisbezoeken door het coaching team). De programma's contrasteren wat betreft de aanleerstrategie, dat wil zeggen hoe de ouders hun kind tijdens de training ondersteunen. In beide programma's geven ouders hun kind instructies en feedback die gericht zijn op de activiteit (dat wil zeggen taakgericht) of het resultaat van de activiteit (dat wil zeggen resultaatgericht). In het expliciete programma worden ouders echter ook geïnstrueerd om exacte instructies en feedback te geven over de motorische uitvoering van de bimanuele activiteiten, terwijl in het impliciete programma het gebruik van beide handen en de juiste motorische uitvoering van de activiteit wordt uitgelokt door het aanpassen van de organisatie van de activiteiten. Met de beschreven protocollen willen we een volgende stap zetten in de ontwikkeling van de hoognodige evidence-based thuistrainingsprogramma's voor kinderen met een UCP.

Hoofdstuk 5 heeft als doel het fenomeen van therapie gerelateerde ouderlijke stress ('therapy-related parental stress' (TRPS)) bij ouders van kinderen met een lichamelijke beperking te conceptualiseren. Drie modellen gerelateerd aan ouderlijke stress worden beschouwd, namelijk algemene ouderlijke stress, belasting door zorgverlening bij ouders van kinderen met een beperking en ervaringen van deze ouders met de therapie van hun kind. We definiëren TRPS als de subjectieve stress en daaropvolgende veranderingen in functioneren en gezondheid ervaren door een ouder van een kind met een lichamelijke beperking als reactie op paramedische therapieën (fysiotherapie, ergotherapie en/of logopedie). Een theoretisch model wordt voorgesteld om het proces van TRPS te beschrijven. Beschikbare vragenlijsten zullen hoogstwaarschijnlijk niet valide en responsief zijn om de (veranderingen in) stressbeleving van ouders in verband met therapie die hun kind krijgt vast te leggen. Dit Hoofdstuk biedt een eerste definitie van TRPS en een theoretisch model om de processen met betrekking tot dit onderwerp te visualiseren. Empirisch testen van de gepresenteerde componenten en hun samenhang is nodig om het model te bevestigen of te verbeteren. Een vragenlijst die specifiek het construct van TRPS meet is nodig, evenals evaluatie van TRPS in de klinische praktijk en onderzoek. 
Hoofdstuk 6 beschrijft de ontwikkeling van een Nederlandse vertaling van de'Lifestyle Assessment Questionnaire for children with cerebral palsy' (LAQ-CP), aangepast voor cross-culturele verschillen. Het vertaalproces bestond uit 6 fasen, volgens een richtlijn voor cross-culturele aanpassingen, namelijk dubbele 'forward- en back-vertalingen', beoordeling door een expertgroep, een pilot-test en een procesaudit. Er waren verschillende aanpassingen aan de vragenlijst nodig vanwege cross-culturele verschillen. Als resultaat van de pilottest werd de lay-out aangepast aan de wensen van de gebruikers. De procesauditeur verklaarde dat het proces uitgebreid was en waardeerde de kwaliteit van het werk. Het project resulteerde in een Nederlandse vertaling van de LAQ-CP, aangepast voor cross-culturele verschillen. Validatie van de vertaalde vragenlijst is vereist voor gebruik in de klinische praktijk en onderzoek wordt aanbevolen.

Veel onderzoeken slagen er niet in het beoogde aantal deelnemers te includeren, waardoor wetenschappelijke en ethische problemen ontstaan. De COAD-studie naar thuistrainingsprogramma's voor kinderen met een UCP ervoer wervingsproblemen, hoewel de door ouders verleende thuisbenadering voldoet aan de recente ontwikkelingen in de gezondheidszorg in $\mathrm{Ne}$ derland. Het project gepresenteerd in hoofdstuk $\mathbf{7}$ beoogde de belemmeringen voor de werving in de COAD-studie te identificeren. Deze summatieve, multidimensionale evaluatie bestond uit informele gespreksinterviews waarin betrokkenen reflecteerden op de factoren die succesvolle werving van deelnemers aan de COAD-studie belemmerden. Belemmeringen voor implementatie en werving werden geclusterd volgens de constructen van de 'Consolidated Framework for Implementation Research' (CFIR). Member checking valideerde de bevindingen. In totaal hebben 41 stakeholders bijgedragen aan de evaluatie. Belemmeringen voor de implementatie van de thuistrainingsprogramma's werden geïdentificeerd binnen elk domein van de CFIR (interventiekenmerken, externe setting, interne setting, kenmerken van individuen en proces). Door ouders verleende thuistraining werd als zeer complex ervaren en in strijd met de druk op en de behoeften van de ouders. Veel ouders gaven de voorkeur aan de alternatieve groepsinterventies in de centra. De betrokkenheid van een klankbordgroep werd zeer gewaardeerd en er kwamen kansen voor verdere verbeteringen naar voren. Daarnaast werd het belang van onderzoeksconsortia benadrukt. De geschiktheid van de 'randomized controlled trial' (RCT) als studiedesign werd bekritiseerd. De bevindingen van deze studie zijn samengevat in een tool die een dozijn aanwijzingen geeft voor de succesvolle werving van deelnemers voor onderzoek in de kinderrevalidatie.

Het doel van hoofdstuk 8 is om de kind- en ouder-gerelateerde effecten van bimanuele thuistraining, waarbij impliciet of expliciet motorisch leren werd gebruikt, te onderzoeken bij kinderen met een UCP. We presenteren een case series van 14 kinderen (2-7 jaar) die de training voltooiden. Vijf kinderen voerden het impliciete en negen het expliciete programma uit. Beide omvatten doelgerichte, taakspecifieke training gedurende 3,5 uur/week gedurende 12 weken. Een therapeut en orthopedagoog coachten ouders bij het geven van de training. Vooruitgang op bimanuele doelen en TRPS waren van primair belang, gemeten met de 'Canadian Occupational Performance Measure' (COPM) en door ouderinterviews. Gegevens werden verzameld bij baseline (T0) halverwege de training (T1), aan het einde van de training (T2) en na 12 weken (T3). Op de COPM 'performance schaal' werd een klinisch relevante verandering gezien in 50\% (7/14), 86\% $(12 / 14)$ en $85 \%$ (11/13) en, op de 'satisfaction schaal', in 43\% (6/14), 64\% (9/14) en 54\% (7/14) van de kinderen, respectievelijk op T1, T2 en T3. Tijdens de interviews gaven sommige ouders aan dat 
ze stress hadden ervaren vanwege de intensiteit van de training. De secundaire uitkomstmaten toonden een neiging tot vermindering van therapie gerelateerde en generieke ouderlijke stress. Er werd geconcludeerd dat de programma's de bimanuele prestaties van kinderen en ouderlijke stress positief lijken te beïnvloeden.

Een procesevaluatie van de complexe bimanuele thuistrainingsprogramma's is cruciaal om accurate conclusies te trekken en aanbevelingen te doen voor implementatie in de klinische praktijk en verder onderzoek. Hoofdstuk 9 beschrijft het protocol van de procesevaluatie, gericht op het systematisch beoordelen van de getrouwheid van de thuistrainingsprogramma's, het onderzoeken van de mechanismen die bijdragen aan hun effecten op kind-gerelateerde en ouder-gerelateerde uitkomsten en het onderzoeken van de invloed van contextuele factoren. Een 'mixed methods embedded design' werd gebruikt dat voortkomt uit het pragmatisme paradigma. Het kwalitatieve onderdeel behelsde een generieke kwalitatieve benadering. De procesevaluatiecomponenten getrouwheid (kwaliteit), geleverde dosis (volledigheid), ontvangen dosis (blootstelling en tevredenheid), werving en context werden onderzocht. De dataverzameling omvatte de registratie van de aanwezigheid van therapeuten en orthopedagogen bij een cursus met betrekking tot de thuistrainingsprogramma's; een vragenlijst voor de instructeur om deze cursus te evalueren; een rapportageformulier voor de therapeut met betrekking tot de voorbereidingsfase; registratie en video-analyses van de thuistraining; interviews met ouders en vragenlijsten die moesten worden ingevuld door de therapeut en orthopedagoog met betrekking tot het proces van training; en focusgroepen met therapeuten en orthopedagogen, evenals registratie van uitvalpercentages en -redenen, om de gehele thuistrainingsprogramma's te evalueren. Inductieve thematische analyse werd gebruikt om kwalitatieve gegevens te analyseren. Kwalitatieve en kwantitatieve bevindingen werden samengevoegd door middel van meta-inferentie.

Hoofdstuk 10 presenteert de resultaten van de procesevaluatie. De programma's bleken niet volledig geïmplementeerd zoals bedoeld, maar vertoonden een goede therapietrouw van 79\% van de beoogde trainingsintensiteit. Over het algemeen ervoeren ouders de thuistraining als positief. Ze waren goed in staat om de therapie te geven. Activiteiten uit het dagelijks leven werden het gemakkelijkst geoefend. Voor de kinderen was het programma veeleisend. Toch vonden ouders dat het programma de moeite waard was, omdat de bimanuele prestaties van hun kind verbeterden. Bovendien vonden ouders het programma in zijn geheel tijdrovend. De extra druk en tijdsinvestering resulteerden in stress bij enkele ouders. Ouders ervoeren ook de programmaduur van 12 weken als te lang. De gecombineerde coaching door de therapeut en orthopedagoog was een zeer gewaardeerd onderdeel van het programma. De instructiecursus voor zorgprofessionals, de instructievideo's voor ouders, de taakanalyse, de telefonische afspraken en de huisbezoeken droegen allemaal positief bij aan het programma. Desondanks werd de frequentie van de afspraken als te hoog beschouwd wanneer het programma vorderde, de gesprekken via de telefoon hadden beperkingen voor de coaching door de orthopedagoog, alle betrokkenen waren geïriteerd door de problemen met het systeem voor het delen van gegevens en ouders vonden het niet prettig om het registratieformulier in te vullen.

CP-onderzoek wordt gedomineerd door op groepen gebaseerde designs en de RCT werd lang als superieur beschouwd. Het 'single-case experimenteel design'(SCED) biedt een rigoureus alternatief, waarin de heterogeniteit van de populatie in acht wordt genomen. De studie beschre- 
ven in hoofdstuk 11 is gericht op het kritisch evalueren van SCED-onderzoeken uitgevoerd binnen de populatie van kinderen en adolescenten met CP. Er werd een scoping review uitgevoerd. Studies werden geïdentificeerd door elektronische databases te doorzoeken en geselecteerd als ze rapporteerden over een SCED-studie van kinderen en/of adolescenten tot 21 jaar met CP. Demografische, methodologische en statistische gegevens werden geëxtraheerd. Artikelen werden beoordeeld met behulp van de 'Risk of Bias in N-of-1 Trials (RoBiNT) Scale' en beoordeeld op kwaliteit van rapportage met behulp van de 'Consolidated Standards of Reporting Trials (CONSORT) extension for N-of-1 trials (CENT 2015)'. Opmerkingen met betrekking tot sterktes en beperkingen werden geëxtraheerd en geanalyseerd. Studies onderzochten de effecten van een breed scala aan interventies op verschillende uitkomstmaten. De meeste SCED-typen werden in meerdere onderzoeken toegepast. Alle studies gebruikten visuele inspectie in plaats van visuele analyse, vaak aangevuld met basale descriptieve statistiek. Het risico op bias was groot, vooral wat betreft interne validiteit. Veel items van de CENT waren onvoldoende gerapporteerd. Hoewel het aantal onderzoeken in de loop van de tijd was toegenomen, was hun risico op bias niet afgenomen. Verschillende voordelen en beperkingen van SCED werden geïdentificeerd. De conclusies waren dat SCED potentie heeft in de context van gepersonaliseerde, evidence-based geneeskunde bij kinderen en adolescenten met CP, op voorwaarde dat de kwaliteit van het bewijs van de resultaten wordt verhoogd door het risico op bias te verkleinen.

In hoofdstuk 12 wordt een algemene discussie gegeven van de belangrijkste bevindingen van de onderzoeken. Ten eerste wordt een 'logic model'gepresenteerd om de samenhang tussen de resultaten van de verschillende studies te beschrijven. Dit behelst assumpties, bevorderende en beperkende bronnen, programma activiteiten, output en uitkomsten. Ten tweede beschrijven we een nevenproject over de ontwikkeling van de Vragenlijst Ouderlijke Stress als gevolg van Therapie van het kind (VOST). Ten derde pleiten we voor een iteratief proces voor de ontwikkeling en evaluatie van interventies in de complexe setting van de kinderrevalidatie. De SCED maakt klinisch relevant interventie-onderzoek mogelijk. Daarna bespreken we enkele overkoepelende methodologische overwegingen: de 'mixed methods' benadering van het onderzoek, de betrokkenheid van kinderen bij het project, de gebruikte uitkomstmaten en een alternatieve statistische benadering. Concluderend ondersteunen onze resultaten het gebruik van bimanuele thuistraining in de klinische praktijk. Programma's voor kinderen met UCP moeten omvatten: (1) a priori instructie van de zorgprofessionals, (2) een taakanalyse uitgevoerd door de therapeut, (3) uitwisseling van gefilmde trainingssessies en (4) geïndividualiseerde coaching van de ouders door een therapeut en orthopedagoog. Meer werk is noodzakelijk om onze bevindingen te bevestigen en om alternatieve bimanuele thuistrainingsprogramma's te ontwikkelen. Er moet speciale aandacht worden besteed aan het coachingsproces, het welzijn van de ouders tijdens de thuistraining, de manier waarop kinderen de training ervaren en de geschiktheid van motorische leerbenaderingen voor individuele kinderen, ouders en fases van leren. Er zijn aanvullende studies nodig om een volledig beeld te krijgen van de het construct van TRPS en om de beste manier te vinden om dit te meten. Tot slot worden toekomstige studies over de methodologische en statistische procedures van de SCED binnen de kinderrevalidatie bepleit. 


\begin{abstract}
ABOUT THE AUTHOR
Laura Beckers was born on May 12th, 1989 in Brunssum, the Netherlands. She attended pre-university secondary education (VWO) at Gymnasium Trevianum, Sittard. Thereafter, she studied physical therapy at Hogeschool Zuyd, Heerlen, where she obtained her Bachelor of Science degree in 2011. After her graduation she worked as a physiotherapist in geriatrics for a year. Because of her interest in research she applied and was accepted for the Research Master of Health Sciences at Maastricht University (2012-2014). During her study she specialized in clinical epidemiology. While she was performing her research internship at the department of Epidemiology of Maastricht University under the supervision of Dr. Caroline Bastiaenen, she discovered her interest in rehabilitation. She graduated for her Master of Science degree with distinction. In 2015 she was appointed as a PhD candidate at the department of Rehabilitation Medicine of Maastricht University, in collaboration with Adelante Centre of Expertise in Rehabilitation and Audiology (Hoensbroek). She was supervised by Prof. dr. Rob Smeets (Maastricht University), Dr. Yvonne Janssen-Potten (Maastricht University), Dr. Eugène Rameckers (Maastricht University), and Dr. Jan van der Burg (Radboud University, Nijmegen). Her main responsibilities were to develop and conduct the COAD (CO-creation At hanD: the road to independence) project. The results of her work are described in this dissertation, and most of the research has been published in or accepted for international scientific journals. She also wrote several successful grant applications. After finishing her PhD project in 2019, she continued working at the department of Rehabilitation Medicine. She is involved as a post-doc researcher and co-promoter in a project on rehabilitation innovations in chronic musculoskeletal pain.
\end{abstract}





\section{PUBLICATION LIST}

Beckers LWME, Schnackers MLAP, Janssen-Potten YJ, et al. Feasibility and effect of home-based therapy programs for children with cerebral palsy: a protocol for a systematic review. BMJ Open 2017;7:e013687.

Beckers L, Speth L, Rameckers E, et al. Cross-cultural Translation and Adaptation of the Lifestyle Assessment Questionnaire (LAQ-CP) Into Dutch: A Brief Report. Pediatr Phys Ther. 2017;29(3):251-255.

Schnackers M, Beckers L, Janssen-Potten $Y$, et al. Home-based bimanual training based on motor learning principles in children with unilateral cerebral palsy and their parents (the COAD-study): rationale and protocols. BMC Pediatr. 2018;18(1):139.

Beckers L, van der Burg J, Janssen-Potten Y, et al. Process evaluation of two home-based bimanual training programs in children with unilateral cerebral palsy (the COAD-study): protocol for a mixed methods study. BMC Pediatr. 2018;18(1):141.

Beckers LWME, Rameckers EAA, Smeets RJEM, et al. Barriers to recruitment of children with cerebral palsy in a trial of home-based training. (Accepted for publication in Contemporary Clinical Trials Communications)

Beckers LWME, Smeets RJEM, van der Burg JJW. Therapy-related Stress in Parents of Children with a Physical Disability: a Conceptual Analysis. (Submitted for publication)

Beckers LWME, Rameckers EAA, Aarts PBM, et al. Effect of home-based bimanual training in children with unilateral cerebral palsy (the COAD-study): a case series. (Submitted for publication)

Beckers LWME, Stal RA, Smeets RJEM, et al. Single-case experimental design studies in children with cerebral palsy: a scoping review. (Submitted for publication)

Beckers LWME, Geijen MME, Kleijnen J, et al. Feasibility and effectiveness of home-based therapy programs for children with cerebral palsy: a systematic review. (In preparation for submission)

Beckers LWME, Smeets RJEM, de Mooij MAC, et al. Process evaluation of two home-based bimanual training programs in children with unilateral cerebral palsy (the COAD-study): a mixed methods study. (In preparation for submission) 

DANKWOORD (ACKNOWLEDGEMENTS) 
Dit proefschrift is mede tot stand gekomen dankzij het werk en de steun van velen. Daarom wil ik hen in dit laatste deel bedanken.

Allereerst wil ik mijn dank betuigen aan mijn promotor en copromotoren, Prof. dr. Rob Smeets, Dr. Yvonne Janssen-Potten, Dr. Eugène Rameckers en Dr. Jan van der Burg. Ik heb het als een voorrecht ervaren om jullie als promotieteam te mogen hebben. Ik heb me altijd door jullie gesteund, gestimuleerd en gewaardeerd gevoeld. Bedankt voor jullie razendsnelle, onuitputtelijke en constructieve commentaar, zelfs in de avonduren en weekenden. Hiermee ben ik ook dank verschuldigd aan jullie gezinnen, die deze kostbare tijd met jullie hebben moeten opofferen. Het heeft veel voor mij betekend dat jullie mij, toen het onderzoek in zwaar weer verkeerde, de mogelijkheid boden om het traject voort te zetten.

Rob, wat ben jij gedreven, volhardend en een bron van motiverende ideeën. Ik heb het gewaardeerd dat je me de ruimte gaf om zelf mede richting te geven aan de invulling van mijn promotietraject. Ik verheug me erop een vervolg te geven aan onze samenwerking tijdens mijn postdocschap.

Yvonne, ik had me geen betere dagelijkse begeleider kunnen wensen. Je positieve instelling en nuchtere blik werken zeer aanstekelijk. Daarnaast was niets, maar dan ook echt niets, te veel gevraagd.

Eugène, de toewijding en het enthousiasme, waarmee jij talloze projecten en samenwerkingen aangaat, zijn inspirerend. Ik heb veel geleerd van jouw ervaring met en visie op de praktijk.

Jan, de afgelopen jaren heb ik je leren kennen als een bijzonder toegewijde begeleider. Het is bewonderenswaardig hoe grondig je de stukken telkens las en van suggesties voorzag. Daarnaast heb ik het zeer op prijs gesteld dat je oog bleef houden voor hoe het met mij als persoon ging.

Marlous, ook jij verdient een eervolle vermelding. Ik ben trots op onze samenwerking en bewaar hier goede herinneringen aan. Mijn dank is groot voor de bergen werk die we samen hebben verzet, die de basis hebben gevormd van dit proefschrift. Ik vind het bijzonder dat je als paranimf tijdens mijn promotie naast me staat.

Daarnaast wil ik de overige leden van het onderzoeksteam, in de samenstelling van Dr. Pauline Aarts, Prof. dr. Sander Geurts, Dr. Imelda de Groot en Prof. dr. Bert Steenbergen, bedanken voor hun bijdrage aan het COAD-project. Pauline, het was fijn dat je ook in de laatste fase van het onderzoek betrokken wilde blijven.

Tevens wil ik ook alle overige coauteurs, Carolien Bastiaenen, Mellanie Geijen, Jos Kleijnen, Marion de Mooij, Patrick Onghena, Barbara Piškur, en Roos Stal, bedanken voor de inzet, waardevolle feedback en voornamelijk de fijne samenwerking die wij bij de totstandkoming van de diverse hoofdstukken hebben gehad. Mellanie en Roos, dankzij jullie harde werk hebben we de reviews tot een goed einde weten te brengen. Marion, het succesvol afronden van de procesevaluatie heb ik voor een groot deel aan jou te danken. Je bent goud waard! 
Mijn oprechte dank gaat ook uit naar alle kinderen en hun ouders die vol enthousiasme hebben deelgenomen aan het onderzoek. Daarnaast dank ik alle therapeuten, orthopedagogen, revalidatieartsen en andere medewerkers van de deelnemende revalidatiecentra hartelijk. In het bijzonder Anke Defesche, Hanneke Denissen, Danielle Dijkema, Linda Haasjes, Kiki van Heel, Ruth van den Heuvel, Annelies Hillemans, Anne Jensen, Sabine Keus, Marjon Kissels, Jacqueline Koot, Monique Lampe, Odette Maas, Marleen Philippens, Caroline Scheijmans, Jody Sohier, Sylvie Suijkerbuijk, Ingrid van den Tillaar, Eef Vennix en Eeske Vlaar. Jullie betrokkenheid bij de thuistrainingsprogramma's en het project was van onschatbare waarde.

Verder verdienen ook de leden van onze $2^{e}$ kring een plaats in dit dankwoord: Nicole Brouwers, Anke Defesche, Yvonne Geerdink, Bregtje Janssen, Marjon Kissels, Martijn Klem, Denise Martens, Judith van Munster, Bianca Olive, Marleen Philippens, Lucianne Speth en Ingrid van den Tillaar. De kritische meningen vanuit ieders eigen perspectief en de discussies die we hebben mogen voeren, hebben een onmisbare bijdrage geleverd aan het project.

Ine Telgenkamp en Mellanie Geijen, ik ben jullie dank verschuldigd voor het uitvoeren van de vele metingen door heel het land, net als Caroline Bruins, Carmen Ensink en Zoë Sanders voor het beoordelen van de metingen.

De studenten die ik tijdens afstudeerprojecten heb mogen begeleiden, bedankt! Jullie hebben bijgedragen aan mijn persoonlijke ontwikkeling en vaak ook aan het COAD-project.

Les Hearn, thank you for proofreading many of my manuscripts. Michał Sławiński, I am grateful for your dedication in typesetting and designing my dissertation.

De leden van de beoordelingscommissie, Prof. dr. Jeroen Vermeulen, Prof. dr. Jan Willem Gorter, Prof. dr. Marian Jongmans, Dr. Ilse Mesters en Prof. dr. Jeanine Verbunt, ik ben u allen erkentelijk voor uw bereidheid mijn proefschrift te beoordelen.

Leden van de corona, ik waardeer het dat ik met u van gedachten mag wisselen over mijn proefschrift.

Naast de inhoudelijk betrokkenen, wil ik ook graag alle andere (oud)collega's van de afdeling Revalidatiegeneeskunde van de Universiteit Maastricht en van het Adelante kenniscentrum bedanken voor de collegiale sfeer, gezelligheid en adviezen.

Carolien, Charlotte, Cynthia, Hanneke, Ine, Lieke, Mellanie, Reni, Vera B. en Vera M., jullie waren geweldige kamergenootjes. Naast de leuke gesprekken die we hadden, stonden jullie altijd voor me klaar om te sparren en hoogte- en dieptepunten te delen.

De secretariaten, Margareth, Sonja, Veronique en Jolanda, dank voor jullie praktische hulp bij de regelklussen tijdens mijn onderzoek en rondom mijn promotie.

Tenslotte de collega's die een bijdrage hebben geleverd aan mijn ontwikkeling als onderzoeker tijdens de PIMP's, GRASP bijeenkomsten en de denktank kwalitatief onderzoek: bedankt! 
Lieve familie en vrienden, ik ben dankbaar dat jullie deel uitmaken van mijn leven. Jullie oprechte interesse in mijn onderzoek, jullie bemoedigende woorden en de hoognodige afleiding heb ik zeer gewaardeerd. Dymphy, ik ben blij met jou als vriendin en paranimf.

Mam en pap, ik prijs mij gelukkig met jullie onvoorwaardelijke liefde en steun. De vanzelfsprekendheid waarmee jullie mij altijd met raad en daad hebben bijgestaan en hebben meegeleefd tijdens mijn promotietraject is prachtig. Jullie zijn een voorbeeld voor mij.

Micha, voor wie je voor me bent bedank ik je op andere momenten. Je weet hoe dankbaar ik ben dat je mijn promotietraject met me hebt willen doorstaan. Als ik van iemand veel gevraagd heb voor de totstandkoming van dit proefschrift, dan ben jij het. Mijn dank is groot voor al je steun, begrip en liefde. Nu zijn jij en jouw boekje aan de beurt en zal ik er voor jou zijn. Ik beloof je dat ik mijn uiterste best ga doen om net zoveel voor jou te betekenen als jij voor mij hebt gedaan.

\section{Laura}

